\title{
Rollover stability of partially filled heavy-duty elliptical tankers using trammel pendulums to simulate fluid sloshing
}

\author{
Mohamed Ibrahim Salem \\ West Virginia University
}

Follow this and additional works at: https://researchrepository.wvu.edu/etd

\section{Recommended Citation}

Salem, Mohamed Ibrahim, "Rollover stability of partially filled heavy-duty elliptical tankers using trammel pendulums to simulate fluid sloshing" (2000). Graduate Theses, Dissertations, and Problem Reports. 2335.

https://researchrepository.wvu.edu/etd/2335

This Dissertation is protected by copyright and/or related rights. It has been brought to you by the The Research Repository @ WVU with permission from the rights-holder(s). You are free to use this Dissertation in any way that is permitted by the copyright and related rights legislation that applies to your use. For other uses you must obtain permission from the rights-holder(s) directly, unless additional rights are indicated by a Creative Commons license in the record and/ or on the work itself. This Dissertation has been accepted for inclusion in WVU Graduate Theses, Dissertations, and Problem Reports collection by an authorized administrator of The Research Repository @ WVU.

For more information, please contact researchrepository@mail.wvu.edu. 


\title{
Rollover Stability of Partially Filled Heavy-Duty Elliptical Tankers Using Trammel Pendulums to Simulate Fluid Sloshing
}

\author{
Mohamed I. Salem
}

\begin{abstract}
Dissertation submitted to the College of Engineering and Mineral Resources at West Virginia University in partial fulfillment of the requirements for the degree of
\end{abstract}

Doctor of Philosophy in

Mechanical Engineering

\author{
Victor H. Mucino, Ph.D. Chair \\ Mridul Gautam, Ph.D. \\ Gary Morris, Ph.D. \\ Ken Means, Ph.D. \\ Ian Christie, Ph.D.
}

Department of Mechanical and Aerospace Engineering

$$
\begin{gathered}
\text { Morgantown, West Virginia } \\
\mathbf{2 0 0 0}
\end{gathered}
$$

Keywords: Moving Cargo, Lane Change, Road Containers, Semitrailers Copyright 2000 Mohamed I. Salem 


\title{
ABSTRACT \\ Rollover Stability of Partially Filled Heavy-Duty Elliptical Tankers Using Trammel Pendulums to Simulate Fluid Sloshing
}

\author{
Mohamed I. Salem
}

Partially filled tankers undergoing turns or lane change maneuvers have a lower rollover threshold than any other type of vehicle. In order to study and simulate the lateral fluid sloshing effects in partially filled elliptical and cylindrical tankers a trammel pendulum has been utilized. The fundamental basis of selecting the appropriate pendulum parameters have been obtained by matching the pendulum dynamic effects with fluid sloshing dynamic effects obtained using Finite Element (FE) fluid models. Elliptical tankers with different aspect ratios and different levels of fill were considered. The trammel pendulum effects were verified against fluid models from two packages (FLUENT and LS_Dyna 3D) as well as the literature within and beyond the linear range of pendulum oscillation. The trammel pendulum produced up to $20 \%$ more lateral forces and moments than the fluid models beyond the linear range, which renders the pendulum model more conservative from the tanker stability point of view.

Two- and three- dimensional tanker models were used to simulate the tanker's lateral stability using the trammel pendulums. The effects of several parameters such as ellipse ratio, fluid polar moment of inertia, suspension compliance, and the frequency of excitation on tanker stability were studied. The models predicted critical fill levels that agree with the experimental results and the values reported in the literature based on much more complex and time consuming experimental and analytical methods. In addition, the rollover thresholds were predicted with acceptable level of accuracy $(+8 \%)$, especially with the more detailed models. 
In loving Memony
Of my Fathen 


\section{ACKNOWLEDGEMENT}

I would like to express my gratitude to my research advisor Dr. Victor Mucino for his continuous support and advise throughout the years working under his supervision. I would like to thank him for all the opportunities and the brotherly environment you have offered me all the time. I would like also to thank Dr Mridul Gautam for his support and guidance any time I needed it.

Thanks to Dr. Ken Means, Dr Gary Morris and Dr Ian Christie for participating on my Ph.D. committee and for all what I have learned from them. Thanks also are due to Aberdeen Proving Grounds (ATC) for their support and for carrying over the experimental work of this study. Special thanks to Mr. Craig Turner for his help, continuous support and encouragement throughout all the stages of this study.

I would like to express my gratitude to my partners Eric Saunders and Matthew Aquaro for being the members of the best cooperative teamwork with whom I have ever worked. A special thanks to Eric Saunders for his great patience and dedication in running the FE fluid models which have saved me lots of time. Also, I would like to thank him for the brotherhood that we developed over the years working together.

Thanks to Dr. Donald Lyons and the Department of Mechanical and Aerospace Engineering for their support of this study and for the environment, equipment and financial support they have provided throughout this research. 
I know that many pages would not be enough to mention all the people who provided me with different kinds of help, and I express my thanks to all of them, such as my family my friends, and my office mates.

Finally, I would like to thank my late father for his inspiring words before his death; "I wish that I see you a Doctor". I hope that I have fulfilled his dream. 


\section{TABLE OF CONTENTS}

TITLE PAGE

$\underline{\text { Page }}$

$\begin{array}{lll}\text { ABSTRACT } & \text { ii }\end{array}$

$\begin{array}{ll}\text { DEDICATION } & \text { iii }\end{array}$

ACKNOWLEDGEMENT _ iv

TABLE OF CONTENTS Vi vi v v vi

LIST OF TABLES - - xi

LIST OF FIGURES X xiii

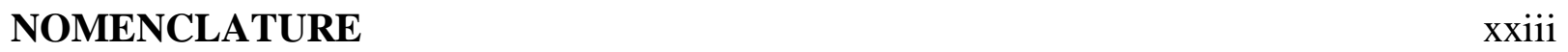

CHAPTER 1. INTRODUCTION AND PROBLEM STATEMENT 1

1.1 Introduction 1

1.2 Problem Statement 1

$\begin{array}{lll}1.3 & \text { Background } & 2\end{array}$

1.4 Objectives 3

$\begin{array}{lll}1.5 & \text { Scope } & 3\end{array}$

CHAPTER 2. LITERATURE REVIEW

2.1 Introduction $\quad 5$

2.2 Fluid-Container Interaction 5

2.3 Heavy-Duty Vehicle Stability 14

2.4 Fluid-Vehicle Interaction 33

2.5 Conclusions Based on the Literature Review 45

CHAPTER 3. EXPERIMENTAL DATA ANALYSIS $\quad 48$ 
$\begin{array}{lll}3.1 & \text { Introduction } & 48\end{array}$

3.2 Vehicle Instrumentation 49

3.3 Road Maneuvers Performed During Tests 50

3.4 Experimental Data Processing $\quad 51$

3.4.1 Units Transformation $\quad 51$

3.4.2 Numerical Differentiation $\quad 52$

3.4.3 Data Filtering $\quad 52$

$\begin{array}{lll}\text { 3.4.4 Spring Characteristics } & 53\end{array}$

3.4.5 Tire-Ground Reactions $\quad 56$

3.4.6 Determining the Experimental Rollover Lateral 62 Acceleration

3.4.7 NATO Lane Change Test Data Processing 68

3.8 Conclusions Based on Experimental Data Analysis 71

CHAPTER 4 MATHEMATICAL VEHICLE-SLOSH MODELS 73

$\begin{array}{lll}4.1 & \text { Introduction } & 73\end{array}$

4.2 Locus of Fluid Volume Center of Gravity 73

4.3 Equation of Motion of the Trammel Pendulum 74

4.4 Pendulum-Fluid Dynamic Effect Matching 76

4.4.1 Pendulum-Fluid Natural Frequency matching 77

4.4.2 Pendulum-Fluid Horizontal Force Component Matching 87

4.4.3 Pendulum-Fluid Static Moment Matching 94

4.5 Trammel Pendulum Fluid Simulation Verification 95

4.6 Trammel Pendulum Behavior Beyond Linearity 106

4.7 Advantages of Trammel Pendulum Model over Dyna and 113 
FLUENT Fluid Models

$\begin{array}{lll}\text { CHAPTER } 5 & \text { VEHICLE-PENDULUM MODELS } & 115\end{array}$

$\begin{array}{lll}5.1 & \text { Introduction } & 115\end{array}$

5.2 Model 1, Vehicle Rigid Body Model with no Support Liftoff 116

$\begin{array}{lll}\text { 5.2.1 } & \text { Model \#1 Applications } & 117\end{array}$

5.2.1.1 Model Frequency Response 123

5.2.1.2 Effect of Changing Aspect Ratio on Rollover 131 Threshold

5.3 Model 2, Vehicle Rigid Body Model, with Support Liftoff 136

5.4 Model 3, Vehicle Plane Model with Tire and Suspension 141 Compliances

5.4.1 Model Description and Inputs 142

5.4.2 Rollover Stability Limits and Effect of Suspension Stiffness 145

5.4.2.1 Self-Induced Rollover 146

5.4.2.2 Tripped-Induced Rollover 148

5.5 Model 4, Finite Element 3-D Vehicle Model Using ANSYS 149

CHAPTER 6 CONCLUSIONS \& CONTRIBUTIONS TO THE FIELD OF 157 ENGINEERING SCIENCE

$\begin{array}{llr}6.1 & \text { Summary } & 157\end{array}$

$\begin{array}{llr}6.2 & \text { Conclusions } & 158\end{array}$

6.3 Contributions to the Field of Engineering 160

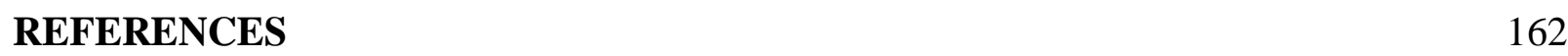

APPENDIX A VEHICLE DATA AND EXPERIMENTAL INSTRUMENTATION 169 INFORMATION 
A.1.1 Weight on Axles at Different Levels of Fill 169

A.1.2 CG Location at Different Levels of Fill 170

$\begin{array}{lll}\text { A.1.3 Tractor Dimensions } & 170\end{array}$

$\begin{array}{lll}\text { A.1.4 Trailer Dimensions } & 173\end{array}$

A.2 Vehicle Experimental Instrumentation Information 176

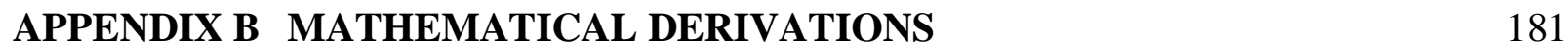

$\begin{array}{lll}\text { B.1 Introduction } & 181\end{array}$

B.2 Calculation of Mass and CG Location of a Fluid Moving 181 in an Elliptical Tank

B.3 Elliptical Trammel Pendulum Equation of Motion 190

B.4 Derivation of Equations of Motion for Vehicle Model \#2 194

B.4.1 Liftoff of the Right Support 195

B.4.2 Liftoff of the Left Support 201

B.5 Polar Mass Moment of Inertia of the Fluid Volume 206

APPENDIX C LIST OF COMPUTER CODES USED IN THIS STUDY 212

$\begin{array}{lll}\text { C.1 Introduction } & 213\end{array}$

$\begin{array}{lll}\text { C.2 The MatLab .m File allfilter.m } & 213\end{array}$

$\begin{array}{lll}\text { C.3 The MatLab .m File spr_dif.m } & 214\end{array}$

$\begin{array}{lll}\text { C.4 The MatLab .m File trammel.m } & 214\end{array}$

C.5 The MatLab Function File equ_mot.m 218

$\begin{array}{lll}\text { C.6 The MatLab.m File reaction.m } & 219\end{array}$

$\begin{array}{lll}\text { C.7 The MatLab.m File ellipse1.m } 220 & 220\end{array}$

$\begin{array}{lll}\text { C.8 The MatLab.m File Basic3.m } 226 & 26\end{array}$ 
C.9 The MatLab.m File mid.m 230

$\begin{array}{ll}\text { C.10 The MatLab Function File Left_s3.m } & 231\end{array}$

C.11 The MatLab Function File Right_s3.m 232

C.12 The 2-D Model ANSYS Log File 233

C.13 The 3-D Model ANSYS Log File 236

$\begin{array}{ll}\text { CURRICULUM VITA } & 246\end{array}$ 


\section{LIST OF TABLES}

$\underline{\text { Table \# }}$

$\underline{\text { Page }}$

4.1 Reduced formulas to evaluate the fundamental lateral frequency of

81

vibration in a half-full cylindrical tank

4.2 Initial pendulum angle at different values of $\mathrm{a} / \mathrm{b}$ for a $5^{\circ}$ fluid surface

90

initial tilt angle

4.3 Values of K, CM, CFV, and CM obtained using FE and FLUENT models At $0.3 \mathrm{~g}$

4.4 Comparison between time costs for Dyna and FLUENT fluid models

114 and their equivalent trammel pendulum models

5.1 Summary of the tanker models utilizing the trammel pendulum

115

5.2 Values of $a$ and $b$ in meters used in model 1 through 4 for constant

119 cross section

5.3 Tanker shell mass at different aspect ratios

A.1 Curb Weight Distribution on the Wheels for the Empty Tanker

A.2 Weight Distribution on the Wheels for the $50 \%$ full Tanker with water

A.3 Weight Distribution on the Wheels for the $75 \%$ full Tanker with water

169

A.4 Weight Distribution on the Wheels for the $100 \%$ full Tanker with water 170

A.5 Center of Gravity Location at Different Loading Condition 170 (M916A1/60PRS)

A.6 Tractor frame rail characteristics for the tractor M916A1

A.7 Fifth wheel dimensions and location

$\begin{array}{lll}\text { A.8 Tractor rear suspension location/dimensions } & 172\end{array}$

A.9 Tractor front suspension location/dimensions 172

$\begin{array}{lll}\text { A.10 Tractor tires and wheel characteristics } & 173\end{array}$

A.11 Main dimensions of tanker shell and rear framerails 174 
$\begin{array}{lll}\text { A.12 Kingpin dimensions and location } & 175\end{array}$

$\begin{array}{lll}\text { A.13 Tractor tire and wheel characteristics } & 175\end{array}$

A.14 Trailer suspension location/dimensions 176

$\begin{array}{llr}\text { A.15 Transducer exact locations } & 180\end{array}$

B.1 Principle polar moment of inertia per meter length of water volume at static 208 Position in a tank equal in area to the ETNYRE tanker at different aspect ratios 


\section{LIST OF FIGURES}

Figure \#

$\underline{\text { Page }}$

2.1 Normalized First and Second Mode Shapes of a Fluid in a Partially

Filled Cylindrical Canal, Budiansky (1960)

2.2 Equivalent mechanical model for a partially filled ellipsoidal tank and the associated variation of sloshing frequencies with liquid depth Rattayya (1965)

2.3 Liquid free surface response in vertical circular tank showing swirl region, Abramson (1966 A)

2.4 Variation of model masses with liquid depth for ellipsoidal tank, Abramson (1966 B)

2.5 Coupled fundamental circular frequency versus wall thickness for a vertical cylindrical tank, Bauer (1972)

2.6 Simple three-unit train used by Schmid (1967)

2.7 Fifth wheel design with eccentricity proposed by Schmid (1967)

2.8 Schematic diagram of five vehicle configurations used by Mallikarjunarao (1978)

2.9 Illustration of $\mathrm{C}_{\mathrm{f}}$ and $\mathrm{K}_{\mathrm{e}}$ for a typical leaf spring using an approximated hysteresis loop, Fancher (1980)

2.10 Spring lash in a variable stiffness leaf spring truck suspension, Ervin (1983 B)

2.11 Roll response of a heavy-duty tractor-semitrailer including spring lash, Ervin (1983 B)

2.12 Simplified vehicle roll model showing the concept of suspension and tire roll centers, Ervin (1983 B)

2.13 Roll response of combination vehicle with three compliant suspensions, Ervin (1983 B)

2.14 A simple suspension model for roll stability analysis used by Winkler (1992)

2.15 Force displacement characteristics of the suspension springs used 
by Sankar (1989)

2.16 Lateral acceleration versus level of fill for a rigid and a liquid cargo elliptical container, Ranganathan (1989)

2.17 Representation of fluid slosh using pendulum model approach, Ranganathan (1993 A)

2.18 Parameters of pendulum analogy, Ranganathan (1993 A)

2.19 Torsional and bending stiffness evaluation of a trailer using FEM, Gautam and Mucino (1999)

2.20 Simple finite element beam model for a tanker tractor-semitrailer developed by Aquaro (1999)

$\begin{array}{lll}3.1 & \text { Tanker used for experimental testing } & 48\end{array}$

3.2 Artificial trailer outriggers to prevent vehicle rollover during tests 50

3.3 Path of the test vehicle during the TOP Lane Change Test 51

3.4 Path of the test vehicle during the NATO Lane Change Test 51

3.5 Calculated and filtered lateral acceleration for the trailer under study, 53 at 50\% full condition, during the TOP lane change test

3.6 Trailer suspension spring characteristics (ETNYRE)

3.7 A variable effective length (stiffness) spring, Spring Design Manual 54 (1996)

3.8 Vertical acceleration of the left-hand side of the trailer rear axle measured by an accelerometer before and after filtering

3.9 Walking-Beam type suspension, Winkler $1992 \quad 58$

3.10 Tractor front wheels reactions during the TOP lane change test 60 (50\% full tanker)

3.11 Tractor rear wheels reactions for axles 2 and 3 summed together during 60 the TOP lane change test (50\% full tanker)

3.12 Trailer front wheels reactions during the TOP lane change test (50\% full tanker) 
3.13 Trailer rear wheels reactions during the TOP lane change test (50 full tanker)

3.14 Illustration of the cause of the dip in the reaction on the positive side of the trailer axles

3.15 Tractor lateral acceleration normal to the path during the TOP lane change test obtained using equation (3.4)

3.16 Trailer lateral acceleration normal to the path during the TOP lane change test obtained using equation (3.4)

3.17 Tractor lateral acceleration normal to the path during the TOP lane change test obtained using accelerometers' data

3.18 Trailer lateral acceleration normal to the path during the TOP lane change test obtained using accelerometers' data

3.19 Tractor front wheels reactions during the NATO lane change test (75\%full tanker)

3.20 Tractor rear wheels reactions for axles $2 \& 3$ summed together during the NATO lane change test (75\%full tanker)

3.21 Trailer front wheels reactions during the NATO lane change test (75\%full tanker)

3.22 Trailer rear wheels reactions during the NATO lane change test (75\%full tanker)

3.23 Tractor rear right side spring deflection measured during the TOP lane change test $50 \%$ full and the NATO lane change test $75 \%$ full

4.1 Contour of C.G. location for a given volume of fluid in an elliptical container

4.2 C.G. of fluid volume contour drawn by an elliptical trammel mechanism

4.3 Parameters used in the analysis of the elliptical pendulum motion

4.4 Natural frequency of an elliptical pendulum as a function of the pendulum swing angle using different aspect ratios using the ETNYRE tanker outer shell dimensions

4.5 Trammel pendulum parameters required for dynamic matching of fluid lateral sloshing effects 
4.6 Phase trajectory plot for a trammel pendulum oscillating at small angles

4.7 Initial fluid position and after 0.5 cycle models using Ls-Dyna-3D positive $\mathrm{X}$ direction using trammel pendulum.

4.8 Horizontal " $\mathrm{X}$ " coordinate location as a function of time used to obtain natural frequency of fluid oscillation

4.9 Natural frequency vs. fill level for a fluid volume in an elliptical tank with different values of $\mathrm{a} / \mathrm{b}$ at initial fluid surface angle $=5^{\circ}$ obtained using FEM

4.10 FEM results for $\bar{b} / b$ vs. fill level at different values of $a / b$ at initial fluid angle $=5^{\circ}$

4.11 Results for $\bar{b} / b$ vs. fill level at different values of $a / b$ at initial fluid surface angle $=5^{\circ}$ obtained using equation 4.13

4.12 Error \% in estimating for $\bar{b} / b$ using equation 4.13

$4.13 \bar{b} / b_{c g}$ for different levels of fill and a/b varying from 1 to 2

4.14 Dimensionless parameter $B_{n}$ for a circular tank, Budiansky (1960)

4.15 Maximum horizontal component of sloshing force vs. fill level using water in an 0.1 elliptical slice

4.16 Maximum horizontal component of sloshing force per unit fluid weight vs. fill level in an 0.1 thick elliptical slice

4.17 $\quad \mathrm{M}_{\mathrm{p}} / \mathrm{M}_{\mathrm{t}}$ vs. fill level obtained from FE results at different values of $\mathrm{a} / \mathrm{b}$

4.18 $\quad \mathrm{M}_{\mathrm{p}} / \mathrm{M}_{\mathrm{t}}$ vs. fill level obtained from Equation 4.19 at different values of $\mathrm{a} / \mathrm{b}$

4.19 Error \% in estimating $\mathrm{M}_{\mathrm{p}} / \mathrm{M}_{\mathrm{t}}$ using Equation 4.19

$4.20 \mathrm{~h}_{\mathrm{o}} / \mathrm{b}$ vs. fill level at different values of $\mathrm{a} / \mathrm{b}$ obtained using Equation 4.21

4.21 FE model used to match the moment around point " $\mathrm{D}$ " before and after applying the lateral acceleration

4.22 Comparison between the roll moment around point " $\mathrm{D}$ " for $\mathrm{a} / \mathrm{b}=1.25$ and Fill levels 30\% and 70\% using FE and trammel pendulum models 
4.23 Peak values of roll moment at point " $D$ " at constant fluid surface angle using fluid FE models

4.24 Peak values of roll moment at point " $D$ " at constant fluid surface angle using trammel pendulum models

4.25 Peak moment values at point "D" at constant lateral acceleration equal $0.05 \mathrm{~g}$

4.26 Frequency parameter vs. fill level for a/b varying from 1 to 2 at constant surface angle (input acceleration is given by equation 4.20)

4.27 Frequency parameter vs. fill level for $\mathrm{a} / \mathrm{b}$ varying from 1 to 2 at constant lateral acceleration $=0.05 \mathrm{~g}$

4.28 Vertical force coefficient at constant fluid surface angle equals 5 degrees

4.29 Vertical force coefficient at constant lateral acceleration equals $0.05 \mathrm{~g}$

102

4.30 Horizontal force coefficient at constant fluid surface angle equals 5 degrees

103

4.31 Horizontal force coefficient at constant lateral acceleration equals $0.05 \mathrm{~g}$

103

4.32 Rolling moment coefficient at constant fluid surface angle equals $5^{\circ}$

104

4.33 Rolling moment coefficient at constant lateral acceleration equals $0.05 \mathrm{~g} \quad 104$

4.34 Change of rolling moment vs. fill level at different values of lateral 107 acceleration

4.35 Change of the frequency parameter vs. fill level at different values of

108 lateral acceleration

4.36 Change of the vertical force vs. fill level at different values of lateral $\quad 108$ acceleration

4.37 Change of the horizontal force coefficient vs. fill level at different values of lateral acceleration

4.38 Change of the horizontal force coefficient (According to Sankar's definition) vs. fill level at different values of lateral acceleration

4.39 Change of the rolling moment coefficient vs. fill level at different values of lateral acceleration 
4.40 Change of the rolling moment coefficient (according to Sankar's definition) $\quad 110$ vs. fill level at different values of lateral acceleration

5.1 A schematic diagram of vehicle plane model \#1

116

5.2 ANSYS simple plane beam model (Modified from Aquro 1999)

5.3 Reactions on supports A \& B obtained analytically using Equations 5.1

118 and 5.2

5.4 Reactions on supports A \& B obtained using ANSYS FEM

5.5 Change of $\bar{a}$ as a function of fill level

$\begin{array}{lll}\text { 5.6 Change of } \bar{b} \text { as a function of fill level } & 120\end{array}$

5.7 Change of fluid total mass as a function of fill level $\quad 121$

5.8 Change of pendulum mass as a function of fill level $\quad 121$

5.9 Change of fixed fluid mass as a function of fill level 122

5.10 Change of the height of the fixed mass from ground (Tank CL height 122 is equal to $2.47 \mathrm{~m}$ )

5.11 Change of trammel pendulum base natural frequency as a function of fill level 123 using the ETNYRE tanker data

5.12 Support reactions of model \#1 for a 50\% full circular tank at lateral acceleration 124 of $0.01 \mathrm{~g}$ and frequency of $1.2393 \mathrm{rad} / \mathrm{sec}$

5.13 Support reactions of model \#1 for a 50\% full circular tank at lateral acceleration 125 of $0.01 \mathrm{~g}$ and frequency of $3.917 \mathrm{rad} / \mathrm{sec}$

5.14 Support reactions of model \#1 for a 50\% full circular tank at lateral acceleration 126 of $0.05 \mathrm{~g}$ and frequency of $3.917 \mathrm{rad} / \mathrm{sec}$

5.15 Support reactions of model \#1 for a 50\% full elliptical tank $(\mathrm{a} / \mathrm{b}=2)$ at lateral 127 acceleration of $0.05 \mathrm{~g}$ and frequency of $2.8025 \mathrm{rad} / \mathrm{sec}$

5.16 Normalized maximum and minimum support reactions at $0.01 \mathrm{~g}$ and $0.05 \mathrm{~g}$ lateral 128 acceleration for $50 \%$ full $(\mathrm{a} / \mathrm{b}=2)$

5.17 Weight transfer percent at $0.01 \mathrm{~g}$ and $0.05 \mathrm{~g}$ lateral acceleration for $50 \%$ full $(\mathrm{a} / \mathrm{b}=2) 128$ 
5.18 Normalized weight transfer at $0.01 \mathrm{~g}$ and $0.05 \mathrm{~g}$ lateral accelerations for $50 \%$ full $(\mathrm{a} / \mathrm{b}=2)$

5.19 Normalized maximum and minimum support reactions at $0.05 \mathrm{~g}$ lateral Acceleration

5.20 Decrease of tank shell perimeter with the decrease of $\mathrm{a} / \mathrm{b}$ from 2 to 1

5.21 Minimum support reactions vs. lateral acceleration at different levels of fill $(\mathrm{a} / \mathrm{b}=1)$

5.22 Rollover threshold at different fill levels for the ETNYRE tanker assuming 133 constant CG height

5.23 Rollover threshold at different fill levels for the ETNYRE tanker assuming constant overall height

5.24 Rollover threshold at different fill levels for the ETNYRE tanker assuming 134 constant ground clearance

5.25 Support reactions for the 50\% full ETNYRE tanker obtained using ATC lateral acceleration input during the TOP lane change test

5.26 Model 2, vehicle rigid body model with support liftoff 136

5.27 Absolute and relative rollover thresholds of vehicle model \#2 at different weight 138 and inertia considerations

5.28 Ratio between fluid polar moment of inertia and tanker polar moment of inertia around the support point of rotation at different levels of fill

5.29 Vehicle roll angle near the absolute rollover threshold (case for a 50\% full tanker 139 with weight considered and no inertia

5.30 Pendulum angle during a rollover of a 50\% full tanker with tanker weight 140 considered

5.31 Effect of vehicle and fluid polar moment of inertia on the rate of change of vehicle 141 roll angle

5.32 Model \#3, Vehicle plane model with tire and suspension compliances 142

5.33 Stabilization of wheel reactions with stabilization damper after increasing the 144 suspension stiffness (case for $75 \%$ full, axles $2 \& 3$

5.34 Effect of tire damping on wheel reactions (75\% full rear tractor suspension model 145 
at $0.25 \mathrm{~g}$ )

5.35 Comparison between rollover stability limits obtained using the ETNYRE plane 146 slice models and the experimental results at constant radius tests

5.36 Effect of changing suspension stiffness on the self induced rollover threshold of 147 the tanker (section at axles $2 \& 3$ under $75 \%$ full condition)

5.37 Rollover lateral impact speed for the tanker plane slice models at different levels 148 of fill

5.38 Effect of changing suspension stiffness on tripped-induced rollover speed (75\%full axles $2 \& 3$ )

5.39 Model 4, Modified Aquaro's 3-D vehicle ANSYS beam model 150

5.40 Total reactions on the tanker wheels during the TOP lane change maneuver 151

5.41 Comparison of reactions on axle \#1 during the top lane change maneuver 151

5.42 Comparison of reactions on axles \# $2 \& 3$ during the top lane change maneuver 152

5.43 Comparison of reactions on axles \# 2\&3 during the top lane change maneuver 152

5.44 Tractor and trailer roll angles during the TOP lane change maneuver 153

5.45 Effect of using a wide track on the reactions of axles $4 \& 5$ during the TOP lane 154 change maneuver

5.46 Comparison between reactions on axles $4 \& 5$ with and without pendulum action 155 during the TOP lane change test

5.47 Effect of increasing model support beam stiffness on the reactions of axles $4 \& 5 \quad 156$ during the TOP lane change maneuver

$\begin{array}{lll}\text { A.1 Main Dimensions of the Test Tractor (M916A1) } & 171\end{array}$

$\begin{array}{lll}\text { A.2 Main Dimensions of the Test Trailer (60PRS) } & 173\end{array}$

$\begin{array}{lll}\text { A.3 Main dimensions of trailer leaf spring } & 175\end{array}$

A.4 Oscillation Gyrometer to measure the Yaw rate of the tractor and 177 lateral speed sensor

A.5 Oscillation Gyrometer to measure the Yaw rate of the trailer at the its

CG 
A.6 3 perpendicular accelerometers and a potentiometer to measure the acceleration all 3 directions and the spring deflection respectively fitted on all axles (Figure for tractor front axle)

A.7 3 perpendicular accelerometers on both sides of tractor frame 178 and potentiometer to measure spring deflection on the tractor rear suspension

A.8 Circular dial to measure the steering angle and hence the slip angle 179 of the tires can be determined (device was not needed in this study but could be used later)

A.9 Pezoelectric pressure measurement strips installed in the front-most 179 compartment to measure fluid level.

B.1 Elliptical tank partially filled at initial position

B.2 Fluid tilted with an angle $\theta$ in an elliptical tank 184

B.3 The two sub-arias $A_{1}$ and $A_{2}$ used in the analysis 186

B.4 Location of fluid CG at different levels of fill and different angles of tilt 188

B.5 Illustration of Symbols Used In Equations (B15) and (B16), Rakheja (1993)

B.6 Ratio $\mathrm{R} / \mathrm{b}$ as a function of fill level and ellipse ratio $\mathrm{a} / \mathrm{b}$

B.7 The trammel pendulum is the initial and final set of coordinates after 180 a negative displacement $\mathrm{x}$.

B.9 Trammel pendulum angular position and angular velocity at 30 degrees initial angle

B.9 Trammel pendulum angular position and angular velocity at 60 degrees initial angle

B.10 Trammel pendulum angular position and angular velocity at 90 degrees initial angle

B.11 Trammel pendulum angular position and angular velocity at 120 degrees initial angle

B.12 Trammel pendulum angular position and angular velocity at 150 degrees initial angle 
B.13 Trammel pendulum angular position and angular velocity at 179.9 degrees initial angle

B.14 Right support liftoff of model \#2, fluid inertia fixed to the frame (modified from 195 Karam, 1999)

B.15 Left support liftoff of model \#2, fluid inertia fixed to the frame (modified from Karam, 1999)

B.16 Normalized polar moment of inertia vs. fill level at different levels and different 209 Tank aspect ratios

B.17 Variation of the principle polar moment of inertia per meter length of a fluid 210 Volume as a function of the fluid surface angle for a 50\% full ETNYRE tanker

B.18 Normalized polar moment of inertia $\left(\mathrm{I}_{\theta} / \mathrm{I}_{0}\right)$ for a $50 \%$ full ETNYRE tanker

B.19 Error in evaluating the principle angular polar moment of inertia of a fluid 211 volume using an elliptical relation 


\section{NOMENCLATURE}

\section{English Symbols}

\section{Symbol}

a Half Width of an Elliptical tank

$\bar{a} \quad$ Major Axis of the Trammel Pendulum

$a_{\mathrm{cg}} \quad$ Half Width of Elliptical Path of the Fluid Volume CG

A Fluid cross section area (used by Bohn 1981)

$\mathrm{A}_{\mathrm{n}} \quad$ Non-Dimensional Constant used by Budiansky (1960)

$\mathrm{B}_{\mathrm{n}} \quad$ Non-Dimensional Constant used by Budiansky (1960)

b Leaf Spring Width or Half Height of an Elliptical tank

$\bar{b} \quad$ Minor Axis of the Trammel Pendulum

$b_{c g} \quad$ Half Height of Elliptical Path of the Fluid Volume CG

$\mathrm{CFH}$ Horizontal Force Coefficient

CFV Vertical Force Coefficient

CM Rolling Moment Coefficient

E Modulus of Elasticity (equal $209 * 10^{9}$ for leaf spring material)

Eu Euler Number $=\frac{P_{o}}{\rho W_{o}^{2}}$

$\mathrm{F}_{\mathrm{A}} \quad$ Reaction at Left Support

$\mathrm{F}_{\mathrm{B}} \quad$ Reaction at Right Support

$\mathrm{F}_{\mathrm{H}} \quad$ Horizontal Sloshing Force Component

Fr Froude Number $=\frac{W_{o}^{2}}{g L_{o}}$

$\mathrm{F}_{\mathrm{s}} \quad$ Lateral Sloshing Force in Budiansky's Study (1960)

$\mathrm{F}_{\mathrm{t}} \quad$ Total Reaction on Both Left and Right Supports

H Fluid Fill Level From Tank Lowermost Point

$\mathrm{h}_{1} \quad$ Height of Vehicle Curb Mass from Ground Level

\section{Unit}

$\mathrm{m}$

$\mathrm{m}$

$\mathrm{m}$

$\mathrm{m}^{2}$

Unitless

Unitless

$\mathrm{m}$

m

m

Unitless

Unitless

Unitless

$\mathrm{N} / \mathrm{m}^{2}$

Unitless

$\mathrm{N}$

$\mathrm{N}$

$\mathrm{N}$

Unitless

$\mathrm{N}$

$\mathrm{N}$

m

m 


\begin{tabular}{|c|c|c|}
\hline $\mathrm{h}_{\mathrm{ls}}$ & Leaf Spring thickness & $\mathrm{m}$ \\
\hline $\mathrm{h}_{\mathrm{cg}}$ & Height of Vehicle C.G. from the Ground Level & $\mathrm{m}$ \\
\hline $\mathrm{h}_{\mathrm{o}}$ & Height of Fixed Fluid Mass from Bottom Point of the Tank & $\mathrm{m}$ \\
\hline $\mathrm{I}_{\mathrm{f}_{-} \mathrm{o}}$ & Mass Roll Moment of Inertia of the Fluid around Support Point of Rotation & $\mathrm{kg} \cdot \mathrm{m}^{2}$ \\
\hline $\mathrm{I}_{\mathrm{f}}$ & Mass Roll Moment of Inertia of the Fluid around Fluid Volume CG & $\mathrm{kg} \cdot \mathrm{m}^{2}$ \\
\hline $\mathrm{I}_{\text {Tanker_o }}$ & Mass Roll Moment of Inertia around Support Point of Rotation & $\mathrm{kg} \cdot \mathrm{m}^{2}$ \\
\hline $\mathrm{I}_{\text {tanker }}$ & Mass Roll Moment of Inertia of Tanker around Its CG & $\mathrm{kg} \cdot \mathrm{m}^{2}$ \\
\hline $\mathrm{I}_{\mathrm{c}}$ & Instantaneous Center of Rotation of a Trammel Pendulum & \\
\hline $\mathrm{K}$ & Frequency Parameter Defined in Equation 4.23 & Unitless \\
\hline $\mathrm{K}_{\mathrm{s}}$ & Suspension Vertical Stiffness & $\mathrm{N} / \mathrm{m}$ \\
\hline $\mathrm{K}_{\mathrm{t}}$ & Tire Vertical Stiffness & $\mathrm{N} / \mathrm{m}$ \\
\hline $\mathrm{k}$ & Spring Stiffness & $\mathrm{N} / \mathrm{m}$ \\
\hline $\mathrm{L}_{\mathrm{o}}$ & Characteristic Length & $\mathrm{m}$ \\
\hline 1 & Half the Distance between the Leaf Spring Supports) & $\mathrm{m}$ \\
\hline $\mathbf{M}$ & Rolling moment around tank base point & N.m \\
\hline $\mathrm{M}_{\mathrm{f}}$ & Fixed Portion of Fluid Mass & $\mathrm{kg}$ \\
\hline $\mathrm{M}_{\mathrm{L}}$ & Liquid Mass used by Budiansky (1960) & $\mathrm{kg}$ \\
\hline $\mathrm{M}_{\mathrm{p}}$ & Pendulum (Sloshing) Mass of the Fluid & $\mathrm{kg}$ \\
\hline $\mathrm{M}_{\mathrm{t}}$ & Total Fluid Mass & $\mathrm{kg}$ \\
\hline $\mathrm{M}_{\text {tanker }}$ & Tanker Curb Mass & $\mathrm{kg}$ \\
\hline $\mathrm{n}$ & Total Number of leaves, or mode number & Unitless \\
\hline$n^{\prime}$ & The number of Leaves at the Ends of a Leaf Spring & Unitless \\
\hline $\mathrm{P}$ & Tank Perimeter & $\mathrm{m}$ \\
\hline$\overline{\mathrm{P}}$ & Position Vector of Pendulum Mass from Tank Base Point & Vector \\
\hline $\operatorname{Re}$ & Reynolds number $=\frac{L_{o} W_{o}}{v}$ & Unitless \\
\hline $\mathrm{r}$ & $\begin{array}{l}\text { Elliptical Pendulum Radius Measured from Geometrical Center } \\
\text { of the thank }\end{array}$ & $\mathrm{m}$ \\
\hline$\ddot{\mathrm{r}}$ & Acceleration Vector of Pendulum Mass & Vector \\
\hline $\mathrm{St}$ & Strouhal number $=\frac{L_{o}}{t_{o} W_{o}}$ & Unitless \\
\hline
\end{tabular}


T Vehicle Track $\quad \mathrm{m}$

$\mathrm{T}_{\mathrm{n}} \quad$ Periodic Time of Pendulum Oscillation $\quad$ sec

$\mathrm{t}_{\mathrm{o}} \quad$ Characteristic time $=\sqrt{L_{o} / g} \quad$ sec

$\ddot{U} \quad$ Lateral Acceleration used by Budiansky (1960) $\mathrm{m} / \mathrm{s}^{2}$

$\overline{\mathrm{V}} \quad$ Half width of fluid surface at the static position $\quad \mathrm{m}$

W Vehicle Weight $\quad \mathrm{N}$

$\mathrm{W}_{\mathrm{o}} \quad$ Characteristic velocity $=\sqrt{g L_{o}} \quad \mathrm{~m} / \mathrm{s}$

$\ddot{X} \quad$ Vehicle Lateral Acceleration $\mathrm{m} / \mathrm{s}^{2}$

$\mathrm{x}_{\mathrm{h}} \quad \mathrm{X}$ Coordinate of the Points of Intersection of Horizontal Fluid Surface with Tank m

Shell

$\mathrm{Y}_{\mathrm{AB}} \quad$ Height of Tank Geometrical Center from Ground $\mathrm{m}$

$\mathrm{Y}_{\mathrm{Mf}} \quad$ Height of Fluid Fixed Mass from the Ground Level m

$\mathrm{Y}_{\mathrm{cg}} \quad$ Height of Fluid Volume CG from Bottom of the Tank m

\section{Greek Symbols}

Symbol

$\alpha \quad$ Angle between pendulum Radius "r" and Vertical Axis

$\Lambda \quad$ Dimensionless length ratio between tank minor axis and "b"

$\theta \quad$ Pendulum Angle Measured from Vertical Tank Axis

$\theta_{\mathrm{v}} \quad$ Roll Angle of the Vehicle Sprung Mass Relative to the Horizontal Level

$\Phi \quad$ Dimensionless Mass Ratio $\mathrm{M}_{\mathrm{p}} / \mathrm{M}_{\mathrm{t}}$

$\phi \quad$ Angle Between Pendulum Arm and Tank Vertical Axis

$\phi_{1} \quad$ Sprung Mass Roll Angle at which Restoring Moment Starts

Taking Place

$\xi \quad$ Normalized Coordinate used by Budiansky (1960)

$\omega_{\mathrm{n}} \quad$ Natural Frequency (of Fluid in Budiansky's Study 1960, and of Pendulum in this Study)

$\Omega \quad$ Circular Frequency of Fluid Sloshing
Unit

Radians

Unitless

Radians

Radians

Unitless

Radians

Radians

Unitless

Radians/s

Radians/s 


\section{CHAPTER 1}

\section{INTRODUCTION AND PROBLEM STATEMENT}

\section{$\underline{1.1-\text { Introduction }}$}

Rollover accidents affect strongly highway safety, especially rollover of heavy-duty tankers carrying fuels and chemicals, which might result in explosions and/or catastrophic chemical spills. This fact made the study of rollover stability of various vehicles, especially heavy-duty tankers, a major concern for many vehicle manufacturers, organizations, and researchers. The rollover of a partially filled military truck tractor and semi-trailer with an elliptical tank shape in Fort Rucker, Alabama, in 1996 was the motivating factor to reopen the file of studying the rollover phenomena of such vehicles. A joint research project between West Virginia University (WVU) and Aberdeen Proving Grounds (ATC) was triggered to study this problem, which was the source of funds and information for this research.

\section{2- Problem Statement}

Previous investigations and analysis of statistical data related to rollover accidents indicated that heavy-duty trucks carrying shifting cargoes such as liquids, livestock, and hanging carcasses have greater tendency to rollover earlier (i.e. at lower lateral accelerations) than any other vehicles. The study of rollover of partially filled heavy-duty tanker trucks during various road maneuvers (such as lane change and/or breaking) has an exceptionally high degree of difficulty due to the interaction between fluid and vehicle structure. Fluid sloshing in the tanker exerts lateral forces and moments promoting earlier rollover than other types of vehicles, which attracted the focus of many researchers to study the rollover of those tankers specifically. 


\section{$\underline{\text { 1.3- Background }}$}

Fluid sloshing inside partially filled containers has been investigated extensively in the aerospace field in the 1950's and 1960's. The purpose was to study the effect of partially filled fuel tanks of different shapes on the stability of rockets and airplanes. Several mathematical models as well as equivalent mechanical models have been developed to simulate fluid sloshing effects in partially filled containers. The use of simple and compound pendulums has been proved successful in simulating fluid-sloshing effects in partially filled tankers of various shapes, especially spherical and cylindrical.

The focus on studying the fluid slosh effects in partially filled road tankers started in the late 1970's. Experimental investigations of tankers' rollover was found to have major drawbacks, such as; high cost, excessive noise in measuring instruments, lack of repeatability of test results, and finally difficulty of reaching the rollover limit of a full-scale vehicle without causing major damage to the vehicle. Analytical studies followed two tracks; the first was to integrate a fluid slosh model into a vehicle dynamics model such as the work done by Bohn et. al (1981) and Sankar et. al (1992). This approach proved to be expensive in terms of computational time and in the case of elliptical-shape tanks, it produced inaccurate results (Bohn et. al 1981). The second approach was to integrate the pendulum models used earlier to simulate fluid sloshing by the researchers in the aerospace industry into the vehicle models. This approach was successful in case of a cylindrical tank (Ranganathan et. al 1993 A and Rakheja et. al 1993).

So far, no model has been proved successful to simulate and predict with adequate accuracy the rollover threshold of a partially filled elliptical tanker. Also, experience indicates that a tanker trailer combination does not behave as a system of rigid bodies during a rollover 
accident. The effect of the torsional and bending elasticity of a long shell tanker and other components such as the king-pin of a tractor-trailer has been only considered for the first time by Aquaro (1999) who used a Finite Element Model (FEM) to simulate a vehicle rollover. The results obtained by Aquaro show that further investigations on the effect of elasticity of different vehicle components on rollover stability can be done using finite element structural models.

\section{4- Objectives}

The objectives of this study are:

- Develop a tanker-slosh model that can effectively predict rollover threshold of partially filled elliptical tankers.

- Conduct parametric studies to assess the effect of various features and parameters on the rollover stability of partially filled elliptical tankers.

- Establish a rational basis for selecting pendulum parameters to simulate sloshing in generic elliptical tankers with arbitrary level of fill.

- Develop a case study in which all of the above is applied and compare the results to experiments.

- Provide a tool that can be used by designers to improve tanker's rollover stability.

\section{5- Scope}

The scope of this study is:

- Develop and verify models that can accurately predict the rollover threshold of a partially filled elliptical tanker undergoing any prescribed steering maneuver without braking on a level road. 
- Establish the fundamental bases for selecting a pendulum that can simulate the effects of lateral fluid sloshing in a partially filled elliptical tank.

- Once the parameters (masses, arm lengths, etc.) of this pendulum are obtained, its dynamic behavior are verified against real fluid behavior obtained using simple finite element models.

- Once the dynamic performance of this pendulum is verified, it is used in progressively detailed mathematical and/or FE models that are used to study the effect of several parameters on rollover stability of tankers.

- Use the pendulum-tanker models to predict the rollover threshold of partially filled elliptical tankers. Inputs to the models from experimental data based on circular paths, TOP and NATO lane change tests are used to obtain comparable results with the experimental outputs to verify the models. Several unknown parameters such as the tire and suspension lateral stiffness are expected to influence the results of the models when compared to the experimental results. Thus, a $10 \%$ error is considered quite acceptable by researchers working in this field. 


\section{CHAPTER 2}

\section{LITERATURE RIVIEW}

\section{1- Introduction}

The problem of fluid sloshing in partially filled containers and its effect on vehicle stability concerned researchers for a long time. Much work has been done to study the fluid sloshing effect on various kinds of vehicles such as aerospace vehicles (rockets and planes), ships carrying liquid cargo such as crude oil, and finally, heavy-duty mobile tankers.

An extensive literature review has been done to cover all the work done in the past, that relates to the problem of stability of partially filled heavy duty tankers during various road maneuvers. Topics such as; fluid sloshing in moving containers, parameters affecting heavy duty vehicle stability, steering characteristics of heavy duty vehicles, including the suspension types and characteristics, were covered in this literature review. Given below is a categorized summary for the work done related to the problem of partially filled heavy-duty vehicle rollover stability.

\section{2- Fluid-Container Interaction}

Rayleigh (1876) was the first who tackled the problem of fluid sloshing in partially filled containers. Rayleigh used a pendulum to simulate the dynamic effects of sloshing and obtained the correct lengths for regular pendulums, that matched the fluid natural frequencies. Lamb (1945) was the first to analytically obtain the transverse mode fundamental frequency $\omega_{n}$ of a $50 \%$ filled horizontal cylindrical tank of diameter $\mathrm{D}$, which was given by:

$$
\omega_{n}=1.169 \sqrt{g / D}
$$


Later, The problem of fluid sloshing in moving containers became a major concern for the researchers working in the aerospace industry during the 50's and 60's. Graham and Rodriguez (1952) simulated and duplicated exactly rectangular tank-surface forces and moments given by linearized hydrodynamic theory using spring-bobs, a fixed mass, and a rotary inertia. Abramson et. al (1960) and Anderson (1960) made a similar simulation for tank-surface forces using a circular tank. Bauer (1958 A) studied the inertia of a fluid in completely filled circular cylindrical tank and compared the moment of inertia of an ideal fluid (incompressible and nonviscous) with the moment of inertia of the same fluid in a frozen state. He found out that they can be considerably different. Bauer (1958 B) also found out that the moment of inertia of a liquid in a partially filled tank is strongly frequency dependant and that it is different from the moment of the same amount of fluid in a frozen state. Also, Bauer (1958C) studied the effect of damping on fluid inertia in a partially filled circular tank and found that the damping effect on the calculated inertia is negligible at high levels of fill.

Budiansky (1960) estimated the natural frequencies, mode shapes, and forces exerted on the walls of a partially filled circular canal and spherical tanks due to lateral excitation resulting in small-amplitude oscillations of the fluid. Budiansky considered the antisymmetric modes only since the symmetrical modes would not be induced by transverse motion of the tank. He also assumed an irrotational inviscid fluid, which is a good approximation for a deep container. Budiansky carried out the analysis using an arbitrary velocity potential on the surface and solving the continuity and the linearized Bernoulli equation to obtain homogeneous Fredholm integral equation describing the surface fluid motion (Equation 2.2).

$$
\omega(\xi)=(\lambda a) \int_{0}^{1} G(\xi, \bar{\xi}) \omega(\bar{\xi}) d \bar{\xi}
$$


Budiansky used a conformal mapping technique to find the kernel function $G(\xi, \bar{\xi})$ and then solved equation 2.2 numerically for different values of fluid depth to obtain the eigenvalues (Natural Frequencies), and the eigenvectors (mode shapes), at different values of fluid depth. Budiansky provided also in his study two equations to calculate the forces and moments on the circular canal surfaces that were used later by Ranganathan (1993) to establish the equivalent parameters of a regular circular pendulum to simulate the fluid sloshing. Those two equations are:

The differential equation of motion derived using Lagrange's method;

$$
\ddot{\xi}+\omega_{n}^{2} \xi_{n}=-\left(\lambda_{n} a\right)\left(B_{n} / A_{n}\right) \ddot{U}
$$

And the sloshing force of the fluid is given by:

$$
F_{s}=-M_{L} \ddot{U}-2 \rho_{L}(a R)^{2} \sum B_{n} \xi_{n}
$$

The first 2 mode shapes of fluid vibration in a circular canal according to Budiansky are shown in Figure 2.1.
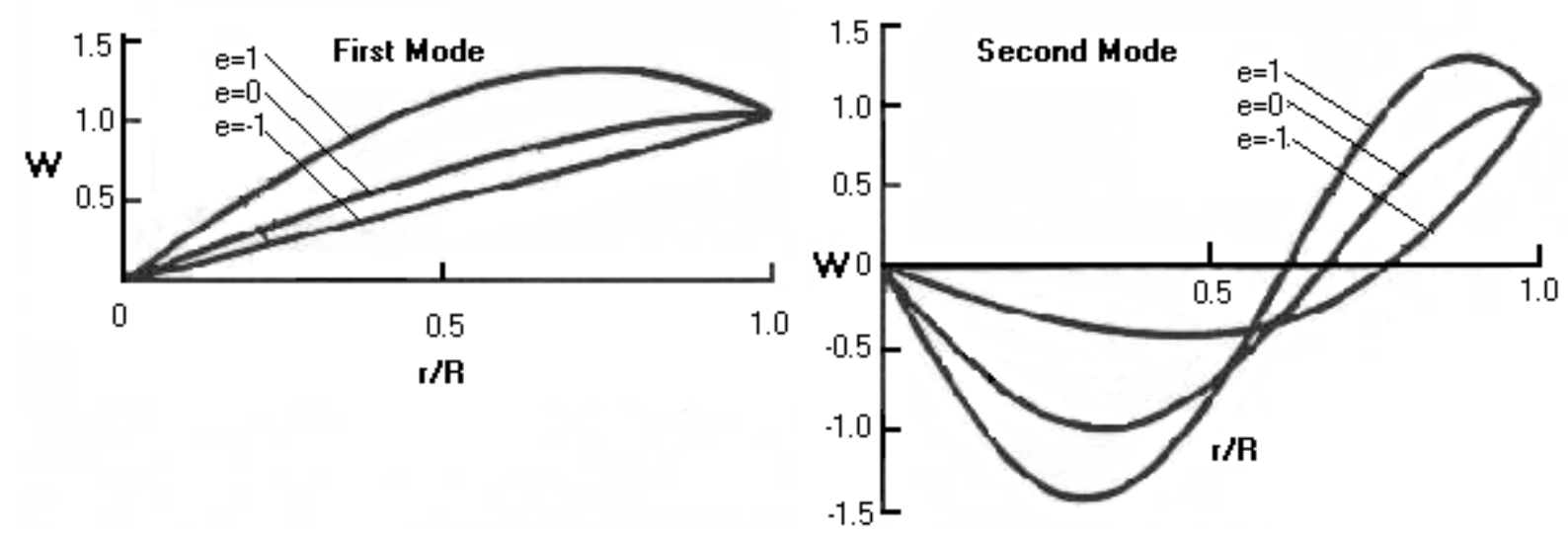

Figure 2.1: Normalized First and Second Mode Shapes of a Fluid in a Partially Filled Cylindrical Canal, Budiansky (1960) 
In Figure 2.1, $\mathrm{W}$ is the normalized fluid height, and e is a parameter representing the fill level, e equal -1 for empty canal, e equal 0 for half full, and e equal 1 for a full canal

Warner (1961) made an experimental study to evaluate two mathematical models, (the regular pendulum model and the spring-bob model) that simulate fuel sloshing in a vertical cylindrical tank subjected to small lateral oscillations. Although Warner stated that the pendulum model is not free of error like the spring-bob model in simulating the fluid sloshing, the experimental results showed that both models provide excellent simulation of the stiffness and inertia terms for fuel sloshing.

Rattayya (1965) evaluated the natural frequencies and mode shapes of a fluid in a partially filled ellipsoidal tank. He also simulated the first two modes of fluid vibration using a fixed mass and two laterally vibrating masses on springs. Figure 2.2 shows the equivalent mechanical model and the associated chart used to find the parameters of this model. It can be seen that the first two natural frequencies of the fluid sloshing reach infinity at all tank aspect ratios when the level of fill approaches the complete full condition. It worth mentioning that this fact holds for any tank geometry.

Abramson (1966 A) studied the nonlinear lateral sloshing of fluids in partially filled cylindrical containers. One of the main objectives of Abramson study was to define the range at which swirl occurs in a partially filled circular and spherical tanks subjected to lateral excitation. 

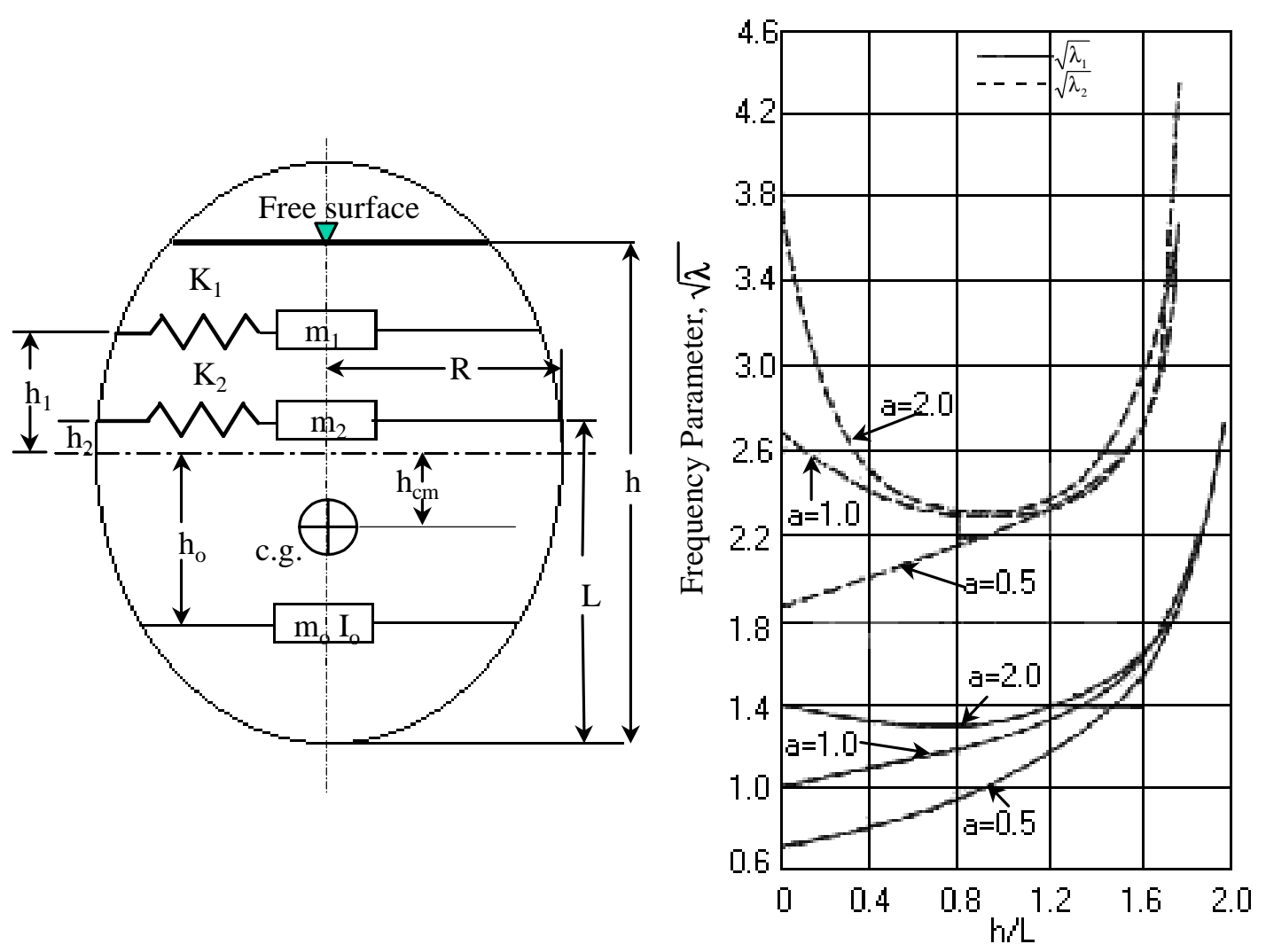

Figure 2.2: Equivalent mechanical model for a partially filled ellipsoidal tank and the associated variation of sloshing frequencies with liquid depth Rattayya (1965)

In Figure 2.2, $\lambda$ on the vertical axis represents the frequency parameter given by;

$$
\lambda=\frac{\omega_{\mathrm{n}}^{2} \mathrm{R}}{\mathrm{g}}
$$

"a" is the ratio $\mathrm{L} / \mathrm{R}, \mathrm{g}$ " is the gravitational acceleration, " $\mathrm{R}$ " is the tank radius as shown in Figure 2.2, and $\omega_{\mathrm{n}}$ is the n'th natural frequency of the fluid lateral slosh.

Figure 2.3 shows the swirl zone for a vertical circular tank subjected to lateral excitation at different levels of fill. 


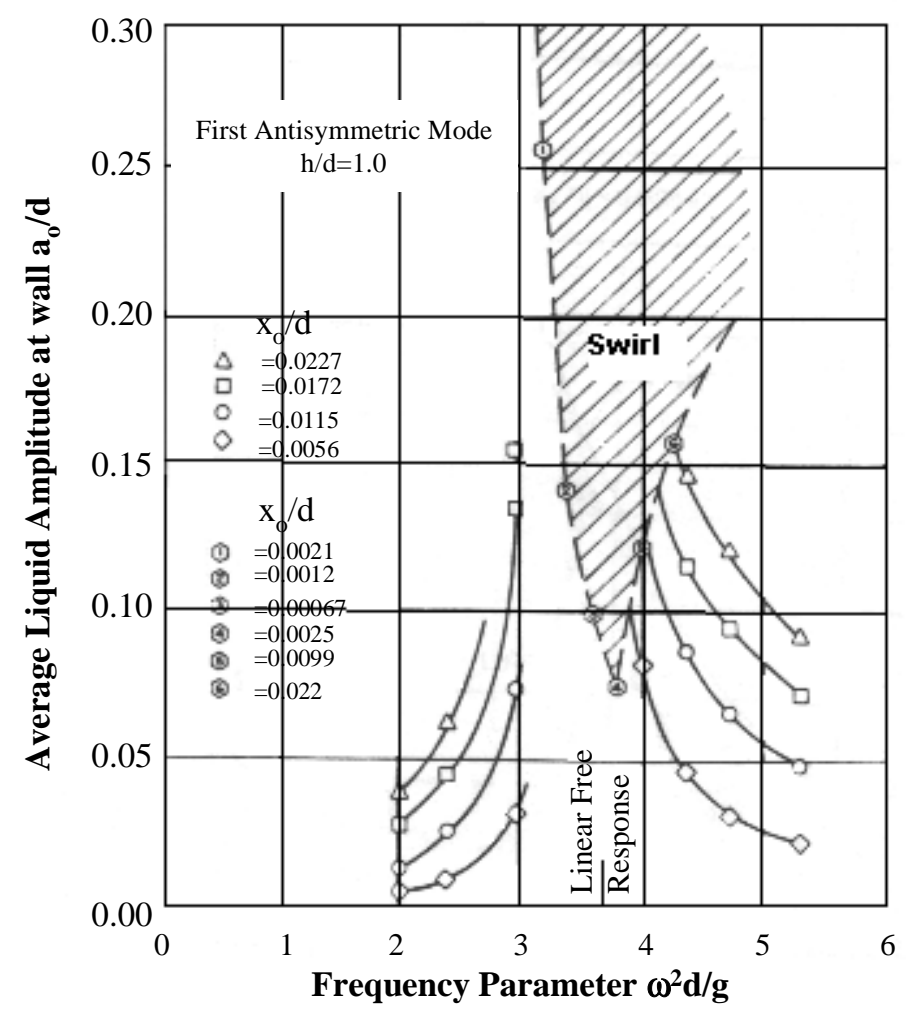

Figure 2.3: Liquid free surface response in vertical circular tank showing swirl region, Abramson (1966 A)

It can be seen that the swirl happens when the excitation frequency is close to the first natural frequency of the fluid. At low excitation frequencies, no swirl was detected. Abramson stated also that it has customarily been thought that the first antisymmetric slosh mode is characterized only by the rocking motion of the fluid. However, Abramson (1966 A) noticed that the fluid surface consisted of a combination of the first antisymmetric mode at the frequency of excitation and the first symmetric mode occurring as a superharmonic at double the frequency of excitation.

Abramson (1966 B) collected all the work done in the field of fluid sloshing tell 1966 both experimentally and analytically in one single document titled "The Dynamic Behavior of 
Liquids in Moving Containers". Abramson concluded that the first mode of vibration of fluid sloshing in any partially filled tank is the most dominant. He supported his conclusion by comparing the value of the mass $\mathrm{m}_{2}$ required to simulate the second mode of vibration to the mass $m_{1}$ required to simulate the first mode in the same ellipsoidal tank used by Rattayya (1965), see Figure 2.4 for details. He also showed that almost all the researchers in this field preferred to simulate at most the first two modes of vibration of a fluid in a partially filled container.

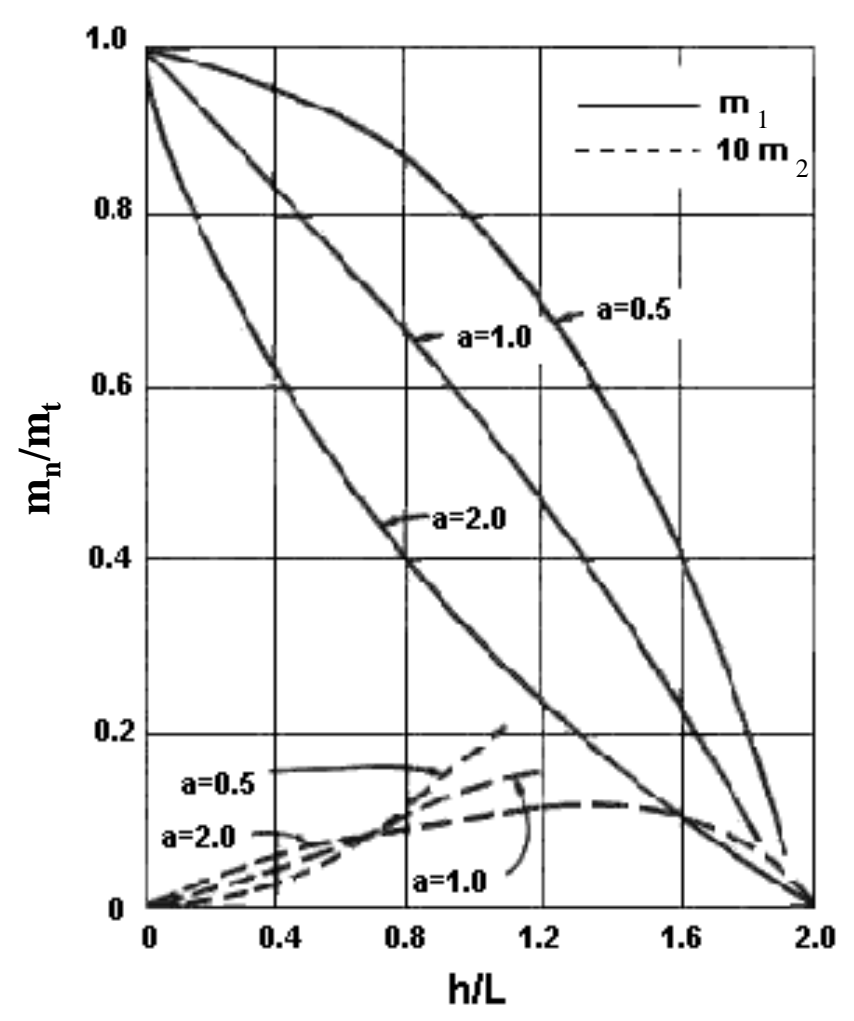

Figure 2.4: Variation of model masses with liquid depth for ellipsoidal tank, Abramson (1966 B)

It should be noted that although Abramson (1966 B) listed many models and analyses for various tank shapes, the elliptical tank shape under concern in this study was not included. Perhaps the reason is that this shape is not used in the aerospace industry, to which Abramson's document was related. 
Bauer (1972) studied the destabilizing effect of liquids in various vehicles. Bauer considered the interaction between the fluid and the elasticity of the walls of the tank. Bauer considered both rectangular and cylindrical tanks in vertical upright positions. He assumed the bottom of the tank to be either a membrane or a clamped elastic thin plate with thickness $\overline{h_{P}}$. Bauer evaluated the coupled circular frequency as a function of the cylinder wall thickens. Figure 2.5 shows the plot of one coupled fundamental circular frequency versus wall thickness for a vertical cylindrical tank.

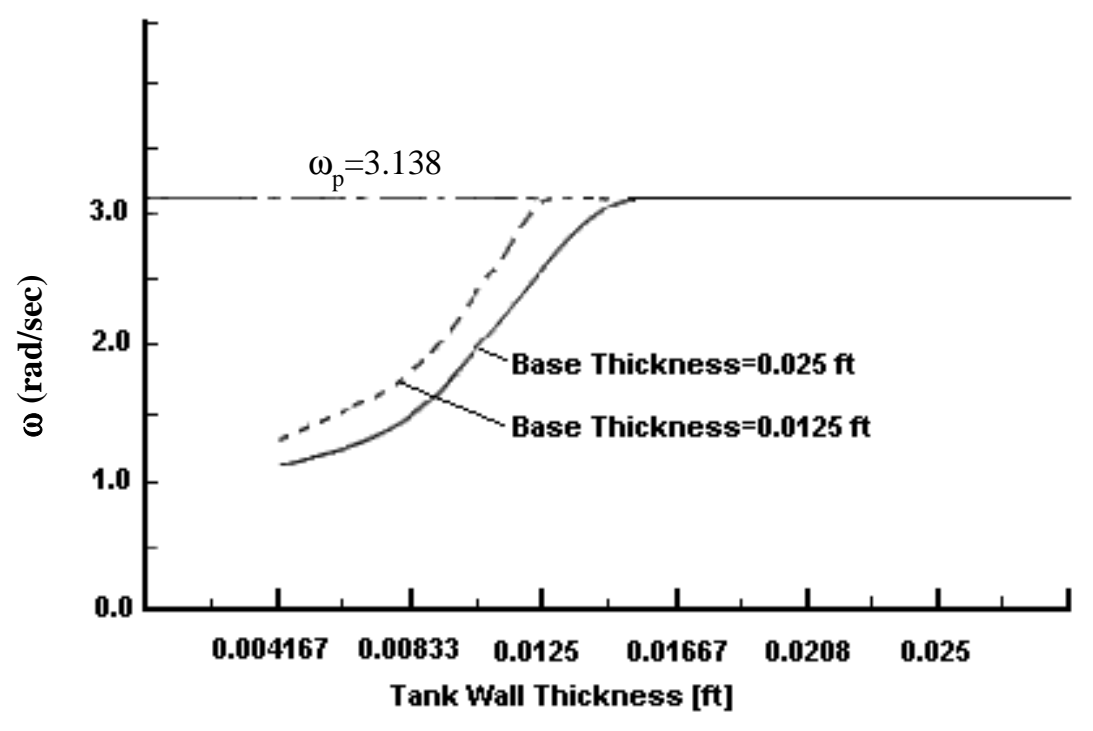

Figure 2.5 Coupled fundamental circular frequency versus wall thickness for a vertical cylindrical tank, Bauer (1972)

It can be seen that with increasing wall thickness, the coupling effect of the elastic plate bottom is diminishing and the liquid frequency in a complete rigid tank is approached rapidly.

Also, Bauer (1981) extended his study about the interaction between the elastic walls of a partially filled container and the fluid inside to cover more tank shapes. Although Bauer studied 
many tank shapes (rectangular, cylindrical, containers with inclined side walls, paraboloid and inverted paraboloid), the elliptical shape was not mentioned in his work.

Komatsu (1987) presented a nonlinear sloshing analysis of liquids in tanks with arbitrary geometries neglecting the viscous effect. Shimizu and Hayama (1987) studied nonlinear sloshing in rectangular tanks, basing their analysis on shallow water theory for an inviscid fluid.

Sankar et. al (1992 A) considered the non-linearities of fluid sloshing in an elliptical container. Sankar stated that all the theories used before to investigate the fluid sloshing were linear theories and they are in good agreement with the experiments, but only over a small range of motion amplitude. They mentioned many parameters that are commonly used, and gave ranges of these parameters which if exceeded, the nonlinear effects can not be neglected. One of the most important parameters is the lateral acceleration which should not exceed $0.2 \mathrm{~g}$ for fill levels of about $50 \%$ for a close to circular cross section tank. They solved the following sets of equations to determine the non-linear fluid behavior in an elliptical tank:

Navier-Stokes equations for an incompressible fluid in 2-D:

$$
\begin{aligned}
& S t \frac{\partial U}{\partial T}+\frac{\partial U^{2}}{\partial X}+\frac{\partial U V}{\partial Y}=\frac{G_{x}}{F r}-E u \frac{\partial P}{\partial X}+\frac{1}{\operatorname{Re}} \nabla^{2} U \\
& S t \frac{\partial V}{\partial T}+\frac{\partial U V}{\partial X}+\frac{\partial U^{2}}{\partial Y}=\frac{G_{y}}{F r}-E u \frac{\partial P}{\partial Y}+\frac{1}{\operatorname{Re}} \nabla^{2} Y
\end{aligned}
$$

The continuity equation:

$$
\frac{\partial U}{\partial X}+\frac{\partial V}{\partial Y}=0
$$

The free surface equation: 


$$
S t \frac{\partial H}{\partial T}=V-U \frac{\partial H}{\partial X}
$$

Popov et. al, (1993 B) studied the dynamics of liquid sloshing in baffled and compartmented rectangular road containers during uniform braking and steady state cornering. The influence of the number of separating walls, size and location of orifices in the separating walls was studied. The transient response of the fluid was obtained by numerically solving 2-D Navier-Stokes, continuity, and free surface equation using the modified Marker-and-Cell technique described in details by Popov (1991). The braking or acceleration maneuver was presented by a constant change in deceleration or acceleration. This approach was proved to be valid by Popov et. al 1992).

Popov et. al, (1993 C) conducted a study to obtain the optimal shape of a rectangular road container. They integrated the static momentum equations of a fluid in 2-D to obtain the objective function represented by the overturning moment around the middle point of the container. The objective function was minimized with respect to the container height and fill level, considering the lateral acceleration as a parameter.

\section{3- Heavy-Duty Vehicle Stability}

The problem of heavy-duty vehicle stability (regardless of its cargo) undergoing different road maneuvers has been addresses by many researchers. Topics such as jackknifing, pendular motion of the trailer(s), and rollover stability have been addressed.

Williams (1952) conducted a simplified analysis to study the snaking of a two-wheel trailer behind a towing vehicle. Later, Slibar and Paslay (1957) considered the mechanical 
response of a two-wheel trailer by itself when its tow point moves at a constant velocity with a lateral perturbation.

Jindra (1963) and Ellis (1966) studied the jackknifing problem using linearized equations of motion and constant forward velocity. However, the results obtained from their models were not readily apparent to relate to jackknifing of a real vehicle. Hales (1963) and Ellis (1963) have considered the effect of braking but the consequence of the simplification of their models were still uncertain, and the question as to whether or not jackknifing can take place without the locking of one or more sets of wheels has not been answered.

Jindra (1964) investigated the lateral oscillations of a long trailer train composed of a lead vehicle and several identical four-wheel trailers. Jindra (1965) extended his research to study the effect of change of different parameters on the directional stability of a tractor-trailer combination consisting of a tractor, semitrailer, and a full trailer. Effect of fifth wheel location, semitrailer length, semitrailer overhang and many other parameters on the directional stability were considered.

Barter and Hales (1966) studied experimentally the jackknifing and trailer swing during tractor-trailer breaking. They were not able to produce jackknifing without use of the brakes during these experiments.

Schmid (1967) studied the stability of a truck and tractor train using a simplified model, which consisted of a three-unit train system (Figure 2.6). Schmid assumed a fixed steering angle through his analysis and neglected bounce, roll, and pitch of individual bodies. Schmid also 
ignored the change of the wheel loads in curves as well as aligning torque, toe-in, camber and the effect of the wheel suspension. He assumed that the coupling joints between the vehicle units are frictionless. Schmid stated that the wheel locking at the tractor rear axle is found to be the cause of jack-knifing.

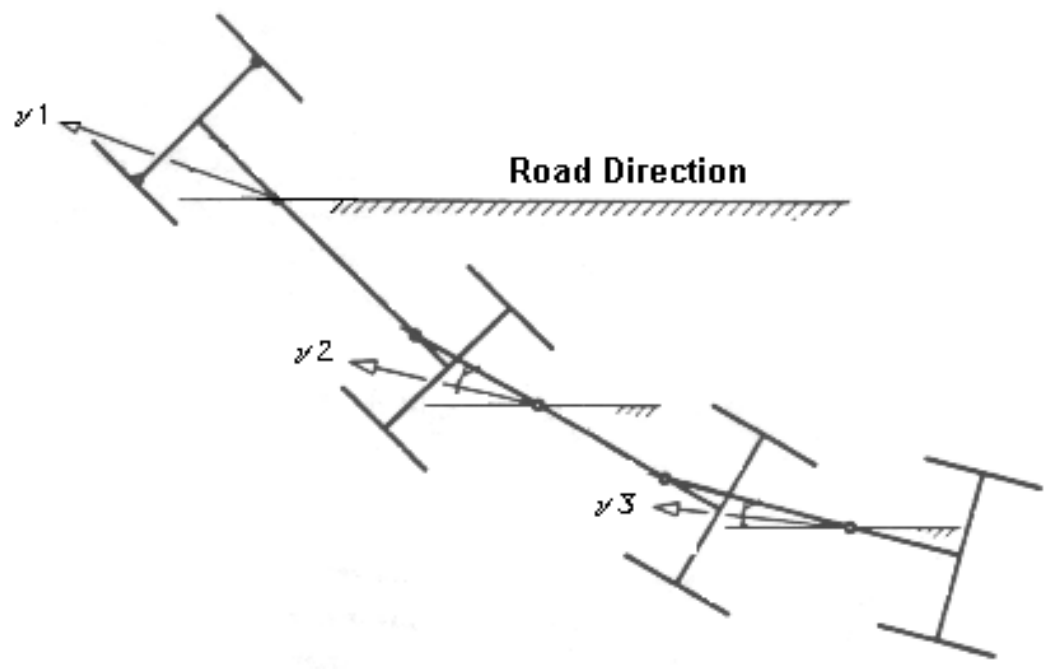

Figure 2.6: Simple three-unit train used by Schmid (1967)

Schmid's model had only the tire side forces, propulsion and braking forces as external forces acting on it. Schmid stated that the lateral movements of the vehicle units are composed of several elementary movements either periodic or aperiodic. Schmid used the Routh criterion to distinguish between stability and instability. Schmid discussed the influence of various parameters and distribution of braking forces applied to wheels. And finally giving constructional recommendations to improve the vehicle stability on roads. The following were Schmid's main conclusions:

1- The amplitude of the pendular movements increase as increases the travelling speed.

2- The pendular movements can be reduced by a wider wheel-base.

3- The frequency of oscillation decreases as the moment of inertia of the trailer increases.

4- The tendency of pendular movements increases with the number of trailers. 
5- Dynamically load dependant braking force distribution is preferable to prevent jack-knifing.

6- An eccentricity "e" in the fifth wheel between the load point and the wheel center line (see Figure 2.7) leads to one-sided loading of the frame in curves, which is desirable to a certain extent, since it acts against the slope of the vehicle.

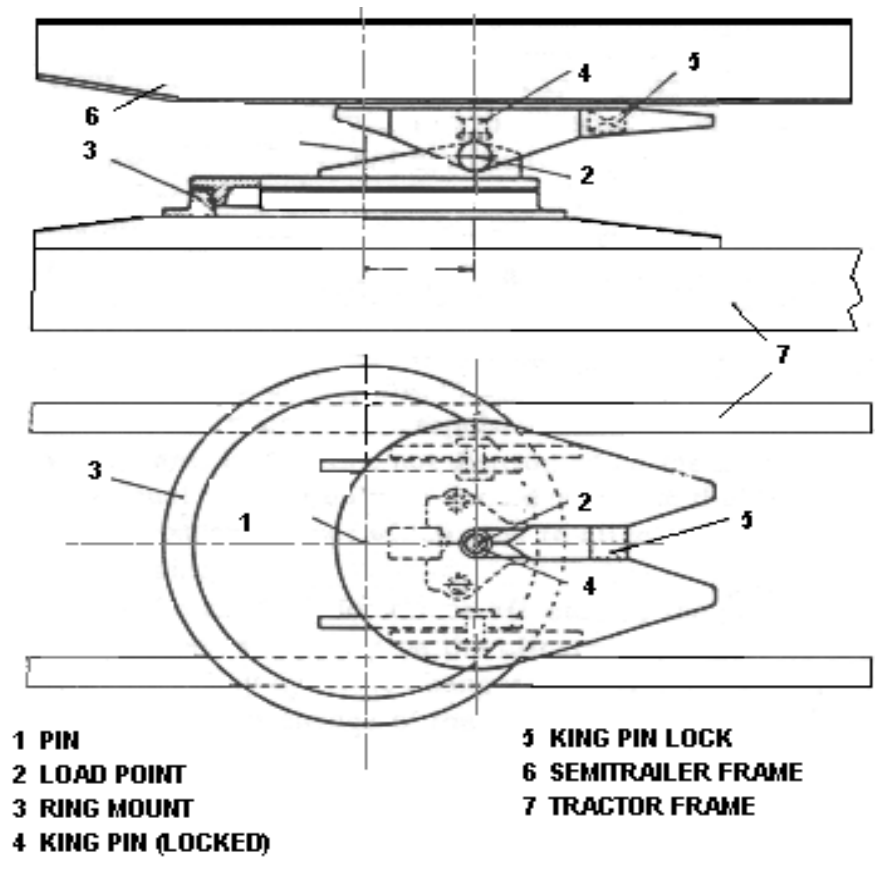

Figure 2.7: Fifth wheel design with eccentricity proposed by Schmid (1967)

Bundorf (1967) conducted an analytical study on a passenger car-travel trailer combination. Bundorf's study included evaluation of wind disturbance responses for the cartrailer combination. Also, Beauvais (1967) studied the aerodynamic interference between a car and a trailer by means of wind tunnel tests.

Mikulcik (1968, 1971) studied the jackknifing problem by studying the dynamics of tractor-semitrailers in more detail. Mikulcik provided for time variant steering inputs, as are variable braking forces at each wheel, provision was also made for coulomb and viscous damping at the hitch. Both linear and nonlinear equations of motions for the vehicle were 
considered in Mikulcik's model and the results were compared. The results demonstrated the occurrence of jackknifing of the nonlinear model, even without application of braking forces for certain inputs, while the linearized model was stable. Mikulcik recommended that the load should be placed towards the front of the semitrailer whenever possible to provide for a maximum availability of traction at the rear tractor wheels, and these wheels should be equipped with tires which provide a high coefficient of friction with the road surface.

Bidwell (1970) listed most of the work related to vehicle control and road holding in one document. Topics such as; Air resistance, rolling resistance, tire compliance, vehicle-driver interaction braking, and tractor-trailer handling were included. A list of 120 references containing work done in these fields until 1970 was given.

Leucht (1970) studied the directional dynamics of tractor-semitrailers during braking. Leucht investigated the effect of loading conditions, brake-torque distribution, brake application times, and load sensitive brake-torque control on the directional response of tractor-semitrailers during braking.

Krauter (1972) enhanced the model established by Mikulcik (1971) to allow translation, yaw, roll, and pitch of both tractor and semitrailer. Lateral and fore-and-aft weight transfer was considered, including the wheel dynamics. The ability of this model to describe accurately a real vehicle was studied by using the model to simulate a full-scale experimental test. The model was also used to study two types of proportional braking for a tractor-semitrailer executing a large-radius turn on a wet asphalt track. The effect of tire properties and dynamics were 
considered in this study and were used to calculate the tire vertical and lateral forces, which were used later to simulate braking torque proportional to the wheel load.

Hazemoto (1973) studied the lateral stability of doubles in terms of frequency response and characteristic roots using simple models. Hazemoto found that the frequency response determined by his calculation was in rough agreement with the value determined experimentally. He also found that the vehicle train is most unstable when the second trailer is loaded while the first trailer is empty and that the stability of each trailer increases in proportion to its wheelbase.

Ervin et. al (1975), Ervin and Wild (1976) and Ervin et. al (1976) studied the longitudinal and lateral properties of heavy-duty vehicles' tires and their effect on the dynamic vehicle behavior. Ervin et. al (1976) documented the significant difference in heavy-tire characteristics and passenger cars' tire characteristics.

Ervin et. al (1978) studied the stability of heavy-duty double tankers. They hypothesized and then demonstrated that a typical configuration of double tanker has a peculiarly low level of rollover resistance. Furthermore, it was shown that the second trailer (the so-called "pup trailer") has a propensity for rollover in obstacle-avoidance maneuvers.

Mallikarjunarao (1978) extended the linear models established by Jindra (1965), Hazemoto (1973), and Hales (1975) to study the lateral stability of heavy-duty double tankers under different fill conditions. Mallikarjunarao included multiple axles on the various units of the vehicle. The tankers were assumed to be fully compartmented, and the percent of fill was established by assuming a different configuration of completely filled compartments (to avoid 
fluid sloshing effects). Figure 2.8 shows the different vehicle configurations considered by Mallikarjunarao.

Mallikarjunarao found that the pup trailer would have unstable oscillations at highway speeds when the rear compartment of the pup trailer is loaded and the other compartments of the pup trailer are empty. Also, rigidizing the connection at the pintle hook improves the directional performance of the double tanker.
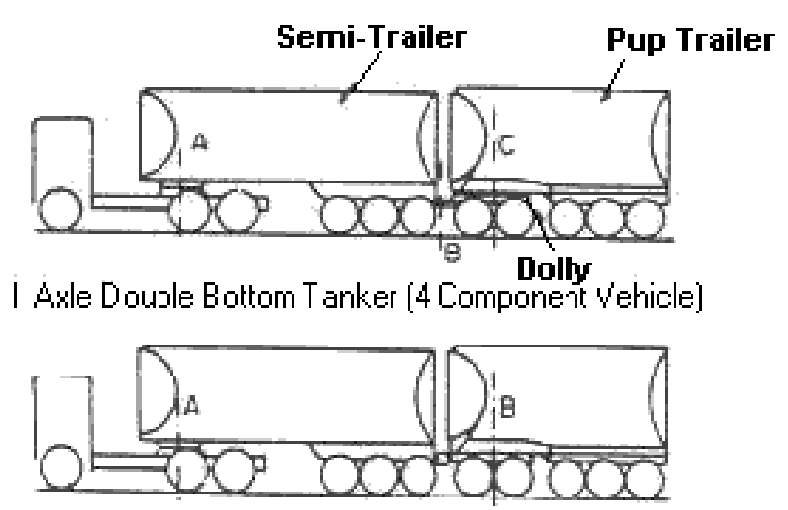

Modefied II Axle Double Bottom Tanker With Rigid Pinlle Hook [3 Zomponert Vehicle]

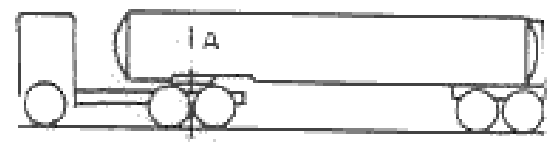

5- Axle Single Tanker

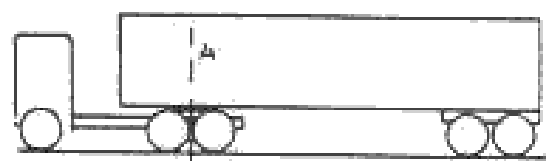

Tractor with Conventional Van Semi-Trailer

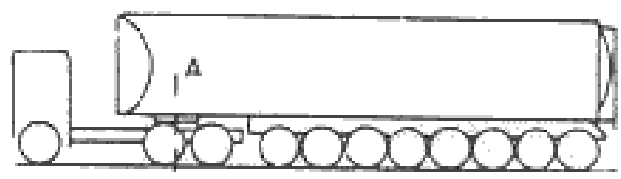

II - Axle Single Tanker

Figure 2.8 Schematic diagram of five vehicle configurations used by Mallikarjunarao (1978) 
MacAdam (1979) approximated the characteristics of leaf spring suspensions by the equation 2.10, which can be used to simulate leaf spring force-deflection characteristics at different time steps based on data that governs the boundaries of the spring hysteresis loop.

$$
F_{i}=F_{E N V_{i}}+\left(F_{i-1}-F_{E N V_{i}}\right)^{-\left|\delta_{i}-\delta_{i-1}\right| / \beta}
$$

Where:

$\mathrm{F}_{\mathrm{i}} \quad$ is the suspension force at the current simulation time step

$\mathrm{F}_{\mathrm{i}-1} \quad$ is the suspension force at the last simulation time step

$F_{E N V_{i}}$ is the force corresponding to the upper and lower boundaries of the envelope of the measured spring characteristics at the deflection $\delta_{\mathrm{i}}$

$\delta_{\mathrm{i}} \quad$ is the suspension deflection at the current simulation time step

$\delta_{\mathrm{i}-1} \quad$ is the suspension deflection at the last simulation time step

$\beta \quad$ is an input parameter used for describing the rate at which the suspension force within a hysteresis loop approaches the outer boundary of the envelope. Different values of $\beta$ for increasing versus decreasing deflection may be specified.

Ervin et. al, (1979) studied the yaw stability of tractor-semitrailer during pure cornering with no braking. They stated that tractor yaw stability might be threatened before the rollover threshold is reached. They studied the effect of several design parameters on the tractor yaw stability. Some of these parameters were not commonly changed, such as the torsional stiffness of the tractor frame and its front suspension. They stated that the tractor frame stiffening alone produced negligible change in tractor behavior, while the suspension stiffness proved to be the most significant parameter affecting vehicle yaw stability. 
Fancher et. al (1980) measured the mechanical properties of truck leaf springs and presented them in a way suitable for analytical modeling. They mentioned that the results from low frequency (quasi-static) tests of typical tandem suspensions have indicated that truck leaf springs are complicated force-producing mechanisms. These leaf springs may exhibit varying levels of; (a) Effective spring rate and (b) Damping, coulomb friction, or hysteresis, depending upon the loading of the spring and the amplitude of the oscillation studied.

They studied five types of leaf-spring suspensions; (a) Multi-leaf front spring (b) Tapered-leaf front spring, (c) Multi-leaf rear spring with torque rod, (d) one spring from a fourspring suspension with torque rods and (e) one spring from a four-spring suspension with torque leaves. They considered the inertial force component developed from the effective mass of the spring $\left(M_{e} \ddot{Z}\right)$ and subtracted it from the total spring force to obtain a spring characterization, which applies directly to the definitions of component functions typically used in vehicle simulations.

They tested the leaf springs mentioned above at frequencies varying from $0.5 \mathrm{~Hz}$ to 15 $\mathrm{Hz}$, and found out that the quasi-static force-deflection behavior such as at $0.5 \mathrm{~Hz}$ constitutes a good representation of the average or "smoothed" behavior up to $15 \mathrm{~Hz}$. They mentioned also that although the characteristics of these tested springs were not frequency dependent, the results indicted that the force-deflection properties of truck leaf springs depend upon both the amplitude of motion and the nominal load. 
They defined an average coulomb damping force " $\mathrm{C}_{\mathrm{F}}$ " and an average effective spring rate $" \mathrm{~K}_{\mathrm{e}}$ " (see Illustration in Figure 2.9) that can be used to describe leaf spring characteristics. They found out that the effective leaf spring rate decreases as deflection amplitude increases. This "inverse trend of $\mathrm{K}_{\mathrm{e}}$ is necessary for the approximating hysteresis loop to have both the same force amplitude and area as the measured data. Finally, they used equation 2.9 to fit the data of the suspension springs mentioned above.

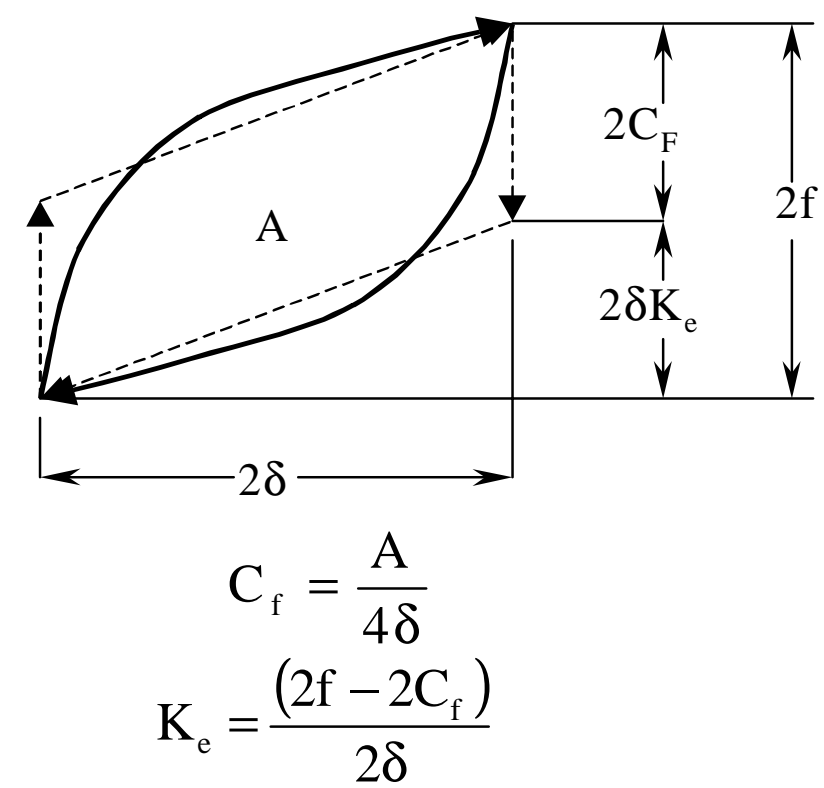

Figure 2.9: Illustration of $\mathrm{C}_{\mathrm{f}}$ and $\mathrm{K}_{\mathrm{e}}$ for a typical leaf spring using an approximated hysteresis loop, Fancher et. al (1980)

Ervin et. al (1980) related the rollover accidents of heavy tractor-semitrailers to their nominal roll stability from statistical data. Then, Ervin (1983 A \& B) studied the influence of size and weight variables on the roll stability of heavy trucks using both tests and simulation methods. He investigated the effect of the suspension spring lash on the rollover stability. Figure 2.10 shows a spring lash in a typical variable stiffness leaf spring suspension, while Figure 2.11 shows the effect of the spring lash on roll response of a vehicle. 


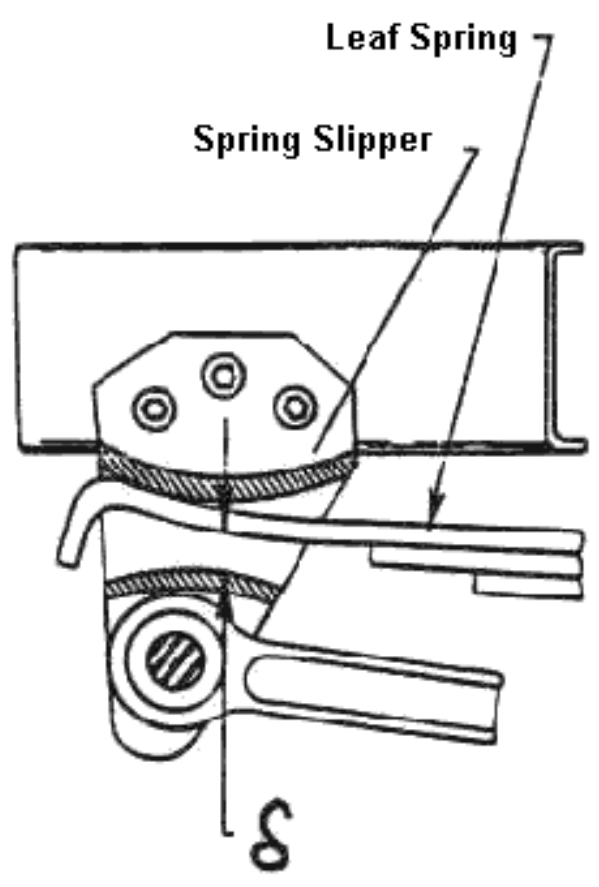

Figure 2.10: Spring lash in a variable stiffness leaf spring truck suspension, Ervin et. al (1983 B)

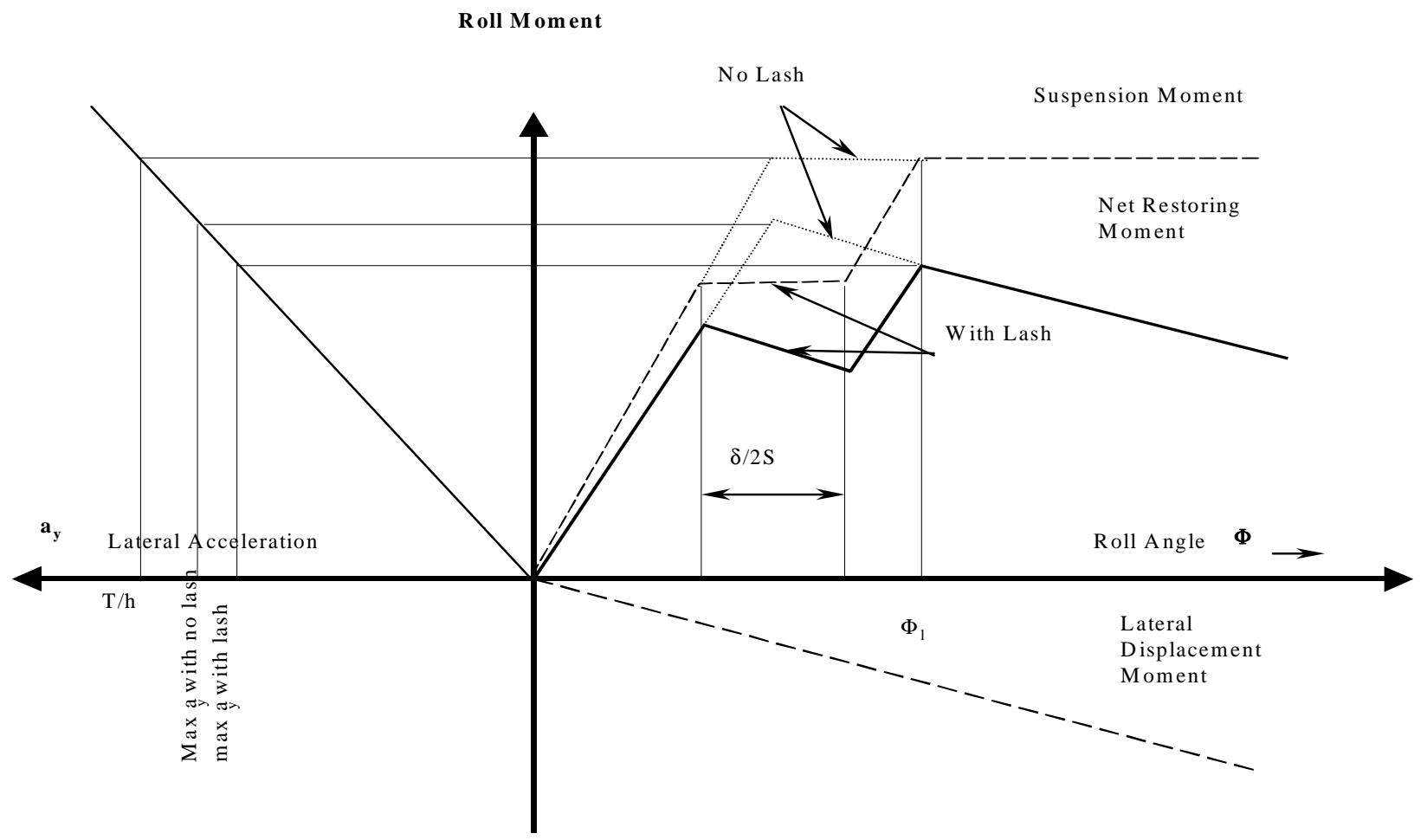

Figure 2.11: Roll response of a heavy-duty tractor-semitrailer including spring lash, Ervin et. al(1983 B) 
Ervin et. al (1983 B) stated that the road irregularities or deformability of the surface at the roadside could introduce vertical inputs, which promote a roll response. Also, the nominal ground plane can become rather inclined with respect to the horizontal They also stated that when a vehicle conducts a cornering maneuver while travelling on a paved highway, the only significant inputs promoting rollover are the lateral traction forces of the tires, which are generally capable of achieving lateral acceleration levels approaching $0.8 \mathrm{~g}$ 's.

They mentioned that for typical heavy vehicles, approximately $2 / 3$ of the total roll angle subtended by the sprung mass (up to the wheel liftoff condition) involves rotation about the suspension roll center (see Figure 2.12). The other 1/3 involves rotation about the tire roll center located in the ground plane. He also stated that the wheel liftoff angles are much larger than the suspension unloading angle values.

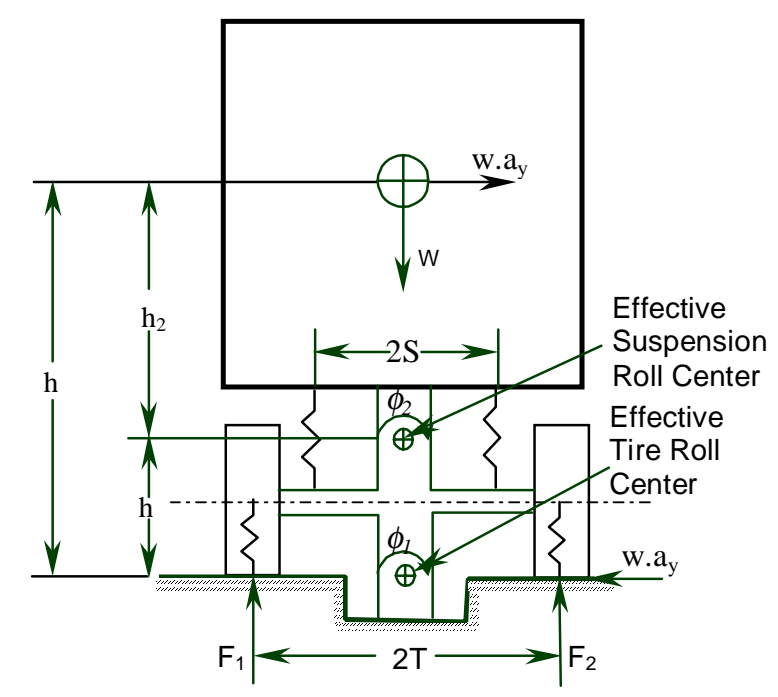

Figure 2.12: Simplified vehicle roll model showing the concept of suspension and tire roll centers, Ervin et. al (1983 B)

Ervin et. al (1983 B) found out that since the front suspension is less effective in developing the restoring moment followed by the tractor rear suspension, the trailer rear 
suspension is more likely to liftoff first in a rollover incident followed by the tractor rear axles and finally the tractor front axle. Figure 2.13 illustrates the sequence at which axles of a typical tractor-trailers would liftoff the ground. It can be seen that the roll angle at which the rear axle of the trailer starts lifting off " $\phi_{13}$ " is less than that of the tractor rear axle " $\phi_{12} "$, which in turn is less than the tractor front axle " $\phi_{11} "$.

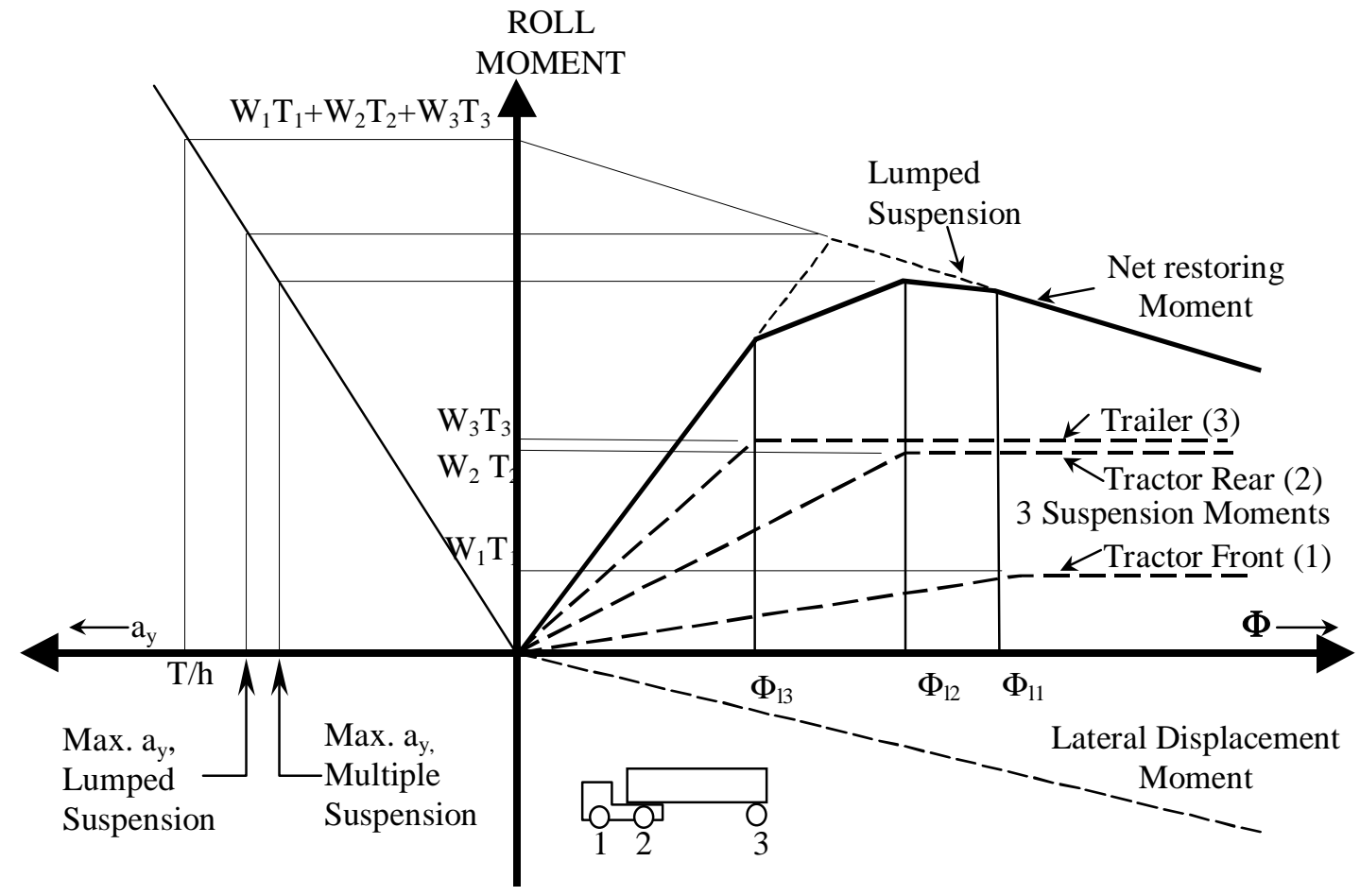

Figure 2.13: Roll response of combination vehicle with three compliant suspensions, Ervin et. al (1983 B)

Recently developed mathematical methods [Holmes (1981), and Carr (1981), Guckenheimer (1983), and Arnold (1983)] helped researchers such as Troger (1984) and Stribersky (1989) to proceed the study conducted by Schmid (1967) in the nonlinear range. Troger used the bifurcation theory to investigate the loss of stability of equilibrium position of a tractor-semitrailer in steady state motion, while Stribersky extended the same study for a B-train 
vehicle. Both Troger and Stribersky empathized the importance of modeling the lateral forces of tires. While Troger used a nonlinear expression that was given by Zeman (1982) to describe the tire side forces, Stribersky used an adhesion/sliding approach given by Schallamach and Turner (1960).

Dill (1985) developed a computer code to study heavy-duty vehicle stability based on the Static Roll Model (SRM) developed by Mallikarunarao (1982 A and B) for a tractor-trailer. This model included torsional springs representing the torsional stiffness of the tractor and the trailer frames.

Pflug (1986) investigated the lateral dynamic behavior of tractor-trailer combinations under the influence of change in mass and yaw moment of inertia. He showed that increasing the yaw inertia of the trailer relative to its mass deteriorated its lateral dynamic behavior.

Tilt table tests have been used to regulate rollover threshold for buses. While an angle of 35 degrees for single decker busses and of 28 degrees of double decker busses were set [White (1986)], the tilt table procedure and angle for heavy duty trucks is under study (SAE J2180, 1990).

Nalecz and Genin (1984) conducted a literature survey on all the work done prior to 1984 related to the topic of dynamic stability of heavy articulated vehicles. About 79 articles were reviewed in their review. The reviewers highlighted the significance of pneumatic tires on the performance of heavy vehicle dynamics and the incorporation of nonholonomic constraints being incorporated recently at that time in the models. 
Nalcez $(1988,1989)$ proposed the concept of "Rollover Prevention Energy Reserve" (RPER) to quantify the tripped rollover threshold. The RPER is defined as the difference between the potential energy at rollover threshold (when the vehicle's CG aligns vertically above the outer tire) and the sum of the instantaneous potential energy and rolling kinetic energy. Also, Aurell and Edlund (1989) studied the influence of steered axles on the dynamic stability of heavy vehicles.

El-Gindy and Hosamel-deen (1989) used (SRM) to conduct sensitivity analysis on a tractor and a trailer. They considered the tractor and the trailer stiffness as well as the fifth wheel compliance in their study.

Winkler et. al (1990) studied the rollover accident rates as a function of rollover threshold. They introduced an analysis of payload of fixed C.G. height vs. fixed density.

Hinch et. al (1992) presented a Rollover Prevention Matrix (RPM) to predict tripped rollover stability. The RPM is defined as the difference between the initial lateral translation kinetic energy and the rotational kinetic energy after the impact divided by the initial energy.

Gerorge (1992) provided statistical analysis of actual vehicle rollover and discussed static and dynamic considerations. Winkler et. al (1992) considered the lateral stiffness of heavy-duty vehicle's suspension and its effect on the vehicle stability. They broke down the roll-stability properties of commercial vehicles into two major components: The rigid vehicle stability factor (composed of half-track width and CG height), and the roll lateral compliances of the vehicle. Figure 2.14 illustrates the concept of suspension lateral stiffness and its effect on CG lateral shift 
and hence, roll stability. They also listed typical values for various leafspring suspension properties including suspension roll and lateral stiffnesses. Later, Karamihas and Winkler (1997) published tables that contain typical lateral suspension characteristics for 5 different configurations of heavy-vehicle suspensions, namely; 4-spring suspension, walking beam, 2spring suspension, trailing arm suspension, and 2-stage suspension. These suspension properties are useful for modeling heavy-duty vehicles.

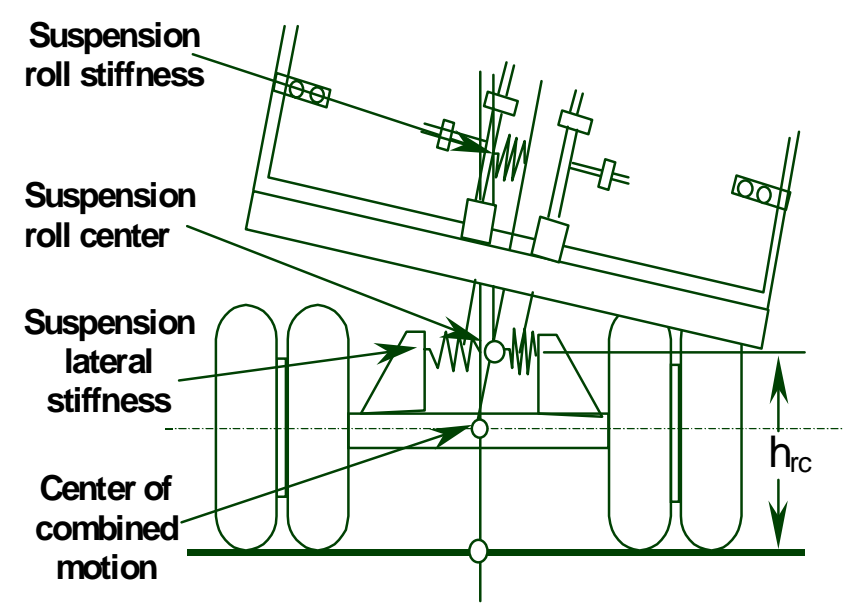

Figure 2.14: A simple suspension model for roll stability analysis used by Winkler et. al (1992)

Das et. al (1993) estimated the rollover threshold of a commercial bus using low speed experimental data. They used a simple plane model developed earlier by Verma-Gillespie (1980) with some minor modifications (using dampers instead of coulomb friction). They used lateral experimental acceleration in calculating the input force and stated that this approach bypasses the modeling complexity of tire for lateral force generation. They used the concept of "Rollover Prevention Energy Reserve (RPER) which is defined as the difference between the potential energy at rollover threshold (when the vehicle's CG aligns vertically above the outer tire) and the sum of the instantaneous potential energy and rolling kinetic energy. The RPER 
was proposed earlier by Nalcez $(1988,1989)$ to quantify the rollover threshold. They explained the need of using an extrapolation technique to obtain the rollover threshold since it was not possible to reach the point of rollover experimentally. The reason was that outriggers were not used due to their high cost, effect on vehicle's inertial parameters, and poor repeatability.

Sankar and Surial (1994) utilized the static roll model established earlier by Dill (1985) to provide rollover sensitivity analysis for a variety of tanker-trailer parameters. However, they didn't report results on variation of tractor and trailer stiffness.

Jansen and Lupker (1994) proposed many simplifications to tractor-semitrailer models (such as reducing the total number of axles with axle suspension reduction to roll suspension only) to reduce CPU-time.

Although the model developed by Lieh (1994) was directed towards studying the ride comfort of heavy duty trucks, Lieh provided a very useful survey of the coordinate systems used by earlier researchers to study heavy-duty vehicle dynamics. Those coordinate systems are namely: the generalized coordinate system, the Cartesian Coordinates, Euler parameters, and natural coordinates. Many studies related to heavy-duty trucks ride comforts and vibration response can also be found in Lieh's study.

Ranganathan and Aia (1995) used MATLAB/SIMULINK to build an interactive model to study the yaw response of a tractor-semitrailer linear model. The model was accomplished in a modular form to accommodate interactive re-runs of the model with design variations. The results of their model were validated against other software packages. They indicated the 
importance of using SIMULINK in heavy-vehicle modeling since it's also a control package that can be installed once fully developed to provide active control and corrective action during vehicle instability.

El-Gindy (1995) indicated two different dynamic performance measures for heavy-duty tractor-trailer combinations. The first was in terms of Load Transfer Ratio (LTR), which is defined as the ratio of the absolute value of the difference between the sum of right wheel loads and the sum of the left wheel loads to the sum of all wheel loads. The second is the rearward amplification ratio, which is defined as the peak lateral acceleration response of the rearmost trailer to that of the tractor.

Lund and Bernard (1995) furthered the study on tripped rollover of vehicles and proposed a criterion for tripped rollover referred to as Critical Sliding Velocity (CSV). The CSV is defined as the measure of the minimum lateral velocity required to initiate tripped rollover.

Rakheja et. al (1995) studied the influence of articulation damping on the yaw and lateral dynamic response of a tractor-semitrailer. Two dampers were installed between the tractor and the semitrailer and a mathematical yaw-plane model was developed to simulate the dynamic behavior of this system. Results showed that the peak amplitudes of yaw and lateral oscillations were reduced significantly.

Sayers and Riley (1996) developed a multibody simulation program called TruckSim to simulate the behavior of heavy-trucks in yaw and roll. They provided full details of the parameters and assumptions of their model, such as; steering system compliance, tire and 
suspension compliances, hitch moment, ground geometry, etc. The assumptions were tested using the AUTOSIM multibody code generator developed Kortüm et. al, (1993). A fleet of truck simulation programs for straight-line trucks and tractor-semitrailers with different axle configurations were built and assembled into a software package called TruckSim. The results of the TruckSim were successfully compared against the results of the earlier Yaw-Roll model developed by Mallikarjunarao (1982 A) and Gillespie, and MacAdam (1982).

Liu et. al, (1997) studied the rollover threshold of different configurations of heavy-duty vehicles. They classified the rollover based on its nature to; tripped rollover and maneuver induced rollover. They concluded that maneuver-induced rollovers are more likely to occur than tripped-rollover accidents based on a survey conducted by Wolkowicz (1986) of highway accidents involving heavy vehicles. The survey revealed that $77 \%$ of rollover accidents occurred on dry pavement. They also classified the roll instability to; relative instability and absolute instability. They provided detailed definition of both forms of instabilities. A tractor-semitrailer and an eight axle A-train double were analyzed to establish the dynamic rollover threshold based upon relative rollover instability criterion. Also differences between A-train, B-train and C-train doubles in terms of modeling were highlighted in their study.

Dahlberg and Vàgstedt (1997) developed a simple 9DOF roll axis model using "DADS" (Dynamic Analysis and Design System) to study the yaw response of a tractor-semitrailer combination. The yaw response of the tractor and the trailer obtained using their model was compared against field test results as well as the results obtained using a more complex DADS model that included FE-models of the frames. The results of the simple models using DADS were in good agreement with the results of the complex model and the real time performance. In 
addition, the simple model required $0.8 \mathrm{CPU}$ calculation effort of the real time performance compared to $250 \mathrm{CPU}$ time effort required by the complex model. The researchers concluded that their simple model is more suitable for application in vehicle control systems.

Vàgstedt and Dahlberg (1997) used a "bicycle model" to obtain the yaw response and lateral axle data for a tractor-semitrailer. They verified the results of their model with experimental testing of a $4 \times 2$ tractor fully air suspension towing a three axle Euro-trailer.

Ruhl L. and Ruhl R., (1997) Used Microsoft Excel's nonlinear equation solution capabilities to develop a model to study the rollover stability of flat bed tractor- trailers. They included the effect of the stiffness of the trailer in their model. They found out that the torsional stiffness of a flat bed trailer is a more important parameter than typical suspension and tire stiffness in studying the roll behavior of such flat bed tractor-trailers.

\section{$\underline{\text { 2.4- Fluid-Vehicle Interaction }}$}

Strandberg et. al (1975) studied the overturning risk of heavy-duty truck-trailer combinations both analytically and experimentally. They compared the drivers' estimated overturning risks with the computed risk and found out that the drivers tend to overestimate the possible cornering capacity of the vehicle at low speeds, and underestimate it at high speeds irrespective of loading condition. They also conducted some fluid sloshing tests on partially filled, scaled models of glass tanks of circular, elliptical, and supper elliptical cross-sections using a laboratory test rig that provides different lateral excitations. They provided recommendations to improve vehicle controllability and lateral stability. Some of these recommendations were related to road regulations, some to driver education, and some to vehicle 
design. They suggested reducing the number of articulations and installing a piston arrangement to completely eliminate fluid slosh effects in partially filled cylindrical tankers.

Slibar and Troger (1977) characterized the sloshing liquid cargo in the roll plane by two lumped masses coupled through linear spring and a viscous damper. Later, Strandberg (1978) investigated the fundamental slosh frequency of partially filled tanks using dynamically similar scale model of the tank.

Bohn et. al (1981) studied the effect of cargo shifting on articulated vehicles performing breaking and/or cornering maneuvers. Bohn et. al (1981) were the first to derive the fluid equations of motion in a partially filled elliptical tank using Lagrange's equation. The fluid slosh model was integrated into a very well known vehicle dynamics simulation program called TDVS (Three Dimensional Vehicle Simulation).

They compared the simulation results with field test results obtained from testing cylindrical and elliptical tankers, with or without baffles. Both dry and wet surface conditions were considered. Bohn mentioned that the TDVS path-following algorithm unfortunately failed and became unstable when simulation on low friction surfaces was attempted. Rendering maneuvers on wet surfaces impossible to simulate. Also the results obtained from the slosh dynamics augmented TDVS simulation was considered a qualitative, not a quantitative, analysis tool. The lack of experimental wave data was notable and significantly degraded the slosh model transient response. They also commented on their study that "While good agreement could be obtained from the braking profiles, the steer profile information left much to be desired, specifically, path and another parametric deviations were unacceptably large". The DSI test path 
specifications used in this study were obtained from Culley et. al (1978). These specifications were found to be too severe and resulted in vehicle instabilities at unrealistically low speed. Since Bohn's study was the closest to the current study, careful attention has to be paid to the problems he encountered in his model and the recommendations they made. They gave recommendations regarding both experimental measurements and modeling. About 5 pages of conclusions and recommendations were given by Bohn et. al (1981). Perhaps the most important recommendations were:

1- A vehicle handling simulation algorithm should be able to handle arbitrary path trajectories.

2- The slosh model used by Bohn et. al (1981) should be more extensively verified, especially the effect of ellipticity on stability.

3- The suspension limits should be correctly implemented.

4- Experimental test data must initiate from steady state.

5- Probabilistic and frequency analysis techniques should be investigated for experimental/simulation data correlation.

Other recommendations related to breaking tests and the tire model used were given, for further information, refer to Bohn et. al (1981).

Bechtold (1983) listed typical values for rollover threshold of different cargo vehicles. For a rigid vehicle, Bechtold mentioned that the rollover threshold is in the order of $0.6 \mathrm{~g}$. Compliant tires lead to a lower value of rollover threshold (about $0.5 \mathrm{~g}$ ), while combined compliant tires and suspension results in a rollover threshold about $0.34 \mathrm{~g}$. For a typical $50 \%$ full elliptical tank vehicle, the rollover threshold can be well below $0.2 \mathrm{~g}$ during lane change maneuvers. 
Rakheja and Sankar (1986) reviewed accident reports involving tank vehicles. They stated that these reports demonstrate the poor controllability and associated potential risks of highway operation of tank vehicles.

Many models have been derived in the late eighties and nineties which incorporate both fluid and vehicle dynamics. Rakheja et. al, (1988) investigated the rollover immunity of articulated cylindrical tank vehicles using a static roll model. They analyzed influence of tank compartments on steady state turning roll response of the vehicle. They also discussed an optimal sequence of unloading the compartmented tank.

Murnane (1989) conducted a statistical study on rollover accidents of tank semitrailers involving spills. Murnane stated that tank vehicles are more frequently involved in single vehicle highway accidents than rigid cargo vehicles. He reported that $69 \%$ of road accidents involving tank trucks were single vehicle accidents. Nearly $50 \%$ of the single vehicle accidents and almost $80 \%$ of multiple vehicle accidents involved at least one fatality. While majority of single vehicle accidents occurred during cornering, $52 \%$ resulted in rollover.

Rakheja et. al (1989) studied the influence of tank design factors on the rollover threshold of partially filled tank vehicles. Rakheja mentioned that considering the spring lash would reduce the rollover threshold of a heavy-duty vehicle from $0.44 \mathrm{~g}$ to $0.34 \mathrm{~g}$. This is about $25 \%$ reduction, which is a considerable value. Rakheja studied different tank shapes assuming that the fluid surface is a straight line and that the mass of the fluid is concentrated at the filled crosssection CG For the elliptical tank, he found out that the rollover threshold acceleration varies 
from $0.38 \mathrm{~g}$ for a $83 \%$ filled tank to $0.35 \mathrm{~g}$ for a $34 \%$ filled tank. Rakheja stated that the comparison between different tank cross-sections revealed that vehicles with modified oval and modified square tanks can rollover at lower values of lateral accelerations compared to the circular and elliptical cross-sections.

Sankar et. al (1989) developed a quasi-dynamic roll plane model of a partially filled circular tank and integrated this model to a 3-dimensional model of an articulated vehicle. The non-linear force displacement characteristics of the suspension springs were tabulated and included in the model. Figure 2.15 shows the force displacement characteristics of the suspension springs used by Sankar et. al (1989). The spring lash in the trailer spring at the negative deflection side as well as the reduction in stiffness with deflection in the positive side for the tractor rear spring can be recognized.

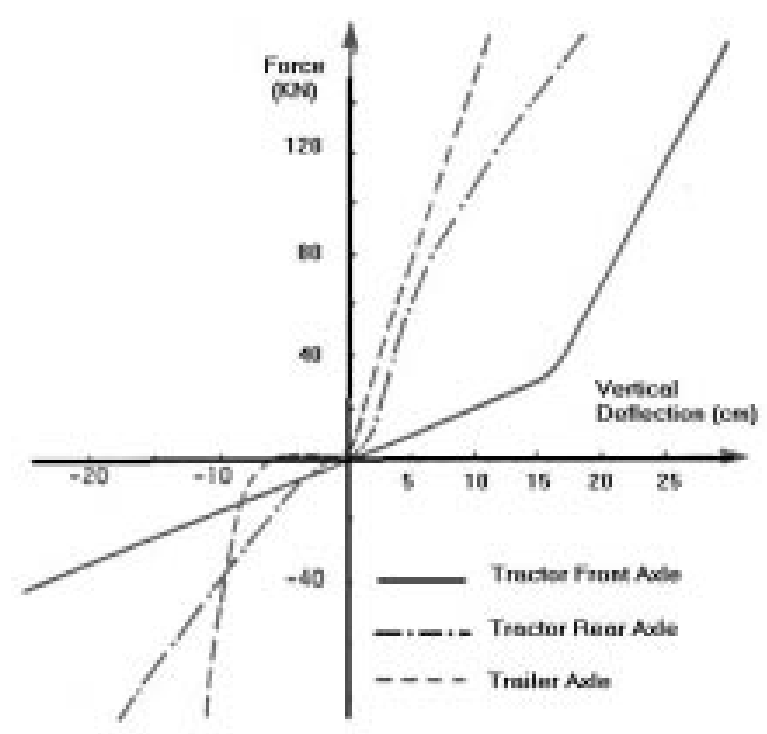

Figure 2.15: Force displacement characteristics of the suspension springs used by Sankar et. al (1989)

They used Equation 2.11 to obtain the gradient of the fluid free surface as a function of a given lateral acceleration. Sankar used his model to compare the directional response 
characteristics of a vehicle carrying rigid cargo, and the same vehicle carrying liquid cargo under steady and transient steering input.

$$
\Phi=\tan ^{-1}\left[\frac{\theta_{\mathrm{s}}-\mathrm{a}_{1}}{1+\mathrm{a}_{1} \theta_{\mathrm{s}}}\right]
$$

Where:
$\mathrm{a}_{1}$ is the lateral acceleration
$\Phi$ is the fluid free surface angle relative to the horizontal plane
$\theta_{\mathrm{s}}$ is the sprung mass tilt angle

Ranganathan et. al (1989) and Ranganathan (1990) developed a roll plane model of a partially filled tank to study the instantaneous location of the fluid volume as a function of lateral acceleration for various tank shapes. They also used this model to compare rollover lateral acceleration of different tank shapes at different level of fills with the equivalent rigid cargo shape. Figure 2.16 shows a comparison between liquid and rigid cargo rollover lateral acceleration at different level of fill for an elliptical tank.

Rakheja and Sankar (1991) constructed a semi-transparent cylindrical tank and mounted it on a truck. The motion of the free surface of the fluid has been recorded during constant radius turns, lane change maneuvers and was compared to the straight line assumption used by Sankar $(1989,1990)$. The deviation from the straight-line assumption was minimal confirming the free surface equation (equation 2.11) used by Sankar et. al (1989). 


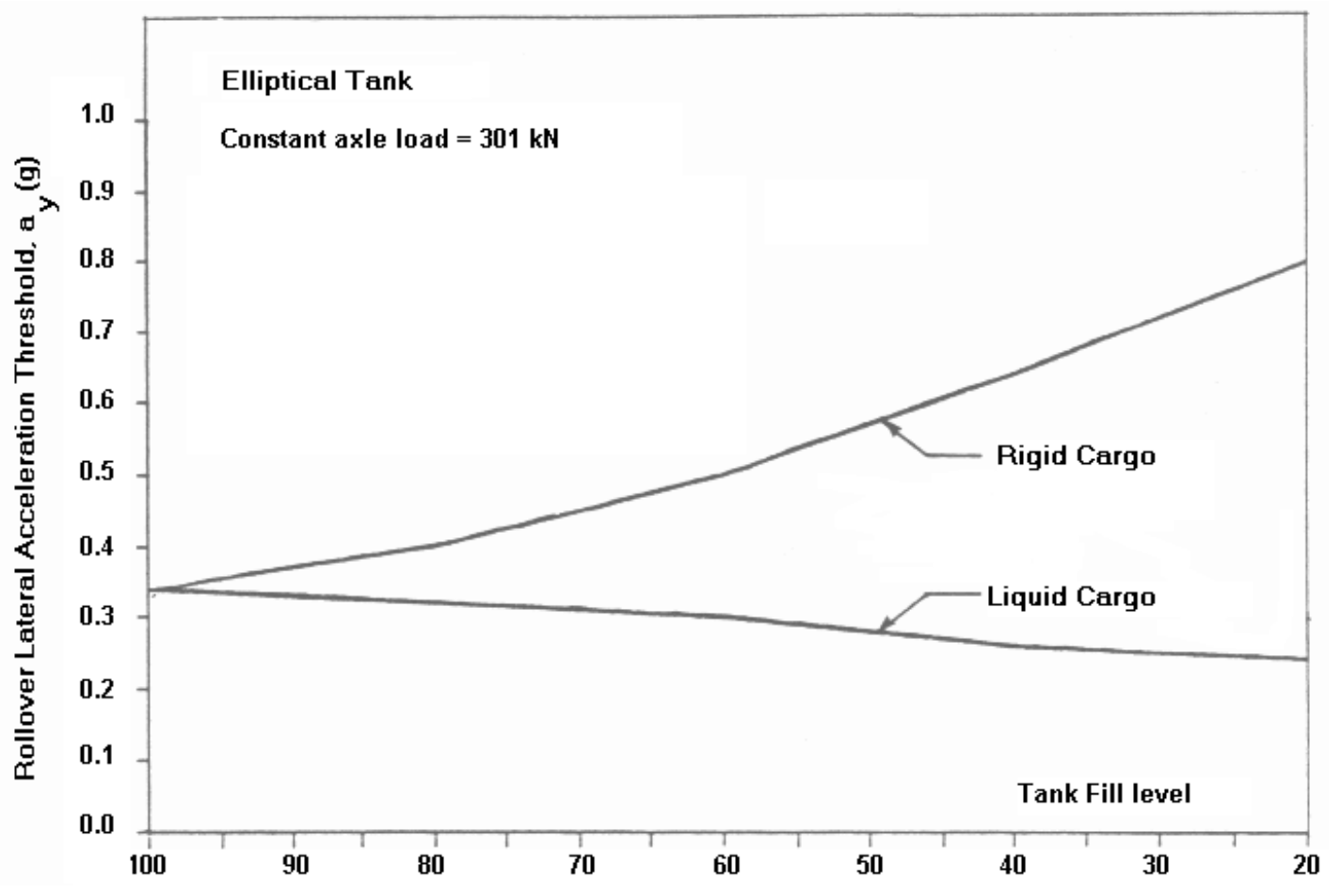

Figure 2.16: Lateral acceleration versus level of fill for a rigid and a liquid cargo elliptical container, Ranganathan et. al (1989)

Later, Rakheja et. al, (1992) used the same vehicle to study the directional dynamics of a partially filled tank truck and compared the results of the field tests with those obtained using the analytical roll plane model developed earlier by Ranganathan et. al (1989). They verified that the quasi-dynamic analytical model can accurately predict the fluid slosh loads.

Sankar (1992 B) integrated the non-linear fluid slosh equations (equations 2.6 through 2.9) to a three-dimensional vehicle dynamics model to obtain the most comprehensive model ever built for a cylindrical tanker. The model was used to evaluate the directional response characteristics of tank vehicles. The results of this were compared with the results obtained using a steady state fluid model and were found to exhibit oscillations around the steady state values. Sankar concluded that a quasi-dynamic model can predict the directional response of 
tank vehicles quite close to that evaluated using the comprehensive fluid slosh model during various highway maneuvers.

Popov et. al (1993) studied the dynamics of liquid sloshing in horizontal cylindrical containers. Both steady state solution derived analytically from hydrostatic equations and transient solution obtained from numerical integration of Navier-Stokes, continuity, and free surface equation were used to obtain the natural frequencies of liquid vibrations and the liquid slosh loads. Rectilinear and rotational motion of the container were both considered as well as the effect of change of fluid viscosity. One of their most important findings was that the first damped frequency of fluid sloshing for the same level of fill decreases with the increase applied lateral acceleration $\left(\mathrm{K}=1.198\right.$ at $\mathrm{G}_{\mathrm{x}}=-0.1$ and $\mathrm{K}=0.883$ at $\left.\mathrm{G}_{\mathrm{x}}=-0.5\right)$.

Where $G_{x}$ is the lateral acceleration ratio to $\mathrm{g}, \Omega$ is the circular frequency of fluid vibration, and $\mathrm{K}$ is the normalized frequency of fluid slosh given by:

$$
\mathrm{K}=\frac{\Omega}{\left[2 \mathrm{~g}\left(1+\mathrm{G}_{\mathrm{x}}^{2}\right)^{1 / 2} / \mathrm{D}\right]^{1 / 2}}
$$

Ranganathan et. al (1993 A) simulated the fluid sloshing in a cylindrical tank with a regular pendulum and a fixed mass. Ranganathan mentioned that the pendulum mass should simulate the sloshing effects of the first mode of the part of the fluid participating in the slosh. The fixed mass should simulate the Inertia and the weight of the remaining part of the fluid. To obtain the parameters of the pendulum, Ranganathan matched the equations describing the dynamic fluid effects (equations 2.3 and 2.4) given by Budiansky (1960) and the equations describing the dynamic effect of a single mass pendulum. Figure 2.17 shows the model used by 
Ranganathan, while Figure 2.18 shows the chart obtained from the analogy of the equations mentioned before.

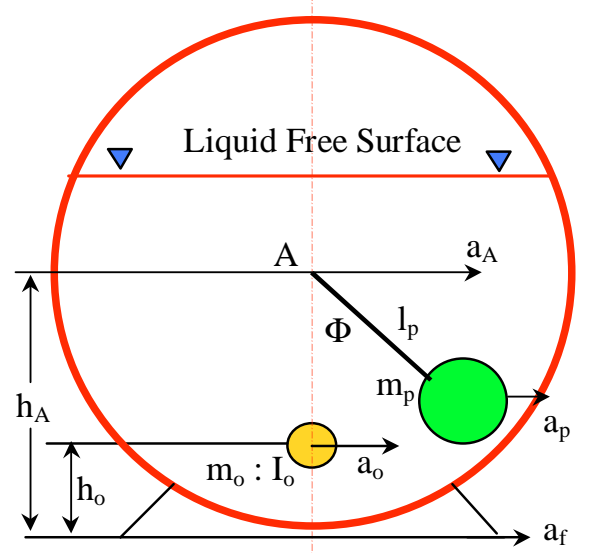

Figure 2.17: Representation of fluid slosh using pendulum model approach, Ranganathan et. al (1993 A)

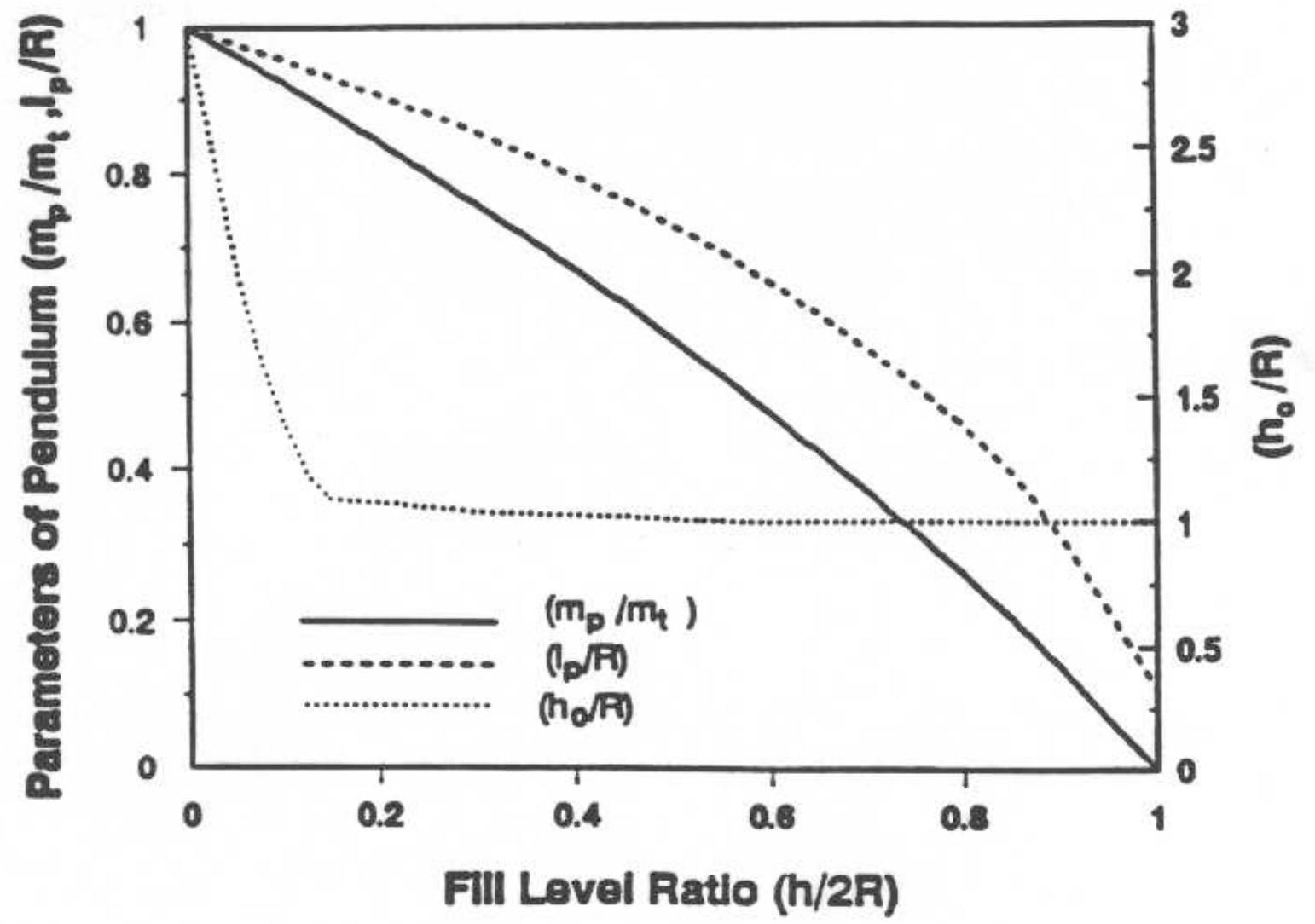

Figure 2.18: Parameters of pendulum analogy, Ranganathan et. al (1993 A) 
Rakheja and Ranganathan (1993) used two different methods to determine the rollover threshold of partially filled tanker of a general configuration. The first was based on the study done earlier by Ervin et. al (1983 B) where the rollover threshold is determined by balancing the total overturning and restoring moments using a single roll degree-of freedom model of rigidly suspended tank vehicle. The compliant properties of the suspension and the tires were incorporated in the analysis through a compliance factor. In the second method, the roll moments arising from the multiple-axle suspension and tires, individual axle loads, and lateral displacements were computed and integrated in the analysis. The comparison of the results show that the first simplified method can provide accurate prediction of rollover threshold for constant as well as varying payloads.

Ranganathan et. al (1993 B) studied the directional response of a B-train vehicle combination of a circular cross section tank carrying liquid cargo. The trailer and the pup-trailer were both assumed unbaffled. Thus, unlike the study done by Mallikarjunarao (1978), the fluid slosh effects on the stability of the vehicle were considered. The results for liquid cargo were compared to those of an equivalent rigid cargo.

Ranganathan et. al, (1994) studied the dynamic behavior of liquids in partially filled tank vehicles during typical straight line braking. They used an equivalent mass-spring system to model the fluid longitudinal slosh effect obtained using potential flow theory for longitudinal oscillations. They integrated the fluid model into a pitch plane model of a tank vehicle. Fluid damping was neglected and the axles load results were compared with the results of the steady state fluid model developed by Yang (1992). The axle loads obtained by Ranganathan et. al 
(1994) fluctuated about the steady state values obtained by Yang and continued to oscillate about the static load value after the vehicle came to a complete stop because of lack of damping.

Zhanqi et. al (1995) studied the influence of partition location on the braking performance of partially-filled elliptical tank trucks using an analytical model and experimental tests. The response characteristics of a truck equipped with a compartmented tank were evaluated in terms of dynamic load transfer, stopping distance, braking time, and time lag between front and rear axle wheel lockup. The results clearly demonstrated that equal length compartments yield minimal longitudinal load transfer under straight-line braking.

Popov et. al (1996) conducted a study to obtain an optimum shape of elliptical road containers in terms of minimizing the overturning moment resulting in a vehicle rollover. They found that an elliptical tank of $\mathrm{b} / \mathrm{a}=0.625$ to 0.325 is very suitable for lateral accelerations varying from 0.1 to $1 \mathrm{~g}$.

Gautam and Mucino (1999) were the first to study the rollover stability of partially filled heavy-duty elliptical tankers using a finite element approach. The advantage of this approach is that the elasticity of the tanker can be taken into consideration. A trammel pendulum was used for the first time to follow an elliptical path to simulate the lateral motion of the fluid. This work has the first attempt to include the effect of the elasticity of different vehicle components on the rollover stability of the vehicle. A detailed finite element model of the vehicle simulated in this study has developed. The torsional and bending stiffness of the trailer were determined by applying torsional and bending loads to a structural model of the tank, and measuring the 
corresponding displacement (see Figure 2.19). The calculated stiffness was matched to the stiffness of the trailer used in the simple FEM model they used in their study.
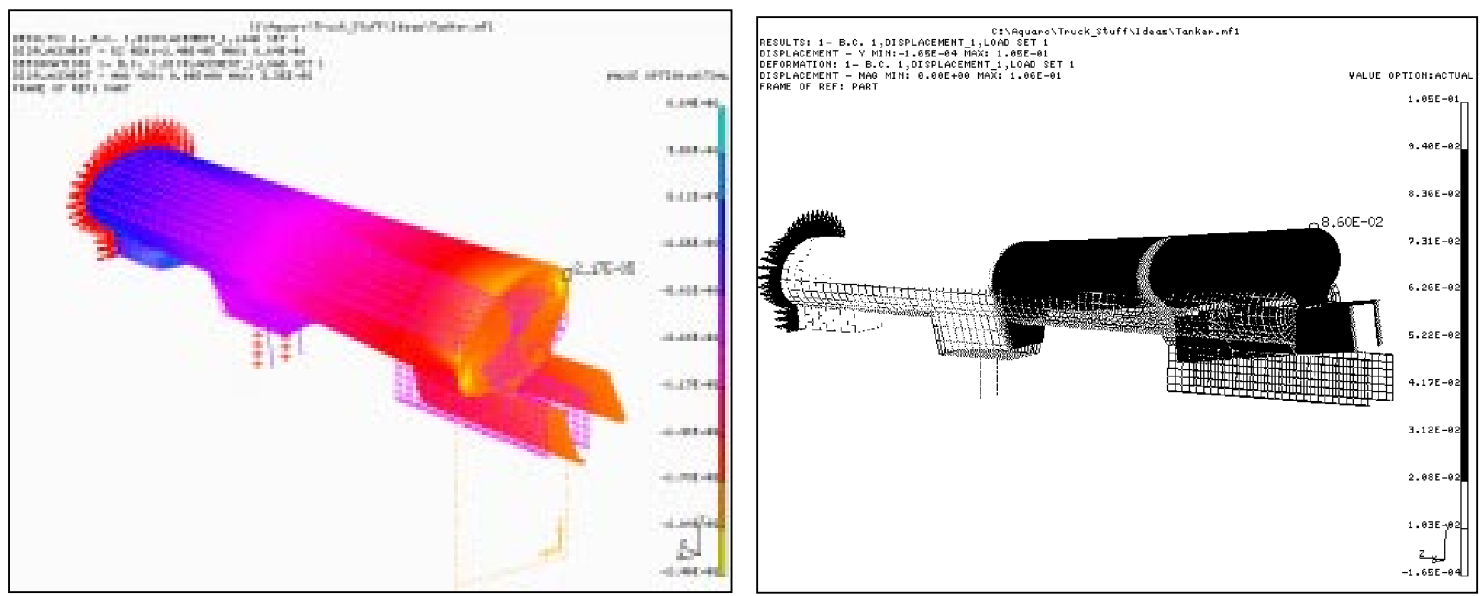

Figure 2.19: Torsional and bending stiffness evaluation of a trailer using FEM, Gautam and Mucino (1999)

Aquaro (1999) established a simplified 3-D finite element model of mainly beam elements (shown in Figure 2.20) to simulate the fluid slosh effects to calculate the reactions at the wheels of a tanker going through a lane change maneuver.

Aquaro used experimental data obtained from a standard TOP Lane Change maneuver as well as a cycloidal path to validate his model. There was an error of about $10 \%$ of the rollover threshold prediction since Aquaro used the cylindrical pendulum chart provided by Ranganathan (1993 A), and shown in Figure 2.18 to obtain the parameters of the elliptical pendulum. Also, the model didn't allow wheel liftoff due to problems with gap elements and the rotation of the coordinate system, to which the trammel pendulum constraints are related . 


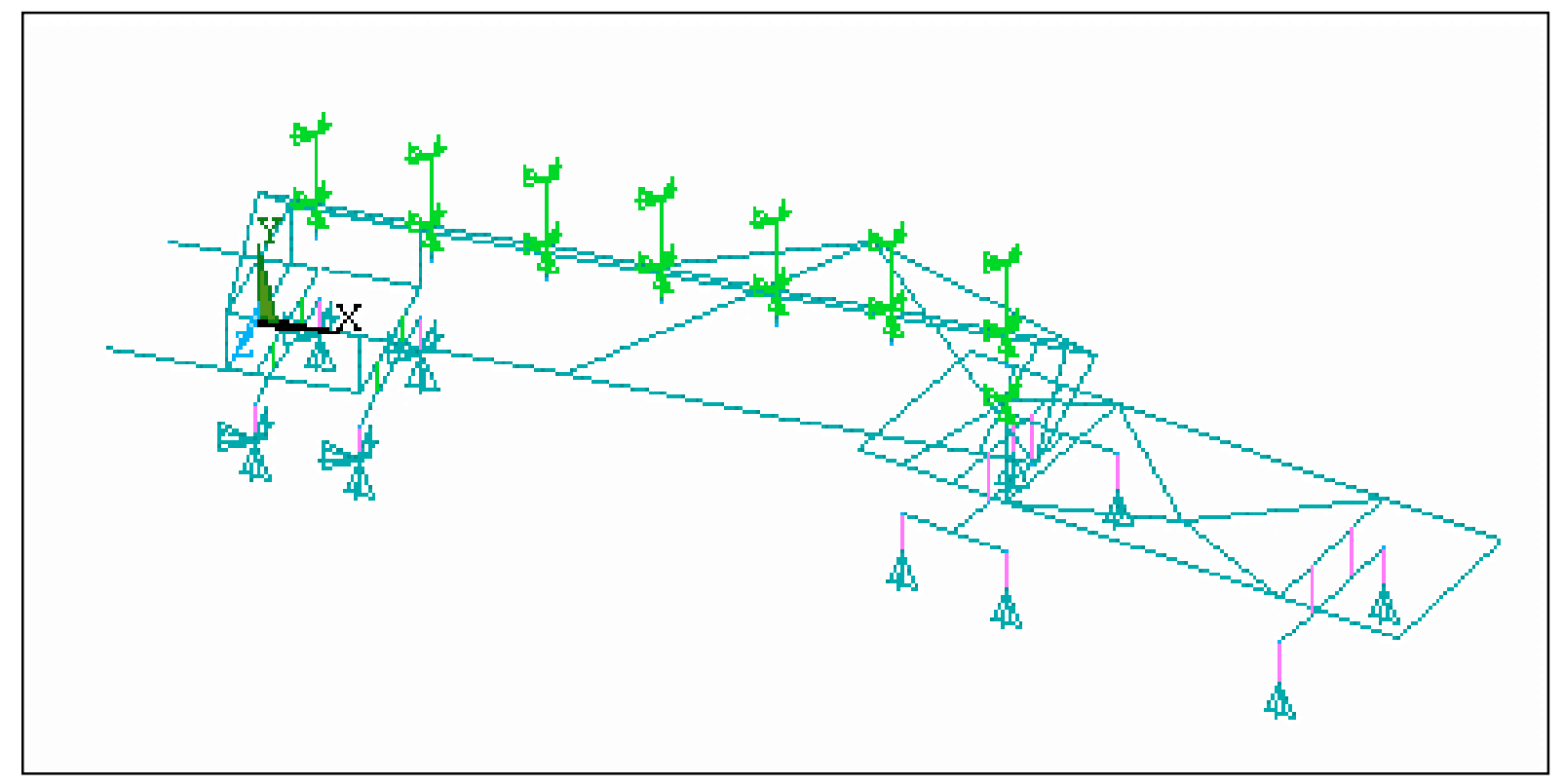

Figure 2.20: Simple finite element beam model for a tanker tractor-semitrailer developed by Aquaro (1999)

\section{5- Conclusions based on the Literature Review}

The following conclusions can be drawn from the literature review, which will help in understanding the effect of each parameter and different assumptions in the models. Those conclusions should help also in interpreting the results of subsequent models.

1. The first mode of lateral vibration of a fluid is the most significant and can closely simulate the fluid slosh effect of a partially filled fluid container, if other modes were neglected (see Figure 2.4), Abramson (1966 B).

2. The first mode of lateral vibration of a fluid in partially filled tank can be assumed to have a tilted flat surface (see Figure 2.1), Budiansky (1960), Sankar et. al (1989) Sankar and Rakheja (1990), and Rakheja and Sankar (1991). 
3. The dynamic effect of first mode of lateral vibration of a fluid in a partially filled tank can be closely simulated with a simple pendulum and a fixed mass or a spring-bob model (see Figure 2.2), Warner (1961).

4. Fluid swirl effects can be neglected at low frequency of excitation (see Figure 2.3), Abramson (1966 A).

5. The effect of the elasticity of a fluid container on the slosh natural frequencies and mode shapes can be neglected if the thickness of the walls of the container exceeds $4.77 \mathrm{~mm}$ (0.01567 ft), see Figure 2.5, Bauer (1972).

6. Some of the parameters can have significant effect on the rollover problem and are very hard to obtain such as; moment of inertia of the truck and the trailer around the 3 axis, lateral stiffness of tires and suspension springs. Some other geometrical details are very hard to include in a mathematical model such as, fifth wheel eccentricity (see Figure 2.7) or suspension spring lash (see Figure 2.10).

7. Lateral sideslip of the tires can be neglected and the wheels can be assumed sticking in the lateral direction on a dry highway if the lateral acceleration doesn't exceed $0.8 \mathrm{~g}$, Ervin et. al (1983 B).

8. Two thirds of the total roll angle is subtended by the sprung mass (up to the wheel liftoff condition, while one third of the total rolling takes place around the tire roll center located in the ground plane, Ervin et. al (1983 B).

9. The wheel liftoff for a typical tractor-semitrailer starts at the trailer rear axle, then at the tractor rear axles, and finally takes place at the tractor front axle (see Figure 2.13), Ervin et. al (1983 B). 
10. The availability of experimental lateral acceleration data during a certain maneuver would simplify a vehicle rollover model since there will be no need to incorporate a complex tire model to obtain the lateral forces acting on the model, Das et. al (1993).

11. The rollover threshold acceleration for the elliptical tank should be around $0.35 \mathrm{~g}$ to $0.38 \mathrm{~g}$ for a typical elliptical tanker, Rakheja et. al (1989).

12. The most critical level of fill is expected to be close to $60-65 \%$ full for supper elliptical tankers similar to the tanker considered in this study, Sankar et. al (1992).

13. The elasticity of the tanker was not included in any study except the finite element model established by Aquaro (1999) which didn't allow for wheel liftoff.

14. The recommendations given by Bohn et. al (1981) regarding the experimental data and the fluid model needs to be considered as much as possible since his work is the closest to this effort.

15. The first model simulating the sloshing of fluid in an elliptical tank using a pendulum that follows an elliptical path was proposed by Gautam and Mucino (1999). It was also the first model that considered the elasticity of the tanker shell on the rollover stability. 


\section{CHAPTER 3}

\section{EXPERIMENTAL DATA ANALYSIS}

\section{1-Introduction}

Experimental testing has been performed by Aberdeen Test Center (ATC) using a fullscale army tanker truck (M916A1/Entyre 60 PRS 6000-Gallon Water Tanker) shown in Figure 3.1. Different lane change maneuvers as well as constant radius turns were used as test paths. The dimensions of the tanker required to establish the mathematical models were measured and are given in Appendix A. Two sets of data were sent as a sample to West Virginia University to verify the analytical models.

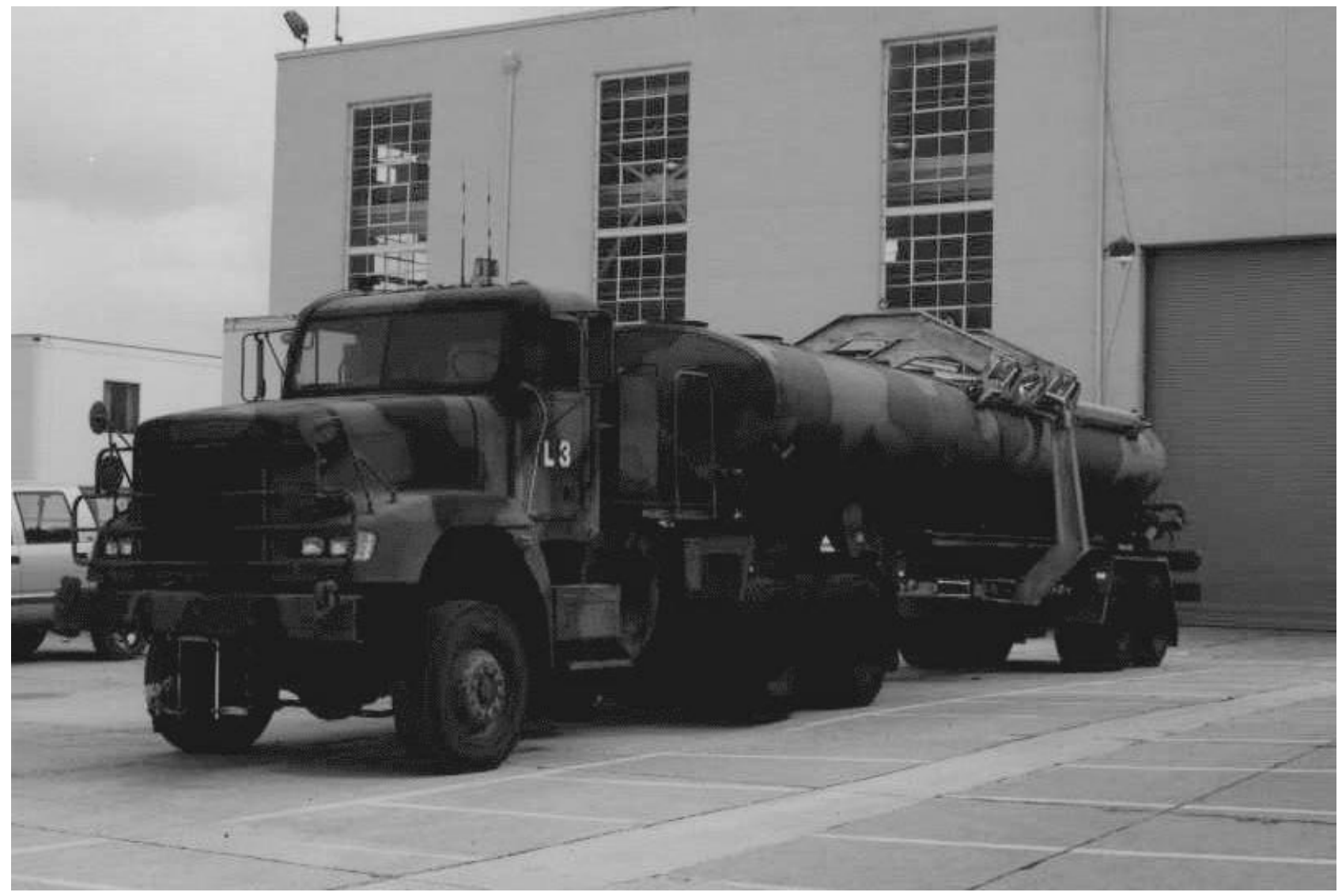

Figure 3.1: Tanker used for experimental testing 


\section{$\underline{\text { 3.2-Vehicle Instrumentation }}$}

The truck has been instrumented with several test instruments to provide adequate data to define the path of the vehicle, fluid level, accelerations in different directions, and the deflections of the springs. Figures showing all instrumentation are given in Appendix A.

Accelerations in all 3 coordinates at the CG location of both tractor and trailer as well as the accelerations at the axles on both sides were measured using 3 perpendicular accelerometers. Exact locations of the accelerometers are given in table A.15 in Appendix A.

Deflection of all suspension springs have been measured by means of wire potentiometers attached between the frame of the vehicle and the to middle point of each spring. The zero value of deflection is calibrated at vehicle curb weight.

The Yaw angle of the tractor and the trailer is determined by means of motion gyros. While the steering angle was measured using a dial indicator installed on the steering wheel. The fluid level was measured in the front most compartment by means of a strip piezoelectric pressure indicator. A 100 channel data acquisition system was used to collect the data measured by all the measurement instruments during the tests.

Artificial outriggers were manufactured and installed on the trailer side (See Figure 3.2) to prevent the vehicle from rolling over during the tests when it reaches the point of rolling instability. These outriggers will have some effects on the calculated reactions that will be discussed later in this chapter. 


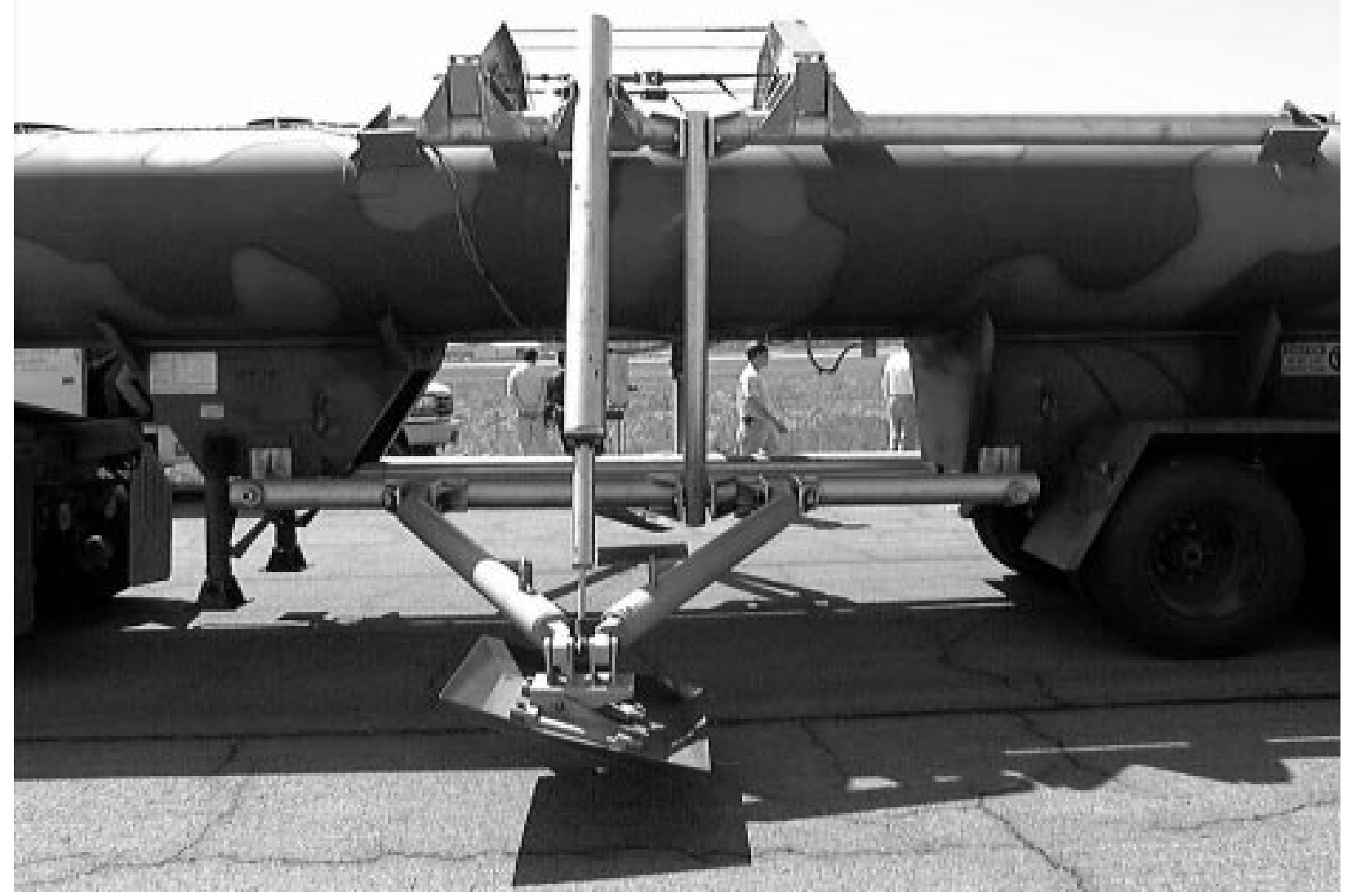

Figure 3.2: Artificial trailer outriggers to prevent vehicle rollover during tests

\section{3- Road Maneuvers Performed During Tests}

Several test runs has been conducted by ATC for the test vehicle at different levels of fill. Mainly, the TOP 2-2-002 lane change test and the NATO (AVTP 03-160W) lane change tests were used for testing. Also, 100 and $75 \mathrm{ft}$ constant radius turns were used. Figures 3.3 and 3.4 show the path of the tested vehicle during two samples of tests using the TOP lane change test and the NATO lane change test provided by ATC respectively. 


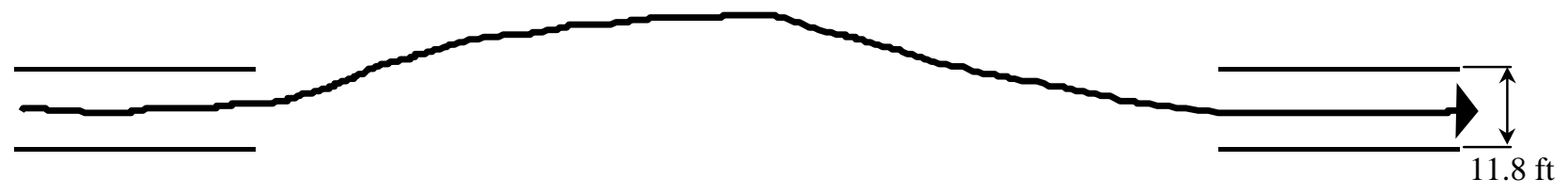

$49.2 \mathrm{ft}$

$1.5 \mathrm{x}$ Min Turn Dia $=115.1 \mathrm{ft}$

$1.5 \times$ Min Turn Dia $=115.1 \mathrm{ft}$

$49.2 \mathrm{ft}$

Figure 3.3: Path of the test vehicle during the TOP Lane Change Test

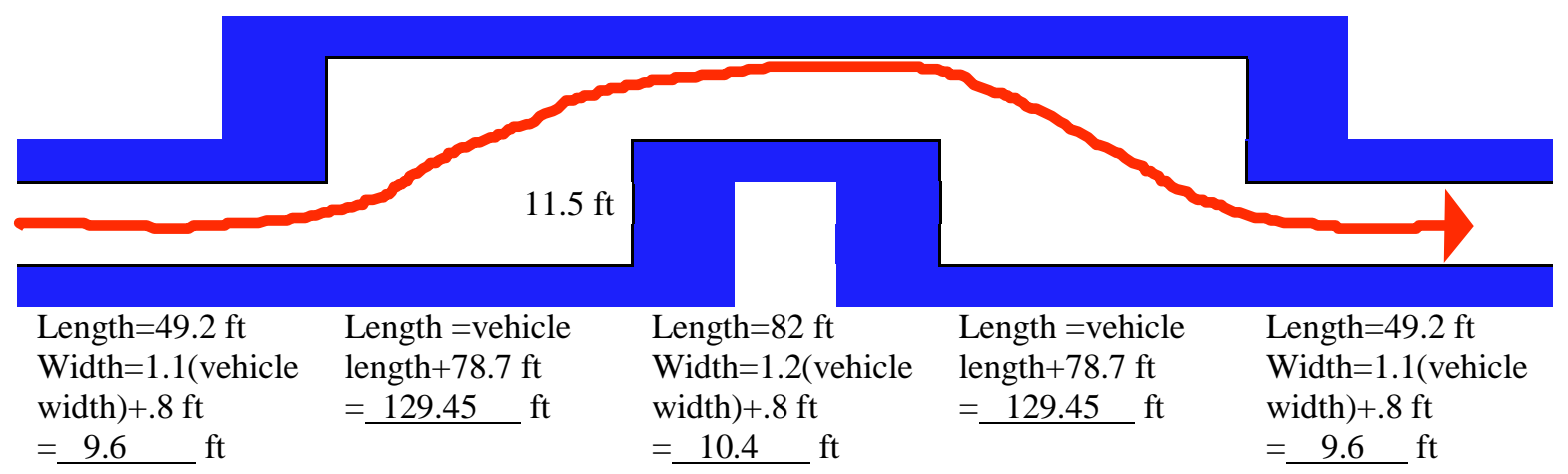

Figure 3.4: Path of the test vehicle during the NATO Lane Change Test

\subsection{Experimental Data Processing}

The data provided by ATC to WVU during the two sample tests needed several data processing operations to extract data that can be used as inputs for the mathematical models derived in this study. Also, test results that can be used for comparison with the results of these models (such as wheel reactions during the tests) needed to be extracted.

The following operations were performed on the data to obtain the required inputs for the mathematical models and the reactions on the wheels during the tests:

\subsubsection{Units Transformation}

All data provided by ATC has been transformed from British to SI units. 


\subsubsection{Numerical Differentiation}

The lateral acceleration of the trailer has been obtained by differentiating numerically the $Y$ coordinate of the $C G$ of the trailer twice with respect to time using the central difference method described by equation (3.1).

$$
V_{n}=\frac{X_{n+1}-X_{n-1}}{2 \Delta t}
$$

This lateral acceleration can be used as an input for all plane models developed in this study.

\subsubsection{Data Filtering}

Most of the data provided by ATC required low band pass filtering to get rid of the noise. Also, the calculated lateral acceleration and the Yaw acceleration mentioned above revealed severe noise. A MATLAB special function called "filtfilt" has been used to filter the data using a Buttworth type low band pass filter. Details about the filtering procedure and functions can be found in Etter (1993). A general purpose " $m$ " file has been created that accepts the data in a column format without header, and filter all the required columns of data that need filtering assuming the first column to be the time values. A list of the code used in the filtering process is given in Appendix C. The file works under MATLAB 5.2.

The function "filtfilt" has a unique feature of having no phase distortion of the input signal. This is achieved by first filtering in the forward direction, then the filtered sequence is reversed and run back through the filter. The result has precisely zero phase distortion and magnitude modified by the square of the filter's magnitude response. 
Figure 3.5 shows the calculated lateral acceleration during ATC TOP lane change test for the half-full tanker before and after filtering. It should be noted that the Y direction used in the theoretical analysis is opposite to the Y direction used by ATC. This will not affect the analytical or the models since geometric similarity is assumed across the tanker longitudinal centerline.

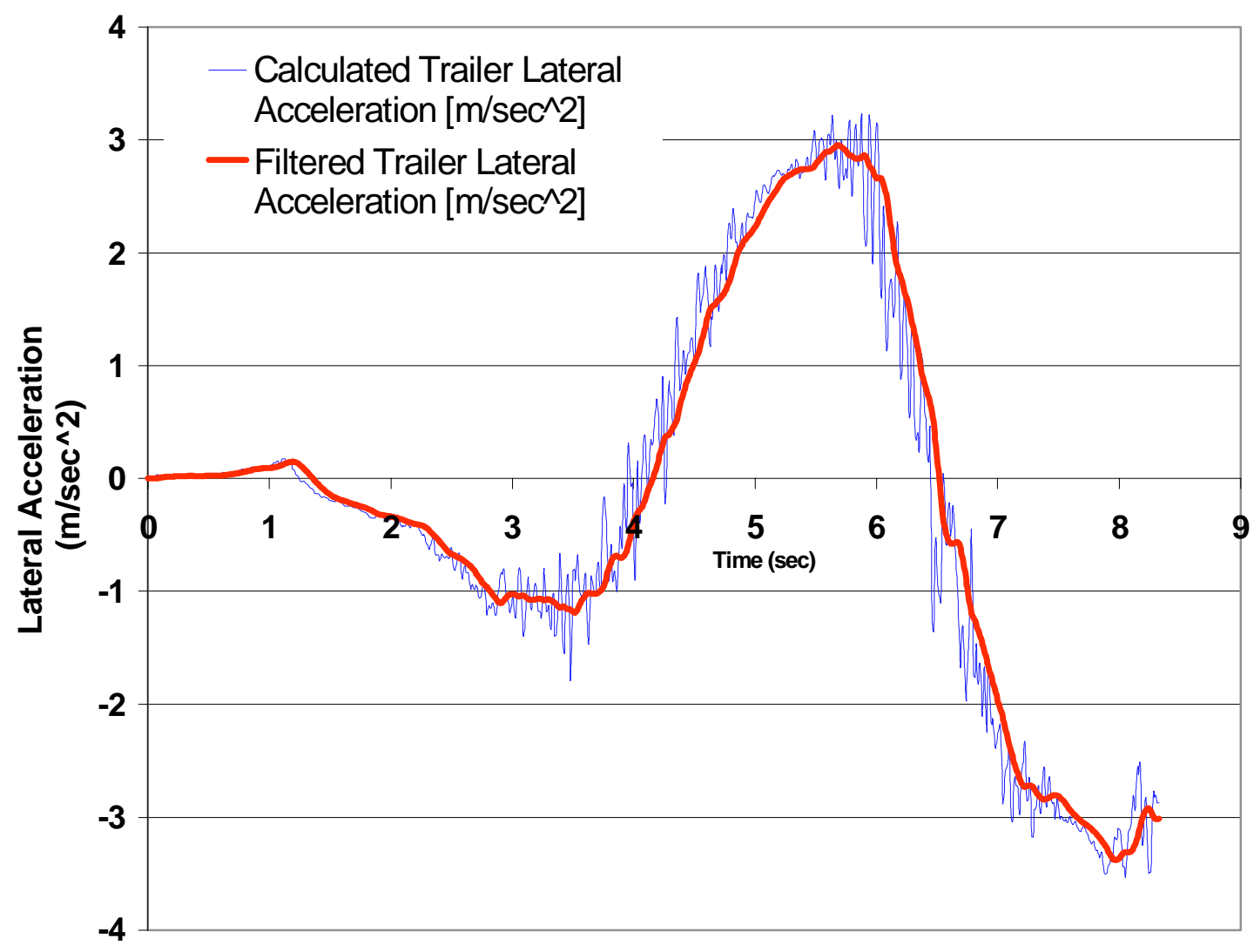

Figure 3.5: Calculated and filtered lateral acceleration for the trailer under study, at $50 \%$ full condition, during the TOP lane change test

\subsubsection{Spring Characteristics}

For the trailer spring, the performance curve in the jounce condition was supplied by the trailer manufacturer (ETNYRE Co.) in British units. The performance curve has been transformed into SI units (See Figure 3.6). The trailer spring performance is non-linear because 
of the spring cam type supports (see Figure 3.7) to produce an increasing stiffness with load. Thus, the trailer suspension performance curve had to be tabulated in a way so that an interpolation technique can be used to find the spring deflection for any given load and visaversa. A cubic spline interpolation technique has been used. For negative deflections (Wheel liftoff), the absolute value of the deflection was used to find the spring force assuming that the characteristics are identical in the opposite direction of loading.

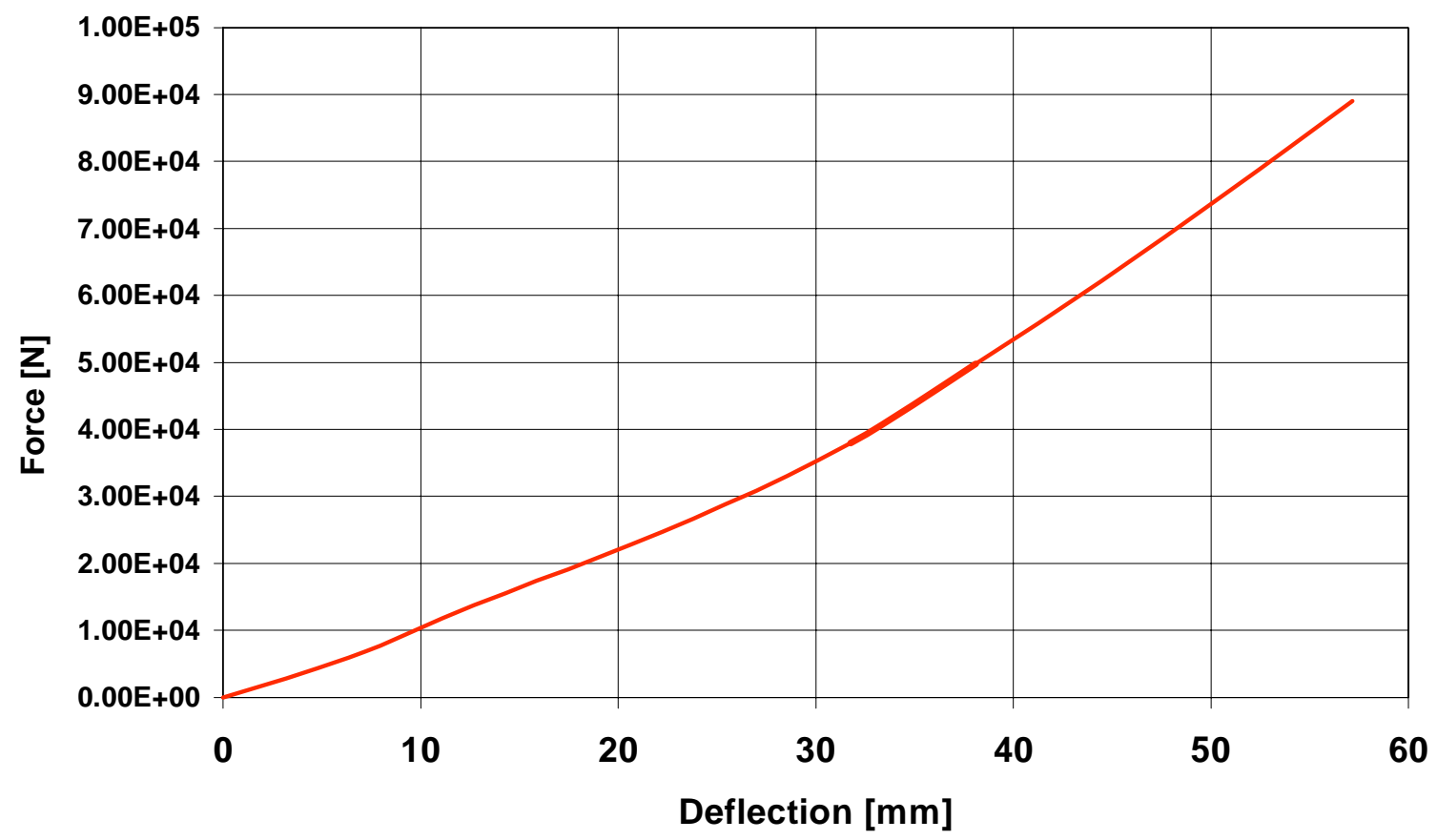

Figure 3.6: Trailer suspension spring characteristics (ETNYRE)

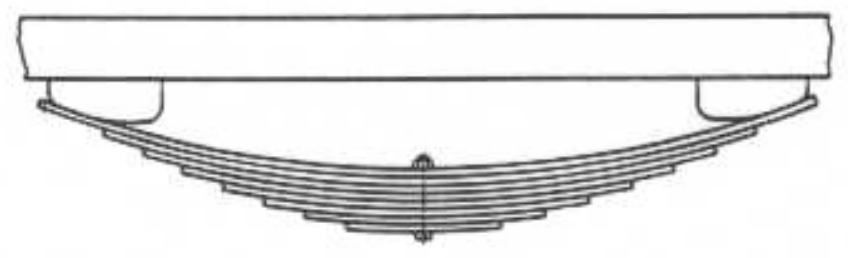

Figure 3.7: A variable effective length (stiffness) spring, Spring Design Manual (1996) 
For the tractor front and rear springs, the load-deflection curves were not available. Equation (3.2) was used initially to estimate the stiffness of tractor suspensions assuming that they have linear characteristics based on the spring parameters. This formula can be found in BOSCH handbook.

$$
\mathrm{k}=\frac{\left(2+\mathrm{n}^{\prime} / \mathrm{n}\right) \operatorname{Enbh}_{\mathrm{ls}}^{3}}{61^{3}}
$$

Based on equation (3.2) and the data provided by ATC, the stiffness of the tractor front suspension is equal to:

$$
\mathrm{k}_{\mathrm{f}}=\frac{(2+1 / 10) \times 206 \times 10^{9} \times 10 \times 0.1016 \times(0.0127)^{3}}{6 \times(0.762)^{3}}=337805 \mathrm{~N} / \mathrm{m}
$$

and for the tractor rear suspension

$$
\mathrm{k}_{\mathrm{r}}=\frac{(2+1 / 13) \times 206 \times 10^{9} \times 13 \times 0.1016 \times(0.0127)^{3}}{6^{*}(0.4573)^{3}}=2017348 \mathrm{~N} / \mathrm{m}
$$

In an attempt to enhance the calculated suspension stiffness for the tractor, another formula was used which accounts for the clamped length of the spring based on SAE leaf spring design recommendations found in SAE Spring Design Manual. The equation is:

$$
\mathrm{k}=1.15 \frac{\operatorname{Enbh}_{\mathrm{ls}}^{3}}{31^{3}}
$$

The calculated stiffnesses using equation 3.3 were; $\mathrm{k}_{\mathrm{f}}=371435 \mathrm{~N} / \mathrm{m}$ and $\mathrm{k}_{\mathrm{r}}=2234027 \mathrm{~N} / \mathrm{m}$.

Thus, the tractor suspensions' calculated stiffnesses are within $10 \%$ of each other using equations 3.2 and 3.3 . 


\subsubsection{Tire-Ground Reactions}

It is very important to calculate the reactions between the tires and the ground during the tests performed by ATC on the tanker under investigation. Since the value of these reactions once turned negative at a certain axle, it indicates that this axle have started to liftoff from the ground signaling the beginning of the rollover threshold. The dynamic deflections of the suspension springs were provided by ATC.

To know the reactions on the tires, the reactions on the springs based on the given deflections has to be added to the dynamic load component resulting from the axle motion (Due to the bouncing and pitching modes of the axles). To know the dynamic loads resulting from the axle motion, the vertical component of the acceleration measured by the accelerometers at the two sides of the axles can be used to calculate the vertical acceleration, as well as the angular (pitching) acceleration. Figure 3.8 shows the vertical acceleration measured at the rear axle of the trailer on the left-hand side before and after filtering.

The figure indicates that the accelerometer was sensing mostly noise from the road. This can be attributed to the fact that the tires' stiffness is much greater than the suspension stiffness, which allows the tire-ground noise to be transmitted easier to the accelerometer than the motion attributed to the maneuver action. 


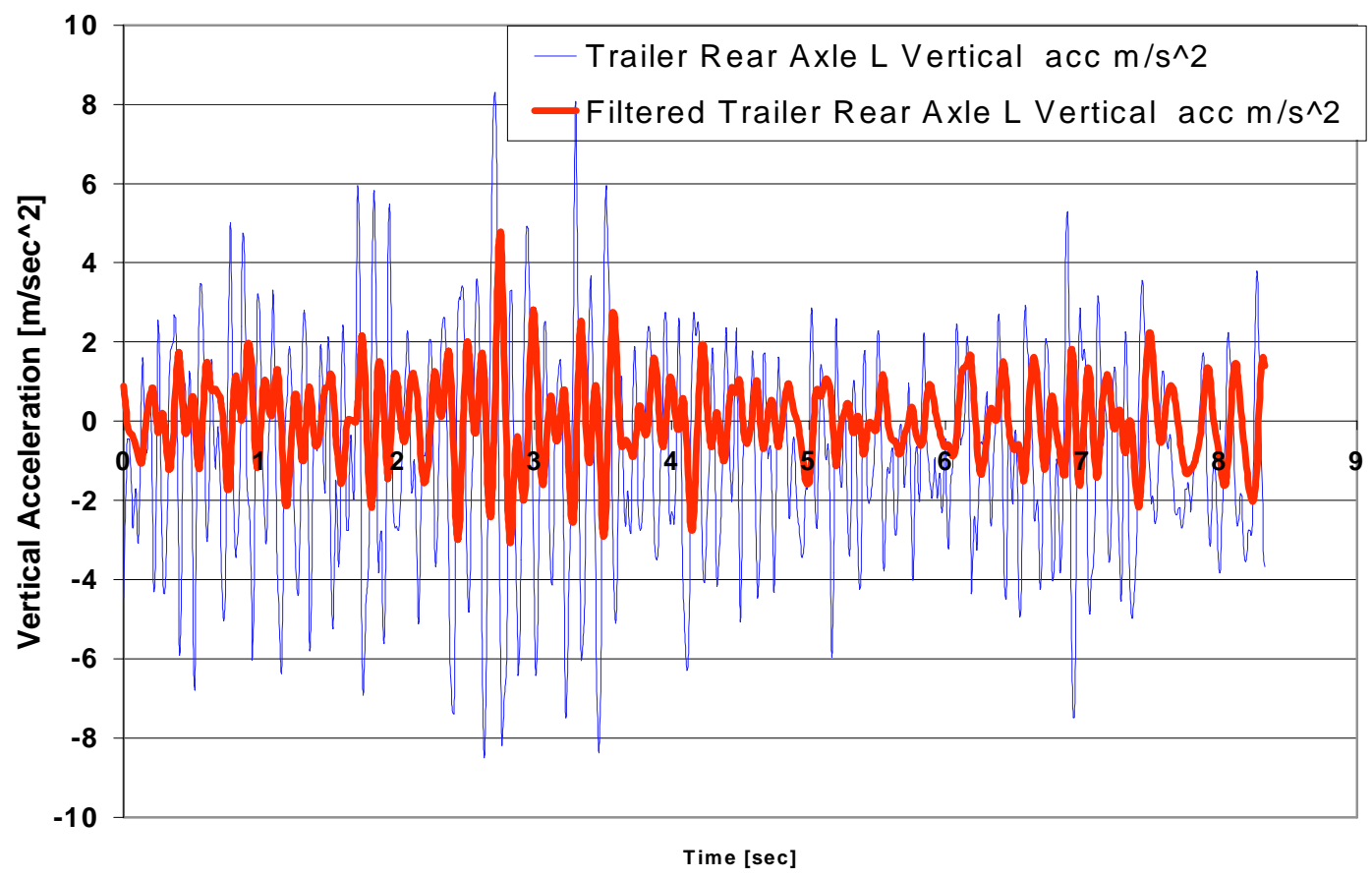

Figure 3.8: Vertical acceleration of the left-hand side of the trailer rear axle measured by an accelerometer before and after filtering

Since the accelerometers vertical acceleration output is too noisy to be considered, in addition to the fact that several accelerometer channels failed during the test (specifically the trailer front axle left side vertical during the TOP lane change test) the contact forces between the tires and the ground was calculated considering the static weight of the axles only in addition to the spring loads.

The steps of calculating the tires' loads during the tests were as follows:

1- Calculate the static load on each spring (Equal static empty load on axle-unsprung weight on this spring.

2- Calculate the static deflection on each spring either by interpolating in the spring performance curve or by dividing the load on the stiffness of this spring. 
3- Add the static deflections calculated in step 2 to the dynamic deflections measured by ATC during the tests.

4- Calculate the force at the base of the springs by interpolating back in the spring performance curve for the trailer suspension or by multiplying the total deflection by the stiffness for the tractor suspensions.

5- Adding the unsprung weight back to the calculated load on each spring.

Care has been taken when treating the rear suspension of the tractor since the walking beam type suspension has two axles supporting one spring (See Figure 3.9). Thus, the load on those two axles has to be combined when calculating the deflection of the spring of that suspension. The details of these calculations are given below.

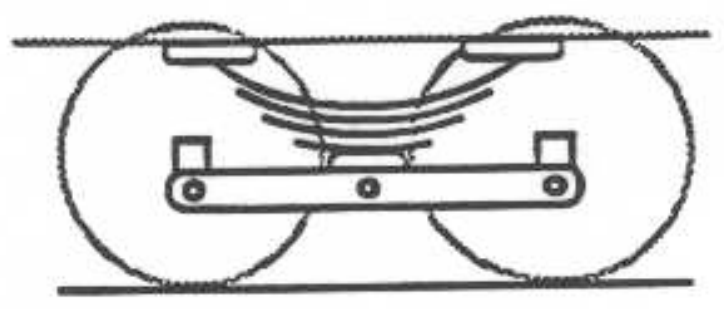

Figure 3.9: Walking-Beam type suspension, Winkler 1992

The masses of the unsprung components of the trailer supplied by E.D. ETNYRE \& CO. were:

Axles, $145 \mathrm{~kg}$ each.

Wheels, $37.2 \mathrm{~kg}$ each.

Tires, $50.3 \mathrm{~kg}$ each.

Hub \& drum, $39.7 * 2$.each.

Thus, the trailer axle mass per side is equal to;

$\mathrm{M}_{\text {us_trailer }_{-}}=\frac{145+4 \times(37.2+39.7+50.3)}{2}=327 \mathrm{~kg}$. 
Assuming that the tractor axles have the same masses per element, thus, the front tractor axle mass per side is equal to $327-50.3-37.2-39.7=200 \mathrm{~kg}$.

The curb weight distribution on the axles of the empty tanker were as given by ATC in table A.1. Based on the data given above and in table A.1, the loads and the static deflections of the suspensions starting from the front axles of the tractor are:-

Tractor front left; $\delta_{1 \mathrm{~L}}=\frac{\mathrm{F}}{\mathrm{k}_{\mathrm{f}}}=\frac{(6930 \times 0.4536-200) \times 9.81}{337805}=\frac{28875}{337805}=0.085 \mathrm{~m}$

Tractor front right; $\delta_{1 \mathrm{R}}=\frac{(7130 \times 0.4536-200) \times 9.81}{337805}=\frac{29765}{337805}=0.088 \mathrm{~m}$

Tractor rear axles left;

$$
\delta_{2 \& 3 \mathrm{~L}}=\frac{[(4540+4240) \times 0.4536-327 \times 2) \times 9.81}{2017348}=\frac{32654}{2017348}=0.0162 \mathrm{~m}
$$

Tractor rear axles right;

$$
\delta_{2 \& 3 \mathrm{r}}=\frac{[(4350+4450) \times 0.4536-327 \times 2) \times 9.81}{2017348}=\frac{32742}{2017348}=0.0162 \mathrm{~m}
$$

Trailer front axle left; Load $=(2430 * 0.4536-327) * 9.81=7605 \mathrm{~N}$, and $\delta_{4 \mathrm{~L}}=0.0078 \mathrm{~m}$

Trailer front axle right; $\operatorname{Load}=(2130 * 0.4536-327) * 9.81=6270 \mathrm{~N}$, and $\delta_{4 \mathrm{R}}=0.0067 \mathrm{~m}$

Trailer rear axle left; Load $=(2670 * 0.4536-327) * 9.81=8673 \mathrm{~N}$, and $\delta_{5 \mathrm{~L}}=0.0087 \mathrm{~m}$

Trailer rear axle right; Load $=(2950 * 0.4536-327) * 9.81=9919 \mathrm{~N}$, and $\delta_{5 \mathrm{R}}=0.0097 \mathrm{~m}$

The file used to find these deflections is a MATLAB .m file called spr_def.m and is listed in Appendix C.

Figures, 3.10 through 3.13 show the reactions on the tires on both sides during the TOP lane change test before and after filtering following the steps mentioned above. 


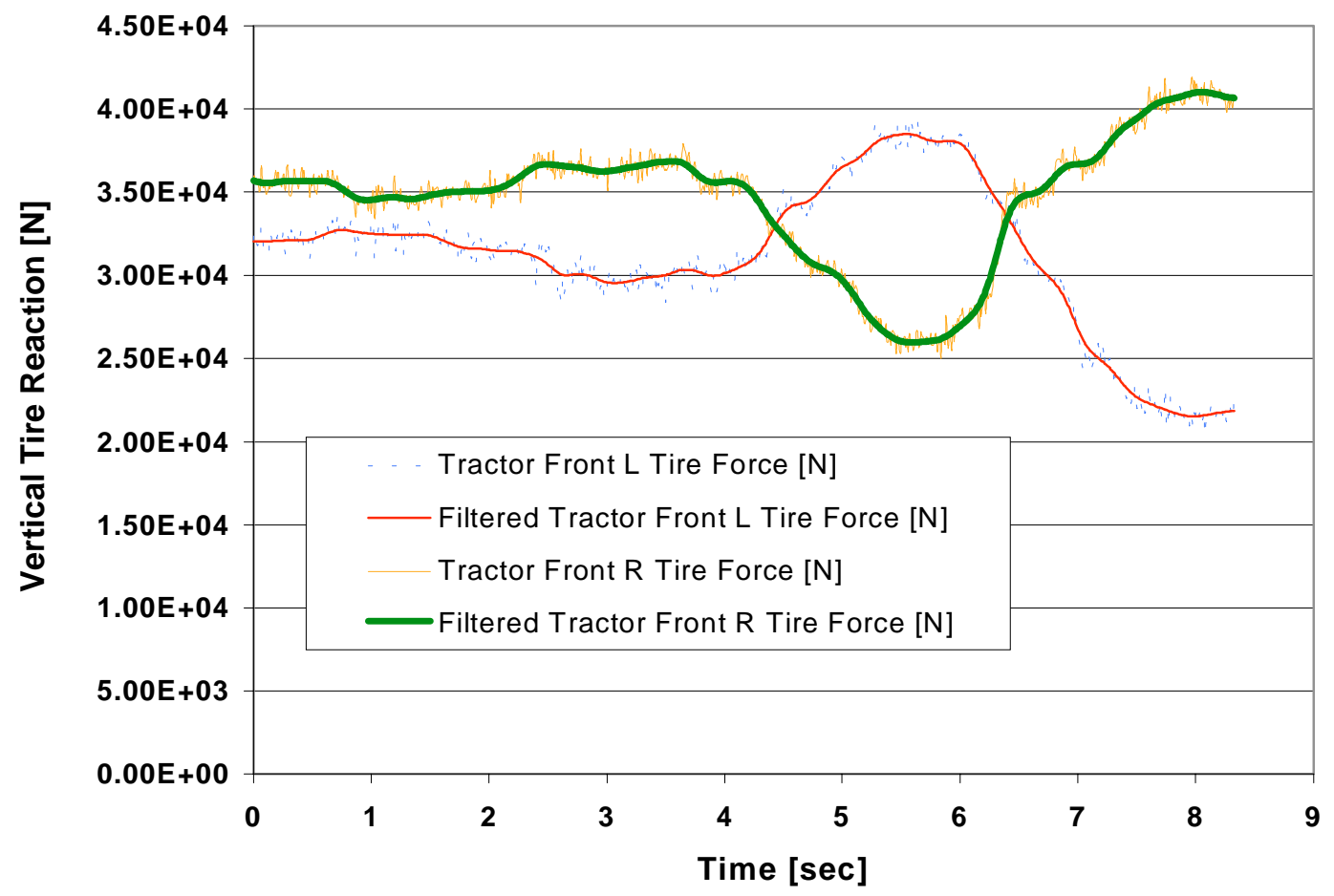

Figure 3.10: Tractor front wheels reactions during the TOP lane change test (50\% full tanker)

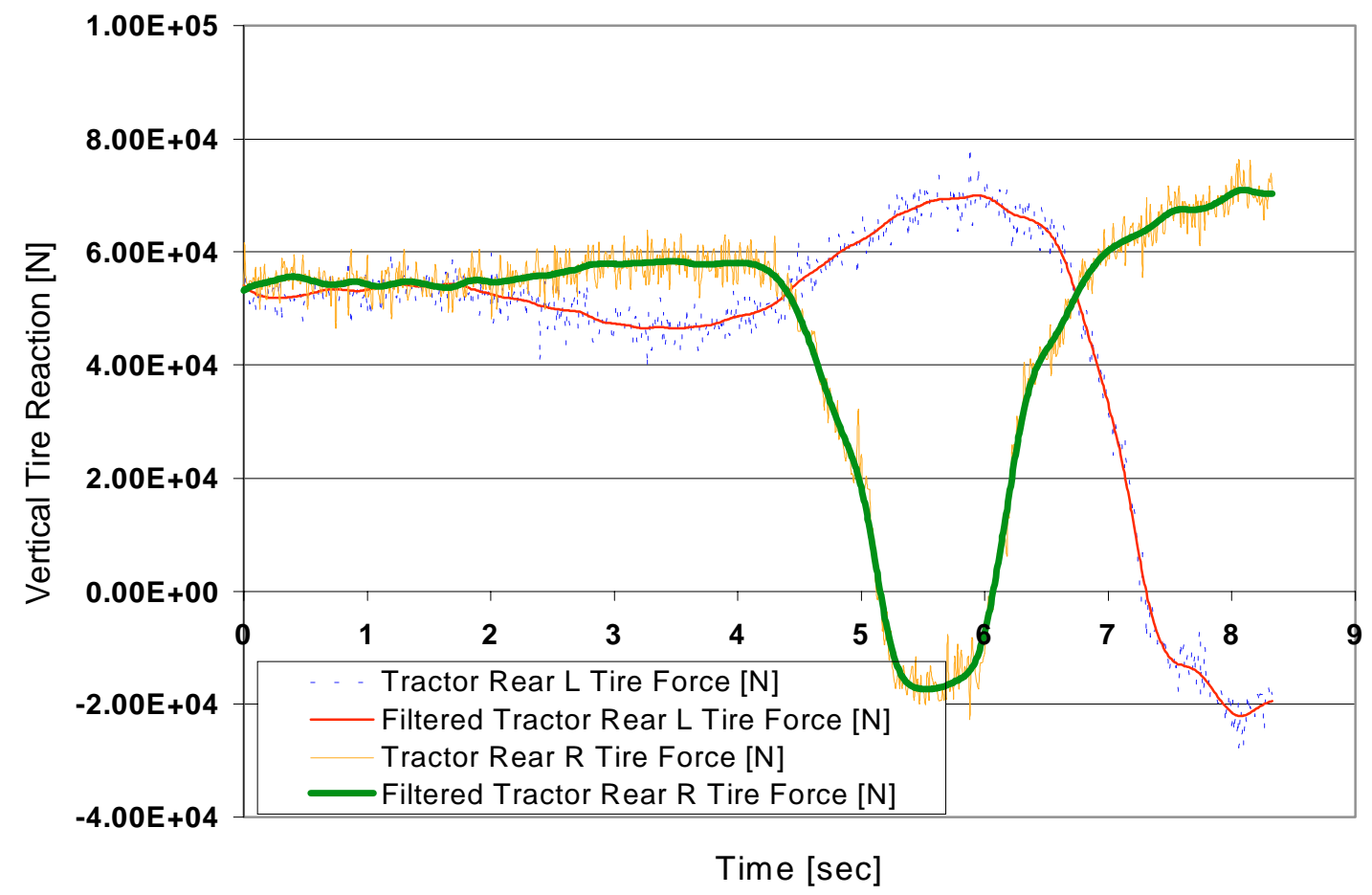

Figure 3.11: Tractor rear wheels reactions for axles 2 and 3 summed together during the TOP lane change test (50\% full tanker) 


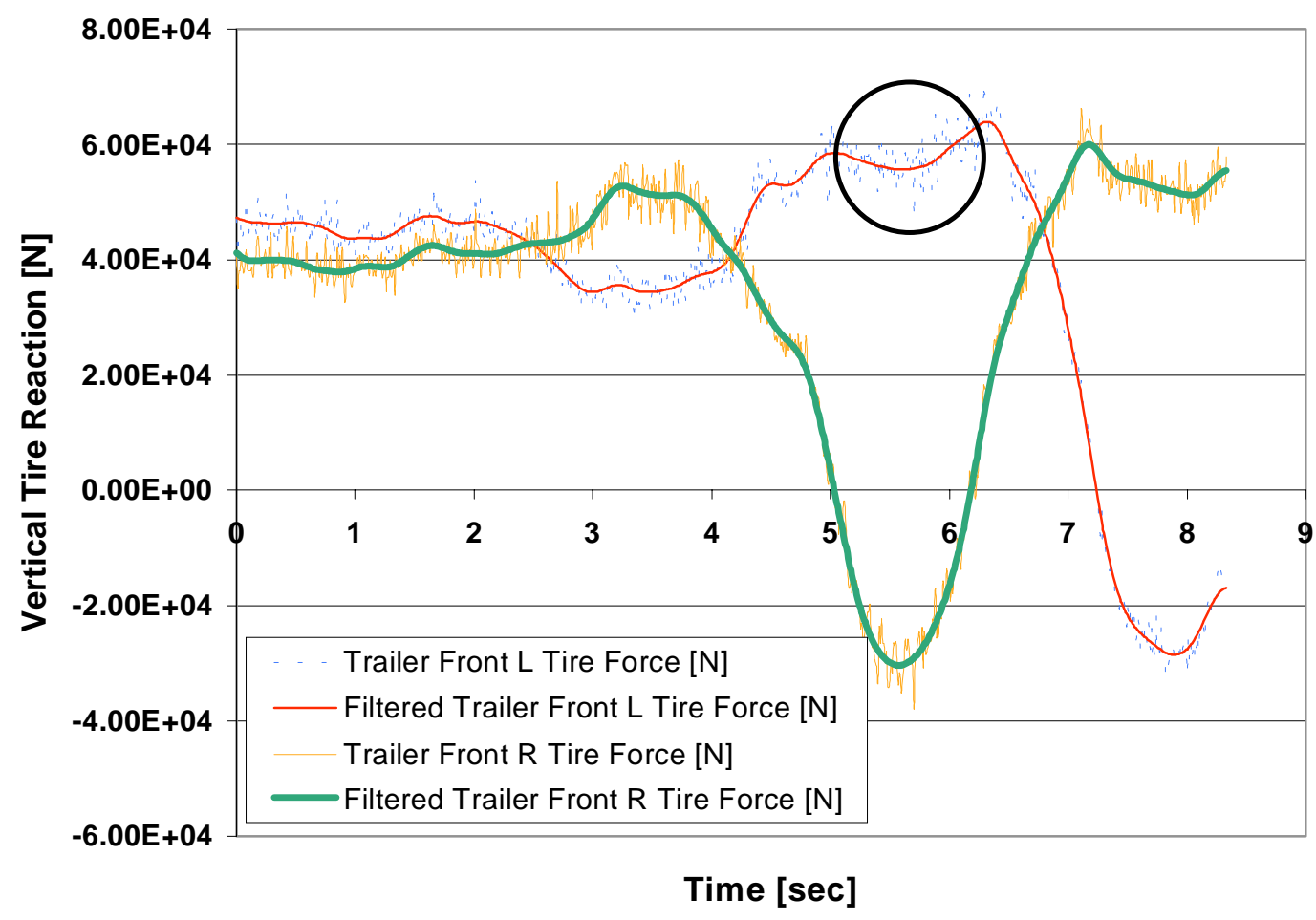

Figure 3.12: Trailer front wheels reactions during the TOP lane change test (50\% full tanker)

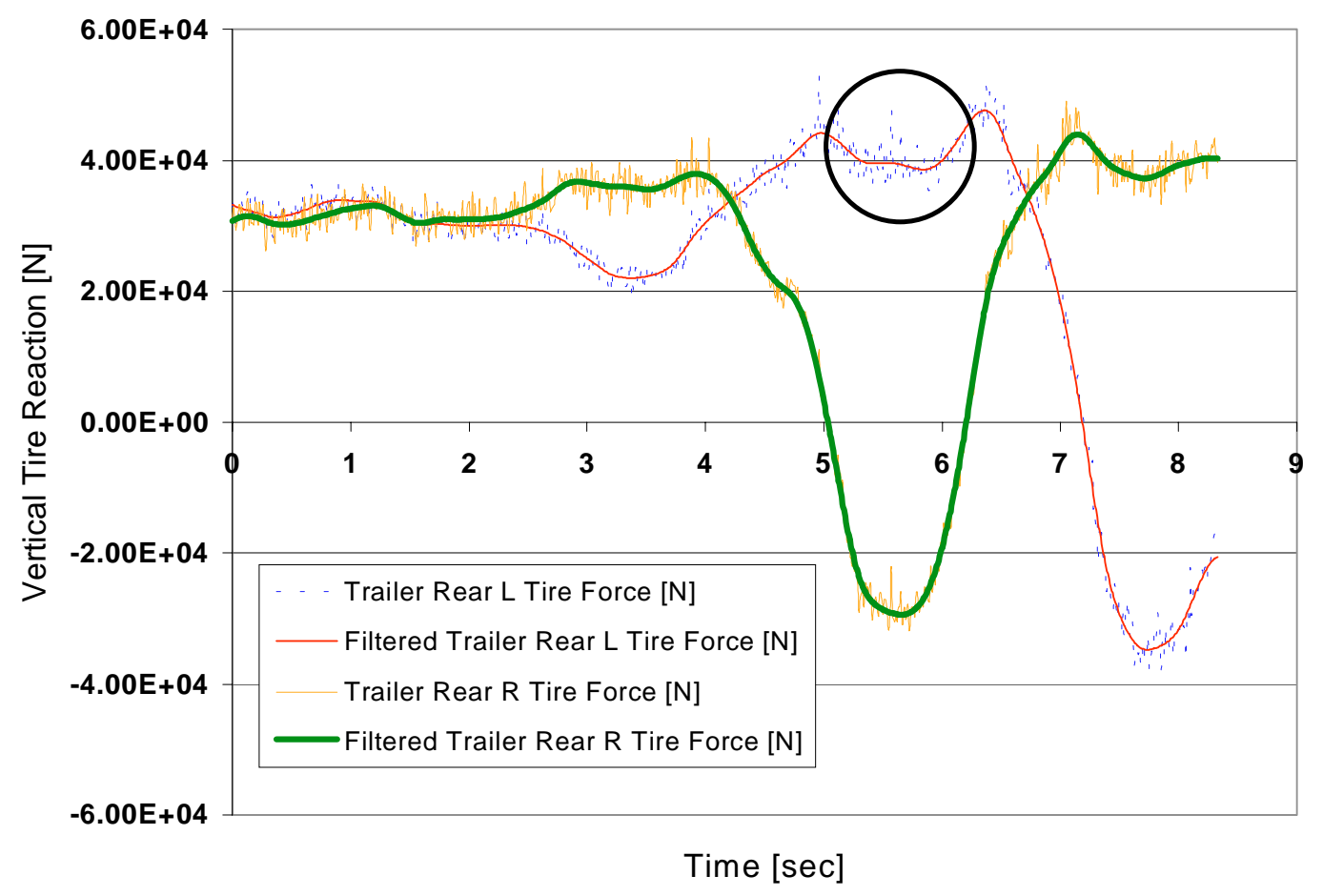

Figure 3.13: Trailer rear wheels reactions during the TOP lane change test (50\% full tanker) 
It can be seen from the graphs that the reaction turns negative first at both right hand side tires of the trailer after exactly 5.04 seconds from the beginning of the test. The tractor rear axles starts lifting off at exactly 5.16 second. This indicates that the rollover starts from the rear most axles and propagates towards the front axles of the tanker. It can be seen also that the reactions never turn negative on the tractor front wheels. It can be seen also that for the tractor front axle, the load on the two sides would add up to a constant value during the maneuver except close to the end, while the case is not the same for the rest of the axles. The reason is that after a certain degree of roll, the artificial supports manufactured by ATC to prevent the tanker from rolling over started to carry the excessive positive reaction. That explains why the negative reactions on the rest of the axles at one side are not reflected as an equal negative reaction on the other side. Close to the end of the test, the liftoff of the left-hand side wheels becomes excessive enough for the side supports to carry some of the load on the right hand side of axle number one.

The dips on the reaction on the positive side circled on the graphs above for both trailer axles reflect the spring relief due to the moment on the axle when the other wheels liftoff the ground. See Figure 16 for illustration of this phenomenon.

\subsubsection{Determining the Experimental Rollover Lateral Acceleration}

The local radius of curvature has been calculated numerically during the TOP lane change test using the provided $\mathrm{x}$ and $\mathrm{y}$ coordinates of the $\mathrm{CG}$ of the tractor and the trailer and equation 3.4 obtained from Korn (1968). 


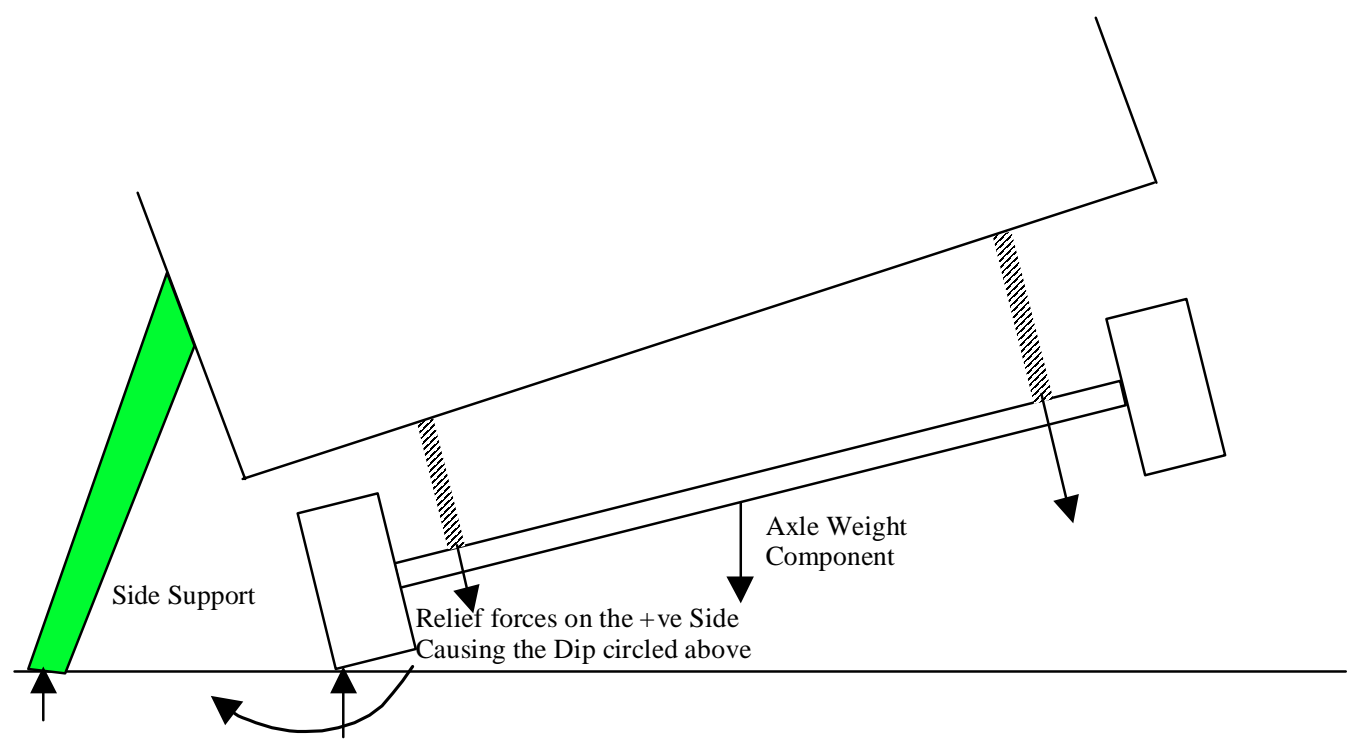

Figure 3.14: Illustration of the cause of the dip in the reaction on the positive side of the trailer axles

$$
\rho=\frac{\left(\mathrm{x}^{.2}+\mathrm{y}^{.2}\right)^{3 / 2}}{\mathrm{x} \cdot \ddot{\mathrm{y}}-\mathrm{y} \cdot \ddot{\mathrm{x}}}
$$

The local lateral acceleration normal to the curve was calculated as a ratio of the gravitational acceleration from the relation; $a=V^{2} /(g * \rho)$. The results are shown in Figures $3.15 \&$ 3.16. From the data used to plot curves 3.15 and 3.16, it was found out that when the trailer's axles started lifting off, i.e. at $\mathrm{t}=5.04 \mathrm{sec}$, the lateral accelerations on the tractor and the trailer were $0.28 \mathrm{~g}$ and $0.25 \mathrm{~g}$ respectively. And when the tractor rear axles started lifting off i.e. at $\mathrm{t}=5.16 \mathrm{sec}$, the lateral accelerations on the tractor and the trailer were $0.283 \mathrm{~g}$ and $0.274 \mathrm{~g}$ respectively. 


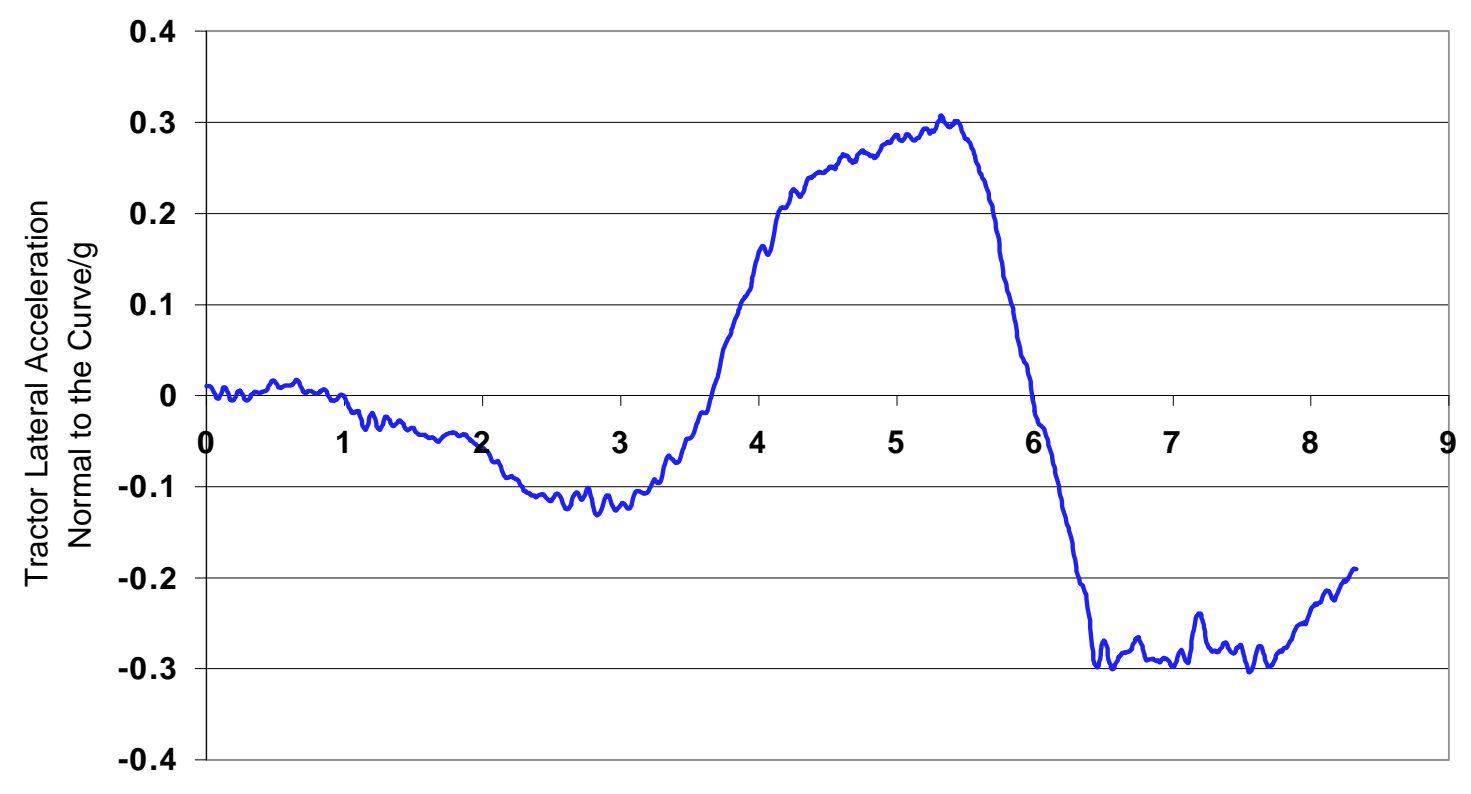

Time $[\mathrm{sec}]$

Figure 3.15: Tractor lateral acceleration normal to the path during the TOP lane change test obtained using equation (3.4)

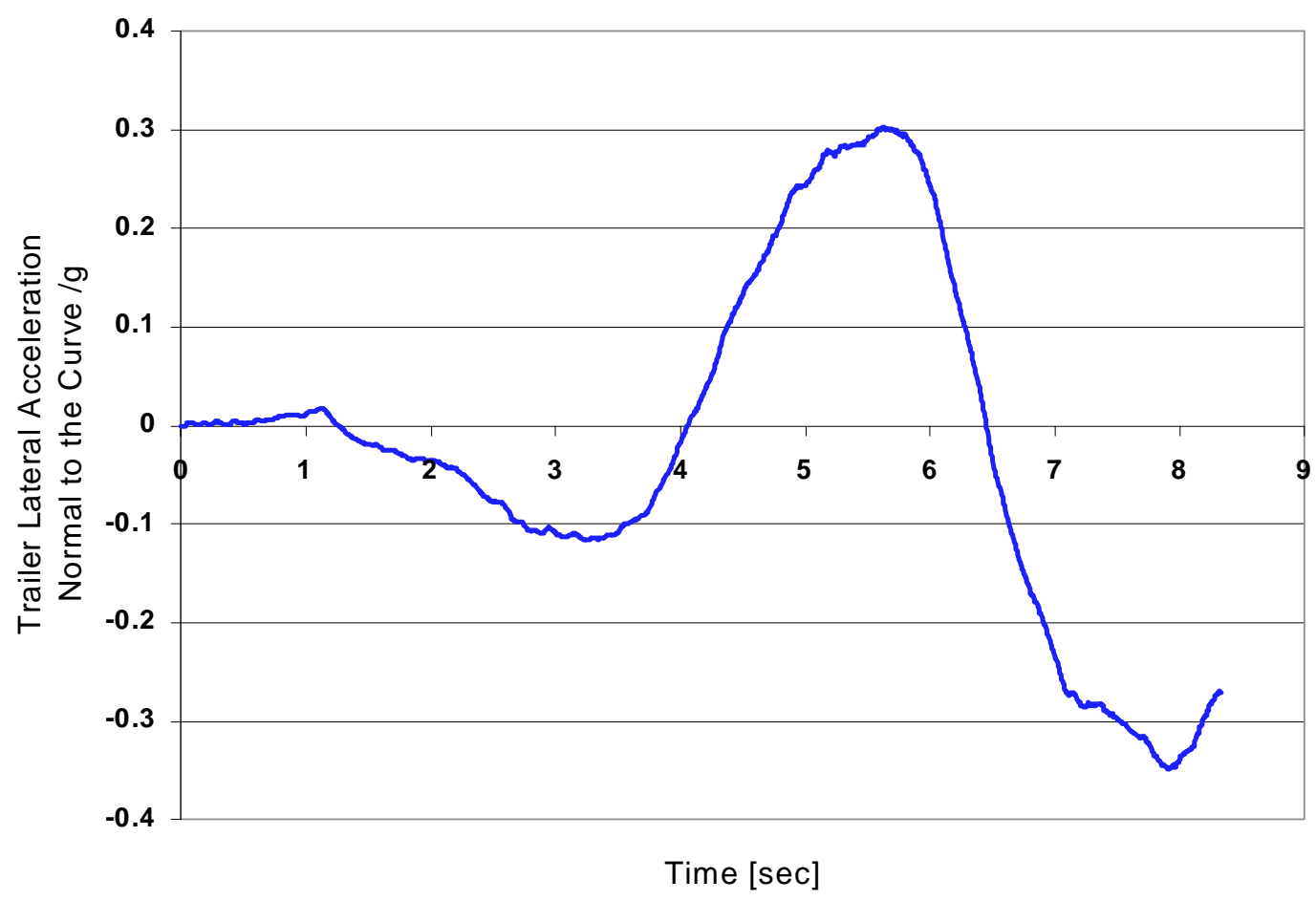

Figure 3.16: Trailer lateral acceleration normal to the path during the TOP lane change test obtained using equation (3.4) 
The results of the constant radius turns show that during the $75 \mathrm{ft}$ radius turn and $50 \%$ full condition, the rollover was triggered at a speed equal to $34 \mathrm{~km} / \mathrm{h}$, which is corespondent to a lateral acceleration of $0.42 \mathrm{~g}$. And for the $100 \mathrm{ft}$ radius turn, the rollover was triggered at a speed of $38 \mathrm{~km} / \mathrm{h}$, which is correspondent to a lateral acceleration of $0.37 \mathrm{~g}$.

Since the results obtained using equation (3.4) were different from the expected results obtained by the constant radius curve, another approach was followed to obtain the lateral acceleration of the tractor and the trailer during the TOP lane change test. The lateral acceleration values provided by ATC from all the valid lateral accelerometer channels were collected ( 8 tractor channels and 5 trailer channels). The average reading during the first 0.3 second of the tractor accelerometer was calculated and the average reading during the first 0.5 second of the trailer was calculated (Duration on which Figures $3.15 \& 3.16$ showed no lateral acceleration for the tractor and the trailer respectively).

These average values were subtracted from each accelerometer reading to get rid of the initial swift in the accelerometer reading. The result signals were passed through the filtering file. The filtered data were averaged for both the tractor and the trailer and multiplied by -1 to change its direction to be constant with the Y direction used in this study. Figures 3.17 and 3.18 show the lateral accelerations for the tractor and the trailer respectively using the technique mentioned above. 


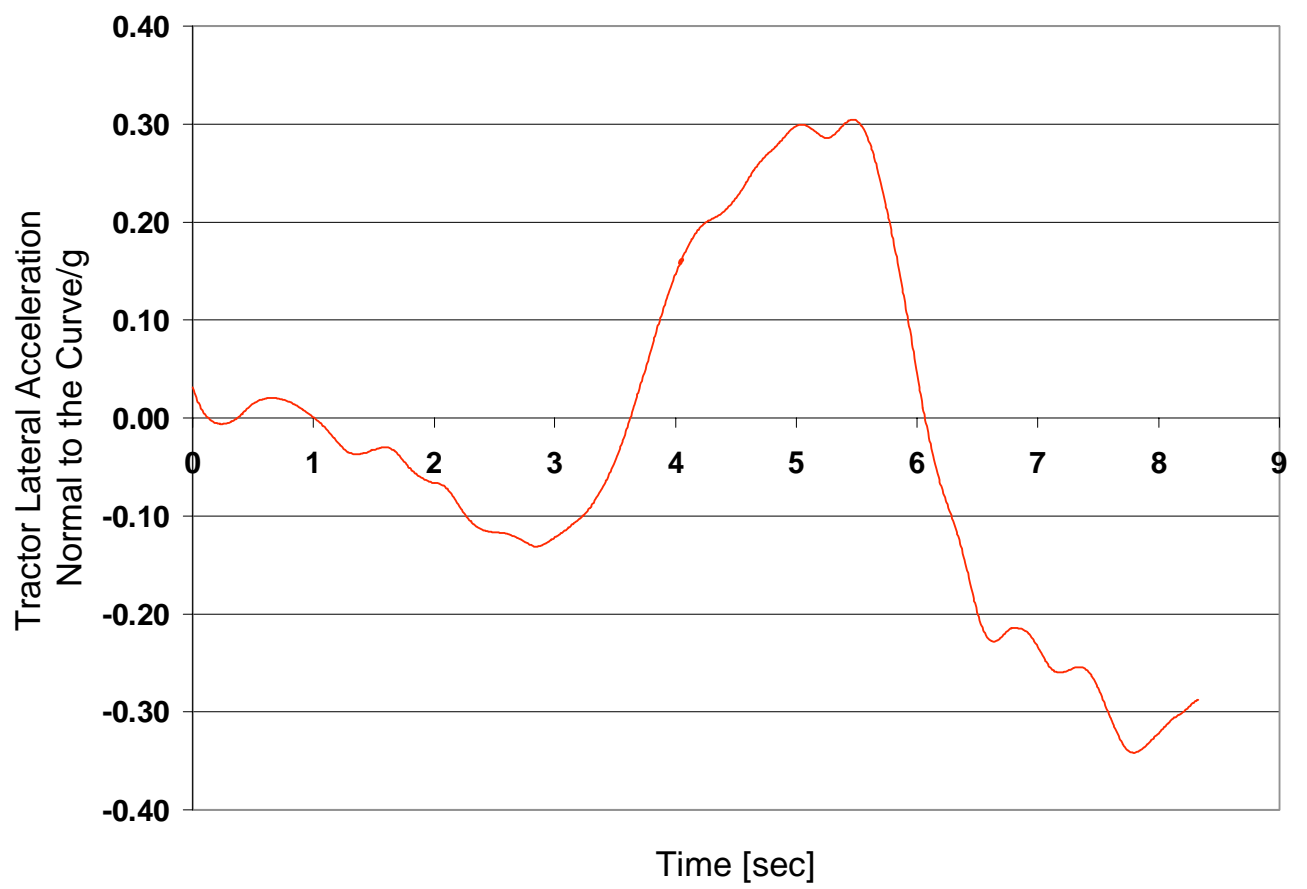

Figure 3.17: Tractor lateral acceleration normal to the path during the TOP lane change test obtained using accelerometer data

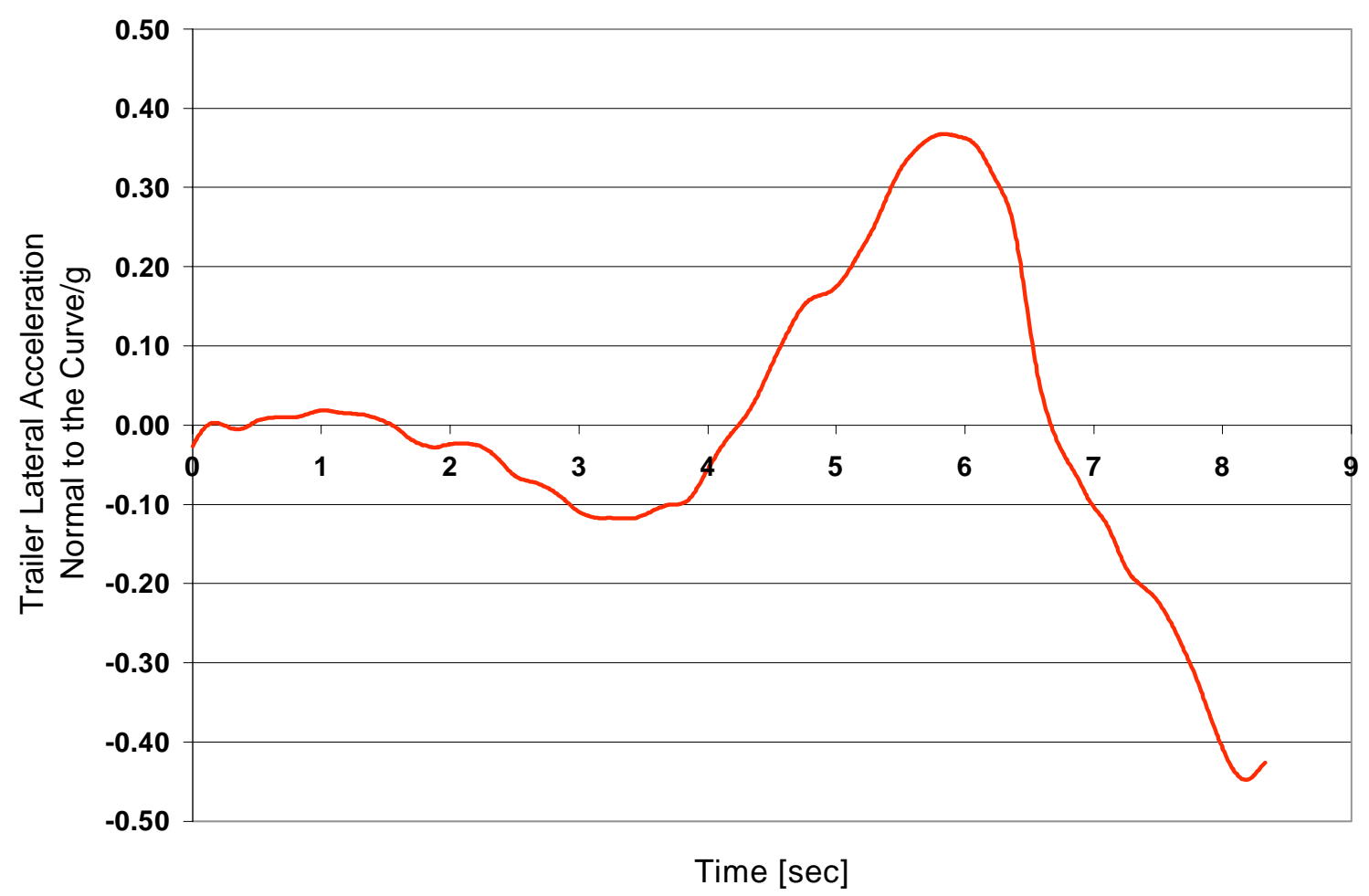

Figure 3.18: Trailer lateral acceleration normal to the path during the TOP lane change test obtained using accelerometer data 
It was found that at $\mathrm{t}=5.04 \mathrm{sec}$., the lateral acceleration of the tractor and the trailer obtained using average filtered corrected accelerometer data were $0.3 \mathrm{~g}$ and $0.182 \mathrm{~g}$ respectively. And at $5.16 \mathrm{sec}$., these values were $0.292 \mathrm{~g}$ and $0.213 \mathrm{~g}$.

It can be seen that the results obtained using the accelerometers' data are close to the results obtained using equation (3.4) for the tractor, while these results shifts towards the undesired direction (less lateral rollover lateral acceleration) for the trailer. It can be seen that the results obtained from the accelerometer data are probably subjected to more error factor, in addition to the fact that those accelerometers don't take into consideration the roll angles of the spring masses. Thus, the results obtained using the $\mathrm{x}$ an $\mathrm{y}$ coordinates of the $\mathrm{CG}$ of the tractor and the trailer and equation (3.4) will be considered.

It should be noted that the acceleration curve for the trailer given in Figure 3.5 is in the $\mathrm{Y}$ direction only and is not normalized by $\mathrm{g}$ and not in the direction normal to the local radius of curvature like the acceleration curve given in Figure 3.16 and 3.18. This curve given in Figure 3.5 is used only as an input parameter for the plane models given in Chapter 5 of this study. 


\subsection{7- NATO Lane Change Test Data Processing}

The results obtained from the NATO lane change test with the $75 \%$ full tanker provided by ATC was processed same way as the data of the TOP lane change test. Figures 3.19 through 3.22 show the reactions at the wheels during this test. It can be seen that the reactions never turned negative at any of the wheels. Thus, the data provided by ATC for the NATO test at $75 \%$ fill tanker and at forward speed of about $68 \mathrm{~km} / \mathrm{h}$ indicate that there was no rollover triggered during that test.

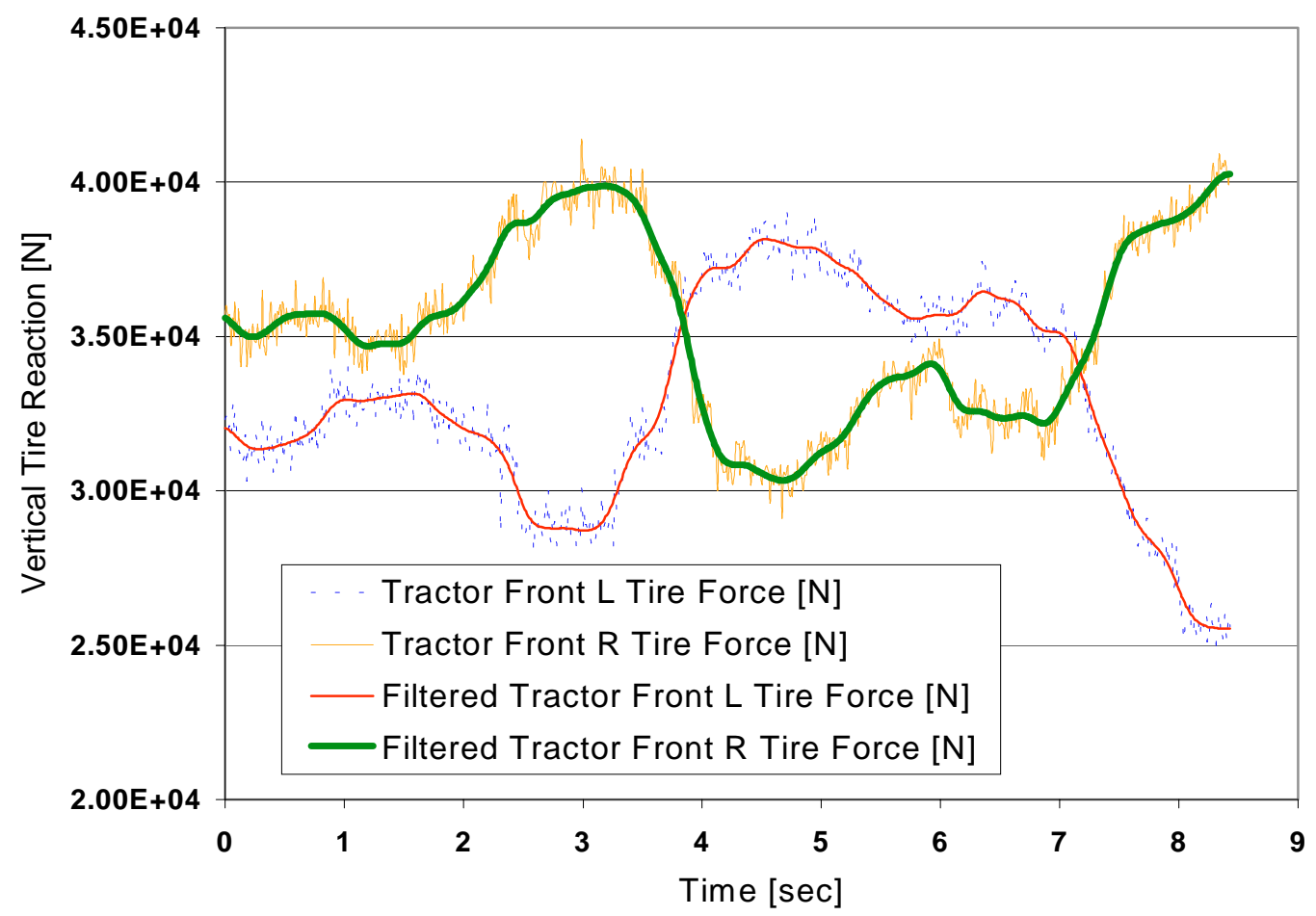

Figure 3.19: Tractor front wheels reactions during the NATO lane change test (75\%full tanker) 


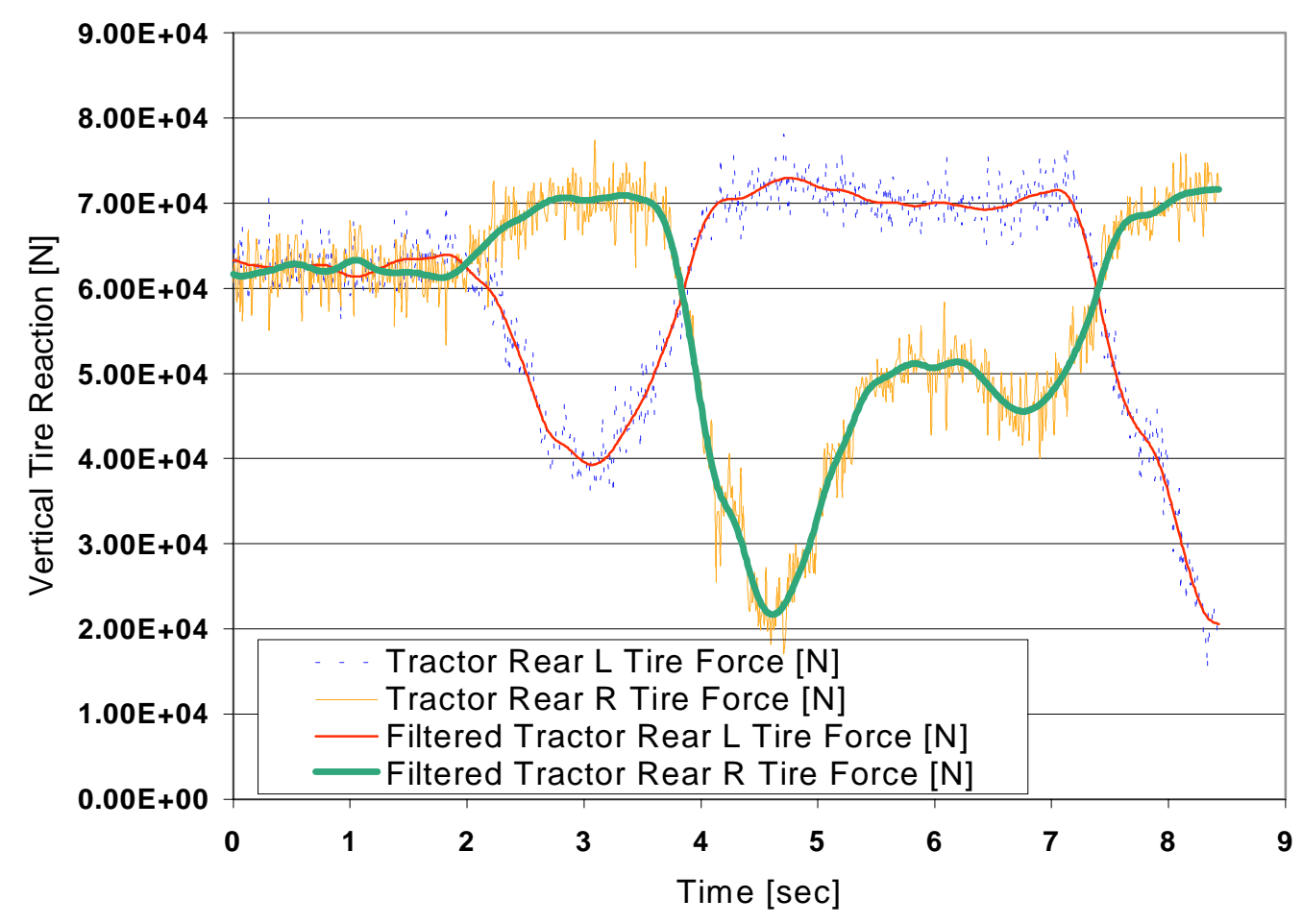

Figure 3.20: Tractor rear wheels reactions for axles $2 \& 3$ summed together during the NATO lane change test (75\% full tanker)

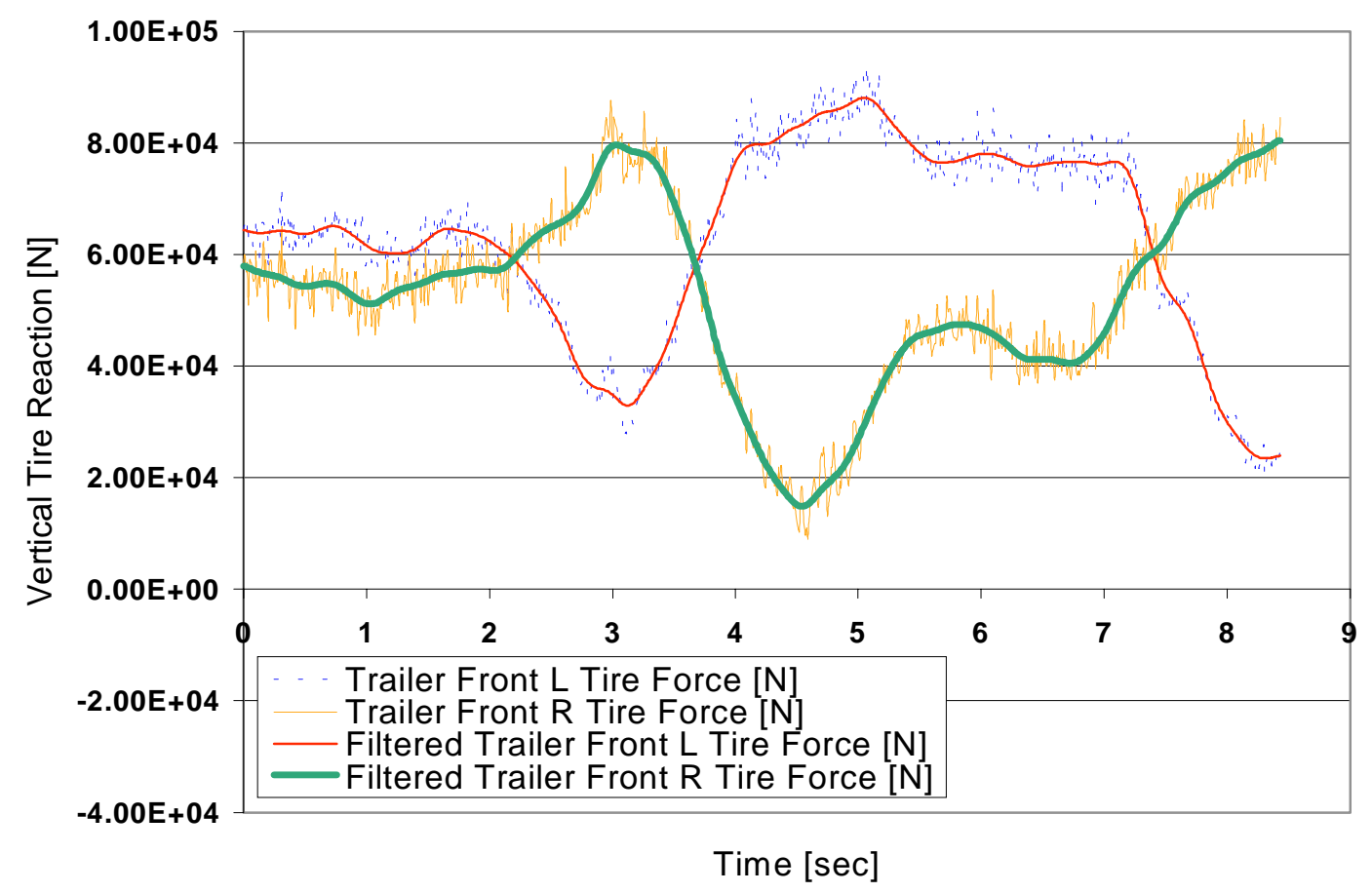

Figure 3.21: Trailer front wheels reactions for axle 4 during the NATO lane change test (75\%full tanker) 


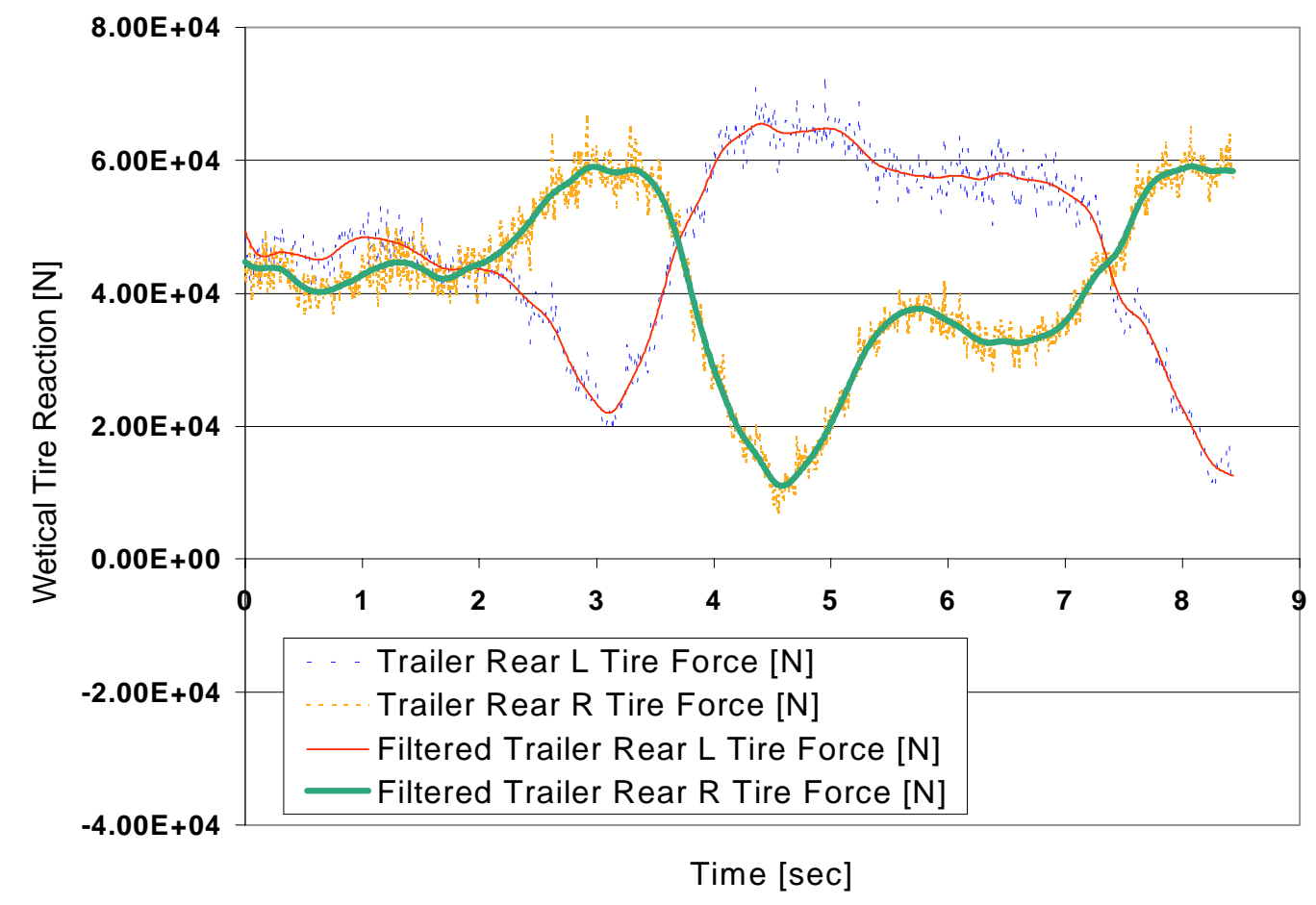

Figure 3.22: Trailer rear wheels reactions for axle 5 during the NATO lane change test (75\%full tanker)

It can be seen from the graphs that the minimum reaction during the NATO test occurs after about 4.5 seconds from the beginning of the test at the rearmost trailer right side wheel. By further investigation of the NATO lane change test data, the lateral acceleration normal to the curvature of the path was obtained for both the tractor and the trailer using equation (3.4), it was found out that the maximum absolute lateral acceleration of the tractor was $0.33 \mathrm{~g}$. While that of the trailer was 0.31g. By plotting the actual spring deflection data of the NATO and the TOP lane change test, it was found out that the maximum positive (lifting) spring deflection during the NATO test is much less than that of the TOP lane change test for all axles. Figure 3.23 shows the plot of the spring deflection of the rear right side wheels on axles $2 \& 3$ of the tractor. It can be seen that the maximum spring positive deflection during the TOP lane change test is more than twice the maximum positive deflection during the NATO lane change test. 


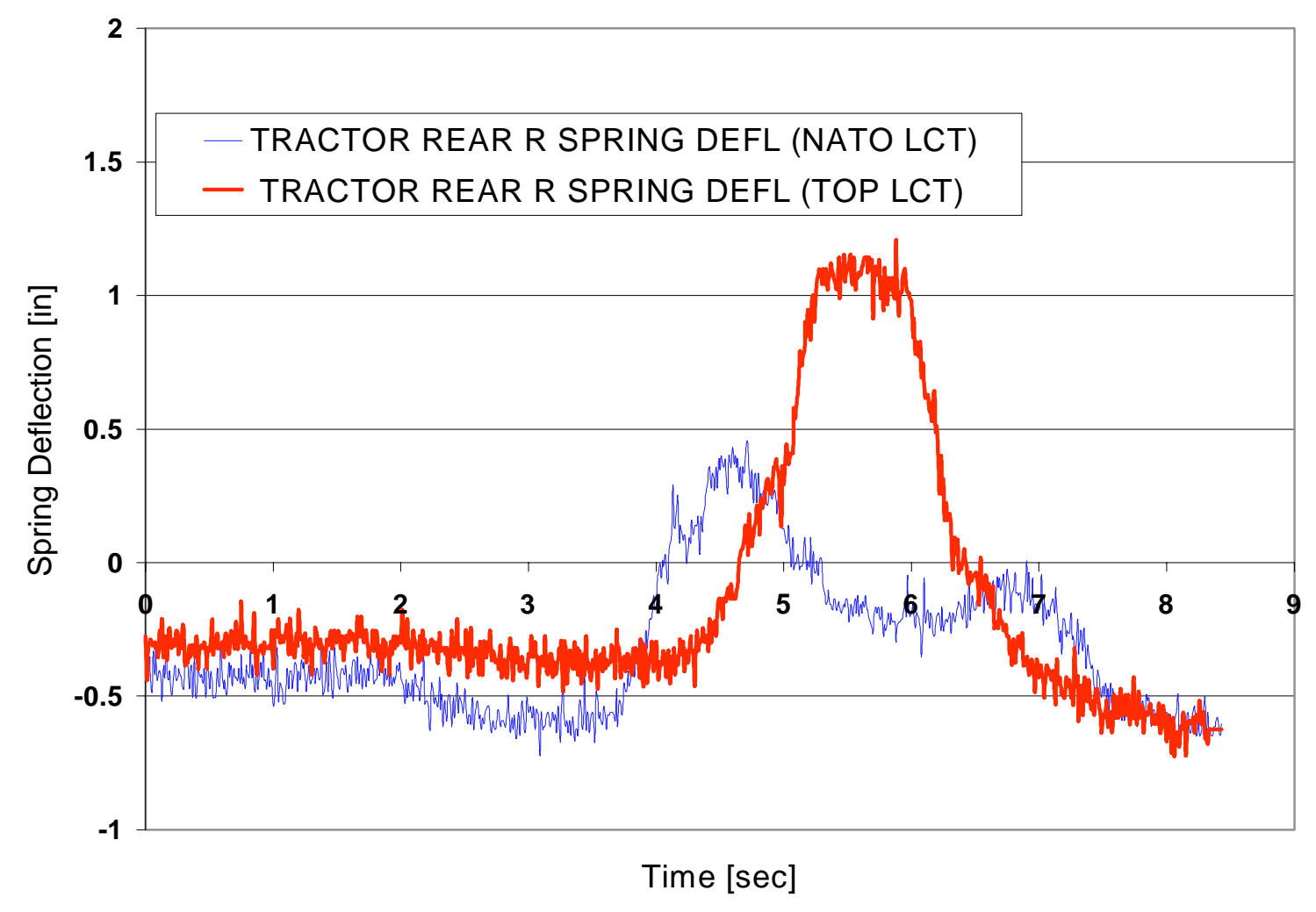

Figure 3.23: Tractor rear right side spring deflection measured during the TOP lane change test $50 \%$ full and the NATO lane change test $75 \%$ full

\section{8- Conclusions based on Experimental Data Analysis}

From the data analysis given above, the following conclusions can be drawn:

1- Because of the high stiffness of the tires relative to that of the suspension springs, the tire-ground noise was more sensible by the accelerometers' vertical channels than the motion attributed to the maneuver action.

2- The rollover starts from the rear most axle of the trailer and transfers to the rear axles of the tractor.

3- For the 50\% full tanker under study, the rollover starts after 5.04 seconds from the start of the TOP lane change test going at a speed of $56.2829 \mathrm{~km} / \mathrm{h}$. 
4- There are some details in the reaction plots of the wheels that are attributed to the side support manufactured by ATC and would not be expected to appear in the plots resulted from the mathematical models.

5- The lateral acceleration at which the rollover is triggered at the same loading condition has a wide range of variation $(0.25 \mathrm{~g}$ to $0.42 \mathrm{~g})$ and depends on the test path.

6- The data provided by ATC for the $75 \%$ full tanker during the NATO test indicated no rollover during the test. 


\section{CHAPTER 4}

\section{TRAMMEL PENDULUM PARAMETERS}

\section{$\underline{4.1}$ - Introduction}

In this chapter, it has been verified that the locus of the CG of a fluid volume moving in a rocking motion in a tank such that the fluid surface is flat follows an exact elliptical path. Thus, a pendulum that follows a similar path was proposed (a trammel pendulum) to simulate the fluid lateral dynamic effect. The equation of motion of the pendulum was derived to study its dynamic performance, natural frequency, angular displacement, velocity and acceleration diagrams under certain inputs.

\section{2- Locus of Fluid Volume Center of Gravity}

From the literature review given in chapter 2, it has been concluded that the most important sloshing mode for a fluid in a partially filled container is the first mode. This mode can be described by the rocking motion of the fluid surface (approximately flat). Based on this fact, study has been performed to find out the location of the center of gravity of a given fluid volume in an elliptical container (Details can be found in Appendix B). It was found out that the $\mathrm{CG}$ of the fluid volume falls on an elliptical contour parallel to the tank wall (see Fig. 4.1). This contour is such that

$$
\frac{\overline{\mathrm{a}}_{\mathrm{cg}}}{\overline{\mathrm{b}}_{\mathrm{cg}}}=\frac{\overline{\mathrm{a}}}{\overline{\mathrm{b}}}=\frac{\mathrm{a}}{\mathrm{b}} .
$$




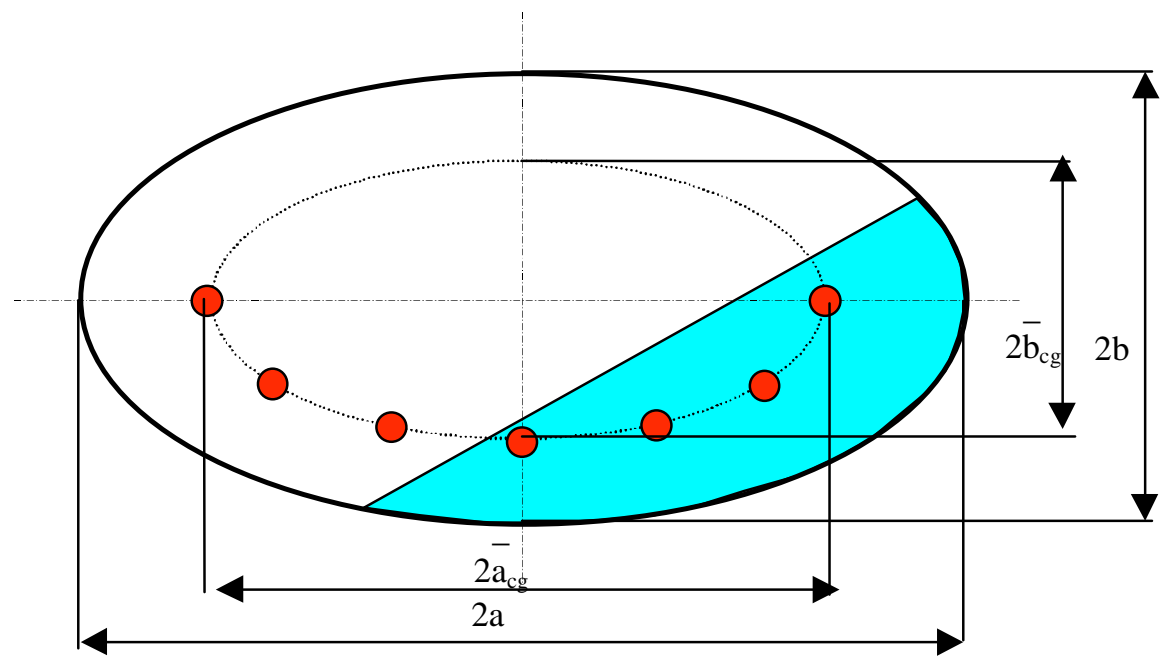

Figure 4.1: Contour of C.G. location for a given volume of fluid in an elliptical container

\section{3- Equation of Motion of the Trammel Pendulum}

A trammel mechanism, which draws an elliptical path, was considered to simulate an elliptical pendulum (Figure 4.2). The dynamic effects of this pendulum were investigated to determine its parameters (Mp, $\bar{a}, \bar{b}$ and the location of the fixed mass).

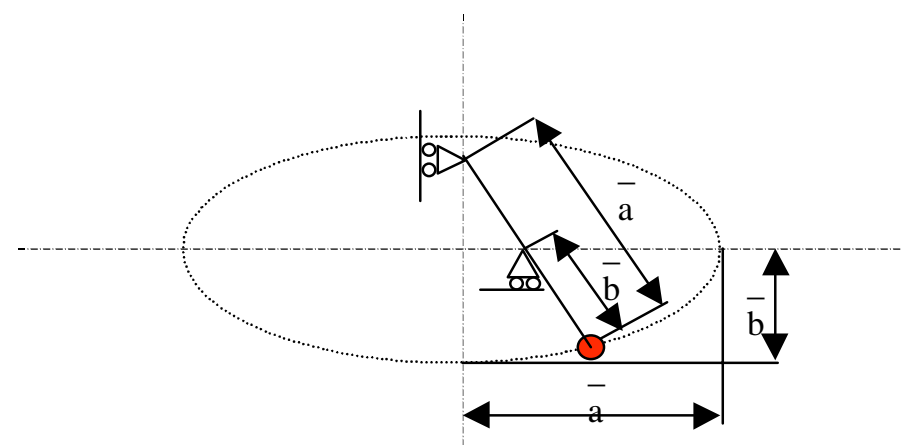

Figure 4.2: C.G. of fluid volume contour drawn by an elliptical trammel mechanism

Figure 4.3 shows the important notations used in deriving the equation of motion of the pendulum and finding the forces exerted due to its motion (Details of deriving the trammel pendulum's equation of motion can be found in Appendix B). 


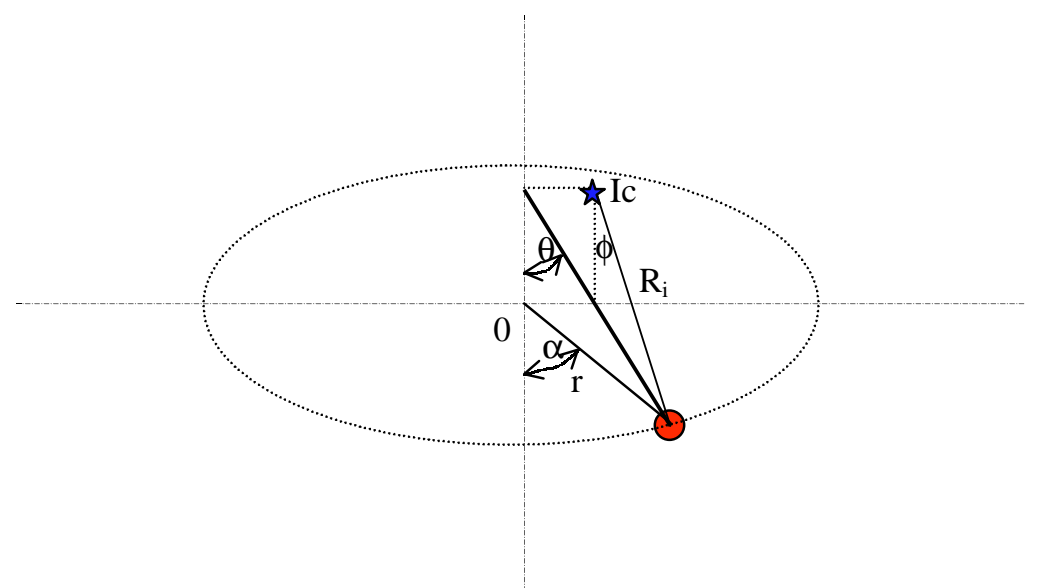

Figure 4.3: Parameters used in the analysis of the elliptical pendulum motion

Since the pendulum arm was found to be perpendicular to the fluid surface, the angle $\theta$ was taken as the parameter used in deriving the equation of motion of the pendulum. The equation of motion for the elliptical pendulum due to an external horizontal acceleration $\ddot{X}$ was found to be:

$$
\left(\overline{\mathrm{a}}^{2} \cos ^{2} \theta+\overline{\mathrm{b}}^{2} \sin ^{2} \theta\right) \ddot{\theta}+0.5\left(\overline{\mathrm{b}}^{2}-\overline{\mathrm{a}}^{2}\right) \theta^{0^{2}} \sin 2 \theta+\mathrm{g} \overline{\mathrm{b}} \sin \theta+\overline{\mathrm{a}} \cos \theta \ddot{\mathrm{x}}=0
$$

This is a nonlinear second order differential equation. Equation (B19) has been solved numerically to find $\theta, \dot{\theta}$ and $\ddot{\theta}$. The natural frequency for this pendulum was calculated and was found to have a range dependent on the value of the initial angle of swing $\theta$. Figure 4.4 shows a plot for the natural frequency for a pendulum at different aspect ratios using the test tanker outer shell contour assuming constant area. It can be seen that for $a / b=1$, the natural frequency drops with the increase of the pendulum angle. On the other hand, the natural frequency increases gradually and peaks at a certain angle for aspect ratios more than 1 . This angle is almost equal to $90^{\circ}$ for $\mathrm{a} / \mathrm{b}=2$. 


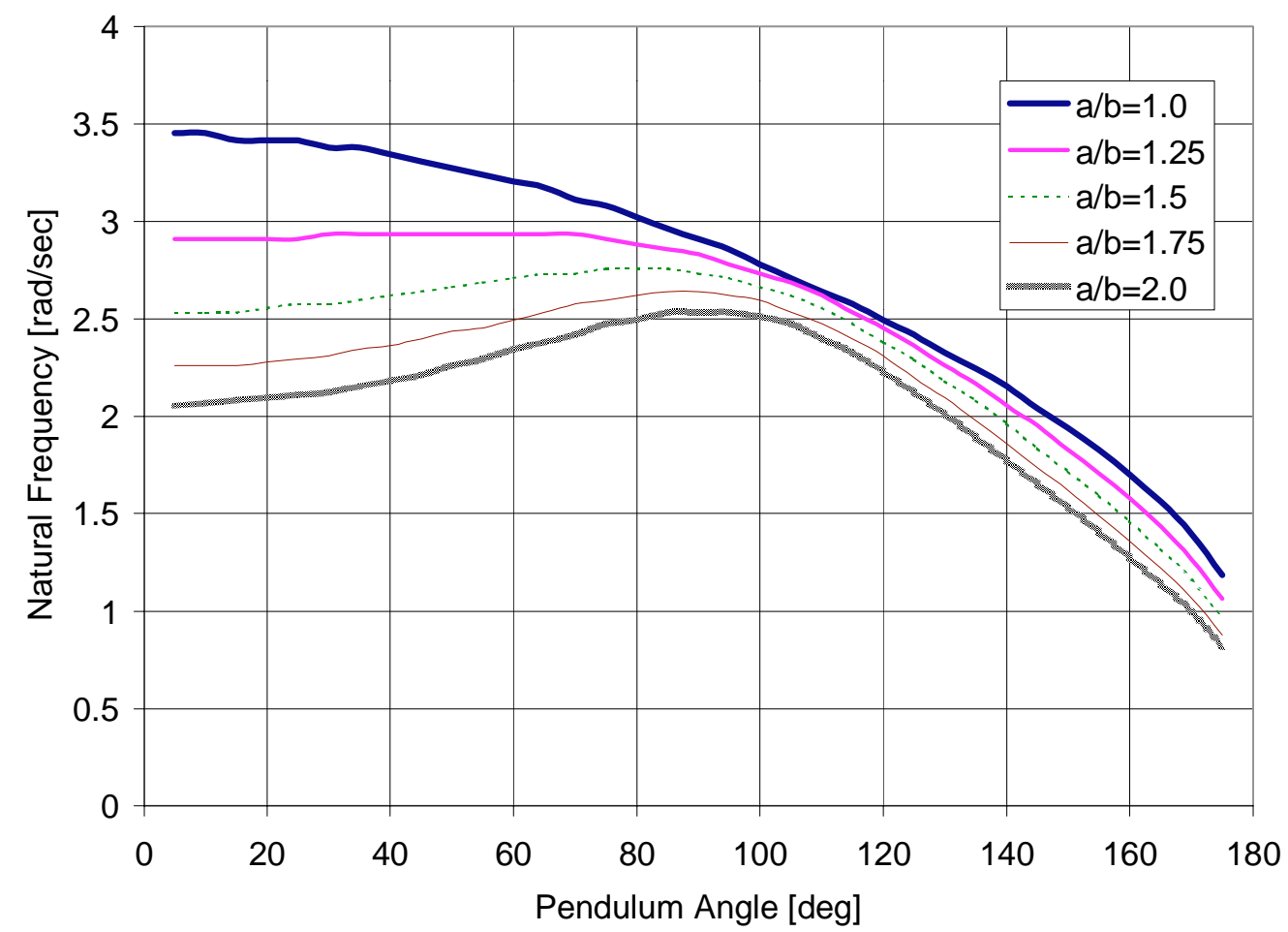

Figure 4.4: Natural frequency of an elliptical pendulum as a function of the pendulum swing angle using different aspect ratios using the ETNYRE tanker outer shell dimensions

It was found out that the values of $M_{p}, a_{c g}$, and $b_{c g}$, are not equal to $M_{\text {fluid, }}$ $\bar{a}$, and $\bar{b}$ respectively. The reason was due to the fact that not all the mass of the fluid volume contributes in the sloshing process.

\section{4- Pendulum-Fluid Dynamic Effect Matching}

All efforts to match pendulums or spring-bob models to the dynamic effects of sloshing of a fluid in a partially filled containers in the past were based on linearized equations of motion for the mechanical systems to the linearized equations of motion for the fluid. Based on this fact, equation B19 modified for small trammel pendulum angles and unforced vibration will be: 


$$
\ddot{\theta}+\left(\frac{\overline{\mathrm{b}}^{2}-\overline{\mathrm{a}}^{2}}{\overline{\mathrm{a}}^{2}}\right) \dot{\theta}^{2} \theta+\frac{\mathrm{g} \overline{\mathrm{b}}}{\overline{\mathrm{a}}^{2}} \theta=0
$$

Figure 4.5 shows the pendulum parameters that needs to be evaluated to achieve a complete simulation of the dynamic effect of fluid lateral sloshing in a partially filled elliptical tank. The four parameters are; the arms of the pendulum $\overline{\mathrm{a}}$ and $\overline{\mathrm{b}}$, the pendulum mass $\mathrm{M}_{\mathrm{p}}$ and the fixed mass $M_{f}$, and the height at which the fixed mass is located relative to the tank base point $h_{o}$. The polar moment of inertia of the fluid will take in effect when the tank itself starts rotating around a certain point. This parameter will be discussed when applicable.

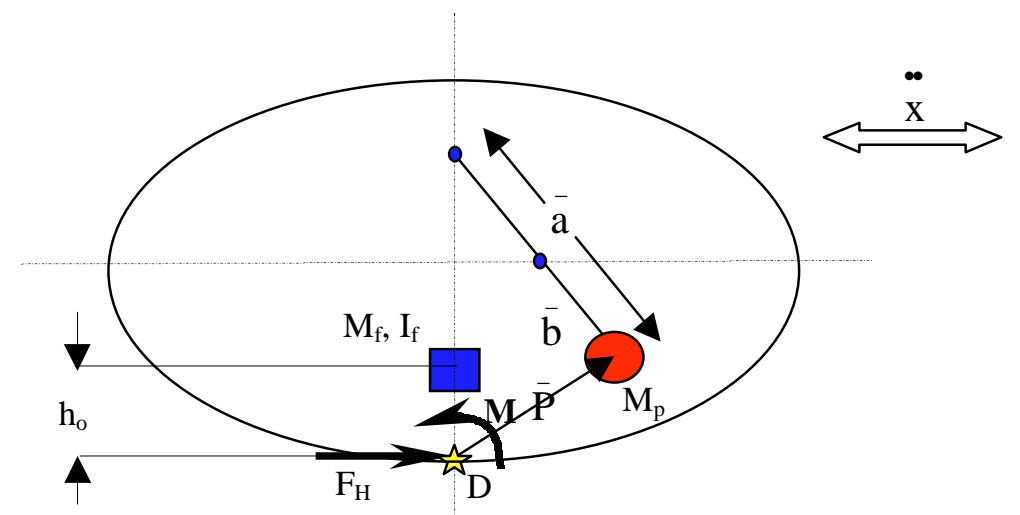

Figure 4.5: Trammel pendulum parameters required for dynamic matching of fluid lateral sloshing effects

The details of the trammel pendulum-fluid dynamic matching are given in details in the next section.

\subsection{1- Pendulum-Fluid Natural Frequency Matching}

The first step in matching the pendulum dynamic effect to the fluid slosh dynamic effect is to match the first mode of natural frequency of oscillation. Since the natural frequency of the trammel pendulum is independent on the mass, equations 4.1 and 4.2 should be sufficient to 
obtain the values of $\bar{a}$ and $\bar{b}$ required to match the natural frequencies of the fluid and mechanical systems. Unfortunately, the middle term of the left-hand side of equation 4.2 still renders this equation as a nonlinear, second order differential equation with no closed form analytical solution. Thus, unlike a simple cylindrical pendulum, the natural fundamental natural frequency is not clearly described in the equation of motion.

To obtain the fundamental frequency of vibration for the trammel pendulum, equation 4.2 is transferred to the phase-plane trajectory equation as follows:

Let $\theta=\mathrm{x}$, then let $\frac{d x}{d t}=y$

Then from equation $4.2, \frac{\mathrm{dy}}{\mathrm{dt}}=\mathrm{C}_{1} \mathrm{y}^{2} \mathrm{x}-\mathrm{C} 2 \mathrm{x}$

Where $C 1=\left(\frac{\bar{b}^{2}-\bar{a}^{2}}{\bar{a}^{2}}\right)$, and $\mathrm{C} 2=\frac{\mathrm{g} \overline{\mathrm{b}}}{\overline{\overline{\mathrm{a}}}^{2}}$

From equation 4.3 and 4.4 , the direction field equation of the trammel pendulum is given

by: $\quad \frac{d y}{d x}=\frac{C 1 y^{2} x-C 2 x}{y}$

The solution of equation 4.5 is the phase-plane trajectory equation:

$$
y=\frac{ \pm \sqrt{\mathrm{Cl}\left(\mathrm{C} 2+\mathrm{C} 1 \mathrm{Ae}^{\mathrm{ClX} \mathrm{X}^{2}}\right)}}{\mathrm{C} 1}
$$

Where " $\mathrm{A}$ " is an integration constant that can be found from the condition that at $\mathrm{x}=\mathrm{x}_{\max }, \mathrm{y}=0$ Thus, the phase trajectory equation for the trammel pendulum is:

$$
y=\frac{ \pm \sqrt{\mathrm{Cl}\left(\mathrm{C} 2-\mathrm{Cle}^{\mathrm{Cl}\left(\mathrm{x}^{2}-\mathrm{x}_{\max }^{2}\right)}\right)}}{\mathrm{Cl}}
$$


The phase trajectory plot for the trammel pendulum is shown in Figure 4.6 for $\bar{a}=1.2$ and $\bar{b}=0.6$

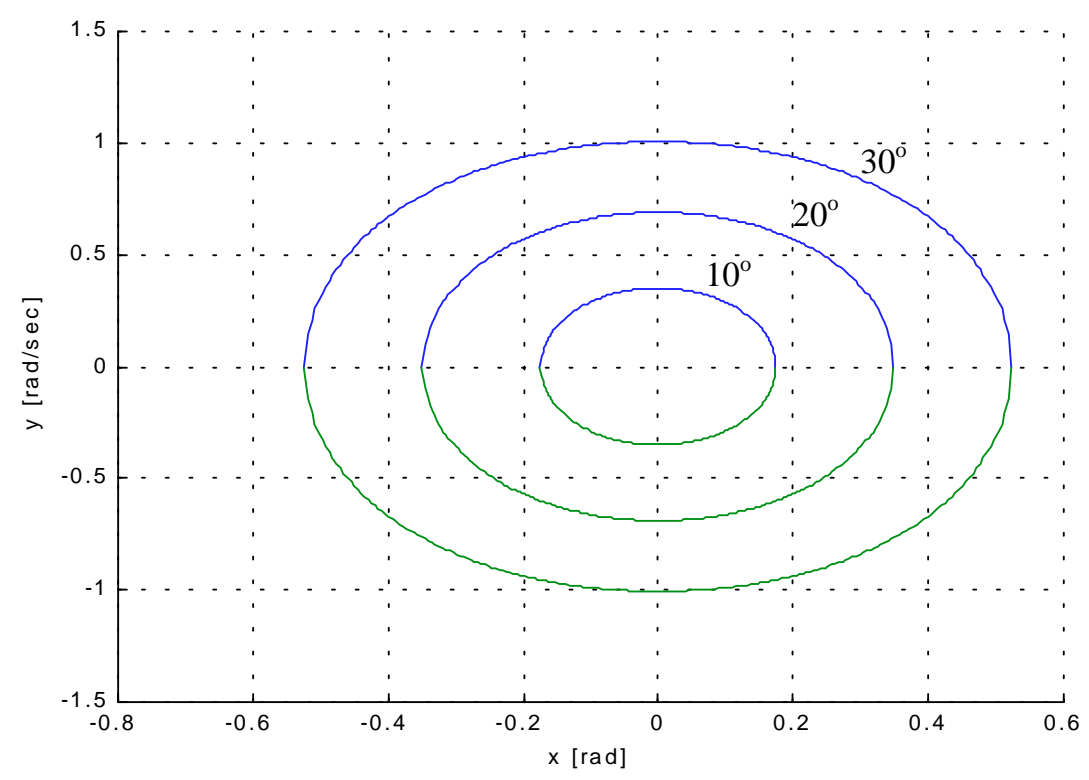

Figure 4.6: Phase trajectory plot for a trammel pendulum oscillating at small angles

Equation 4.7 can be reduced to the form $\pm \sqrt{\mathrm{C} 3\left(1-\mathrm{e}^{\mathrm{Cl}\left(\mathrm{x}^{2}-\mathrm{x}_{\max }^{2}\right)}\right)}$, where $\mathrm{C} 3=\mathrm{C} 2 / \mathrm{C} 1$

Let $\mathrm{z}=C 1\left(x^{2}-x_{\max }^{2}\right)$

Thus, Equation 4.7 can be reduced to: $y=C 4\left(1-e^{z}\right)^{1 / 2}$

Where $\mathrm{C} 4=$ the square root of $\mathrm{C} 3$

Expanding $\mathrm{e}^{\mathrm{z}}$ using Taylor series expansion in Equation 4.8 yields:

$$
y=C 4 \sqrt{-\left(z+\frac{z^{2}}{2 !}+\frac{z^{3}}{3 !}+\cdots\right)}
$$

Since $\mathrm{z}$ is small at small pendulum angles of oscillation, then higher order terms can be neglected. Substituting $\mathrm{z}$ back in Equation 4.9 yields:

$$
\mathrm{y}=\mathrm{C} 4 \sqrt{\mathrm{Cl}\left(\mathrm{x}_{\max }^{2}-\mathrm{x}^{2}\right)}
$$


By comparing Equation 4.10 with the phase trajectory equation of a simple pendulum having small oscillations, $y=\omega \sqrt{x_{\max }^{2}-x^{2}}$, it can be concluded that the fundamental frequency of natural vibration of a trammel pendulum going through small angular oscillations is given by:

$$
\omega=\sqrt{\mathrm{C} 3 \mathrm{Cl}}=\sqrt{\mathrm{C} 2}=\sqrt{\frac{\mathrm{g} \overline{\mathrm{b}}}{\overline{\mathrm{a}}^{2}}}
$$

Equation 4.11 was verified numerically for small trammel pendulum angles by comparing its results with the results obtained from equation B.19.

Given equation 4.1 and 4.11, and knowing the fundamental natural frequency of fluid sloshing in an elliptical tank, the values of $\bar{a}$ and $\bar{b}$ can be calculated.

Bohn (1981) stated that the first natural frequency of fluid oscillation in a partially filled elliptical container is given by:

$$
\omega_{\mathrm{n}}=\sqrt{\frac{3 \mathrm{Ag}}{2 \overline{\mathrm{V}}^{3}}}
$$

One of Bohn's recommendations was to verify the fluid model he derived, because of the discrepancy of analytical results from experimental results during turning for an elliptical tanker in his study. Equation 4.12 was checked using a half full cylindrical tank (which is a special case of a partially filled elliptical tank) that has been studied before by Rayleigh 1876, Lamb 1945, and Budiansky 1960). Also, the results of equation 4.12 were checked against a finite element model for a sloshing fluid in half full cylindrical tank using LS-Dyna 3D. Table 4.2 illustrates the results obtained using each method. 
Table 4.1: Reduced formulas to evaluate the fundamental lateral natural frequency of vibration in a half-full cylindrical tank.

\begin{tabular}{|c|c|}
\hline $\begin{array}{c}\text { Model or Method to Obtain The Fundamental } \\
\text { natural Frequency In a Half Full Cylindrical Tank }\end{array}$ & Formula \\
\hline Rayleigh (1876) & $\omega_{n}=1.1644 \sqrt{\frac{g}{R}}$ \\
\hline Lamb (1945) & $\omega_{n}=0.8266 \sqrt{\frac{g}{R}}$ \\
\hline Budiansky (1960) & $\omega_{n}=1.169 \sqrt{\frac{g}{R}}$ \\
\hline Bohn (1981) & $\omega_{n}=1.535 \sqrt{\frac{g}{R}}$ \\
\hline WVU LS-Dyna 3D Fluid Model & $\omega_{n}=1.1 \sqrt{\frac{g}{R}}$ \\
\hline
\end{tabular}

It can be seen that Bohn reduced formula for the fundamental natural frequency of lateral fluid sloshing in a half full cylindrical tank is biased towards a higher value of natural frequency from any other method (at least 30\% higher from the closest method). Thus, equation 4.12 obtained by Bohn is questionable.

It has been found that the results obtained using Ls-Dyna 3D agrees with the literature. Thus, FE models for an elliptical tank with the same cross section area as the test tanker and a/b varying from 1 to 2 were used to determine the fundamental natural frequency of fluid slosh in a partially filled elliptical tank at different levels of fill. A small initial surface angle of $5^{\circ}$ was given to the fluid surface to maintain the linear assumption used to derive equation 4.11. Figures $4.7 \mathrm{a}$ and $\mathrm{b}$ show a sample of the fluid models used to obtain the natural frequencies at the initial state and after 0.5 cycle respectively. Figure 4.8 shows the " $\mathrm{X}$ " location coordinate during this sample run. The time between two successive amplitudes on the "X" coordinate plots was used to calculate the natural frequencies. 


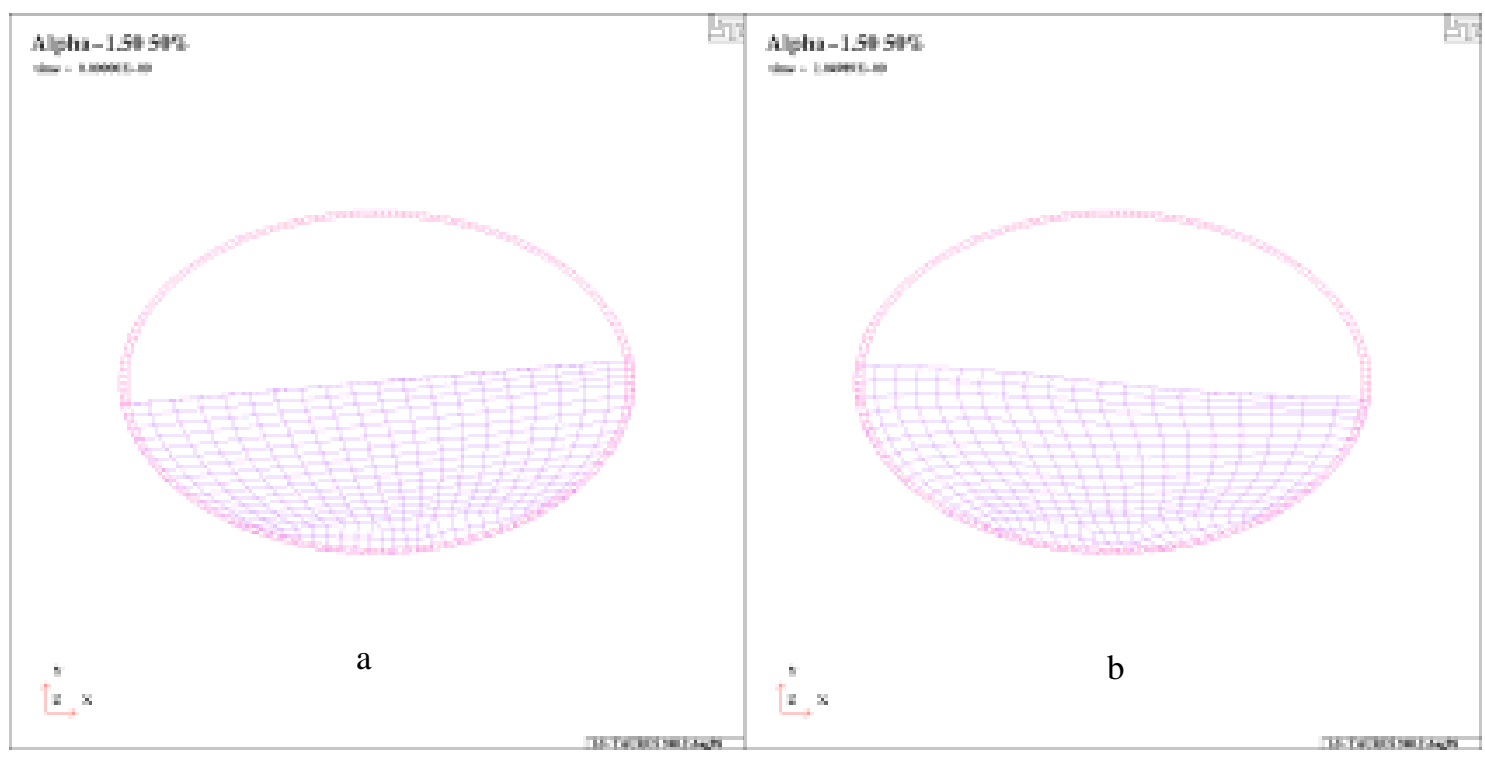

Figure 4.7: Initial fluid position and after 0.5 cycle models using LS-Dyna-3D

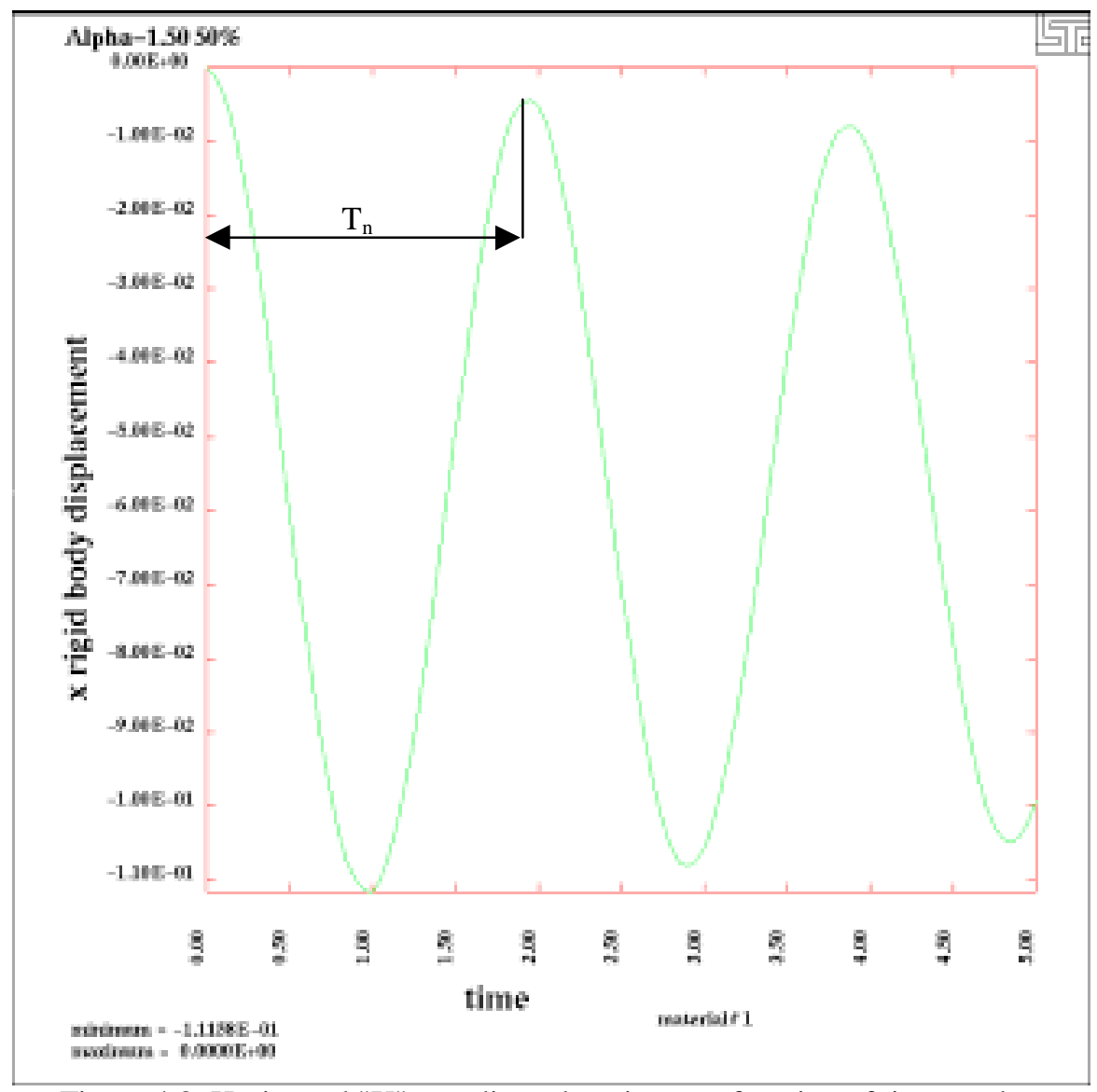

Figure 4.8: Horizontal "X" coordinate location as a function of time used to obtain natural frequency of fluid oscillation 
The natural frequencies obtained from these FE models are shown in Figure 4.9 and were used together with equations 4.1 and 4.11 to get $\bar{a}$ and $\bar{b}$.

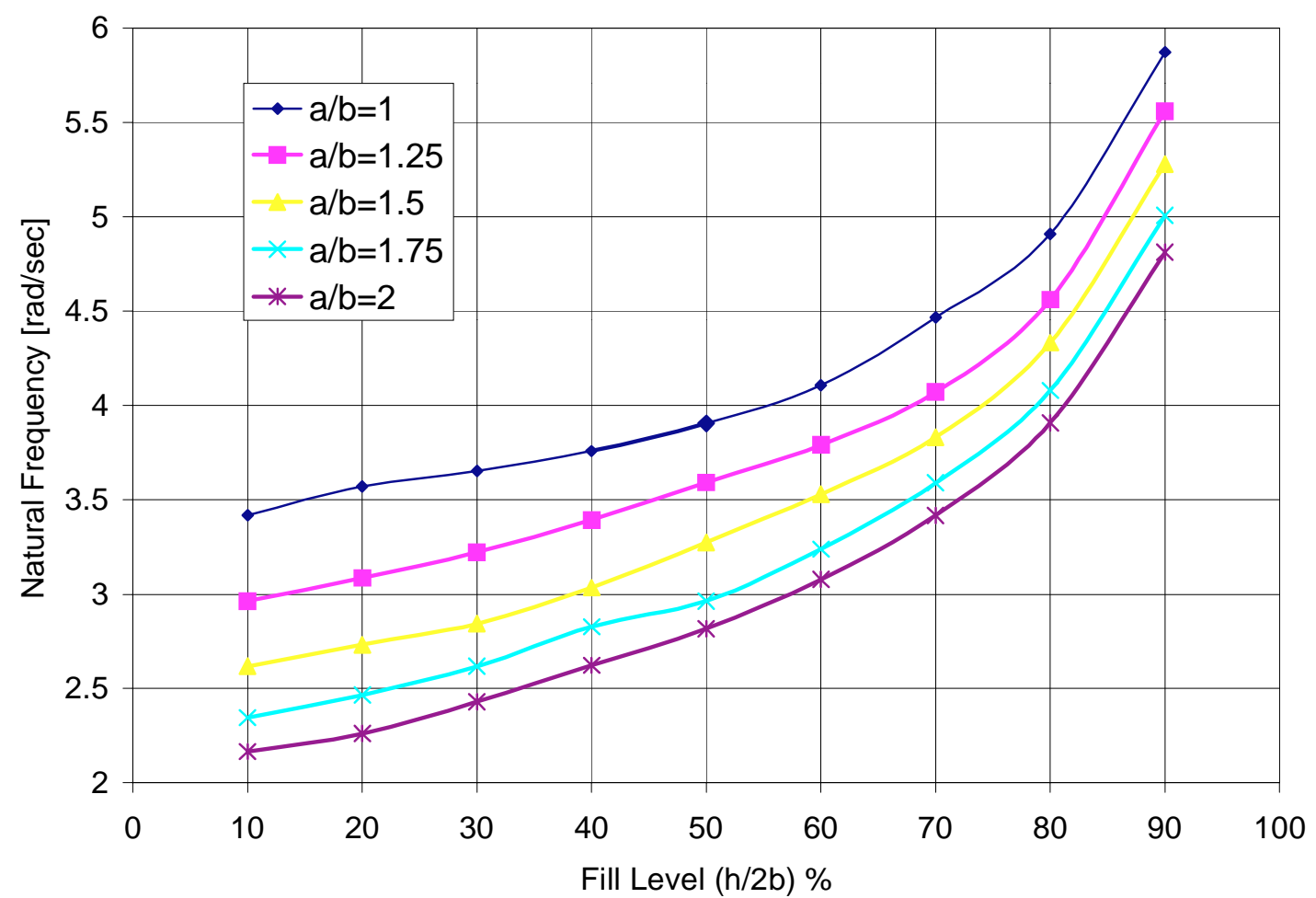

Figure 4.9: Natural frequency vs. fill level for a fluid volume in an elliptical tank with different values of $\mathrm{a} / \mathrm{b}$ at initial fluid surface angle $=5^{\circ}$ obtained using FEM.

Figure 4.10 show the values of $\bar{b} / \mathrm{b}$ obtained using equation 4.11 and the natural frequencies shown in Figure 4.9.

A family of parabolas has been used to best fit the data shown in Figure 4.10. Equation 4.13 was showed a coefficient of correlation of 0.98 in representing the values of $\bar{b} / \mathrm{b}$ for different values of $\mathrm{a} / \mathrm{b}$ up to 0.9 fill level, which is well above any reported critical level of fill $(0.5-0.75)$ for any tanker. 


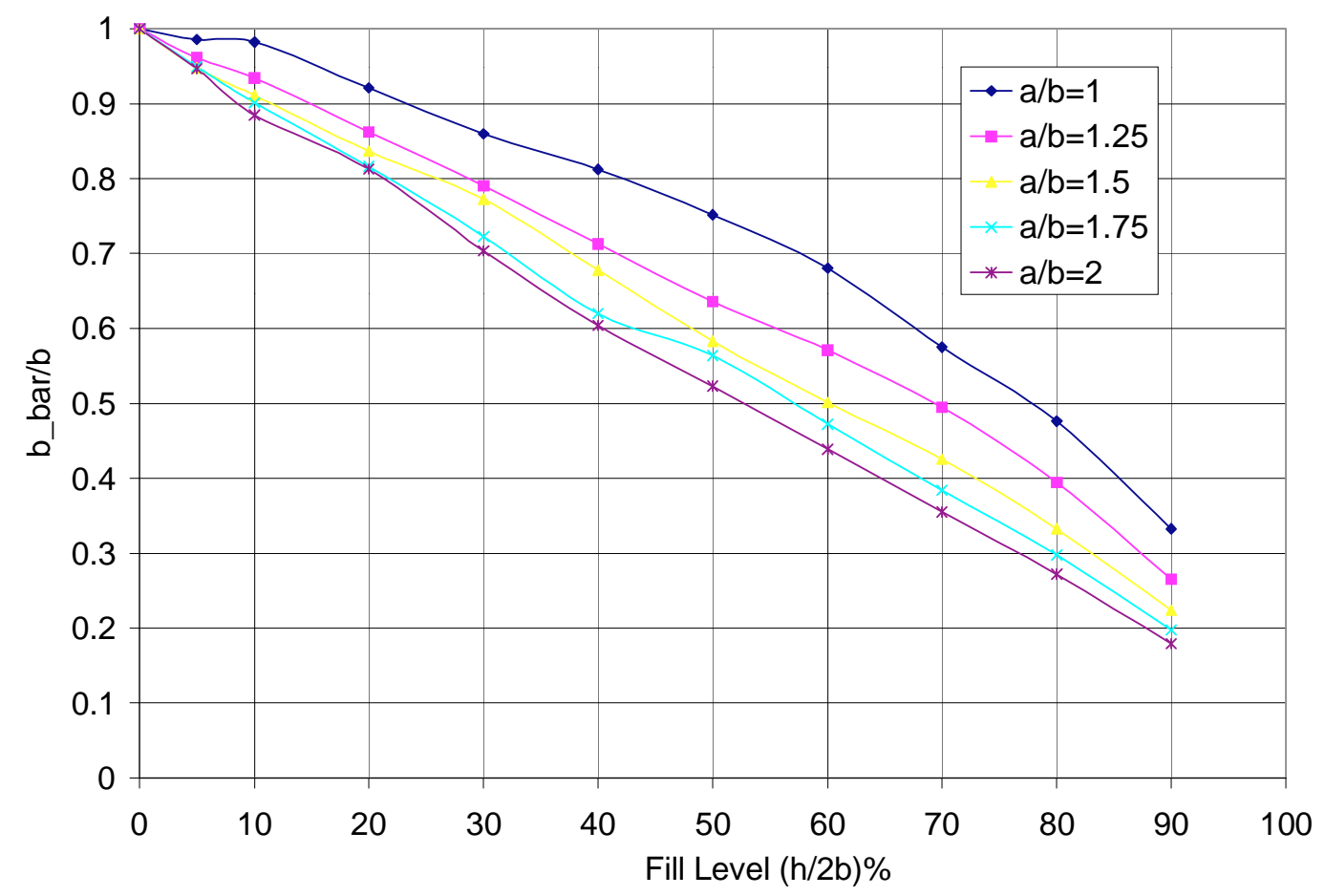

Figure 4.10: FEM results for $\bar{b} / \mathrm{b}$ vs fill level at different values of a/b at initial fluid surface angle $=5^{\circ}$

$$
\frac{\bar{b}}{\mathrm{~b}}=1+\left(1.780896+\frac{1.542048}{(\mathrm{a} / \mathrm{b})}\right)\left(\frac{\mathrm{h}}{2 \mathrm{~b}}\right)+\left(0.7726259-\frac{1.304727}{(\mathrm{a} / \mathrm{b})}\right)\left(\frac{\mathrm{h}}{2 \mathrm{~b}}\right)^{2}=\Lambda
$$

Once $\bar{b}$ is found from equation 4.13 , $\bar{a}$ can be found from equation 4.1.

Equation 4.13 is plotted in Figure 4.11. It can be seen that the curve corresponding to $\bar{b} / \mathrm{b}$ at $\mathrm{a} / \mathrm{b}=1$ (circular tank) is in excellent agreement with the $1_{\mathrm{p}} / \mathrm{R}$ curve obtained by Ranganathan et. al. (1993) and shown in Figure 2.18. 


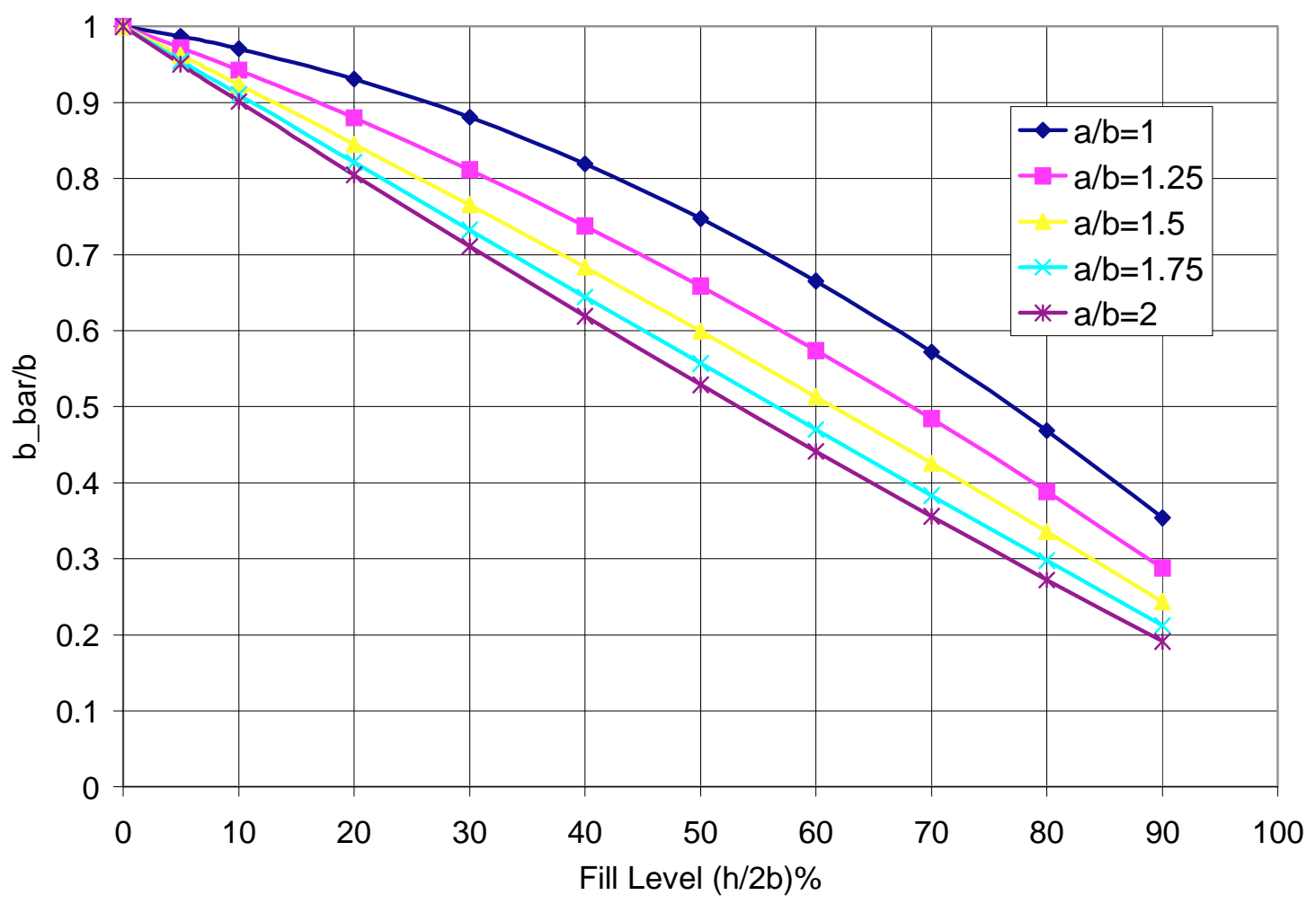

Figure 4.11: Results for $\bar{b} / \mathrm{b}$ vs fill level at different values of a/b at initial fluid surface angle $=5^{\circ}$ obtained using equation 4.13

It was expected that the family of the curves obtained should pass at $\bar{b} / \mathrm{b}=0$ at $\mathrm{h} / 2 \mathrm{~b}=100$. However, the curve for $1_{\mathrm{p}} / \mathrm{R}$ obtained by Ranganathan et. al. (1993) shows that this is not the case. Also, problems that are related to the FEM stability in LS-Dyna 3D prevented the extension of the results beyond the $90 \%$ level of fill.

Figure 4.12 shows the error $\%$ between the $\bar{b} / \mathrm{b}$ value obtained using the FE models and the values predicted using equation 4.13. It can be seen that the maximum absolute value of the error at the critical zone of level of fills (50\% to $75 \%$ ) is below $4 \%$ 


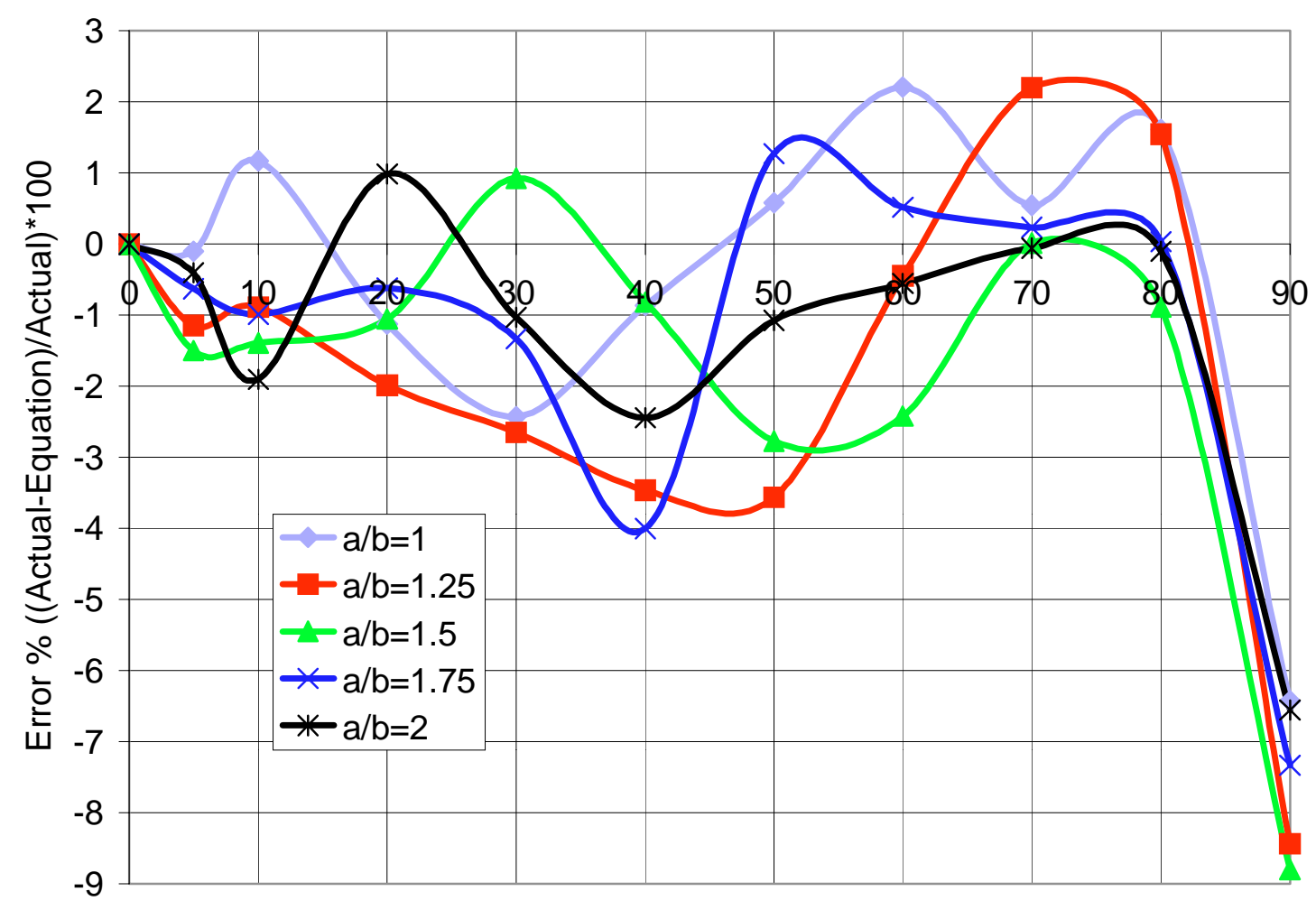

Fill Level (h/2b) \%

Figure 4.12: Error \% in estimating $\bar{b} / \mathrm{b}$ using equation 4.13

Figure 4.13 shows the plot of $\bar{b} / \mathrm{b}_{\mathrm{cg}}$ for $\mathrm{a} / \mathrm{b}$ varying from 1 to 2 and fill levels from 0 to $90 \%$. It can be seen that the value of $\bar{b}$ required to obtain the same natural frequency is always higher than that of the $\mathrm{CG}$ of the fluid mass and reaches its maximum value at higher levels of fill. 


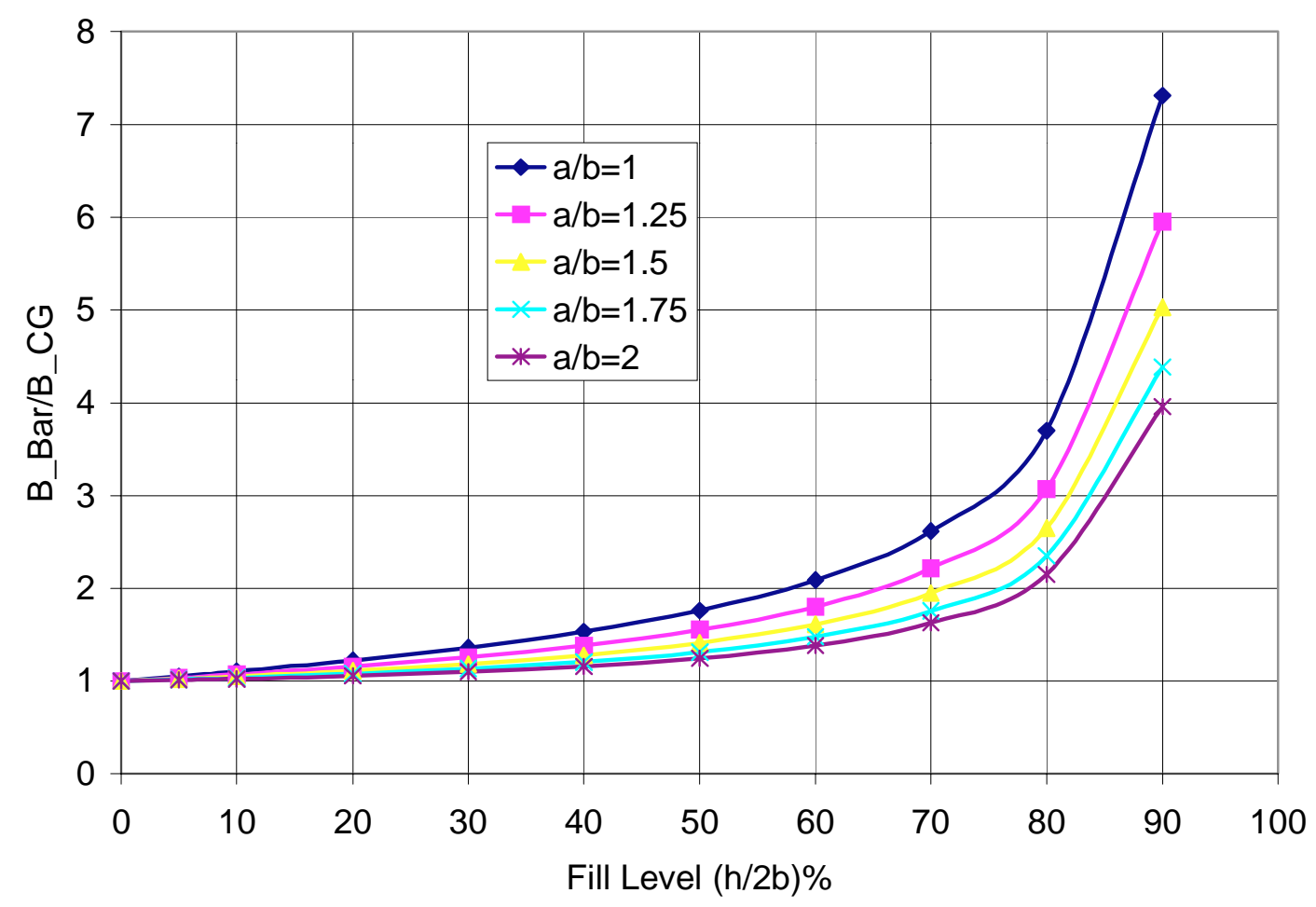

Figure 4.13: $\bar{b} / \mathrm{b}_{\mathrm{cg}}$ for different levels of fill and $\mathrm{a} / \mathrm{b}$ varying from 1 to 2

\subsection{2- Pendulum-Fluid Horizontal Force Component Matching}

The second step in the Pendulum-Fluid slosh dynamic matching is to match the lateral force equation to get the pendulum mass $\left(\mathrm{M}_{\mathrm{p}}\right)$.

The total lateral force due to fluid sloshing in a partially filled elliptical tank according Bohn is given by:

$$
\mathrm{F}_{\mathrm{x}}=\frac{\mathrm{WTF}_{2}}{\overline{\mathrm{V}}}
$$

Where WT is the fluid weight and $\mathrm{F}_{2}$ is the fluid slosh wave height given by the following ODE:

$$
\ddot{F}_{2}+\frac{2 g l \bar{V}}{3 C_{2}^{2}(V o l)} F_{2}=-\frac{\ddot{X}}{C_{2}}
$$


Where $\mathrm{C}_{2}=\frac{2 l \bar{V}}{3(\mathrm{Vol})}$

Substituting for $\mathrm{C}_{2}$ in 4.15 and solving for $\mathrm{F}_{2}$ yields:

$$
\mathrm{F}_{2}=-\left(\frac{\overline{\mathrm{V}}}{\mathrm{g}} \ddot{\mathrm{X}}+\frac{21 \overline{\mathrm{V}}^{3}}{3 \mathrm{~g}(\mathrm{Vol})} \ddot{\mathrm{F}}_{2}\right)
$$

Substituting for $\mathrm{F}_{2}$ in 4.15 and knowing that $\mathrm{WT}=\mathrm{m}_{1} \mathrm{~g}$ and that $\frac{m_{l}}{V o l}=\rho_{l}$ yields:

$$
F_{x}=-\left(m_{l} \ddot{X}+\frac{2}{3} \rho_{l} \bar{V}^{2} \ddot{F}_{2}\right)
$$

Equation 4.16 describes the lateral force component due to first mode of fluid sloshing in a partially filled elliptical tank. Again, this equation was checked by assuming a cylindrical tank case as a special case of an elliptical tank, which has an expression derived earlier by Budiansky (1960) in equation 2.4.

For a cylindrical tank, $\bar{V}=a R$ and $\mathrm{F}_{2}=\xi_{1}$. Thus the lateral force due to the first mode of fluid sloshing in a partially filled cylindrical tank according to Bohn's approach (equation 4.16) is given by:

$$
F_{x}=-\left(m_{l} \ddot{X}+\frac{2}{3} \rho_{l}(a R)^{2} \ddot{\xi}_{1}\right)
$$

By comparing equation 4.17 and equation 2.4 , it can be concluded that $\mathrm{B}_{1}$ is equal to $1 / 3$ in equation 4.17. It can also be seen that the value of $B_{1}$ is independent of the fluid level in equation 4.17, unlike the value of $\mathrm{B}_{1}$ in equation 2.4 (see Figure 4.14). This constitutes another concern about the lateral force equation derived by Bohn (equation 4.16). 


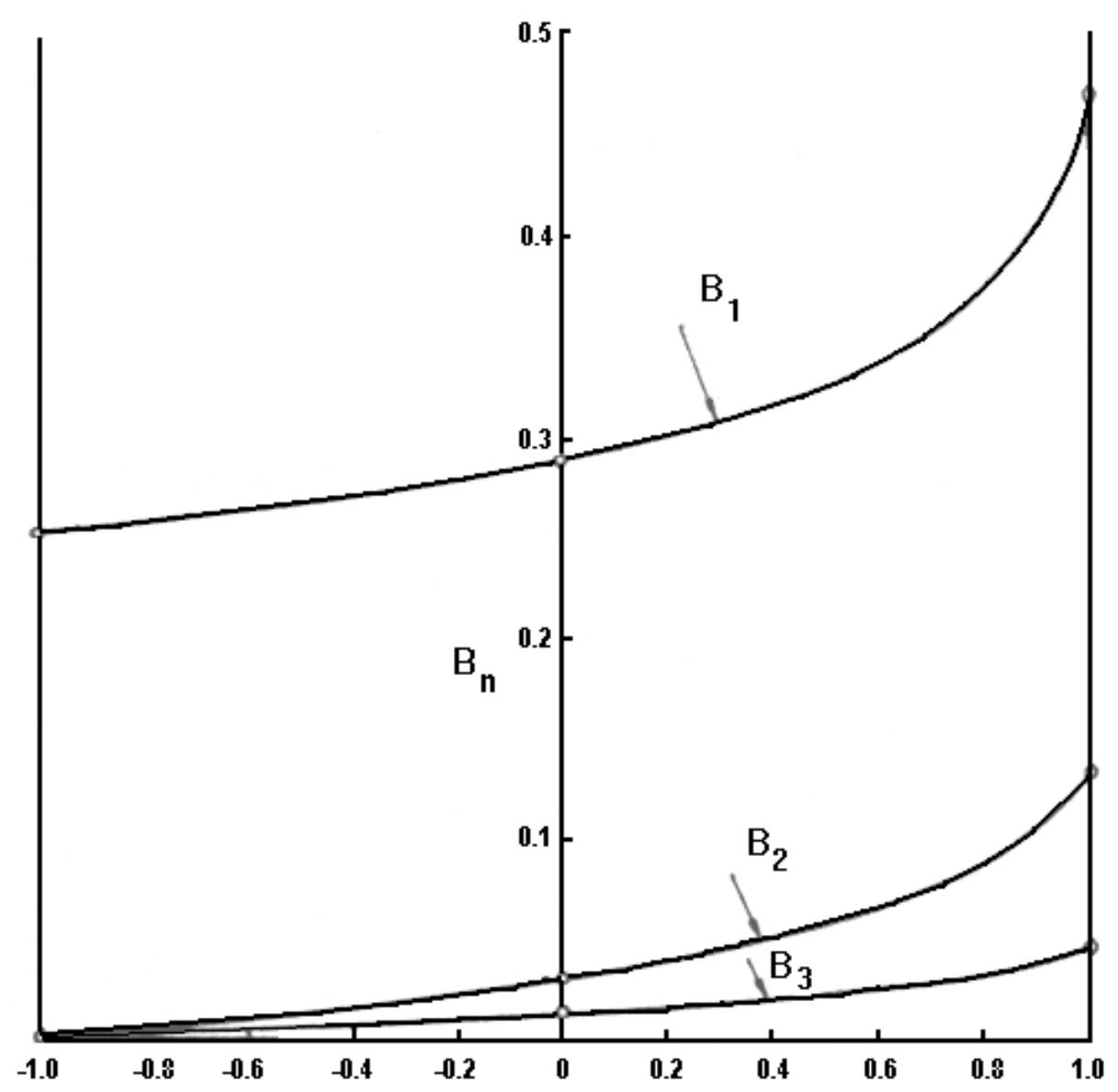

Figure 4.14: Dimensionless parameter $B_{n}$ for a circular tank, Budiansky (1960)

Since the values of $\bar{b}$ and $\bar{a}$ are already known from equations 4.13 and 4.1 respectively, the value of the pendulum mass $\mathrm{M}_{\mathrm{p}}$ has been found by matching the maximum horizontal force resulting from FE fluid slosh models and the maximum horizontal force resulting from the pendulum motion at point "D" (see Figure 4.5). Same FE models shown in Figure 4.7 were used with an initial value for the fluid surface angle of tilt $=5^{\circ}$. The fluid was lift to oscillate freely under gravity to eliminate the effect of an external acceleration on the fixed part of the fluid mass. The maximum horizontal forces from these models were evaluated and compared to the value maximum force resulting from equivalent free oscillation of the trammel pendulum. The fact that the initial angle of the trammel pendulum is dependent on the value of $a / b$ since it's defined by the location of the fluid volume CG was taken into consideration. Table 4.2 shows 
the values for $\theta_{\mathrm{cg}}$ equivalent to a fluid surface tilt of $5^{\circ}$ at different values of a/b (see Figures 4.3 and B.2)

Table 4.2: Initial pendulum angle at different values of $\mathrm{a} / \mathrm{b}$ for a $5^{\circ}$ fluid surface initial tilt angle

\begin{tabular}{|c|c|c|c|c|c|}
\hline $\mathrm{a} / \mathrm{b}$ & 1 & 1.25 & 1.5 & 1.75 & 2 \\
\hline$\alpha$ & 5 & 7.7801 & 11.1341 & 14.9676 & 18.7265 \\
\hline$\theta_{\mathrm{cg}}=\theta=\tan ^{-1}\left(\frac{\overline{\mathrm{b}}}{\overline{\mathrm{a}}} \tan \alpha\right)$ & 5 & 6.238 & 7.4777 & 8.6858 & 9.7733 \\
& & & & & \\
\hline
\end{tabular}

When the pendulum is left to swing freely assuming that the total mass of the fluid is at the tip of the pendulum, the horizontal component of force is given by: $\mathrm{F}_{\mathrm{H}}=\mathrm{M}_{\mathrm{t}} * \mathrm{a}_{\mathrm{x}}$ Where $\mathrm{a}_{\mathrm{x}}$ is the horizontal component of acceleration at the tip of the pendulum and is given by the "i" component of the acceleration vector $\ddot{r}$ given in equation B17 when $\ddot{x}=0$.

Thus, by equating the maximum horizontal force from the FE models with the maximum horizontal force of the pendulum, $\mathrm{F}_{\mathrm{H} \_\mathrm{FE} \_\max }=\max \left(\mathrm{M}_{\mathrm{p}} \mathrm{a}_{\mathrm{x}}\right), \therefore \mathrm{F}_{\mathrm{H} \_\mathrm{FE} \_\max } / \mathrm{F}_{\mathrm{H}}=\mathrm{M}_{\mathrm{p}} / \mathrm{M}_{\mathrm{t}}$.

Figure 4.15 shows the maximum force obtained from the $\mathrm{FE}$ results using a $0.1 \mathrm{~m}$ thickness elliptical slice filled with water with same cross section as the model tank. It can be seen that the maximum horizontal force is around $60 \%$ level of fill for all values of a/b varying from 1 to 2 . Figure 4.16 shows the value of the maximum force obtained from the FE models normalized over the weight of the fluid. It indicates that the lower the level of fill, the higher the horizontal force per unit weight of the fluid, i.e. the more the sloshing portion of the mass of the fluid.

The results of equation 4.18 are plotted in Figure 4.17. Best fit has been performed on the data shown in Figure 4.14, which resulted in the equation 4.19. 


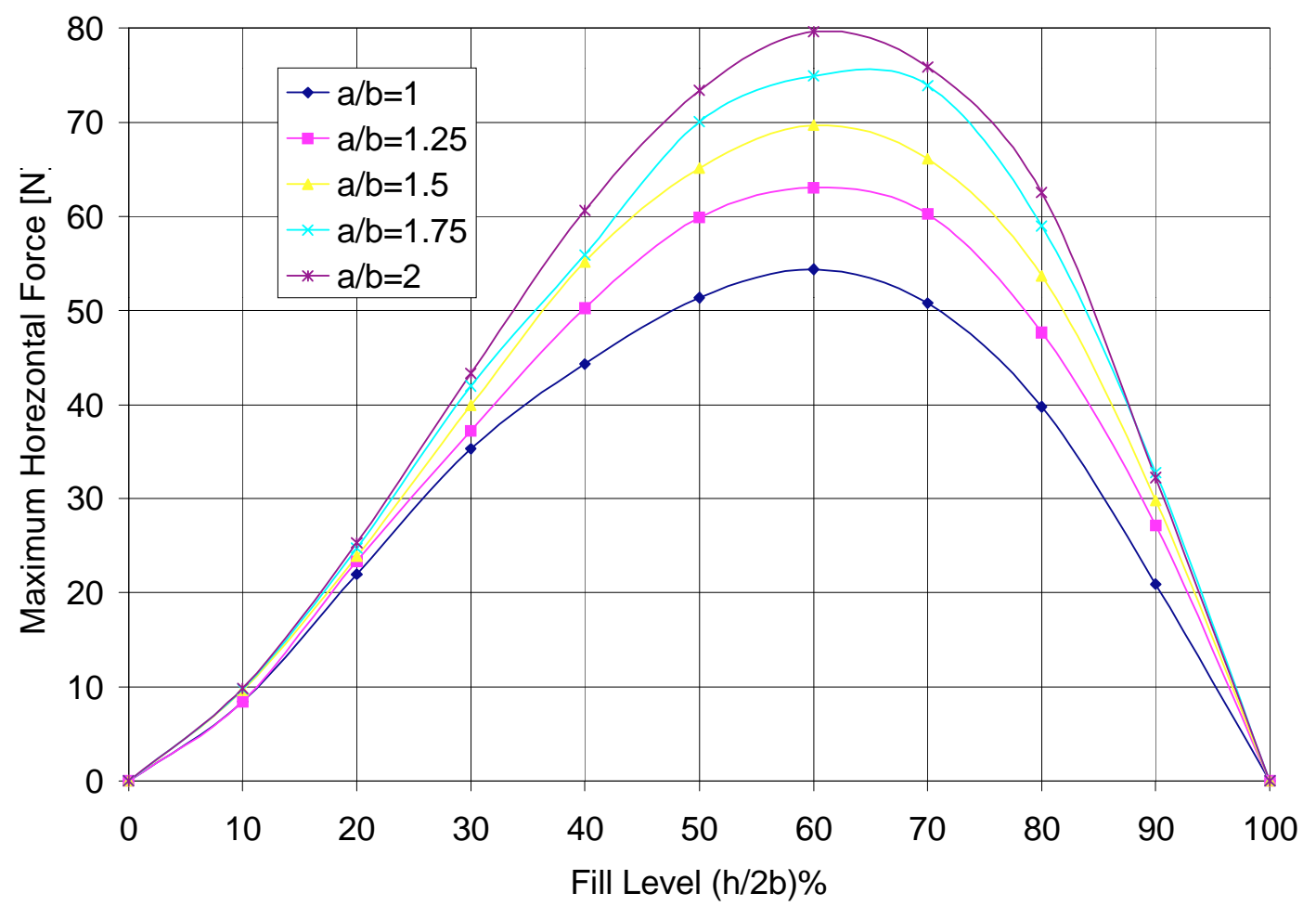

Figure 4.15: Maximum horizontal component of sloshing force vs fill level using water in an 0.1 thick elliptical slice

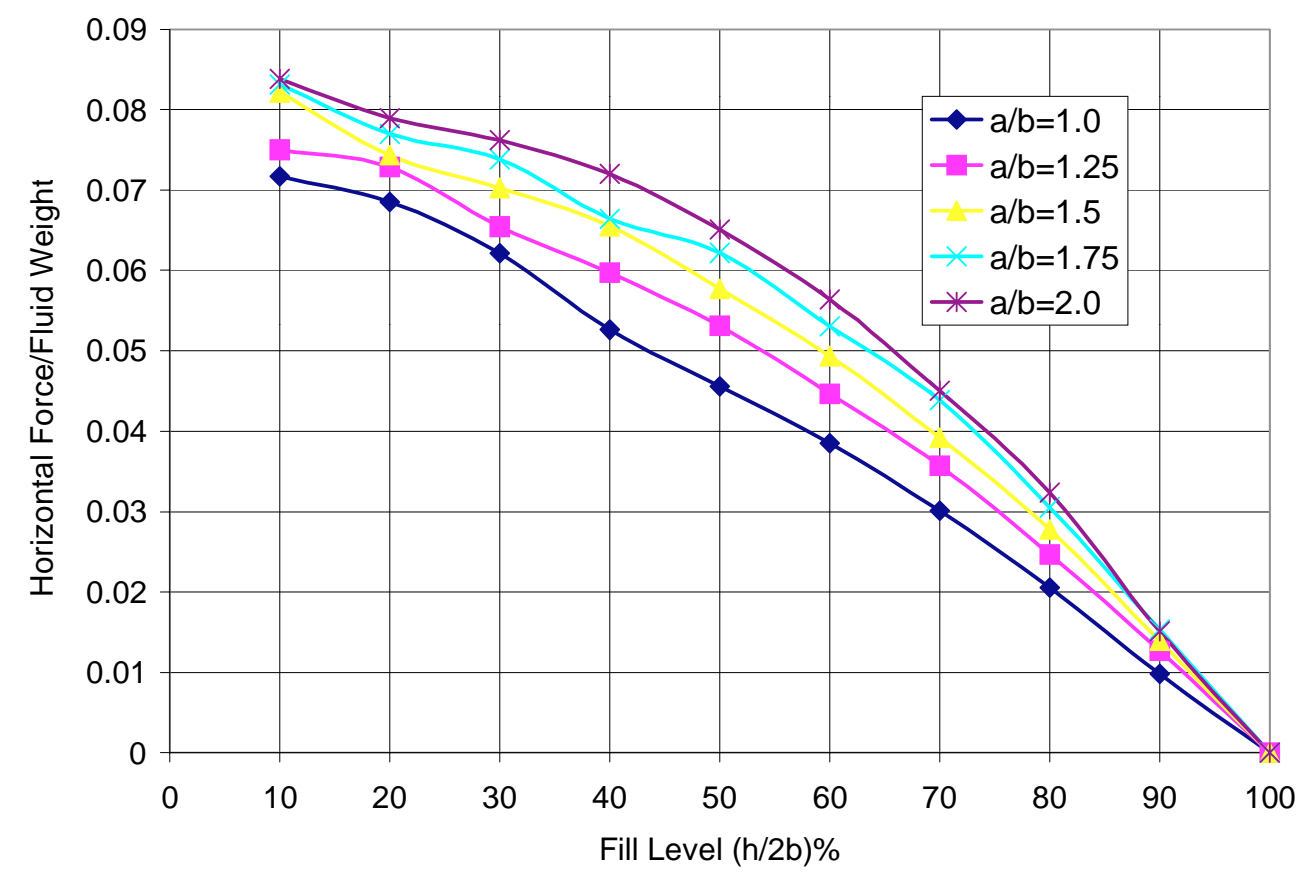

Figure 4.16: Maximum horizontal component of sloshing force per unit fluid weight vs fill level in an 0.1 thick elliptical slice 


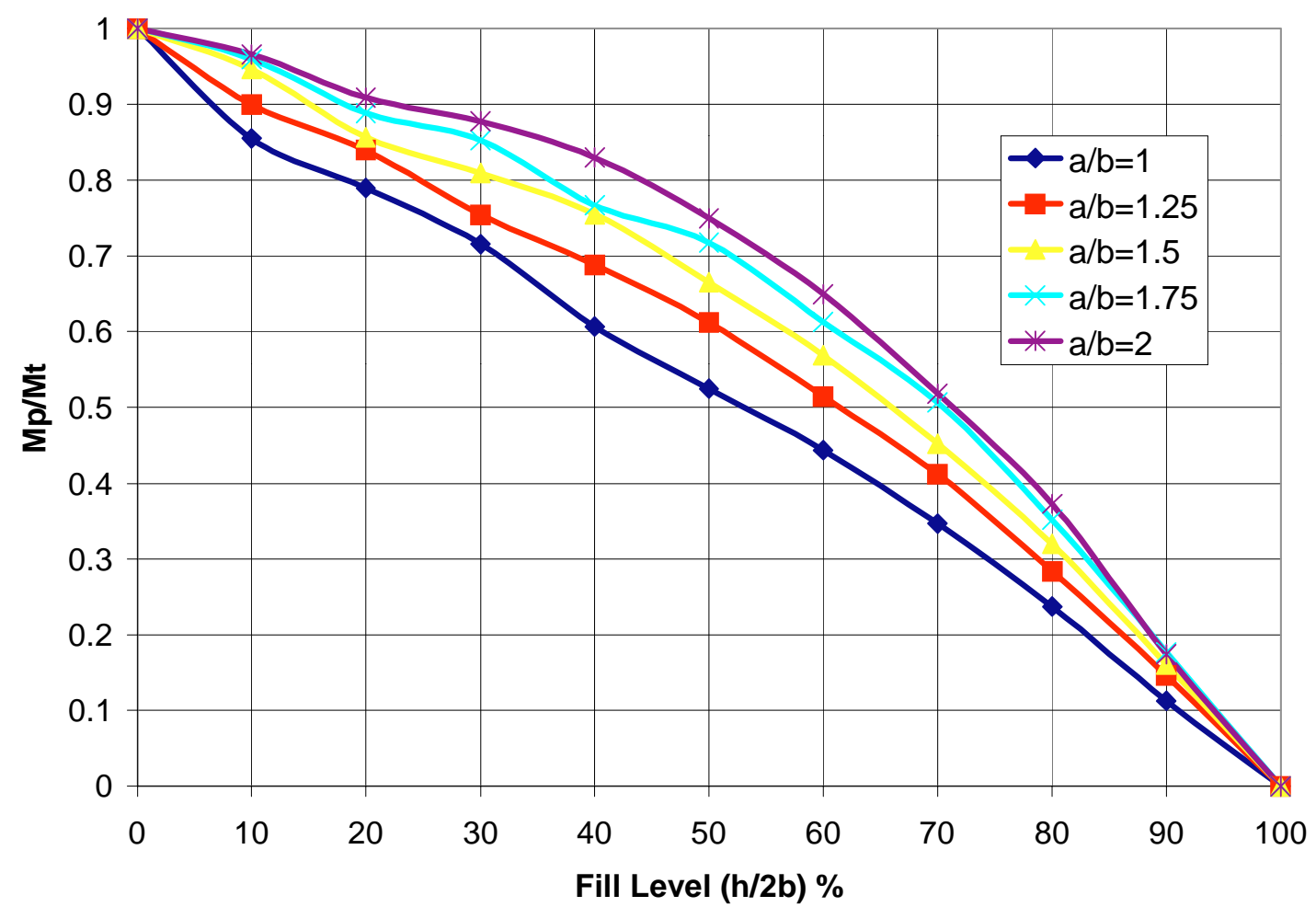

Figure 4.17: $\mathrm{M}_{\mathrm{p}} / \mathrm{M}_{\mathrm{t}}$ vs fill level obtained from FE results at different values of $\mathrm{a} / \mathrm{b}$

$$
\frac{\mathrm{M}_{\mathrm{P}}}{\mathrm{M}_{\mathrm{t}}}=1+\left[-0.863+1.237 \ln \left(\frac{\mathrm{a}}{\mathrm{b}}\right)\right]\left(\frac{\mathrm{h}}{2 \mathrm{~b}}\right)-\left[0.1226+1.2489 \ln \left(\frac{\mathrm{a}}{\mathrm{b}}\right)\right]\left(\frac{\mathrm{h}}{2 \mathrm{~b}}\right)^{2}=\Phi
$$

Equation 4.19 has a minimum coefficient of correlation of 0.9983 in presenting the data shown in Figure 4.17.

A plot for the results of equation 4.19 is given in Figure 4.18 for a/b varying from 1 to 2 . The curve for $a / b=1$ is in excellent agreement with the $M_{p} / M_{t}$ curve obtained by Ranganathan et. al. (1993) and is shown in Figure 2.8.

Figure 4.19 shows the error $\%$ between the predicted values of Mp/Mt using the LSDyna-3D runs and equation 4.19. It can be seen that the maximum error in the critical zone of level of fills (50\% to $75 \%)$ is below $6 \%$. 


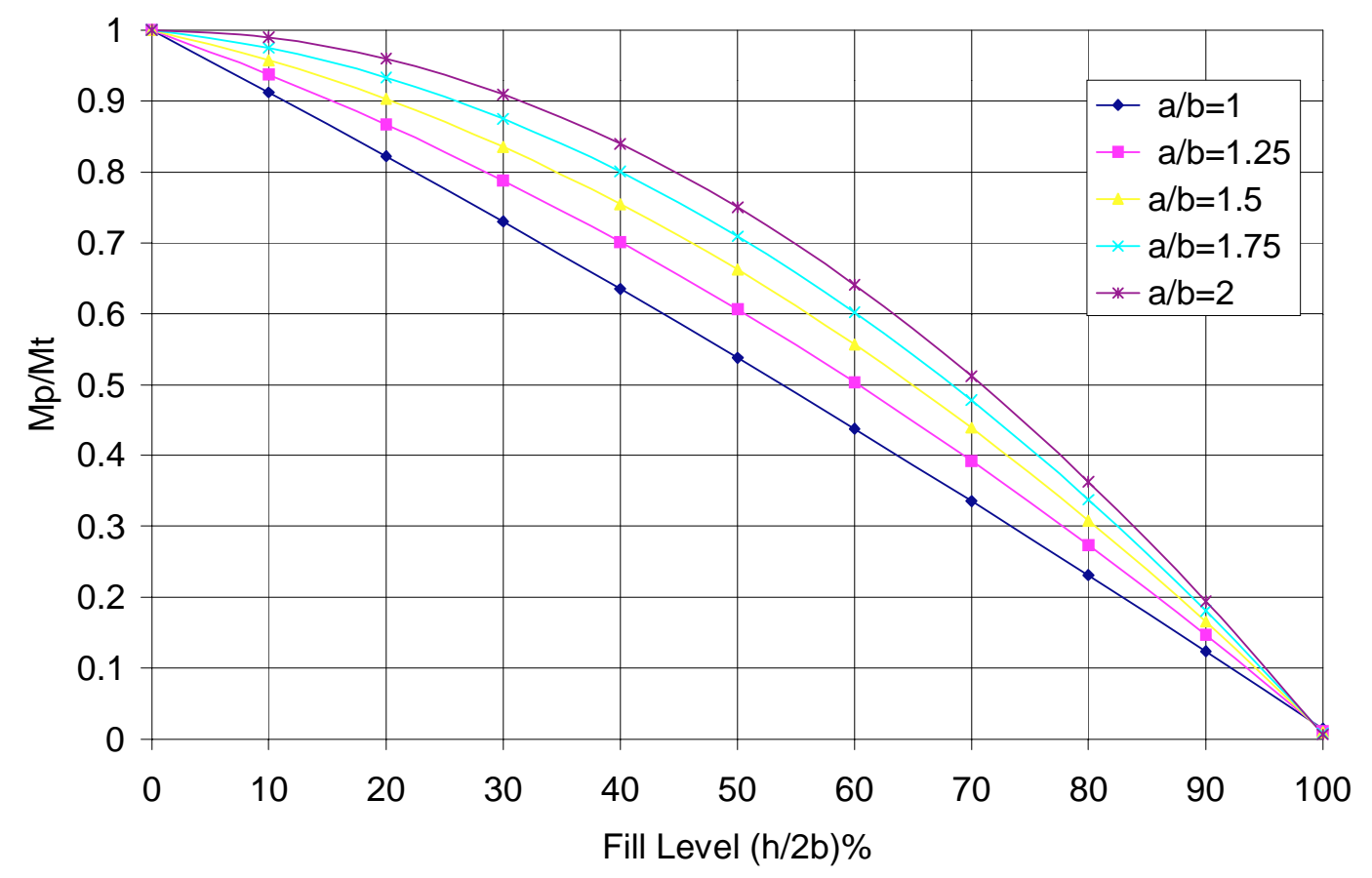

Figure 4.18: $\mathrm{M}_{\mathrm{p}} / \mathrm{M}_{\mathrm{t}}$ vs fill level obtained from Equation 4.19 at different values of $\mathrm{a} / \mathrm{b}$

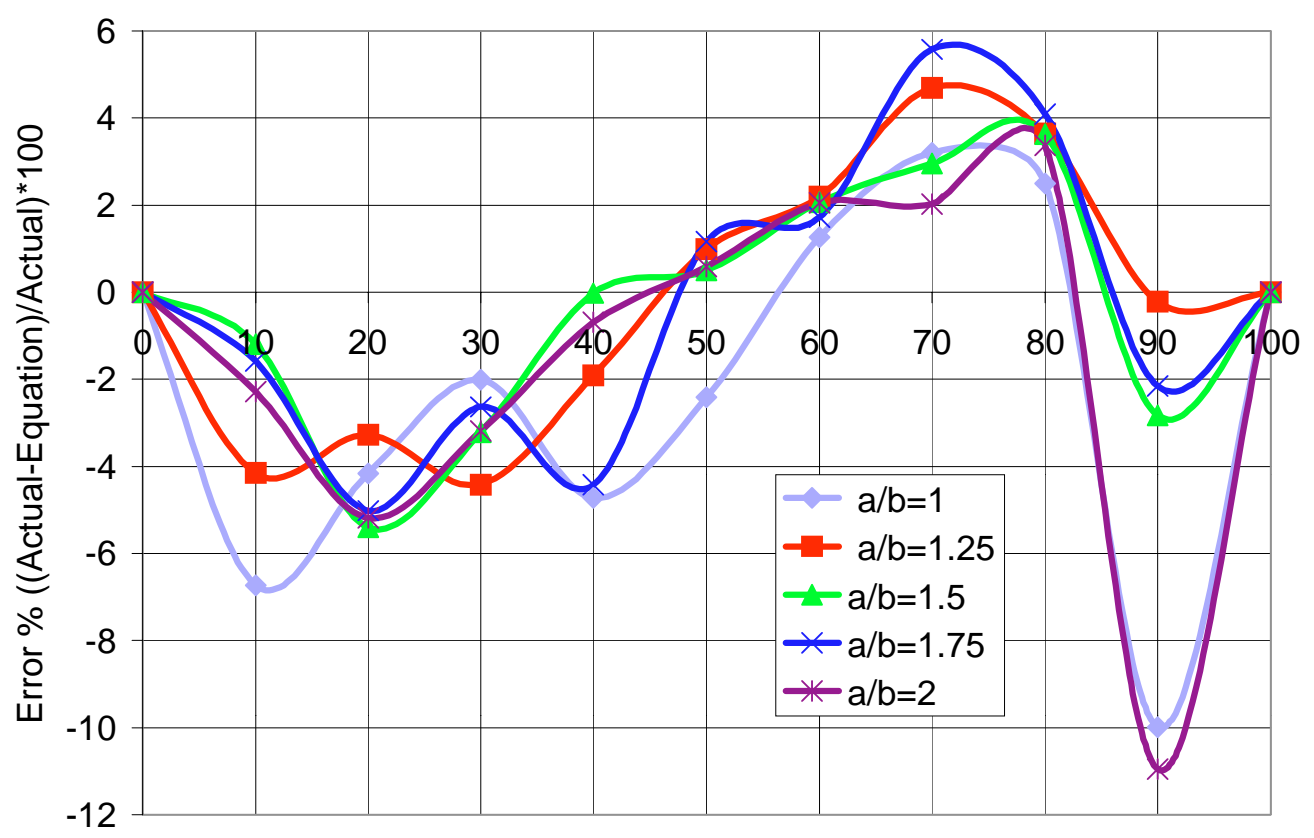

Fill Level \% (h/2b)

Figure 4.19: Error \% in estimating Mp/Mt using Equation 4.19 


\subsection{3- Pendulum-Fluid Static Moment Matching}

To find the height of the fixed mass " $\mathrm{h}_{\mathrm{o}}$ " from the base point of the tank, Abramson (1966) suggested that the static moment of the tank when rolled at a small angle should be the matched. In other words, the CG of the fixed mass and the pendulum mass at the static location should coincide with the fluid's CG.

By taking the mass moment around point "D" gives:

$$
M_{f} h_{o}+M_{p}(b-\bar{b})=M_{t} Y_{C G}
$$

Where $\mathrm{Y}_{\mathrm{CG}}$ is given by equations $\mathrm{B} 6$ and $\mathrm{B} 8$.

By substituting for $\bar{b}=b \Lambda$ (see equation 4.13), $M_{f}=M_{t}-M_{p}$, and $M p=M_{t} \Phi$ (see equation 4.19) in equation 4.20 , rearranging and dividing by b, equation 4.20 can be written as:

$$
\frac{\mathrm{h}_{\mathrm{o}}}{\mathrm{b}}=\frac{\mathrm{Y}_{\mathrm{CG}}-\Phi \mathrm{b}(1-\Lambda)}{\mathrm{b}(1-\Phi)}
$$

The values of $h_{o} / b$ have been evaluated using equation 4.21 at levels of fill varying from $0 \%$ too $100 \%$ for different values of $\mathrm{a} / \mathrm{b}$ and are plotted in Figure 4.20 . It can be seen that the results obtained for $a / b=1$ as well as the other values for different ratios of $a / b$ agree with the trend obtained by Raganathan et. al (1993) shown in Figure 2.18 which is $h_{o} / b \cong 1$ except for very low levels of fill.

There is no need to curve fit the data shown in Figure 4.20 since they have been obtained analytically. Also the fact that the results obtained for $h_{o} / b$ are very close to " 1 " verifies that the curve fitting used for the pendulum parameters (equations 4.13 and 4.19) is a suitable choice. 


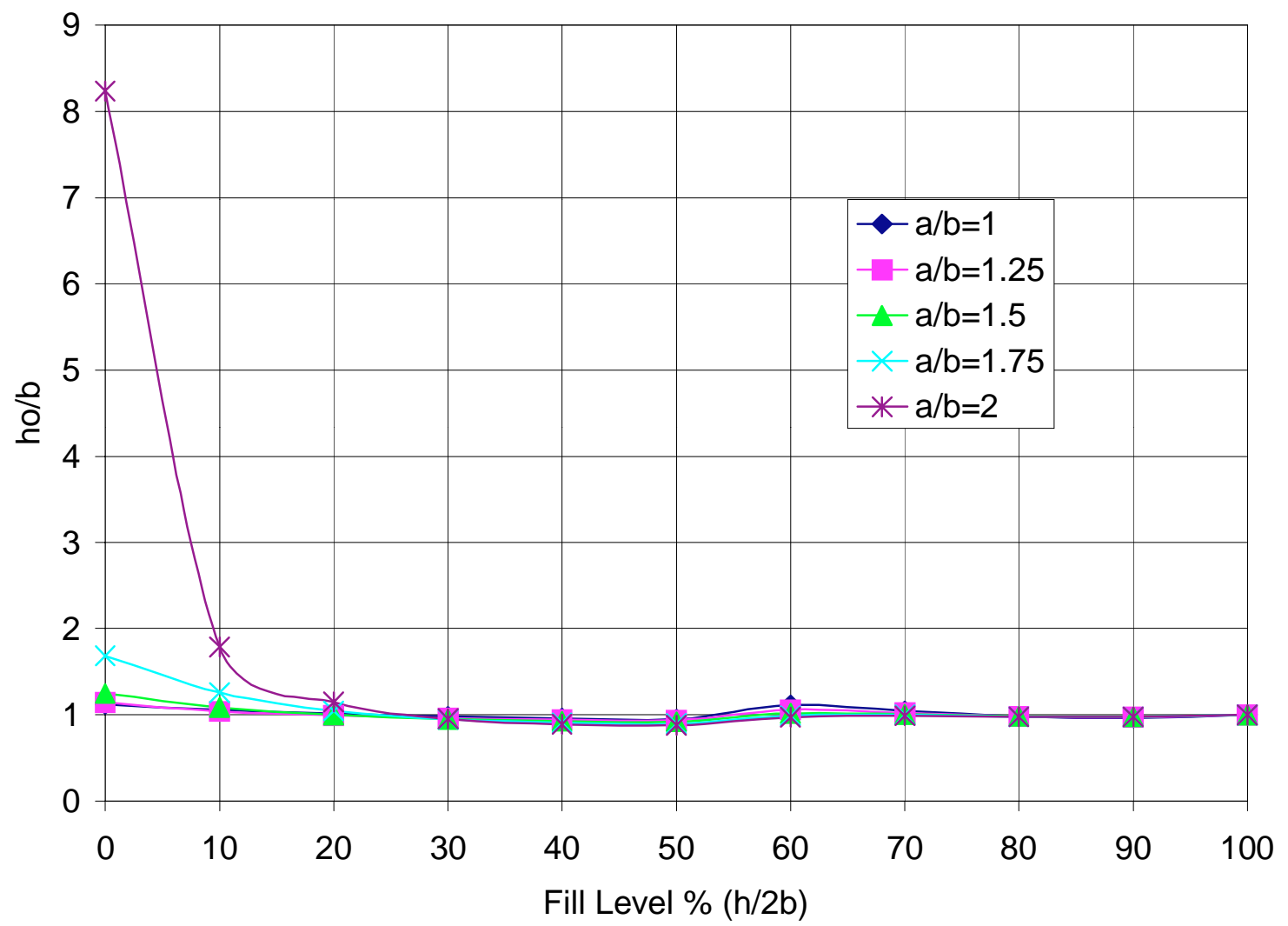

Figure 4.20: $h_{o} / b$ vs fill level at different values of a/b obtained using Equation 4.21

\section{5- Trammel Pendulum Fluid Simulation Verification.}

Since all the pendulum parameters have been obtained in the previous parts, the next step is to verify how close can a trammel pendulum simulate fluid sloshing effects on a partially filled elliptical tank. The roll moment about point " $\mathrm{D}$ " has been selected for this purpose. This was achieved by applying a certain acceleration to the tank and compare the maximum moment due to fluid sloshing obtained using LS_Dyna-3D "M" around point D with that of the trammel pendulum. Figures $4.21 \mathrm{a}$ and $\mathrm{b}$ show the FE model used to conduct this analysis at the initial state and after applying the acceleration values. 


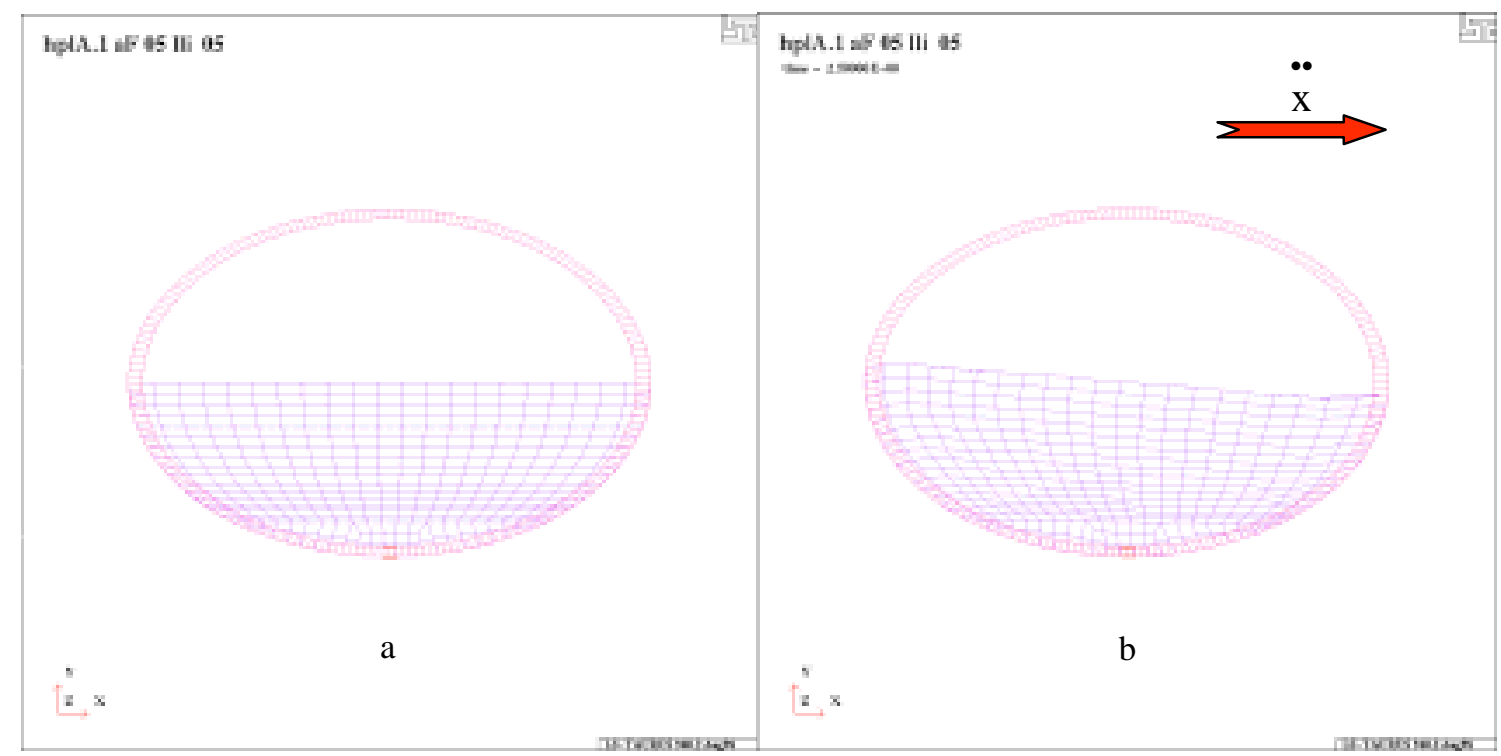

Figure 4.21: FE model used to match the moment around point "D" before and after applying the lateral acceleration

The lateral acceleration chosen was such that the steady state angle around which the fluid surface will rock around is $5^{\circ}$. This angle is independent on the fill level and is given by:

$$
\ddot{x}=g\left(\frac{\tan 5}{a / b}\right)
$$

The moment around point "D" from the pendulum model during applying a lateral acceleration $\ddot{x}$ has two components; the constant moment component due to the fixed mass inertia $=-\mathrm{M}_{\mathrm{f}} \ddot{\mathrm{x}} \mathrm{h}_{\mathrm{o}}$, and the variable moment component coming from the pendulum mass and is given by:

$$
\mathrm{M}_{\mathrm{v}}=-\mathrm{M}_{\mathrm{p}}(\ddot{\mathrm{r}}+\mathrm{g}) \times \overline{\mathrm{P}}
$$

Where $\ddot{r}$ is the acceleration vector of the pendulum mass given by equation B.17 and P is the position vector of the pendulum mass from point "D" (see Figure 4.5). P is given by:

$$
\overline{\mathrm{P}}=\mathrm{r}-\mathrm{x} \overline{\mathrm{i}}+\mathrm{bj}=(\mathrm{a} \sin \theta) \mathrm{i}+(\mathrm{b}-\overline{\mathrm{b}} \cos \theta) \mathrm{j}
$$


Thus, the cross product of equation 4.21 yields:

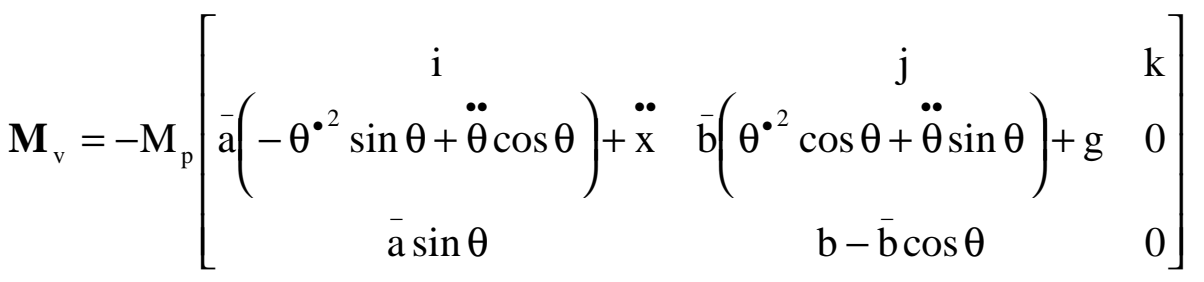

$$
\begin{aligned}
& \therefore \mathbf{M}_{\mathrm{v}}=\mathrm{M}_{\mathrm{p}}\left\{\left[\overline{\mathrm{a}}\left(\theta^{\cdot 2} \sin \theta-\ddot{\theta} \cos \theta\right)-\ddot{\mathrm{x}}\right](\mathrm{b}-\overline{\mathrm{b}} \cos \theta)+\overline{\mathrm{a}} \sin \theta\left[\overline{\mathrm{b}}\left(\theta^{\cdot 2} \cos \theta+\ddot{\theta} \sin \theta\right)+\mathrm{g}\right]\right\}
\end{aligned}
$$

Thus, the maximum moment around point " $\mathrm{D}$ " is given by:-

$$
M_{\max }=\operatorname{Max}\left(M_{v}-M_{f} \ddot{x} h_{o}\right)
$$

Elliptical slice models with the same properties used in the horizontal force matching models and given the same values of acceleration were used to compare the moment values around point "D". Figure 4.22 shows a comparison between the rolling moment values around point " $\mathrm{D}$ " as a function of time at $\mathrm{a} / \mathrm{b}=1.25$ for $30 \%$ and $70 \%$ full obtained using FE fluid models and trammel pendulum models. It can be seen that the frequency of the moment is in good agreement. However, the pendulum models produce slightly higher values of roll moment than the fluid models. This can be attributed to the fact that LS_Dyna 3d Assigns a default low value of damping to the materials used if the damping was assumed zero, or to the filtering process used to filter the FE results from noise due to numerical errors.

Figures 4.23 and 4.24 show the peak absolute values of the moments using FE fluid models and the trammel pendulum models respectively. It can be seen that the trends of the two sets of moments are in excellent agreement. However, the trammel pendulum produces generally higher values of rolling moment, which renders the pendulum models more conservative than the fluid models. 


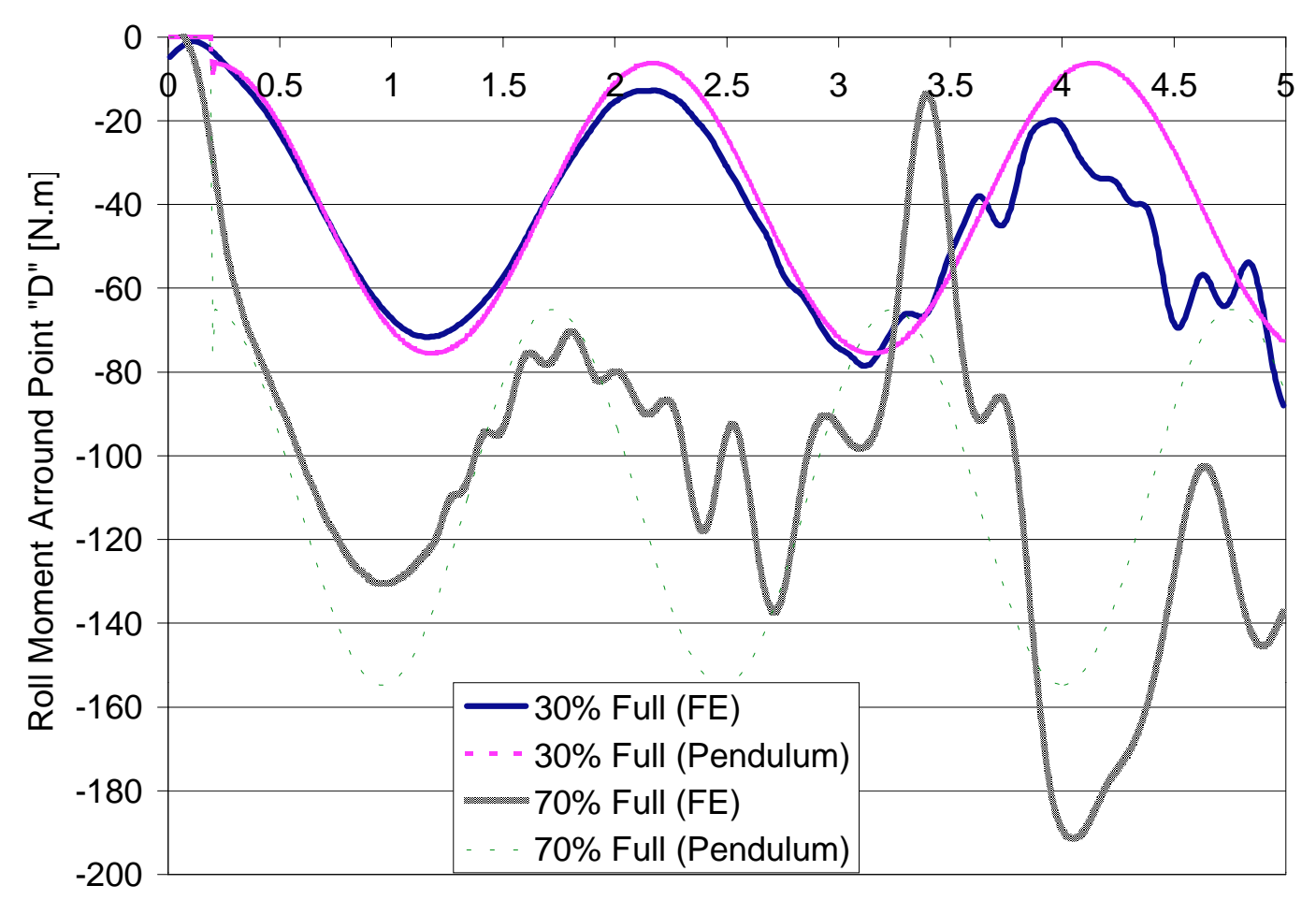

Time [sec]

Figure 4.22: Comparison between the roll moment around point " $D$ " for $\mathrm{a} / \mathrm{b}=1.25$ and fill levels $30 \%$ and $70 \%$ using FE and trammel pendulum models

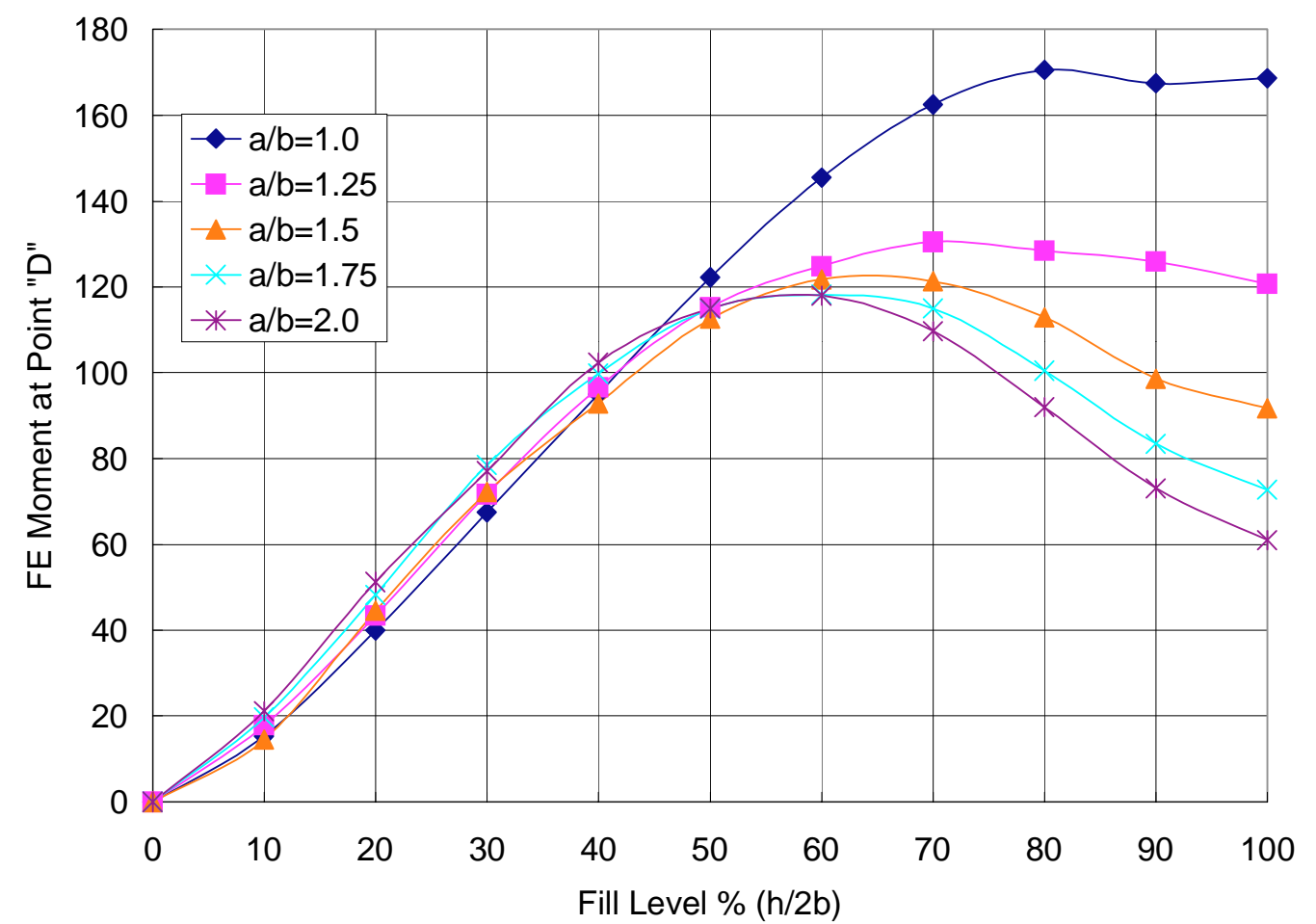

Figure 4.23: Peak values of roll moment at point "D" at constant fluid surface angle using fluid FE models 


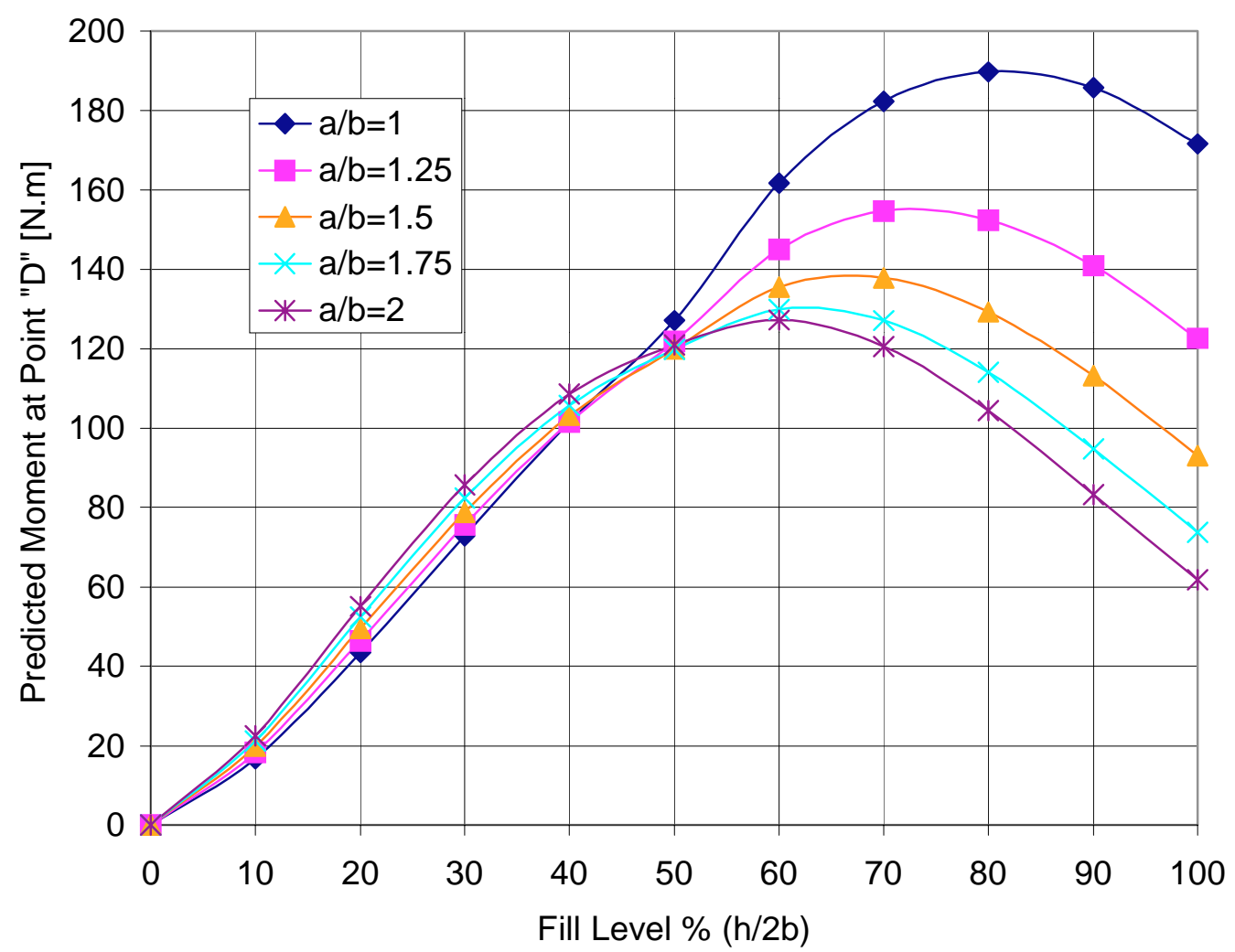

Figure 4.24: Peak values of roll moment at point "D" at constant fluid surface angle, using trammel pendulum models

It can be seen that the peek value of the rolling moment is higher for the circular tank $(\mathrm{a}=\mathrm{b})$, and is around $83 \%$ full, and it drops gradually with the increase of a/b until it becomes at $60 \%$ full a superelliptical tank $(\mathrm{a} / \mathrm{b}=2)$. It can be seen also the peak value of the moment for the circular tank is less than that of an elliptical tank for fill levels less than $45 \%$.

Figure 4.25 shows the maximum moment at point "D" when applying constant acceleration equal $0.05 \mathrm{~g}$ on the trammel pendulum models. It can be seen that the rolling moment is less for circular tanks for fill levels below $83 \%$. Thus, it can be concluded from that that the circular tank is favorable over an elliptical tank if partially filled conditions is expected, while the elliptical tank is favorable for high fill levels and completely full conditions. This conclusion agrees with the literature (Sankar et. al. 1992 A). 


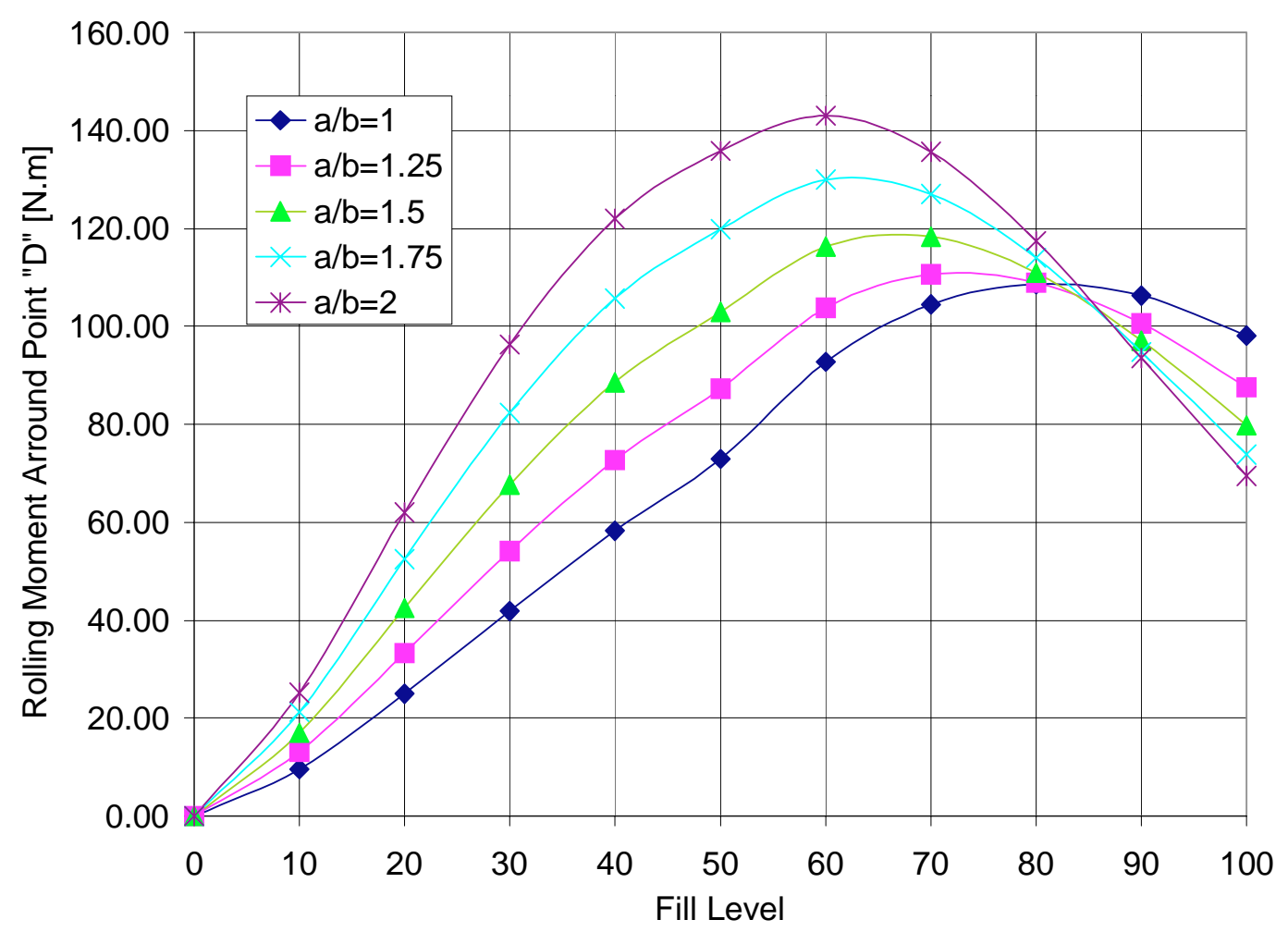

Figure 4.25: Peak moment values at point "D" at constant Lateral acceleration equal $0.05 \mathrm{~g}$

Figures 4.26 through 4.333 show the plots of several other parameters that can shed further light on the trammel pendulum behavior and its ability to simulate fluid sloshing in a partially filled elliptical tank at both constant fluid surface angle 4qual 5 degrees, and constant acceleration equal 0.05g. Namely, those parameters are; the frequency parameter "K", the vertical force coefficient "CFV", the horizontal force coefficient " $\mathrm{CFH}$ " and the rolling moment coefficient "CM". The frequency parameter was defined by Popov et. al. 1993A as:

$$
\mathbf{K}=\frac{\Omega}{\sqrt{g\left[1+\left(\frac{\ddot{x}}{g}\right)^{2}\right] / a}}
$$

Is a normalized parameter that has been used by researchers to relate the sloshing frequency to the input acceleration. 


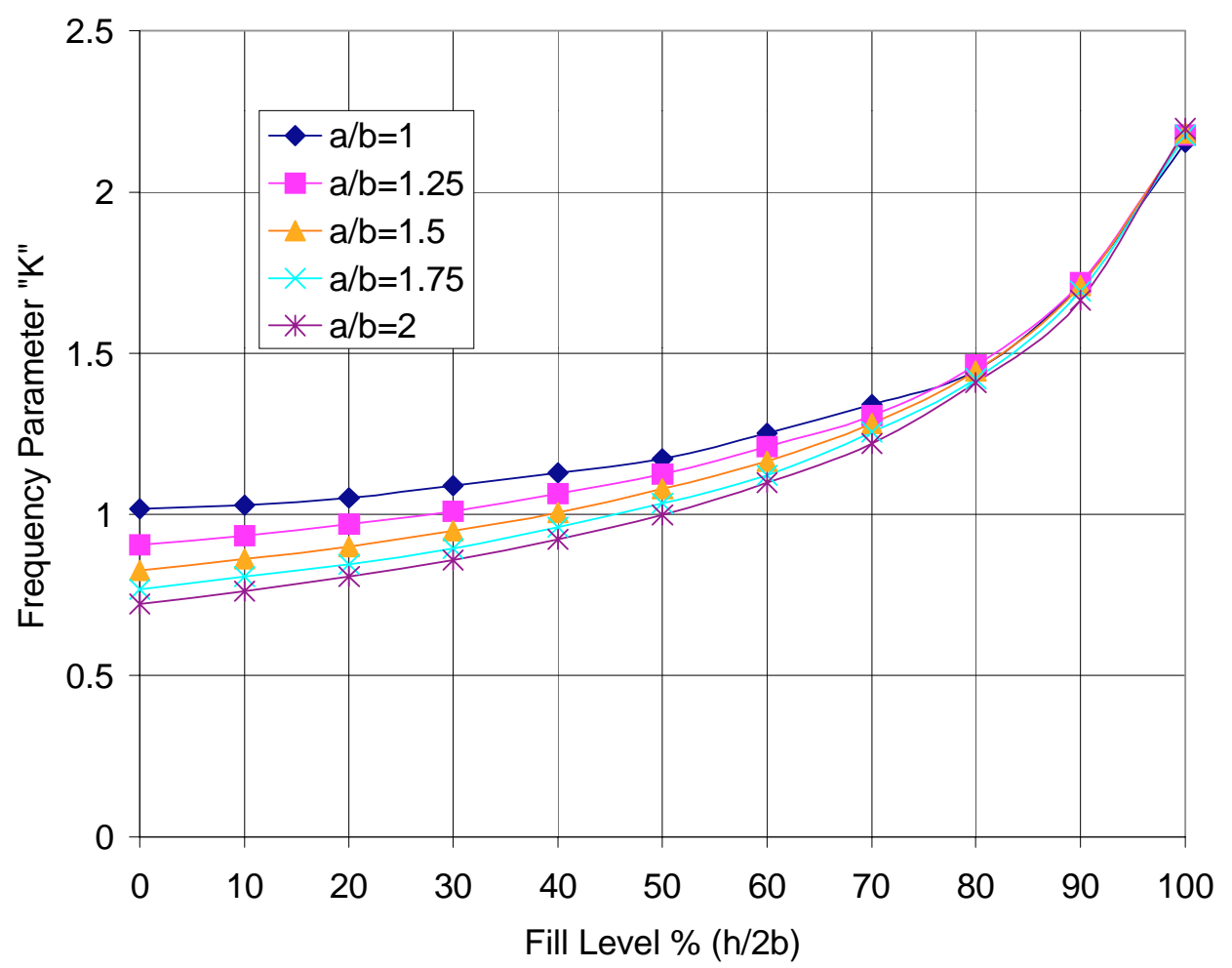

Figure 4.26: Frequency parameter vs fill level for a/b varying from 1 to 2 at constant surface angle (Input acceleration is given by Equation 4.20)

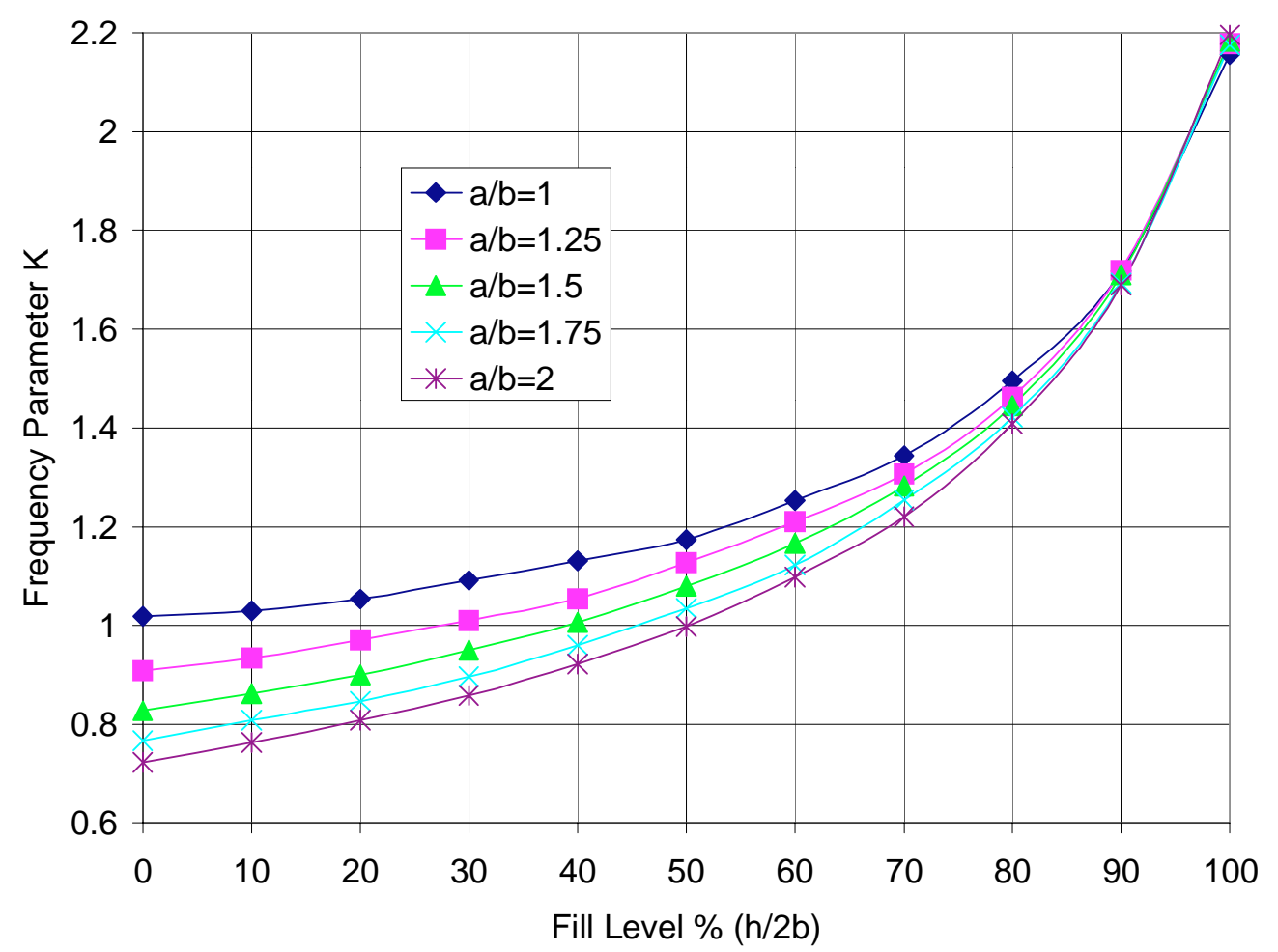

Figure 4.27: Frequency parameter vs fill level for a/b varying from 1 to 2 at constant lateral acceleration $=0.05 \mathrm{~g}$ 


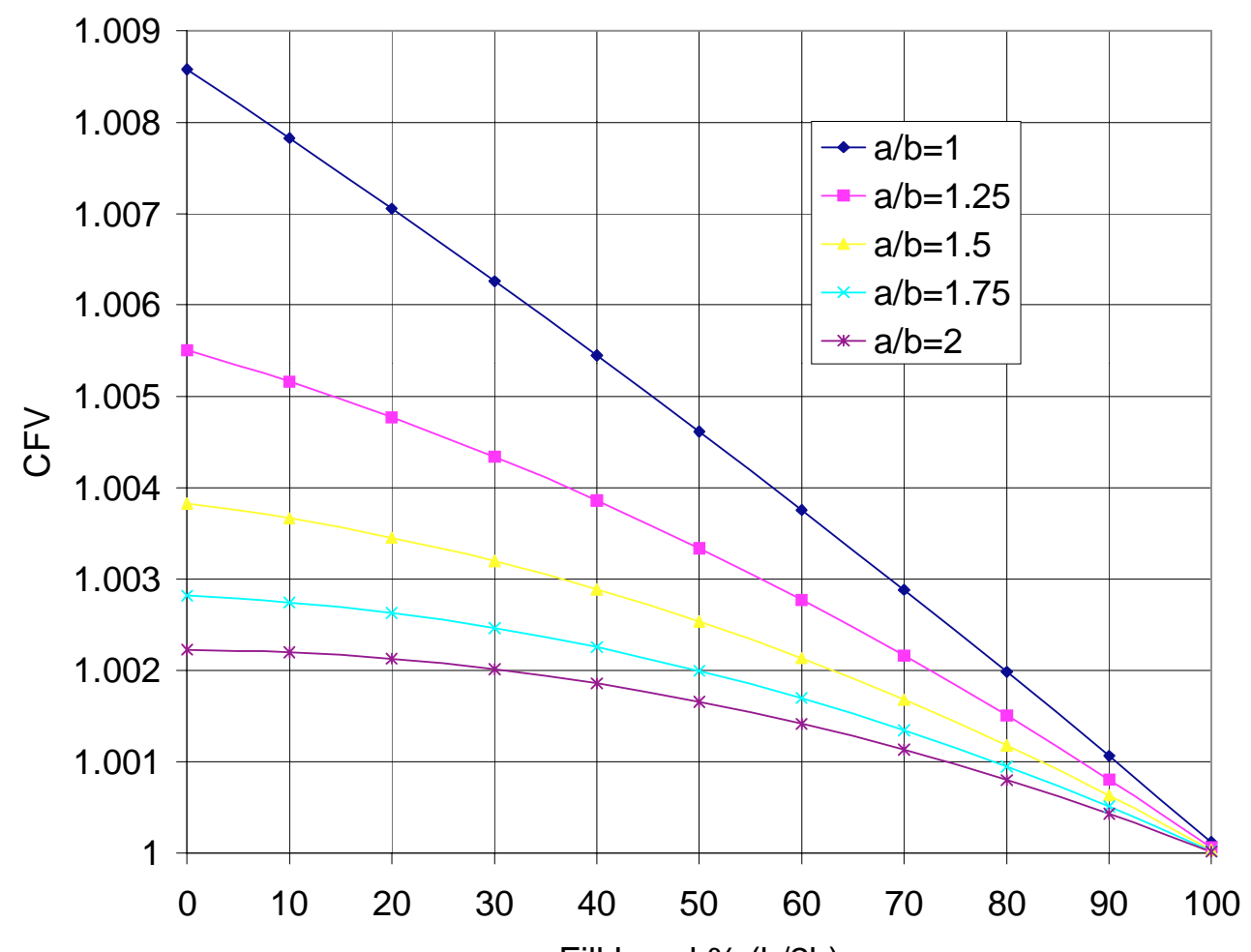

Fill Level \% (h/2b)

Figure 4.28: Vertical force coefficient at constant fluid surface angle equals 5 degrees

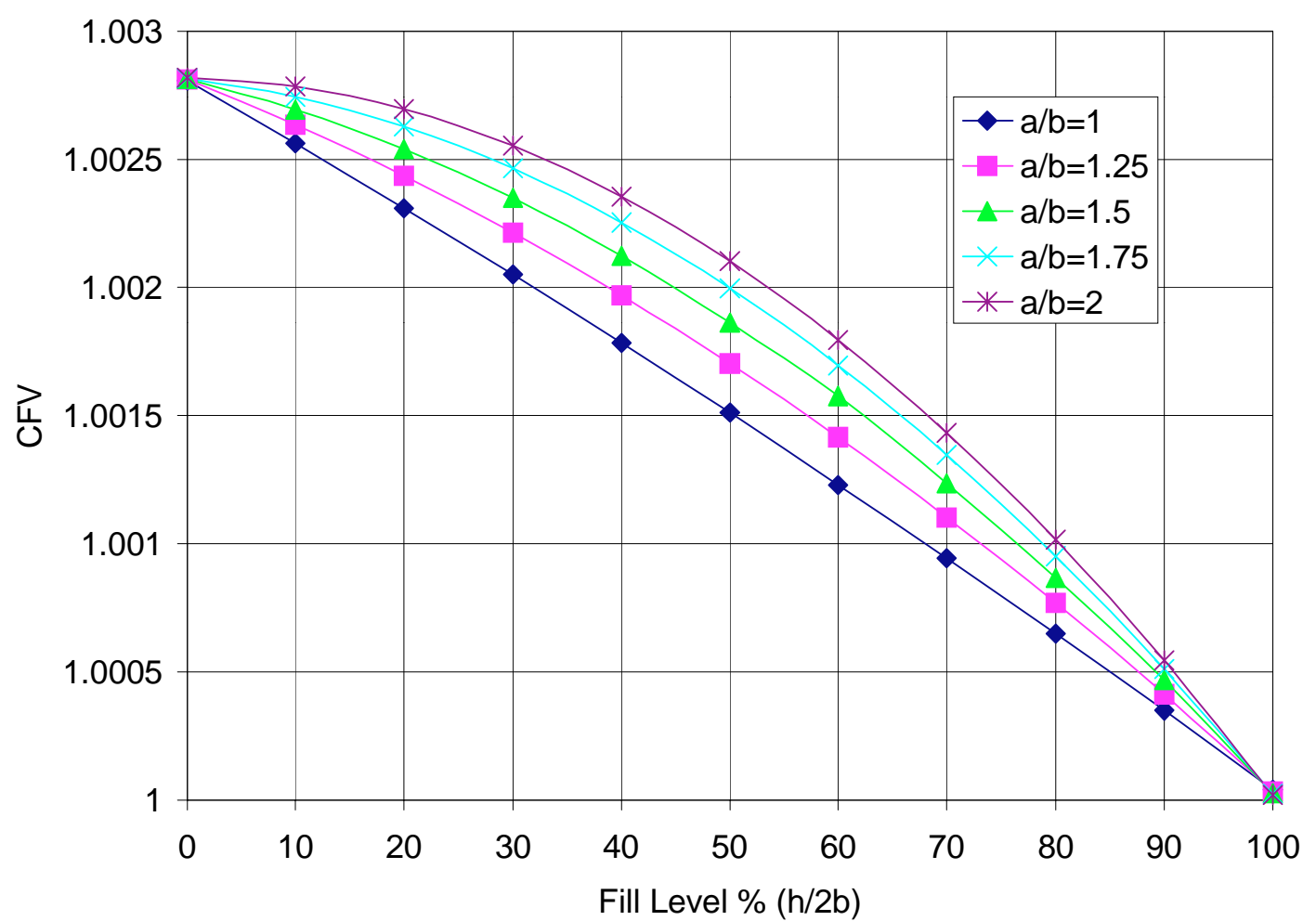

Figure 4.29: Vertical force coefficient at constant lateral acceleration equals $0.05 \mathrm{~g}$ 


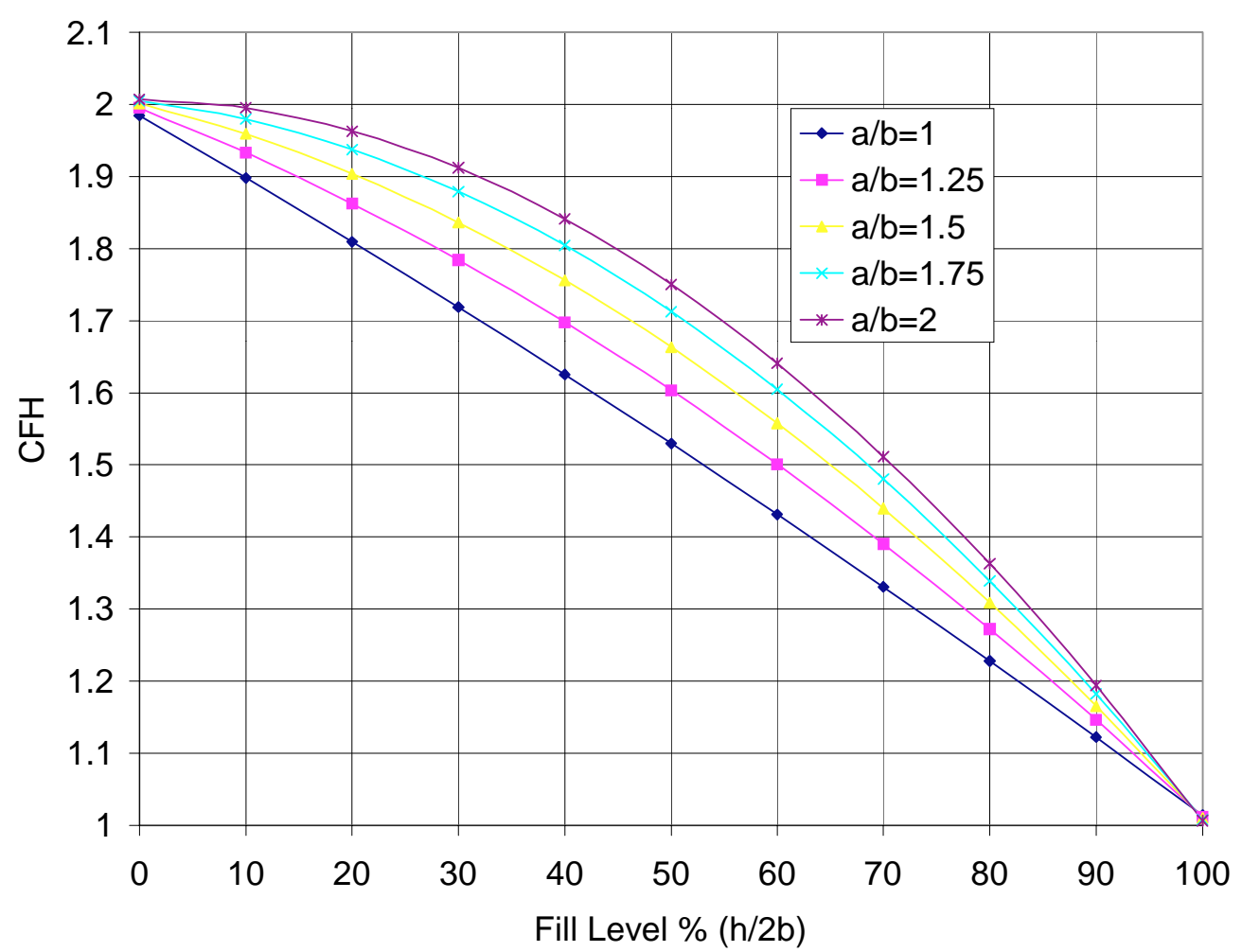

Figure 4.30: Horizontal force coefficient at constant fluid surface angle equals 5 degrees.

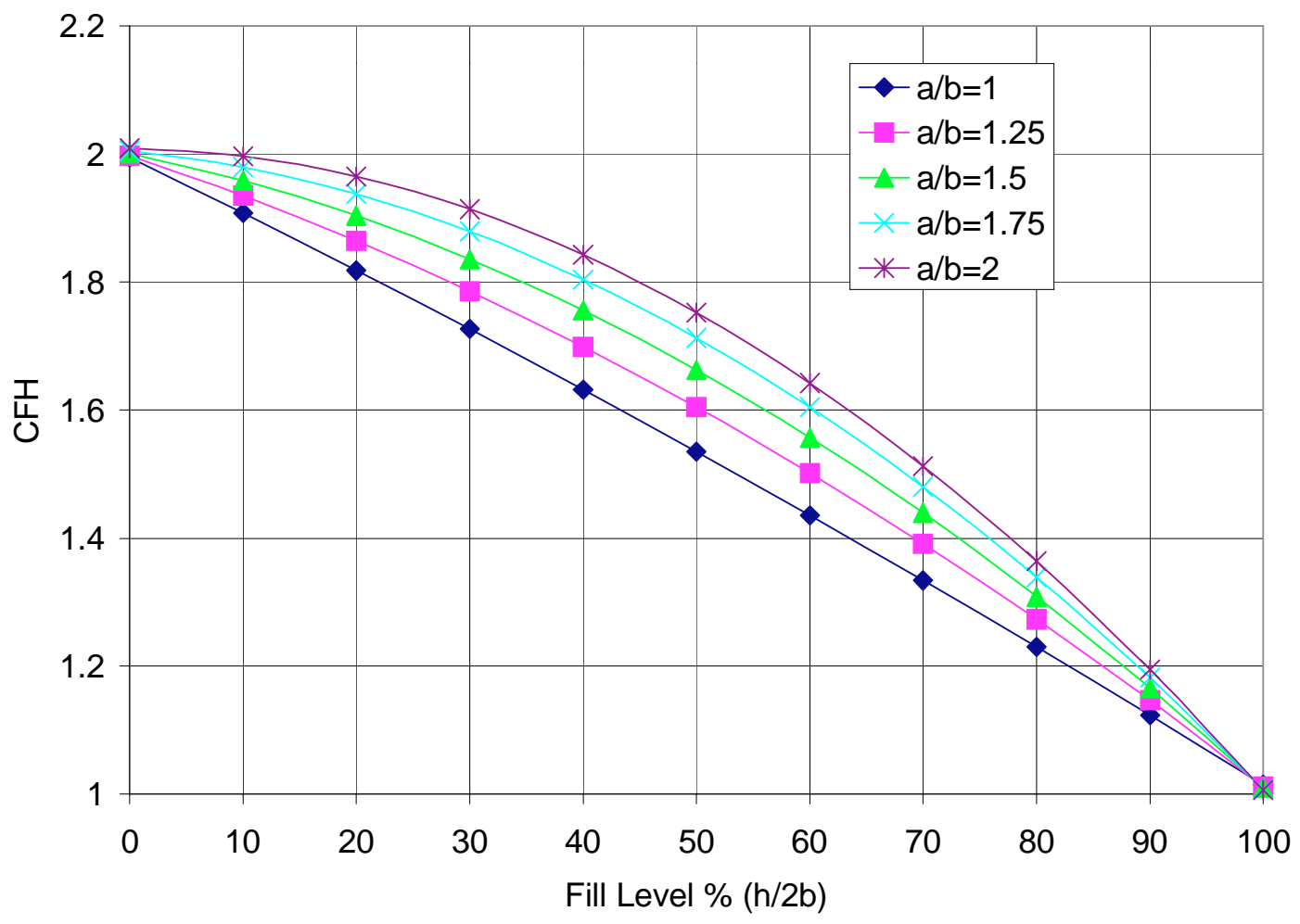

Figure 4.31: Horizontal force coefficient at constant lateral acceleration equals $0.05 \mathrm{~g}$. 


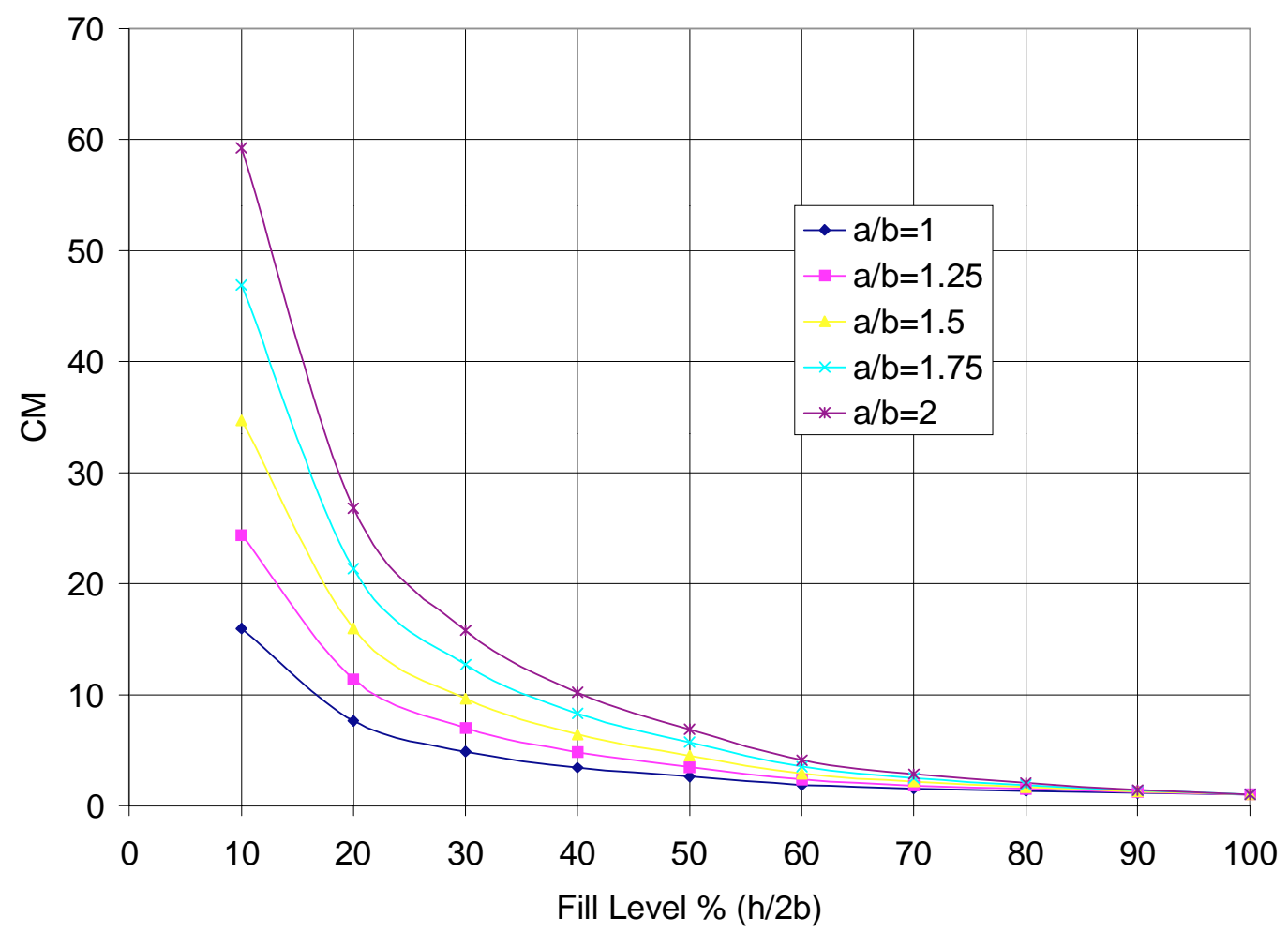

Figure 4.32: Rolling moment coefficient at constant fluid surface angle equals 5 degrees

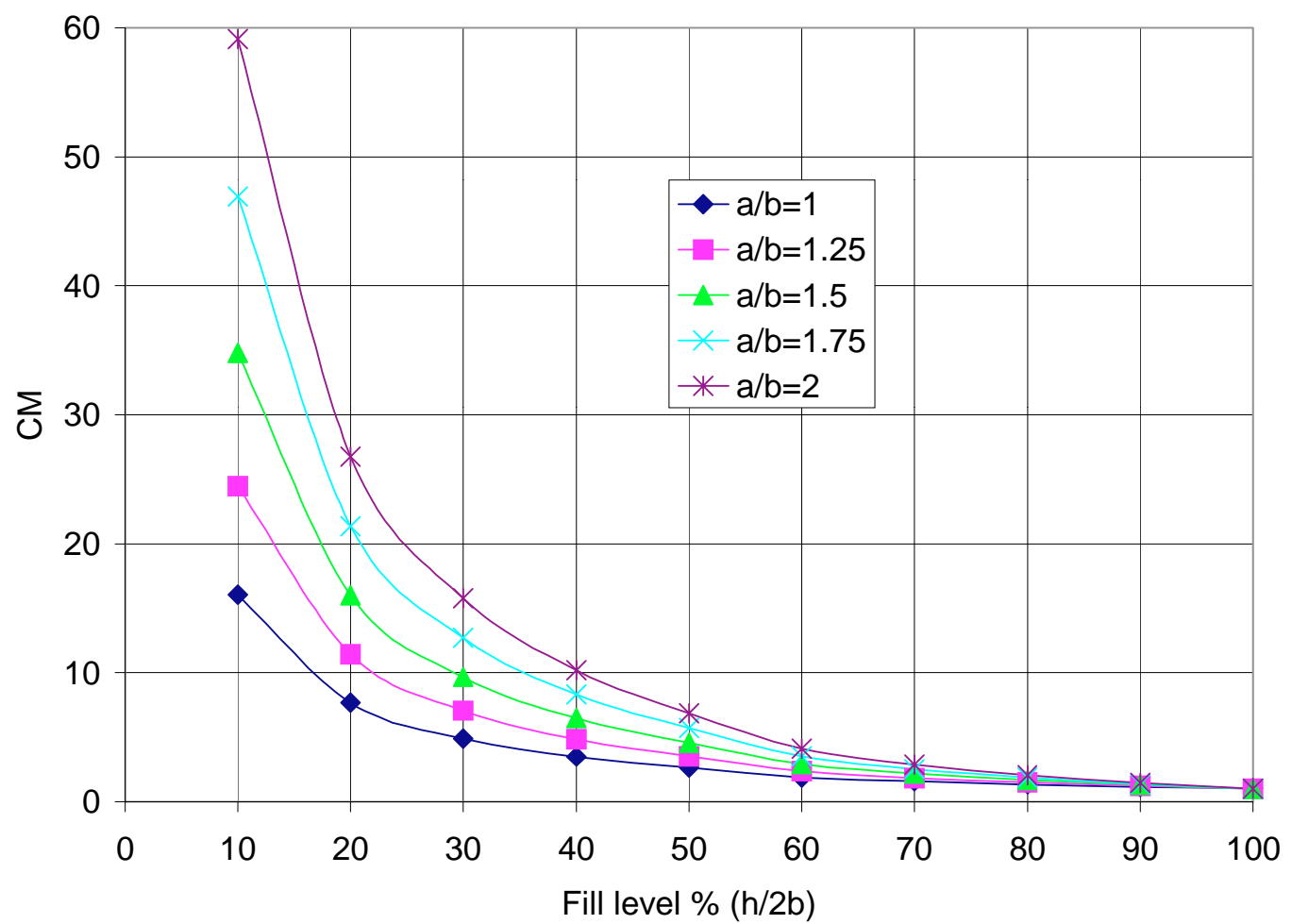

Figure 4.33: Rolling moment coefficient at constant lateral acceleration equals $0.05 \mathrm{~g}$. 
It can be seen that the frequency parameter increases with the level of fill for both cases of lateral acceleration, with the circular tank having the highest value of the frequency parameter until 95\% full. In other words, the lower the value of $a / b$, the higher frequency of sloshing for a given lateral acceleration. The frequency parameter reaches about 2.2 for all tank geometry at $100 \%$ full.

The vertical, horizontal, and moment coefficients are defined as the ratio between the vertical force, horizontal force, and rolling moment peak values due to sloshing effects divided by the fluid equivalent rigid body vertical force, horizontal force, and the rolling moment respectively. Figure 4.28 and 4.29 show that the change in the horizontal vertical component is negligible (less than $0.9 \%$ ) due to the low values of lateral acceleration applied. Moreover, the change drops to zero for a $100 \%$ full tank. Figure 4.28 shows that a circular tank has the maximum change in the vertical force component for the same fluid surface angle. This is because the circular tank is subjected to more lateral acceleration according to equation 4.20 since it has less value of $a / b$ than the elliptical tanks. For the constant acceleration case, all the tank shapes have the same value of the vertical force coefficient at the $0 \%$ and the $100 \%$ full cases. However, the vertical force coefficient increases with the increase of $\mathrm{a} / \mathrm{b}$ indicating more increase in the vertical force component due to fluid sloshing with the increase of $a / b$.

Figures 4.30 and 4.31 show the change in the horizontal force coefficient with fill level for constant fluid surface angle equal 5 degrees and constant acceleration equal $0.05 \mathrm{~g}$ respectively. It can be seen that both curves follow the same trend with $\mathrm{CFH}=1$ at $100 \%$ full and $\mathrm{CFH}=2$ for $0 \%$ full for all values of $\mathrm{a} / \mathrm{b}$. It can be seen also that the $\mathrm{CFH}$ increases with the increase of $\mathrm{a} / \mathrm{b}$ for all fill levels. In other words, elliptical tanks have more change in the 
horizontal force component than circular tanks due to fluid sloshing for the same fill level and same lateral acceleration.

Figures 4.32 and 4.33 show the change in the moment coefficient "CM" with fill level for constant fluid surface angle equal 5 degrees and constant acceleration equal $0.05 \mathrm{~g}$ respectively. It can be also seen that both curves follow the same trend with $\mathrm{CM}=1$ at $100 \%$ full, and $\mathrm{CM}$ increases exponentially to infinity at $0 \%$ full. It can also be seen that the tanks with higher values of $\mathrm{a} / \mathrm{b}$ has the maximum change in the rolling moment relative to the rigid body especially at low fill levels.

\section{6- Trammel Pendulum Behavior Beyond Linearity}

The trammel pendulum properties derived in the previous sections were based on the fact that the pendulum oscillates around a small steady state angle equal 5 degrees. This value agrees with the small angle assumption used to derive the natural frequency of the pendulum oscillation (equation 4.11). It also agrees with the linear pendulum behavior used by all the previous researchers who developed pendulum or spring-bub models to simulate fluid sloshing. However, the values of lateral acceleration which a tank mounted on a vehicle can be subjected to can reach a value up to $0.5 \mathrm{~g}$. Such a value of lateral acceleration will result in a steady state pendulum angle equals 26.5 degrees for a circular tank $(\mathrm{a} / \mathrm{b}=1)$ and equals 45 degrees for a superelliptical tank $(\mathrm{a} / \mathrm{b}=2)$, with the peak pendulum angle as twice as much as these values. Such large angles of oscillation result in a non-linear behavior of the trammel pendulum during simulating actual road maneuvers. Thus, it's essential to study the behavior of the trammel pendulum beyond the linear range. 
Since Sankar et. al. (1992) provided some results for fluid sloshing in an elliptical slice that can be compared the trammel pendulum behavior beyond the linear range, the inputs and definitions they used will be applied in this section.

An elliptical slice of an area equal $3.008575657 \mathrm{~m}^{2}$ is used in this section. Lateral acceleration values ranging from $0.1 \mathrm{~g}$ to $0.5 \mathrm{~g}$ were applied on the equivalent trammel pendulum to Sankar's model to obtain the variation of $\mathbf{K}, \mathrm{CFV}, \mathrm{CFH}$, and $\mathrm{CM}$ as a function of the lateral acceleration beyond the linear range. Figures 4.34 through 4.40 show a sample of the results obtained for an elliptical slice with $\mathrm{a} / \mathrm{b}=2,(\mathrm{a}=1.38395 \mathrm{~m}$ and $\mathrm{b}=0.691875 \mathrm{~m})$, having a thickness of $0.1 \mathrm{~m}$ and filled with water. In Figures 4.38 and $4.40, \mathrm{CFH}$, and $\mathrm{CM}$ were redefined according to Sankar for the purpose of comparison as the ratio between the peek values of the horizontal force and rolling moment to that of the steady state (fluid moving cargo with no sloshing) values respectively.

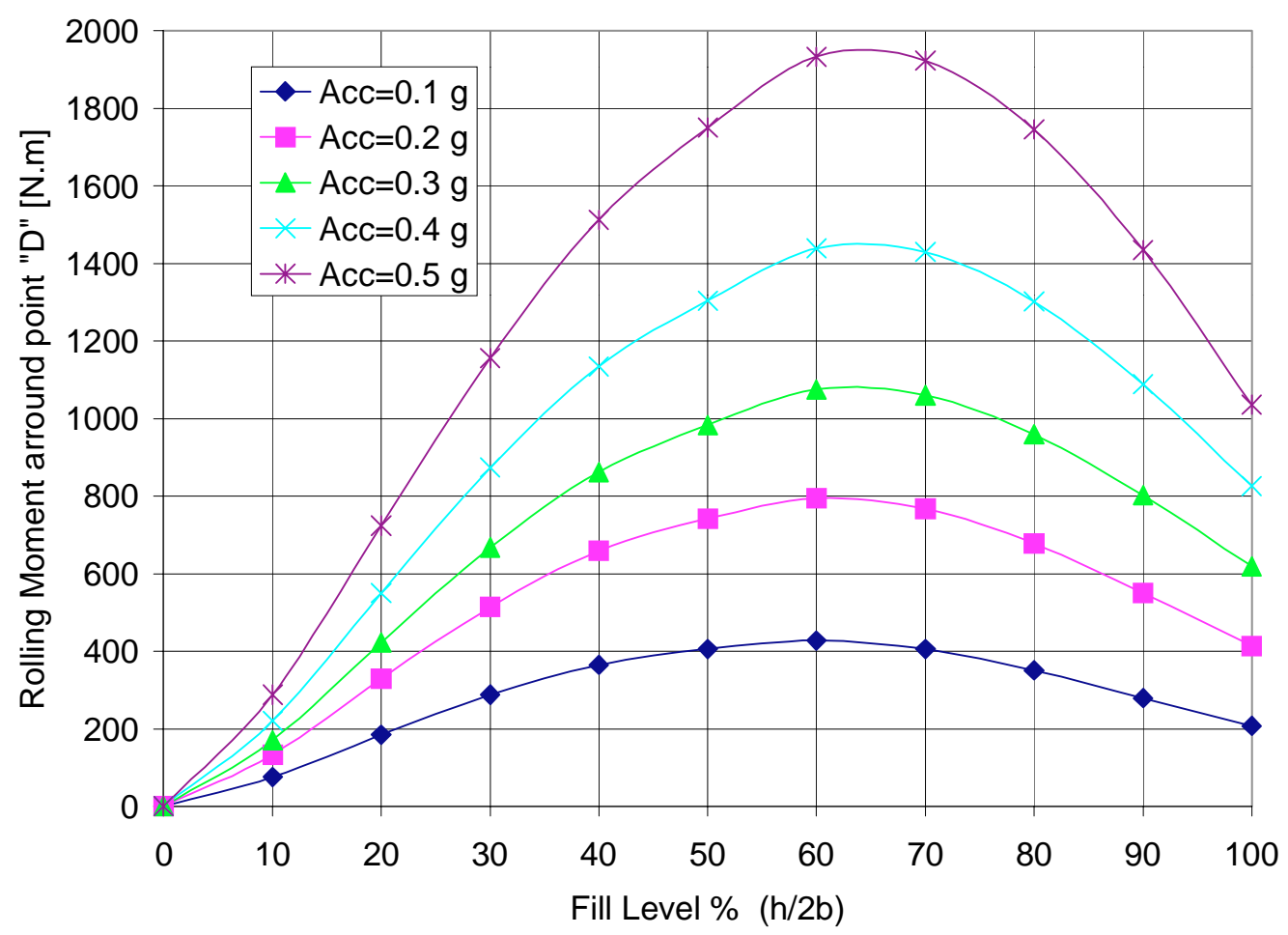

Figure 4.34: Change of rolling moment vs. fill level at different values of lateral acceleration 


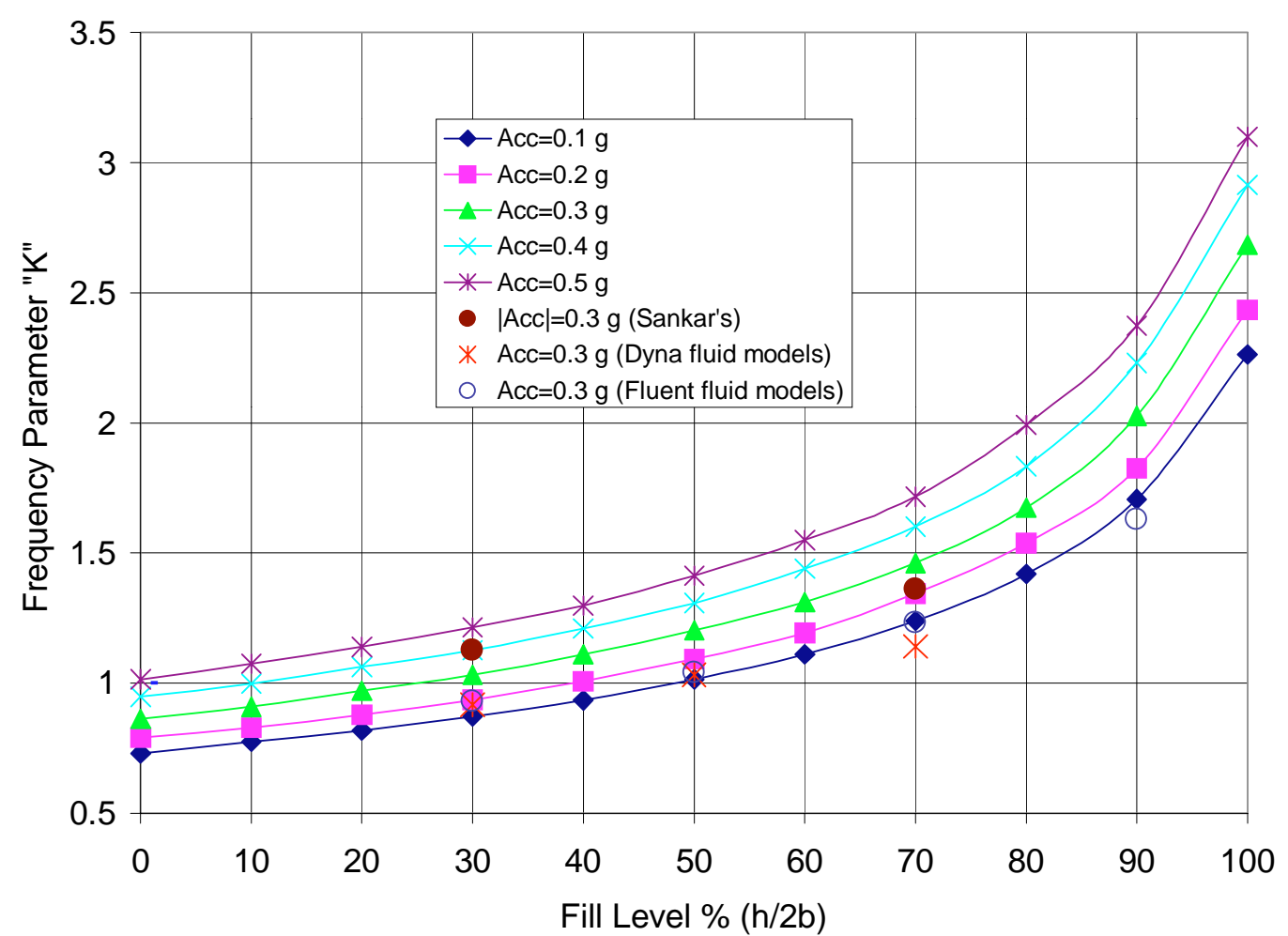

Figure 4.35: Change of the frequency parameter vs. fill level at different values of lateral acceleration

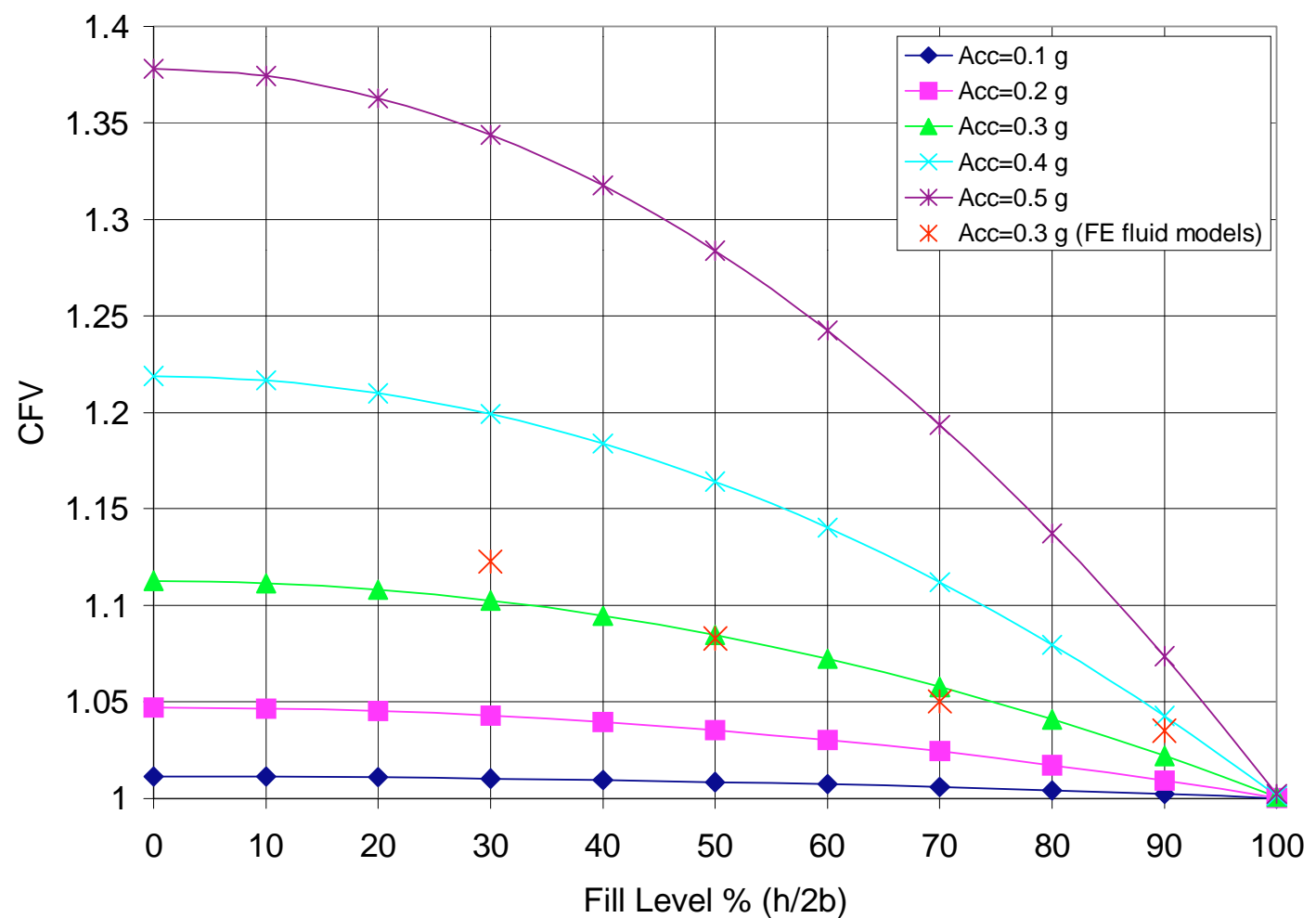

Figure 4.36: Change of the vertical force coefficient vs. fill level at different values of lateral acceleration 


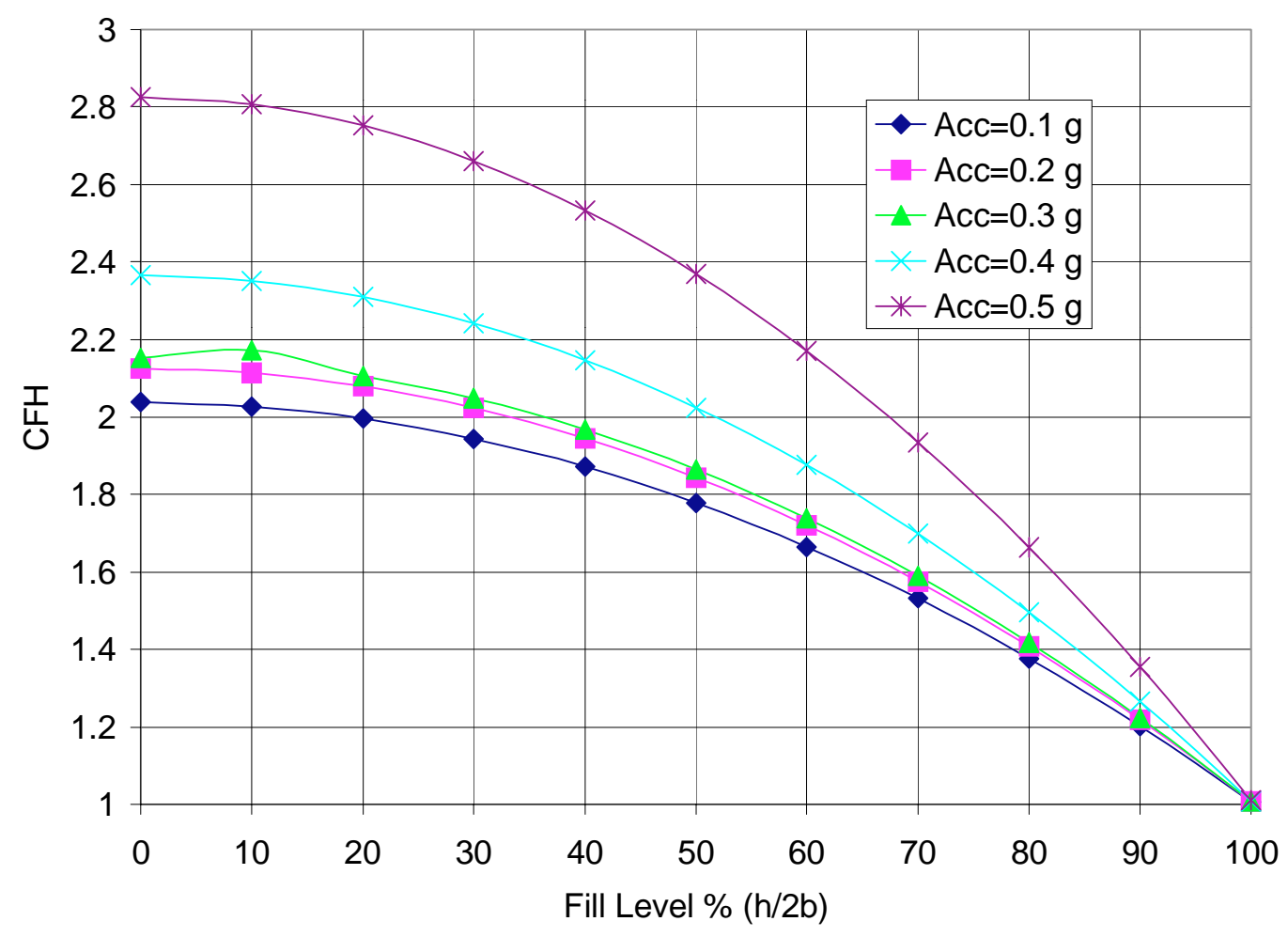

Figure 4.37: Change of the horizontal force coefficient vs. fill level at different values of lateral acceleration

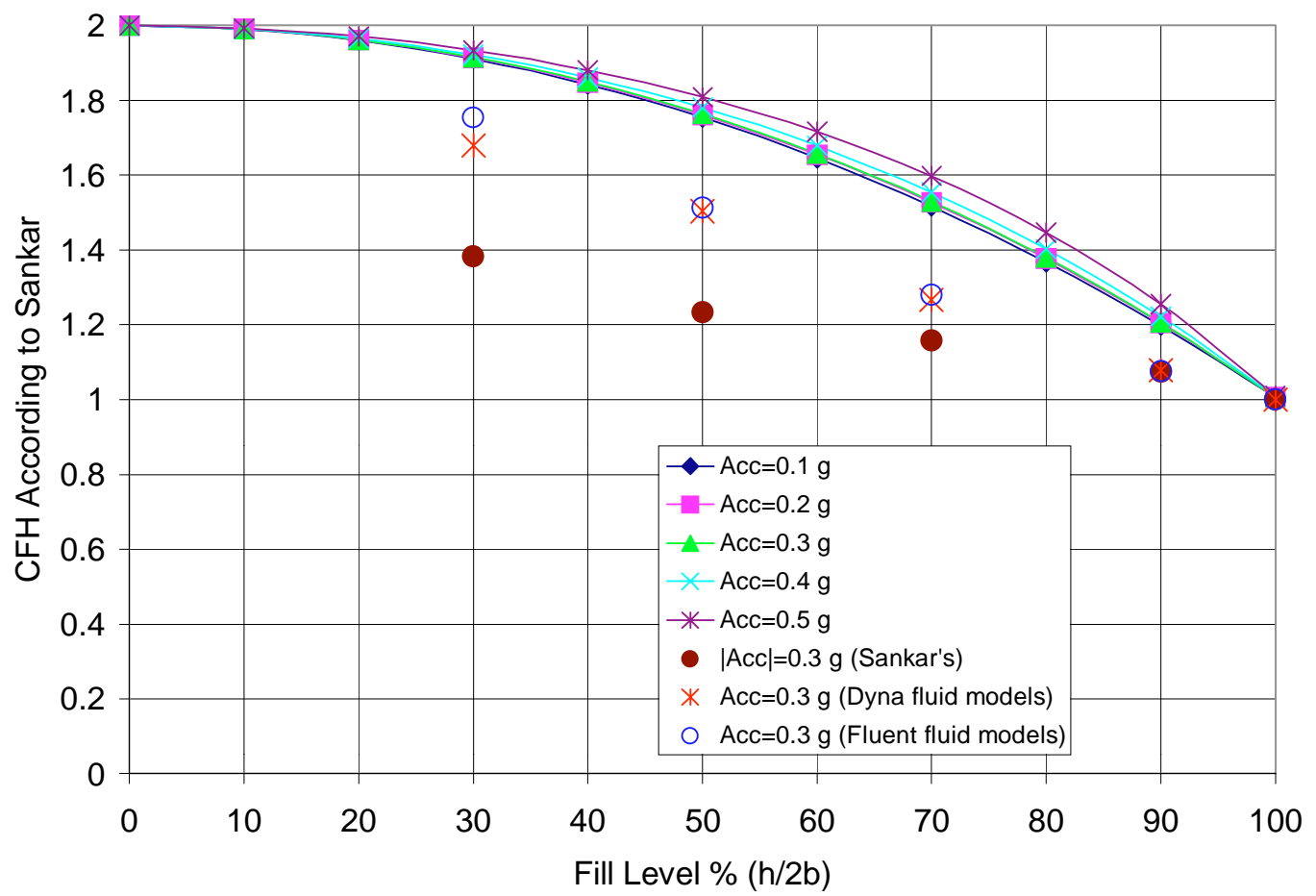

Figure 4.38: Change of the horizontal force coefficient (According to Sankar's Definition) vs. fill level at different values of lateral acceleration 


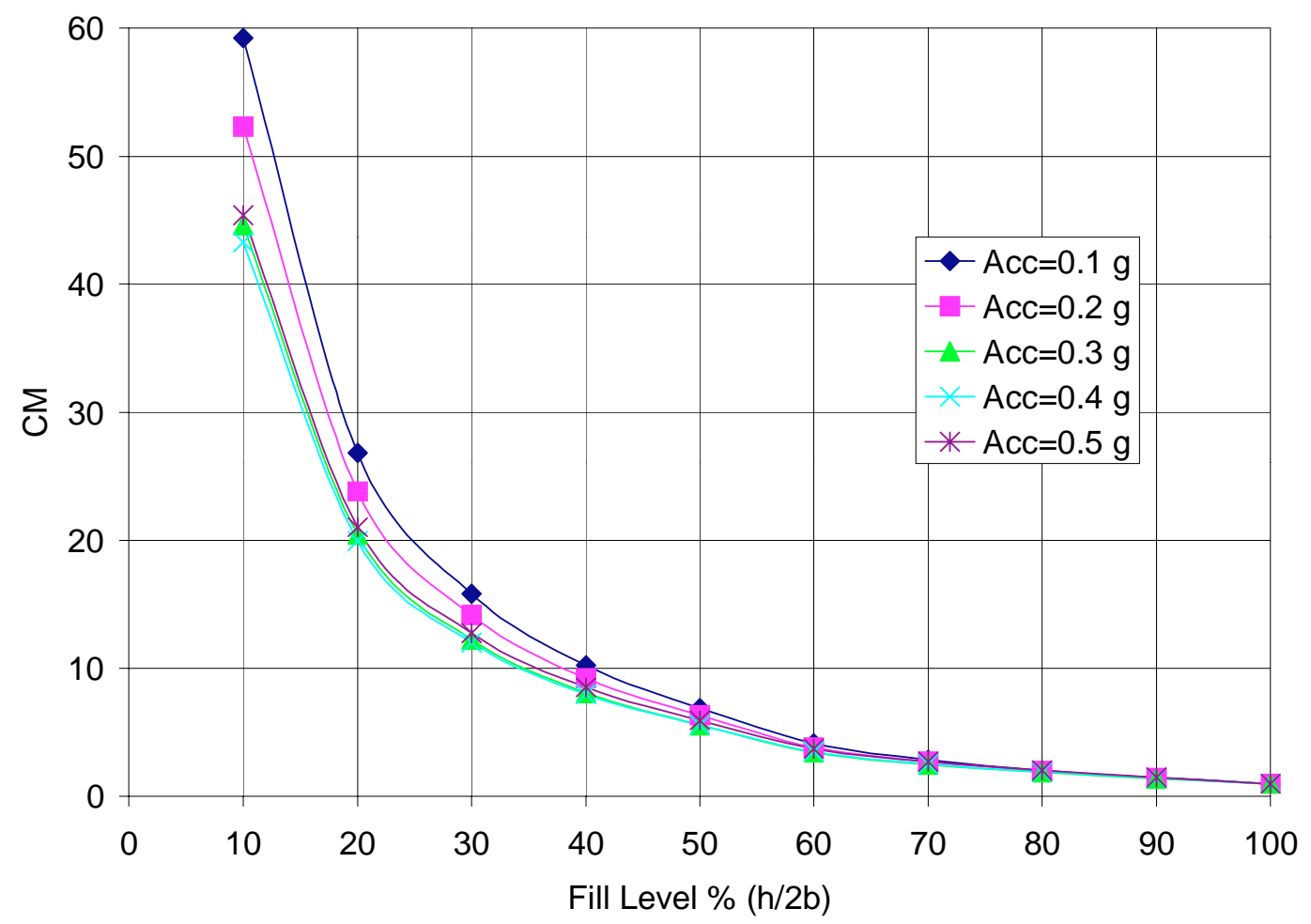

Figure 4.39: Change of the rolling moment coefficient vs. fill level at different values of lateral acceleration

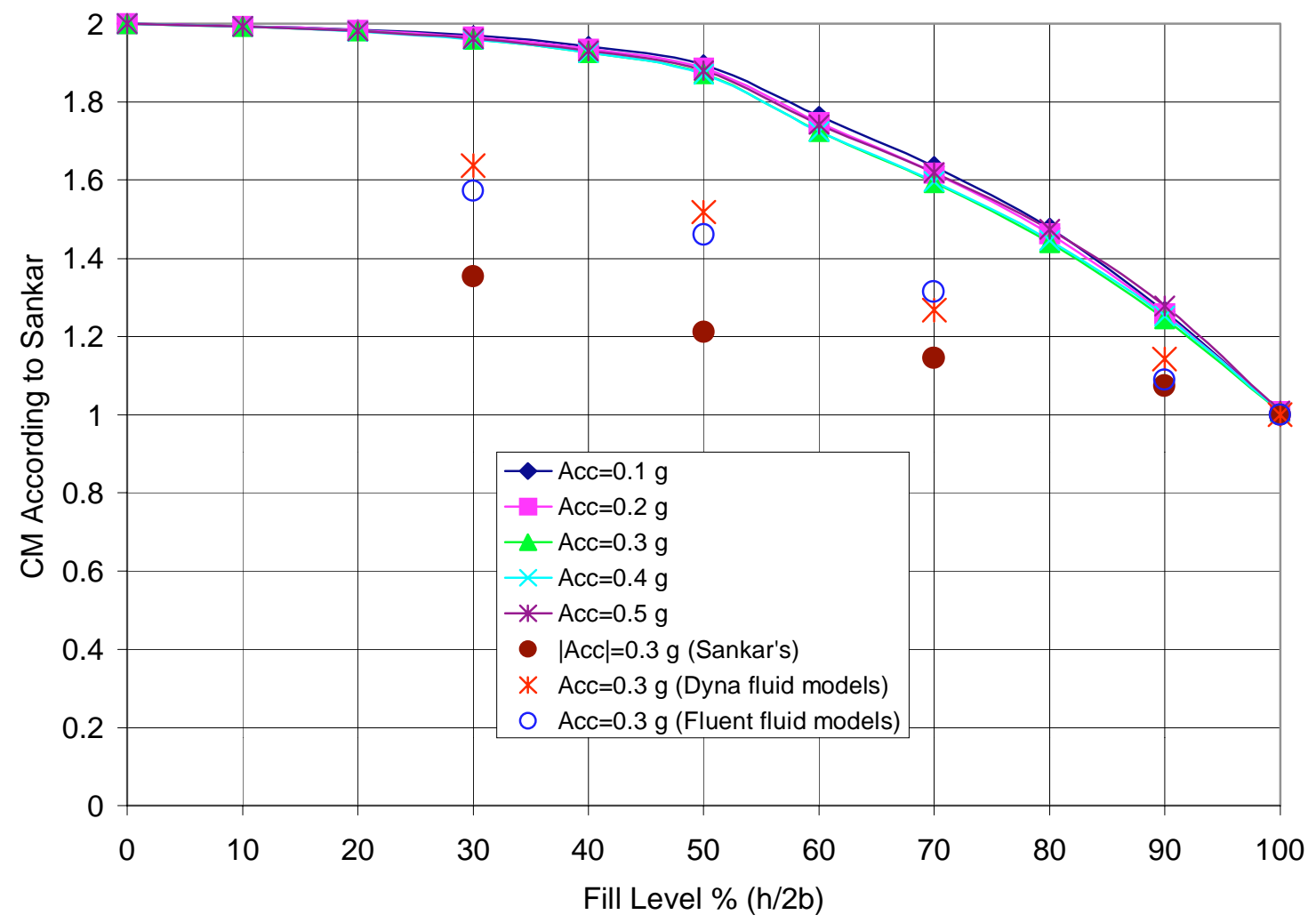

Figure 4.40: Change of the rolling moment coefficient (according to Sankar's definition) vs. fill level at different values of lateral acceleration 
Figure 4.34 shows the roll moment around point "D" vs. fill level at different values of lateral acceleration. It can be seen that the peek value of the rolling moment shifts gradually from $60 \%$ full at $0.1 \mathrm{~g}$ to $65 \%$ full at $0.5 \mathrm{~g}$.

Figure 4.35 shows that the frequency parameter increases with the increase of lateral acceleration, fill level, as well as a/b. This indicates that the frequency of the trammel pendulum oscillation increases with the increase of an applied lateral acceleration ranging from 0 to $0.5 \mathrm{~g}$, which agrees with the increasing portion curve shown in Figure 4.4.

Figure 4.36 shows the change of the vertical force coefficient vs. fill level at different values of lateral acceleration. It can be seen that the vertical force coefficient changes more rapidly at elevated values of lateral acceleration.

Figure 4.37 and 4.38 show the change in the horizontal force coefficient according to the definition given in this study and in Sankar's respectively. It can be seen that the value of CFH can reach more than 2.8 at zero fill levels, and that it's different for different values of lateral acceleration according to this study. However, according to Sankar's definition, CFH can only reach a maximum value of 2 at zero fill level regardless of the applied lateral acceleration. In addition, the change in CFH according to Sanakr's definition is almost negligible for higher level of fill.

Figures 4.39 and 4.40 show the change in rolling moment coefficient according to the definition given in this study and in Sankar's respectively. It can be seen that the value of CM is higher for lower lateral accelerations in both Figures and that all the values of CM for different levels of fill is equal to unity at the $100 \%$ full condition. However, the values of CM increases 
exponentially to infinity with the drop of the fill level according to this study's definition, while it reaches a maximum value of 2 at $0 \%$ fill level according to Sankar's definition. This is expected because the rigid body moment approaches zero at $0 \%$ fill condition, while the steady state fluid sloshing moment drops to a low value always equal half the peak moment value at $0 \%$ fill condition.

Unfortunately, Sankar et. al. (1992) provided a limited set of data for comparison at a lateral acceleration equal $-0.3 \mathrm{~g}$ only. This data is illustrated as the dark circular dots on Figures 4.35, 4.38 and 4.40. It can be seen that the values of CFH and CM obtained by Sankar are much lower than those obtained by the trammel pendulum. Also, the value or $\mathbf{K}$ is more for the $30 \%$ level of fill and less for the $70 \%$ level of fill than those of the trammel pendulum results.

Since the values of $\mathbf{K}, \mathrm{CM}$, and $\mathrm{CFH}$ obtained using the trammel pendulum are considerably different from those obtained by Sankar et. al (1992), FE Dyna_3D fluid models as well as FLUENT fluid models were used to simulate Sankar's models. The results of these models are used as a second reference to verify whither the trammel pendulum really behaves quite differently from the sloshing fluid behavior beyond the linear range or not. Table 4.3 lists the values of $\mathbf{K}, \mathrm{CFH}$, and CM obtained using the Dyna_3D and FLUENT fluid models.

Table 4.3: Values of K, CFH, CFVand CM obtained using Fluent and Dyna models at 0.3g

\begin{tabular}{|c|c|c|c|c|}
\hline Fill Level & K(Dyna/Fluent) & CFH(Dyna/Fluent) & CFV(Dyna/Fluent) & CM(Dyna/Fluent) \\
\hline 30 & $0.916 / 0.93$ & $1.68 / 1.753$ & $1.12266 /$ N.A. & $1.637 / 1.573$ \\
\hline 50 & $1.032 / 1.04$ & $1.503 / 1.512$ & $1.08276 /$ N.A. & $1.5177 / 1.46$ \\
\hline 70 & $1.141 / 1.232$ & $1.267 / 1.28$ & $1.05 /$ N.A. & $1.2684 / 1.313$ \\
\hline 90 & N.A./1.63 & $1.079 / 1.076$ & $1.035 /$ N.A. & $1.143 / 1.09$ \\
\hline
\end{tabular}


The values of K, CFH and CM obtained using the Dyna_3D fluid models are shown as “ $\times$ ” in Figures 4.35, 4.38 and 4.40 respectively, while the FLUENT results as shown as "O". It can be seen that the values of $\mathrm{K}$ for the trammel pendulum model are still well above the values of K obtained from the fluid models. This can be attributed to the fact that the pendulum natural frequency increases in the range of pendulum angle from 0 to $\pi / 2$ as shown in Figure 4.4 . The values of $\mathrm{CFH}$ and $\mathrm{CM}$ lies between Sankar's results and the trammel pendulum results rendering the trammel pendulum the most conservative model among the 3 models. The values of CFV of the Dyna_3D fluid models are in good agreement with the trammel pendulum results as shown in Figure 4.36. It can be seen also that the results obtained from Dyna_3D and from FLUENT are in good agreement with each other. Thus, either of them can be used as a reference instead of Sankar's results.

\subsection{Advantages of Trammel Pendulum Model over Dyna and FLUENT Fluid Models}

Since the trammel pendulum represents an approximation of the real fluid sloshing behavior, it is worth mentioning the advantages of applying such a simple mechanical model in simulating the sloshing problem.

The trammel pendulum model is much simpler to integrate in a rigid body vehicle model or even in an FE model that will be used to simulate the lateral stability problem of partially filled elliptical tanker. This fact is illustrated in the next chapter.

In terms of simulation time, it has been found that the trammel pendulum model requires much less time in preparation and running than the Dyna_3D of FLUENT fluid models. Table 
4.4 illustrates a comparison between the time used to obtain the data points of Figures 4.23 and 4.24 using FE fluid models and equivalent trammel pendulum models respectively.

Table 4.4: Comparison between time costs for Dyna and Fluent fluid models and their equivalent trammel pendulum models

\begin{tabular}{|c|c|c|c|}
\hline Point of Comparison & Dyna fluid Models & FLUENT fluid Models & $\begin{array}{l}\text { Equivalent Trammel } \\
\text { Pendulum Models }\end{array}$ \\
\hline $\begin{array}{l}\text { Model Setup and } \\
\text { preparation time }\end{array}$ & 2 hours per fill level & 1 hour per fill level & $\begin{array}{l}20 \text { seconds to feed } \\
\text { the inputs }\end{array}$ \\
\hline Model Run Time & $\begin{array}{l}2.5 \text { to } 3.5 \text { hours per } 1 \\
\text { second of simulation } \\
\text { depending on the fill } \\
\text { level (higher for } \\
\text { higher levels of fill), } \\
\text { on a Dec Alfa } \\
\text { 600AU work station }\end{array}$ & $\begin{array}{l}3 \text { min per } 1 \text { second of } \\
\text { simulation, independent } \\
\text { on the fill level. On a } \\
366 \mathrm{MHz} \text { PC }\end{array}$ & $\begin{array}{l}0.5 \text { second per } 1 \\
\text { second of simulation, } \\
\text { independent on the } \\
\text { level of fill, on a } 366 \\
\text { MHz PC }\end{array}$ \\
\hline $\begin{array}{l}\text { Probability of } \\
\text { Crashing and/solution } \\
\text { diversion }\end{array}$ & $10 \%$ to $20 \%$ & None & None \\
\hline
\end{tabular}

It also worth mentioning that the Dyna_3D FE fluid models starts becoming gradually unstable and produces useless results after few cycles of fluid oscillation, while the equivalent trammel pendulums are always steady. This fact is illustrated in Figure 4.22 


\section{CHAPTER 5 \\ VEHICLE-PENDULUM MODELS}

\section{$\underline{5.1 \text { - Introduction }}$}

This chapter contains the models used to investigate the rollover stability of a partially filled heavy-duty elliptical tanker using the trammel pendulum studied in the previous chapter. Four models have been utilized to for this purpose. Table 5.1 shows a summarized description of those models.

Table 5.1: Summary of the tanker models utilizing the trammel pendulum

\begin{tabular}{|c|c|c|c|c|}
\hline Mode \# & Model Name & Type & Purpose & $\begin{array}{c}\text { Main } \\
\text { parameters } \\
\text { Considered }\end{array}$ \\
\hline 1 & $\begin{array}{l}\text { Vehicle rigid body } \\
\text { model, with no } \\
\text { support liftoff }\end{array}$ & $\begin{array}{l}\text { Analytical } \\
\text { 2-D model }\end{array}$ & $\begin{array}{l}\text { Studying the frequency } \\
\text { response of the pendulum, } \\
\text { resonance effect, and } \\
\text { effect of changing aspect } \\
\text { ratio. }\end{array}$ & $\begin{array}{l}\text { Fluid fixed mass, } \\
\text { fluid sloshing } \\
\text { mass, and vehicle } \\
\text { mass }\end{array}$ \\
\hline 2 & $\begin{array}{l}\text { Vehicle rigid body } \\
\text { model, with } \\
\text { support liftoff }\end{array}$ & $\begin{array}{l}\text { Analytical } \\
\text { 2-D model }\end{array}$ & $\begin{array}{l}\text { Studying the effect of the } \\
\text { tanker and the fluid polar } \\
\text { moment of inertia and the } \\
\text { absolute instability. }\end{array}$ & $\begin{array}{l}\text { Fluid fixed mass, } \\
\text { fluid sloshing } \\
\text { mass, fluid and } \\
\text { vehicle polar } \\
\text { moment } \\
\text { inertia. }\end{array}$ \\
\hline 3 & $\begin{array}{l}\text { Vehicle plane } \\
\text { model with tire } \\
\text { and suspension } \\
\text { compliances }\end{array}$ & $\begin{array}{l}\text { Rigid body } \\
\text { 2-D model } \\
\text { Using } \\
\text { Working } \\
\text { Model }\end{array}$ & $\begin{array}{l}\text { Study the effect of } \\
\text { suspension compliance on } \\
\text { stability as well as the } \\
\text { tripped rollover limits }\end{array}$ & $\begin{array}{l}\text { Same as "2" plus } \\
\text { the suspension } \\
\text { and the tire roll } \\
\text { centers, the axles } \\
\text { weight and polar } \\
\text { moment of } \\
\text { inertia, the } \\
\text { suspension and } \\
\text { tire compliances }\end{array}$ \\
\hline 4 & $\begin{array}{l}\text { 3-D full vehicle } \\
\text { model }\end{array}$ & $\begin{array}{l}\text { FEM 3-D } \\
\text { model using } \\
\text { ANSYS }\end{array}$ & $\begin{array}{l}\text { Comparing the results } \\
\text { obtained from the } \\
\text { pendulum model with the } \\
\text { experimental results. }\end{array}$ & $\begin{array}{lr}\text { All } & \text { possible } \\
\text { factors } & \text { and } \\
\text { parameters } & \end{array}$ \\
\hline
\end{tabular}




\section{2- Model 1, Vehicle Rigid Body Model with no Support Liftoff}

Figure 5.1 shows the rigid body model used to calculate the reactions at the supports analytically by means of utilizing the charts given in chapter 4 to find the values of, $\mathrm{M}_{\mathrm{o}}, \mathrm{M}_{\mathrm{p}}, \bar{a}$ , $\bar{b}$ and $\mathrm{Y}_{\mathrm{Mo}}$. The purpose of this simple model is to study the effects of changing tank aspect ratio on the rollover threshold and to study the pendulum behavior near resonance.

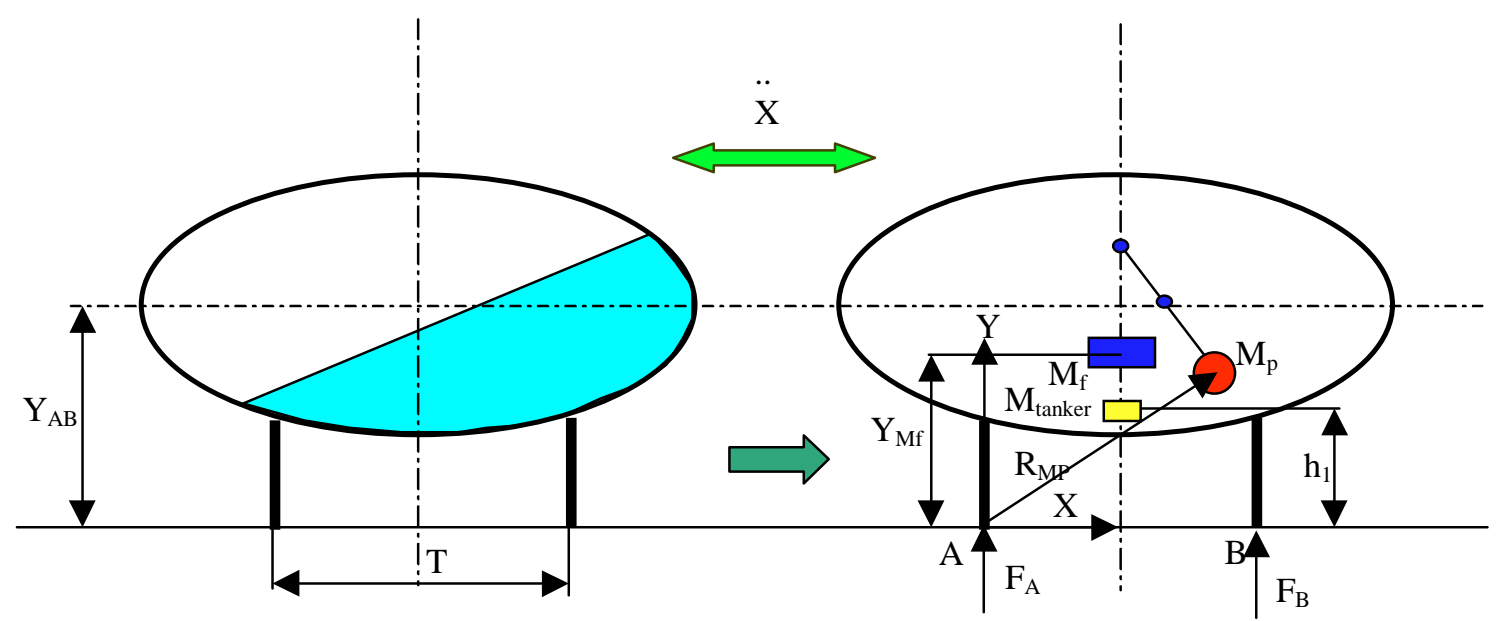

Figure 5.1: A schematic diagram of vehicle plane model \#1

The reactions at $A$, and $B$ can be calculated by taking the moments about point A. Thus, the reactions are given by:

$$
\begin{gathered}
\mathrm{F}_{\mathrm{t}}=\mathrm{F}_{\mathrm{A}}+\mathrm{F}_{\mathrm{B}}=\left(\mathrm{M}_{\mathrm{t}}+\mathrm{M}_{\text {tan ker }}\right) \mathrm{g}+\mathrm{Mp} \overline{\mathrm{b}}\left(\theta^{\bullet^{2}} \cos \theta+\ddot{\theta} \sin \theta\right) \\
\mathrm{F}_{\mathrm{B}}=\left(\left(\mathrm{M}_{\mathrm{f}}+\mathrm{M}_{\text {tanker }}\right) \mathrm{g} \frac{\mathrm{T}}{2}+\mathrm{M}_{\mathrm{p}}(\ddot{\mathrm{r}}+\mathrm{gj}) \times \mathrm{R}_{\mathrm{MP}}-\left(\mathrm{M}_{\mathrm{f}} \mathrm{Y}_{\mathrm{Mf}}+\mathrm{M}_{\text {tan ker }} \mathrm{h} 1\right) \ddot{\mathrm{x}}\right)
\end{gathered}
$$

Where $R_{M P}=\left(\bar{a} \sin \theta+\frac{T}{2}\right) j+\left(Y_{A B}-\bar{b} \cos \theta\right) j$ 


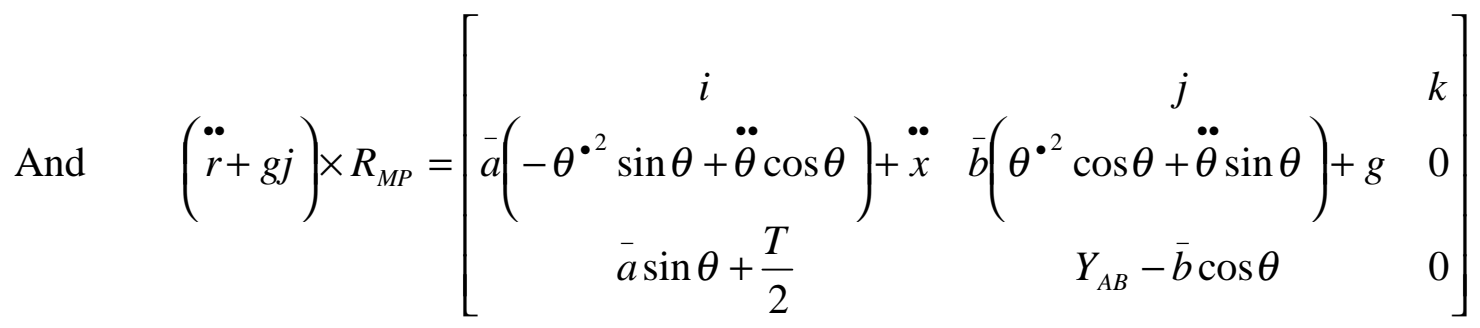

Equations 5.1 and 5.2 were used to find the reactions at the two supports A \& B. Results were compared against an equivalent ANSYS FE shown in Figure 5.2 for verification.

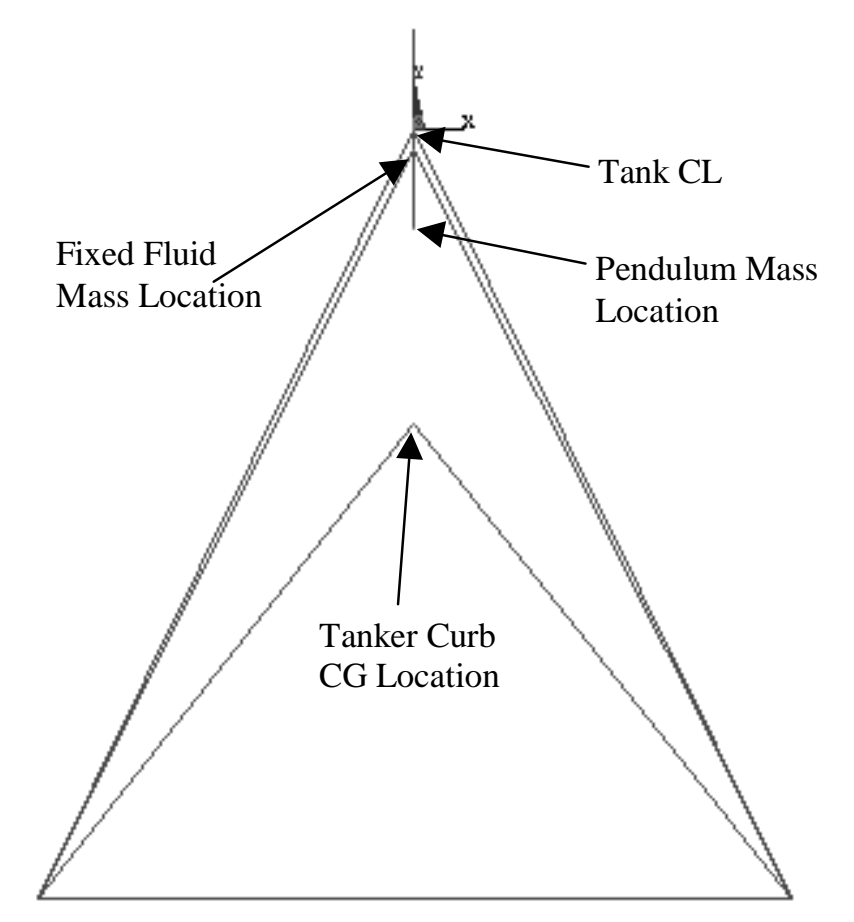

Figure 5.2: ANSYS simple plane beam model (Modified from Aquaro 1999)

Figures 5.3 and 5.4 show two samples of the analytical code using MatLab to solve for the reactions using equations 5.1 and 5.2 and ANSYS results receptively for. Case is for 50\% full ETNYRE tanker subjected to lateral acceleration $=0.3 \mathrm{~g}$.

\subsubsection{Model \#1 Applications}

Since this model is a simple rigid body model, it's very easy to utilize this model to study the effect of changing some parameters on tanker relative lateral stability. Such as tank ellipse 
ratio $(\mathrm{a} / \mathrm{b})$, or the frequency of the applied lateral acceleration. In all the following models, the area is assumed always constant and equal to the ETNYRE tanker cross section area. Table 5.2 lists the values of $a$ and $b$ at different aspect ratios that will yield the same area as the ETNYRE tanker. The fluid is water, and the total length is taken equal to $9.8783 \mathrm{~m}$.

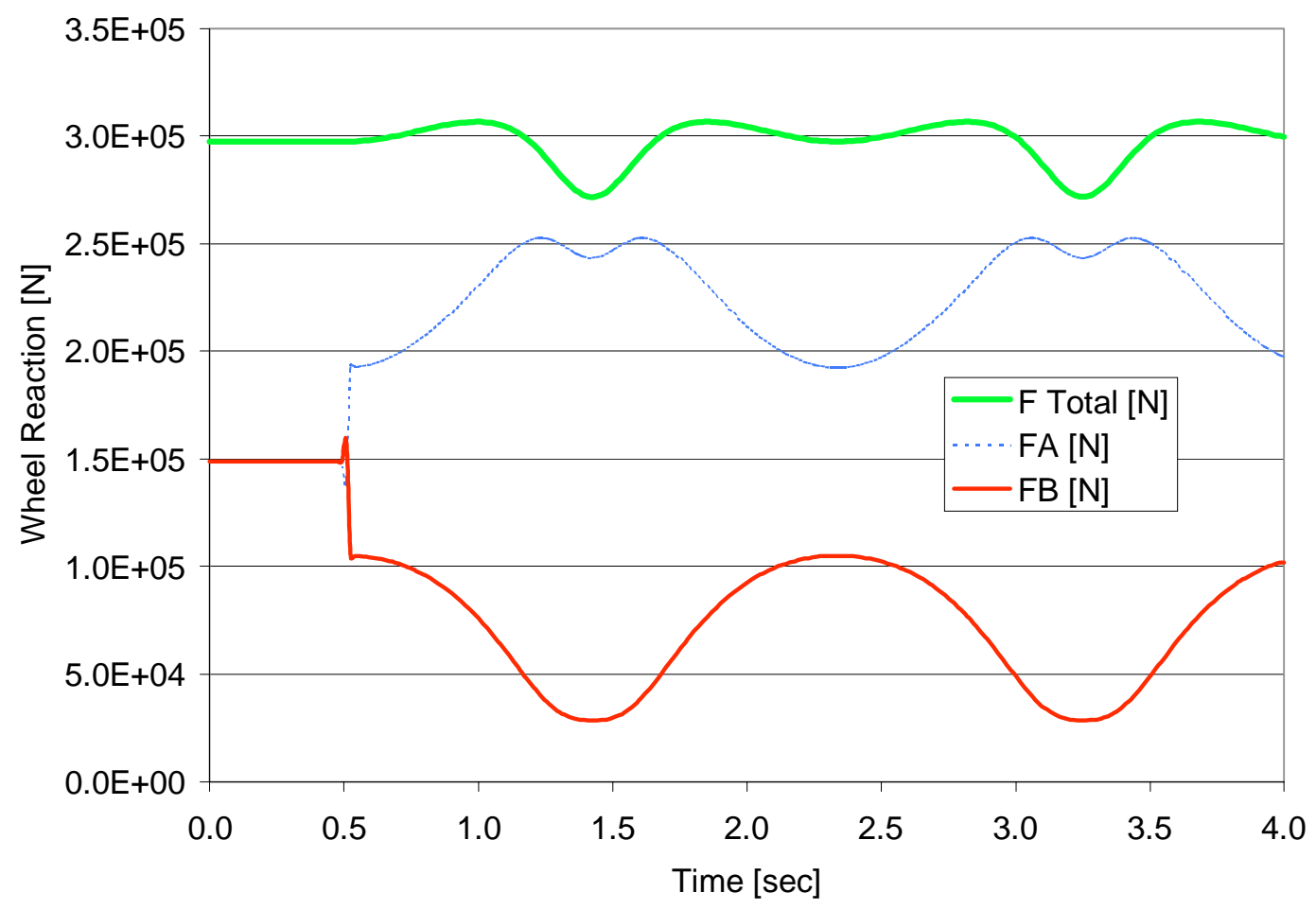

Figure 5.3: Reactions on Supports A \& B obtained analytically using Equations 5.1 and 5.2 


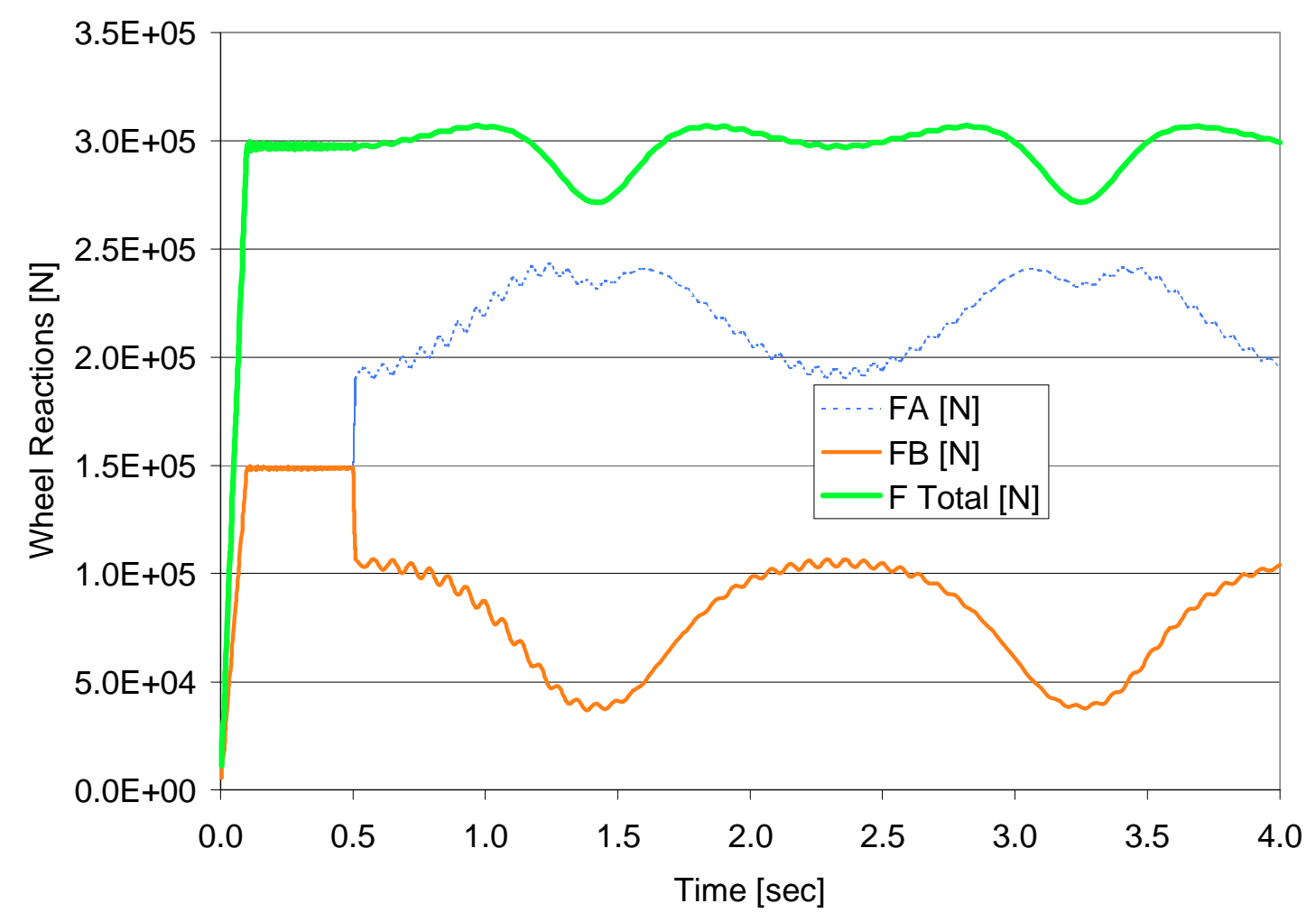

Figure 5.4: Reactions on supports A \& B obtained using ANSYS FEM

Table 5.2: Values of $\mathrm{a}$ and $\mathrm{b}$ in meters used in models 1 through 4 for constant cross section.

\begin{tabular}{|c|c|c|c|c|c|}
\hline & $\mathrm{a} / \mathrm{b}=1.0$ & $\mathrm{a} / \mathrm{b}=1.25$ & $\mathrm{a} / \mathrm{b}=1.5$ & $\mathrm{a} / \mathrm{b}=1.75$ & $\mathrm{a} / \mathrm{b}=1.9688 \cong 2.0$ \\
\hline $\mathrm{a}$ & 0.8552 & 0.956 & 1.0474 & 1.131 & 1.2 \\
\hline $\mathrm{b}$ & 0.8552 & 0.765 & 0.6982 & 0.6465 & 0.6095 \\
\hline
\end{tabular}

Figures 5.5 through 5.10 show the values of the pendulum parameters used in all the following models at different levels of fill and aspect ratios. 


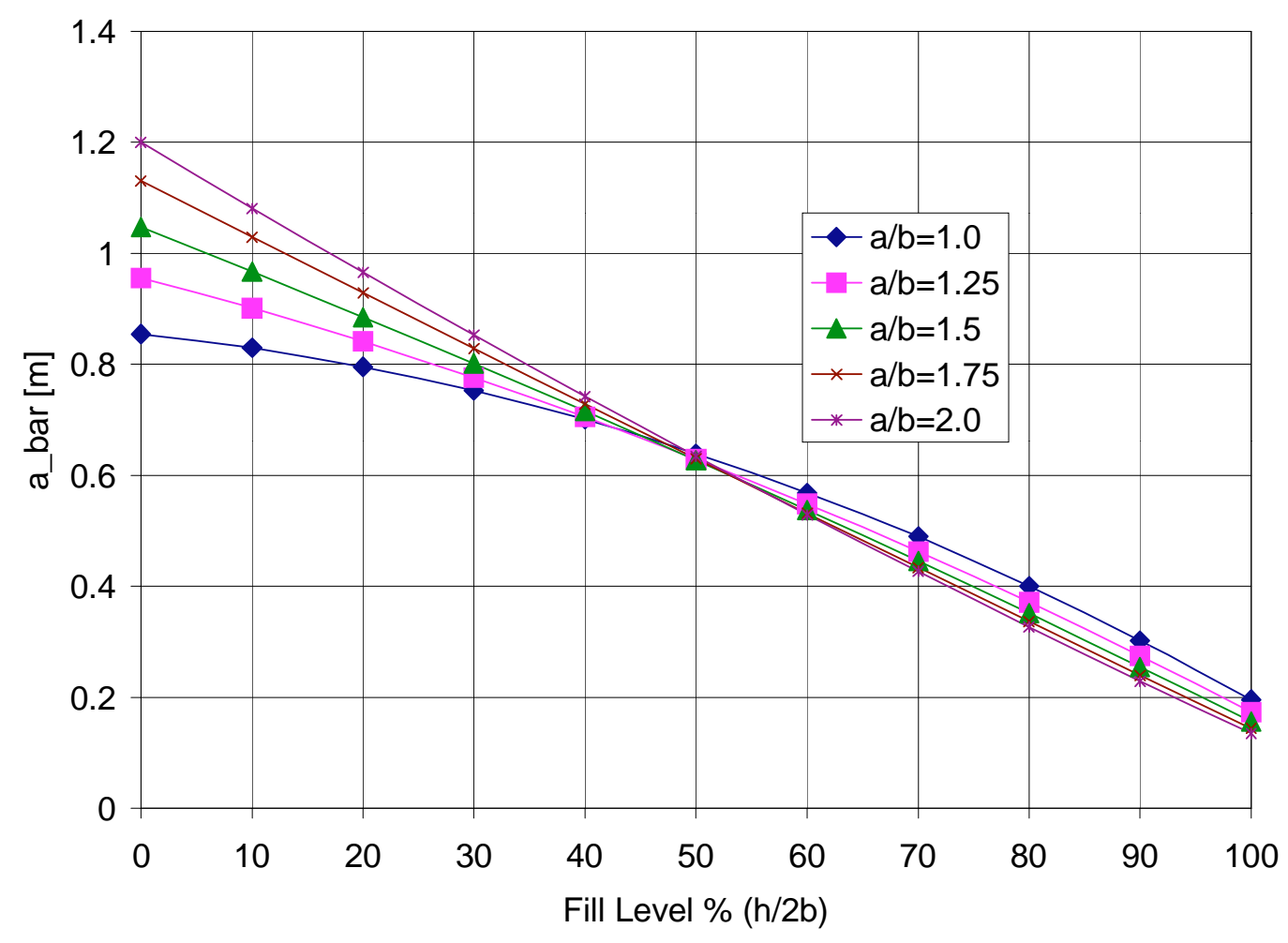

Figure 5.5: Change of $\bar{a}$ as a function of fill level

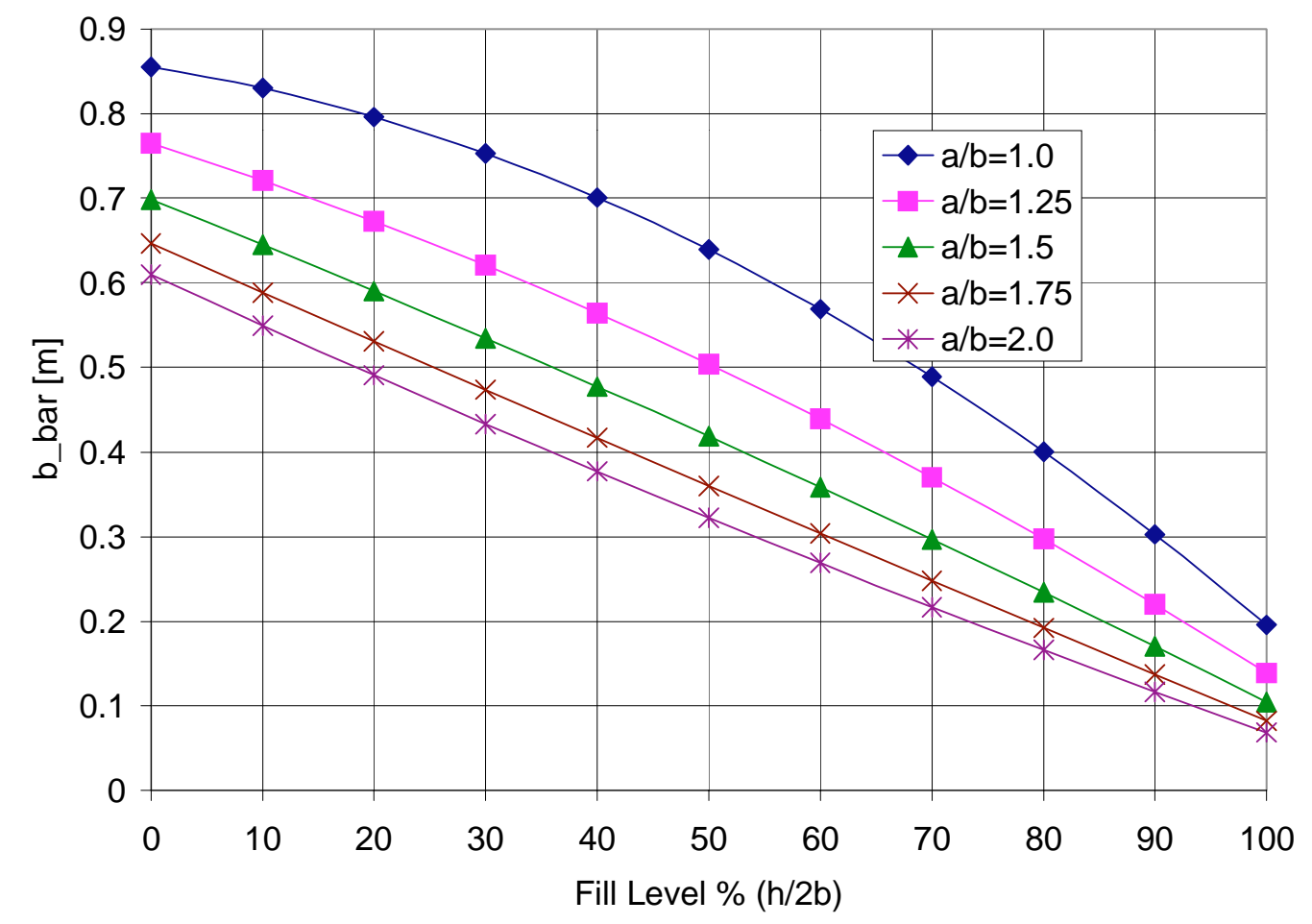

Figure 5.6: Change of $\bar{b}$ as a function of fill level 


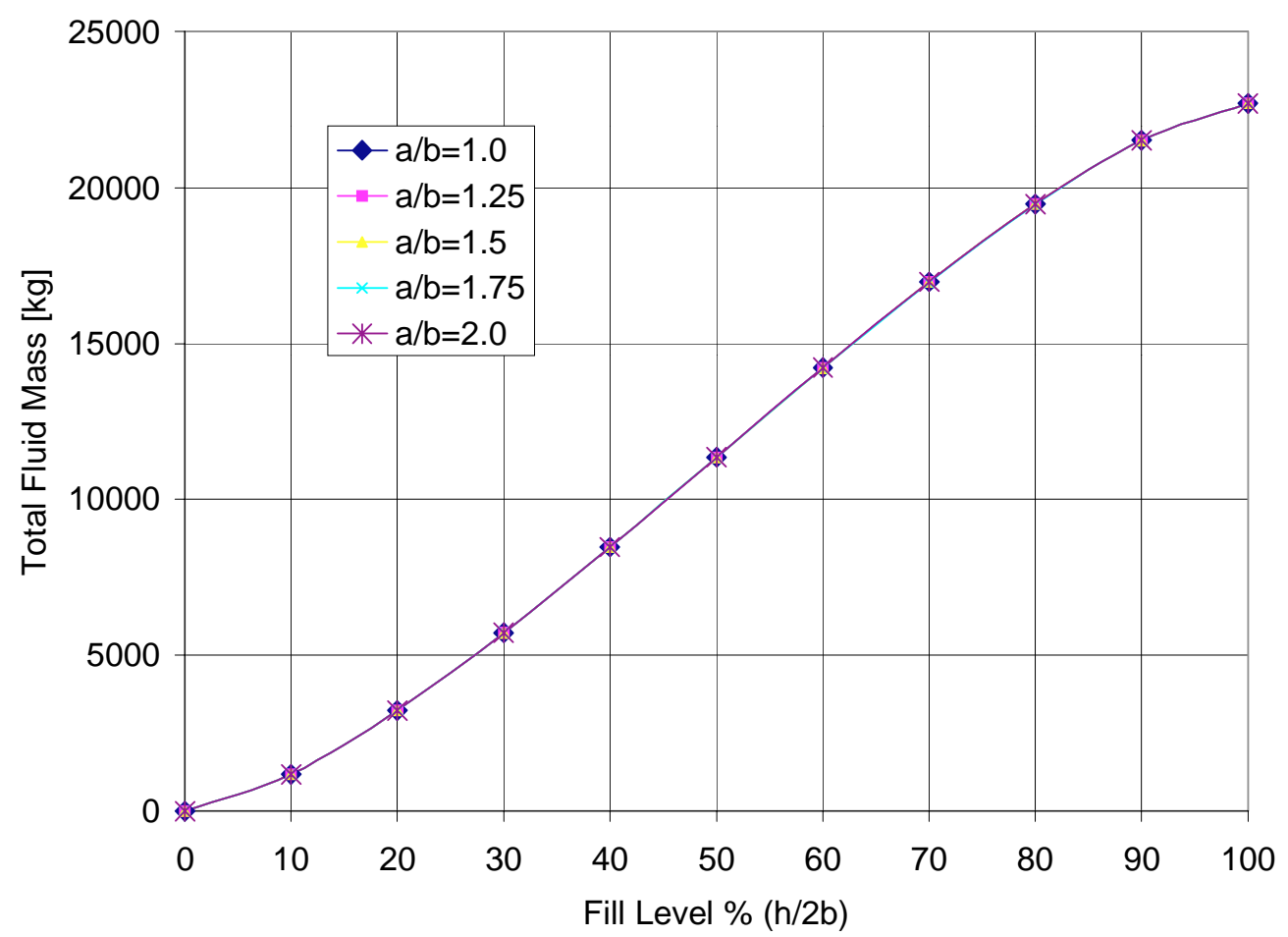

Figure 5.7: Change of fluid total mass as a function of fill level

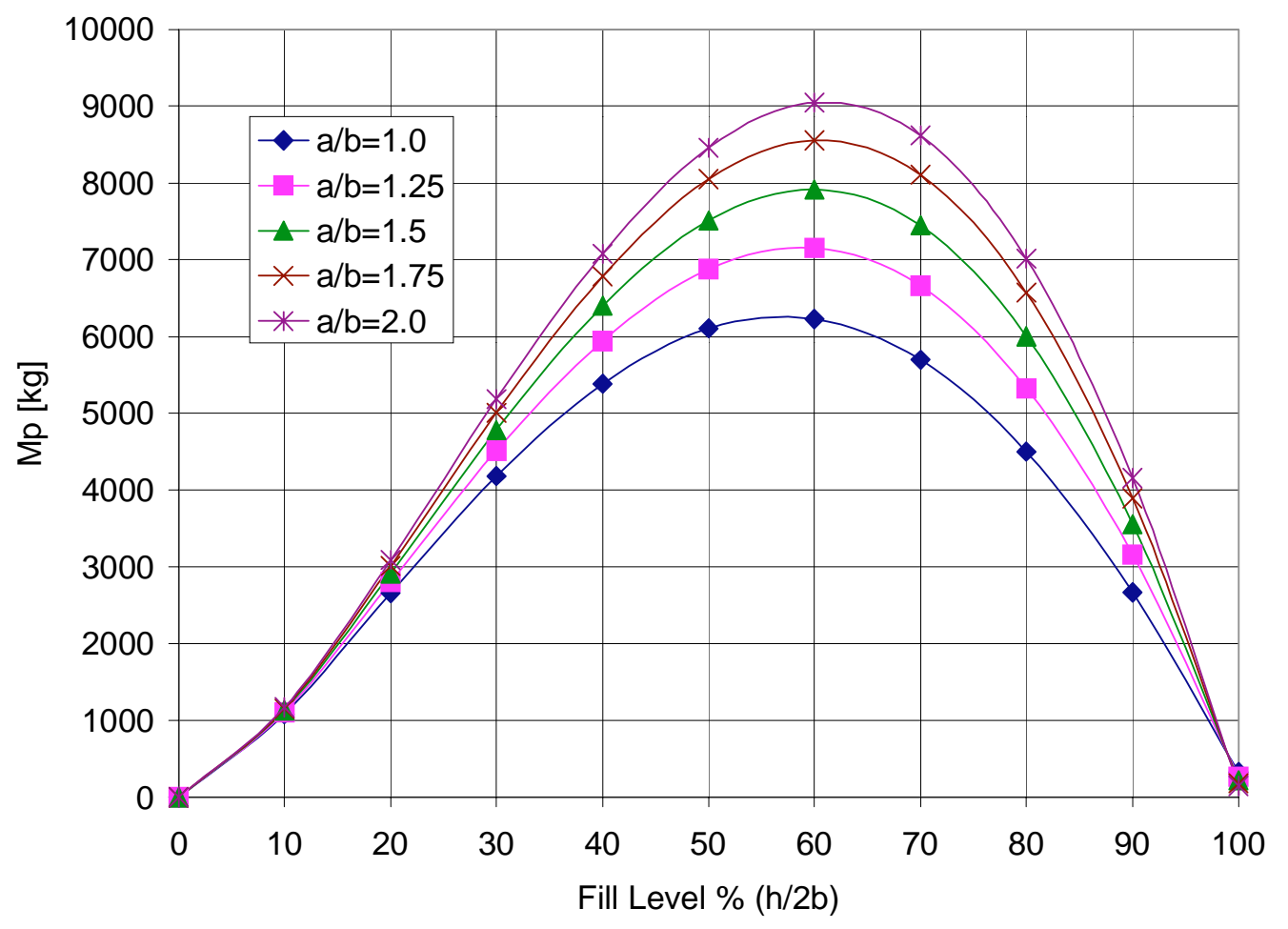

Figure 5.8: Change of pendulum mass as a function of fill level 


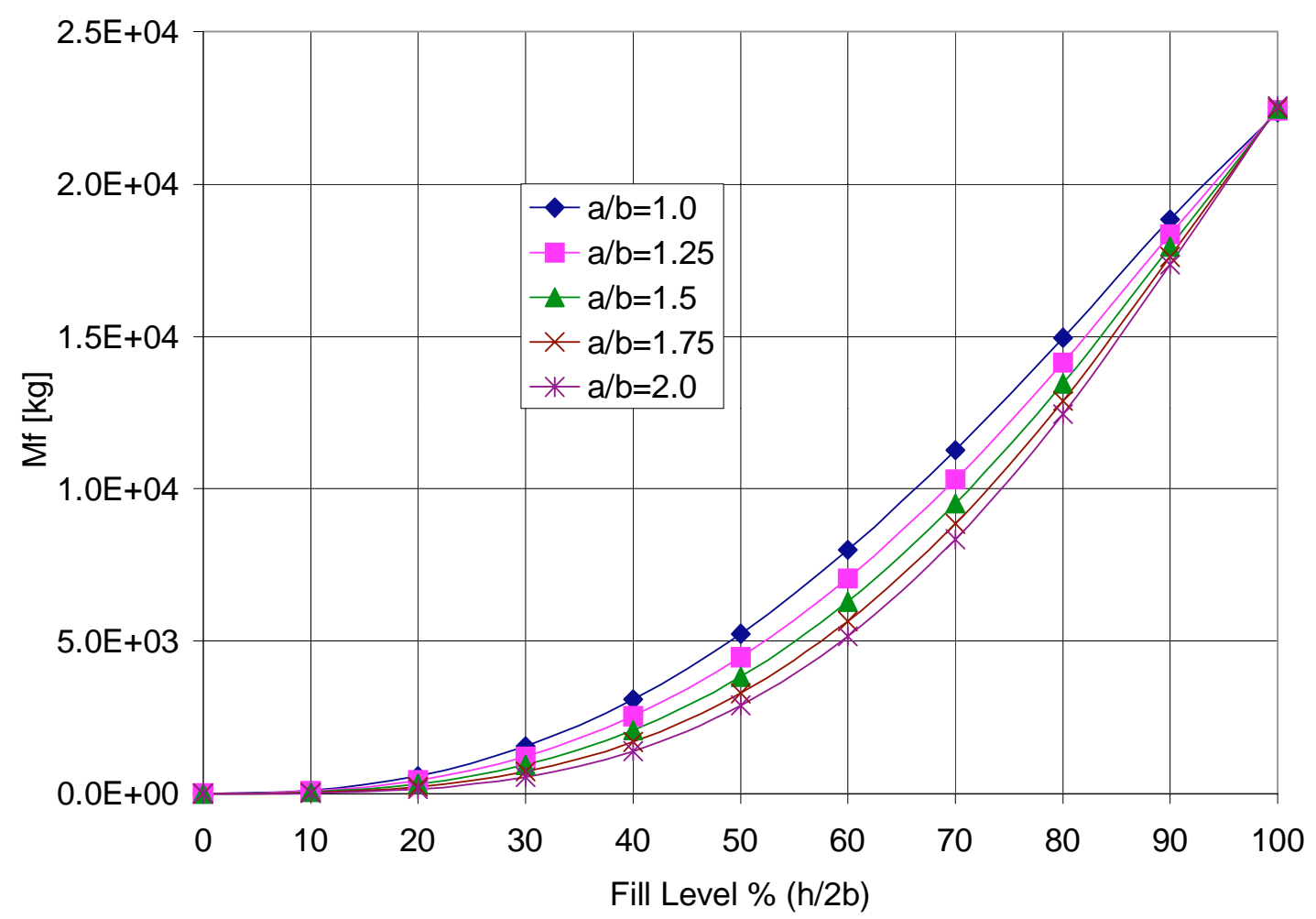

Figure 5.9: Change of fixed fluid mass as a function of fill level

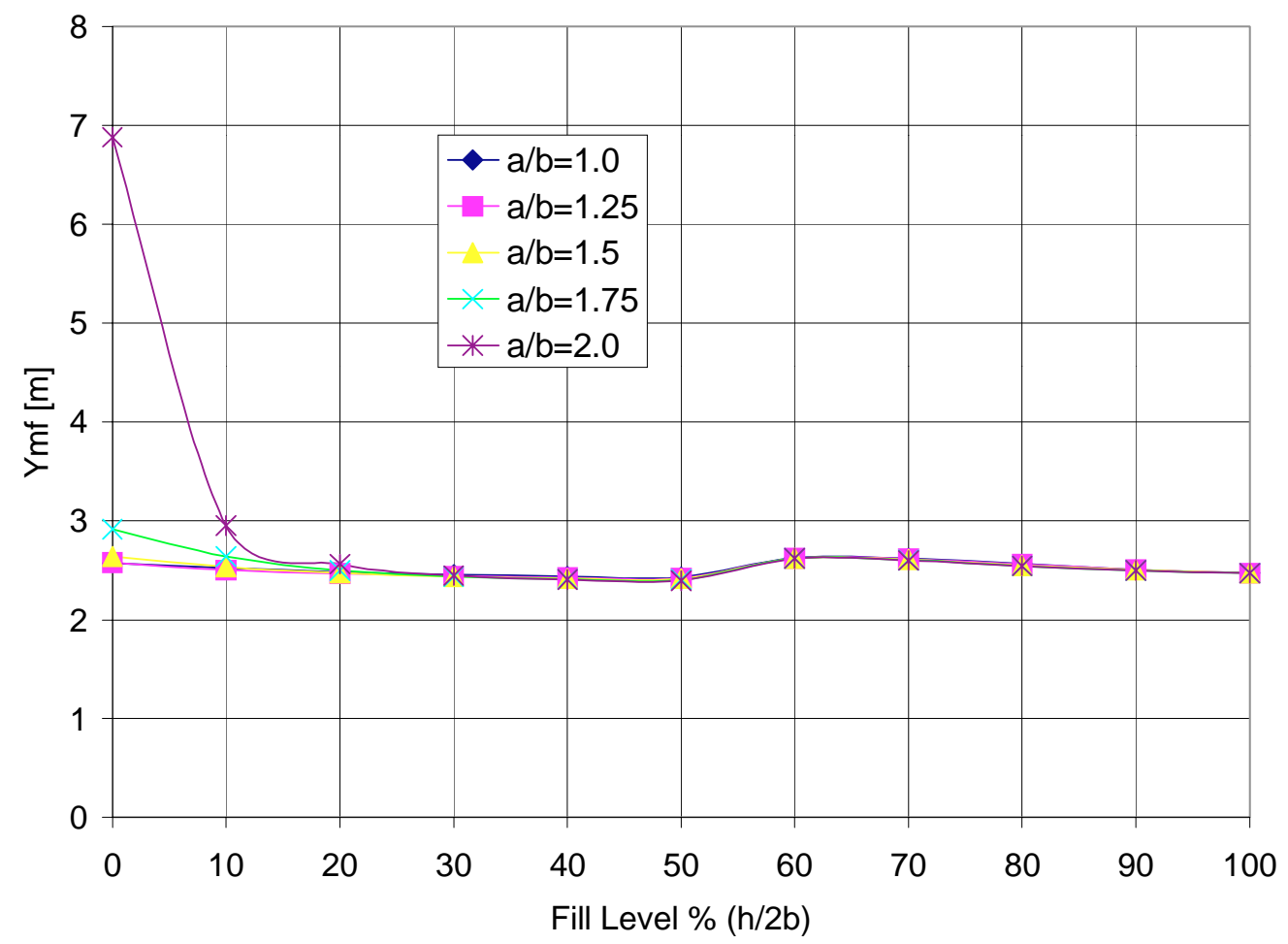

Figure 5.10: Change of the height of the fixed fluid mass from ground (Tank CL height is equal to 3.38m) 
It can be seen from Figure 5.7 that the total mass is the same for different aspect ratios of the ellipse. This important fact is very useful if an attempt is to be made to modify all the results obtained in this chapter and in chapter 4 to be related to the volumetric fill level instead of the fluid height.

\subsubsection{Model Frequency Response.}

The first application of model \#1 was to study the frequency response of the trammel pendulum and its behavior near lateral excitation close to the pendulum base natural frequency given by Equation 4.11. Figure 5.11 shows the plot of the trammel pendulum natural frequency as a function of fill level for the values of $\bar{a}$ and $\bar{b}$ given in Figures 5.5 and 5.6, respectively.

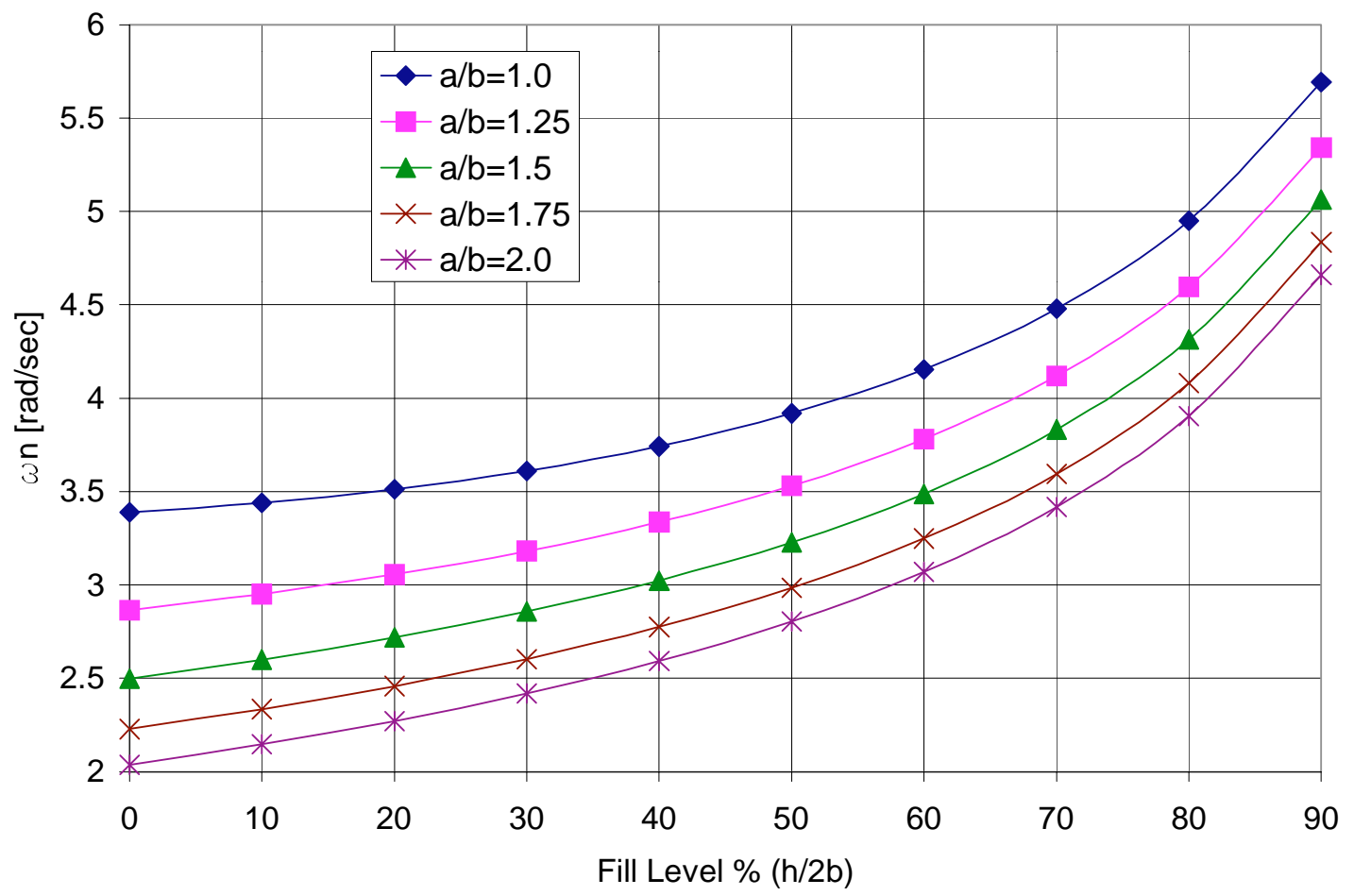

Figure 5.11: Change of trammel pendulum base natural frequency as a function of fill level using the ETNYRE tanker data 
Figures 5.12 shows the response of model 1, as it was exited with low value of lateral acceleration $(0.01 \mathrm{~g})$ at a frequency equal to that obtained from the TOP lane change maneuver given in Figure $3.16(1.2393 \mathrm{rad} / \mathrm{sec})$. On the other hand, Figure 5.13 shows the response of model 1 under the same lateral acceleration and the base natural frequency $(3.917 \mathrm{rad} / \mathrm{sec})$, both cases are for a circular tank. It can be seen that the TOP lane change maneuver frequency is far from the resonance frequency. However, applying the base natural frequency results in a gradual increase in the fluctuation of the support reaction due to the increase of the pendulum angle. Figure 5.14 shows the response of model 1 when applying a lateral acceleration of $0.05 \mathrm{~g}$ with the same base natural frequency mentioned above.

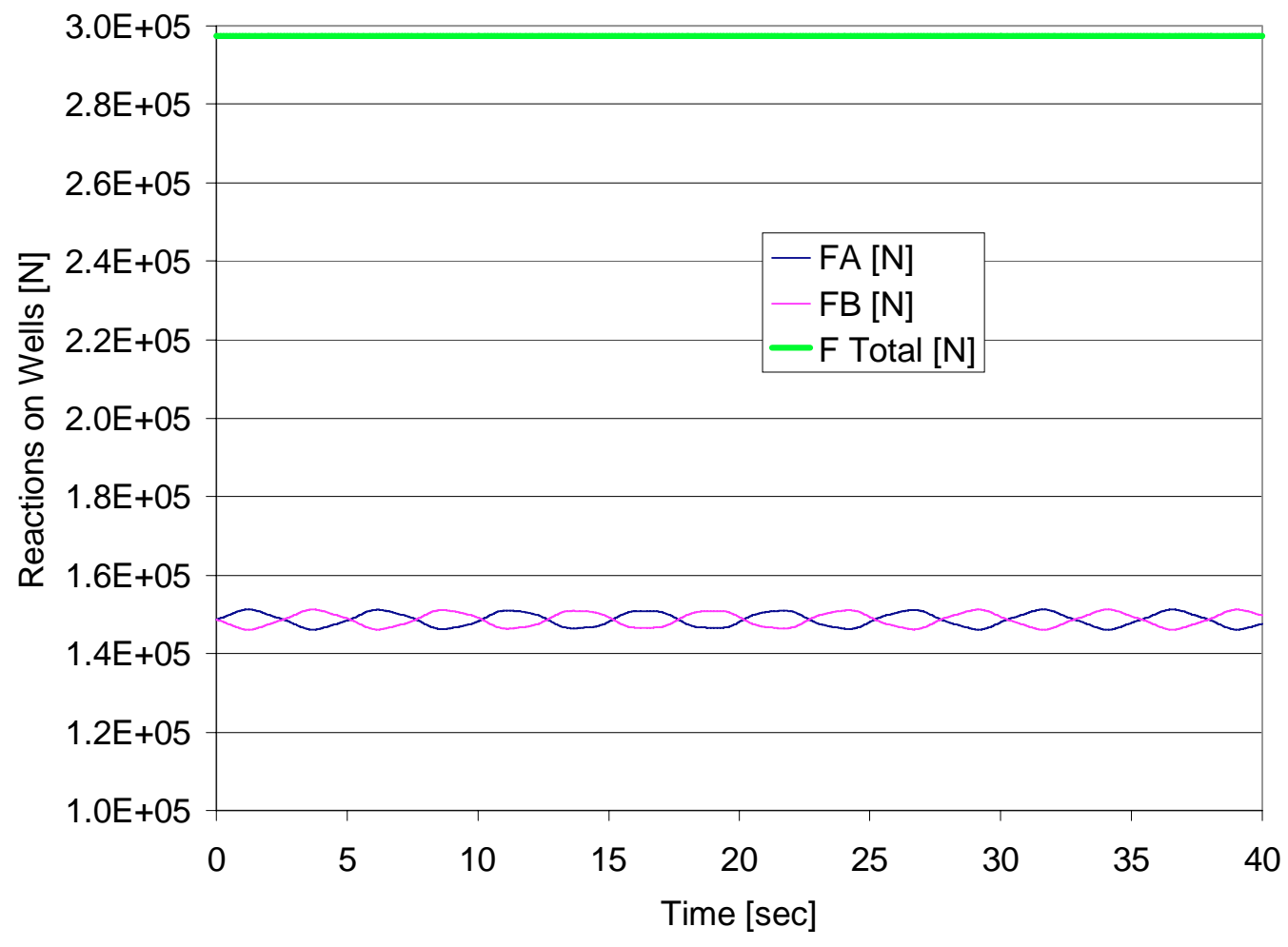

Figure 5.12: Support reactions of model \#1 for a 50\% full circular tank at lateral acceleration of $0.01 \mathrm{~g}$ and frequency of $1.2393 \mathrm{rad} / \mathrm{sec}$ 


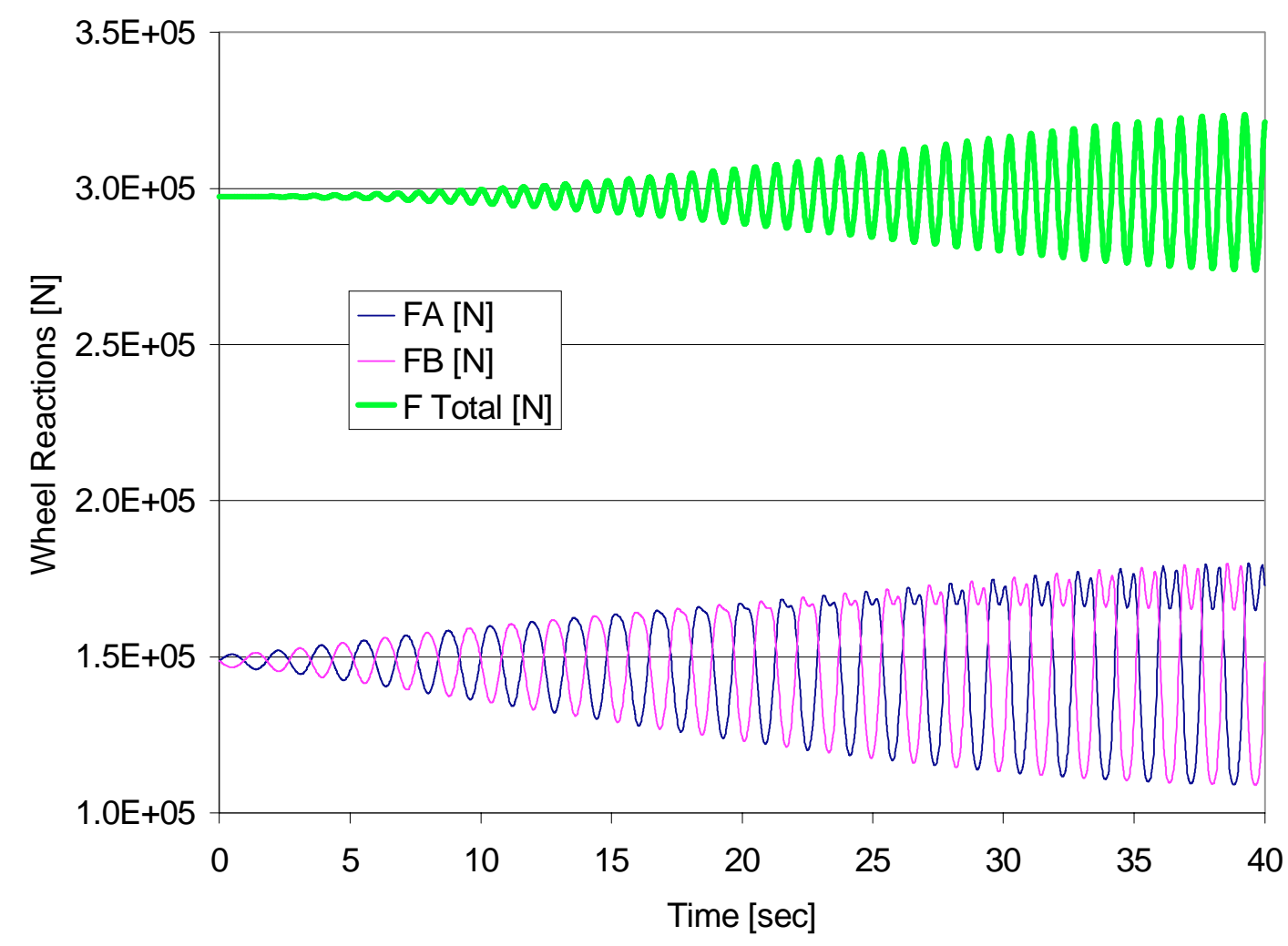

Figure 5.13: Support reactions of model \#1 for a 50\% full circular tank at lateral acceleration of $0.01 \mathrm{~g}$ and frequency of $3.917 \mathrm{rad} / \mathrm{sec}$

It can be seen that the support reactions start also fluctuating with increased amplitude. However, the fluctuations are similar in behavior to the beats ( 2 frequencies with small difference). This can be explained by referring to Figure 4.4. It can be seen that as the pendulum angle increases in the range from 0 to 90 degrees, the frequency of oscillation decreases (for $\mathrm{a} / \mathrm{b}=1$ ). Thus, when applying a lateral excitation equal to the base natural frequency, the pendulum oscillation angle starts increasing gradually. This increase results in more deviation of the input frequency from the natural frequency of oscillation. As this deviation increases with time, the amplitude of pendulum oscillation starts dropping and the same cycle starts again. This phenomenon takes more time when applying a lower lateral acceleration (Figure 5.13) since the initial angle of pendulum oscillation is smaller. 


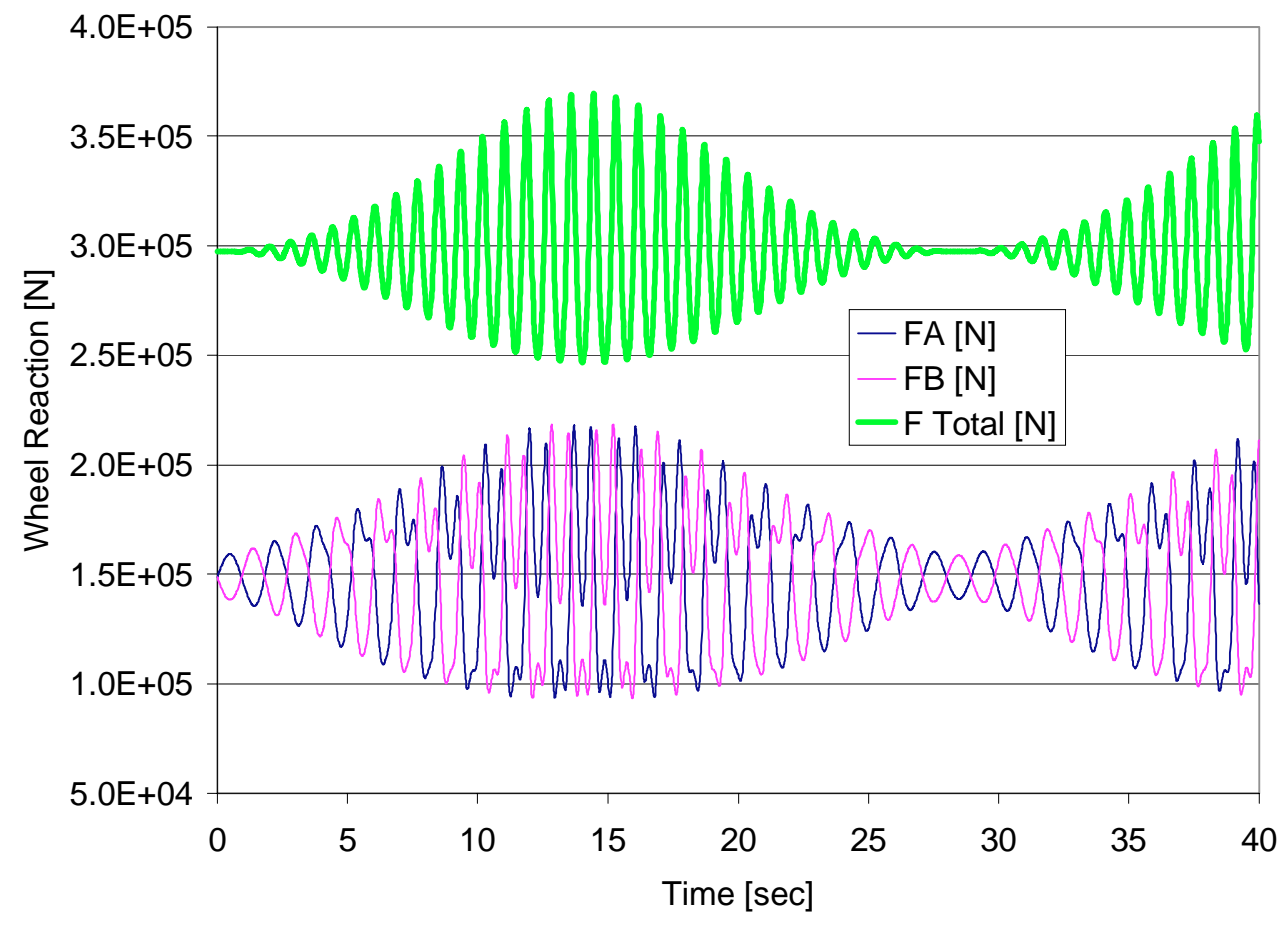

Figure 5.14: Support reactions of model \#1 for a 50\% full circular tank at lateral acceleration of $0.05 \mathrm{~g}$ and frequency of $3.917 \mathrm{rad} / \mathrm{sec}$

Figure 5.15 shows the support reactions when applying a lateral acceleration equal to $0.05 \mathrm{~g}$ on an elliptical tank $(\mathrm{a} / \mathrm{b}=2)$ at the base natural frequency (in this case equals 2.8025 $\mathrm{rad} / \mathrm{sec}$ ). By comparing the results with those for a circular tank shown in Figure 5.14, it can be seen that the time of the beat has increased. 


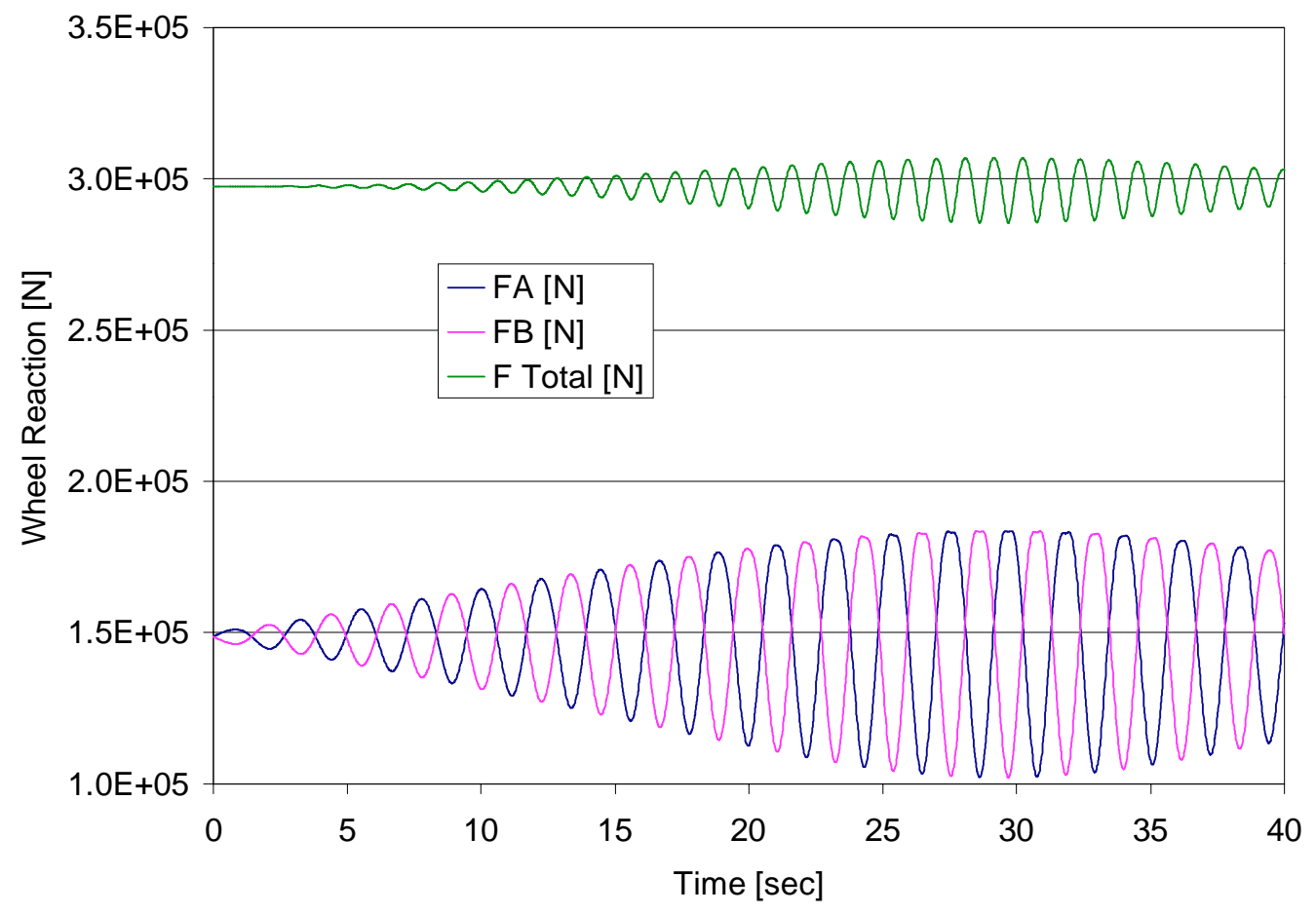

Figure 5.15: Support reactions of model \#1 for a 50\% full elliptical tank $(\mathrm{a} / \mathrm{b}=2)$ at lateral acceleration of $0.05 \mathrm{~g}$ and frequency of $2.8025 \mathrm{rad} / \mathrm{sec}$

Next, the frequency response was calculated and plotted in Figures 5.16 through 5.18. Two cases are shown; one at $0.01 \mathrm{~g}$ and the other at $0.05 \mathrm{~g}$. Figure 5.16 shows the normalized maximum and minimum support reactions over the equivalent steady state values of these reactions vs. the normalized input frequency over the base natural frequency. Figure 5.17 shows the percent weight transfer from one side to the other, the star on the graph indicates the point of relative instability at $100 \%$ weight transfer. 


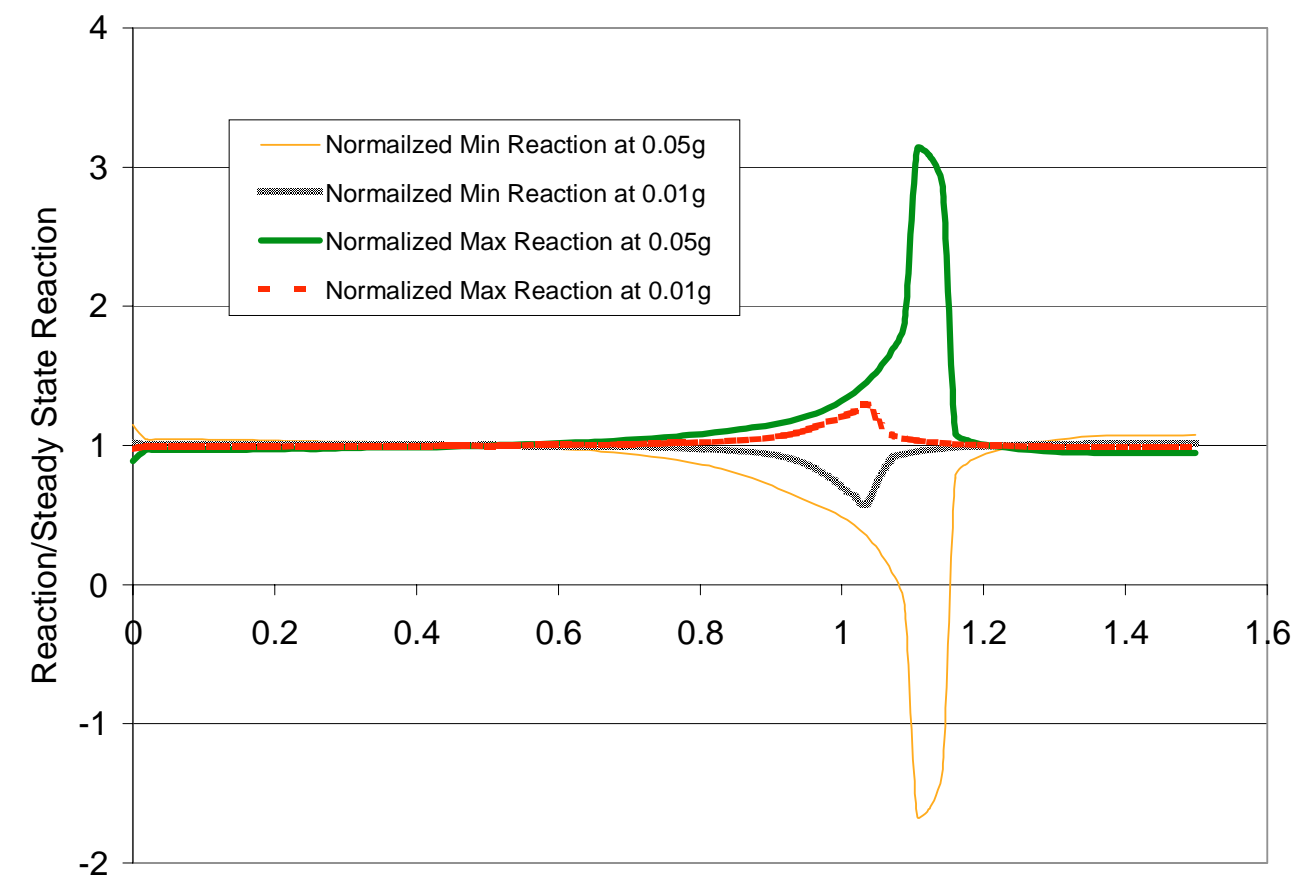

$\omega / \omega \mathbf{n}$

Figure 5.16: Normalized maximum and minimum support reactions at $0.01 \mathrm{~g}$ and $0.05 \mathrm{~g}$ lateral accelerations for $50 \%$ full $(\mathrm{a} / \mathrm{b}=2)$

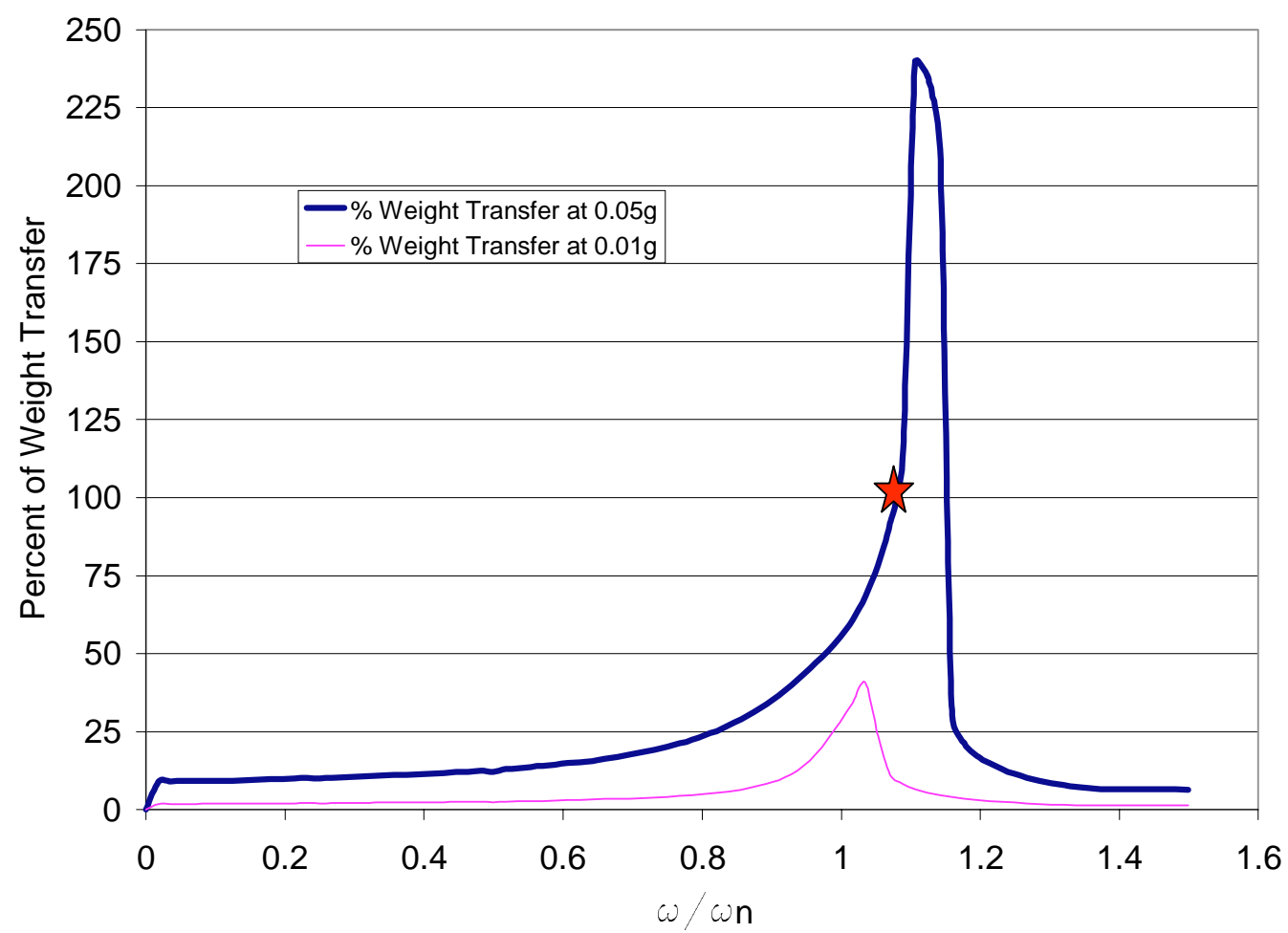

Figure 5.17: Weight transfer percent at $0.01 \mathrm{~g}$ and $0.05 \mathrm{~g}$ lateral accelerations for $50 \%$ full $(\mathrm{a} / \mathrm{b}=2)$ 
Figure 5.18 shows the normalized percent weight transfer over the steady state percent weight transfer. It can be seen that the ratio has reached more than 18 times the steady state.

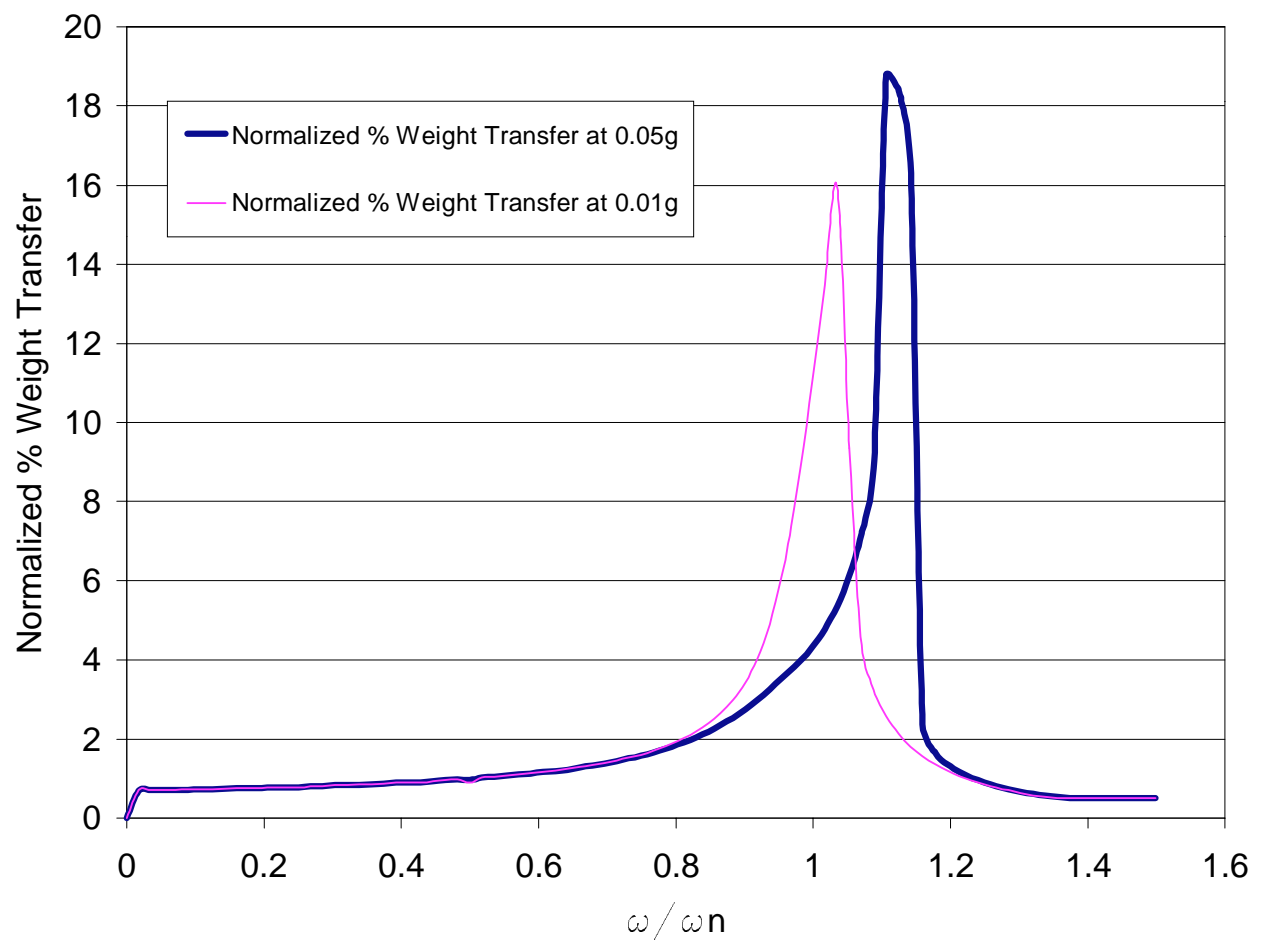

Figure 5.18: Normalized Weight transfer percent at $0.01 \mathrm{~g}$ and $0.05 \mathrm{~g}$ lateral accelerations for $50 \% \mathrm{full}(\mathrm{a} / \mathrm{b}=2)$

Two features can be recognized in the frequency response given above:

The first, it can be seen that the peek value of reaction occurs at a higher value than $\omega=\omega_{\mathrm{n}}$ and that this peak shifts towards a higher ratio of $\omega / \omega_{n}$ with the increase of the input lateral acceleration. This is because for an aspect ratio equals 2, as the angle of oscillation increases, the natural frequency increases (See Figure 4.4). Thus, when applying an input frequency slightly higher that the base natural frequency, the frequency of oscillation will increase and catch up with this input value resulting in more resonance of the pendulum. Thus, an opposite trend should be obtained for the circular tank, which is illustrated in Figure 5.19. 


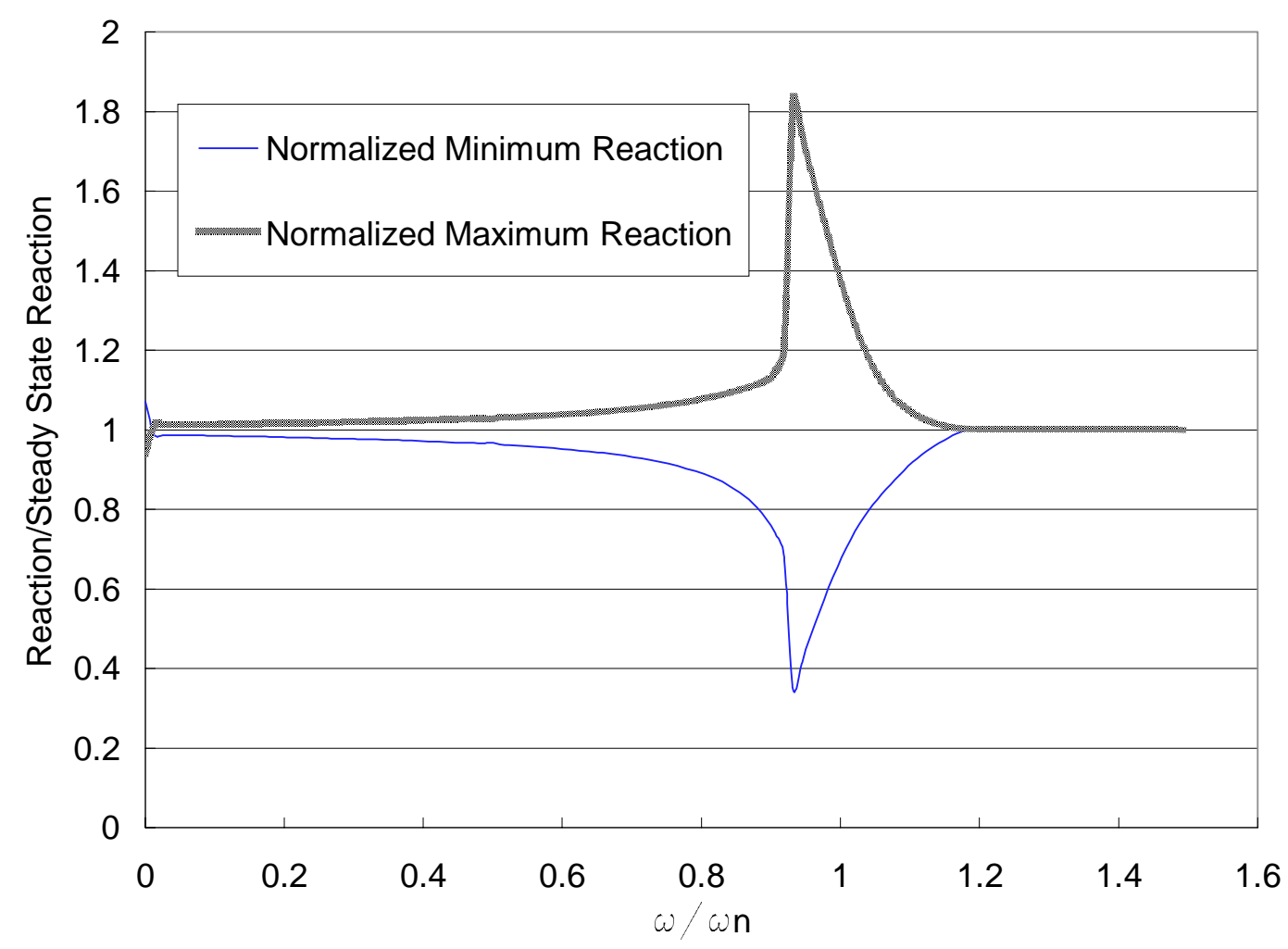

Figure 5.19: Normalized maximum and minimum support reactions at $0.05 \mathrm{~g}$ lateral accelerations for $50 \%$ full $(\mathrm{a} / \mathrm{b}=1)$

The second is that the rate at which the amplitude increases as the frequency approaches the base natural frequency, is much less than the rate of amplitude decrease when the input frequency starts exceeding the base natural frequency for $a / b=2$. Again, an opposite trend is recognized for $\mathrm{a} / \mathrm{b}=1$.

From the above discussion, it can be concluded that the critical frequency shifts to higher value from the base natural frequency as the aspect ratio increases. On the other hand, it can shift to a lower value than the base natural frequency for a circular tank. Also, the model requires long time to reach critical amplitudes of oscillation under low lateral acceleration values. Such behavior is rather to be achieved when a vehicle is going in a straight line and 
having lateral inputs due to driver corrective action or due to parametric instabilities (Snaking) not during lane change maneuvers.

It should be also kept in mind that the above discussion holds for the pendulum model and not for the fluid behavior. Such trends need further experimental or FEM validation.

\subsubsection{Effect of Changing Aspect Ratio on Rollover Threshold.}

The first effect that was considered in changing the aspect ratio of the tank on the rollover threshold is the change of the tank weight it self. It's known that for an elliptical shape, the perimeter is given by:

$$
\begin{gathered}
\mathrm{P}=\pi(a+b)\left[1+\sum_{n=1}^{\infty}\left(\prod_{k=1}^{n} \frac{2 k-3}{2 k}\right)^{2} \lambda^{2 n}\right] \\
\lambda=\frac{a-b}{a+b}
\end{gathered}
$$

The percent change of the perimeter length was calculated for the ETNYRE tanker as the aspect ratio changes from 2 to 1 . Figure 5.20 shows that the length can decrease by $8 \%$ for a circular tank of an equivalent are. The mass of the tank was calculated based on the perimeter, length, density of steel and the given shell thickness $(4.36 \mathrm{~mm})$ and is listed in table 5.3.

Table 5.3: Tanker shell mass at different aspect ratios.

\begin{tabular}{|c|c|c|c|c|c|}
\hline $\mathrm{a} / \mathrm{b}$ & 1 & 1.25 & 1.5 & 1.75 & 2 \\
\hline Shell Mass & $1805 \mathrm{~kg}$ & $1821 \mathrm{~kg}$ & $1860 \mathrm{~kg}$ & $1911 \mathrm{~kg}$ & $1961 \mathrm{~kg}$ \\
\hline
\end{tabular}

However, the shell's mass is only $20 \%$ of the total trailer mass. Thus, the maximum change in weight from the elliptical shape to the circular shape will be less than $2 \%$ for the ETNYRE tanker, which can be neglected. Although for compressed liquids tanks the thickness 
of the shell can reach more than $10 \mathrm{~mm}$, and the change of perimeter can be significant, these tanks are hardly of an elliptical shape.

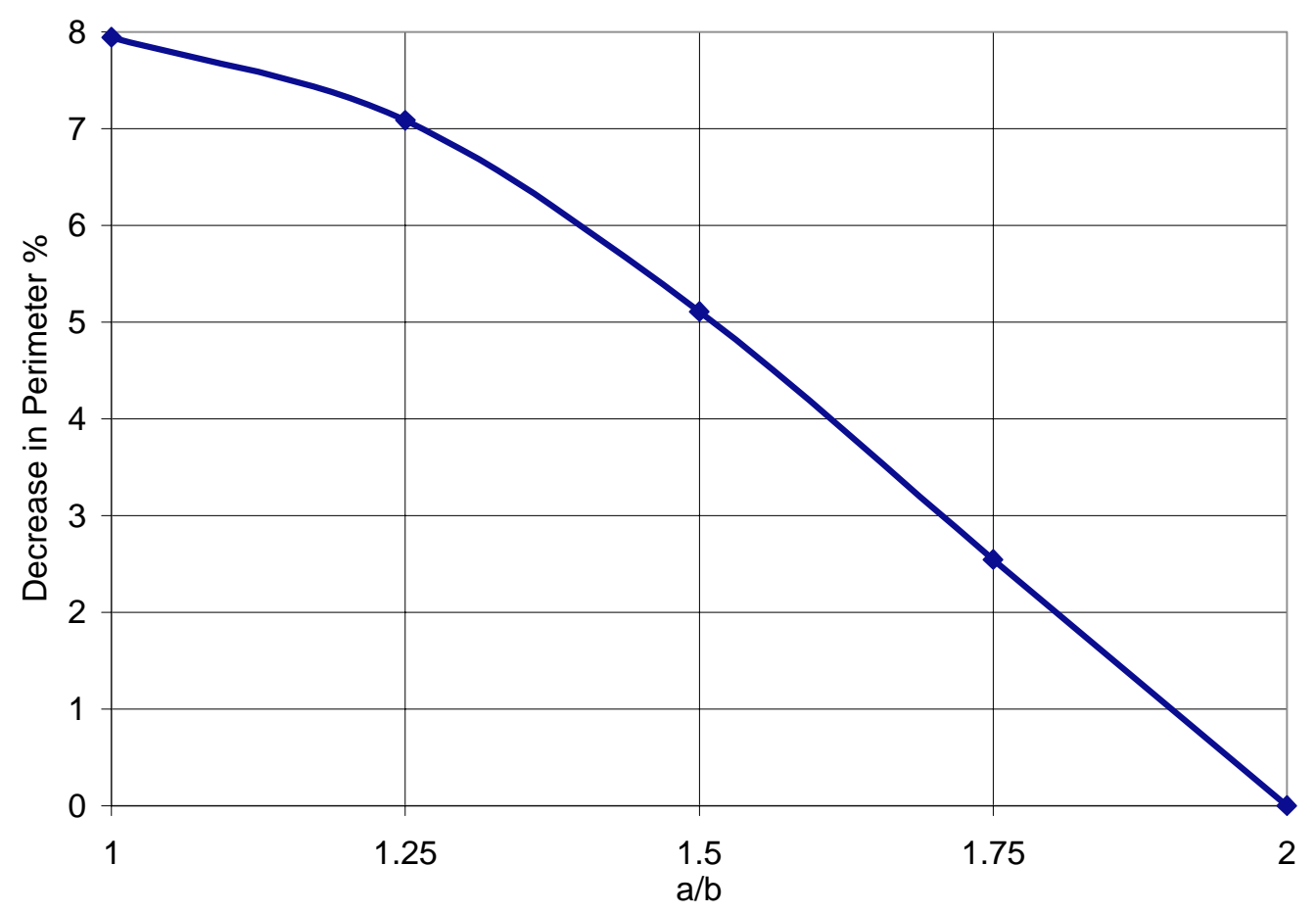

Figure 5.20: Decrease of tank shell perimeter with the decrease of a/b from 2 to 1

The change in the aspect ratio can be considered many different ways. Probably, the most three significant aspects are; constant tanker CG height, constant tanker overall height, or constant ground clearance. Choosing which method to use depends on many parameters such as road regulations, tractor fifth wheel height, ground clearance, stability, etc. All three assumptions have been analyzed, to determine the rollover threshold at different levels of fill. Figure 5.21 shows a sample of the plots used to determine the rollover threshold at different levels of fill. Figures 5.22, 5.23 and 5.24 show the rollover threshold at different fill levels considering CG height, constant overall height, and constant ground clearance, respectively. 


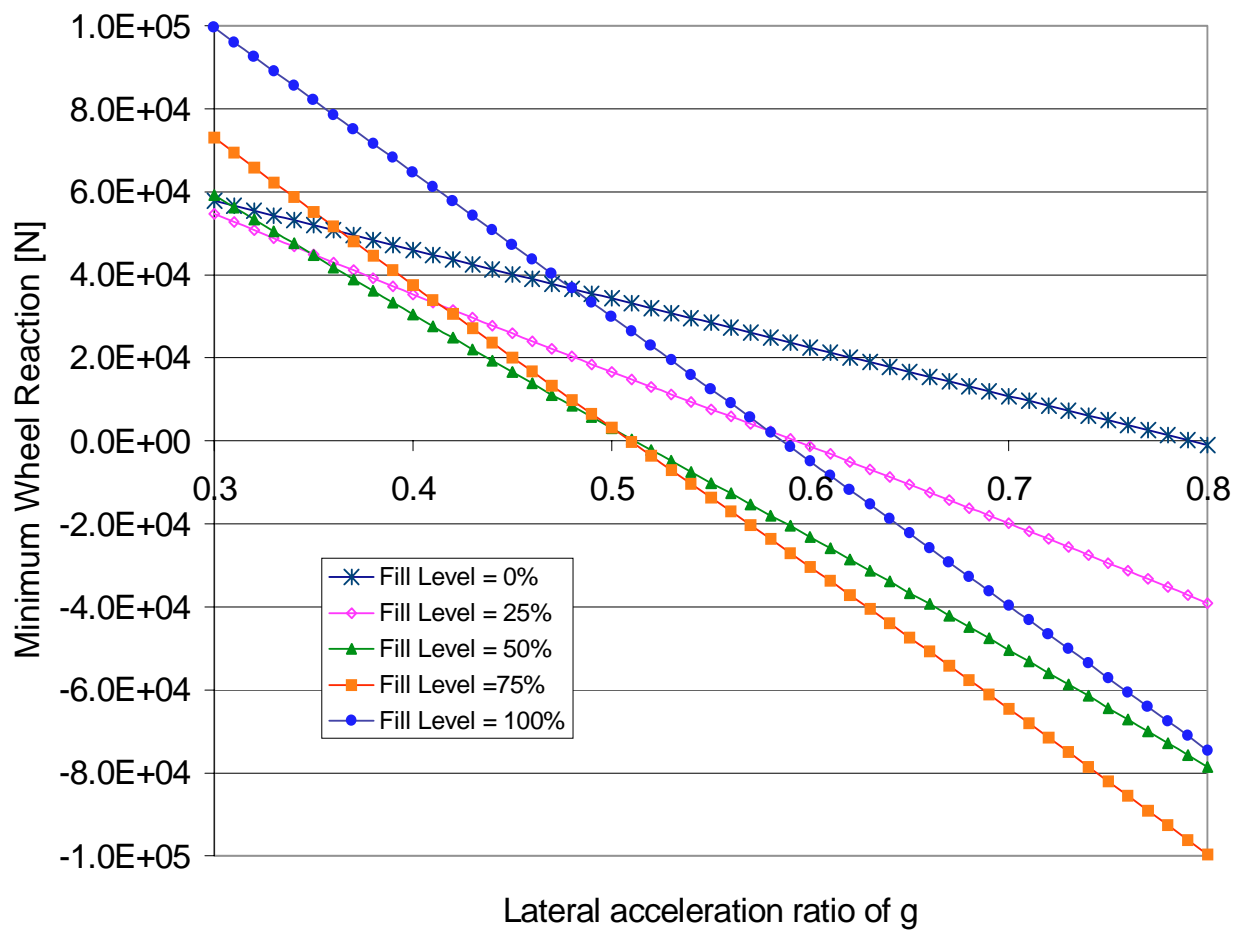

Figure 5.21: Minimum support reactions vs. lateral acceleration at different levels of fill $(\mathrm{a} / \mathrm{b}=1)$

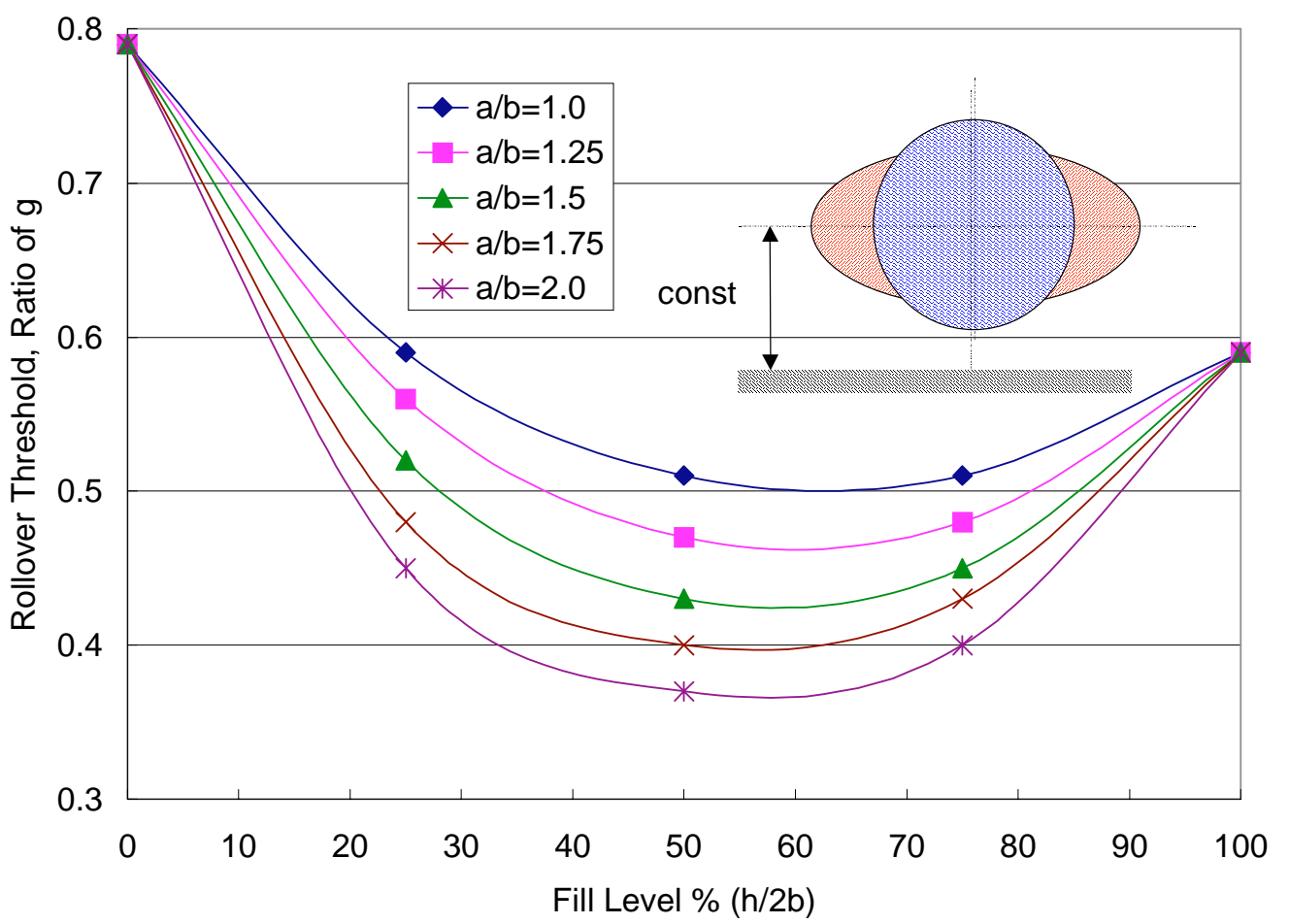

Figure 5.22: Rollover threshold at different fill levels for the ETNYRE tanker assuming constant CG height 


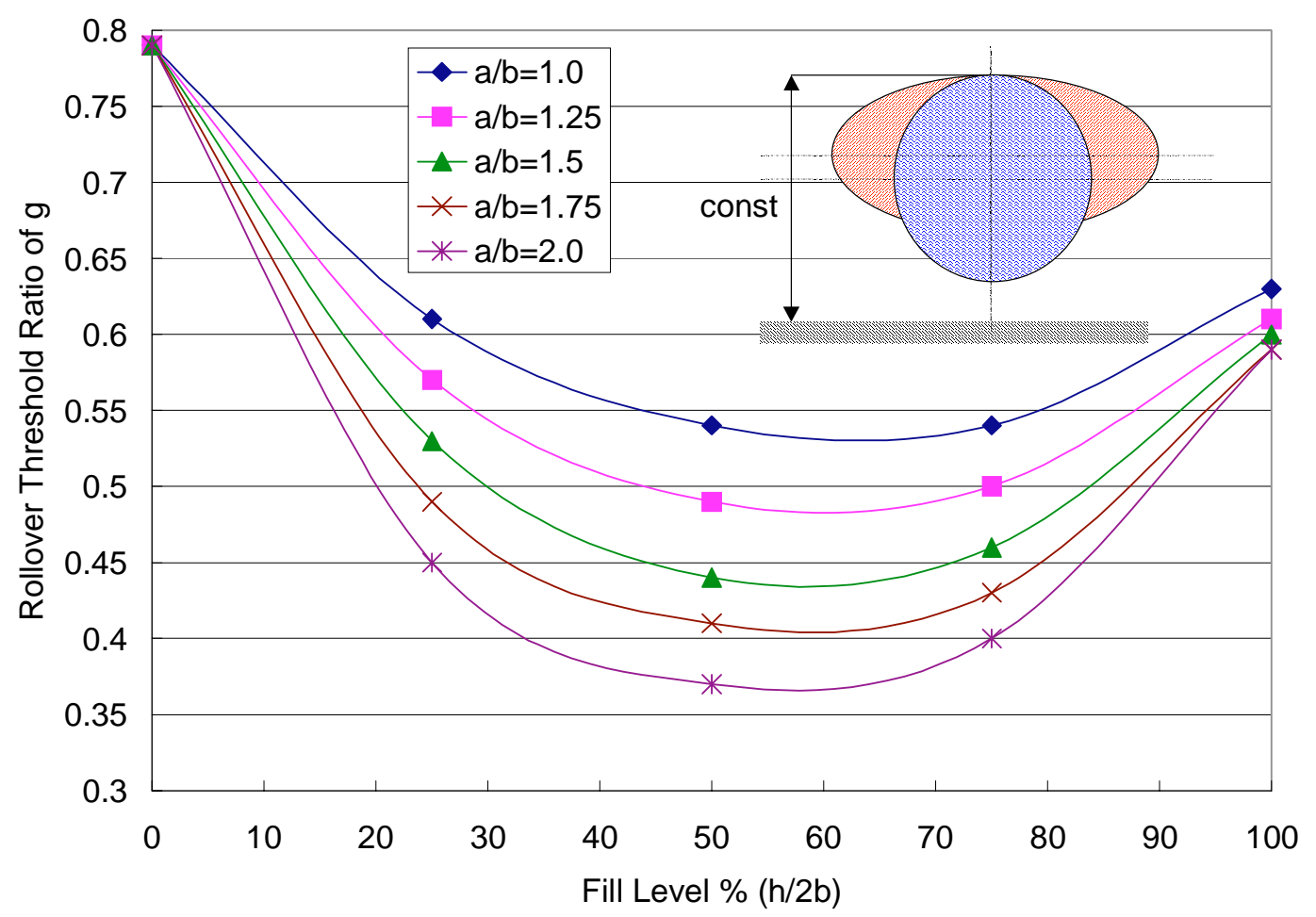

Figure 5.23: Rollover threshold at different fill levels for the ETNYRE tanker assuming constant overall height

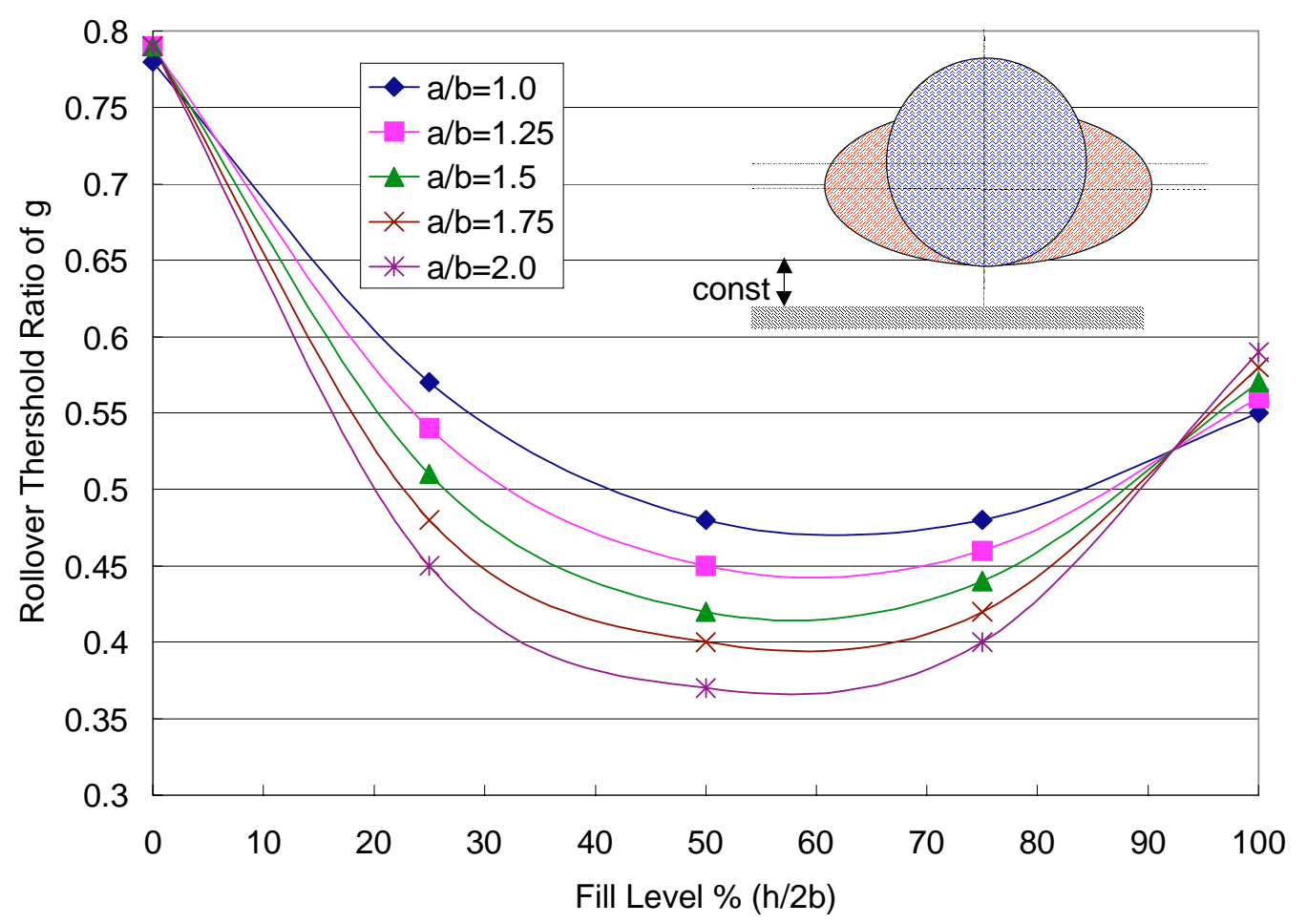

Figure 5.24: Rollover threshold at different fill levels for the ETNYRE tanker assuming constant ground clearance 
It can be seen that for the constant CG height case and the constant overall height case, the more the aspect ratio of the tank, the less the rollover threshold at all levels of fill except at $0 \%$. It can also be seen that the critical level of fill is around $58 \%$ for the tanker with $\mathrm{a} / \mathrm{b}=2$ and increases gradually to $64 \%$ when $\mathrm{a} / \mathrm{b}=1$. The minimum rollover threshold improves from $0.36 \mathrm{~g}$ for the constant overall height to $0.38 \mathrm{~g}$ for the constant $\mathrm{CG}$ assumption. It can be seen from Figure 5.24 that the constant ground clearance assumption resulted in a more stable elliptical tank than a circular tank for fill levels above 92\% and below 3\% (nearly full and empty conditions). Figures 5.22 through 5.24 provide guides for the inputs of model \#2 to test the support liftoff. Figure 5.25 shows the results of model \#1 when subjected to the TOP lane change test lateral acceleration (see Figure 3.16). It can be seen that model \#1 didn't indicate instability of the tanker. Thus, these inputs will not be used with model \#2.

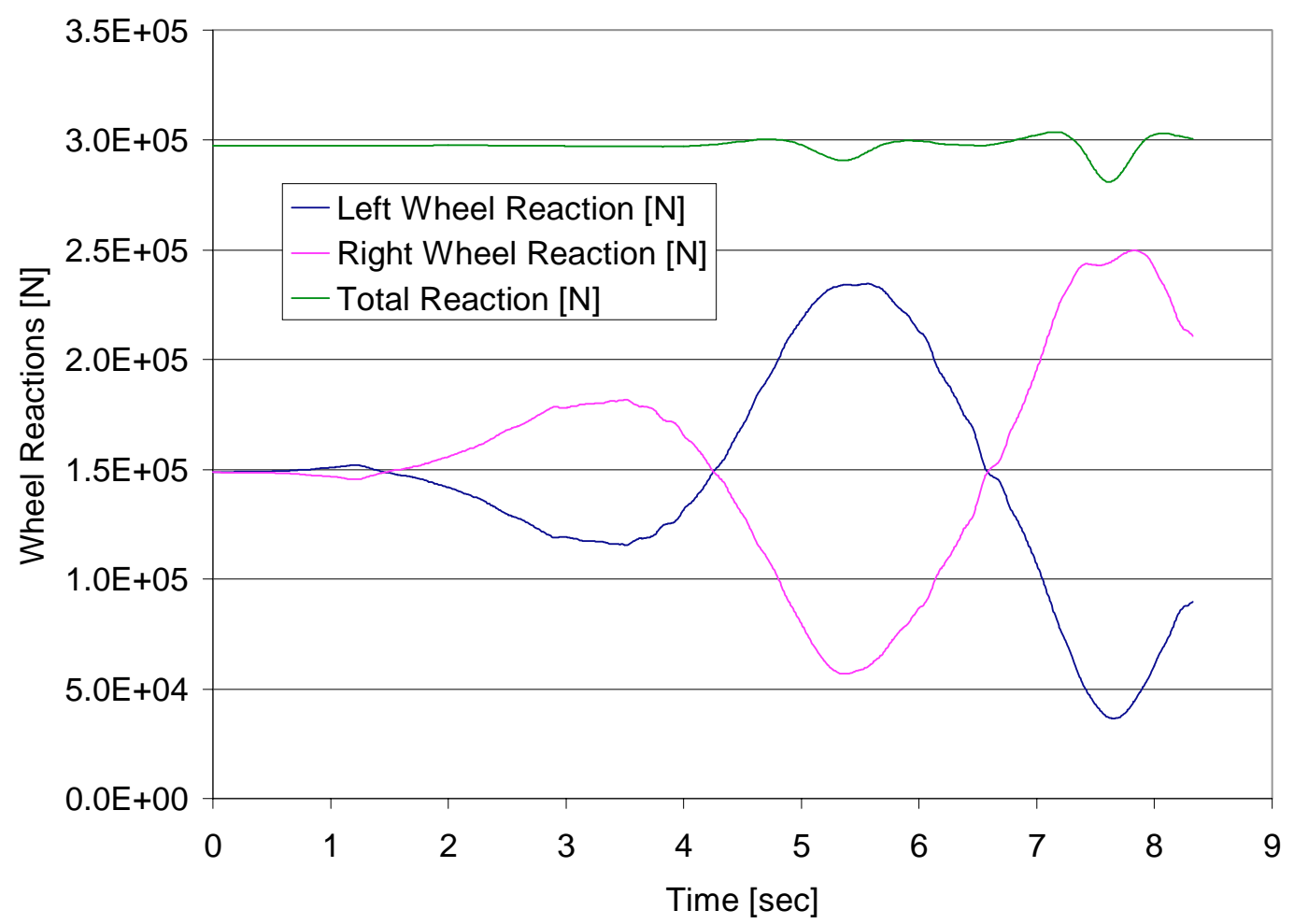

Figure 5.25: Support reactions for the 50\% full ETNYRE tanker obtained using ATC lateral acceleration input during the TOP lane change maneuver 


\section{3- Model 2, Vehicle Rigid Body Model, with Support Liftoff.}

This model is similar in geometry and all assumptions to model number one, except for the fact that the supports are allowed to liftoff. The rotation is assumed to take place about one of the two supports representing the wheels (unilateral support case) assuming no slip with the ground. Since the vehicle mass will rotate, the polar moment of inertia of the vehicle and the fluid volume was considered. Figure 5.25 shows a schematic diagram of this model.

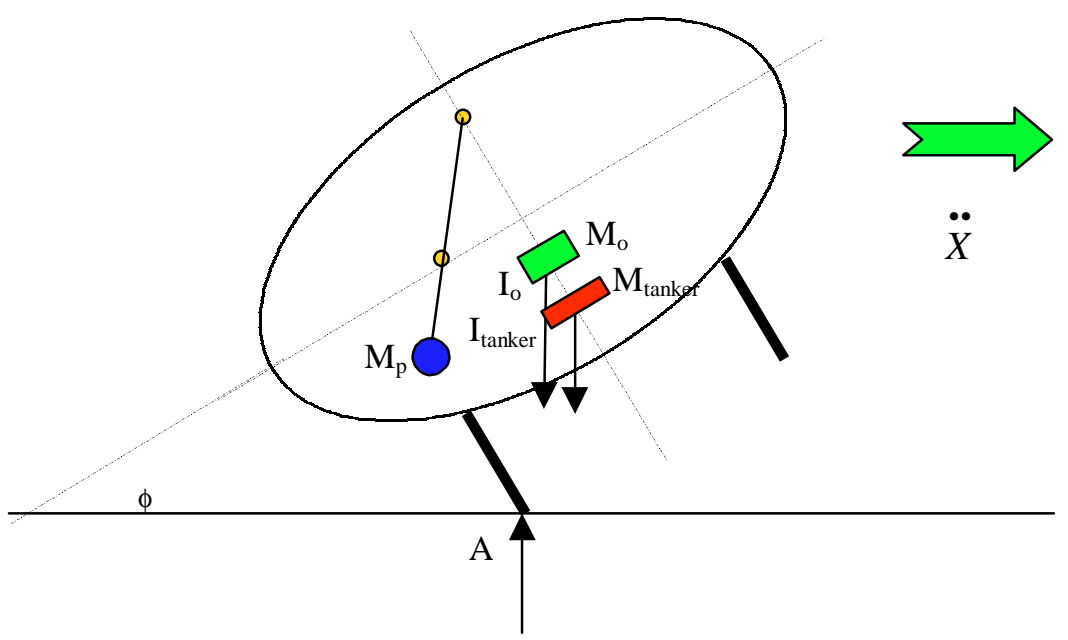

Figure 5.26: Model \#2, vehicle rigid body model with support liftoff

It should be noted that the moment of inertia of the tanker was obtained from the FE model developed by Gautam and Mucino (shown in Figure 2.19) and was found equal to $4.91 * 10^{4} \mathrm{~kg} \mathrm{~m}^{2}$. The principle moment of inertia of the fluid was calculated analytically and using FEM. The details of calculating the polar moment of inertia of the fluid are given in section B5 in Appendix B. In addition, since the tank will rotate and the fluid cross section shape will change, the variation in the principle polar moment of inertia with analyzed in section B5. It should be also noted that the term "fixed mass" is inaccurate in describing the non- 
sloshing portion of the fluid inside the tank since it should move in a counter direction to the rolling angle of the vehicle to remain at the bottom of the tank under the action of gravity.

Bearing in mind the facts about the inertia and the fixed mass given in the previous paragraph, the equations of motion of model \# 2 were driven assuming constant fluid polar moment of inertia and a fixed fluid mass fixed to the tanker body. The derivation of the equations of motion of model \#2 is given in section B4 of appendix B. A computer code called "basic3.m" was written using MatLab to solve the equations of motion and obtain the behavior of model \#2. The code uses the minimum wheel reaction criterion to identify the beginning of the relative rollover and switches between different sets of differential equations based on which reaction side becomes less than zero.

Figure 5.27 shows the rollover threshold when neglecting the vehicle mass, considering the vehicle mass, considering the vehicle polar moment of inertia, and considering the fluid inertia. Both relative and absolute stability limits are shown. It can be seen that for the weightless vehicle, the more the fill level, the more the roll stability. In addition, it's clear that neglecting the vehicle weight reduces the rollover threshold considerably. It can be seen that when the weight of the vehicle is considered, the absolute rollover threshold can be much higher than the relative rollover especially at the middle levels of fill. On the other hand, when considering the polar moment of inertia of the tank, the absolute rollover threshold limits had a negligible increase. Figure 5.28 shows the polar moment of inertia of the fluid as a ratio of the ETNYRE tanker polar moment of inertia around the support point of rotation. 


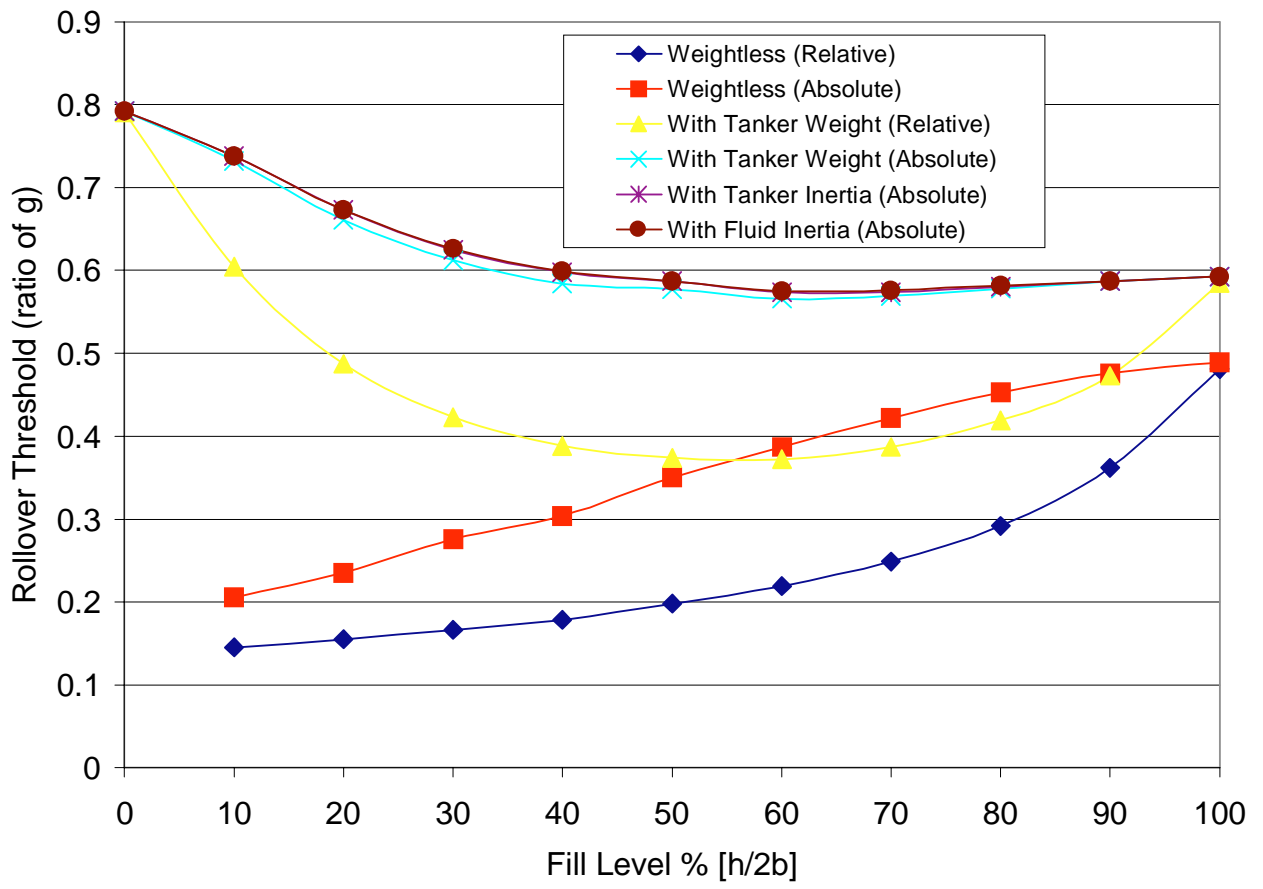

Figure 5.27: Absolute and relative rollover thresholds of vehicle model \#2 at different weight and inertia considerations

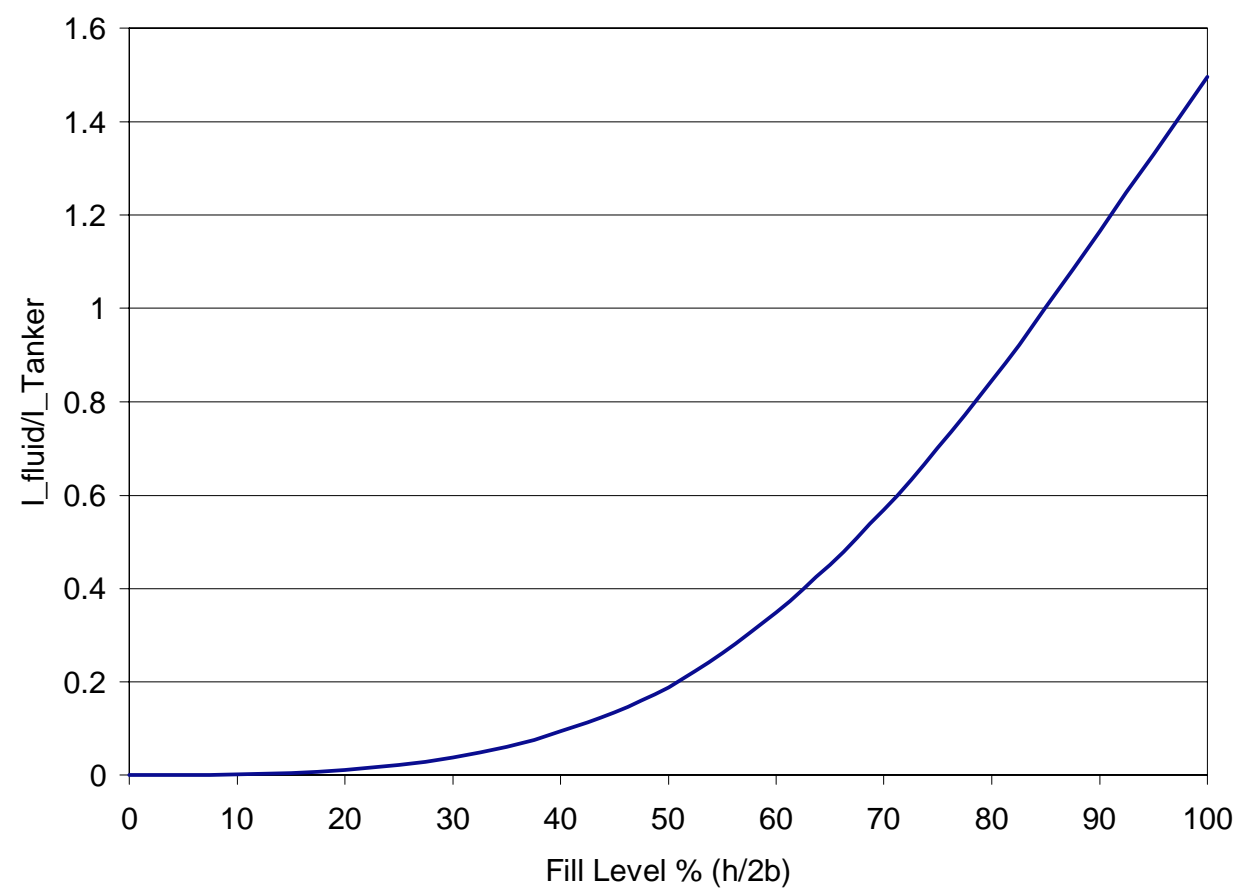

Figure 5.28: Ratio between fluid polar moment of inertia and tanker polar moment of inertia around the supports point of rotation at different levels of fill 
It can be seen from Figure 5.28 that the fluid polar moment of inertia reaches a maximum value of 1.5 the tanker inertia at the $100 \%$ full condition. This explains why the fluid inertia also has negligible effect on the rollover stability limits.

Figure 5.29 shows the vehicle toll angle as it approaches the absolute rollover stability limits for a $50 \%$ full tanker considering the tanker weight. It can be seen that although the difference between the absolute and the relative rollover threshold is considerable, once the vehicle roll angle reaches few degrees, the vehicle rolls over within a very narrow window $(0.009 \mathrm{~g})$.

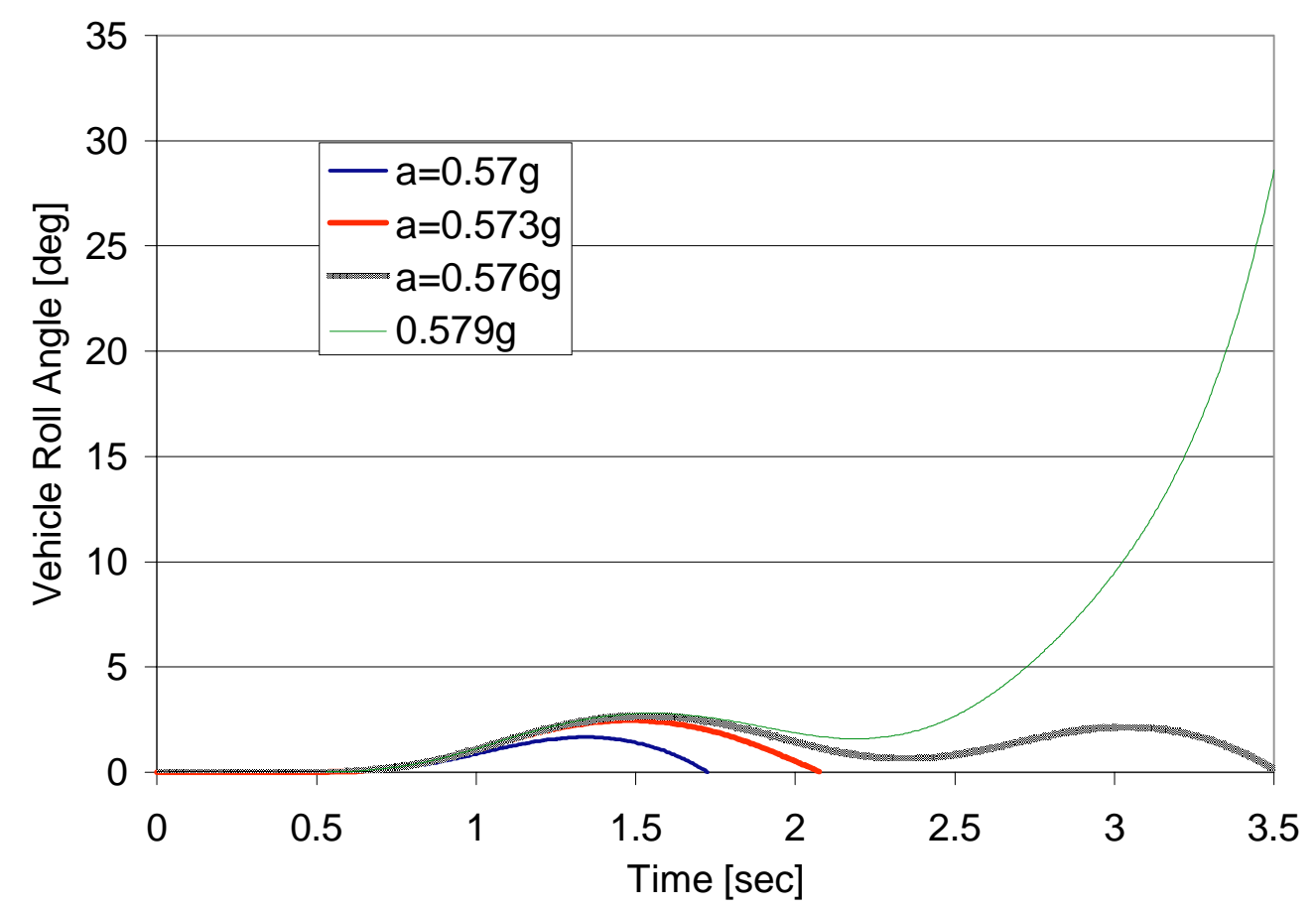

Figure 5.29: Vehicle roll angle near the absolute rollover threshold (Case for a 50\% full tanker with weight considered and no inertia)

To see how the pendulum behaves near rollover, Figure 5.30 shows the pendulum angle plotted on top of the vehicle roll angle. Note that the pendulum starts from $270^{\circ}$ based on the reference frame given in Figures B13 and B14 and that it's measured relative to the vehicle. 


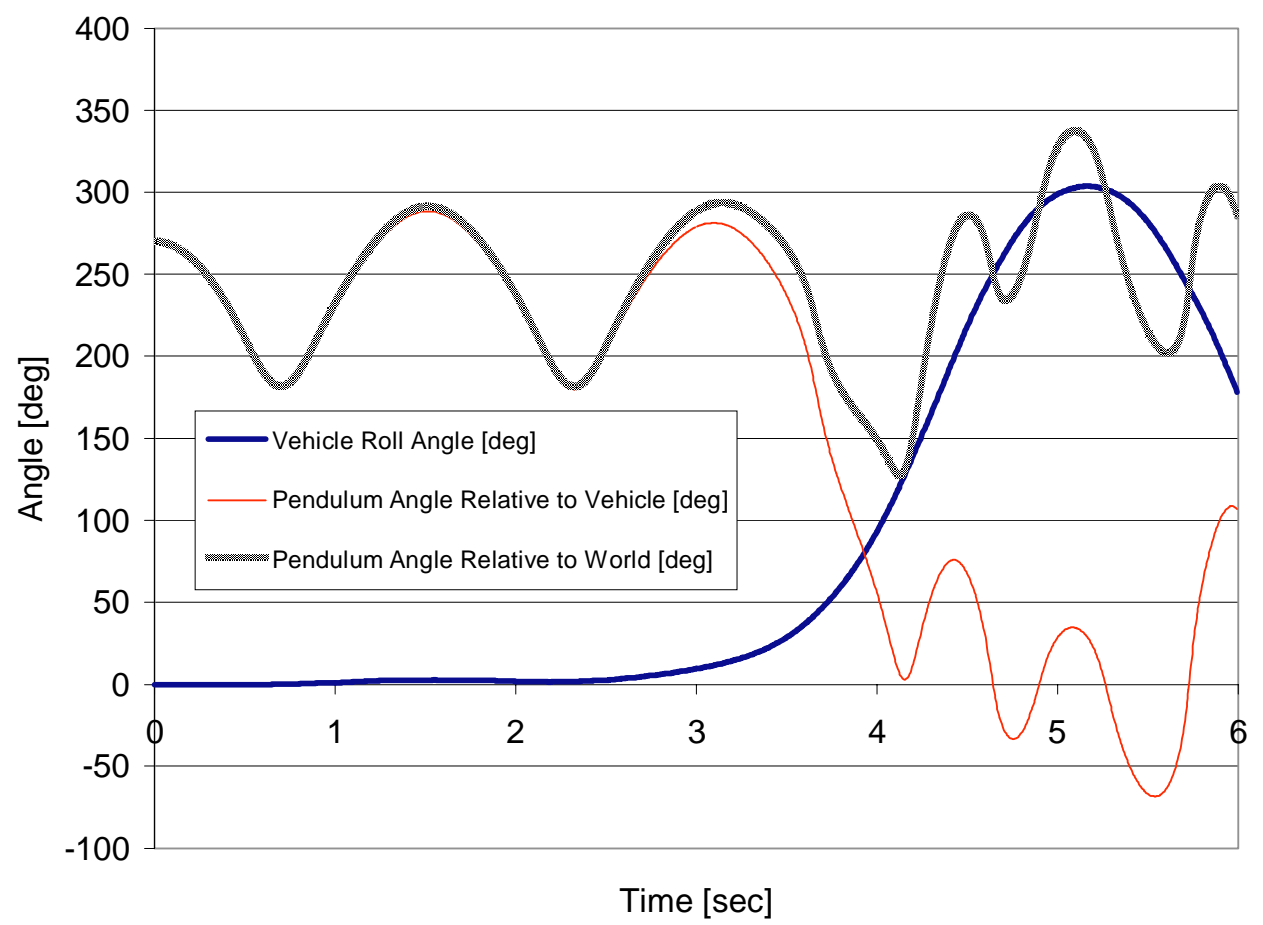

Figure 5.30: Pendulum angle during a rollover of a 50\% full tanker with tanker weight considered

It can be seen that the pendulum angle fluctuates with gradually increasing amplitude until when the rollover occurs around the middle of the fourth second. Then the pendulum angle relative to the world reference frame indicates that the fluid doesn't rotate with the tanker and remains always towards the ground.

To further investigate the effect of the inertia on the rollover behavior of the tanker, Figure 5.31 shows a comparison between the change in the vehicle roll angle for a $100 \%$ full tanker at different weight and inertia considerations. It can be seen that although the three cases indicate the initiation of the rollover at the same time, the rate at which the roll angle increases, decreases when the inertia is considered. For example, Figure 5.31 indicates that the vehicle roll angle will reach theoretically $90^{\circ}$ after $3.5,5.3$, and 6.5 seconds with no inertia, tanker inertia and fluid inertia considerations respectively. 


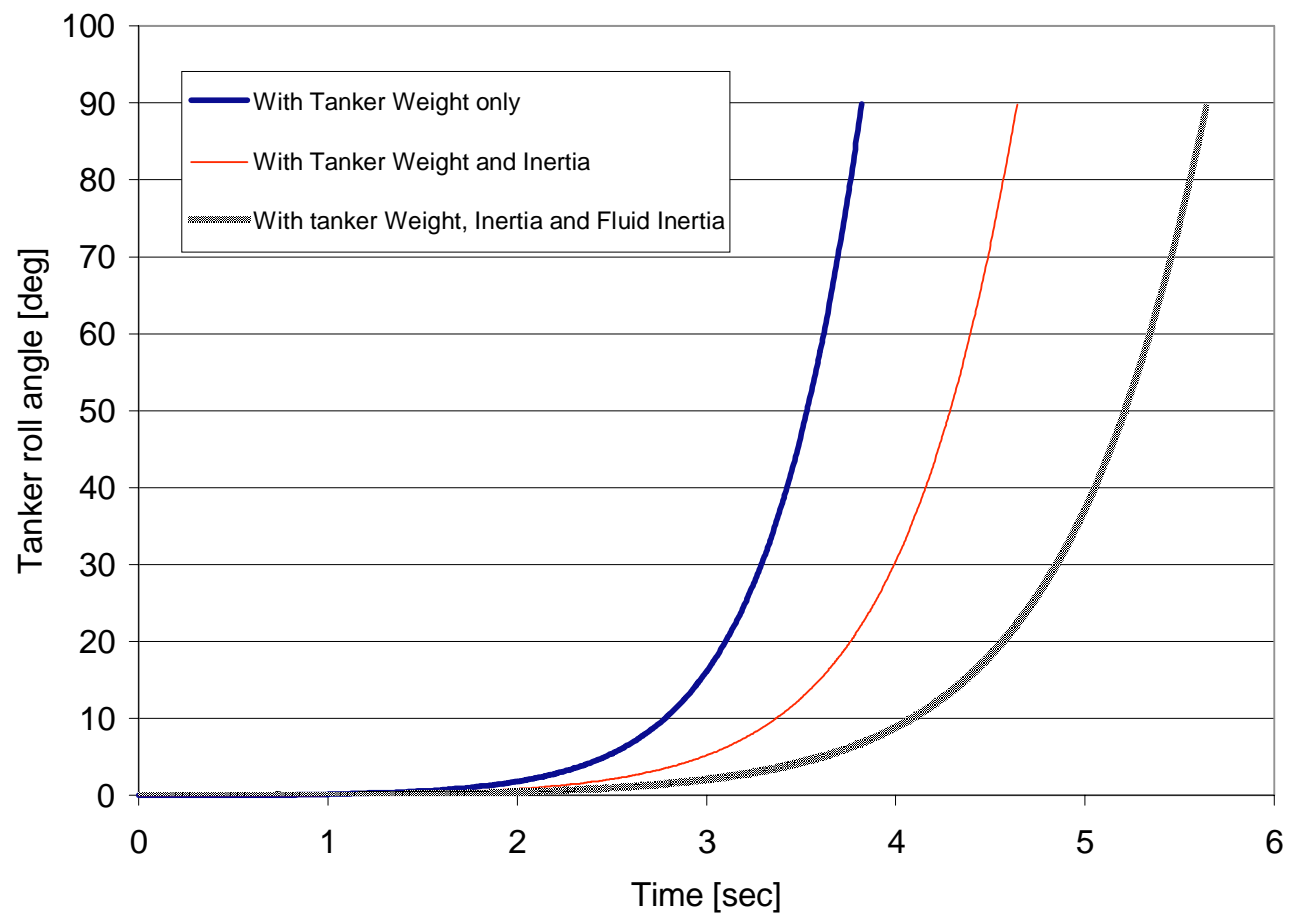

Figure 5.31: Effect of vehicle and fluid polar moment of inertia on the rate of change of vehicle roll angle

\section{$\underline{\text { 5.4- Model 3, Vehicle Plane Model with Tire and Suspension Compliances }}$}

Working Model $^{\mathrm{TM}}$ package version 2.01 was used to build model \#3. Figure 5.32 shows the details of model \# 3. Tire and suspension compliances as well as the effective suspension and tire roll centers have been considered. This model is a 5-degrees of freedom model, namely, bounce and roll of the sprung mass, bounce and roll of the unsprung mass, and the pendulum swing angle. 


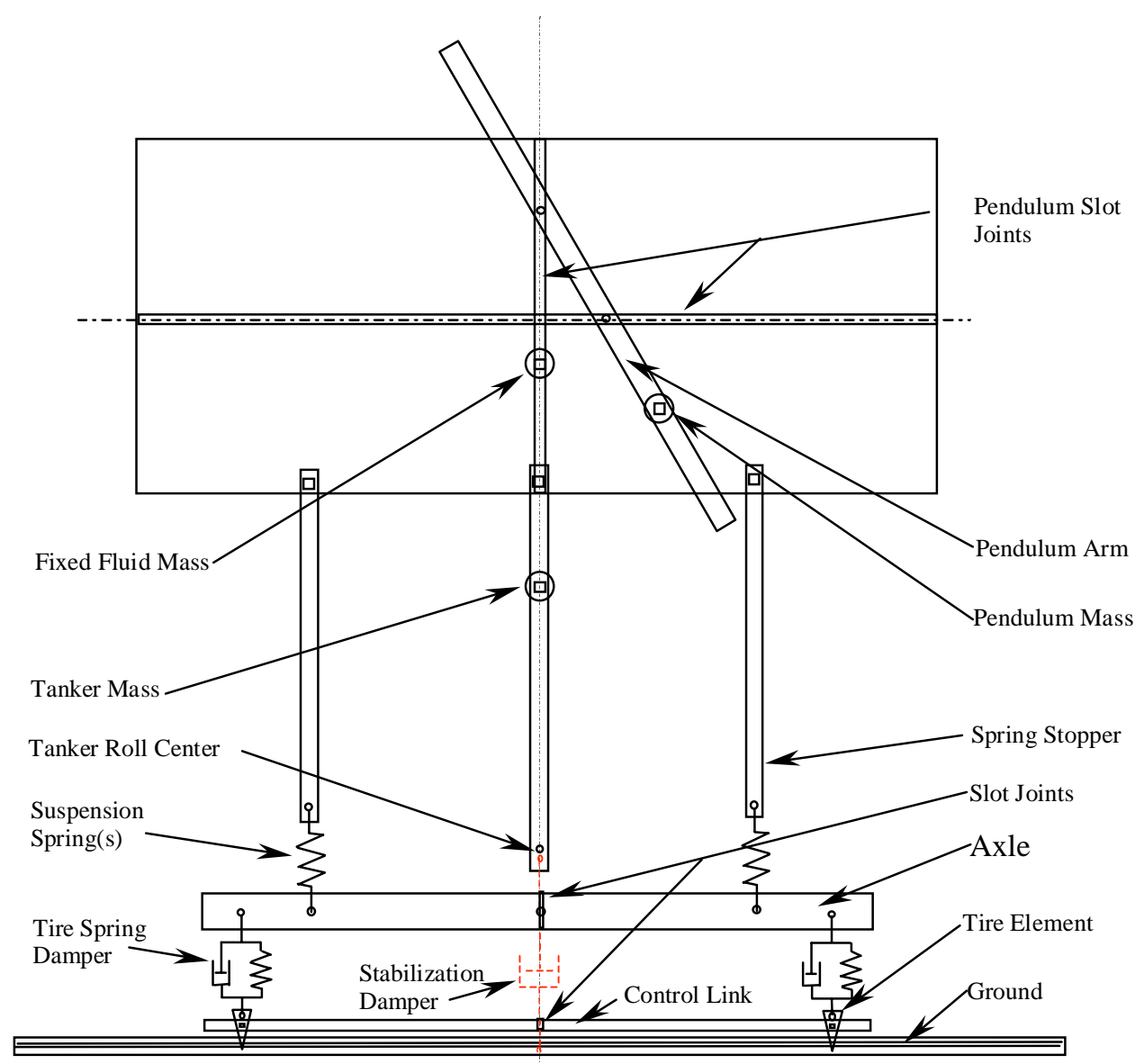

Figure 5.32: Model \#3, Vehicle plane model with tire and suspension compliances

\subsubsection{Model Description and Inputs}

The model is constructed mainly from weightless links. The tire elements are attached to the ground in slot joints that allow them to move in the $\mathrm{X}$ direction only. This was done because the model was numerically unstable if the tires were allowed to liftoff. The model was mainly driven through the control element with velocity and acceleration inputs. The slot joint in the control element is required to guide the axle center so that the tire spring and damper element don't act like a four bar link mechanism. On the other hand, the slot joint in the axle guides the sprung mass roll center so that the suspension springs don't act like a four bar link mechanism. 
The sprung mass roll center is assumed to be at the mid-span of the suspension springs. Impact prevented between all masses except the spring stoppers and the axle to speedup the solution. All masses are concentrated in very small volume circular shapes keyed to the model to minimize the polar moment of inertia effect. The trammel pendulum is simulated as a weightless link moving in a horizontal and vertical slots in the sprung mass.

The model was used to represent two-slice plane model at the tanker body. The first is taken at the tractor rear axles $(2 \& 3)$ using all the suspension, tire and reaction inputs given at this location. The second was taken at the middle of the trailer rear axles (4\&5) and also has all the suspension, tire and reaction inputs given at this location (see tables A.1 through A.4).

A typical tire damping of $3000 \mathrm{~N} . \mathrm{s} / \mathrm{m}$ and a typical tire stiffness of $809 * 10^{3} \mathrm{~N} / \mathrm{m}$ were used in all models (Wong 1993). It was found that the tire damping increases the solution time considerably when considered by about 4 to 10 times for the same model without damping.

For the section at axles $2 \& 3$, the suspension stiffness was considered linear and is given by equation 3.3 , which is equal to $2234027 \mathrm{~N} / \mathrm{m}$. For the section at axles $4 \& 5$, the suspension stiffness given in Figure 3.6 was fitted using a second degree polynomial and was found to be:

$$
\mathrm{k}=-0.08+847430 \delta+12296523 \delta^{2}
$$

The first factor was neglected and two parallel springs were used in the model to simulate the nonlinear characteristics of the trailer suspension. The first spring is a linear spring that has a stiffness equals to $2 * 847430 \mathrm{~N} / \mathrm{m}$ ( 2 leaf springs per side), the other is a quadratic spring that has a stiffness equals to $2 * 12296523 \mathrm{~N} / \mathrm{m}$. 
When the model starts simulating any maneuver, it was found that bouncing takes place for a long time due to the sudden application of gravity load on the model and the low value of damping of the tires. Thus, a stabilization damper was used to reach a steady state reaction in a short time which had a damping coefficient varying from $8 * 10^{5} \mathrm{~N} . \mathrm{s} / \mathrm{m}$ to $8 * 10^{4} \mathrm{~N} . \mathrm{s} / \mathrm{m}$ depending on the initial weight (level of fill). Figure 5.33 shows the effect of the stabilization damper on obtaining steady state reactions after stiffness change (axles $2 \& 3$ ). This damper is used whenever the overall mass changes (fill level) and/or the suspension stiffness changes. Then, it's taken out after the model reaches a steady state under gravity load only. The boundary conditions are then set to start from this stable point at the beginning of any simulation.

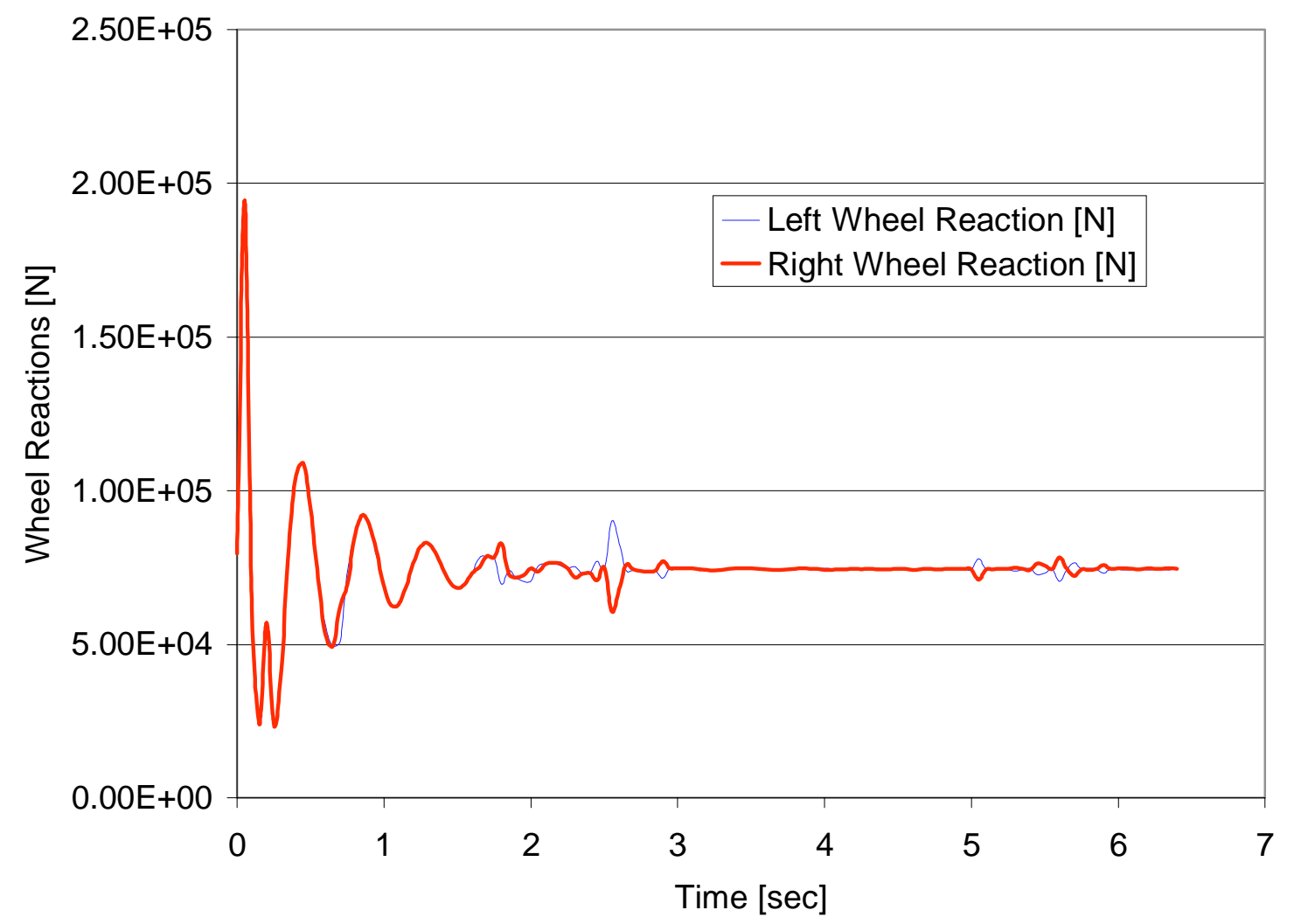

Figure 5.33: Stabilization of wheel reaction with the stabilization damper after increasing the suspension stiffness (Case is for $75 \%$ full, axles $2 \& 3$ ) 


\subsubsection{Rollover Stability Limits and Effect of Suspension Stiffness}

Several simulations have been used to obtain the stability limits of the two ETNYRE tanker plane models. The deflections of the tire springs were used to obtain the reactions at different values of lateral accelerations. Figure 5.34 shows the effect of the tire damping in the reactions obtained for a $75 \%$ full axles $4 \& 5$ plane model subjected to a lateral acceleration equals $0.25 \mathrm{~g}$.

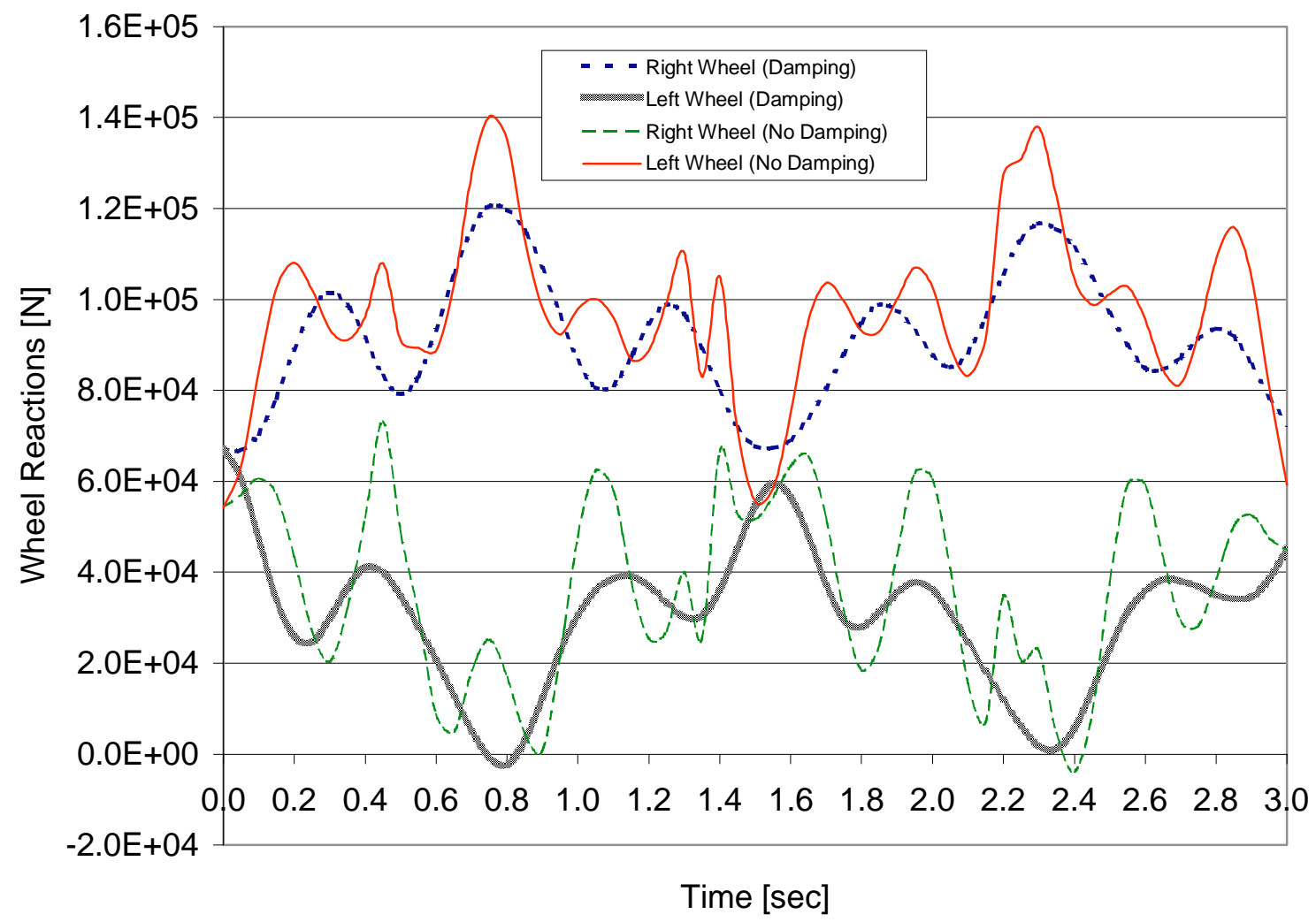

Figure 5.34: Effect of tire damping on wheel reactions (75\% full rear tractor suspension model at $0.25 \mathrm{~g}$ )

It can be seen from Figure 5.34 that there are two distinguished frequencies, the higher is the roll mode of the sprung mass (tanker and the fixed fluid mass). The lower is the pendulum oscillation frequency. It can be seen that the tire damping (though small) has reduced the 
fluctuations in the reactions considerably without having almost no effect on the minimum wheel reaction at the left wheel (where rollover is expected).

\subsubsection{Self Induced Rollover}

By running several simulations for the front and the rear slice plane models of the suspension and obtaining the minimum wheel reaction at different values of lateral acceleration, the rollover threshold has been obtained for different levels of fill and is given in Figure 5.35 .

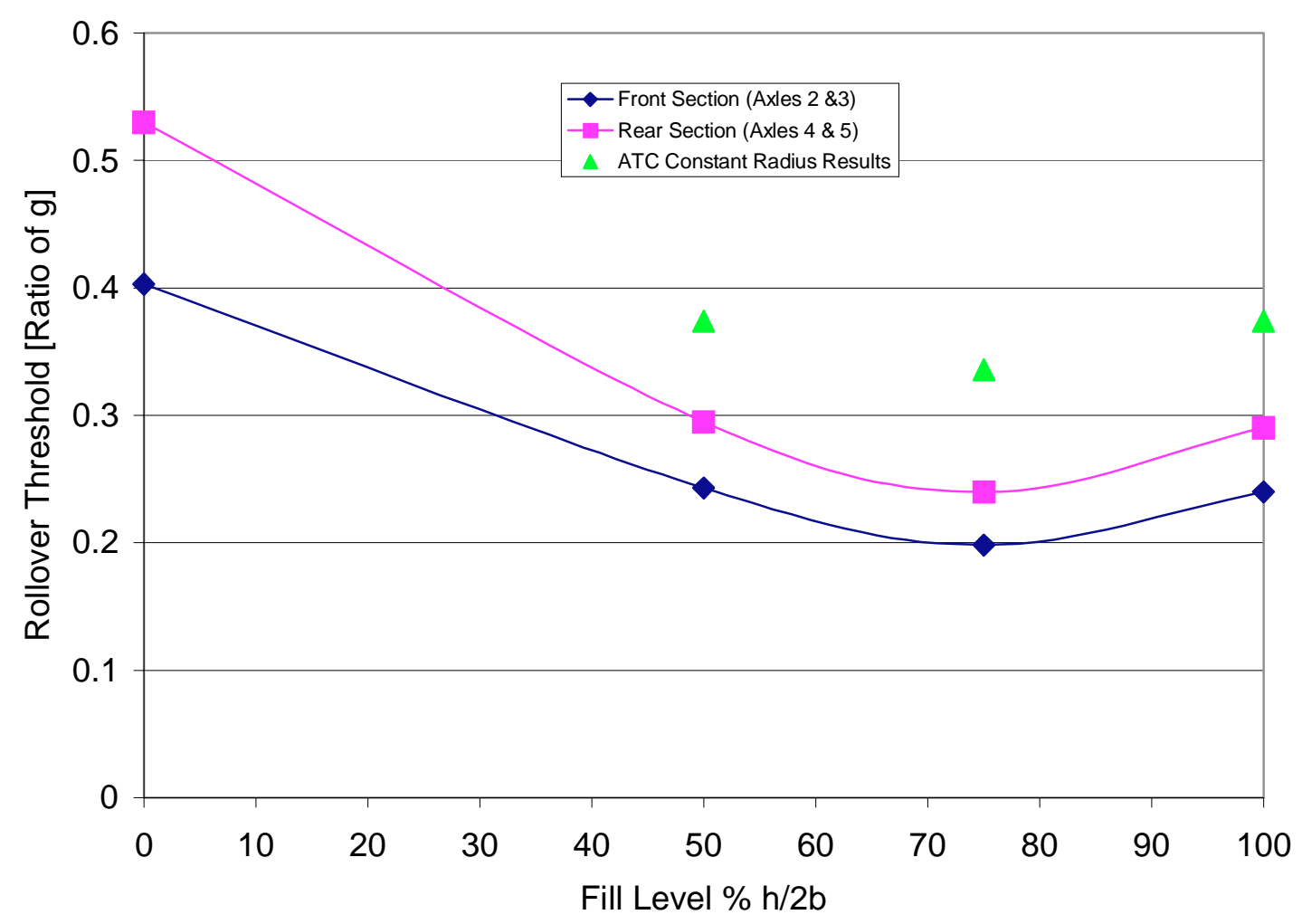

Figure 5.35 : Comparison between rollover stability limits obtained using the ETNYRE plane slice models and the experimental results at constant radius tests

It can be seen that the most critical level of fill is $75 \%$ for both sets of axles. It can be also seen that the front axles $2 \& 3$ have less rollover threshold than the rear axles $4 \& 5$. The reason is attributed to several factors, such as: the suspension stiffness at axles $4 \& 5$ is higher 
than axles $2 \& 3$ when the deflection accedes $22 \mathrm{~mm}$ due to its nonlinear characteristics; higher lateral spring spacing at axles $4 \& 5$, and less tire diameter. It can be seen also that the rollover threshold obtained from the two plane sections is less than that obtained experimentally. The reason is that the weight of the tractor, the polar moment of inertia of the tanker and the trailer, as well as the tanker stiffness add to the rollover stability of the tanker and are neglected in the plane models.

Figure 5.36 shows the effect of changing the suspension stiffness on the self-induced rollover stability of the tanker (section at axles $2 \& 3$ ). It can be seen that increasing the suspension stiffness enhances the self-induced rollover stability of the tanker, which agrees which the literature (Ervin et. al. 1983 B).

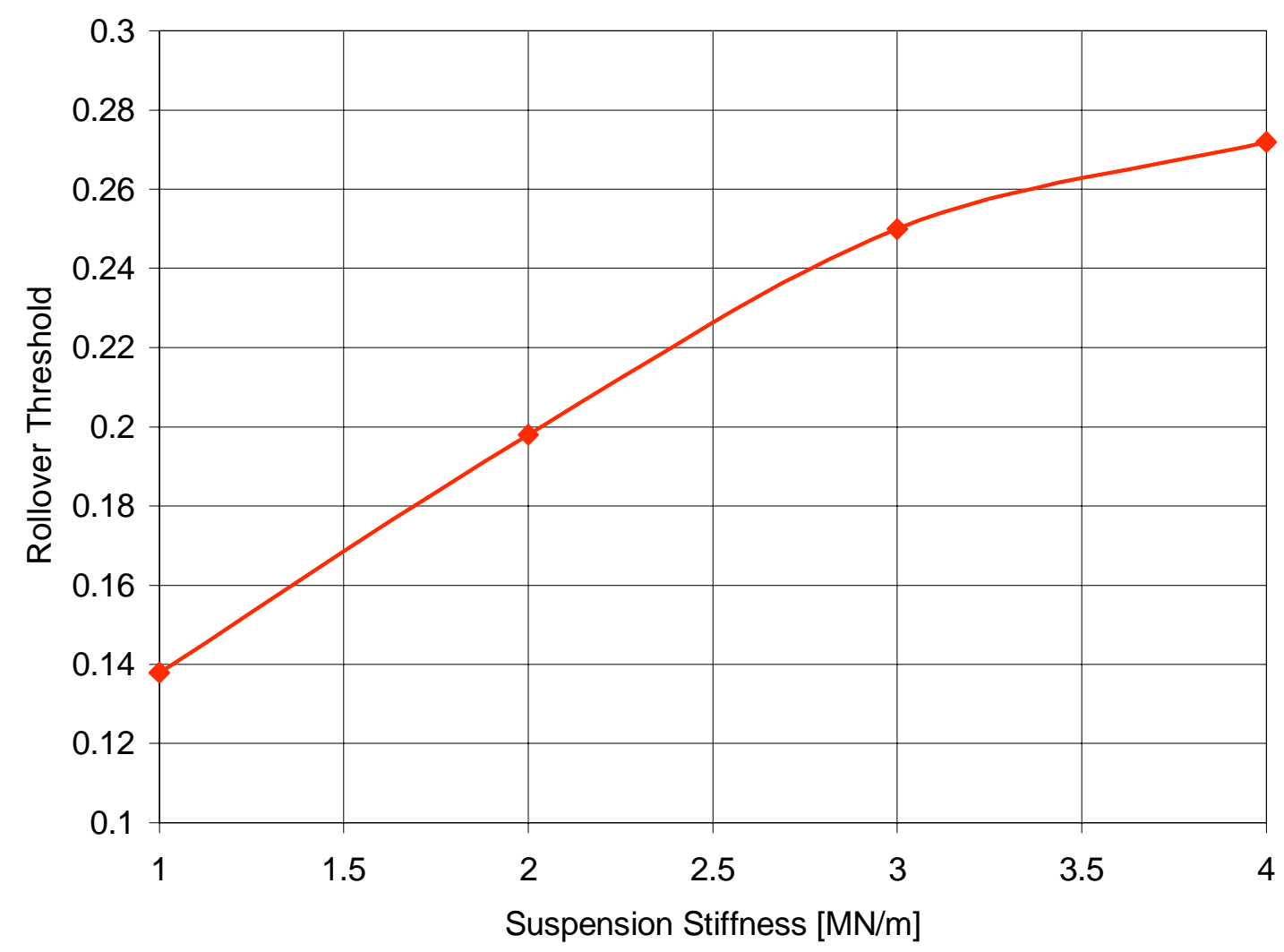

Figure 5.36: Effect of changing suspension stiffness on the self induced rollover threshold of the tanker (section at axles $2 \& 3$ under $75 \%$ full condition) 


\subsubsection{Tripped-Induced Rollover}

Model \#3 has been used also to study the tripped-induced lateral stability limits of the tanker. The plane slice models were subjected to an inelastic impact with a curb or an obstacle that has a height of $10 \mathrm{~cm}$. The speeds at which the reaction at the opposite wheel turns negative were recorded and are shown in Figure 5.37 for different levels of fill.

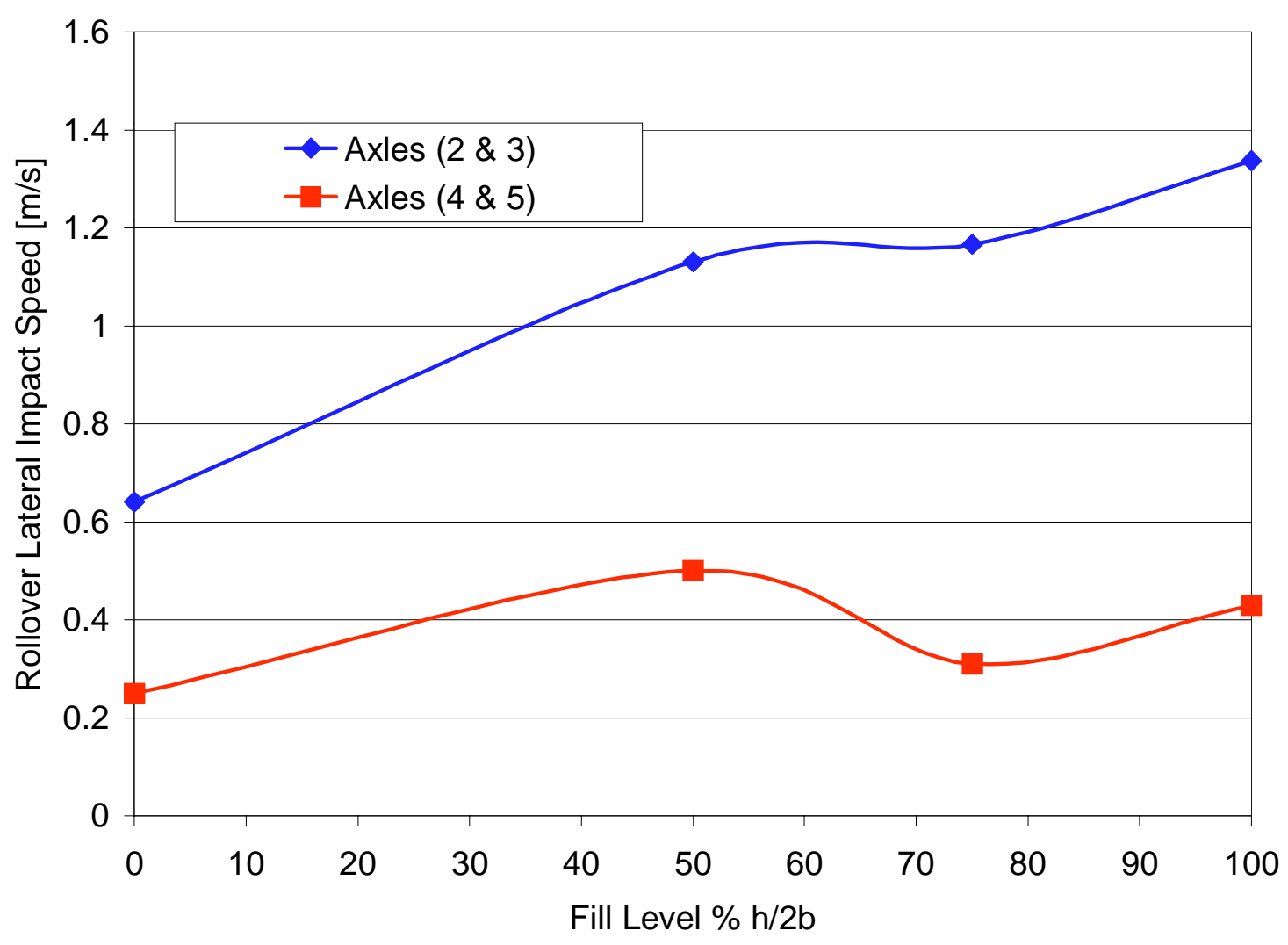

Figure 5.37: Rollover lateral impact speed for the tanker plane slice models at different levels of fill.

It can be seen that unlike the self induced case, the section at axles $4 \& 5$ is less stable.

The reason is that axles $4 \& 5$ are carrying more weight and they require more kinetic energy to be lifted of the ground. Also, the increasing stiffness of axles $4 \& 5$ increases the effect of the impact on the weight transfer from one side to the other. To confirm this concept, the effect of changing the suspension stiffness on tripped rollover stability has been studied and is shown in 
Figure 5.38 for a $75 \%$ full slice model at axles $2 \& 3$. It can be seen that the higher the suspension stiffness, the less the lateral speed at which tripped induced rollover takes place.

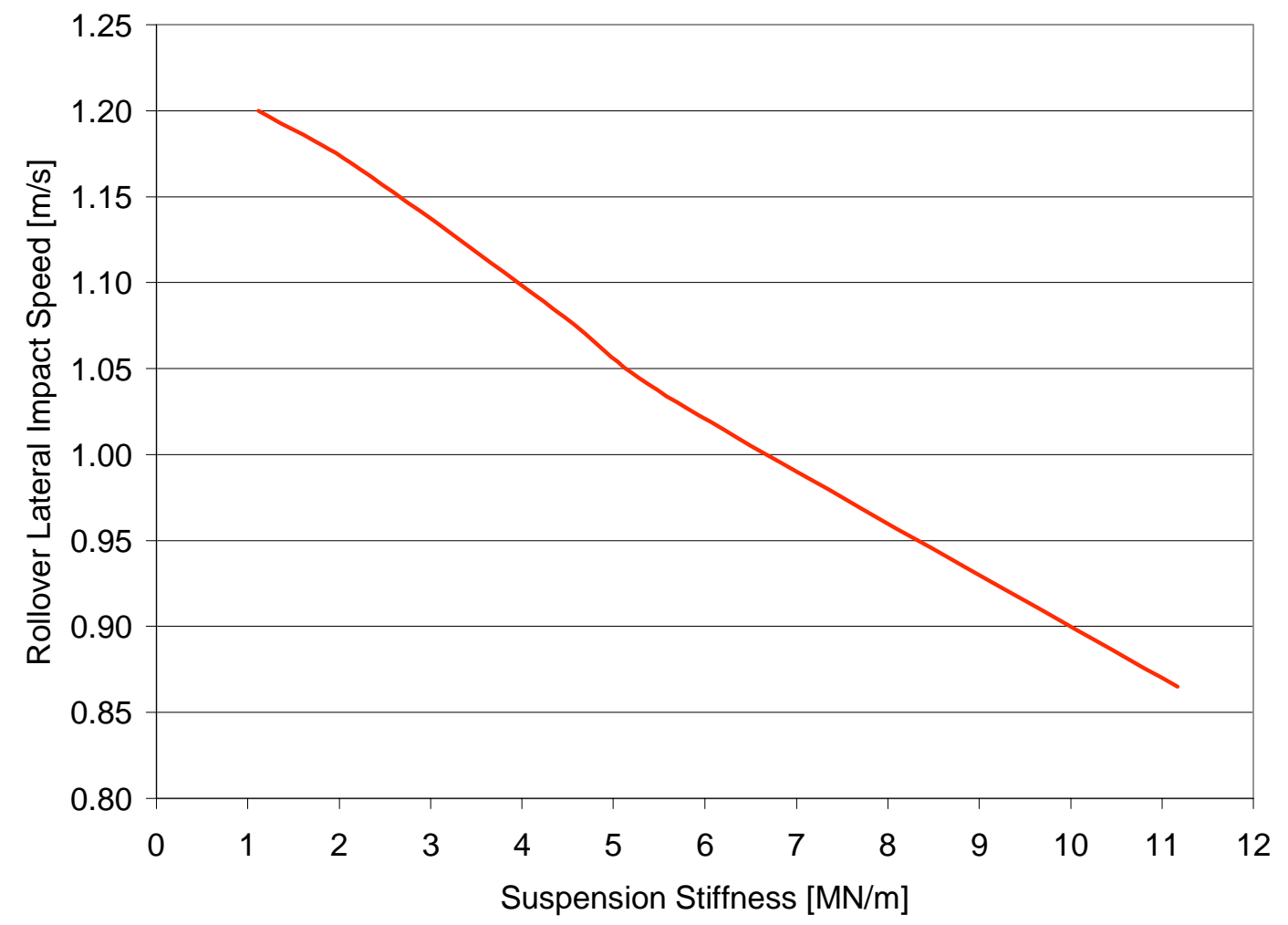

Figure 5.38 Effect of changing suspension stiffness on tripped-induced rollover speed (75\% full axles $2 \& 3$ )

\subsection{Model 4, Finite Element 3-D Vehicle Model Using ANSYS}

The model developed by Aquaro (1999) for the ETNYRE tanker and is shown in Figure 2.20 was modified and used in this analysis. Aquaro's model lacked the parameters of the trammel pendulum because they were not available. Also the model needed several modifications to increase the accuracy of the simulation. Figure 5.39 shows the modified version of Aquaro's model. Stiffing beam elements were added at the trammel pendulum support points to reduce the vibrations of the pendulum- carrier link and obtain less noise. The damping of the tires in Aqauro's model was over estimated, thus, it was reduced to a reasonable value. 


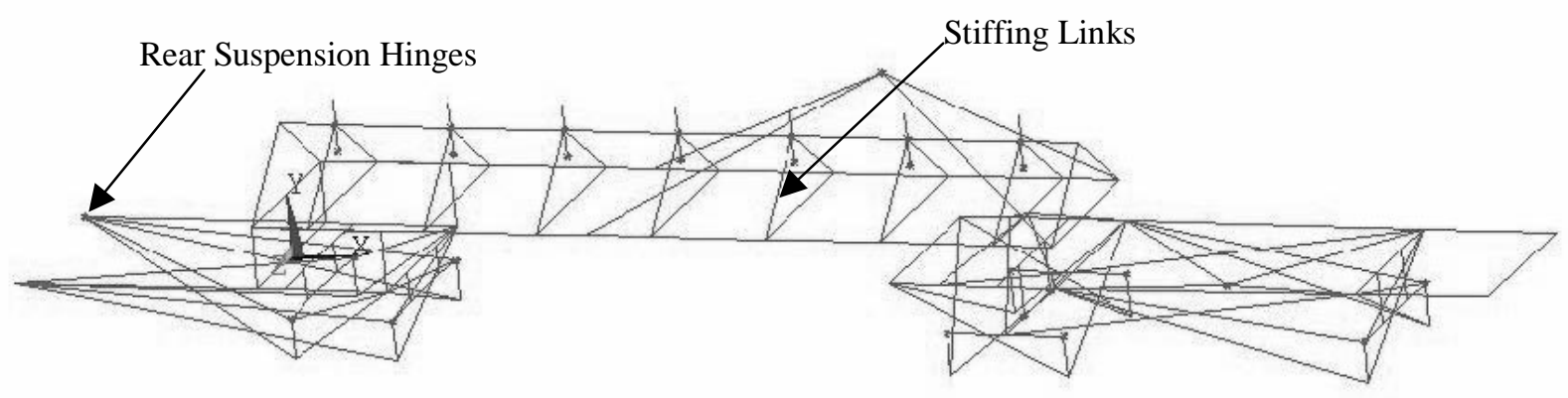

Figure 5.39: Model \#4, Modified Aquaro's 3-D vehicle ANSYS beam model

Several other modifications were added to enhance the performance of the model such as: Dragging the two rear suspension hinges backwards to increase the rear suspension linear stroke; Adjusting the tractor front suspension lateral spacing, adjusting the overall mass and the reactions on the wheels to match as close as possible those of the test tanker at steady state. Figure 5.40 shows the total reaction on the wheels during the TOP lane change test for the modified model, Aquaro's model, and the experimental results. It can be seen that Aquaro's model has an error of $-9.2 \%$ relative to the experimental while the modified FE model has $+2.5 \%$ error at the beginning of the maneuver. It also can be seen that the dips in the reactions obtained experimentally are magnified due to the reactions absorbed by the outriggers.

Figures 5.41 through 5.43 show the reactions on the tanker axles 1, $2 \& 3$ combined, and $4 \& 5$ combined respectively. It can be seen that the trends of the two models and the experimental results are the same. 


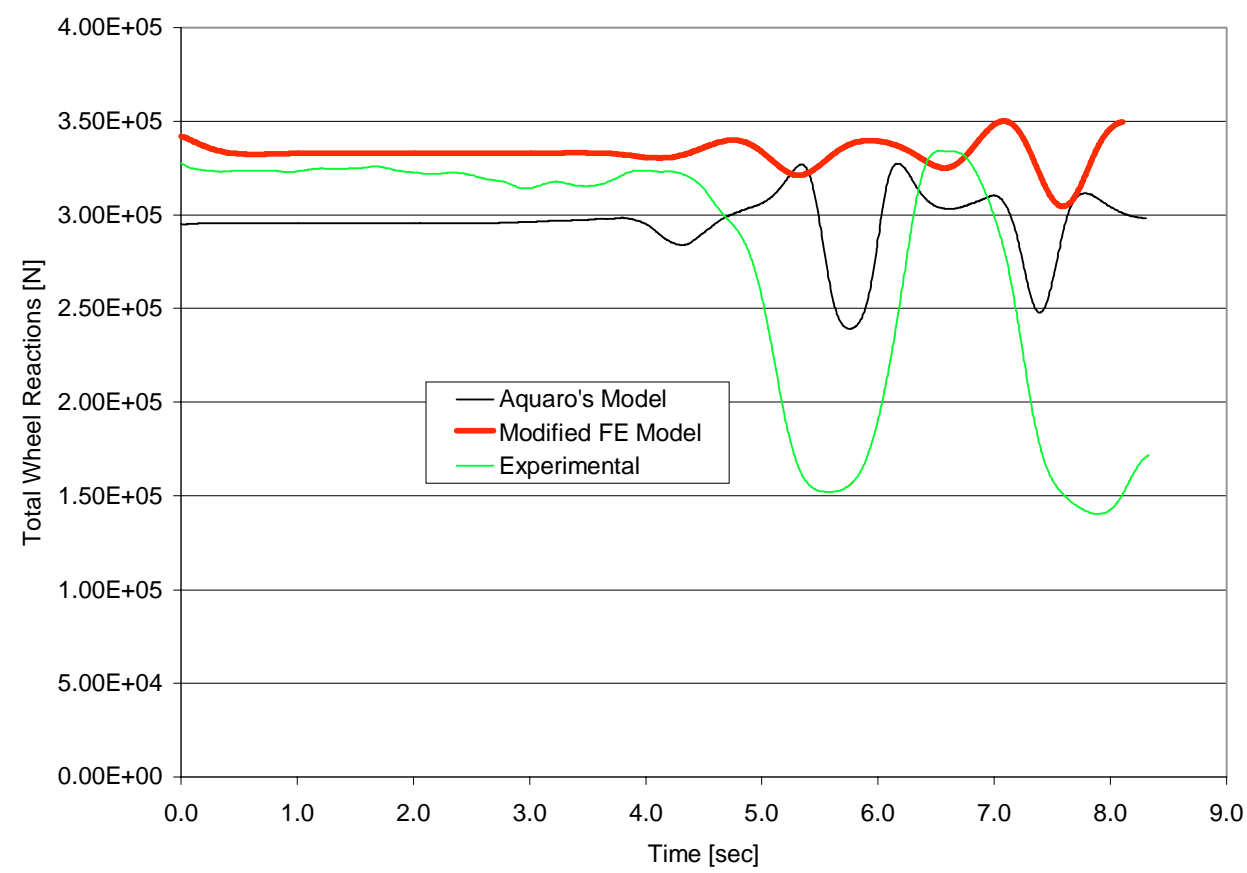

Figure 5.40 :Total reactions on the tanker wheels during the TOP lane change maneuver

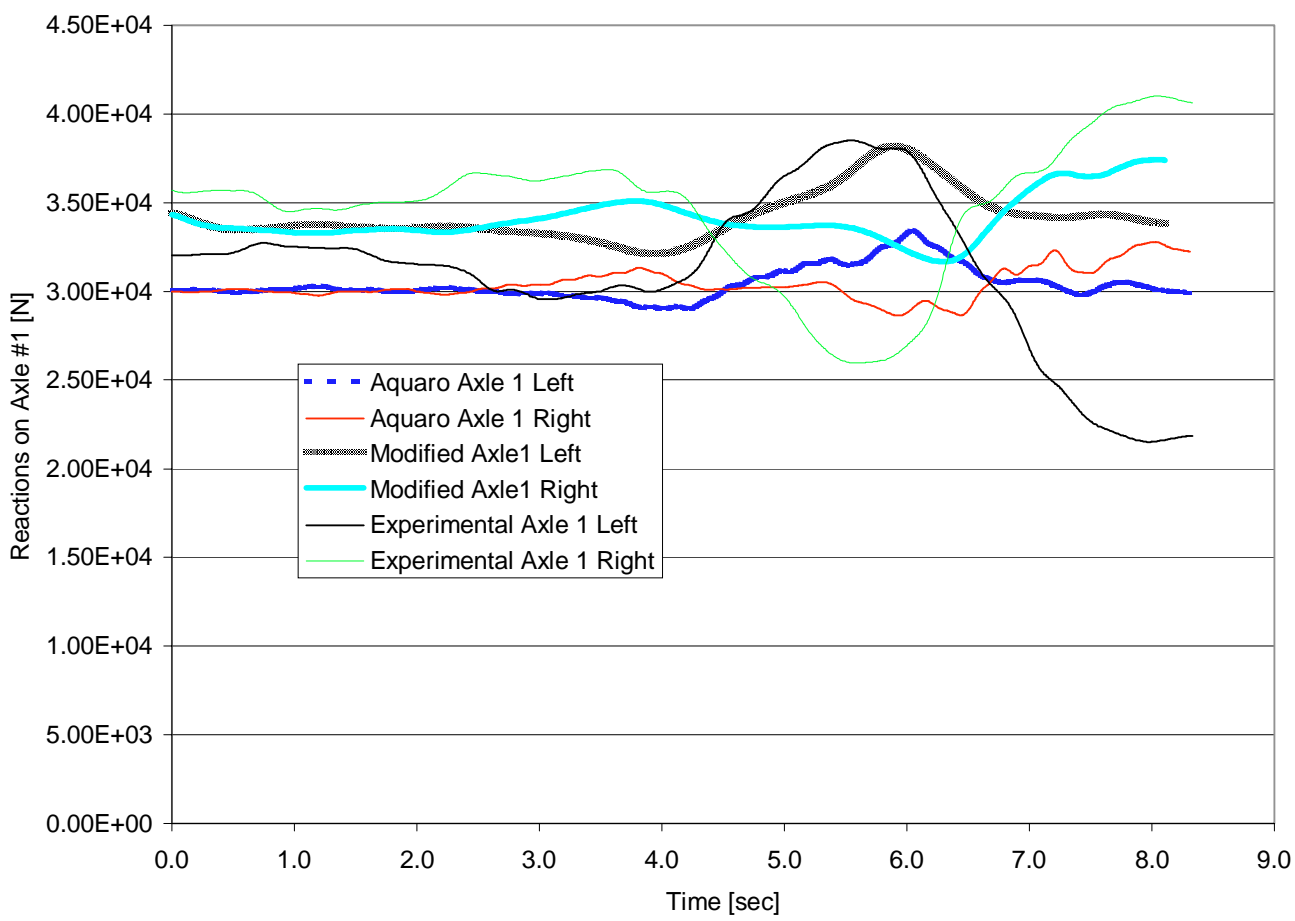

Figure 5.41: Comparison of reactions on axle \# 1 during the top lane change maneuver 


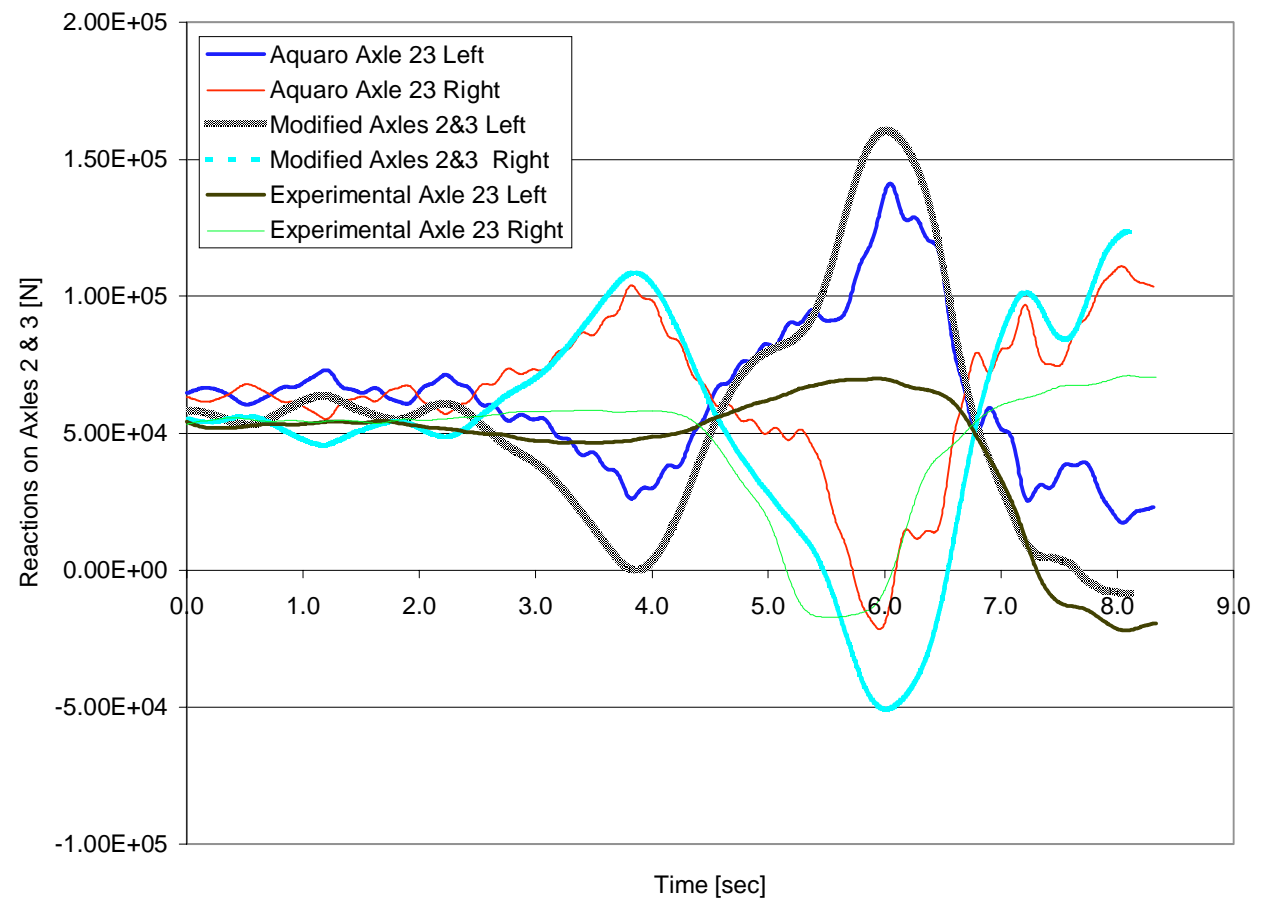

Figure 5.42: Comparison of reactions on axles \# $2 \& 3$ during the top lane change maneuver

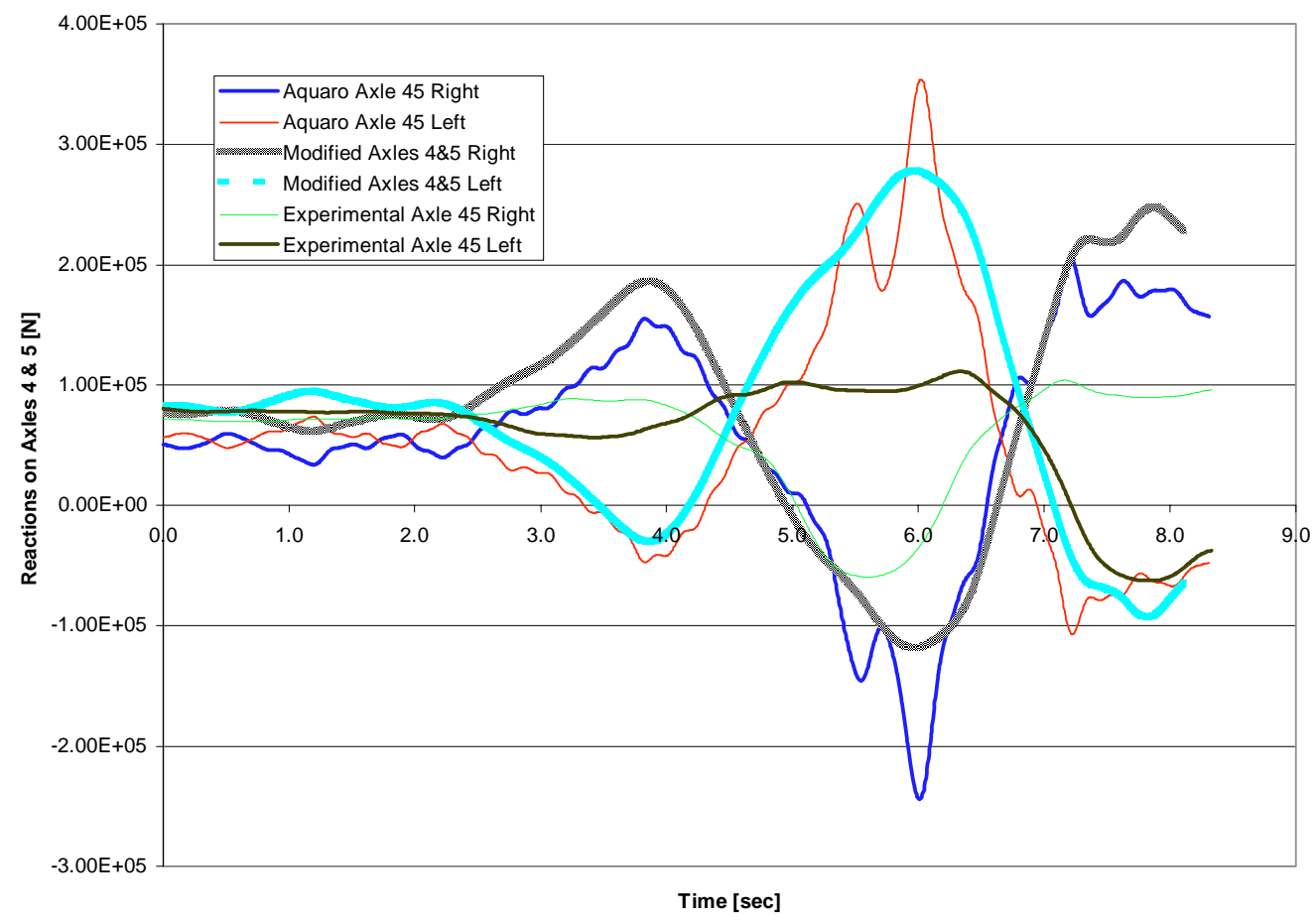

Figure 5.43: Comparison of reactions on axles \# $4 \& 5$ during the top lane change maneuver 
However, the same problem that Aquaro encountered in his model remained, which is the early peak of weight transfer at the forth second in axle sets $2 \& 3$, and $4 \& 5$. This peak indicates early rollover at axles \# 4\&5. To investigate the cause of this problem, the roll angle of the tractor and the trailer was checked and are plotted in Figure 5.44. It can be seen that the trend of the angle of roll of the trailer doesn't indicated excessive roll at the forth second, which agrees with the spring deflection measurements. Also by checking the lateral acceleration at the time when the early rollover is predicted, it was found to be equal to $0.12 \mathrm{~g}$, which is too low to cause rollover for the ETNYRE tanker. Thus, it was concluded that the problem is in the model and not in the experimental data.

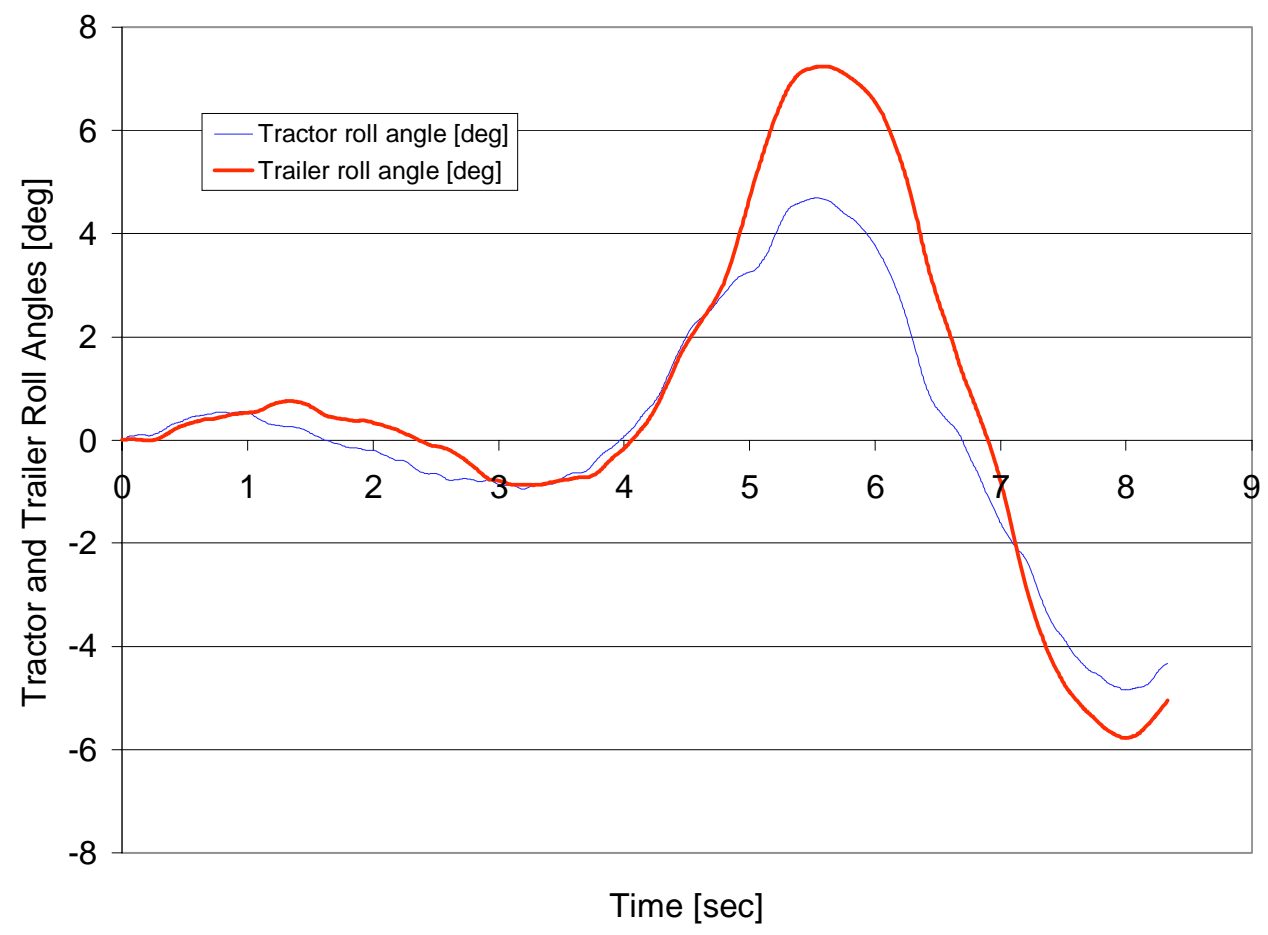

Figure 5.44: Tractor and trailer roll angles during the TOP lane change maneuver

Several parameters were considered to find the problem in model \#4. The wheel track at which the tanker rolls over was increased from the middle of the two outer wheels to the outer most point. Figure 5.45 shows that this change had negligible effect on the wheel reaction. 


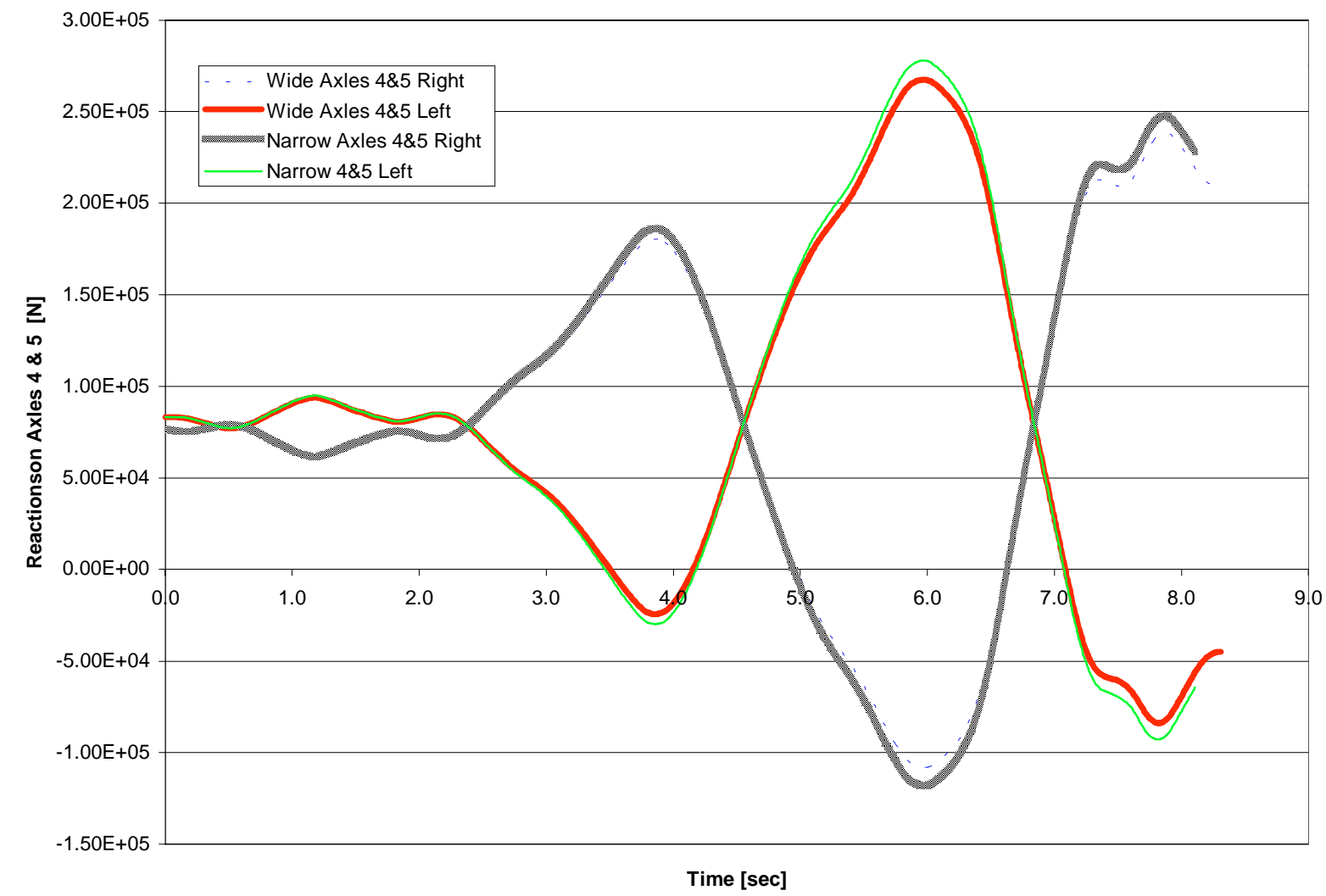

Figure 5.45: Effect of using wide track on the reactions of axles $4 \& 5$ during the TOP lane change maneuver

The nonlinear characteristics of the trailer suspension were considered throughout all its range of operation and the spring lash was considered. This change also showed almost no effect. Most importantly, the pendulum action was suppressed and the pendulums were assumed rigid. Figure 5.46 shows the effect of fixing the pendulums on the reactions of axle $4 \& 5$. It can be seen that the pendulums' action has almost no effect on the reactions, which indicates that the fluid sloshing has almost no effect on the stability. This agrees with the findings of ATC during conducting the experimental test. This negligible effect of fluid sloshing is attributed to the special feature of the very high roll center of the ETNYRE tanker. 


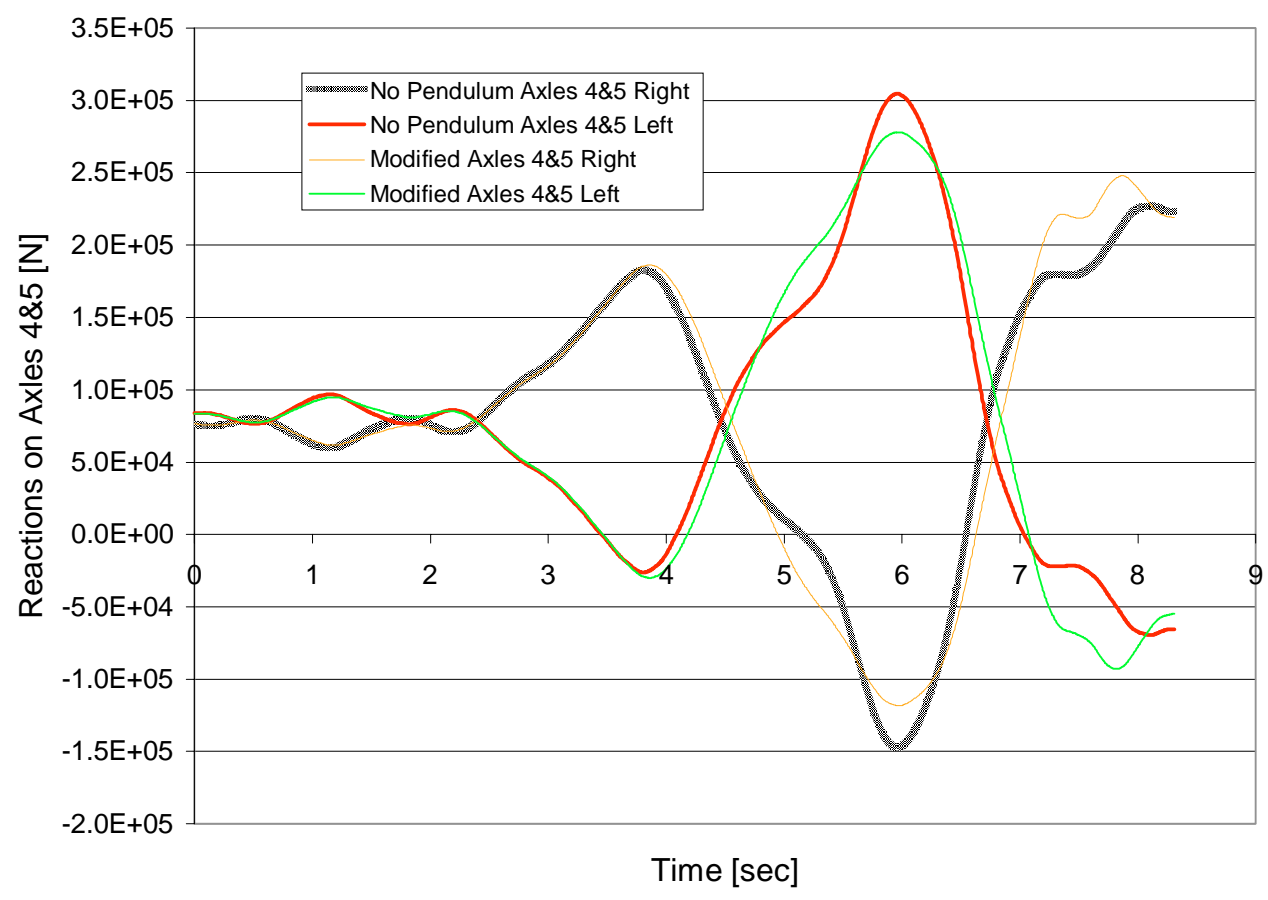

Figure 5.46: Comparison between reactions on axles $4 \& 5$ with and without pendulum action during the TOP lane change maneuver

Finally, the lateral stiffness of the tire and axle beam elements in the model were increased. Figure 5.47 shows the effect of this increase on the reactions of axle $4 \& 5$. It can be seen that this modification reduced the weight transfer considerably bringing the model results close to the experimental results. It should be noted that increasing the bending stiffness has been achieved by increasing the values of $I_{x x}$ and $I_{y y}$ of the cross section of the support beams. These values are underlined in the in the program list given in Appendix $\mathrm{C}$ part 13 . It can be seen that the first peak of weight transfer doesn't indicate rollover any more since the reactions doesn't go below zero. The rollover takes place at $\mathrm{t}=5.14$ second according to the model and at $\mathrm{t}=5.04 \mathrm{sec}$ according to the experimental results. The equivalent lateral accelerations at those two times are $0.254 \mathrm{~g}$ and $0.235 \mathrm{~g}$ respectively. Thus, the error in estimating the rollover lateral acceleration is $+8 \%$. 


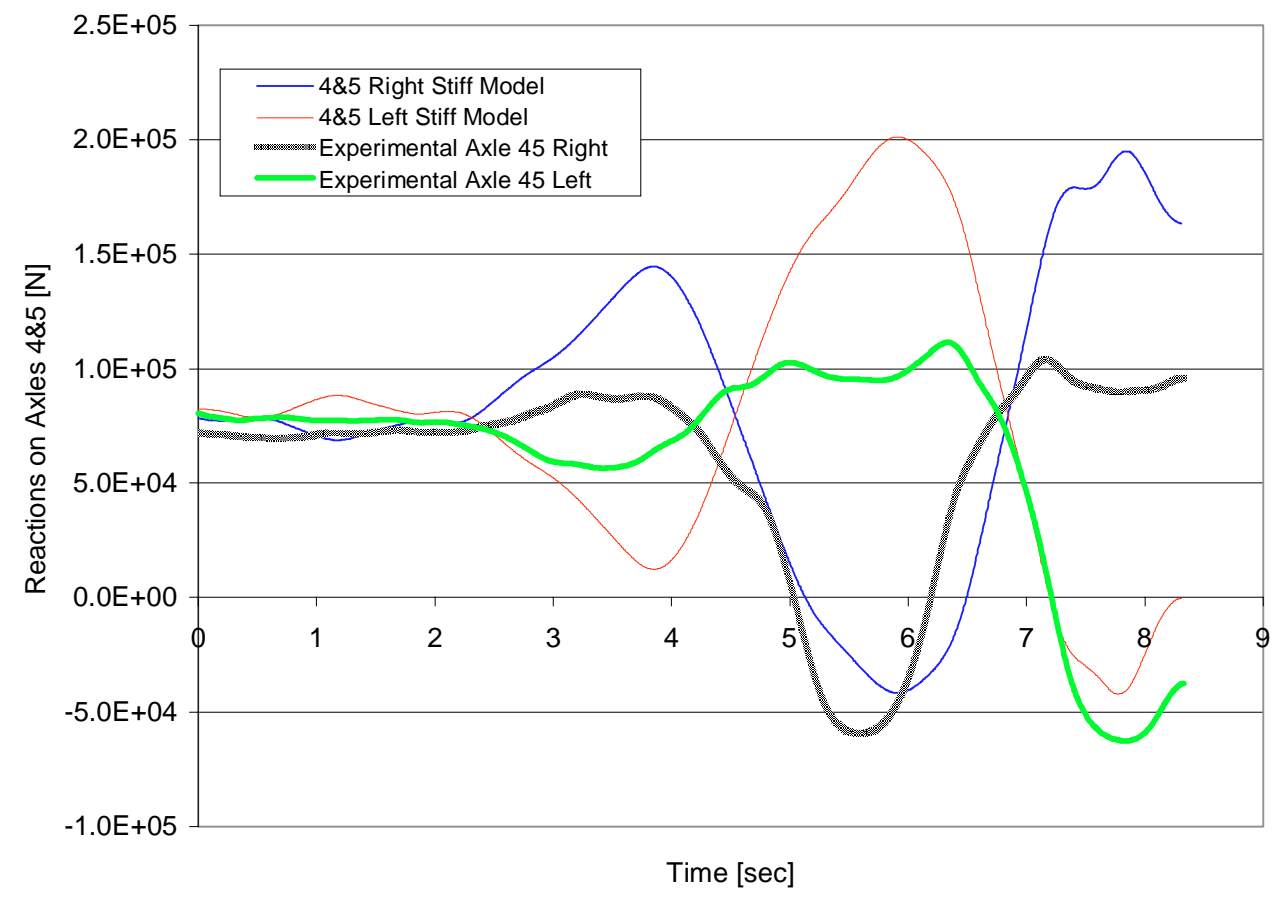

Figure 5.47: Effect of increasing model support beam stiffness on the reactions of axles $4 \& 5$ during the TOP lane change maneuver.

Although the absolute value of the predicted rollover threshold has been reduced, it's very clear that model 4 is very sensitive to modeling vehicle-related parameters than the pendulum parameters. 


\section{CHAPTER 6 \\ CONCLUSIONS \\ $\&$ \\ CONTRIBUTIONS TO THE FIELD OF ENGINEERING SCIENCE}

\subsection{Summary}

In this study, steps have been taken to study and simulate the rollover stability of partially filled heavy-duty elliptical tankers using trammel pendulums. First a literature review was conducted to collect all the information necessary to approach the problem and know all the governing factors and parameters regarding heavy-duty vehicle stability and fluid sloshing. Then, experiments were carried over on a partially filled heavy-duty tanker to provide data necessary to compare with sloshing models. The experimental data were processed to obtain acceleration and angular inputs for the mathematical and FE models and also to obtain reaction results to compare with.

The equation of motion of the trammel pendulum was derived and the dynamic effect of that pendulum was matched to the dynamic effect of fluid sloshing FE models built by LS-Dyna3D. The pendulum parameters were obtained for different levels of fill and different aspect ratios in the practical range of road containers $(\mathrm{a} / \mathrm{b}=1$ to 2$)$. Namely those parameters were; the pendulum arm length(s), the pendulum mass, the fixed mass, and the location of the fixed mass. The dynamic effect of the pendulum was checked against fluid models using LS-Dyna-3D and FLUENT. The behavior of the pendulum was studied beyond the linear range (small angles of oscillation). Results were compared with fluid models as well as the literature and the pendulum 
models proved to be more conservative by up to $20 \%$ in concluding the lateral sloshing forces and the rollover moment.

The trammel pendulum parameters (once obtained) were included in four successively detailed vehicle models to study the sloshing effects on tanker's lateral stability. The effects of several vehicle parameters were also changed to study their effect on tanker stability in the simulation of fluid sloshing with the trammel pendulums. Results have shown that yield the same critical levels of fill and the rollover threshold were obtained with more accuracy when the more detailed models were utilized.

\section{$\underline{6.2 \text { Conclusions }}$}

From this study, the following conclusions can be drawn:

1- The trammel pendulum can be used to simulate the fluid sloshing in partially filled elliptical tankers with reasonable accuracy.

2- The trammel pendulum produces more lateral force and rollover moment than the equivalent fluid sloshing by up to $20 \%$ maximum rendering the trammel pendulum more conservative form the vehicle stability point of view. This error reduces to 0 at $0 \%$ and $100 \%$ fill levels.

3- The trammel pendulum models are more stable from the numerical stability point of view than the fluid models and has much less solution time. It is much also easier to integrate a trammel pendulum model with a vehicle model.

4- The resonance behavior of the pendulum shows that the critical frequency of excitation falls beyond the base natural frequency obtained from the liner theories for elliptical tankers. 
This frequency reduces as the ratio $\mathrm{a} / \mathrm{b}$ drops until it becomes less than the base natural frequency for $\mathrm{a} / \mathrm{b}=1$.

5- The effect of change of the tanker shell weight on rollover stability is negligible as the aspect ratio changes from 1 to 2 .

6- A partially filled cylindrical tanker is more stable than a partially filled elliptical tanker except when constant ground clearance is considered and near very high levels of fill.

7- Critical levels of fill predicted with the trammel pendulum agree with the literature (50\% to $75 \%$ for elliptical tankers and raises with the reduction of the aspect ratio) and the experimental data ( $75 \%$ for the ETNYRE test tanker).

8- The polar moment of inertia of the tanker and the fluid has negligible effect on the rollover threshold and can be neglected in modeling. Other studies neglected it without explanation and others included it as a constant.

9- Plane slice models of a tanker will predict higher stability limits if the suspension and tire compliances were not considered (rigid plane slices), while they will predict lower stability limits when the suspension and tire compliances are considered.

10-Increasing the spring stiffness will enhance the self-induced rollover threshold but will deteriorate the tripped induced rollover thresholds.

11- The tripped induced rollover threshold increases with the increase of the level of fill.

12- Tire damping (though small) helps damping the vehicle vibration and the fluid sloshing.

13-If the tractor rear axle has less stiffness than the trailer axles, a plane model taken at the tractor axle will indicate earlier rollover than the trailer axles. This is because the tractor weight and the stiffness of the shell add to the stability of the tractor rear axles and are not included in the plane models. 
14- The vehicle used by ATC to conduct the experiments in this study had an exceptionally high ground clearance and roll center that resulted in negligible effects of the fluid sloshing.

15- The 3-D beam model (model \#4) although predicted the rollover threshold value almost at the right time of the simulation, is more sensitive to the vehicle parameters than the pendulum parameters when using the ETNYRE tanker inputs. Thus, it's very hard to use this model to verify the pendulum effect under the current model configuration and the available experimental results.

16-Important vehicle parameters such as tire and suspension lateral stiffnesses are very important to obtain accurate sloshing effect simulation and need to be simulated carefully in an FE model. Since these values are hard to obtain experimentally, typical values can be used from literature.

\subsection{Contributions to the Field of Engineering}

1- A thorough literature review has been conducted on the issues of heavy-duty tanker stability and fluid sloshing. This review has led to several conclusions (listed on pages 45 through 47) that help the researchers tackle the problem of heavy-duty partially filled tanker stability of knowing what is the work that has been done in the filed as well as the important parameters to be considered and what to neglect. This review has been published by Salem et. al. (1999).

2- The recommendations of Bohn et. al. (1981) regarding checking their fluid sloshing model have been conducted. Flaws and drawbacks in the model in predicting the sloshing base natural frequency and the lateral sloshing force have been identified. 
3- The fundamental bases of selecting a trammel pendulum to simulate the lateral fluid sloshing in a partially filled elliptical tanker have been developed. Charts and equations have been provided to obtain the values of these parameters at different levels of fills and different practical tank aspect ration (from 1 to 2 ).

4- The behavior of this pendulum beyond the linear range has been analyzed including implicitly Ranganathan's (1993) circular pendulum as a special case of the trammel pendulum (when $a=b=1$ ). Deviation from real fluid behavior in terms of lateral force and roll moments have been clearly stated when the vehicle is subjected to non-linear practical range lateral accelerations.

5- Clear analysis of the pendulum frequency response has been provided. This analysis highlights the critical frequencies of excitation, which was found to be different from the base natural frequency derived from small pendulum oscillations.

6- The effect of tanker shell weight change with aspect ratio, the polar moment of inertia of the tanker and the fluid was considered for the first time. It has been found that the effect of these factors on vehicle roll stability can be neglected.

7- Effect of suspension and tire compliances on tripped rollover stability of a partially filled tank has been studied for the first time. 


\section{REFERENCES}

Abramson, H., N., Chu, W., and Ransleben, G., E., Jr., "Representation of Fuel Sloshing in Cylindrical Tanks by an Equivalent Mechanical Model," Technical Report No. 8, Southwest Research Institute, June 30, 1960.

Abramson, H., N., Chu, W., H., and Kana, D., D., "Some Studies of Nonlinear Lateral Sloshing in Rigid Containers," Journal of Applied Mechanics, December 1966 A.

Abramson, H. Norman, "The Dynamic Behavior of Liquids in Moving Containers," NASA SP106, 1966 B.

Anderson, G. W., "Sloshing Parameters for Cylindrical Tanks," Report SM-37477, Douglas Aircraft Co., Santa Monica Div., August 26, 1960.

Aquaro, M., "Stability of Partially Filled Tanker Trucks Using a Finite Element Modeling Approach," Masters Thesis, West Virginia University, April 1999.

Arnold, V., I., "Geometrical Methods in the Theory of Ordinary Differential Equations," Springer Verlag, Berlin, Heidelberg, New York, 1983.

Artobolevsky I., I., Mechanisms in Modern Engineering Design, Vol. II, Mir Publishers, Moscow, 1976.

Barter, N., F., Hales, F., D., "The Lateral Stability of Articulated Vehicles under Braking Conditions," Motor Industry Research Association, Report No. 166/3, January 1966.

Bauer, H., F., "The Moment of Inertia of A Liquid in a Circular Cylindrical Tank," Army Ballistic Missile Agency, April 1958 A.

Bauer, H., F., "Fluid Oscillations in a Cylindrical Tank," Army Ballistic Missile Agency, Report DA-TR-4-58, 1958 B.

Bauer, H., F., "Fluid Oscillations in a Cylindrical Tank with Damping," Army Ballistic Missile Agency, Report DA-TR-4-58, 1958 C.

Bauer, H., F., "On the Destabilizing Effects of Liquids in Various Vehicles," Vehicle System Dynamics 1 (1972), pp. 227-260, 1972.

Bauer, H., F., "Dynamic Behavior of an Elastic Separating Wall in Vehicle Containers," Journal of Vehicle Design, Vol.2 No. 1, 1981.

Beauvais, F., N., "Aerodynamics of a Car-Trailer Combination," SAE Paper No. 670100, 1967. 
Bidwell, J., B., "State-of-the-Art- Vehicle Control and Road Holding," SAE Paper No. 700366, 1970.

Bohn, P., F., Butler, M., C., Dunkle, H., D., and Eshleman, R., L., "Computer Simulation of the Effect of Cargo Shifting on Articulated Vehicles Performing Braking and Cornering Maneuvers," Vol. 2, Technical Report, The John Hopkins University, May 1981.

BOSCH, Automotive Handbook, $3^{\text {rd }}$ Edition, SAE, ISBN 1-56091-372-X, 1993.

Brien, O., O., "Information on the Model 60PRS Water Distributor," E.D. ENTYRE \& CO., fax dated January 23, 1998.

Budiansky, B., "Sloshing of Liquids in Circular Canals and Spherical Tanks," Journal of the Aero/Space Sciences, V 27, Number 3, pp 161-173, March 1960.

Bundorf, R., T., "Directional Control Dynamics of Automobile Travel Trailer Combinations," SAE Transactions, Vol. 76, Paper No. 670099, 1967.

Carr, J., "Applications of Center Manifold Theory," Applied Mathematical Sciences, N). 35, Springer-Verlag, Berlin, Heidelberg, New York, 1981.

Culley, C., Anderson, R., L., "Effect of Cargo Shifting on Vehicle Handling," FHWA-RD-78-76, Dynamic Science Inc., March 1978.

Dahlberg, E., and Vagstedt, N., "The Advantages of a Simple Approach Modeling Heavy Vehicle Handling," SAE Paper No. 973264, 1997.

Das, N., S., Suresh, B., A., and Wambold, J., C., "Estimation of Dynamic Rollover Threshold of Commercial Vehicles Using Low Speed Experimental Data," SAE Paper No. 932949, 1993.

Ellis, J., R., "The Dynamics of Vehicles During Braking," Symposium on Control of Vehicles During Braking and Cornering, Proceedings, London, 1963.

Ellis, J., R., "The Ride and Handling of Semi-Trailer Articulated Vehicles, " Automobile Engineer, Vol. 56, pp523-529, 1966.

Ervin, R.D., MacAdam, C., C., and Fancher, P., S., "The Longitudinal Traction Characteristics of Truck Tires as Measured on Dry Pavements," Report \# UM-HSRI-PF-75-3, Highway Safety Research Institute, University of Michigan, February, 1975.

Ervin, R., D., and Wild, R., E., "The Noise and Traction Characteristics of Bias-Ply Truck Tires," Report \# UM-HSRI-PF-76-2-1, Highway Safety Research Institute, University of Michigan, January, 1976. 
Ervin, R., D., Winkler, C., B., Bernard, J., E., and Gupta, R., K., "Effects of Tire Properties on Truck and Bus Handling," Final Report, Contract No. DOT-HS-4-00943, Highway Safety Research Institute, University of Michigan, December, 1976.

Ervin, R., D., et al., "Ad Hoc Study of Certain Safety-Related Aspects of Double-Bottom Tanker," Final Report prepared for Office of Highway Safety Planning, Michigan Department of State Police, Contract No. MPA-78-002A, Highway Safety Research Institute, University of Michigan, Report No. UN-HSRI-78-18, May 7, 1978.

Ervin, R., D., Mallikarunaroa, C., and Gillespie, T., D., "Future Configuration of Tank Vehicles Hauling Flammable Liquids in Michigan," Final Report to Michigan Department of Transportation, Agreement \# 78-2230, Highway Safety Research Institute, University of Michigan, Report No. UM-HSRI-80-73, December 1980.

Ervin, R., D., et al., "Influence of Size and Weight Variables on the Stability and Control Properties of Heavy Trucks," Final Report, No. UMTRI-83-10, March 1983.

Ervin, R., D., "The Influence of the Size and Weight Variables on the Roll Stability of Heavy Duty Trucks," SAE Paper No. 831163, 1983 B.

Etter, D., M., "Engineering Problem Solving With Matlab". Prentice Hall, Englewood Cliffs, New Jersey 07632. ISBN 0-13-280470-0, 1993.

Fancher, P., S., Ervin, R.,D., MacAdam, C., C., and Winkler, C., B., "Measurement and Representation of the Mechanical Properties of Truck Leaf Springs," SAE paper No. 800905, 1980.

Gautam, M., Mucino, V., Salem, M., Saunders, E., and Aquaro, M., "Automotive Stability of Heavy-Duty Truck Tractor-Tanker Combination," Final Report, Fredrick Manufacturing, March, 1999.

Graham, E. W. and Rodriguez, A., M., "The Characteristics of Fuel Motion which Affect Airplane Dynamics,” Journal of Applied Mechanics, Vol. 19, pp.381-388, September 1952.

Guckenheimer, J., \& Holmes, P., J., "Nonlinear Oscillations, Dynamical Systems, and Bifurcations of Vector Field," Applied Mathematical Sciences, No. 42, Springer-Verlag, New York, Berlin, Heidelberg, Tokyo, 1983.

Hales, F., D., "The Lateral Stability of a Simplified Articulated Vehicle," Symposium on Control of Vehicles During Braking and Cornering, Proceedings, London, 1963.

Hales, F., D., "The Rigid Body Dynamics of Road Vehicle Trains," The Dynamics of Vehicles on Roads and Tracks, Proceedings of IUTAM Symposium, Delft, August 1975.

Hazemoto, T., "Analysis of Lateral Stability for Doubles," SAE paper No. 730688, June 1973. 
Holmes, P., J., "Center Manifold, Normal Forms and Bifurcation of Vector Fields with Application to Coupling between Periodic and Steady Motions," Physica, 2D, pp449-481, 1981.

Jerry, A. J., Introduction to Integral Equations with Applications, Marcel Bekker, Inc. NY, ISBN 0-8247-7293-8, 1985.

Jindra, F., "Tractor and Semi-Trailer handling," Automobile Engineer, Vol. 52 pp. 438-446, 1963.

Jindra, F., "Handling Characteristics of Tractor-Trailer Combinations," SAE paper No. 650720, 1965.

Karam, H. N., "Dynamic Stability Of An Elliptical Pendulum With Unilateral Simple Supports;" Ms. Thesis, Mechanical and Aerospace Engineering Department, College of Engineering and Mineral Resources, West Virginia University, May 1999.

Karamihas, S., M., and Winkler, C., B., "Ride Performance of Heavy-Vehicle Suspensions: Data Tables," SAE paper No. 973208, 1997.

Korn G., A., and Korn, T. M., Mathematical Handbook for Scientists and Engineers, $2^{\text {nd }}$ Edition, Mc-Graw Hill, ISBN 0-07-035370-0, 1968.

Krauter, A., I., and Wilson, R., K., "Simulation of Tractor-semitrailer Handling," SAE paper No. $720922,1972$.

Lamb, M., Hydrodynamics, Section 259, Dover Publications, N.Y., 1945.

Leucht, P., M., "The Directional Dynamics of the Commercial Tractor Semitrailer Vehicle During Breaking," SAE paper 700371, 1970.

Lieh, J., “A Multibody Dynamics Program for Truck Simulation,” SAE paper No. 942303, 1994.

MacAdam, C., C., "Computer Simulation and Parameter Sensitivity Study of a Commercial Vehicle During Antiskid Braking," $6^{\text {th }}$ VSD- $3^{\text {rd }}$ IUTAM Symposium, Berlin, Germany, September, 1979.

Mallikarjunarao, C., and Fancher, P., "Analysis of the Directional Response Characteristics of Double Tankers,” SAE paper No. 781064, 1978.

Mikulcik, E., C., "The Dynamics of Tractor-Semitrailer Vehicles: The Jackknifing Problem," Ph.D. Thesis, Cornell University, Ithaca, N.Y., June 1968.

Mikulcik, E., C., "The Dynamics of Tractor-Semitrailer Vehicles: The Jackknifing Problem," SAE paper No. 710045, 1971. 
Waterloo Maple Inc., Maple V R5 Learning Guide, Springer-Verlag, NY, NY, ISBN 0-38798399-6, 1998.

Nalecz, A., G., and Genin, J., "Dynamic Stability of Heavy Articulated Vehicles," International Journal of Vehicle Design, Vol. 5, No. 4, 1984.

Nalecz, A., G., "Intermediate Maneuver Induced Vehicle Rollover Simulation," Final Report, TSC-U.S. DOT, Contract No. DTRS57-88-P-82668, 1988.

Nalecz, A., G., et al., "Development and Analysis of Intermediate Tripped Vehicle Rollover Simulation," ITRS Final Report, NHTSA-U.S., DOT, Contract No., DTNH22-87-D-27174, 1989.

Olmstead, J.C., Test Engineer, Aberdeen Test Center, document titled "Stability Model Data M916A1/Entyre 60PRS 6000 Gallon Water Tanker," 1996.

Popov, G., Sankar, S., Sankar, T. S., and Vatistas, G. H., "Dynamics of Liquid Sloshing in Horizontal Cylindrical Road Containers," Proc. Inst. Of Mechanical Engineers ImechE, Vol 207, Part C: Journal of Mechanical Engineering Science, pp 399-406, 1993 A.

Popov, G., Sankar, S., and Sankar, T. S., "Dynamics of Liquid Sloshing in Baffled and Compartmented Road Containers," Journal of Fluids and Structures, Vol. 7, pp. 803-821, 1993 B.

Popov., G., Sankar, S., and Sankar, T., S., "Optimal Shape of a Rectangular Road Container," Journal of Fluid and Structures Vol. 7, pp. 75-86, 1993 C.

Papov, G., Sankar, S., and Sankar, T., S., "Shape Optimization of Elliptical Road Containers Due to Liquid Load in Steady-State Turning," Vehicle System Dynamics, Vol. 25, pp. 203-221, 1996.

Rakheja, S., Sankar, S., and Ranganathan, R., "Influence of Truck Design Factors on the Rollover Threshold of Partially Filled Tank Vehicles," SAE paper No. 892480, 1989.

Rakheja, S., and Sankar, S., "Field Testing of a Tank Truck and Study of Fluid Slosh," SAE paper No. 912679, 1991.

Rakheja, S., Ranganathan, R., and Sankar, S., "Field Testing and Validation of Directional Dynamics Model of a Tank Truck," International Journal of Vehicle Design, Vol. 13, No. 3, 1992.

Rakheja, S., and Ranganathan, R., "Estimation of the Rollover Threshold of Heavy Vehicles Carrying Liquid Cargo: a Simplified Approach," Heavy Vehicles Systems, International Journal of Vehicle Design, Vol. 1, No, 1, 1993. 
Ranganathan, R., Rakheja, S., and Sankar, S., "Kineto-Static Roll Plane Analysis of Articulated Tank Vehicles with Arbitrary Tank Geometry," International Journal of Vehicle Design, Vol. 10, No. 1, 1989.

Ranganathan, R., Rakheja, S., and Sankar, S., "Influence of Liquid Load Shift on the Dynamic Response of Articulated Tank Vehicles," Vehicle System Dynamics, Vol. 19, pp. 177-200, 1990.

Ranganathan, R., Ying, Y., Miles J. B., “Analysis of Fluid Slosh in Partially filled Tanks and Their Impact on the Directional Response of Tank Vehicles," SAE paper 932942, 1993 A.

Ranganathan, R., Rakheja, S., and Sankar, S., "Directional Response of a B-Train Vehicle Combination Carrying Liquid Cargo," Journal of Dynamic Systems, Measurement, and Control, Vol. 115, pp. 133-139, 1993 B.

Ranganathan, R., and Aia, A., "Development of Heavy Vehicle Dynamic Stability Analysis Model Using MATLAB/SIMULINK,” SAE paper No. 952638, 1995.

Rattayya, Jasti V., "Sloshing of Liquids in Axisymmetric Ellipsoidal Tanks," AIAA paper No. 65-114, $2^{\text {nd }}$ Aerospace Science Meeting, Jan. 1965.

Rayleigh, Lord, “On Waves. Phil. Mag.,” Series 5, Vol.1, No. 4, pp 257-279, 1876.

SAE J2189, "A Tilt Table Procedure for Measuring Static Rollover Threshold for Heavy Trucks," (draft), !990.

Salem, M., Mucino, V., Aquaro, M., and Gautam, M., "Review of Parameters Affecting Stability of Partially Filled Heavy-Duty Tankers," SAE Truck and Bus Conference, Paper No. 1999-013709, November 15-17, 1999.

Sankar, S., Rakheja, S., and Ranganathan, R., "Directional Response of Partially Filled Tank Vehicles," SAE paper No. 892481, 1989.

Sankar, S., and Rakheja, S., "Liquid Tanker Stability," Final Report, Transport Canada, Transportation Development Center Report No. TP 10690 E., November 1990.

Sankar, S., Sankar, T., S., and Popov, G., "Dynamic of Liquid Sloshing in Elliptical Road Containers," CSME, 1992 A.

Sankar, S., Ranganathan, R., and Rakheja, S., "Impact of Dynamic Fluid Slosh Loads on the Directional Response of Tank Vehicles," Vehicle System Dynamics, Vol. 21, pp. 385-404, 1992 B.

Schmid, I., "Engineering Approach to Truck and Tractor Train Stability," SAE paper No. 670006, 1967.

Schallamach, A., Turner, D., M., "The wear of Slipping Wheels," Wear, Vo. 3, pp. 1-25, 1960. 
Spring Design Manual, $2^{\text {nd }}$ Edition, SAE, ISBN 1-56091-680-X, 1996.

Stribersky, A., and Fancher, P., S., "The Nonlinear Behavior of Heavy-Duty Truck Combinations with Respect to Straight-line Stability," Journal of Dynamic Systems, Measurement, and Control, Vol. 111, pp.577-582, 1989.

Tabarrok, B., and Tong, X., "A computer Simulation Analysis of Road Dynamics of Log Hauling Trucks," SAE paper No. 942305, 1994.

Troger, H., and Zeman, K., "A nonlinear Analysis of the Generic Types of Loss of Stability of the Steady State Motion of a Tractor-Semitrailer," Vehicle System Dynamics, 13, pp 161-172, 1984.

Vagstedt, N., and Dahlberg, E., "Determination of Lateral Axle Data of Heavy Vehicles Combinations," SAE paper No. 973188, 1997.

Verma, M., K., et al., "Roll Dynamics of Commercial Vehicles," Vehicle System Dynamics, Vol. 9, pp1-17, 1980.

Warner,R., W., and Caldwell, J., T., "Experimental Evaluation of Analytical Models for the Inertias and Natural Frequencies of Fuel Sloshing in Circular Cylindrical Tanks," National Aeronautics and Space Administration, May, 1961.

White, D., et al., "Bus Rollover Stability," Imech E., Paper No. C225/86, 1986.

Winkler, C., B., Karamihas, S., M., and Bogard, S., E., "Roll-Stability Performance of HeavyVehicles Suspensions," SAE paper No. 922426, 1992.

Wong, J., Y., Theory of Ground Vehicles $2^{\text {nd }}$ Edition, John Wiley \& Sons, Inc., ISBN 0-47152496-4, 1993.

Zeman, K., Troger, H., and Scheidl, R., "Bifurcation Theory and Vehicle Dynamics-with Application to the Tractor-Semitrailer," A., H., Wickens (ed.) Proceedings of the $7^{\text {th }}$ IAVSDSymposium, Cambridge, U., K., 1981, Swets and Zeitlinger B., V., 1982. 


\section{APPENDIX A}

\section{Vehicle Data and Experimental Instrumentation Information}

\section{$\underline{\text { A.1- Vehicle Data }}$}

All data given for this appendix are for the tractor-trailer combination M916A1/60PRS when the fifth wheel is in the forward position.

\section{A.1.1- Weights on Axles at Different Levels of Fill}

Table A.1: Curb Weight Distribution on the Wheels for the Empty Tanker

\begin{tabular}{|c|c|c|c|}
\hline Axle & Left Side $[\mathrm{lbs} / \mathbf{N}]$ & Right Side $[\mathrm{lbs} / \mathbf{N}]$ & Both Sides $[\mathrm{lbs} / \mathbf{N}]$ \\
\hline 1 & $6930 / \mathbf{3 0 8 3 7}$ & $7130 / \mathbf{3 1 7 2 7 . 1 9}$ & $14060 / \mathbf{6 2 5 6 4 . 4 1}$ \\
\hline 2 & $4540 / \mathbf{2 0 2 0 2 . 1 6}$ & $4350 / \mathbf{1 9 3 5 6 . 7}$ & $8890 / \mathbf{3 9 5 5 8 . 8 6}$ \\
\hline 3 & $4240 / \mathbf{1 8 8 6 7 . 2 2}$ & $4450 / \mathbf{1 9 8 0 1 . 6 8}$ & $8690 / \mathbf{3 8 6 6 8 . 9}$ \\
\hline 4 & $2430 / \mathbf{1 0 8 1 3 . 0 5}$ & $2130 / \mathbf{9 4 7 8 . 1 0 8}$ & $4560 / \mathbf{2 0 2 9 1 . 1 6}$ \\
\hline 5 & $2670 / \mathbf{1 1 8 8 1 . 0 1}$ & $2950 / \mathbf{1 3 1 2 6 . 9 6}$ & $5620 / \mathbf{2 5 0 0 7 . 9 7}$ \\
\hline Total & $20810 / \mathbf{9 2 6 0 0 . 6 7}$ & $21010 / \mathbf{9 3 4 9 0 . 6 3}$ & $41820 / \mathbf{1 8 6 0 9 1 . 3}$ \\
\hline
\end{tabular}

Table A.2: Weight Distribution on the Wheels for the $50 \%$ full Tanker with water

\begin{tabular}{|c|c|c|c|}
\hline Axle & Left Side $[\mathrm{lbs} / \mathbf{N}]$ & Right Side $[\mathrm{lbs} / \mathbf{N}]$ & Both Sides $[\mathrm{lbs} / \mathbf{N}]$ \\
\hline 1 & $7040 / \mathbf{3 1 3 2 6 . 7}$ & $7440 / \mathbf{3 3 1 0 6 . 6 3}$ & $14480 / \mathbf{6 4 4 3 3 . 3 4}$ \\
\hline 2 & $7168 / \mathbf{3 1 8 9 6 . 2 8}$ & $6890 / \mathbf{3 0 6 5 9 . 2 3}$ & $14058 / \mathbf{6 2 5 5 5 . 5 1}$ \\
\hline 3 & $6852 / \mathbf{3 0 4 9 0 . 1 4}$ & $6914 / \mathbf{3 0 7 6 6 . 0 3}$ & $13766 / \mathbf{6 1 2 5 6 . 1 7}$ \\
\hline 4 & $4636 / \mathbf{2 0 6 2 9 . 3 5}$ & $6004 / \mathbf{2 6 7 1 6 . 7}$ & $10640 / \mathbf{4 7 3 4 6 . 0 4}$ \\
\hline 5 & $6290 / \mathbf{2 7 9 8 9 . 3 4}$ & $7462 / \mathbf{3 3 2 0 4 . 5 3}$ & $13752 / \mathbf{6 1 1 9 3 . 8 7}$ \\
\hline Total3 & $31986 / \mathbf{1 4 2 3 3 1 . 8}$ & $34710 / \mathbf{1 5 4 4 5 3 . 1}$ & $66696 / \mathbf{2 9 6 7 8 4 . 9}$ \\
\hline
\end{tabular}

Table A.3: Weight Distribution on the Wheels for the $75 \%$ full Tanker with water

\begin{tabular}{|c|c|c|c|}
\hline Axle & Left Side $[\mathrm{lbs} / \mathbf{N}]$ & Right Side $[\mathrm{lbs} / \mathbf{N}]$ & Both Sides $[\mathrm{lbs} / \mathbf{N}]$ \\
\hline 1 & $7240 / \mathbf{3 2 2 1 6 . 6 7}$ & $7440 / \mathbf{3 3 1 0 6 . 6 3}$ & $14680 / \mathbf{6 5 3 2 3 . 3}$ \\
\hline 2 & $8504 / \mathbf{3 7 8 4 1 . 2 4}$ & $8590 / \mathbf{3 8 2 2 3 . 9 2}$ & $17094 / \mathbf{7 6 0 6 5 . 1 5}$ \\
\hline 3 & $7781 / \mathbf{3 4 6 2 4 . 0 2}$ & $8442 / \mathbf{3 7 5 6 5 . 3 5}$ & $16223 / \mathbf{7 2 1 8 9 . 3 6}$ \\
\hline 4 & $6968 / \mathbf{3 1 0 0 6 . 3 2}$ & $7292 / \mathbf{3 2 4 4 8 . 0 6}$ & $14260 / \mathbf{6 3 4 5 4 . 3 8}$ \\
\hline 5 & $8065 / \mathbf{3 5 8 8 7 . 7 7}$ & $7911 / \mathbf{3 5 2 0 2 . 4 9}$ & $15976 / \mathbf{7 1 0 9 0 . 2 6}$ \\
\hline Total & $38558 / \mathbf{1 7 1 5 7 6}$ & $39675 / \mathbf{1 7 6 5 4 6 . 4}$ & $78233 / \mathbf{3 4 8 1 2 2 . 5}$ \\
\hline
\end{tabular}


Table A.4: Weight Distribution on the Wheels for the $100 \%$ full Tanker with water

\begin{tabular}{|c|c|c|c|}
\hline Axle & Left Side $[\mathrm{lbs} / \mathbf{N}]$ & Right Side $[\mathrm{lbs} / \mathbf{N}]$ & Both Sides $[\mathrm{lbs} / \mathbf{N}]$ \\
\hline 1 & $7440 / \mathbf{3 3 1 0 6 . 6 3}$ & $7270 / \mathbf{3 2 3 5 0 . 1 6}$ & $14710 / \mathbf{6 5 4 5 6 . 7 9}$ \\
\hline 2 & $9840 / \mathbf{4 3 7 8 6 . 1 9}$ & $10290 / \mathbf{4 5 7 8 8 . 6 1}$ & $20130 / \mathbf{8 9 5 7 4 . 8}$ \\
\hline 3 & $8710 / \mathbf{3 8 7 5 7 . 9}$ & $9970 / \mathbf{4 4 3 6 4 . 6 7}$ & $18680 / \mathbf{8 3 1 2 2 . 5 6}$ \\
\hline 4 & $9300 / \mathbf{4 1 3 8 3 . 2 9}$ & $8580 / \mathbf{3 8 1 7 9 . 4 2}$ & $17880 / 79562.71$ \\
\hline 5 & $9840 / \mathbf{4 3 7 8 6 . 1 9}$ & $8360 / \mathbf{3 8 1 7 9 . 4 2}$ & $18200 / \mathbf{8 0 9 8 6 . 6 5}$ \\
\hline Total & $45130 / \mathbf{2 0 0 8 2 0 . 2}$ & $44470 / \mathbf{1 9 7 8 8 3 . 3}$ & $89600 / \mathbf{3 9 8 7 0 3 . 5}$ \\
\hline
\end{tabular}

\section{A.1.2- CG Location at Different Levels of Fill}

a- All lateral CG locations are measured from vehicle centerline.

b- All longitudinal CG locations are measured from the trailer trunion centerline or the fifth wheel C.L.

c- All vertical CG locations are measured from ground level.

Table A.5: Center of Gravity Location at Different Loading Condition (M916A1/60PRS)

\begin{tabular}{|c|c|c|c|c|}
\hline Configuration & Weight $[\mathrm{N}]$ & Longitudinal [m] & Vertical [m] & Lateral [m] \\
\hline Tractor Only & 122816 & 2 & 0.782 & 0.005 right \\
\hline $\begin{array}{c}\text { Trailer only } \\
\text { Empty }\end{array}$ & 63320 & 2.108 & 1.524 & 0 \\
\hline $\begin{array}{c}\text { Truck/Trailer } \\
\text { Empty }\end{array}$ & 186136 & 7.484 & 1.018 & 0.005 right \\
\hline $\begin{array}{c}\text { Trailer only } \\
1 / 2 \text { Full }\end{array}$ & 174659 & 3.35 & 1.853 & 0 \\
\hline $\begin{array}{c}\text { Truck/Trailer } \\
1 / 2 \text { Full }\end{array}$ & 297474 & 6.201 & 1.411 & 0.044 right \\
\hline $\begin{array}{c}\text { Trailer Only } \\
\text { Full }\end{array}$ & 275933 & 3.609 & 2.121 & 0 \\
\hline Truck/Trailer & 398748 & 5.657 & 1.709 & 0.008 right \\
\hline
\end{tabular}

\section{A.1.3- Tractor Dimensions}

Figure A.1 shows a drawing for the test tractor M916A1 with some of the major dimensions given in meters. All other measured dimensions are given in tables A.6 through A.10. 


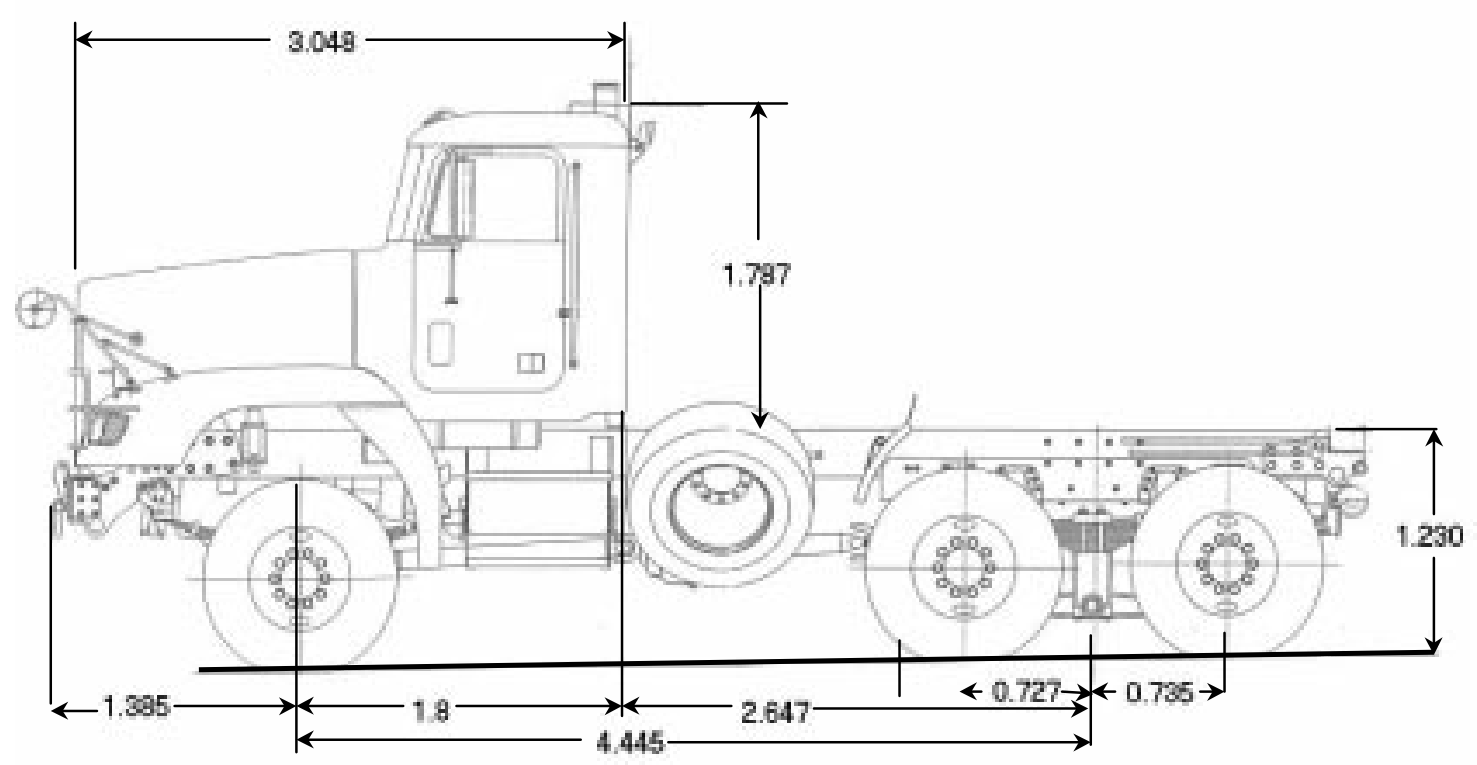

Figure A.1: Main Dimensions of the Test Tractor (M916A1)

Table A.6: Tractor frame rail characteristics for the tractor M916A1

\begin{tabular}{|l|c|}
\hline \multicolumn{1}{|c|}{ Parameter } & Length [m] \\
\hline Overall length of frame rails & 6.863 \\
\hline Height of frame rail cross section & 0.254 \\
\hline Width of frame rail flanges & 0.089 \\
\hline Thickness of frame rail flanges & 0.008 \\
\hline Thickness of frame rail web & 0.008 \\
\hline Frame rail vertical CL - ground Hight & \\
\hline Front, left & 1.214 \\
\hline Front, right & 1.206 \\
\hline Rear, left & 1.138 \\
\hline Rear, right & 1.138 \\
\hline Frame rail - frame rail spacing (outside) & 0.85 \\
\hline
\end{tabular}

Table A.7: Fifth wheel dimensions and location

\begin{tabular}{|l|c|}
\hline \multicolumn{1}{|c|}{ Parameter } & Length [m] \\
\hline Length of fifth wheel plate & 0.61 \\
\hline Width of fifth wheel plate & 0.92 \\
\hline Overall thickness of fifth wheel plate & 0.007 \\
\hline Fifth wheel CL - rear trunion CL & 0.017 \\
\hline Fifth wheel CL - rear of frame rail & 1.494 \\
\hline Fifth wheel CL -front of front bumper & 5.885 \\
\hline Top of fifth wheel - top frame flange & 0.034 \\
\hline
\end{tabular}


Table A.8: Tractor rear suspension location/dimensions

\begin{tabular}{|l|c|}
\hline \multicolumn{1}{|c|}{ Parameter } & Measurement \\
\hline Suspension type & Walking beam, symmetric, F-1 ${ }^{1}$ \\
\hline Number of Leaves & 13 \\
\hline Length of leaves & $\begin{array}{c}\text { Top spring } 0.914 \mathrm{~m} \text {, bottom spring } \\
0.177 \text { m, each spring is 2 to } 2.5 \\
\text { inch shorter }\end{array}$ \\
\hline Leaf width/thickness & $0.1016 / 0.0127 \mathrm{~m}$ \\
\hline Axle tread width (outside-outside) & $2.438 \mathrm{~m}$ \\
\hline Dual wheel spacing (CL-CL) & $0.356 \mathrm{~m}$ \\
\hline Fifth wheel CL - rear axle CL & $0.541 \mathrm{~m}$ \\
\hline \# 2 axle & $0.907 \mathrm{~m}$ \\
\hline \# 3 axle & $0.905 \mathrm{~m}$ \\
\hline Leafspring lateral spacing (CL-CL) & \\
\hline Leafspring mount pin - ground height & $0.838 / 0.841 \mathrm{~m}$ \\
\hline \# 2 left/right & $0.838 / 0.841 \mathrm{~m}$ \\
\hline \# 3 left/right & $0.14 \mathrm{~m}$ \\
\hline Vertical suspension travel @ curb wt & $0.24 \mathrm{~m}$ \\
\hline Ground clearance @ rear differential & \\
\hline
\end{tabular}

Table A.9: Tractor front suspension location/dimensions

\begin{tabular}{|l|c|}
\hline \multicolumn{1}{|c|}{ Parameter } & Measurement \\
\hline Suspension type & Single spring, non-linear, F-1 \\
\hline Number of leaves & $\begin{array}{c}\text { Top spring 1.524 m, bottom spring } \\
0.559 \text { m, each spring is 6 inch } \\
\text { shorter }\end{array}$ \\
\hline Length of leaves & $0.1016 / 0.0127 \mathrm{~m}$ \\
\hline Leaf width & $2.324 \mathrm{~m}$ \\
\hline Axle tread width (outside-outside & $4.244 \mathrm{~m}$ \\
\hline Fifth wheel CL- front axle CL & $0.838 \mathrm{~m}$ \\
\hline Leafspring lateral spacing (CL -CL) & $5.02 \mathrm{~m}$ \\
\hline Fifth wheel CL - front leafspring pins: & $3.495 \mathrm{~m}$ \\
\hline Front, (both sides) & \\
\hline \multicolumn{1}{|c|}{ Rear, (both sides) } & $0.902 \mathrm{~m}$ \\
\hline Leafspring mount pin - ground height: & $1.029 / 1.021 \mathrm{~m}$ \\
\hline Front, (both sides) & $0.114 \mathrm{~m}$ \\
\hline \multicolumn{1}{|c|}{ Rear, left/right } & $0.257 \mathrm{~m}$ \\
\hline Vertical suspension travel @ curb wt & \\
\hline Front ground clearance @ differential & \\
\hline
\end{tabular}

\footnotetext{
${ }^{1}$ For details about suspension types, please refer to SAE spring design Manual 1996

${ }^{2}$ The axles of the tractor trailer are numbered 1 through 5 starting from the tractor front most axle and ending with the last trailer axle.
} 
Table A.10: Tractor tires and wheel characteristics

\begin{tabular}{|l|c|}
\hline \multicolumn{1}{|c|}{ Parameter } & Measurement \\
\hline Tire specifications: & $\begin{array}{c}\text { Michelin X, 315/80R22.5 E-22.5 Pilote XZY, Radial } \\
\text { Regroovable, Tubeless Max Load 8270 lb @ psi, } \\
\text { tread 4 steel plies, sidewall 1 steel ply }\end{array}$ \\
\hline Front & $\begin{array}{c}\text { Goodyear G286 Unisteel, 315/80R22.5, Radial, } \\
\text { Tubeless, Max load Dual 7750 lb @ 120 psi, Max } \\
\text { load single 9000 psi @ 120 psi, tread 5 plies steel } \\
\text { cord, sidewall 1 ply steel cord }\end{array}$ \\
\hline Rear & $1.074 \mathrm{~m}$ \\
\hline Tire overall diameter & $0.267 \mathrm{~m}$ \\
\hline Tire tread width: & $0.254 \mathrm{~m}$ \\
\hline Front & $1.041 \mathrm{~m}$ \\
\hline \multicolumn{1}{|c|}{ Rear } & $0.572 \mathrm{~m}$ \\
\hline $\begin{array}{l}\text { Tire rolling diameter @ curb } \\
\text { weight }\end{array}$ & $0.229 \mathrm{~m}$ \\
\hline Wheel diameter & \\
\hline Wheel rim width & \\
\hline
\end{tabular}

\section{A.1.4- Trailer Dimensions}

Figure A.2 shows a drawing for the teat trailer 60PRS with some of the major dimensions given in meters. All other measured dimensions are given in tables A.11 through A.14.

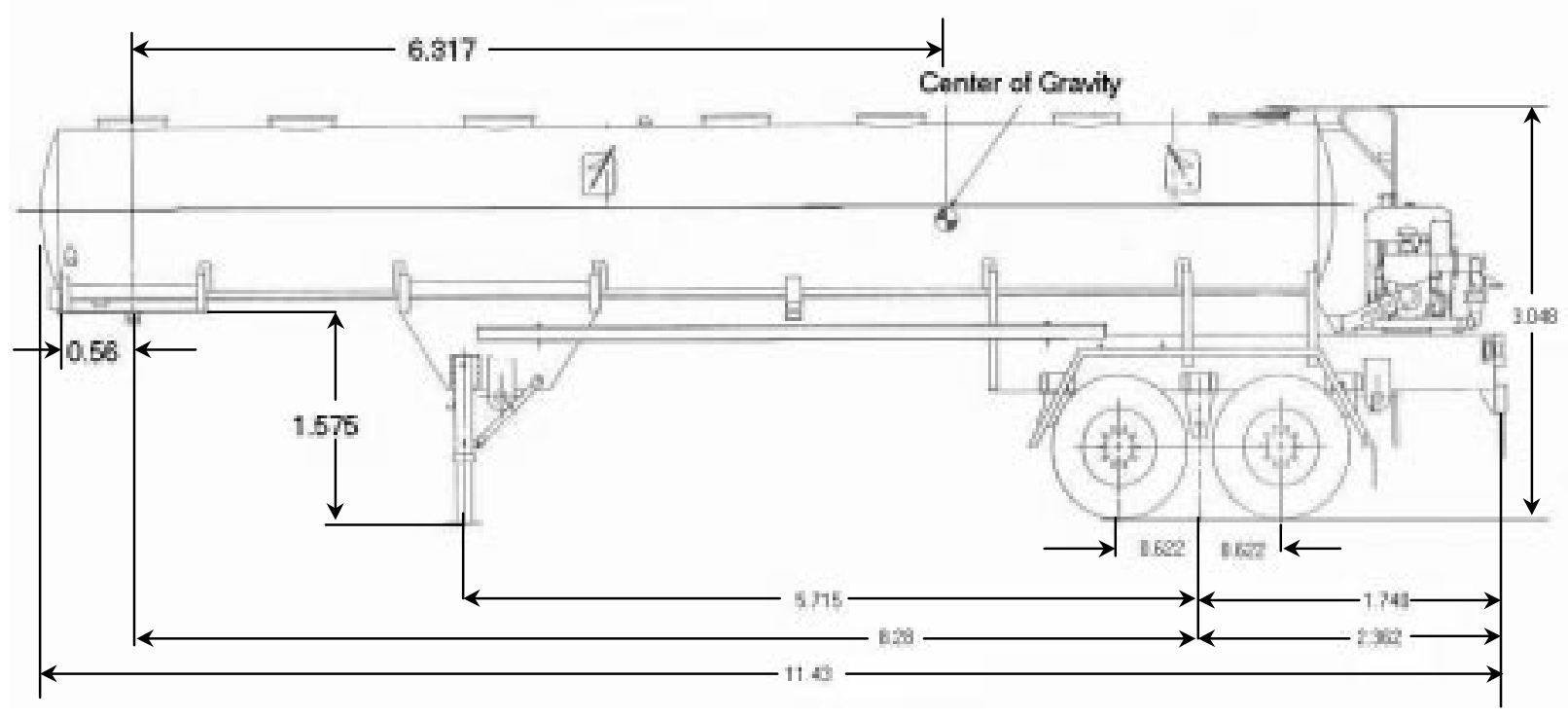

Figure A.2: Main Dimensions of the Test Trailer (60PRS) 
Table A.11: Main dimensions of tanker shell and rear framerails

\begin{tabular}{|c|c|}
\hline Parameter & Measurement \\
\hline Trailer Unsprung Weight & $12837 \mathrm{~N}$ \\
\hline Trailer Sprung weight & $295021 \mathrm{~N}$ \\
\hline Weight on rear tires (empty) & 47613 N/eight tires \\
\hline Weight on rear tires (loaded) & 165800 N/eight tires \\
\hline Shell overall length (outside) & $9.906 \mathrm{~m}$ \\
\hline Shell overall width (outside) & $2.4 \mathrm{~m}$ \\
\hline Shell overall height (outside) & $1.219 \mathrm{~m}$ \\
\hline Projection of front endcap (forward) & $0.13 \mathrm{~m}$ \\
\hline Shell Material (ASTM 569-85 Steel) thickness & $0.00436 \mathrm{~m}$ (\#8 gauge) \\
\hline Head Material (ASTM 569-85 Steel) thickness & $0.00436 \mathrm{~m}$ (\#8 gauge) \\
\hline Baffle Material (ASTM 569-85 Steel) thickness & $0.00357 \mathrm{~m}(\# 10$ gauge $)$ \\
\hline \multicolumn{2}{|l|}{ Front endcap - manhole CL: } \\
\hline$\# 1$ & $0.685 \mathrm{~m}$ \\
\hline \#2 & $1.98 \mathrm{~m}$ \\
\hline$\# 3$ & $3.62 \mathrm{~m}$ \\
\hline \#4 & $5.44 \mathrm{~m}$ \\
\hline$\# 5$ & $6.63 \mathrm{~m}$ \\
\hline \#6 & $8.275 \mathrm{~m}$ \\
\hline \#7 & $9.515 \mathrm{~m}$ \\
\hline Manhole I.D. & $0.49 \mathrm{~m}$ \\
\hline Manhole upper flange O.D. & $0.53 \mathrm{~m}$ \\
\hline Manhole vertical wall height @ longitudinal CL & $0.055 \mathrm{~m}$ \\
\hline Manhole upper flange height & $0.0032 \mathrm{~m}$ \\
\hline Projection of baffle curvature (fwd) & $0.13 \mathrm{~m}$ \\
\hline \multicolumn{2}{|l|}{ Front endcap - baffle CL: } \\
\hline$\# 1$ & $1.255 \mathrm{~m}$ \\
\hline$\# 2$ & $2.805 \mathrm{~m}$ \\
\hline \#3 & $4.305 \mathrm{~m}$ \\
\hline$\# 4$ & $5.815 \mathrm{~m}$ \\
\hline \#5 & $7.345 \mathrm{~m}$ \\
\hline \#6 & $8.865 \mathrm{~m}$ \\
\hline Radius of baffle openings & $0.089 \mathrm{~m}$ \\
\hline Overall length of rear framerails & $3.995 \mathrm{~m}$ \\
\hline Length of body supported on rear frame & $2.545 \mathrm{~m}$ \\
\hline Height of framerail cross section & $0.81 \mathrm{~m}$ \\
\hline Width of framerail flanges & $0.1 \mathrm{~m}$ \\
\hline Thickness of framerail flanges and web & $0.0048 \mathrm{~m}$ \\
\hline Frame rail vertical CL - ground height & $1.34 \mathrm{~m}$ \\
\hline Frame rail - framerail spacing & $0.97 \mathrm{~m}$ \\
\hline
\end{tabular}


Table A.12: Kingpin dimensions and location

\begin{tabular}{|l|c|}
\hline \multicolumn{1}{|c|}{ Parameter } & Length [m] \\
\hline Length of upper kingpin plate & 1.1 \\
\hline Width of upper kingpin plate & 0.97 \\
\hline Thickness of upper kingpin plate & 1.57 \\
\hline Kingpin CL - front of front endcap & 0.71 \\
\hline Kingpin - fifth wheel lateral clearance & 0.002 \\
\hline Kingpin - fifth wheel longitudinal clearance & 0.001 \\
\hline Height of upper plate above ground @ curb weight & 1.59 \\
\hline Kingpin shaft diameter & 0.089 \\
\hline Kingpin shaft height & 0.036 \\
\hline Kingpin upper and lower shoulder diameters & 0.109 \\
\hline Kingpin upper shoulder height & 0.023 \\
\hline Kingpin lower shoulder height & 0.0015 \\
\hline
\end{tabular}

Table A.13: Tractor tire and wheel characteristics

\begin{tabular}{|l|c|}
\hline \multicolumn{1}{|c|}{ Parameter } & \multicolumn{1}{c|}{ Measurement } \\
\hline Tire specifications & $\begin{array}{c}\text { Goodyear, HI-MILLER CS2, 11-22.5, regrovable, load } \\
\text { range F, tread 8 plies Nylon cord, sidewall 6 plies } \\
\text { Nylon cord }\end{array}$ \\
\hline Tire overall diameter & $1.03 \mathrm{~m}$ \\
\hline Tire tread width & $0.19 \mathrm{~m}$ \\
\hline $\begin{array}{l}\text { Tire rolling diameter @ curb } \\
\text { weight }\end{array}$ & $1.011 \mathrm{~m}$ \\
\hline Wheel diameter & $0.572 \mathrm{~m}$ \\
\hline Wheel rim width & $0.21 \mathrm{~m}$ \\
\hline
\end{tabular}

Figure A.3 shows the main dimensions of one of the springs installed on the test trailer, while the rest of the suspension measurements and parameters are given in table A.14

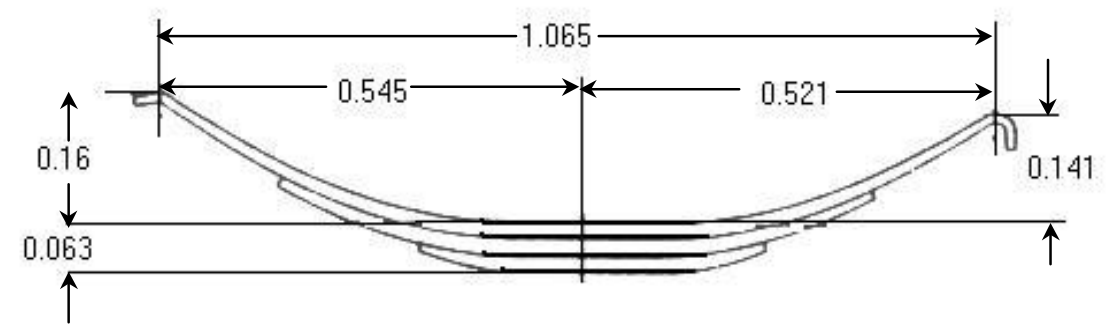

Figure A.3: Main dimensions of trailer leaf spring 
It should be noted that the characteristic curve of the leaf spring shown in Figure A.3 is given during compression and is shown in Figure 3.6.

Table A.14: Trailer suspension location/dimensions

\begin{tabular}{|l|c|}
\hline \multicolumn{1}{|c|}{ Parameter } & Measurement \\
\hline Axle tread width (outside -outside) & $2.35 \mathrm{~m}$ \\
\hline Dual wheel spacing (CL-CL) & $0.343 \mathrm{~m}$ \\
\hline Kingpin CL - front leafspring mount: & $7.054 \mathrm{~m}$ \\
\hline \# 4 axle & $8.222 \mathrm{~m}$ \\
\hline \# 5 axle & $8.222 \mathrm{~m}$ \\
\hline Kingpin CL- rear leafspring mount pt: & $9.431 \mathrm{~m}$ \\
\hline \# 4 axle & $0.97 \mathrm{~m}$ \\
\hline \# 5 axle & \\
\hline Leafspring lateral spacing (CL-CL) & $0.813 \mathrm{~m}$ \\
\hline Leafspring mount pin - ground height: & $0.826 \mathrm{~m}$ \\
\hline \# 4 left & $0.8 \mathrm{~m}$ \\
\hline \# 4 right & $0.788 \mathrm{~m}$ \\
\hline \# 5 left & $0.188 \mathrm{~m}$ \\
\hline \# 5 right & \\
\hline Vertical suspension travel @ curb wt. & $7.6 \mathrm{~m}$ \\
\hline Axle spacing: Kingpin CL- Axle CL: & $8.847 \mathrm{~m}$ \\
\hline \# 4 axle & $0.401 \mathrm{~m}$ \\
\hline \# 5 axle & \\
\hline Ground clearance & \\
\hline
\end{tabular}

\section{$\underline{\text { A.2- Vehicle Experimental Instrumentation Information }}$}

The figures given below show the instrumentation used on the test tanker during the tests performed at ATC. Table A.15 shows indicates the exact location of some of the instruments used. 


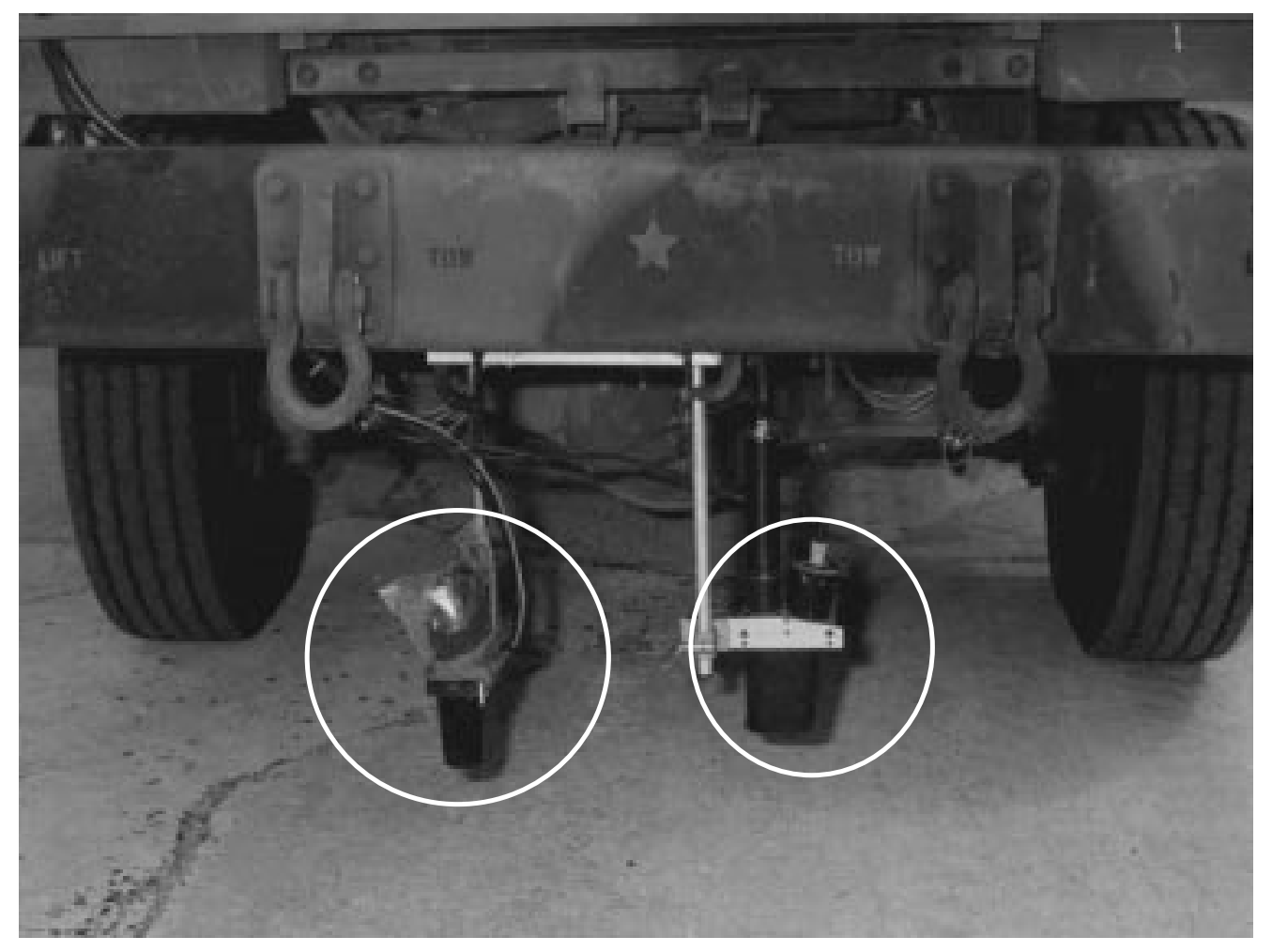

Figure A.4: Oscillation Gyrometer to measure the Yaw rate of the tractor and lateral speed sensor

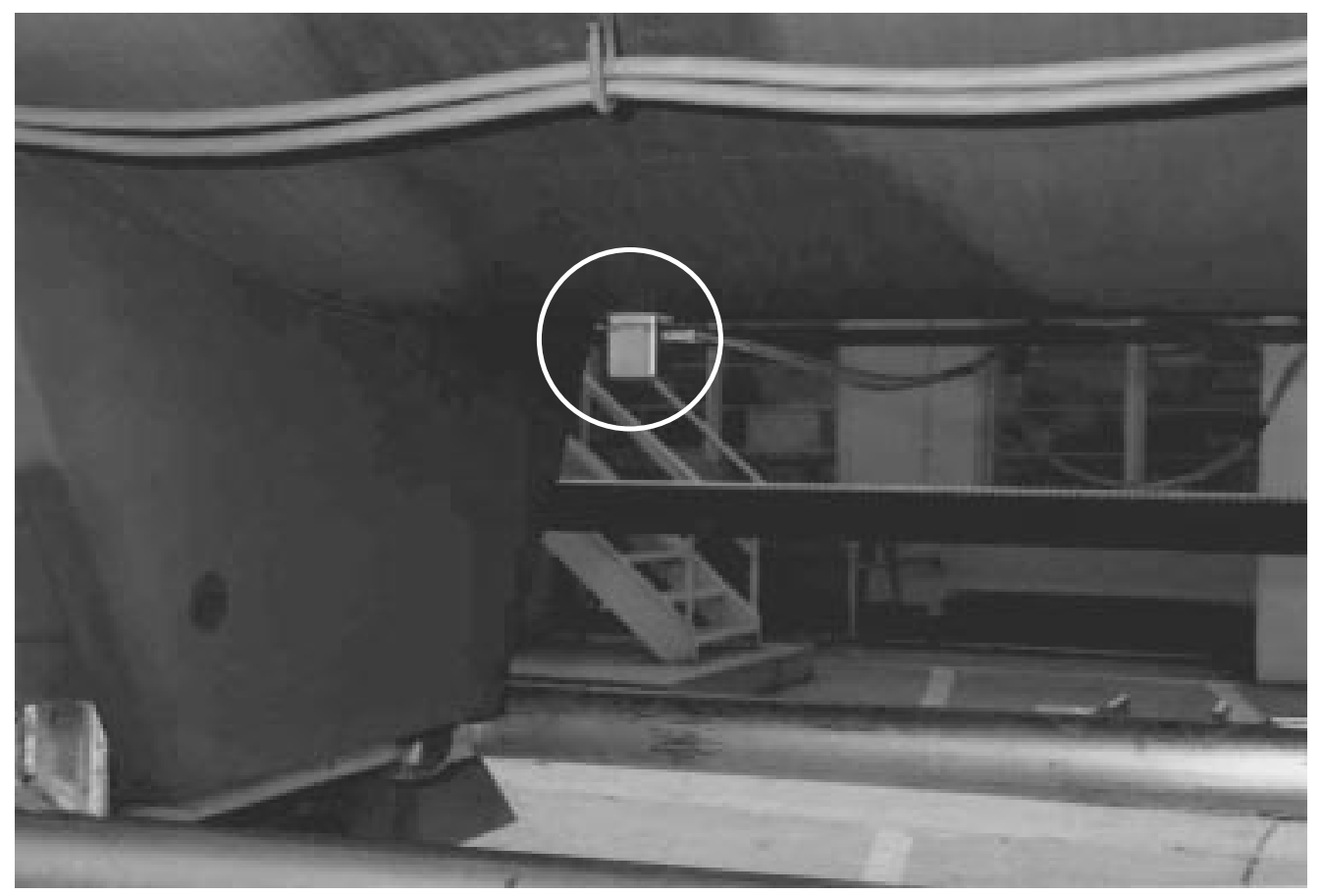

Figure A.5: Oscillation Gyrometer to measure the Yaw rate of the trailer at the its CG 


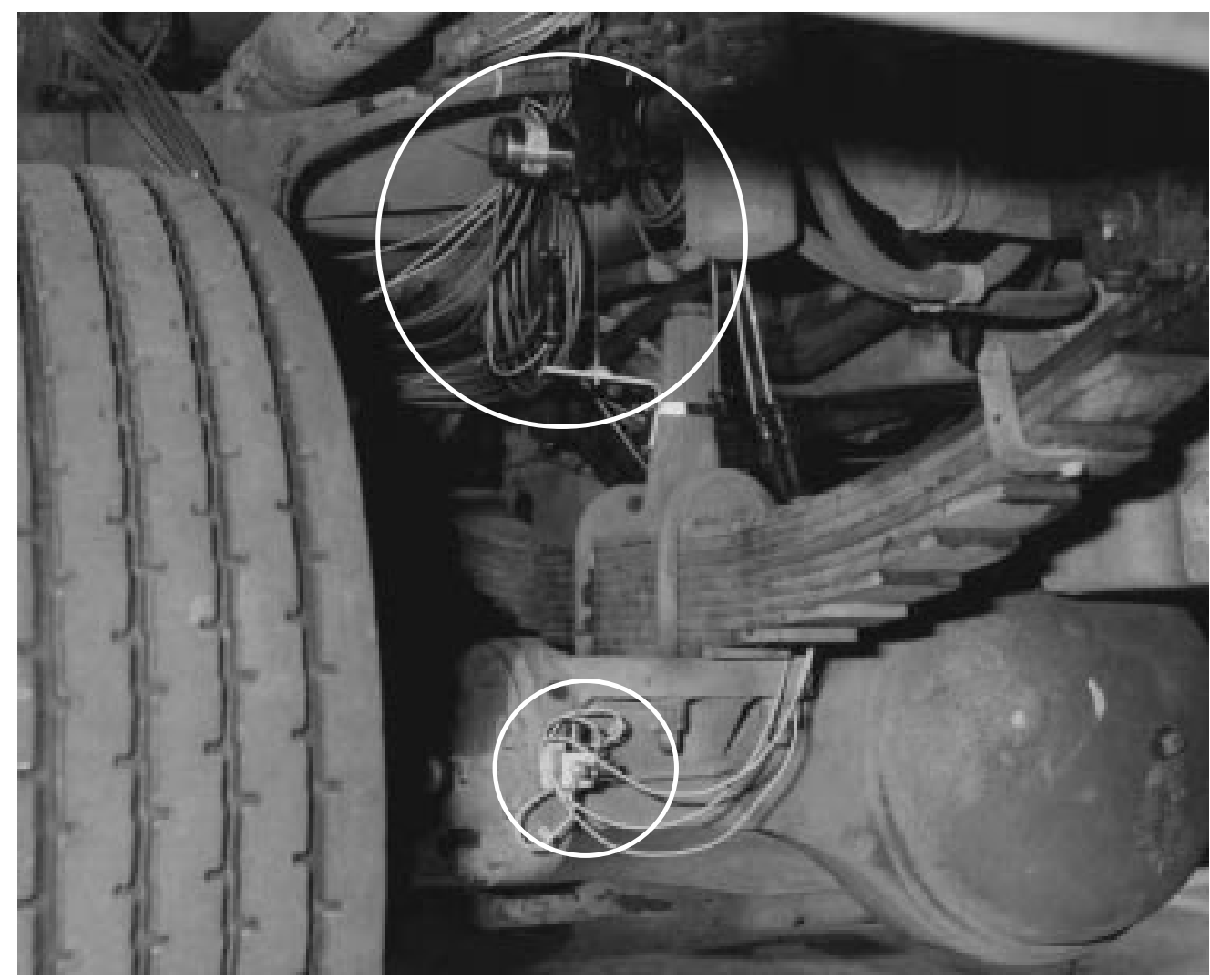

Figure A.6: 3 perpendicular accelerometers and a potentiometer to measure the acceleration all 3 directions and the spring deflection respectively fitted on all axles (Figure for tractor front axle)

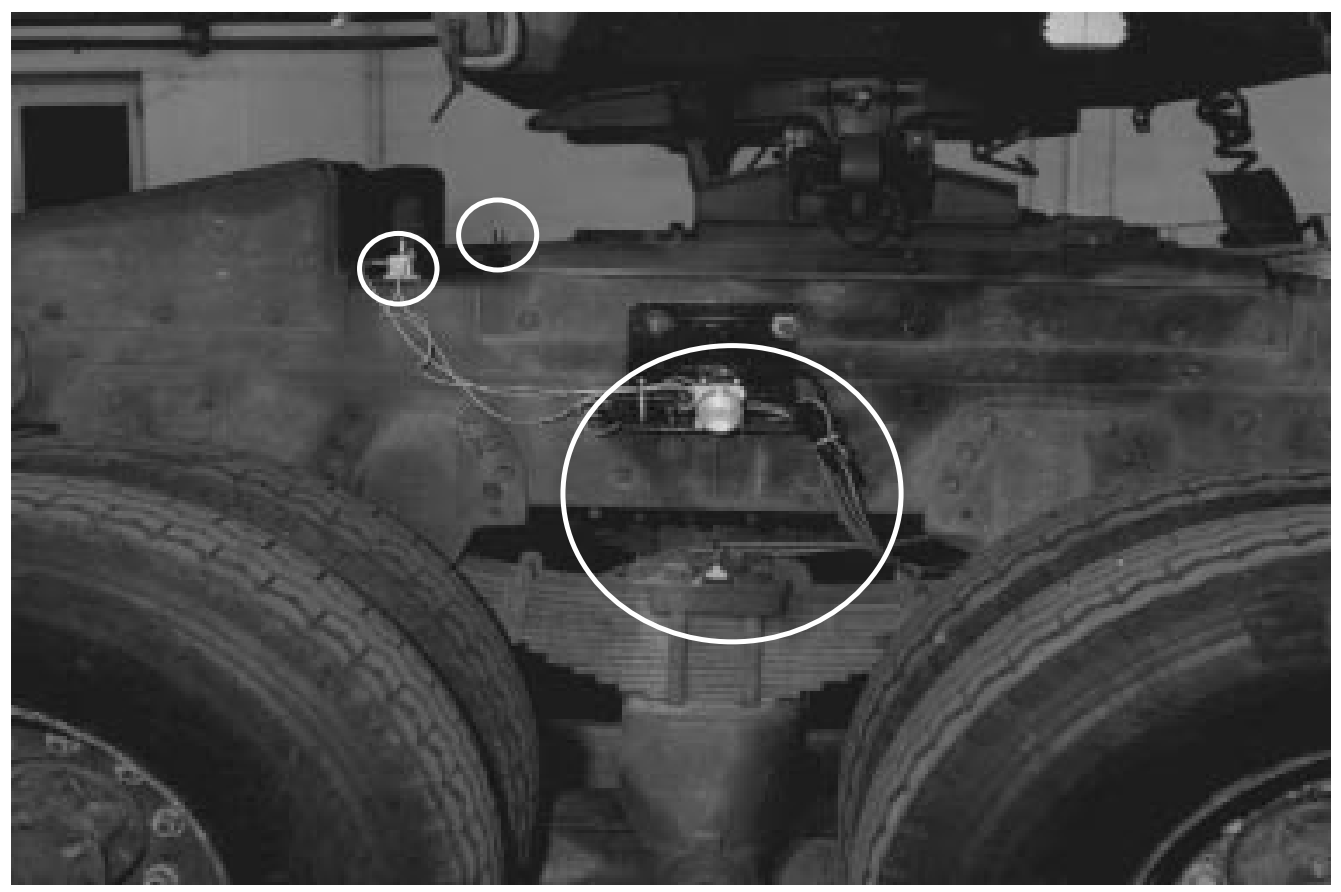

Figure A.7: 3 perpendicular accelerometers on both sides of tractor frame and potentiometer to measure spring deflection on the tractor rear suspension 


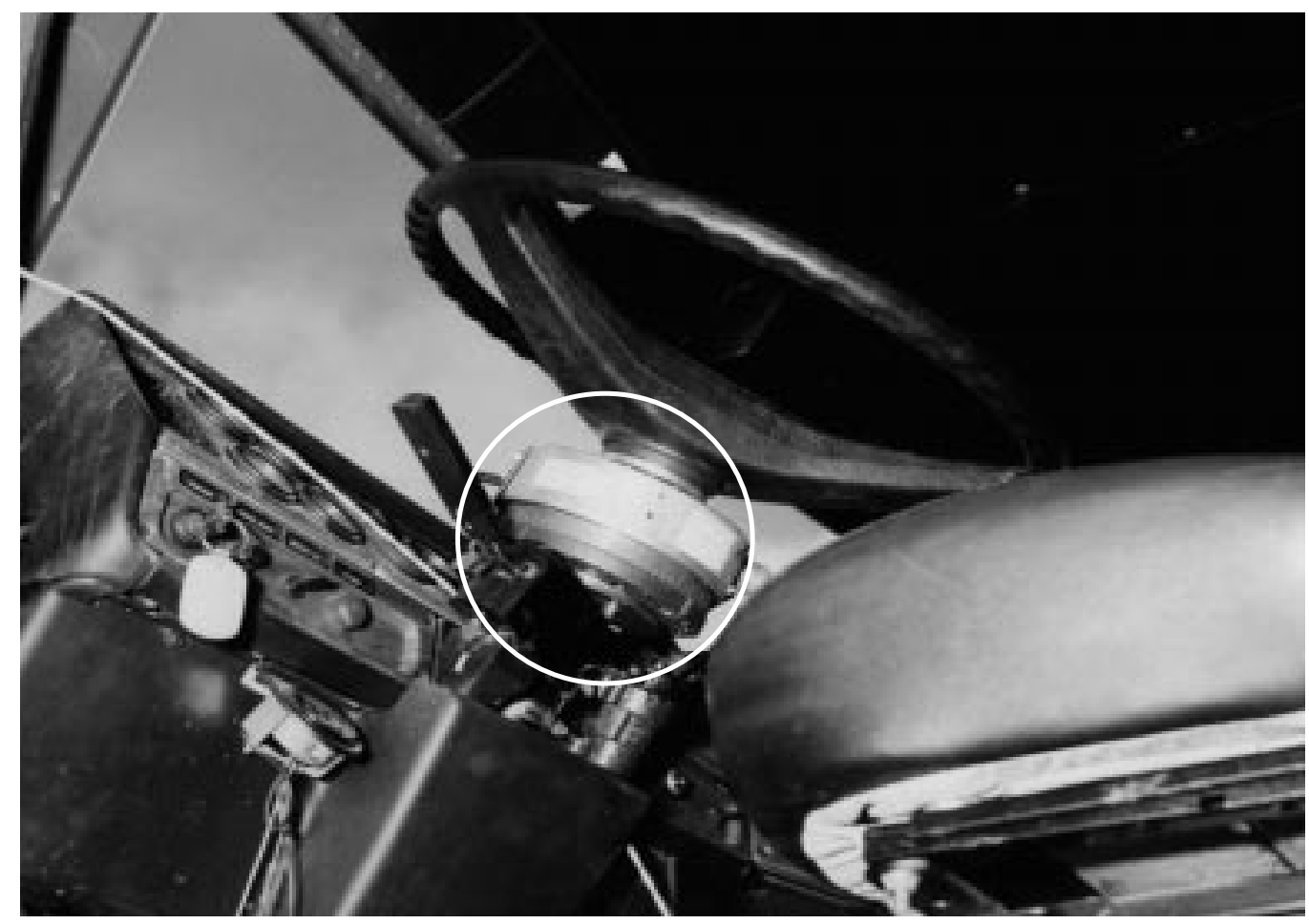

Figure A.8: Circular dial to measure the steering angle and hence the slip angle of the tires can be determined (device was not needed in this study but could be used later)

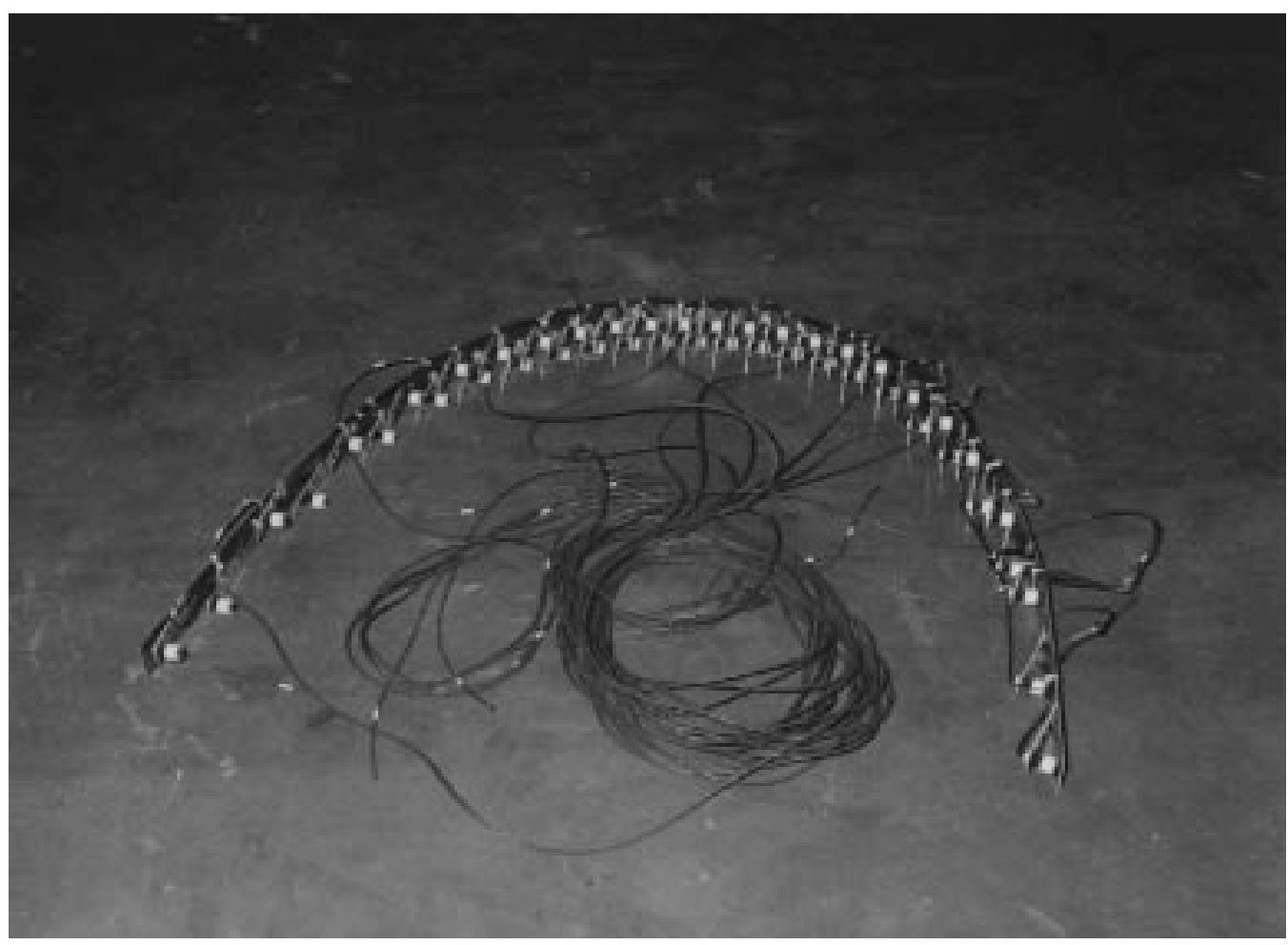

Figure A.9: Piezoelectric pressure measurement strips installed in the front-most compartment to measure fluid level. 
Table A.15: Transducer exact locations

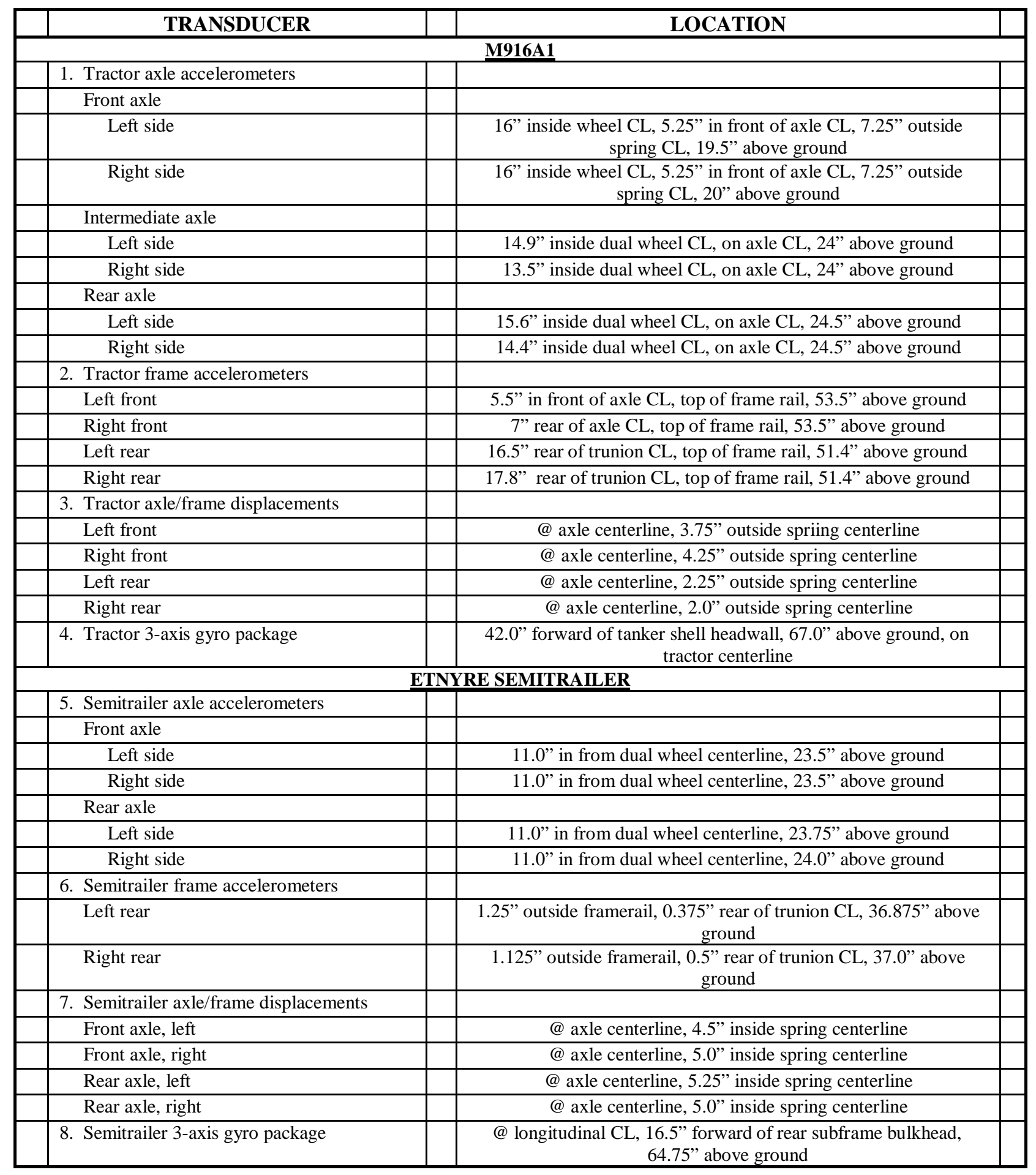




\section{APPENDIX B}

\section{MATHEMATICAL DERIVATIONS}

\section{$\underline{\text { B.1- Introduction }}$}

This Appendix contains all the lengthy mathematical derivations related to this study. The first part has the equations needed to determine the CG of a body of fluid in a partially filled elliptical tank while moving in the vertical (X-Y) plane. It is assumed that the fluid surface remains a straight line and that all the motion is in 2-D. The second part of the Analysis is dedicated to deriving the equation of motion of an elliptical Trammel pendulum simulating the fluid first mode of lateral vibrations. The third part has the derivations of the equations of motion for vehicle model \#2 given in chapter 5 .

\section{B.2- Calculation of Mass and CG Location of a Fluid Moving in an Elliptical Tank}

To obtain the Mass and the CG location representing the fluid motion of an elliptical tank and assuming that the free surface of the fluid remains straight, an iterative approach has been followed. Figure B1 shows the Coordinate position and the initial fluid position used in this analysis.

The equation of the ellipse relative to the coordinate system given above is:-

$$
\frac{x^{2}}{a^{2}}+\frac{(y-b)^{2}}{b^{2}}=1
$$

Which by solving for " $y$ " yields:-

$$
y=b\left(1 \pm \sqrt{1-\left(\frac{x}{a}\right)^{2}}\right)
$$




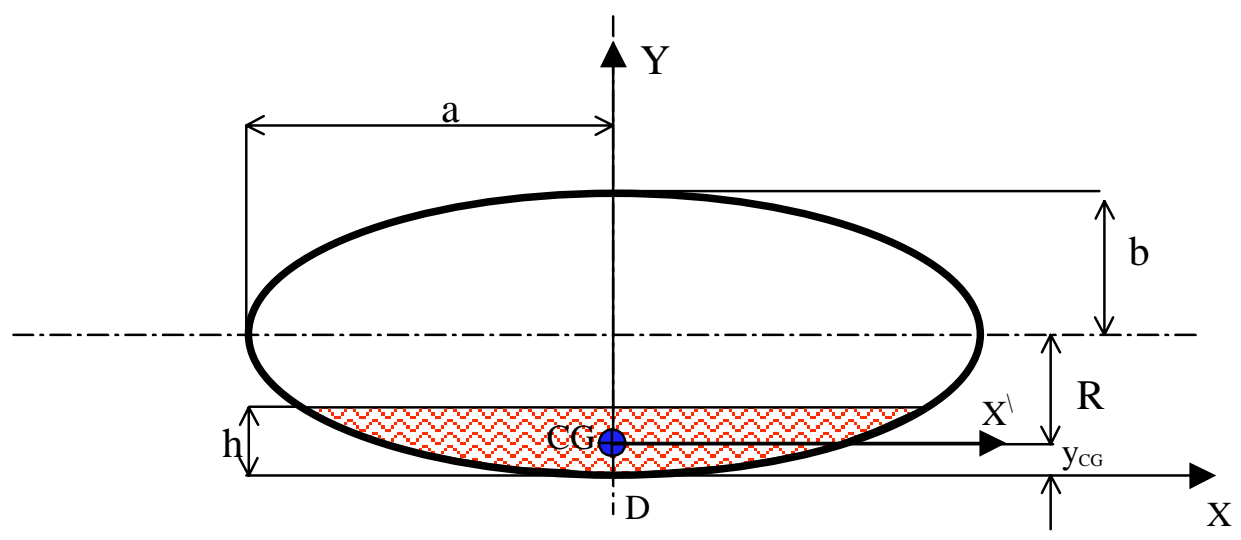

Figure B.1-Elliptical tank partially filled at initial position

Or by solving for " $\mathrm{x}$ " yields:-

$$
x= \pm a \sqrt{\frac{2 y}{b}-\left(\frac{y}{b}\right)^{2}}
$$

Thus, the value of " $\mathrm{x}$ " at a general fluid height "h" is given by:-

$$
x_{h}= \pm a \sqrt{\frac{2 h}{b}-\left(\frac{h}{b}\right)^{2}}
$$

As the fluid surface moves in the $\mathrm{X}-\mathrm{Y}$ plane, the front area of the fluid can be used to represent the whole movement of the fluid to simplify the analysis. This area can be calculated from the following integration:-

$$
A_{h}=2 \int_{0}^{x_{h}} \int_{b *\left(1-\sqrt{1-\left(\frac{x}{a}\right)^{2}}\right)}^{h} d y \cdot d x
$$

$\therefore$ If $\mathrm{h}<\mathrm{b}$

$$
A_{h}=2 *\left\{x_{h} \cdot h-x_{h} \cdot b+\left(\frac{b}{2 a}\right)\left[x_{h} \sqrt{a^{2}-x_{h}^{2}}+a^{2} \sin ^{-1}\left(\frac{x_{h}}{a}\right)\right]\right\}
$$

The "y" position for the CG can be calculated from:- 


$$
\begin{gathered}
\mathrm{y}_{\mathrm{CG}}=\frac{\int_{0}^{\mathrm{x}_{\mathrm{h}}} \mathrm{h} \int_{\mathrm{b}^{*}\left(1-\sqrt{1-\left(\frac{\mathrm{x}}{\mathrm{a}}\right)^{2}}\right)^{\mathrm{y}} \mathrm{y} \cdot \mathrm{dy} \cdot \mathrm{dx}}^{\mathrm{A}_{\mathrm{h}}}}{\therefore y_{C G}=\frac{1}{A_{h}} *\left\{h^{2} x_{h}-2 b^{2} x_{h}+\frac{b^{2}\left[x_{h} \sqrt{a^{2}-x_{h}^{2}}+a^{2} \sin ^{-1}\left(\frac{x_{h}}{a}\right)\right]}{a}+\frac{b^{2} x_{h}^{3}}{3 a^{2}}\right\}}
\end{gathered}
$$

and $\mathrm{R}=\mathrm{b}-\mathrm{y}_{\mathrm{CG}}$

If $h=b$

In this case, the fluid fills a half ellipse, which has an area $A_{h}=\frac{\pi a b}{2}$ and It's CG is at

$$
\mathrm{R}=\frac{4 \mathrm{~b}}{3 \pi}
$$

If $2 b>h>b$

$$
\mathrm{A}_{\mathrm{h}}=\pi \mathrm{ab}-\alpha
$$

Where $\quad \alpha=2 *\left\{x_{h} \cdot b-x_{h} \cdot h+\left(\frac{b}{2 a}\right)\left[x_{h} \sqrt{a^{2}-x_{h}^{2}}+a^{2} \sin ^{-1}\left(\frac{x_{h}}{a}\right)\right]\right\}$

$$
\mathrm{y}_{\mathrm{cg}}=\frac{\pi \mathrm{ab}{ }^{2}-\alpha \cdot \beta}{\mathrm{A}_{\mathrm{h}}}
$$

Where $\beta=2 b-y_{C G(B 6)}$

$$
\mathrm{R}=\mathrm{b}-\mathrm{y}_{\mathrm{CG}(\mathrm{B} 8)}
$$

Now if the fluid tilts with an angle $\theta$ as shown in Figure B2.

To get the $\mathrm{x}$ and $\mathrm{y}$ coordinate of the CG of the fluid area, we have to know the limits of integration in equation (B5) and (B9). 


$$
x_{C G}=\frac{\int_{x 1}^{x 2} h \int_{y 1}^{y 2} x \cdot d y \cdot d x}{A_{h}}
$$

Where $(\mathrm{x} 1, \mathrm{y} 1)$ and $(\mathrm{x} 2, \mathrm{y} 2)$ are the points of intercession between the fluid surface line and the elliptical tank. Those two points can be found by trial and error. The best way to do that is by

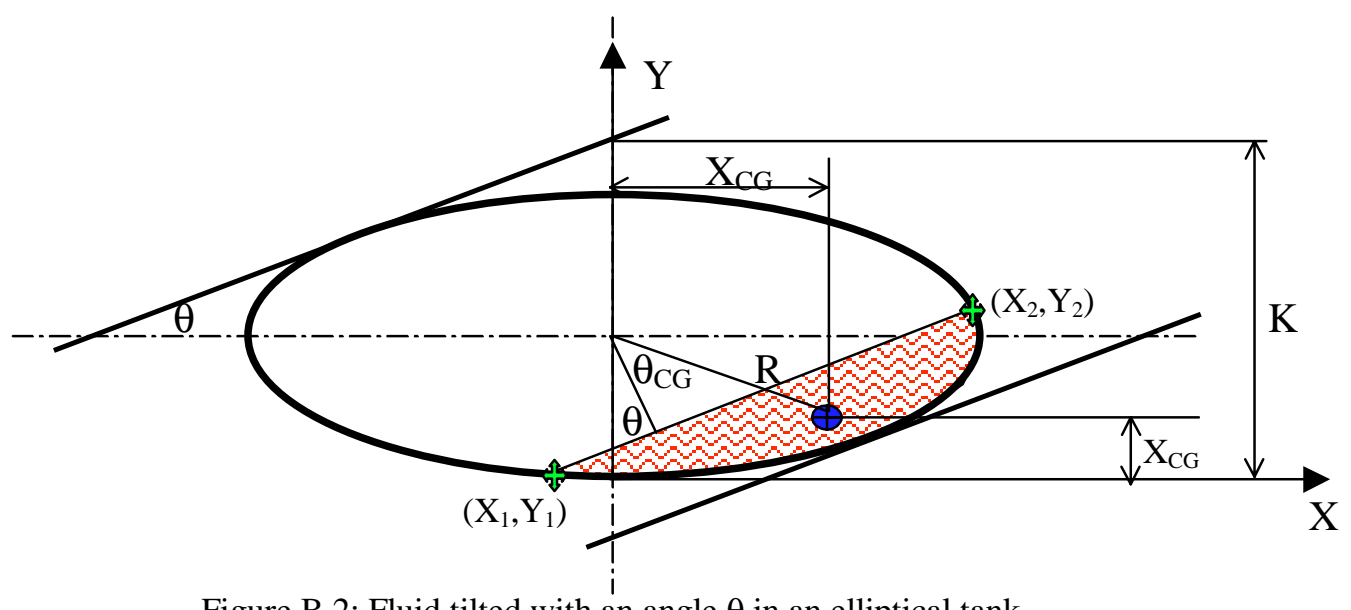

Figure B.2: Fluid tilted with an angle $\theta$ in an elliptical tank

Sweeping a tangent to the ellipse which has an angle of inclination $=\theta$ from the upper tangent point to the lower tangent point, getting the points of intersection at each step, calculating the area and comparing it with the initial calculated area. Once the right area is obtained with a certain acceptable error, the points of intersection are recorded, and the values of $\mathrm{x}_{\mathrm{CG}}, \mathrm{y}_{\mathrm{CG}}, \mathrm{R}$ and $\theta_{\mathrm{CG}}$ can be calculated.

The equation of the tangent is given by:-

$$
\mathrm{Y}=\mathrm{mx}+\mathrm{k}
$$

Solving equations B1 and B10 together for " $\mathrm{x}$ " gives:-

$$
x^{2}+\left[\frac{2 a^{2} m(k-b)}{b^{2}+a^{2} m^{2}}\right] x+\left[\frac{a^{2}(k-b)^{2}-b^{2} a}{b^{2}+a^{2} m^{2}}\right]=0
$$


Equation $\mathrm{B} 11$ is a quadratic equation that has two roots $\mathrm{x} 1$ and $\mathrm{x} 2$, those two roots are equal at the point of tangency. Which yields $\mathrm{B}= \pm \sqrt{2 \mathrm{C}}$

$$
\therefore \frac{2 \mathrm{a}^{2} \mathrm{~m}(\mathrm{k}-\mathrm{b})}{\mathrm{b}^{2}+\mathrm{a}^{2} \mathrm{~m}^{2}}= \pm \sqrt{\left[\frac{\mathrm{a}^{2}(\mathrm{k}-\mathrm{b})^{2}-\mathrm{b}^{2} \mathrm{a}}{\mathrm{b}^{2}+\mathrm{a}^{2} \mathrm{~m}^{2}}\right]}
$$

Solving B12 for k yields:-

$$
\begin{gathered}
\mathrm{k}^{2}-2 \mathrm{bk}-\mathrm{a}^{2} \mathrm{~m}^{2}=0 \\
\therefore \mathrm{k}=\mathrm{b} \pm \sqrt{\mathrm{b}^{2}+\mathrm{a}^{2} \mathrm{~m}^{2}}
\end{gathered}
$$

Equation B13 gives the iteration limits on "k" for the sweeping tangent from the upper tangent point to the lower tangent point.

Depending on the location of the intersection between the fluid surface line and the elliptical surface relative to the $\mathrm{X}-\mathrm{Y}$ axis, the integration limits will change. Three distinct zones can be listed depending of the location of the Y coordinate value of the intersection points:-

Case1:- When $\mathrm{y} 1>=b$

This condition implies that $\mathrm{y} 2$ should be greater than or equal $\mathrm{b}$ automatically according to Figure B2. In this case, the area will be given by:-

$$
\mathrm{A}=\pi \cdot \mathrm{a} \cdot \mathrm{b}-\mathrm{A}_{1}
$$

Where $\quad \mathrm{A}_{1}=\frac{2 \cdot a \cdot b \cdot(\mathrm{x} 2-\mathrm{x} 1)+\mathrm{b} \cdot \mathrm{x} 2 \cdot \sqrt{\mathrm{a}^{2}-\mathrm{x} 2^{2}}-\mathrm{b} \cdot \mathrm{x} 1 \cdot \sqrt{\mathrm{a}^{2}-\mathrm{x} 1^{2}}+\mathrm{m} \cdot \mathrm{a} \cdot\left(\mathrm{x} 1^{2}-\mathrm{x} 2^{2}\right)+\gamma}{2 \mathrm{a}}$

Where

$$
\begin{gathered}
\gamma=\text { b.a } a^{2}\left[\sin ^{-1}\left(\frac{\mathrm{x} 2}{\mathrm{a}}\right)-\sin ^{-1}\left(\frac{\mathrm{x} 1}{\mathrm{a}}\right)\right] \\
\mathrm{x}_{\mathrm{CG}}=\frac{\left(\mathrm{A}_{\mathrm{h}}-\pi \cdot \mathrm{a} \cdot \mathrm{b}\right) \cdot \mathrm{x}_{\mathrm{CG} 1}}{\mathrm{~A}_{\mathrm{h}}}
\end{gathered}
$$

Where $\mathrm{x}_{\mathrm{CG} 1}=\frac{\mathrm{b}}{6 \cdot a \cdot\left(\pi \cdot a \cdot b-\mathrm{A}_{\mathrm{h}}\right)}\left[3 \cdot a \cdot(\mathrm{b}-\mathrm{k}) \cdot\left(\mathrm{x} 2^{2}-\mathrm{x} 1^{2}\right)-2 \cdot m \cdot a \cdot\left(\mathrm{x} 2^{3}-\mathrm{x} 1^{3}\right)+2 \cdot b \cdot\left(\mathrm{a}^{2}-\mathrm{x} 1^{2}\right)^{3 / 2}\right.$

$$
\left.-2 \cdot\left(a^{2}-x 2^{2}\right)^{3 / 2}\right]
$$




$$
\mathrm{y}_{\mathrm{CG}}=\frac{\pi \cdot a \cdot b^{2}-\left(\pi \cdot a \cdot b-\mathrm{A}_{\mathrm{h}}\right) * \mathrm{y}_{\mathrm{CG} 1}}{\mathrm{~A}_{\mathrm{h}}}
$$

where $y_{\mathrm{CG} 1}=\frac{1}{2\left(\pi \cdot a \cdot b-A_{h}\right)}\left[\left(2 b^{2}-k^{2}\right)(x 2-x 1)-m \cdot k \cdot\left(x 2^{2}-x 1^{2}\right)-\frac{\left(b^{2}+a^{2} m^{2}\right) \cdot\left(x 2^{3}-x 1^{3}\right)}{3 a^{2}}+\right.$

$$
\left.\frac{b^{2}}{a}\left(a^{2}\left(\sin ^{-1}\left(\frac{x 2}{a}\right)-\sin ^{-1}\left(\frac{x 1}{a}\right)\right)+x 2 \sqrt{a^{2}-x 2^{2}}-x 1 \sqrt{a^{2}-x 1^{2}}\right)\right]
$$

Case 2:- When $\mathrm{y} 2>=\mathrm{b}$ and $\mathrm{y} 1<\mathrm{b}$

In this case, the area is divided into two areas $\left(A_{1} \& A_{2}\right)$ as shown in Figure B3.

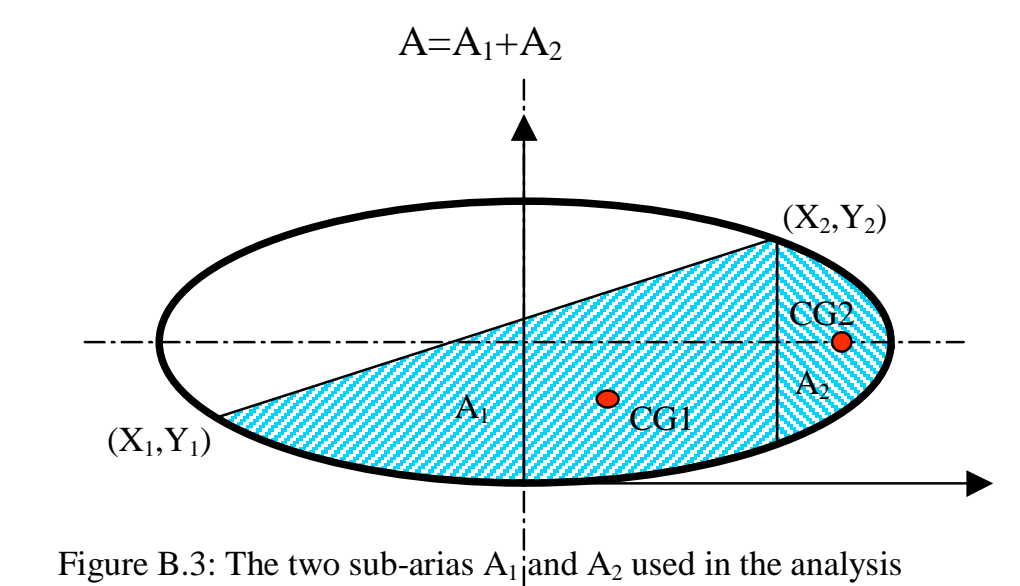

Figure B.3: The two sub-arias $\mathrm{A}_{1}$ and $\mathrm{A}_{2}$ used in the analysis

Where

$$
\begin{aligned}
& \mathrm{A}_{1}=\mathrm{A}_{\mathrm{h}}-\mathrm{A}_{2} \\
& A_{2}=2 b \cdot\left[\frac{a \cdot \pi}{4}-\left(\frac{x 2 \cdot \sqrt{a^{2}-x 2^{2}}+a^{2} \cdot \sin ^{-1}\left(\frac{x 2}{a}\right)}{2 a}\right)\right] \\
& \mathrm{x}_{\mathrm{CG}}=\frac{\mathrm{A}_{1} \cdot \mathrm{x}_{\mathrm{CG} 1}+\mathrm{A}_{2} \mathrm{x}_{\mathrm{CG} 2}}{\mathrm{~A}_{\mathrm{h}}}
\end{aligned}
$$


Where

$$
\begin{gathered}
\mathrm{x}_{\mathrm{CG} 1}=\frac{\mathrm{b}}{6 \cdot \mathrm{a} \cdot \mathrm{A}_{1}}\left[3 \cdot a \cdot(\mathrm{k}-\mathrm{b}) \cdot\left(\mathrm{x} 2^{2}-\mathrm{x} 1^{2}\right)+2 \cdot \mathrm{m} \cdot \mathrm{a} \cdot\left(\mathrm{x} 2^{3}-\mathrm{x} 1^{3}\right)+2 \cdot b \cdot\left(\mathrm{a}^{2}-\mathrm{x} 1^{2}\right)^{3 / 2}-2 \cdot b \cdot\left(\mathrm{a}^{2}-\mathrm{x} 2^{2}\right)^{3 / 2}\right] \\
\mathrm{x}_{\mathrm{CG} 2}=\frac{2 \cdot b \cdot\left(\mathrm{a}^{2}-\mathrm{x} 2^{2}\right)^{3 / 2}}{3 \cdot a \cdot \mathrm{A}_{2}} \\
\mathrm{y}_{\mathrm{CG}}=\frac{\mathrm{A}_{1} \cdot \mathrm{y}_{\mathrm{CG} 1}+\mathrm{A}_{2} \cdot \mathrm{b}}{\mathrm{A}_{\mathrm{h}}}
\end{gathered}
$$

Where

$$
\begin{aligned}
y_{C G 1}= & \frac{-1}{2 A_{1}}\left[\left(2 b^{2}-k^{2}\right)(x 2-x 1)-m \cdot k \cdot\left(x 2^{2}-x 1^{2}\right)-\frac{\left(b^{2}+a^{2} m^{2}\right) \cdot\left(x 2^{3}-x 1^{3}\right)}{3 a^{2}}-\right. \\
& \left.\frac{b^{2}}{a}\left(a^{2}\left(\sin ^{-1}\left(\frac{x 2}{a}\right)-\sin ^{-1}\left(\frac{x 1}{a}\right)\right)+x 2 \sqrt{a^{2}-x 2^{2}}-x 1 \sqrt{a^{2}-x 1^{2}}\right)\right]
\end{aligned}
$$

Case 3:- When y1 and y2 are less than "b"

In this Case, $A=A_{h}$

$$
\begin{gathered}
\mathrm{x}_{\mathrm{CG}}=\frac{\mathrm{b}}{6 \cdot \mathrm{a} \cdot \mathrm{A}_{\mathrm{h}}}\left[3 \cdot \mathrm{a} \cdot(\mathrm{k}-\mathrm{b}) \cdot\left(\mathrm{x} 2^{2}-\mathrm{x} 1^{2}\right)+2 \cdot \mathrm{m} \cdot \mathrm{a} \cdot\left(\mathrm{x} 2^{3}-\mathrm{x} 1^{3}\right)+2 \cdot b \cdot\left(\mathrm{a}^{2}-\mathrm{x} 1^{2}\right)^{3 / 2}-2 \cdot b \cdot\left(\mathrm{a}^{2}-\mathrm{x} 2^{2}\right)^{3 / 2}\right] \\
\mathrm{y}_{\mathrm{CG} 1}=\frac{-1}{2 \mathrm{~A}_{\mathrm{h}}}\left[\left(2 \mathrm{~b}^{2}-\mathrm{k}^{2}\right)(\mathrm{x} 2-\mathrm{x} 1)-\mathrm{m} \cdot \mathrm{k} \cdot\left(\mathrm{x} 2^{2}-\mathrm{x} 1^{2}\right)-\frac{\left(\mathrm{b}^{2}+\mathrm{a}^{2} \mathrm{~m}^{2}\right) \cdot\left(\mathrm{x} 2^{3}-\mathrm{x} 1^{3}\right)}{3 \mathrm{a}^{2}}-\right. \\
\left.\frac{\mathrm{b}^{2}}{\mathrm{a}}\left(\mathrm{a}^{2}\left(\sin ^{-1}\left(\frac{\mathrm{x} 2}{\mathrm{a}}\right)-\sin ^{-1}\left(\frac{\mathrm{x} 1}{\mathrm{a}}\right)\right)+\mathrm{x} 2 \sqrt{\mathrm{a}^{2}-\mathrm{x} 2^{2}}-\mathrm{x} 1 \sqrt{\mathrm{a}^{2}-\mathrm{x} 1^{2}}\right)\right]
\end{gathered}
$$

A computer code was written using MATLAB 5.2 to calculate the mass and the CG of any given fluid for any elliptical tank at different angle of tilt. Figure B4 shows a sample result from this program for different level of fill and at different angels. 
The computer code has been also used to prove that the locus of the CG's of the fluid falls on an exact ellipse. The values of "a" and "b" for those elliptical paths have been extracted from the results at $\theta=0$ and $\theta=90$.

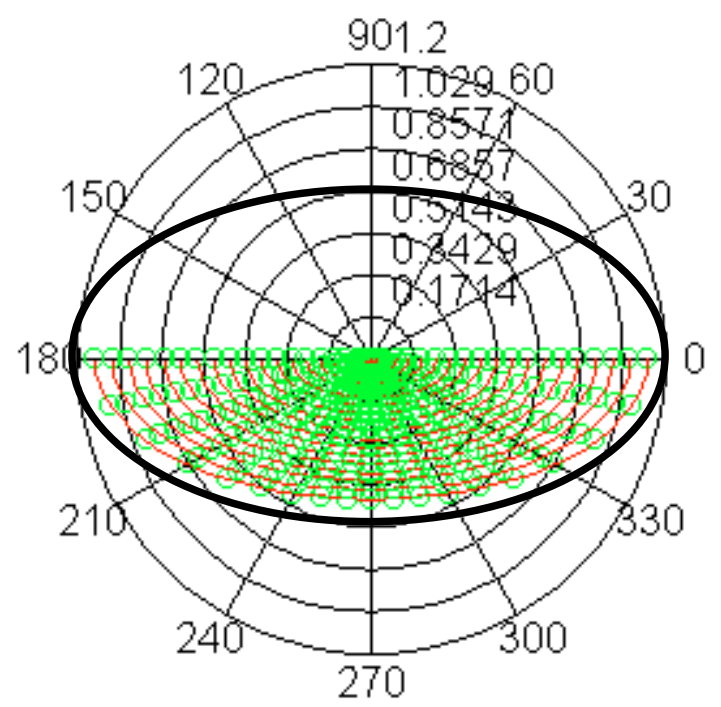

Figure B.4- Location of fluid CG at different levels of fill and different angles of tilt

It has been found that the results of this analysis matches perfectly with the results of obtained using equations (B15) and (B16) given by Rakheja (1993):

$$
\begin{gathered}
Z_{l}=0.5 * H_{2}(1-\cos \phi)+Z_{o} \cos \phi \\
Y_{l}=\left[0.5-\frac{Z_{o}}{H_{2}}\right] * H_{1} \sin \phi
\end{gathered}
$$

It has been found also that the locus of the CG of a fluid in a partially filled elliptical tank assuming flat fluid surface falls on a parallel elliptical path to the container surface such that;

$\frac{a}{b}=\frac{\bar{a}}{\bar{b}}$. 


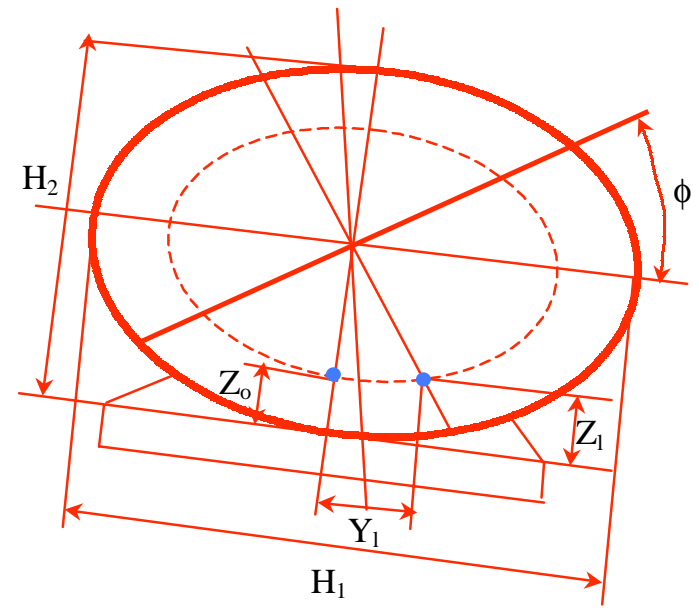

Figure B.5: Illustration of Symbols Used In Equations (B15) and (B16), Rakheja (1993)

Also, it has been found that the angle $\theta_{\mathrm{cg}}$ is constant for different levels of fill for the same angle of fluid surface tilt and the same value of $a / b$ and that the ratio of $R$ to $b$ (see Figure B.1) is always constant regardless of the ratio $\mathrm{a} / \mathrm{b}$ as illustrated in Figure B.6.

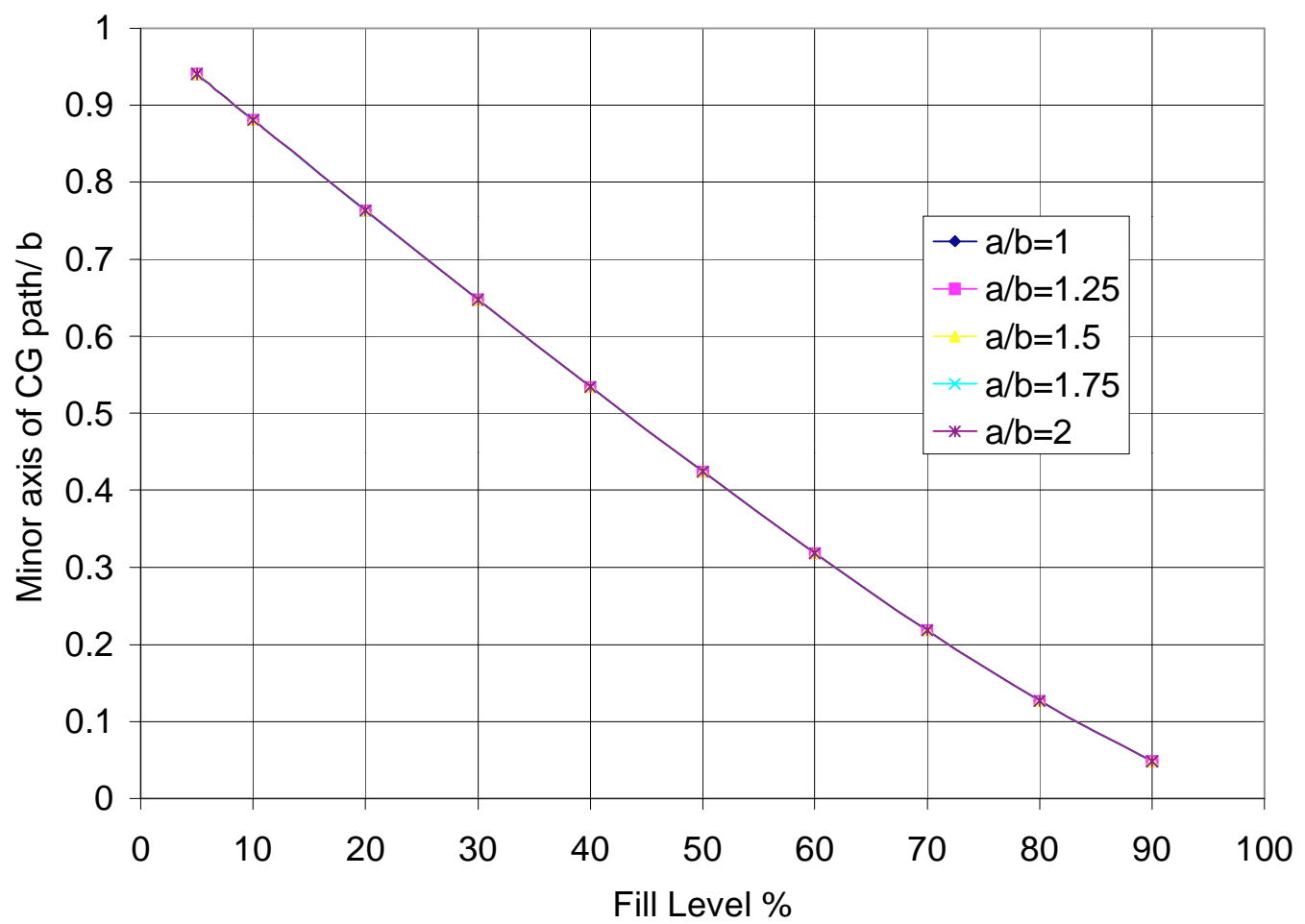

Figure B.6. Ratio $R / b$ as a function of fill level and ellipse ratio $a / b$ 


\section{$\underline{\text { B.3- Elliptical Trammel Pendulum Equation of Motion }}$}

This part contains the derivation of the equation of motion of an elliptical (Trammel)

pendulum under the influence of a lateral acceleration $\ddot{X}$

Figure B7 shows the Trammel pendulum relative to the original axis $\left(\mathrm{X}^{\prime} \& \mathrm{Y}^{\prime}\right)$ and the final axis $(\mathrm{X} \& \mathrm{Y})$.

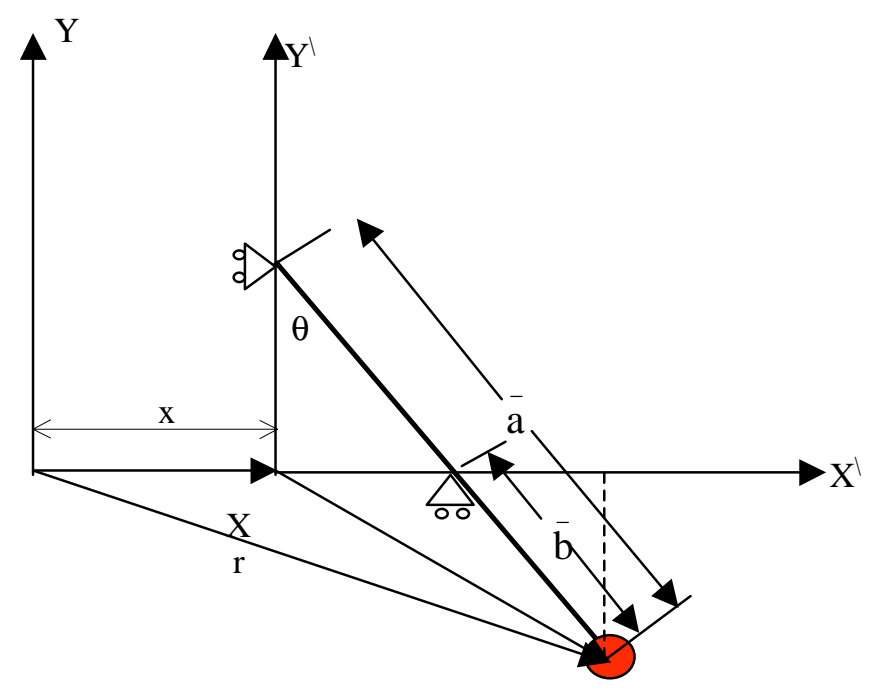

Figure B.7: The trammel pendulum is the initial and final set of coordinates after a negative displacement $\mathrm{x}$.

The position vector $\bar{r}$ is given by:-

$\overline{\mathrm{r}}=(\overline{\mathrm{a}} \sin \theta+\mathrm{x}) \mathrm{i}-\overline{\mathrm{b}} \cos \theta \mathrm{j}$

$\therefore \overline{\mathrm{r}}=\left(\overline{\mathrm{a}} \theta^{\bullet} \cos \theta+\mathrm{x}^{\bullet}\right) \mathrm{i}+\theta^{\bullet} \overline{\mathrm{b}} \sin \theta \mathrm{j}$

and

$$
\ddot{r}=\left[\bar{a}\left(-\theta^{\bullet 2} \sin \theta+\ddot{\theta} \cos \theta\right)+\ddot{x}\right] i+\bar{b}\left(\theta^{\bullet 2} \cos \theta+\ddot{\theta} \sin \theta\right) j
$$

The kinetic energy is given by:

$\mathrm{T}=\frac{1}{2} \mathrm{~m} \overline{\mathrm{r}}^{\bullet 2}=\frac{1}{2} \mathrm{~m}\left(\overline{\mathrm{a}}^{2} \theta^{\bullet 2} \cos ^{2} \theta-2 \overline{\mathrm{a}} \mathrm{x}^{\bullet} \theta^{\bullet} \cos \theta+\mathrm{x}^{\bullet 2}+\theta^{\bullet 2} \overline{\mathrm{b}}^{2} \sin ^{2} \theta\right)$ 
The potential energy $Q$ is given by:

$\mathrm{Q}=\mathrm{m} * \mathrm{~g} * \mathrm{~b} *(1-\cos \theta)$.

The Lagrangian is given by:

$\mathrm{L}=\mathrm{T}-\mathrm{Q}=\frac{1}{2} \mathrm{~m}\left(\overline{\mathrm{a}}^{2} \dot{\theta}^{2} \cos ^{2} \theta-2 \overrightarrow{\mathrm{a}} \ddot{\mathrm{x}} \dot{\theta} \cos \theta+\mathrm{x}^{2}+\dot{\theta}^{2} \overline{\mathrm{b}}^{2} \sin ^{2} \theta\right)-\mathrm{mg} \overline{\mathrm{b}}(1-\cos \theta)$

Since the $\mathrm{x}$ degree of freedom for the pendulum is an outside input, the only degree of freedom is

$\theta$, and the equation of motion for the pendulum will be given by:

$$
\frac{\partial}{\partial \mathrm{t}}\left(\frac{\partial \mathrm{L}}{\partial \dot{\theta}}\right)-\frac{\partial \mathrm{L}}{\partial \theta}=0
$$

Where:

$$
\begin{aligned}
\frac{\partial \mathrm{L}}{\partial \theta}= & \frac{1}{2} \mathrm{~m}\left(-2 \overline{\mathrm{a}}^{2} \theta^{\bullet 2} \cos \theta \sin \theta+2 \overline{\mathrm{b}}^{2} \dot{\theta}^{2} \cos \theta \sin \theta+2 \overline{\mathrm{a}} \mathrm{x} \cdot \theta^{\bullet} \sin \theta\right)-\mathrm{mg} \overline{\mathrm{b}} \sin \theta \\
\frac{\partial \mathrm{L}}{\partial \theta}= & \mathrm{m}\left(\overline{\mathrm{a}}^{2} \theta^{\cdot} \cos ^{2} \theta+\overline{\mathrm{b}}^{2} \theta^{\bullet} \sin ^{2} \theta+\overline{\mathrm{a}} \mathrm{x}^{\bullet} \cos \theta\right) \\
\frac{\partial}{\partial \mathrm{t}}\left(\frac{\partial \mathrm{L}}{\partial \theta}\right)= & \mathrm{m}\left(\overline{\mathrm{a}}^{2} \ddot{\theta} \cos ^{2} \theta-2 \overline{\mathrm{a}}^{2} \theta^{\cdot 2} \sin \theta^{*} \cos \theta+\overline{\mathrm{b}}^{2} \ddot{\theta} \sin ^{2} \theta+2 \overline{\mathrm{b}}^{2} \theta^{\bullet} \sin \theta \cos \theta+\overline{\mathrm{a}} \mathrm{x} \cos \theta+\right. \\
& \left.-\overline{\mathrm{a} x} \cdot \theta^{\bullet} \sin \theta\right)
\end{aligned}
$$

Substituting in B18 yields:

$$
\left(\bar{a}^{2} \cos ^{2} \theta+\bar{b}^{2} \sin ^{2} \theta\right) \ddot{\theta}+0.5\left(\bar{b}^{2}-\bar{a}^{2}\right) \theta^{*^{2}} \sin 2 \theta+\mathrm{g} \bar{b} \sin \theta+\bar{a} \cos \theta \ddot{x}=0
$$

Equation B19 has been reduced to a two first order differential equations and was solved using a MATLAB function called "ODE45" using variable forth-fifth order Rung-Kutta method. 
The function file as well as the .m file used to solve this equation for various inputs of $\ddot{X}$ is given in Appendix C. The solution gives the values of $\theta, \theta^{\bullet}$, and $\ddot{\theta}$.

The trammel pendulum was tested using different values of initial angles and zero initial velocity to study its performance (Velocity, natural frequencies, non linearities effects, etc.). Figures B8 through B13 show the angular displacements and the angular velocities for different values of initial pendulum angle and zero initial velocity $(a=0.5093$, and $b=0.2587)$.
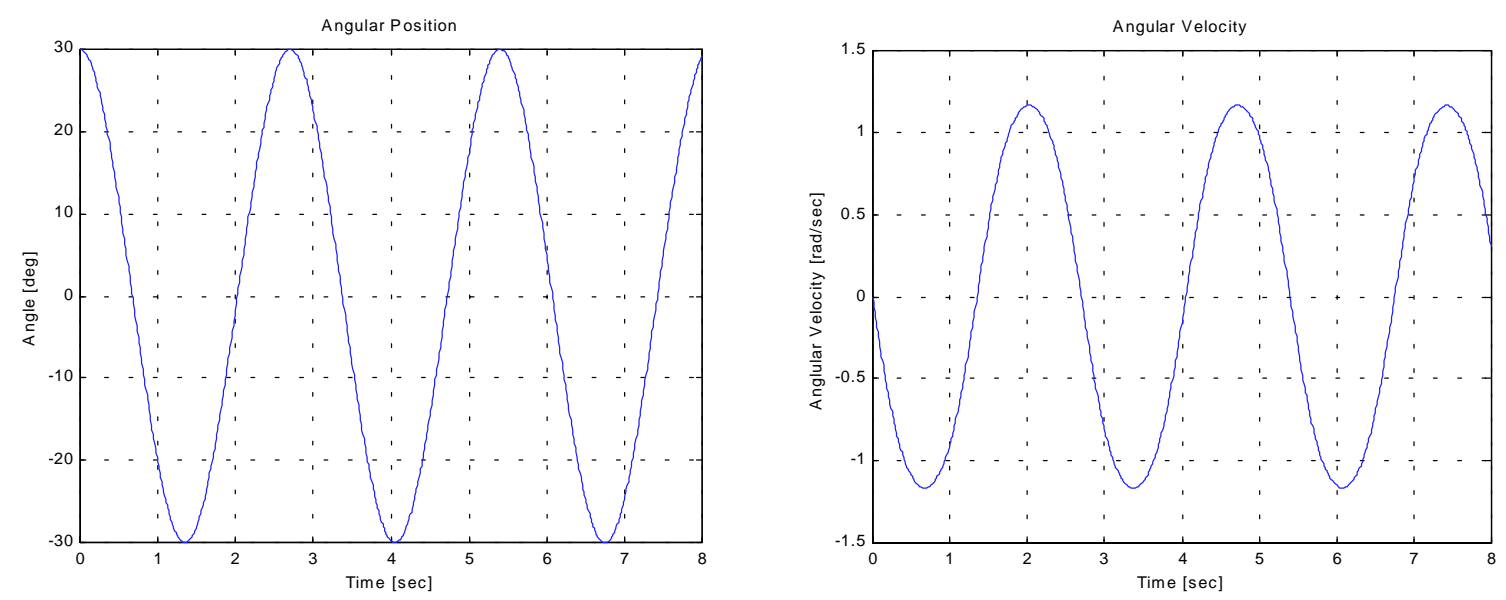

Figure B.8: Trammel pendulum angular position and angular velocity at 30 degrees initial angle
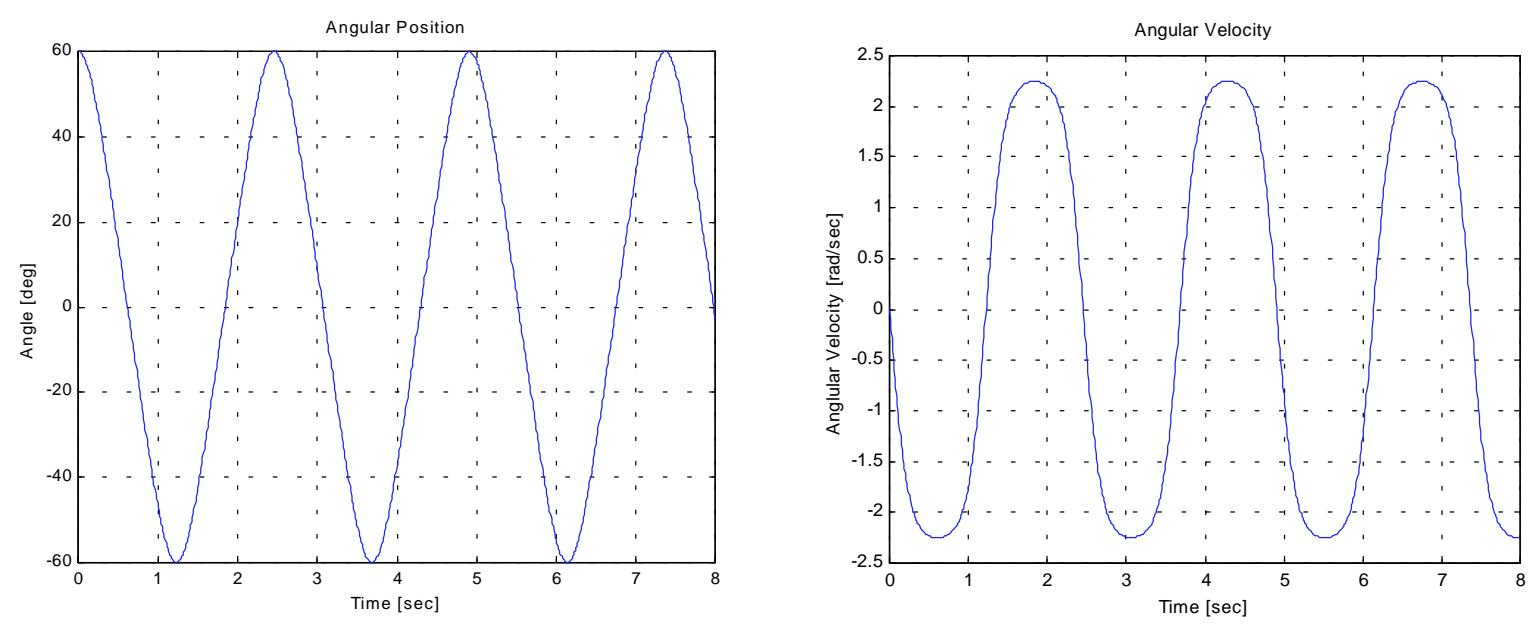

Figure B.9: Trammel pendulum angular position and angular velocity at 60 degrees initial angle 

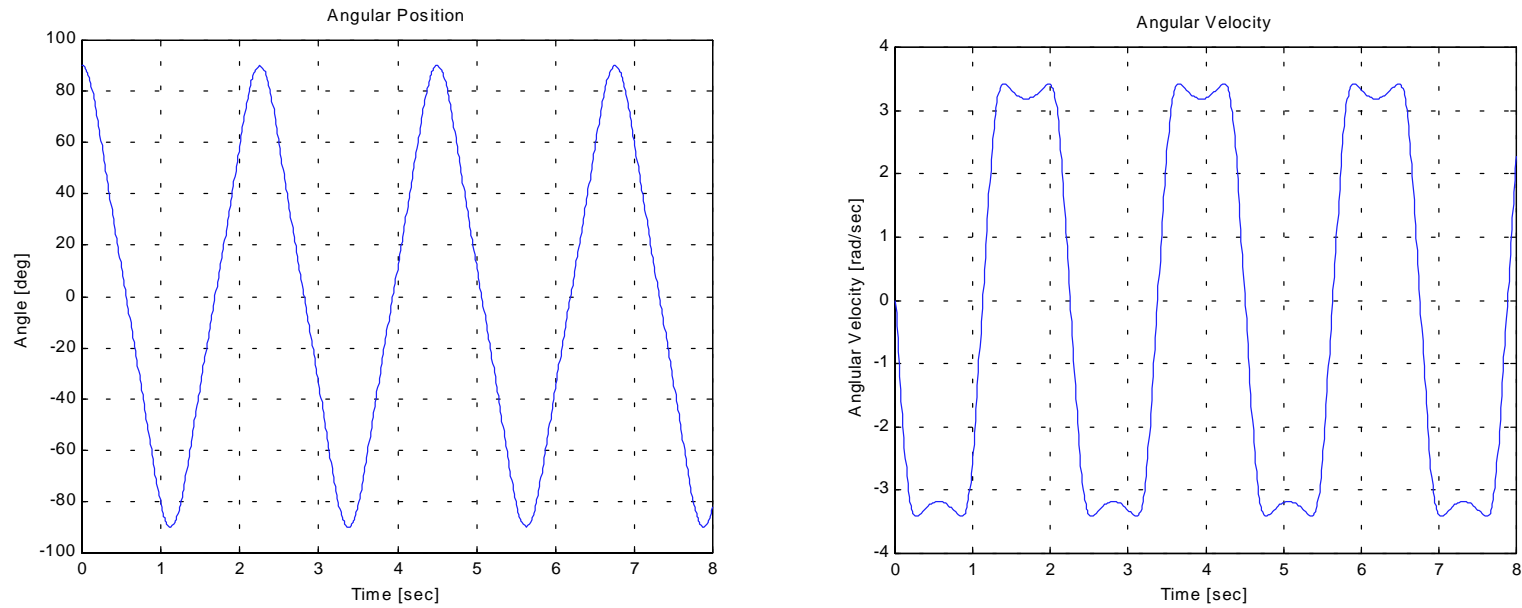

Figure B.10: Trammel pendulum angular position and angular velocity at 90 degrees initial angle
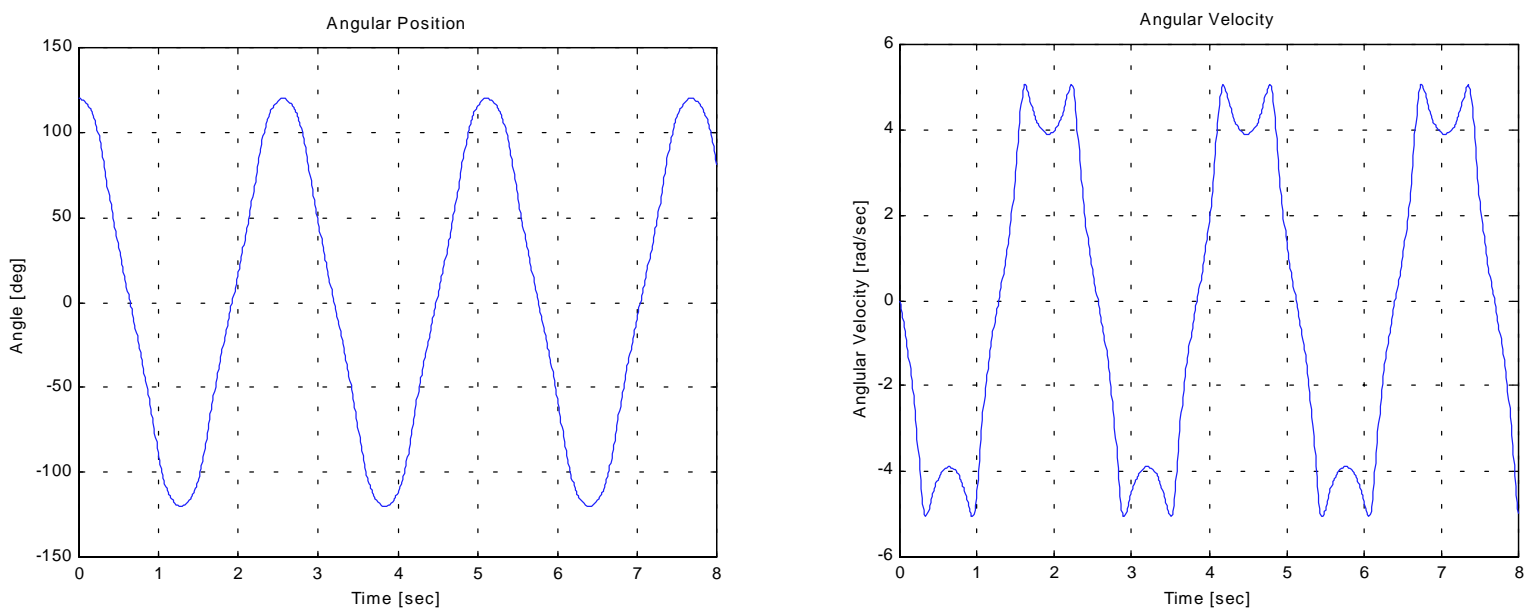

Figure B.11: Trammel pendulum angular position and angular velocity at 120 degrees initial angle
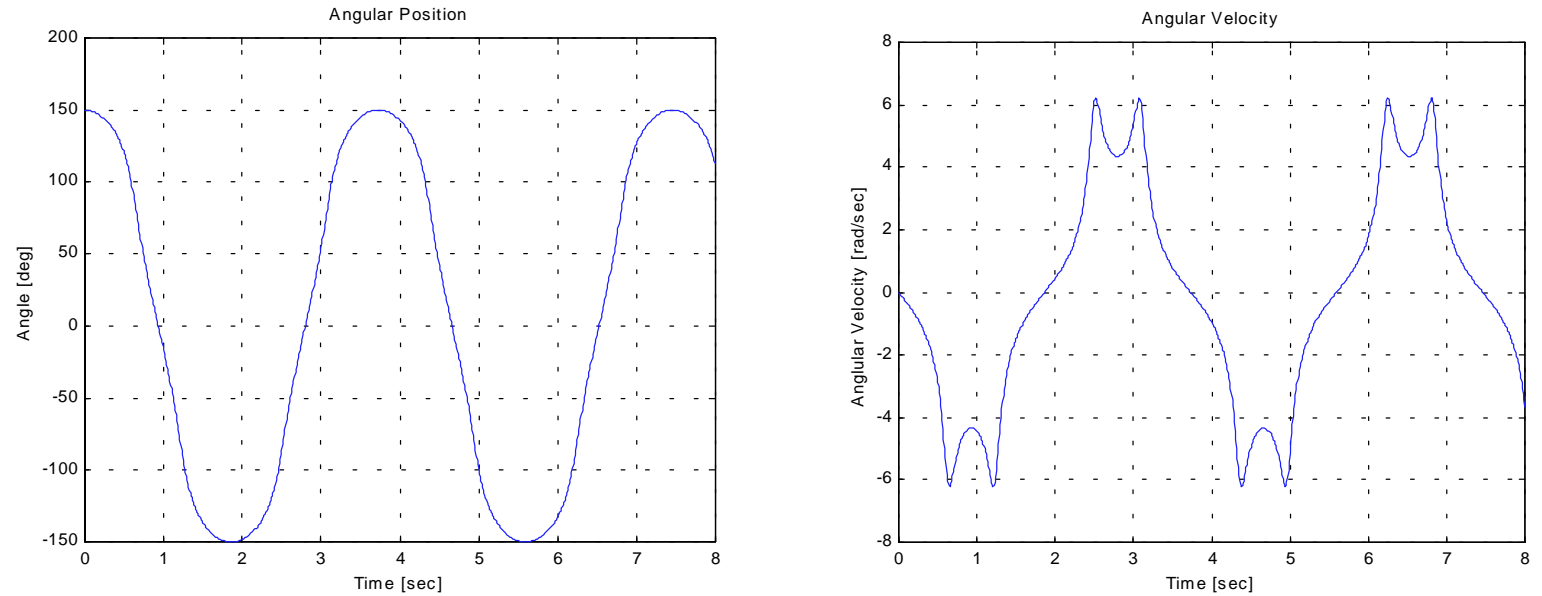

Figure B.12: Trammel pendulum angular position and angular velocity at 150 degrees initial angle 

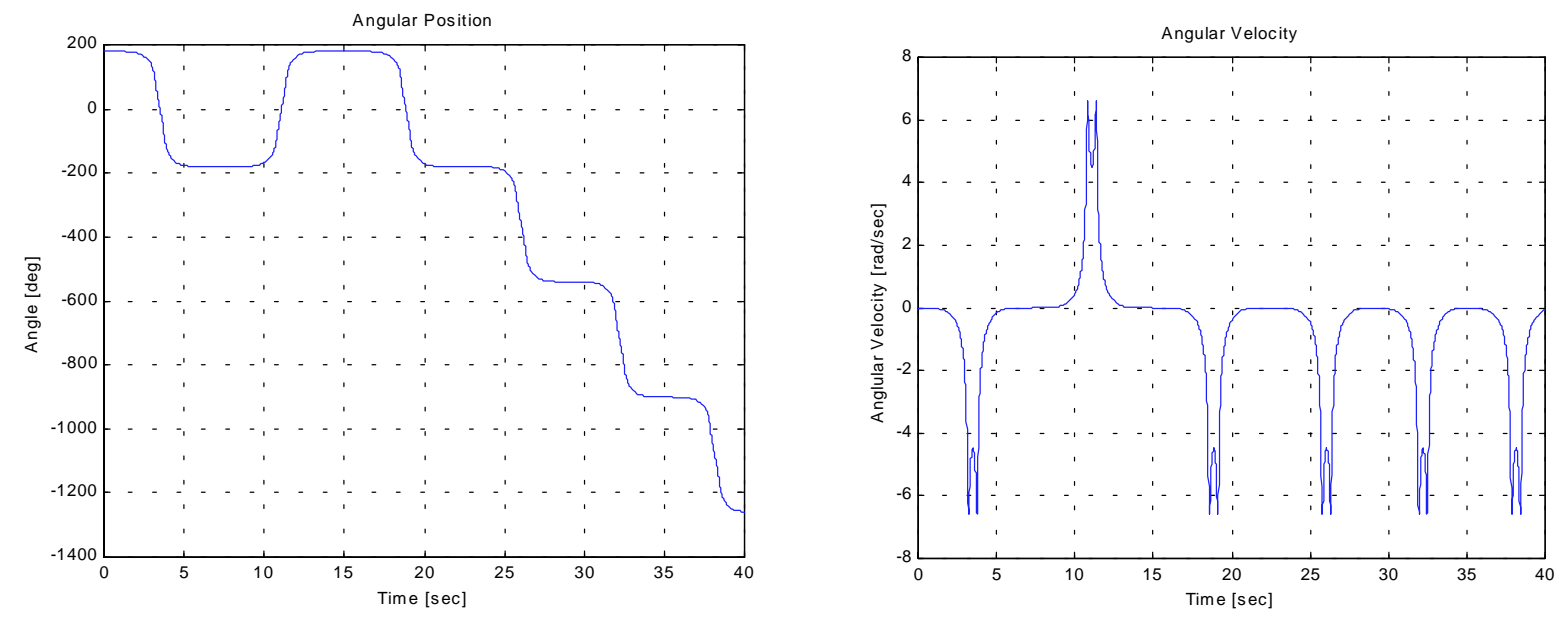

Figure B.13: Trammel pendulum angular position and angular velocity at 179.9 degrees initial angle

It can be seen that that the angular displacement and velocity of the pendulum follows a cosine and a sine shape respectively at low pendulum angles (30,60 degrees). At higher angles (90 and above), the angular position curves starts flatten up at the peek points and the angular velocity starts experiencing a dip at the peek and valley points. These dips increases in magnitude with the increase of the initial pendulum angle and the natural frequency starts decreasing. Finally, at very high pendulum angles (close to 180 degrees), the natural frequency decreases considerably and the pendulum starts wondering about the top most position, whether to complete a full turn or to fluctuate back to its original position. Figure 4.4 shows a plot for the natural frequency versus the initial angle (maximum angle of swing) for the pendulum discussed above.

\section{$\underline{\text { B.4- Derivation of Equations of Motion for Vehicle Model \#2 }}$}

A part of this derivation, which involves the effect of the trammel pendulum effect only, has been given by Karam (1999). In his model, Karam neglected the fixed fluid mass, the polar moment of inertia of the fluid, the vehicle mass, the vehicle polar moment of inertia, and didn't calculate the reactions when the model starts rolling over in either side. His analysis was focused on calculating the pendulum angle and the unilateral support carrying it. Karam divided 
the equations of motion to 3 sets; when the two supports are in contact with the ground, when the right support lifts off, and when the left support lifts off. The first case was included in model \#1 of this study. Thus, the derivation of the equations of motion for the second two cases is given here.

\section{B.4.1- Liftoff of the Right Support}

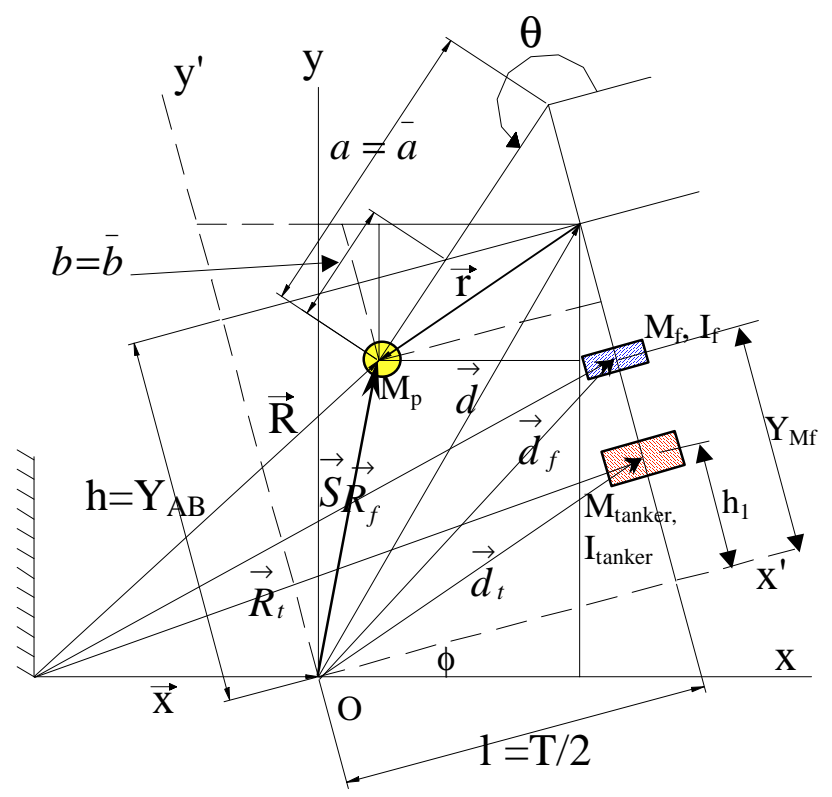

Figure B.13: Right support liftoff of model \#2, fluid inertia fixed to the frame (modified from Karam, 1999)

The displacement equation $\mathrm{R}$ for the above figure is:

$$
\begin{gathered}
\vec{R}=\vec{x}+\vec{d}+\vec{r} \\
\overrightarrow{R_{t}}=\vec{x}+\overrightarrow{d_{t}} \\
\overrightarrow{R_{f}}=\vec{x}+\vec{d}_{f} \\
\vec{x}=x \hat{i}+0 \hat{j} \\
\vec{d}=(l \cos \phi-h \sin \phi) \hat{i}+(h \cos \phi+l \sin \phi) \hat{j}
\end{gathered}
$$




$$
\vec{r}=(a \cos \theta-b \sin \theta \tan \phi) \cos \phi \hat{i}+(b \sin \theta+a \cos \theta \tan \phi) \cos \phi \hat{j}
$$

Thus, $\vec{R}=[x+(l+a \cos \theta) \cos \phi-(h+b \sin \theta) \sin \phi] \hat{i}+[(h+b \sin \theta) \cos \phi+(l+a \cos \theta) \sin \phi] \hat{j}(\mathrm{~B} 20 \mathrm{a})$

$$
\begin{gathered}
\overrightarrow{R_{t}}=\left[x+l \cos \phi-h_{1} \sin \phi\right] \hat{i}+\left[h_{1} \cos \phi+l \sin \phi\right] \hat{j} \\
\overrightarrow{R_{f}}=\left[x+l \cos \phi-Y_{M f} \sin \phi\right] \hat{i}+\left[Y_{M f} \cos \phi+l \sin \phi\right] \hat{j}
\end{gathered}
$$

And $\quad \vec{S}=[(l+a \cos \theta) \cos \phi-(h+b \sin \theta) \sin \phi] \hat{i}+[(h+b \sin \theta) \cos \phi+(l+a \cos \theta) \sin \phi] \hat{j}$

$$
\begin{array}{r}
\dot{\vec{R}}=[\dot{x}-\{(a \sin \theta \cos \phi+b \cos \theta \sin \phi) \dot{\theta}+[(l+a \cos \theta) \sin \phi+(h+b \sin \theta) \cos \phi] \dot{\phi}\}] \hat{i} \\
+[(b \cos \theta \cos \phi-a \sin \theta \sin \phi) \dot{\theta}+[(l+a \cos \theta) \cos \phi-(h+b \sin \theta) \sin \phi] \dot{\phi}] \hat{j} \\
\vec{R}_{t}=\left(\dot{x}-l \sin \phi \dot{\phi}-h_{1} \cos \phi \dot{\phi}\right) \hat{i}+\left(l \cos \phi-h_{1} \sin \phi\right) \dot{\phi} \hat{j} \\
\overrightarrow{\vec{R}}_{f}=\left(\dot{x}-l \sin \phi \dot{\phi}-Y_{M f} \cos \phi \dot{\phi}\right) \hat{i}+\left(l \cos \phi-Y_{M f} \sin \phi\right) \dot{\phi} \hat{j}
\end{array}
$$

$$
\begin{gathered}
\dot{R}^{2}=\dot{x}^{2}-2 \dot{x} \dot{\theta}(a \sin \theta \cos \phi+b \cos \theta \sin \phi)-2 \dot{x} \dot{\phi}[(l+a \cos \theta) \sin \phi+(h+b \sin \theta) \cos \phi] \\
+\dot{\theta}^{2}\left(a^{2} \sin ^{2} \theta+b^{2} \cos ^{2} \theta\right)+2 \dot{\theta} \dot{\phi}(a h \sin \theta+b l \cos \theta+a b) \\
+\dot{\phi}^{2}\left[(l+a \cos \theta)^{2}+(h+b \sin \theta)^{2}\right] \\
\dot{R}_{t}^{2}=\dot{x}^{2}-2 \dot{x} \dot{\phi}\left(l \sin \phi+h_{1} \cos \phi\right)+\left(h_{1}^{2}+l^{2}\right) \dot{\phi}^{2} \\
\dot{R}_{f}^{2}=\dot{x}^{2}-2 \dot{x} \dot{\phi}\left(l \sin \phi+Y_{M f} \cos \phi\right)+\left(Y_{M f}^{2}+l^{2}\right) \dot{\phi}^{2} \\
\ddot{\vec{R}}=\xi \hat{i}+\psi \hat{j}
\end{gathered}
$$

Where: 


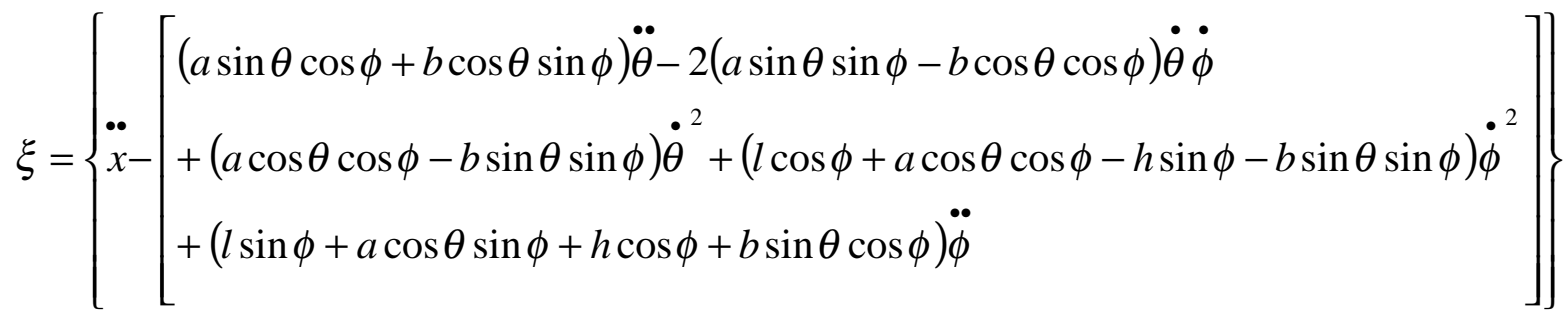

$$
\begin{aligned}
& \psi=\left[\begin{array}{l}
(b \cos \theta \cos \phi-a \sin \theta \sin \varphi) \ddot{\theta}-2(a \sin \theta \cos \phi+b \cos \theta \sin \phi) \dot{\theta} \dot{\phi} \\
-(a \cos \theta \sin \phi+b \sin \theta \cos \phi) \dot{\theta}^{2}-(l \sin \phi+a \cos \theta \sin \phi+h \cos \phi+b \sin \theta \cos \phi) \dot{\phi}^{2} \\
+(l \cos \phi+a \cos \theta \cos \phi-h \sin \phi-b \sin \theta \sin \phi) \ddot{\phi}
\end{array}\right] \\
& \vec{R}_{t}=\left(\ddot{x}-l \sin \phi \ddot{\phi}-l \cos \phi \dot{\phi}^{2}-h_{1} \cos \phi \ddot{\phi}+h_{1} \sin \phi \dot{\phi}^{2}\right) \hat{i}+ \\
& \left(l \cos \phi \ddot{\phi}-l \sin \phi \dot{\phi}^{2}-h_{1} \sin \phi \ddot{\phi}+h_{1} \cos \phi \dot{\phi}^{2}\right) \hat{j} \\
& \ddot{\vec{R}}_{f}=\left(\ddot{x}-l \sin \phi \ddot{\phi}-l \cos \phi \dot{\phi}^{2}-Y_{M f} \cos \phi \ddot{\phi}+Y_{M f} \sin \phi \dot{\phi}^{2}\right) \hat{i}+ \\
& \left(l \cos \phi \ddot{\phi}-l \sin \phi \dot{\phi}^{2}-Y_{M f} \sin \phi \ddot{\phi}+Y_{M f} \cos \phi \dot{\phi}^{2}\right) \hat{j}
\end{aligned}
$$

Lagrange's equation states that:

$$
\frac{\partial}{\partial t}\left(\frac{\partial L}{\partial \dot{q}_{n}}\right)-\frac{\partial L}{\partial q_{n}}=0 \quad n=1,2, \ldots \ldots
$$

Where the Lagrangian is given by:

$$
\mathrm{L}=\mathrm{T}-\mathrm{V}
$$

The kinetic energy equation is such that:

$$
T=1 / 2\left(M_{p} \dot{R}^{2}+M_{\text {Tan } \mathrm{ker}} \dot{R}_{t}^{2}+M_{f} \dot{R}_{f}^{2}+I_{\text {Tan } \mathrm{ker}_{-} o} \dot{\phi}^{2}+I_{f_{-} o} \dot{\phi}^{2}\right)
$$




$$
\Rightarrow T=1 / 2\left[\begin{array}{l}
M_{p} \dot{x}^{2}-2 M_{p} \dot{x} \dot{\theta}(a \sin \theta \cos \phi+b \cos \theta \sin \phi) \\
-2 M_{p} \dot{x} \dot{\phi}[(l+a \cos \theta) \sin \phi+(h+b \sin \theta) \cos \phi] \\
+M_{p} \dot{\theta}^{2}\left(a^{2} \sin ^{2} \theta+b^{2} \cos ^{2} \theta\right)+2 M_{p} \dot{\theta} \dot{\phi}(a h \sin \theta+b l \cos \theta+a b) \\
+M_{p} \dot{\phi}^{2}\left[(l+a \cos \theta)^{2}+(h+b \sin \theta)^{2}\right] \\
+M_{\tan k e r}\left\{\dot{x}^{2}-2 \dot{x} \dot{\phi}\left(l \sin \phi+h_{1} \cos \phi\right)+\left(h_{1}^{2}+l^{2}\right) \dot{\phi}^{2}\right\}+I_{T_{\text {Tan } \text { ker }_{-} o} \dot{\phi}^{2}} \\
+M_{f}\left\{\dot{x}^{2}-2 \dot{x} \dot{\phi}\left(l \sin \phi+Y_{M f} \cos \phi\right)+\left(Y_{M f}^{2}+l^{2}\right) \dot{\phi}^{2}\right\}+I_{f_{-} o} \dot{\phi}^{2}
\end{array}\right]
$$

Moreover, the potential energy is given by:

$$
\begin{aligned}
& V=M_{p} g[(h+b \sin \theta) \cos \phi+(l+a \cos \theta) \sin \phi]+M_{\text {Tan } \mathrm{ker}} g\left(l \sin \phi+h_{1} \cos \phi\right) \\
& +M_{f} g\left(l \sin \phi+Y_{M f} \cos \phi\right)
\end{aligned}
$$

Differentiating the Equation B.23 with respect to $\dot{\theta}$ and then with respect to time yields:

$\frac{\partial L}{\partial \dot{\theta}}=M_{P}\left[\dot{\theta}\left(a^{2} \sin ^{2} \theta+b^{2} \cos ^{2} \theta\right)+\dot{\phi}(a h \sin \theta+b l \cos \theta+a b)-\dot{x}(a \sin \theta \cos \phi+b \cos \theta \sin \phi)\right]$

$\frac{\partial}{\partial t}\left(\frac{\partial L}{\partial \dot{\theta}}\right)=M_{P}\left[\begin{array}{l}\ddot{\theta}\left(a^{2} \sin ^{2} \theta+b^{2} \cos ^{2} \theta\right)+\dot{\theta}^{2}\left(a^{2}-b^{2}\right) \sin 2 \theta+\ddot{\phi}(a h \sin \theta+b l \cos \theta+a b) \\ +\dot{\theta} \dot{\phi}(a h \cos \theta-b l \sin \theta)-\ddot{x}(a \sin \theta \cos \phi+b \cos \theta \sin \phi) \\ -\dot{x} \dot{\theta}(a \cos \theta \cos \phi-b \sin \theta \sin \phi)-\dot{x} \dot{\phi}(b \cos \theta \cos \phi-a \sin \theta \sin \phi)\end{array}\right]$

Now differentiating the equation B.23 with respect to $\theta$ yields:

$$
\begin{aligned}
\frac{\partial L}{\partial \theta}= & 1 / 2 M_{P}\left[\begin{array}{l}
-2 \dot{x} \dot{\theta}(a \cos \theta \cos \phi-b \sin \theta \sin \phi)-2 \dot{x} \dot{\phi}(-a \sin \theta \sin \phi+b \cos \theta \cos \phi)+\dot{\theta}^{2}\left(a^{2}-b^{2}\right) \sin 2 \theta \\
+2 \dot{\theta} \dot{\phi}(a h \cos \theta-b l \sin \theta)+2 \dot{\phi}^{2}\left[-a l \sin \theta+b h \cos \theta-\frac{1}{2}\left(a^{2}-b^{2}\right) \sin 2 \theta\right]
\end{array}\right] \\
& -M_{P} g[b \cos \theta \cos \phi-a \sin \theta \sin \phi]
\end{aligned}
$$

Substituting in Lagrange's equation yields: 


$$
\begin{aligned}
& \ddot{\theta}\left(a^{2} \sin ^{2} \theta+b^{2} \cos ^{2} \theta\right)+\ddot{\phi}(a h \sin \theta+b l \cos \theta+a b)+1 / 2 \dot{\theta}^{2}\left(a^{2}-b^{2}\right) \sin 2 \theta \\
& +\dot{\phi}^{2}\left[a l \sin \theta-b h \cos \theta+\frac{1}{2}\left(a^{2}-b^{2}\right) \sin 2 \theta\right]+g(b \cos \theta \cos \phi-a \sin \theta \sin \phi) \\
& -\ddot{x}(a \sin \theta \cos \phi+b \cos \theta \sin \phi)=0
\end{aligned}
$$

Now, differentiating Equation B.23 with respect to $\dot{\phi}$ and then with respect to time yields:

$$
\begin{aligned}
& \frac{\partial L}{\partial \dot{\phi}}=\left[\begin{array}{l}
M_{p}\{-\dot{x}[(l+a \cos \theta) \sin \phi+(h+b \sin \theta) \cos \phi]+\dot{\theta}(a h \sin \theta+b l \cos \theta+a b) \\
\left.+\dot{\phi}\left[(l+a \cos \theta)^{2}+(h+b \sin \theta)^{2}\right]\right\}+M_{\text {Tan } \text { ker }}\left\{-\dot{x}\left(l \sin \phi+h_{1} \cos \phi\right)+\dot{\phi}\left(h_{1}^{2}+l^{2}\right)\right\} \\
+M_{f}\left\{-\dot{x}\left(l \sin \phi+Y_{M f} \cos \phi\right)+\dot{\phi}\left(Y_{M f}^{2}+l^{2}\right)\right\}+\dot{\phi}\left(I_{\text {Tan } \text { ker } o_{-}}+I_{f_{-} o}\right)
\end{array}\right]
\end{aligned}
$$

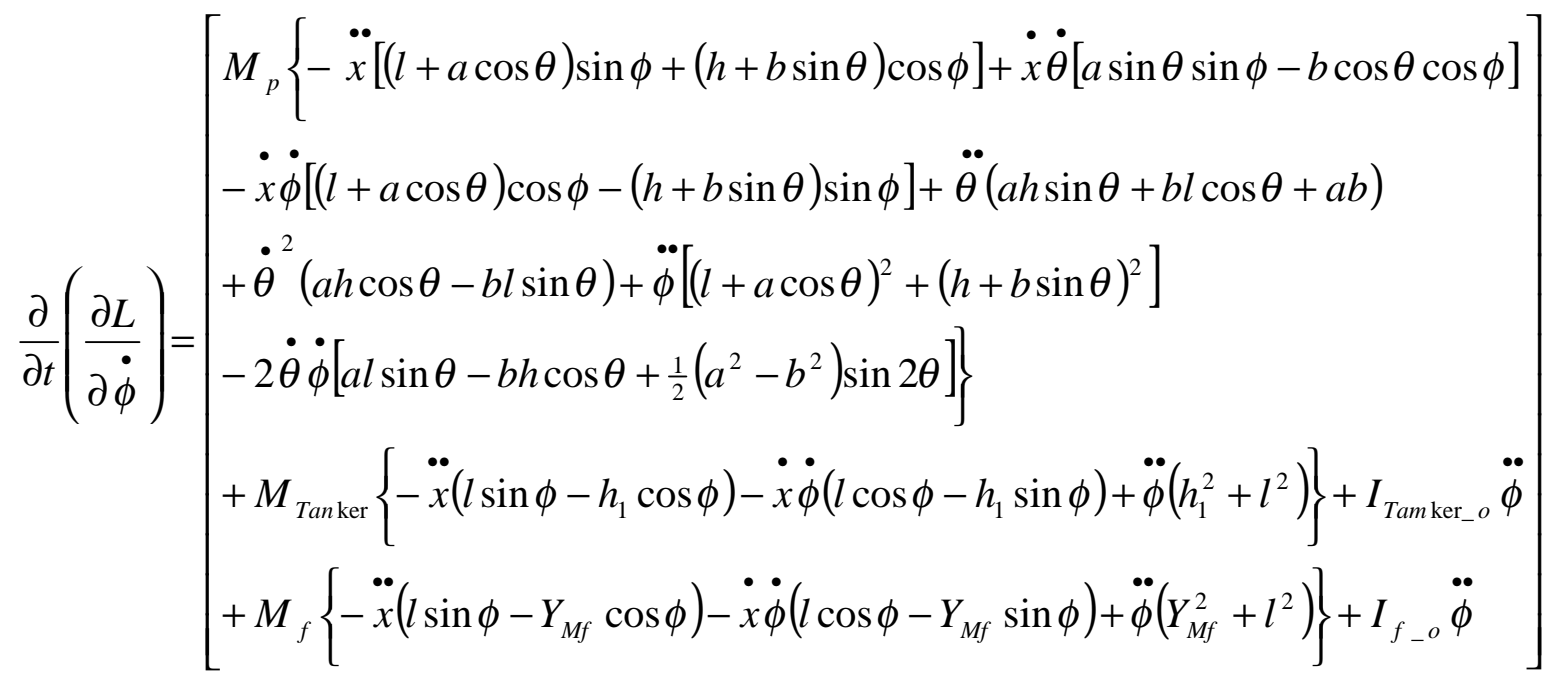

Then, differentiating the Equation B.23 with respect to $\phi$ yields:

$$
\begin{aligned}
\frac{\partial L}{\partial \phi}= & \frac{1}{2} M_{p}[2 \dot{x} \dot{\theta}[a \sin \theta \sin \phi-b \cos \theta \cos \phi]-2 \dot{x} \dot{\phi}[(l+a \cos \theta) \cos \phi-(h+b \sin \theta) \sin \phi]] \\
& -M_{p} g[-(h+b \sin \theta) \sin \phi+(l+a \cos \theta) \cos \phi]+M_{\tan \mathrm{ker}}\left[-\dot{x} \dot{\phi}\left(l \cos \phi-h_{1} \sin \phi\right)\right] \\
& -M_{\text {Tan } \mathrm{ker}} g\left(l \cos \phi-h_{1} \sin \phi\right)+M_{f}\left[-\dot{x} \dot{\phi}\left(l \cos \phi-Y_{M f} \sin \phi\right)\right]-M_{f} g\left(l \cos \phi-Y_{M f} \sin \phi\right)
\end{aligned}
$$


Substituting in Lagrange's Equation yields:

$$
\begin{aligned}
& \ddot{\theta} M_{p}(a h \sin \theta+b l \cos \theta+a b)+\ddot{\phi}\left\{M_{p}\left[(l+a \cos \theta)^{2}+(h+b \sin \theta)^{2}\right]+M_{\text {Tan } \mathrm{ker}}\left(h_{1}^{2}+l^{2}\right)\right. \\
& \left.+M_{f}\left(Y_{M f}^{2}+l^{2}\right)+I_{\text {Tan } \mathrm{ker}_{-} o}+I_{f_{-} o}\right\}-2 \dot{\theta} \dot{\phi} M_{p}\left[a l \sin \theta-b h \cos \theta+\frac{1}{2}\left(a^{2}-b^{2}\right) \sin 2 \theta\right] \\
& +\dot{\theta}^{2} M_{p}(a h \cos \theta-b l \sin \theta)+g\left\{M_{p}[(l+a \cos \theta) \cos \phi-(h+b \sin \theta) \sin \phi]\right. \\
& \left.+M_{\text {Tan } \mathrm{ker}}\left(l \cos \phi-h_{1} \sin \phi\right)+M_{f}\left(l \cos \phi-Y_{M f} \sin \phi\right)\right\}-\ddot{x}\left\{M_{p}[(l+a \cos \theta) \sin \phi+(h+b \sin \theta) \cos \phi]\right. \\
& \left.+M_{\text {Tan } \mathrm{ker}}\left(l \sin \phi+h_{1} \cos \phi\right)+M_{f}\left(l \sin \phi+Y_{M f} \cos \phi\right)\right\}=0
\end{aligned}
$$

Equations B24 and B25 represent the equations of motion for the system shown in Figure B.13. Those two equations are second order non-linear differential equations that can be discreetized to 4 first order differential equations as follows:

Let

$\dot{\theta}=Y$

$\dot{\phi}=Z$

Thus, $\ddot{\theta}=\dot{Y}$ and $\ddot{\phi}=\dot{Z}$

Substituting in B24 and B25 yields:

$$
\begin{aligned}
& \dot{Y}\left(a^{2} \sin ^{2} \theta+b^{2} \cos ^{2} \theta\right)+\dot{Z}(a h \sin \theta+b l \cos \theta+a b)+1 / 2 Y^{2}\left(a^{2}-b^{2}\right) \sin 2 \theta \\
& +Z^{2}\left[a l \sin \theta-b h \cos \theta+\frac{1}{2}\left(a^{2}-b^{2}\right) \sin 2 \theta\right]+g(b \cos \theta \cos \phi-a \sin \theta \sin \phi) \\
& \quad \ddot{x}(a \sin \theta \cos \phi+b \cos \theta \sin \phi)=0
\end{aligned}
$$

and 


$$
\begin{aligned}
& \dot{Y} M_{p}(a h \sin \theta+b l \cos \theta+a b)+\dot{Z}\left\{M_{p}\left[(l+a \cos \theta)^{2}+(h+b \sin \theta)^{2}\right]+M_{\text {Tan } \mathrm{ker}}\left(h_{1}^{2}+l^{2}\right)\right. \\
& \left.+M_{f}\left(Y_{M f}^{2}+l^{2}\right)+I_{\text {Tan } \mathrm{ker}_{-} o}+I_{f_{-} o}\right\}-2 Y Z M_{p}\left[a l \sin \theta-b h \cos \theta+\frac{1}{2}\left(a^{2}-b^{2}\right) \sin 2 \theta\right] \\
& +Y^{2} M_{p}(a h \cos \theta-b l \sin \theta)+g\left\{M_{p}[(l+a \cos \theta) \cos \phi-(h+b \sin \theta) \sin \phi]\right. \\
& \left.+M_{\text {Tan ker }}\left(l \cos \phi-h_{1} \sin \phi\right)+M_{f}\left(l \cos \phi-Y_{M f} \sin \phi\right)\right\} \\
& -\ddot{x}\left\{M_{p}[(l+a \cos \theta) \sin \phi+(h+b \sin \theta) \cos \phi]\right. \\
& \left.+M_{\text {Tan } \mathrm{ker}}\left(l \sin \phi+h_{1} \cos \phi\right)+M_{f}\left(l \sin \phi+Y_{M f} \cos \phi\right)\right\}=0
\end{aligned}
$$

\section{B.4.2- Liftoff of the Left Support}

Similarly, the equations of motion of the trammel pendulum in model \#2 can be driven for the case when the left support lifts off. Figure 5.14 shows this case where the reference coordinate system is placed at the right support. Following the same approach as before yields:

The displacement equation $\mathrm{R}$ for the above figure is:

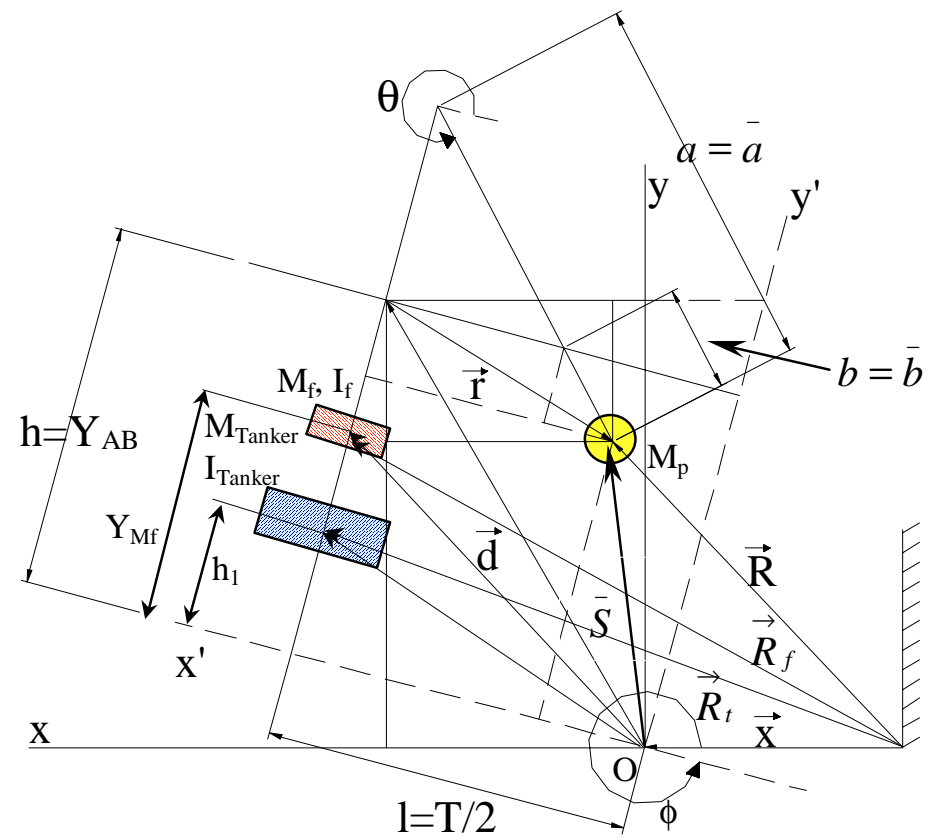

Figure B.14: Left support liftoff of model \#2, fluid inertia fixed to the frame (modified from Karam, 1999)

$$
\vec{R}=\vec{x}+\vec{d}+\vec{r}
$$




$$
\begin{aligned}
& \vec{x}=-x \hat{i} \\
& \vec{d}=(l \cos 180-h \tan \phi) \cos \phi \hat{i}+(h-l \tan \phi) \cos \phi \hat{j} \\
& \vec{R}=[-x-(l-a \cos \theta) \cos \phi-(h+b \sin \theta) \sin \phi] \hat{i}+[(h+b \sin \theta) \cos \phi-(l-a \cos \theta) \sin \phi] \hat{j} \\
& \vec{S}=[-(l-a \cos \theta) \cos \phi-(h+b \sin \theta) \sin \phi] \hat{i}+[(h+b \sin \theta) \cos \phi-(l-a \cos \theta) \sin \phi] \hat{j} \\
& \frac{\partial R}{\partial t}=\dot{\vec{R}}=[-\dot{x}-\{(a \sin \theta \cos \phi+b \cos \theta \sin \phi) \dot{\theta}-[(l-a \cos \theta) \sin \phi-(h+b \sin \theta) \cos \phi] \dot{\phi}\}] \hat{i} \\
& +[(b \cos \theta \cos \phi-a \sin \theta \sin \phi) \dot{\theta}-[(l-a \cos \theta) \cos \phi+(h+b \sin \theta) \sin \phi] \dot{\phi}] \hat{j} \\
& \vec{R}_{t}=-\left(\dot{x}-l \sin \phi \dot{\phi}+h_{1} \cos \phi \dot{\phi}\right) \hat{i}-\left(l \cos \phi+h_{1} \sin \phi\right) \dot{\phi} \hat{j} \\
& \vec{R}_{f}=-\left(\dot{x}-l \sin \phi \dot{\phi}+Y_{M f} \cos \phi \dot{\phi}\right) \hat{i}-\left(l \cos \phi+Y_{M f} \sin \phi\right) \dot{\phi} \hat{j}
\end{aligned}
$$

$$
\begin{gathered}
\dot{R}^{2}=\dot{x}^{2}+2 \dot{x} \dot{\theta}(a \sin \theta \cos \phi+b \cos \theta \sin \phi)-2 \dot{x} \dot{\phi}[(l-a \cos \theta) \sin \phi+(h+b \sin \theta) \cos \phi] \\
+\dot{\theta}^{2}\left(a^{2} \sin ^{2} \theta+b^{2} \cos ^{2} \theta\right)+2 \dot{\theta} \dot{\phi}(a h \sin \theta-b l \cos \theta+a b) \\
+\dot{\phi}^{2}\left[(l-a \cos \theta)^{2}+(h+b \sin \theta)^{2}\right] \\
\dot{R}_{t}^{2}=\dot{x}^{2}-2 \dot{x} \dot{\phi}\left(l \sin \phi-h_{1} \cos \phi\right)+\left(h_{1}^{2}+l^{2}\right) \dot{\phi}^{2} \\
\dot{R}_{f}^{2}=\dot{x}^{2}-2 \dot{x} \dot{\phi}\left(l \sin \phi-Y_{M f} \cos \phi\right)+\left(Y_{M f}^{2}+l^{2}\right) \dot{\phi}^{2}
\end{gathered}
$$

The two components of the pendulum acceleration vector (Equation B22a) will be:

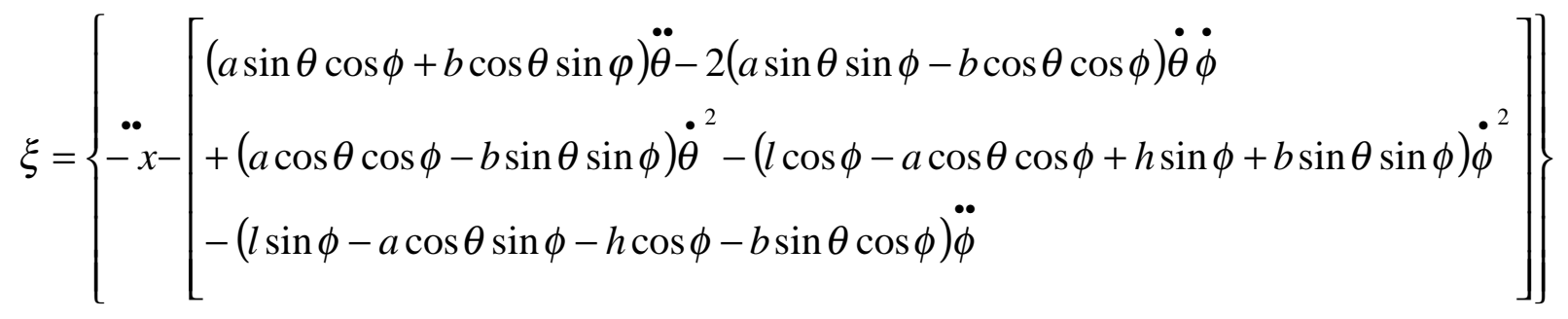


$\psi=\left[\begin{array}{l}(b \cos \theta \cos \phi-a \sin \theta \sin \varphi) \ddot{\theta}-2(a \sin \theta \cos \phi+b \cos \theta \sin \phi) \dot{\theta} \dot{\phi} \\ -(a \cos \theta \sin \phi+b \sin \theta \cos \phi) \dot{\theta}^{2}+(l \sin \phi-a \cos \theta \sin \phi-h \cos \phi-b \sin \theta \cos \phi) \dot{\phi}^{2} \\ -(l \cos \phi-a \cos \theta \cos \phi+h \sin \phi+b \sin \theta \sin \phi) \ddot{\phi}\end{array}\right]$

$$
\begin{aligned}
& \ddot{R}_{t}=-\left(\ddot{x}-l \sin \phi \ddot{\phi}-l \cos \phi \dot{\phi}^{2}+h_{1} \cos \phi \ddot{\phi}-h_{1} \sin \phi \dot{\phi}^{2}\right) \hat{i}+ \\
& -\left(l \cos \phi \ddot{\phi}-l \sin \phi \dot{\phi}^{2}+h_{1} \sin \phi \ddot{\phi}+h_{1} \cos \phi \dot{\phi}^{2}\right) \hat{j}
\end{aligned}
$$

$$
\begin{aligned}
& \stackrel{\vec{R}}{f}=-\left(\ddot{x}-l \sin \phi \ddot{\phi}-l \cos \phi \dot{\phi}^{2}+Y_{M f} \cos \phi \ddot{\phi}-Y_{M f} \sin \phi \dot{\phi}^{2}\right) \hat{i}+ \\
& -\left(l \cos \phi \ddot{\phi}-l \sin \phi \dot{\phi}^{2}+Y_{M f} \sin \phi \ddot{\phi}+Y_{M f} \cos \phi \dot{\phi}^{2}\right) \hat{j}
\end{aligned}
$$

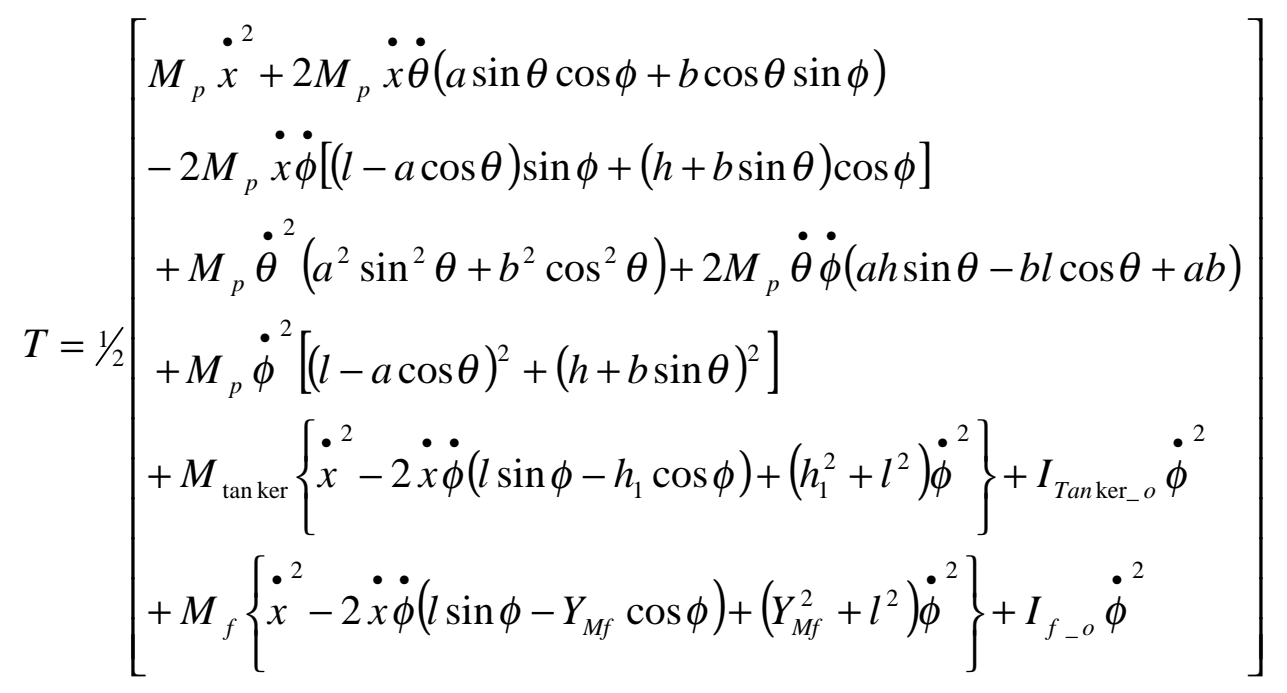

$V=M_{p} g[(h+b \sin \theta) \cos \phi-(l-a \cos \theta) \sin \phi]+M_{\text {Tan } \mathrm{ker}} g\left(-l \sin \phi+h_{1} \cos \phi\right)$

$+M_{f} g\left(-l \sin \phi+Y_{M f} \cos \phi\right)$ 


$$
\frac{\partial L}{\partial \dot{\theta}}=M_{p}\left[\dot{\theta}\left(a^{2} \sin ^{2} \theta+b^{2} \cos ^{2} \theta\right)+\dot{\phi}(a h \sin \theta-b l \cos \theta+a b)+\dot{x}(a \sin \theta \cos \phi+b \cos \theta \sin \phi)\right]
$$

$$
\begin{aligned}
& \frac{\partial}{\partial t}\left(\frac{\partial L}{\partial \dot{\theta}}\right)=M_{p}\left[\begin{array}{l}
\ddot{\theta}\left(a^{2} \sin ^{2} \theta+b^{2} \cos ^{2} \theta\right)+\dot{\theta}^{2}\left(a^{2}-b^{2}\right) \sin 2 \theta+\ddot{\phi}(a h \sin \theta-b l \cos \theta+a b) \\
+\dot{\theta} \dot{\phi}(a h \cos \theta+b l \sin \theta)+\ddot{x}(a \sin \theta \cos \phi+b \cos \theta \sin \phi) \\
+\dot{x} \dot{\theta}(a \cos \theta \cos \phi-b \sin \theta \sin \phi)+\dot{x} \dot{\phi}(b \cos \theta \cos \phi-a \sin \theta \sin \phi)
\end{array}\right] \\
& \frac{\partial L}{\partial \theta}=1 / 2 M_{p}\left[\begin{array}{l}
2 \dot{x} \dot{\theta}(a \cos \theta \cos \phi-b \sin \theta \sin \phi)-2 \dot{x} \dot{\phi}(a \sin \theta \sin \phi-b \cos \theta \cos \phi)+\dot{\theta}^{2}\left(a^{2}-b^{2}\right) \sin 2 \theta \\
+2 \dot{\theta} \dot{\phi}(a h \cos \theta+b l \sin \theta)+2 \dot{\phi}^{2}\left[a l \sin \theta+b h \cos \theta-\frac{1}{2}\left(a^{2}-b^{2}\right) \sin 2 \theta\right]
\end{array}\right] \\
& -M_{p} g[b \cos \theta \cos \phi-a \sin \theta \sin \phi]
\end{aligned}
$$

Thus, the first equation of motion is

$$
\begin{aligned}
\ddot{\theta}\left(a^{2} \sin ^{2} \theta+b^{2} \cos ^{2} \theta\right)+1 / 2 \dot{\theta}^{2}\left(a^{2}-b^{2}\right) \sin 2 \theta+\ddot{\phi}(a h \sin \theta-b l \cos \theta+a b) \\
-\dot{\phi}^{2}\left[a l \sin \theta+b h \cos \theta+\frac{1}{2}\left(a^{2}-b^{2}\right) \sin 2 \theta\right]+g(b \cos \theta \cos \phi-a \sin \theta \sin \phi) \\
\quad+\ddot{x}(a \sin \theta \cos \phi+b \cos \theta \sin \phi)=0
\end{aligned}
$$

By differentiating the Lagrange's equation with respect to $\dot{\phi}$ and then with respect to time we get the following:

$$
\frac{\partial L}{\partial \dot{\phi}}=\left[\begin{array}{l}
M_{p}\{-\dot{x}[(l-a \cos \theta) \sin \phi+(h+b \sin \theta) \cos \phi]+\dot{\theta}(a h \sin \theta-b l \cos \theta+a b) \\
\left.+\dot{\phi}\left[(l-a \cos \theta)^{2}+(h+b \sin \theta)^{2}\right]\right\}+M_{\text {Tan } \text { ker }}\left\{-\dot{x}\left(l \sin \phi-h_{1} \cos \phi\right)+\dot{\phi}\left(h_{1}^{2}+l^{2}\right)\right\} \\
+M_{f}\left\{-\dot{x}\left(l \sin \phi-Y_{M f} \cos \phi\right)+\dot{\phi}\left(Y_{M f}^{2}+l^{2}\right)\right\}+\dot{\phi}\left(I_{\text {Tan } \text { ker }_{-} o}+I_{f_{-} o}\right)
\end{array}\right]
$$




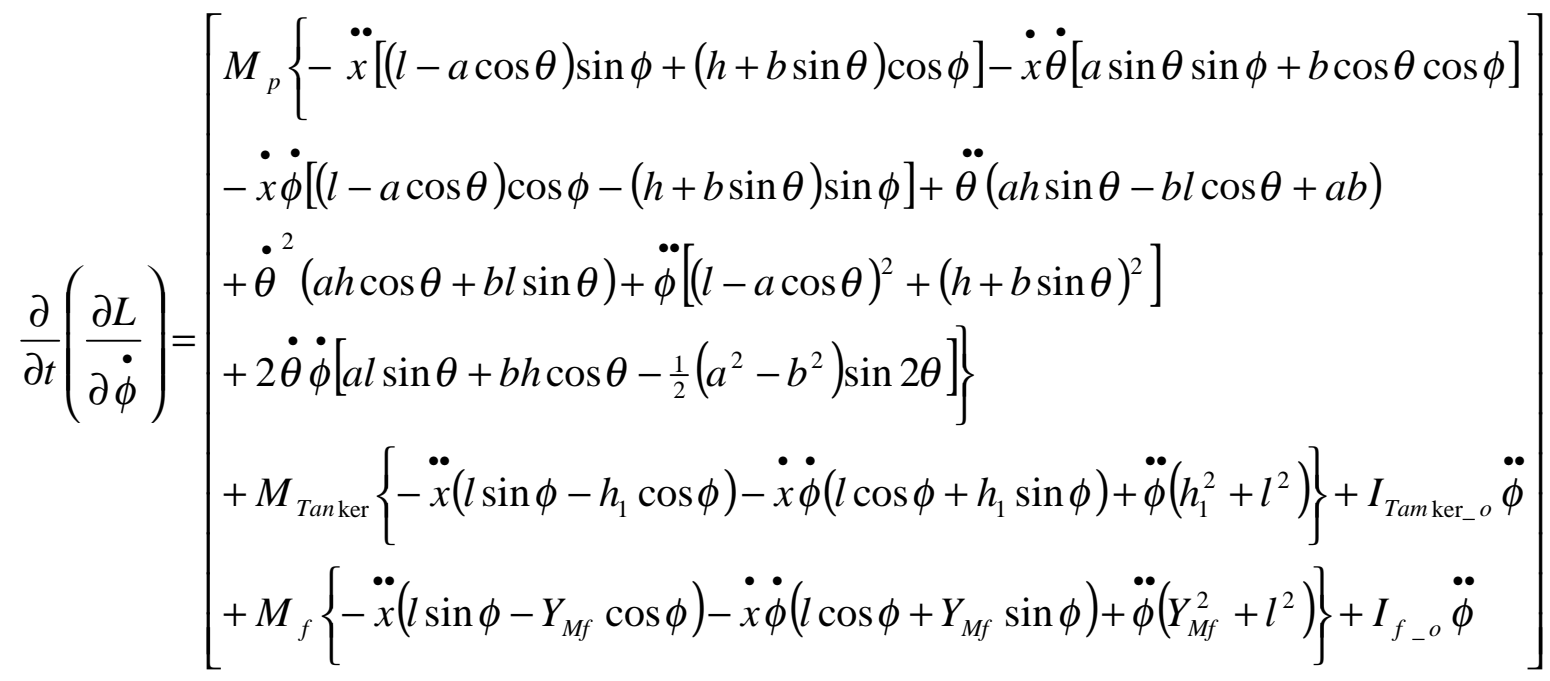

$$
\begin{aligned}
\frac{\partial L}{\partial \phi}= & M_{p}[-\dot{x} \dot{\theta}[a \sin \theta \sin \phi+b \cos \theta \cos \phi]-\dot{x} \dot{\phi}[(l-a \cos \theta) \cos \phi+(h+b \sin \theta) \sin \phi]] \\
& -M_{p} g[-(h+b \sin \theta) \sin \phi-(l-a \cos \theta) \cos \phi]+M_{\tan \mathrm{ker}}\left[-\dot{x} \dot{\phi}\left(l \cos \phi+h_{1} \sin \phi\right)\right] \\
& -M_{\text {Tan ker }} g\left(l \cos \phi+h_{1} \sin \phi\right)+M_{f}\left[-\dot{x} \dot{\phi}\left(l \cos \phi+Y_{M f} \sin \phi\right)\right]-M_{f} g\left(l \cos \phi+Y_{M f} \sin \phi\right)
\end{aligned}
$$

Substituting in Lagrange's Equation yields:

$$
\begin{aligned}
& \ddot{\theta} M_{p}(a h \sin \theta-b l \cos \theta+a b)+\ddot{\phi}\left\{M_{p}\left[(l-a \cos \theta)^{2}+(h+b \sin \theta)^{2}\right]+M_{\text {Tan } \mathrm{ker}}\left(h_{1}^{2}+l^{2}\right)\right. \\
& \left.+M_{f}\left(Y_{M f}^{2}+l^{2}\right)+I_{\text {Tan } \mathrm{ker}_{-} o}+I_{f_{-} o}\right\}+2 \dot{\theta} \dot{\phi} M_{p}\left[a l \sin \theta+b h \cos \theta-\frac{1}{2}\left(a^{2}-b^{2}\right) \sin 2 \theta\right] \\
& +\dot{\theta}^{2} M_{p}(a h \cos \theta+b l \sin \theta)+g\left\{M_{p}[(l-a \cos \theta) \cos \phi-(h+b \sin \theta) \sin \phi]\right. \\
& \left.+M_{\text {Tan } \mathrm{ker}}\left(l \cos \phi+h_{1} \sin \phi\right)+M_{f}\left(l \cos \phi+Y_{M f} \sin \phi\right)\right\}-\ddot{x}\left\{M_{p}[(l+a \cos \theta) \sin \phi+(h+b \sin \theta) \cos \phi]\right. \\
& \left.+M_{\text {Tan } \text { ker }}\left(l \sin \phi-h_{1} \cos \phi\right)+M_{f}\left(l \sin \phi-Y_{M f} \cos \phi\right)\right\}=0
\end{aligned}
$$

Similarly, equations B30 and B31 were discreetized to 4 first order differential equations, which are given below: 
$\dot{\theta}=Y$

$\dot{\phi}=Z$

$\ddot{\theta}=\dot{Y}$ and $\ddot{\phi}=\dot{Z}$

Substituting in B30 and B31 yields:

$$
\begin{aligned}
& \dot{Y}\left(a^{2} \sin ^{2} \theta+b^{2} \cos ^{2} \theta\right)+\dot{Z}(a h \sin \theta-b l \cos \theta+a b)+1 / 2 Y^{2}\left(a^{2}-b^{2}\right) \sin 2 \theta \\
& -Z^{2}\left[a l \sin \theta+b h \cos \theta+\frac{1}{2}\left(a^{2}-b^{2}\right) \sin 2 \theta\right]+g(b \cos \theta \cos \phi-a \sin \theta \sin \phi) \\
& \quad+\ddot{x}(a \sin \theta \cos \phi+b \cos \theta \sin \phi)=0
\end{aligned}
$$

and

$$
\begin{aligned}
& \dot{Y} M_{p}(a h \sin \theta-b l \cos \theta+a b)+\dot{Z}\left\{M_{p}\left[(l-a \cos \theta)^{2}+(h+b \sin \theta)^{2}\right]+M_{\text {Tan } \mathrm{ker}}\left(h_{1}^{2}+l^{2}\right)\right. \\
& \left.+M_{f}\left(Y_{M f}^{2}+l^{2}\right)+I_{\text {Tan } \mathrm{ker}_{-} o}+I_{f_{-} o}\right\}+2 Y Z M_{p}\left[a l \sin \theta+b h \cos \theta-\frac{1}{2}\left(a^{2}-b^{2}\right) \sin 2 \theta\right] \\
& +Y^{2} M_{p}(a h \cos \theta+b l \sin \theta)+g\left\{M_{p}[(l-a \cos \theta) \cos \phi-(h+b \sin \theta) \sin \phi]\right. \\
& \left.+M_{\text {Tan } \mathrm{ker}}\left(l \cos \phi+h_{1} \sin \phi\right)+M_{f}\left(l \cos \phi+Y_{M f} \sin \phi\right)\right\}-\ddot{x}\left\{M_{p}[(l-a \cos \theta) \sin \phi+(h+b \sin \theta) \cos \phi]\right. \\
& \left.+M_{\text {Tan } \mathrm{ker}}\left(l \sin \phi-h_{1} \cos \phi\right)+M_{f}\left(l \sin \phi-Y_{M f} \cos \phi\right)\right\}=0
\end{aligned}
$$

To use the same frame of reference, equation B.19 will be:

$$
\left(\overline{\mathrm{a}}^{2} \cos ^{2} \theta+\overline{\mathrm{b}}^{2} \sin ^{2} \theta\right) \ddot{\theta}+0.5\left(\overline{\mathrm{a}}^{2}-\overline{\mathrm{b}}^{2}\right) \theta^{\bullet^{2}} \sin 2 \theta+\mathrm{g} \overline{\mathrm{b}} \cos \theta-\overline{\mathrm{a}} \sin \theta \ddot{\mathrm{x}}=0
$$

\section{B.5- Polar Mass Moment of Inertia of the Fluid Volume}

Referring to Figure B.1, the polar mass moment of inertia of the fluid volume around point its CG when the fluid is at rest is given by:

$$
I_{C G}=I_{X^{\mid} X^{\mid}}+I_{Y Y}
$$


If $\mathrm{h} \leq \mathrm{b}$ :

$$
\begin{gathered}
I_{Y Y}=2 \int_{0}^{X_{h}} \int_{b\left(1-\sqrt{1-\left(\frac{x}{a}\right)^{2}}\right)}^{h} x^{2} d y d x \\
\therefore I_{Y Y}=\frac{1}{12}\left[3 b a^{3} \sin ^{-1} \frac{x_{h}}{a}+3 b a x_{h} \sqrt{a^{2}-x_{h}^{2}}+8 x_{h}^{3}(h-b)-6 b a^{2} x_{h}\left(\frac{a^{2}-x_{h}^{2}}{a^{2}}\right)^{3 / 2}\right] \\
I_{X X}=2 \int_{0}^{X_{h}} \int_{b}^{h} y^{2} d y d x \\
I_{X X}=\frac{2 b^{3} x_{h}^{3}}{3 a^{2}}+\frac{2}{3}\left(h^{3}-4 b^{3}\right) x_{h}+\frac{b^{3}}{12}\left\{\frac{x_{h}}{a} \sqrt{a^{2}-x_{h}^{2}}\left[17-2\left(\frac{x_{h}}{a}\right)^{2}\right]+15 a \cdot \sin ^{-1} \frac{x_{h}}{a}\right\}
\end{gathered}
$$

If $h \geq b$ :

$$
\begin{aligned}
& I_{Y Y}=\frac{b a^{3} \pi}{4}-2 \int_{0}^{X_{h}} \int_{h}^{b\left(1+\sqrt{1-\left(\frac{x}{a}\right)^{2}}\right.} x^{2} d y d x \\
& \therefore I_{Y Y}=\frac{b a^{3} \pi}{4}-\frac{1}{12}\left[3 b a^{3} \sin ^{-1} \frac{x_{h}}{a}+3 b a x_{h} \sqrt{a^{2}-x_{h}^{2}}+8 x_{h}^{3}(b-h)-6 b a^{2} x_{h}\left(\frac{a^{2}-x_{h}^{2}}{a^{2}}\right)^{3 / 2}\right] \\
& I_{X X}=\frac{\pi a b^{3}}{4}+\pi a b^{3}-\left[2 \int_{0}^{X_{b}} \int_{\left(1-\sqrt{1-\left(\frac{x}{a}\right)^{2}}\right)}^{h} y^{2} d y d x+A_{h}\left(2 b-y_{C G}\right)^{2}\right] \\
& \therefore I_{X X}=\frac{5 \pi a b^{3}}{4}-\frac{2 b^{3} x_{h}^{3}}{3 a^{2}}+\frac{2}{3}\left(h^{3}-4 b^{3}\right) x_{h}-\frac{b^{3}}{12}\left\{\frac{x_{h}}{a} \sqrt{a^{2}-x_{h}^{2}}\left[17-2\left(\frac{x_{h}}{a}\right)^{2}\right]+15 a \cdot \sin ^{-1} \frac{x_{h}}{a}\right\} \\
& -A_{h}\left(2 b-y_{C G}\right)^{2}
\end{aligned}
$$

Where $A_{h}$ and $y_{C G}$ are given by equations $B 4$ and $B 6$ respectively. 
Once the polar moment of inertia of the fluid's volume at the static position is known around its $\mathrm{CG}$ it can be transferred to any point using the parallel axis theory. Table B1 shows the values of the polar moment of inertia per one meter length of water at a tanker equal in cross section to the ETNYRE tanker at different levels of fill and aspect ratios.

Table B1: Principle polar moment of inertia per meter length of water volume at static position in a tank equal in area to the ETNYRE tanker at different aspect ratios

\begin{tabular}{|c|c|c|c|c|c|}
\hline Fill Level \% h/2b & $\begin{array}{c}\text { Polar moment } \\
\text { of Inertia } \\
{\left[\mathbf{k g} \cdot \mathbf{m}^{2]}\right.} \\
\mathbf{a} / \mathbf{b}=\mathbf{1 . 0}\end{array}$ & $\begin{array}{c}\text { Polar moment } \\
\text { of Inertia } \\
{\left[\mathbf{k g} \cdot \mathbf{m}^{\mathbf{2}}\right.} \\
\mathbf{a} / \mathbf{b}=\mathbf{1 . 2 5}\end{array}$ & $\begin{array}{c}\text { Polar moment } \\
\text { of Inertia } \\
{\left[\mathbf{k g} \cdot \mathbf{m}^{\mathbf{2}}\right.} \\
\mathbf{a} / \mathbf{b}=\mathbf{1 . 5}\end{array}$ & $\begin{array}{c}\text { Polar moment } \\
\text { of Inertia } \\
{\left[\mathbf{k g} \cdot \mathbf{m}^{2]}\right.} \\
\mathbf{a} / \mathbf{b}=\mathbf{1 . 7 5}\end{array}$ & $\begin{array}{c}\text { Polar moment } \\
\text { of Inertia } \\
{\left[\mathbf{k g} \cdot \mathbf{m}^{2]}\right.} \\
\mathbf{a} / \mathbf{b}=\mathbf{2 . 0}\end{array}$ \\
\hline $\mathbf{0}$ & 0 & 0 & 0 & 0 & 0 \\
\hline $\mathbf{1 0}$ & 6.639 & 8.21 & 9.798 & 11.38 & 12.79 \\
\hline $\mathbf{2 0}$ & 34.82 & 42.43 & 50.18 & 57.97 & 64.95 \\
\hline $\mathbf{3 0}$ & 88.49 & 106.2 & 124.5 & 143 & 159.6 \\
\hline $\mathbf{4 0}$ & 160.7 & 196.9 & 228.2 & 260.4 & 289.5 \\
\hline $\mathbf{5 0}$ & 267.5 & 308.6 & 353.3 & 399.9 & 442.5 \\
\hline $\mathbf{6 0}$ & 384.4 & 433.6 & 489.4 & 549 & 603.9 \\
\hline $\mathbf{7 0}$ & 511.9 & 562.9 & 625.4 & 694 & 758.4 \\
\hline $\mathbf{8 0}$ & 641 & 687 & 749.9 & 822 & 891.3 \\
\hline $\mathbf{9 0}$ & 759.6 & 793.9 & 850.8 & 920.5 & 989.6 \\
\hline $\mathbf{1 0 0}$ & 840.2 & 861.1 & 910 & 974.6 & 1040 \\
\hline
\end{tabular}

Figure B.15 shows a plot for the normalized principle polar moment of inertia of the fluid volume over the principle polar moment of inertia of the full cross section. 


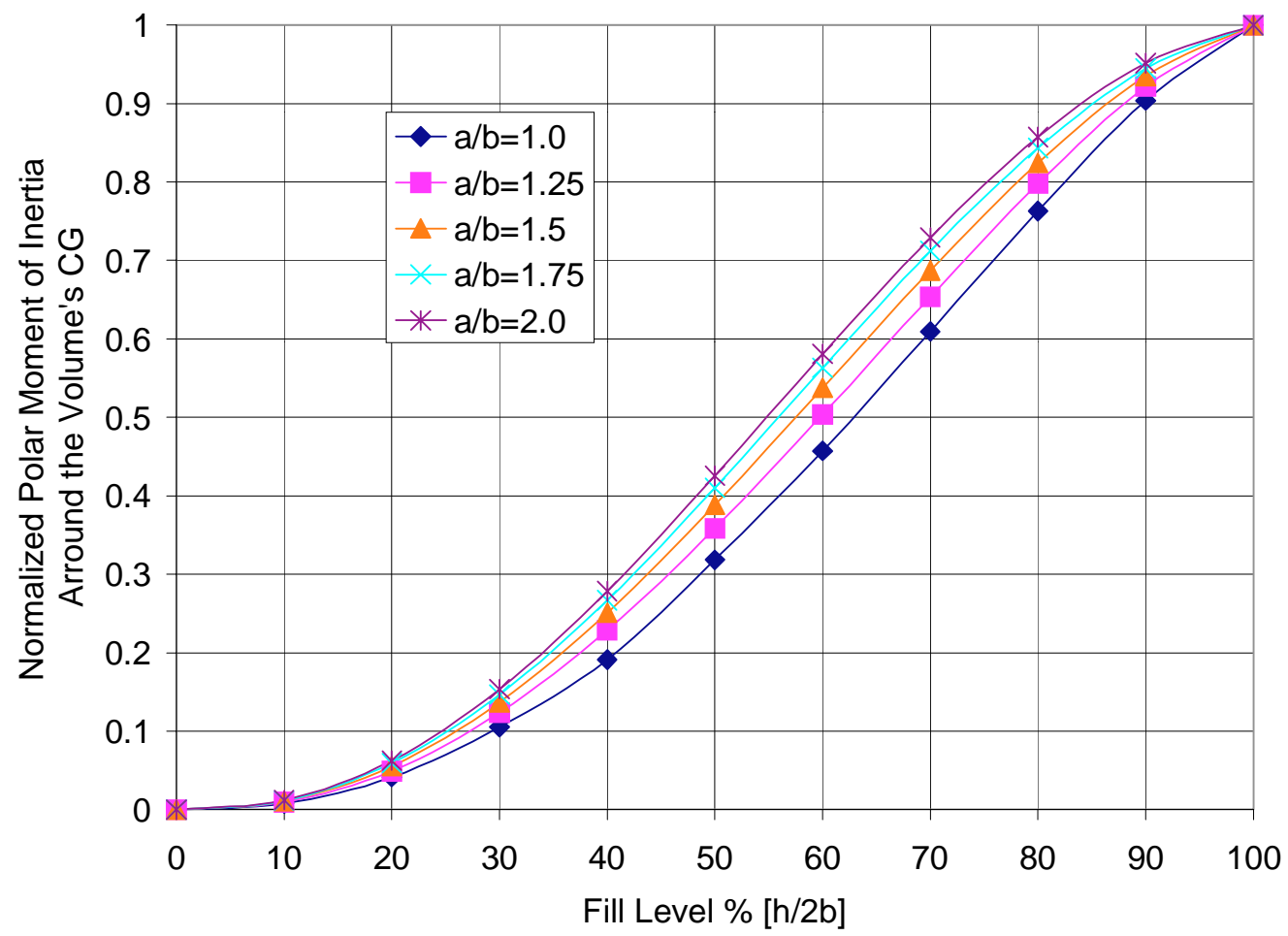

Figure B15: Normalized polar moment of inertia vs. fill level for different levels of fill and different aspect ratios

Since equal areas of different shape has different polar moment of inertia, it's expected that as the fluid moves angularly in an elliptical container, the polar moment of inertia will vary. Figure B.16 shows the variation of the fluid polar moment of inertia for a $50 \%$ full tank as a function of the fluid surface angular position at different levels of fill. It has been found that:

$$
\frac{I_{\text {principle } 0}}{I_{\text {principle } 90}}=\frac{a}{b}
$$

Which can be easily seen from Figure B.17.

However, when assuming that the principle polar moment of inertia varies in an elliptical relation such that B.42 is true, it was found that the principle polar moment of inertia is less than that predicted from that elliptical relation except at 0 and 90 degrees. Figure B.18 shows the error in predicting the principle polar moment of inertia using an elliptical relation. 


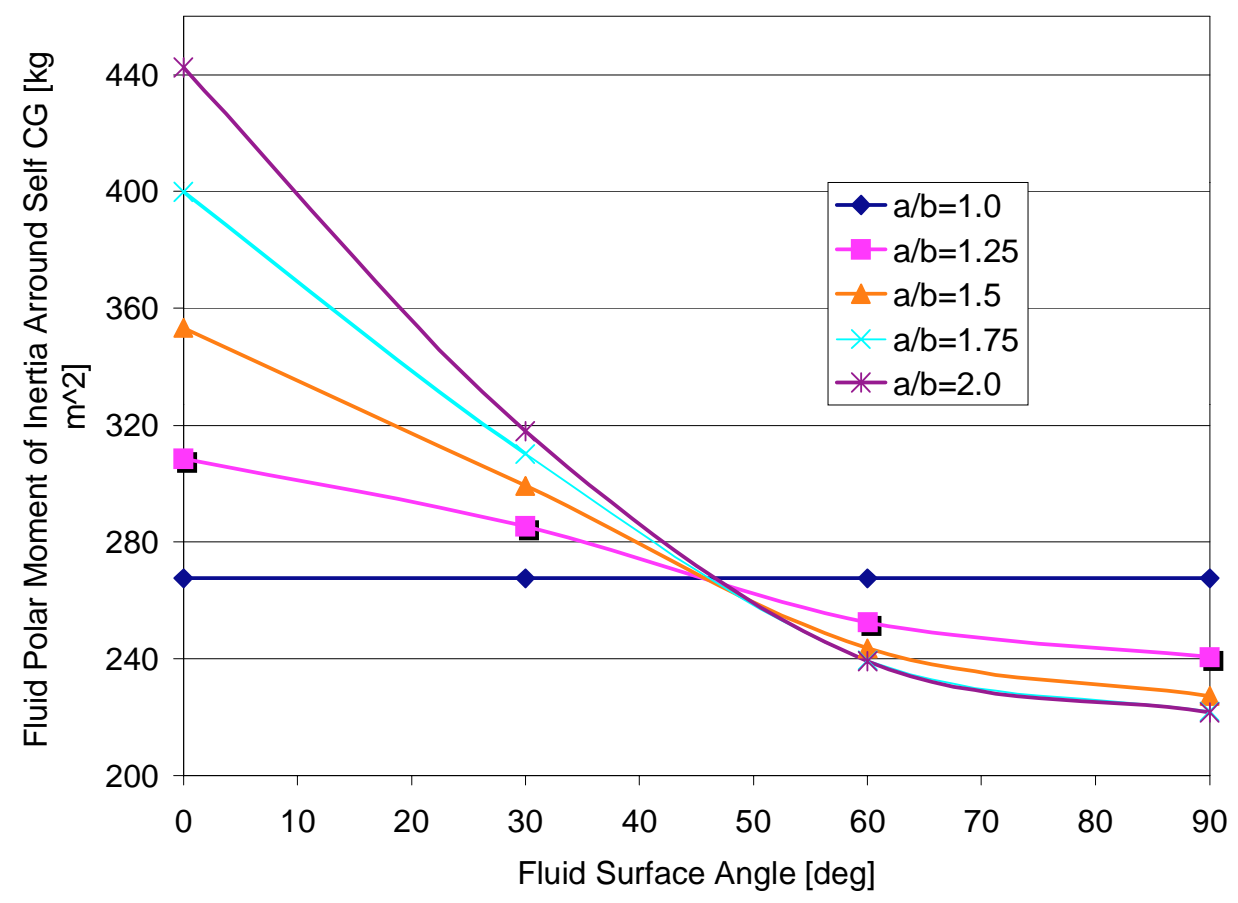

Figure B.16: Variation of the principle polar moment of inertia per meter length of a fluid volume as a function of the fluid surface angle for a 50\% ETNYRE tanker

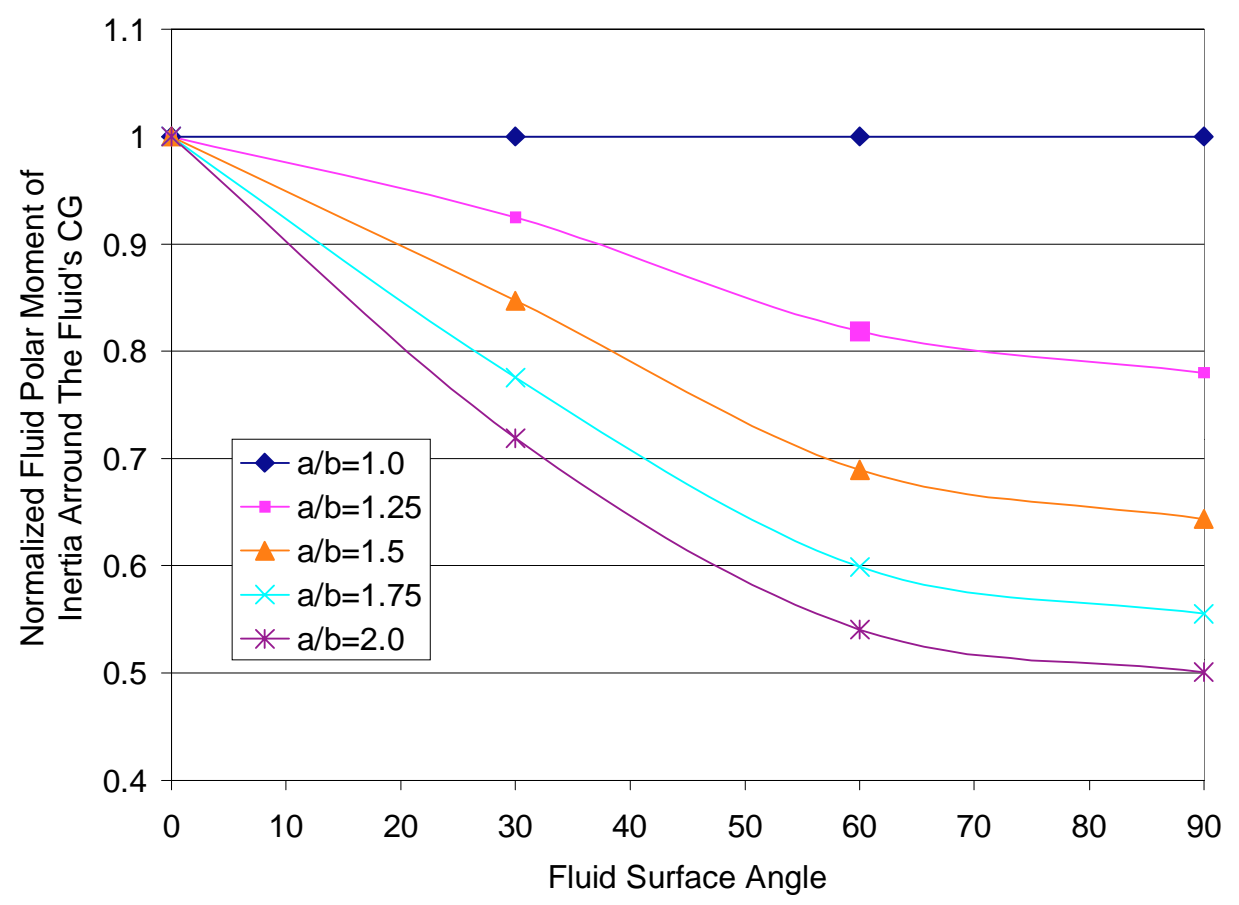

Figure B.17: Normalized polar moment of inertia $\left(\mathrm{I}_{\theta} / \mathrm{I}_{0}\right)$ for a $50 \%$ full ETNYRE tanker 


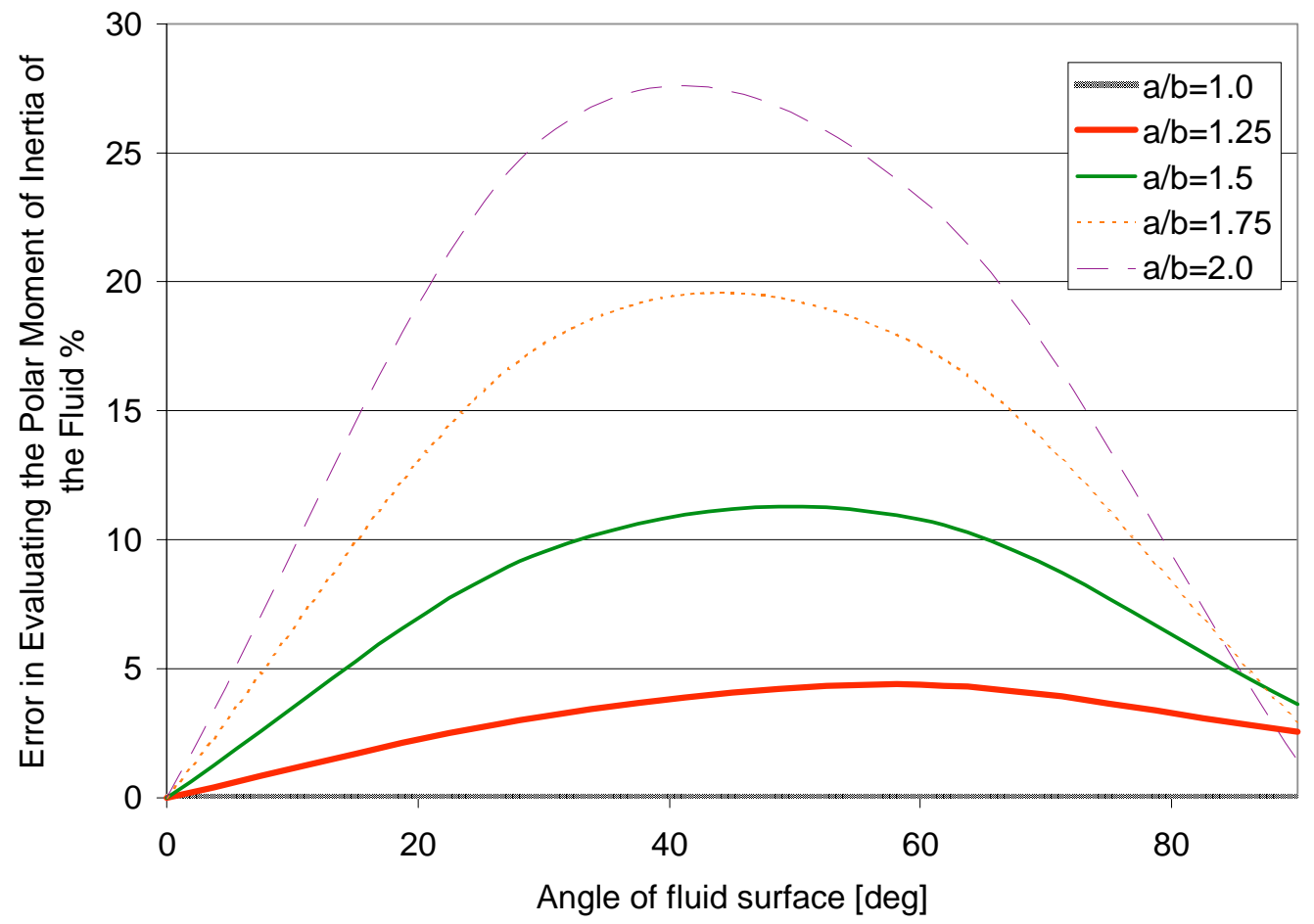

Figure B.18: Error in evaluating the principle angular polar moment of inertia of a fluid volume using an elliptical relation

It can be seen that the maximum error occurs around $45^{\circ}$ and reaches its peak value of about $27 \%$ for the aspect ratio 2:1. 


\section{APPENDIX C \\ LIST OF COMPUTER CODES USED IN THIS STUDY}

\section{C.1- Introduction}

This appendix contains list of the codes used in this study to conduct different tasks as well as comments and directions on how to use these files.

The file allfilter.m accepts data in a column format considering that the first column is time. Thus, no filtering process is applied on the first column of any data file. Care should be taken not to include any text headers at the top of the columns for any data file read by this file.

The filtered data gets saved on a file that has the same name as the input file but with an added " $\mathrm{f}$ " at the end. For example, if the input file is called "acceleration.dat" the output file will be called "accelerationf.dat". All data handled by any .m file in this section should be in a tab delimited text format.

The file spr_dif.m reads the characteristics of the trailer spring from a data file called springchr.dat.

The file trammel.m once asked to read a lateral acceleration data file, it will look for a tab delimited text file with the given name and an extension ".dat". The data in this file should be in columns with the first column being the time (constant time step is recommended for the numerical integration process). This data file should also be without a header text row at the top of the columns. 
The file equ_mot.m is a function that has to be called through the file trammel.m

The file reaction.m although can be called from MATLAB prompt, it will look for data that can only be provided through the file trammel.m.

\section{C.2- The MATLAB .m file allfilter.m}

\%This File Has a general purpose second order Low Band Buttworth Filter

To Filter

$\%$ The experimental Data supplied by ATC

$\%$ By Mohamed Salem (12/31/98)

$\%$ The Input files has to be of a tab delaminated column text format with extension .dat

close all \% Closes All open Windows

clear \% Clears work space

clc \% Clears the screen

Path=input('Input the Path Where your Data Files are (eg C:/Data/)=', 's');

filename=input('Input file name of the data that needs to be filtered without extension ', 's');

eval(['load ',Path,filename, '.dat']); \%oloads the Data File

file=eval(filename); \%will be changed by Filtering

file1=eval(filename); \% will contain the data before filtering

$z=$ size(file,2); $\quad \%$ Reads How many Columns in your Data

clc

fprintf('Your file has \% i columns $\left.\bigvee^{\prime}, z\right)$;

n_filter=input('Which Columns You Want to Filter (Put you Input in Row Format [2,3... ]) ');

$t=$ file $(:, 1)$;

$n=\operatorname{size}\left(n \_\right.$filter, 2$)$;

wn=input('Please Input the Cut-off Frequency or press enter for $\operatorname{default}(0.0<w n<1.0)=$ ');

if $\operatorname{isempty}(w n)==1$

$w n=0.05$;

end

$[B, A]=\operatorname{butter}(2, w n) ; \%$ Create a second order Buttworth Low Band Pass Filter

for $i=1: n$; \% Filter the Chosen Columns of Data

$r=n \_$filter $(i)$;

$x n=$ file $(:, r)$;

$y n=f i l t f i l t(B, A, x n) ; \%$ The filtering Function

plot(t,xn,t,yn) \%Plot the original and the filtered Data

pause

file $(:, r)=y n$;

end

eval(['save ',Path,filename,'f.dat', 'file -ascii']); \%Save the Filtered Data adding "f" to the end of the file

name 


\section{C.3- The MATLAB .m File spr_dif.m}

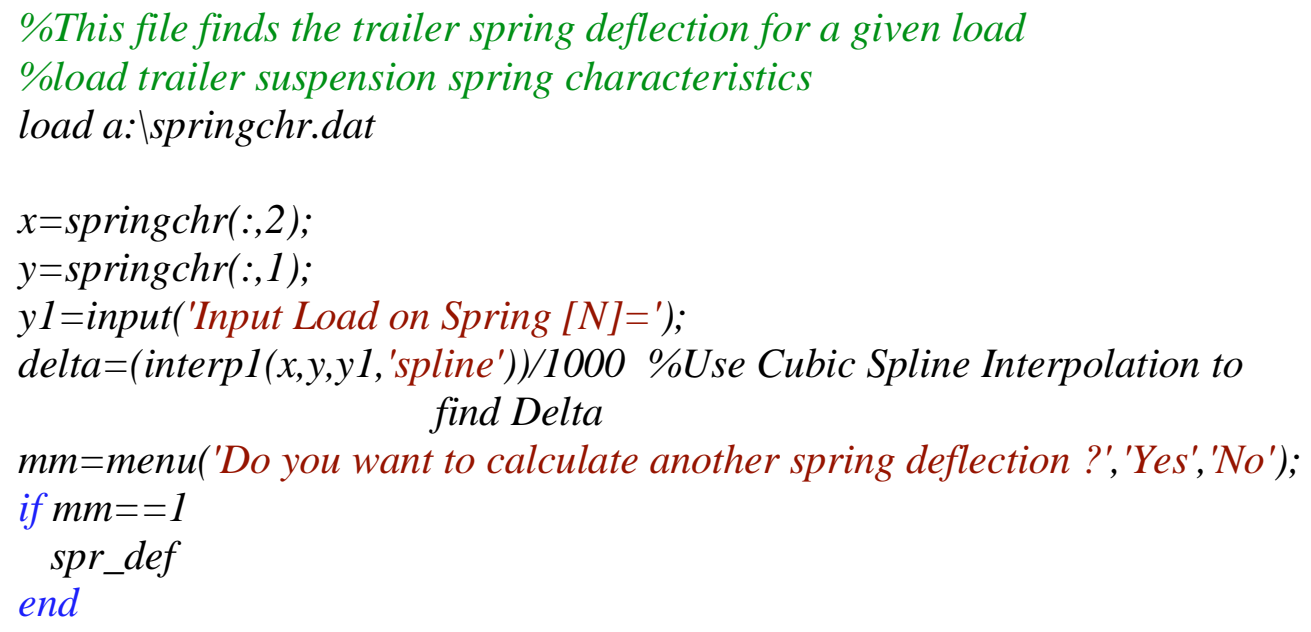

\section{C.4- The MATLAB .m File trammel.m}

$\%$ This file calculates the angular position, velocity, and acceleration of a trammel pendulum $\%$ It calls a function file called equ_mot.m, which contains the equation of motion of the pendulum

$\%$ It passes the results to the $m$ file reaction.m to calculate the reactions at the supports of a plane tank model (Model \#1)

$\%$ Written by Mohamed Salem close all hidden

clear

clc

$\%$ Define Global Variables

global a_barb_baracc_ratio t_start menul z.Acc kg I_frq

$a=$ input('Input the Value of $a=$ ');

$b=$ input('Input the Value of $b=$ ');

Alfa $=a / b$;

fill_level=input('Input Fill Level $h / 2 b=$ ');

Gama $=(0.7726259-1.304727 / A l f a) *(\text { fill_level })^{\wedge} 2+(-1.780896+1.542048 / A l f a) *\left(f i l l \_l e v e l\right)+1$;

b_bar $=b * G a m a$

a_bar=b_bar*Alfa

rho=input('Input fluid density $\left[\mathrm{kg} / \mathrm{m}^{\wedge} 3\right]=$ ');

$L=$ input('Tank Length $[m]=$ ');

$h=$ fill_level $* 2 * b$;

$x h=a *\left(\operatorname{sqrt}\left((2 * h / b)-(h / b)^{\wedge} 2\right)\right)$;

$A h o=2 *\left(x h * h-x h^{*} b+(b /(2 * a)) *\left(x h^{*}\left(\operatorname{sqrt}\left(a^{\wedge} 2-x h^{\wedge} 2\right)\right)+a^{\wedge} 2 * a \sin (x h / a)\right)\right)$;

$Y c g o=\left(h^{\wedge} 2 * x h-2 * b^{\wedge} 2 * x h+\left(b^{\wedge} 2 *\left(x h * \operatorname{sqrt}\left(a^{\wedge} 2-\right.\right.\right.\right.$

$\left.\left.\left.\left.x h^{\wedge} 2\right)+a^{\wedge} 2 * a \sin (x h / a)\right)\right) / a+\left(b^{\wedge} 2 * x h^{\wedge} 3\right) /\left(3 * a^{\wedge} 2\right)\right) / A h o$; 


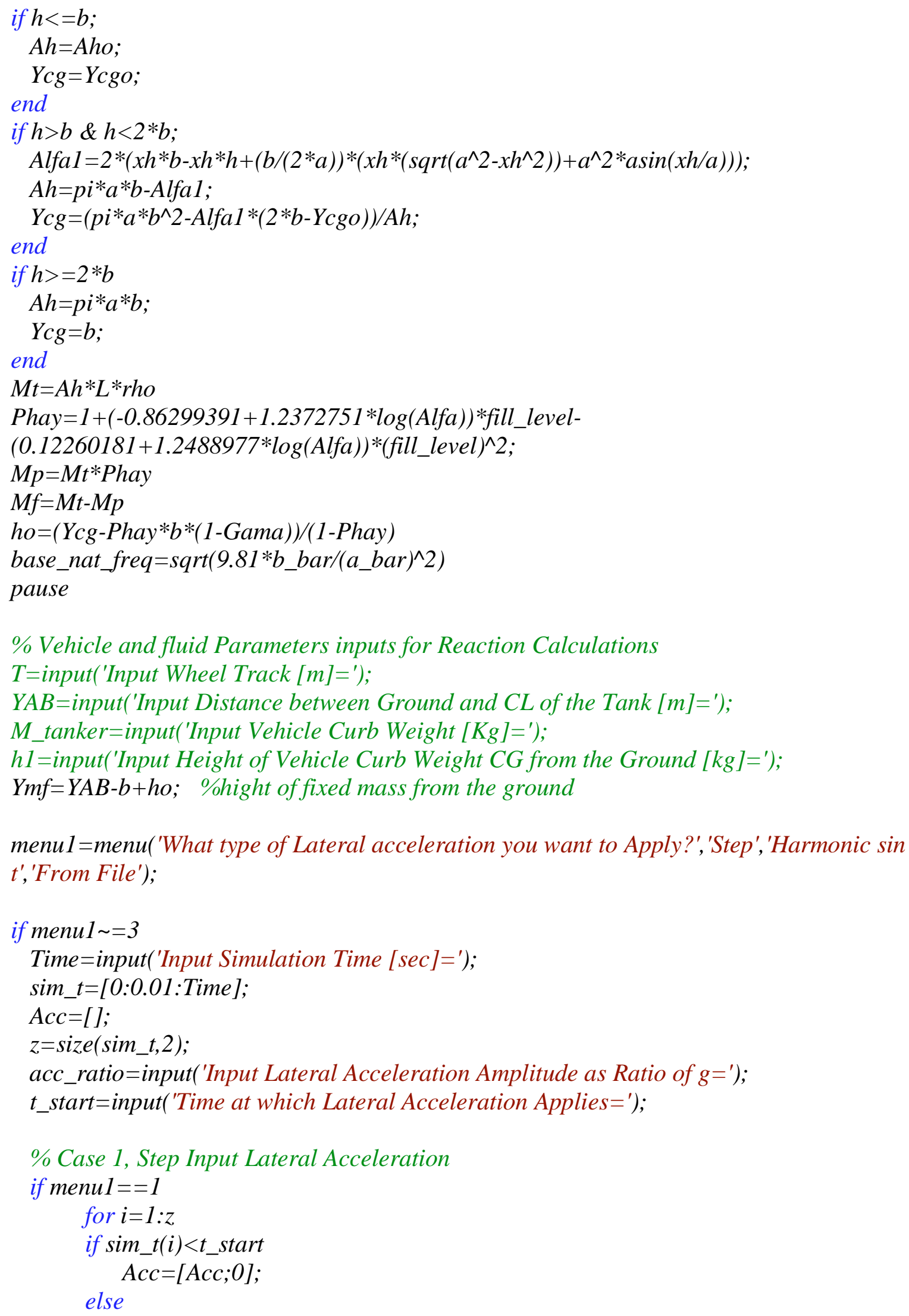




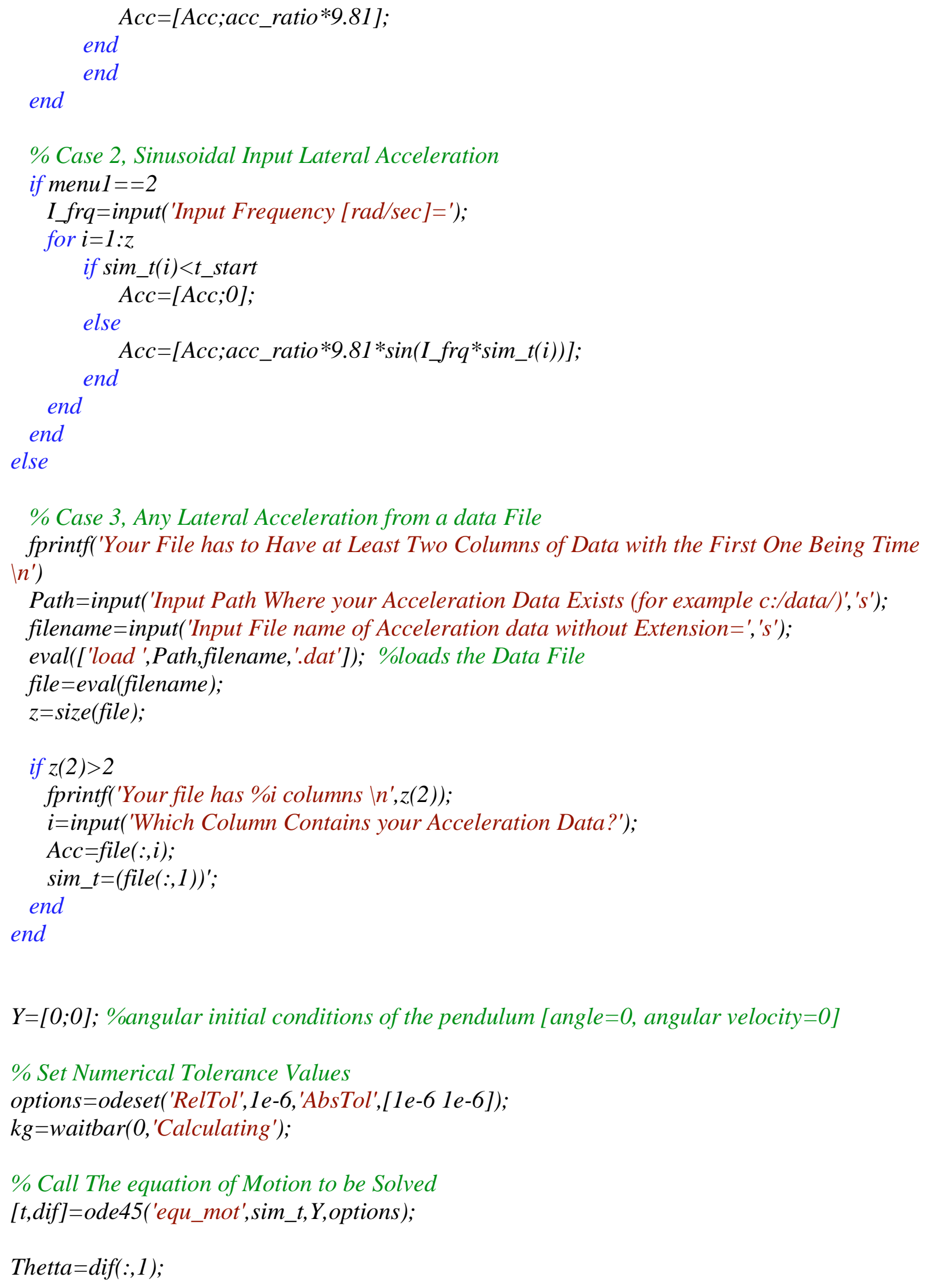




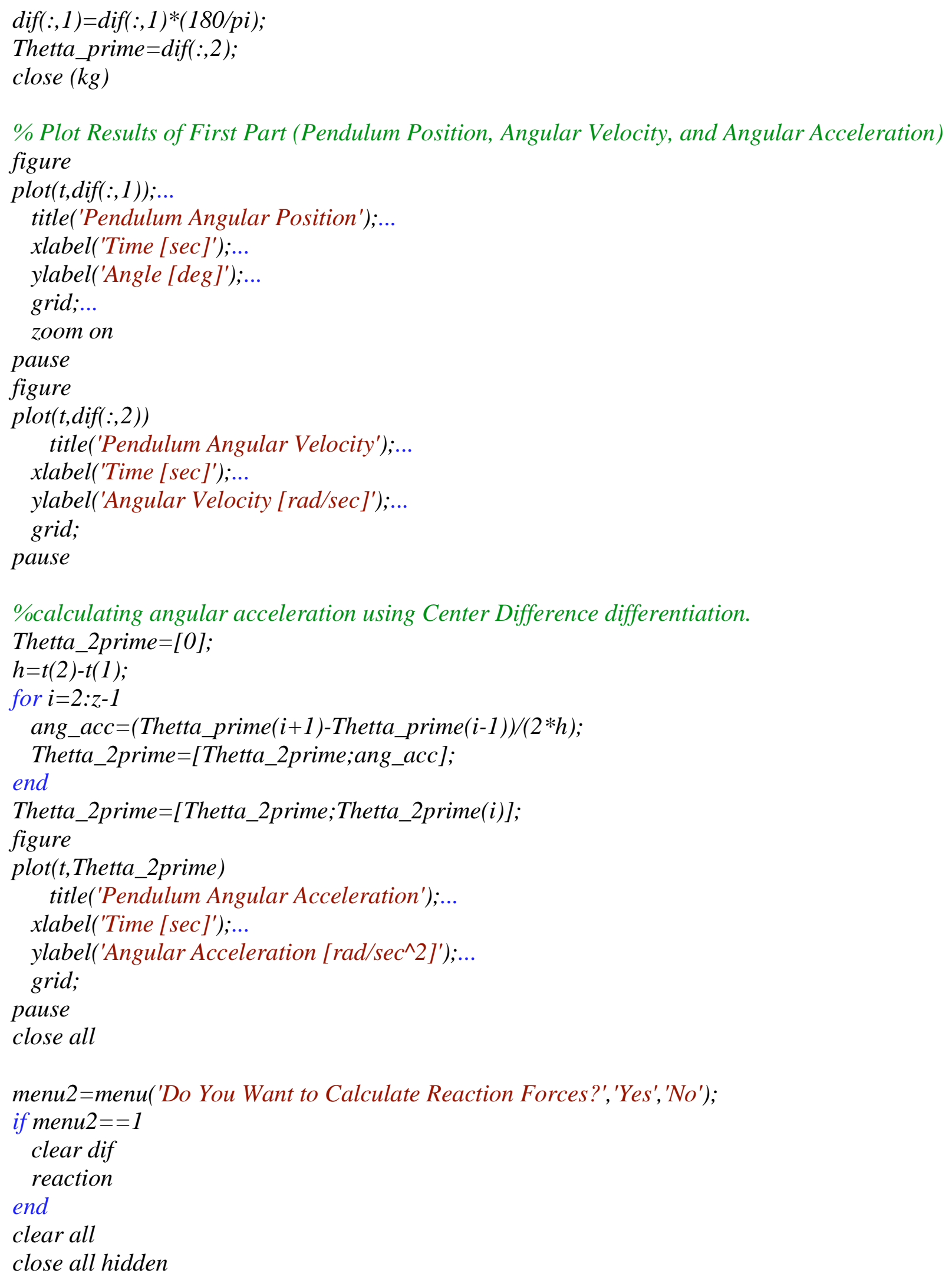




\section{C.5- The MATLAB .m Funtion File equ mot.m}

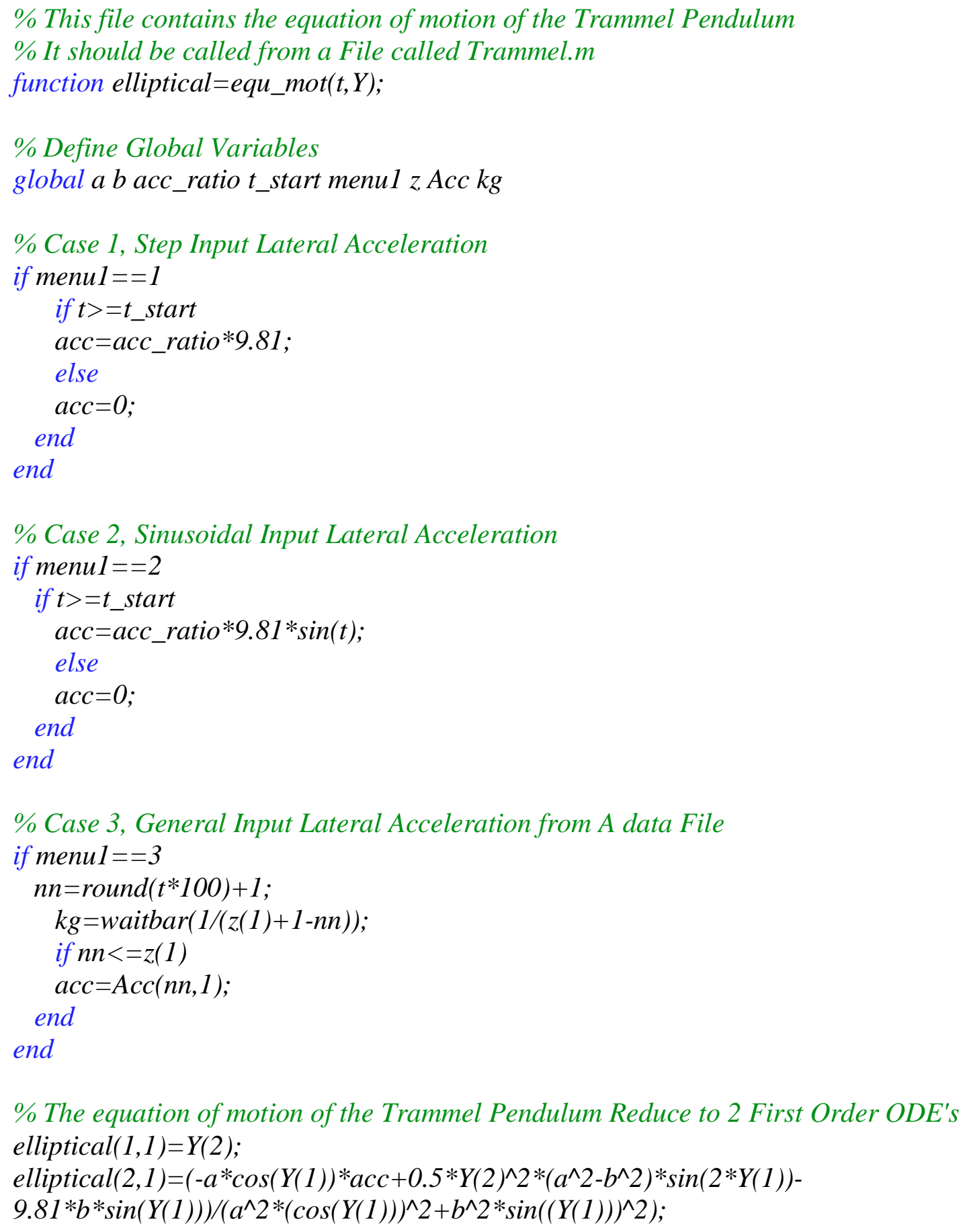




\section{C.6- The MATLAB .m File reaction.m}

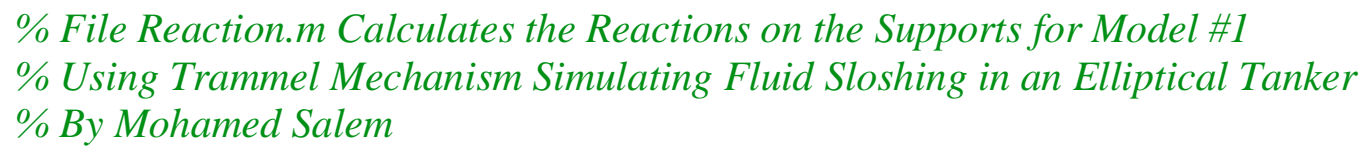


if $m r==1$

trammel

end

\%Saving Results to an Excel File

Column_number=input('Input Column Number=');

Row_number=input('Input Row \#=');

Pos $1=\left[{ }^{\prime}{ }^{\prime}\right.$, num $2 \operatorname{str}($ Row_number +2$),{ }^{\prime} c^{\prime}$, num 2 str $($ Column_number +1$\left.)\right]$;

Pos $2=\left[{ }^{\prime} r^{\prime}\right.$, num $2 \operatorname{str}($ Row_number +2$),{ }^{\prime}{ }^{\prime}$, num $2 s t r($ Column_number +6$\left.)\right]$;

Pos3 $=\left[{ }^{\prime} r\right.$ ',num $2 \operatorname{str}($ Row_number+2), 'c',num2str(Column_number+11)];

Pos4 $=\left[' r\right.$ ',num $2 \operatorname{str}($ Row_number +2$),{ }^{\prime} c^{\prime}$, num 2 str $($ Column_number +16$\left.)\right]$;

Pos5 $=\left[{ }^{\prime} r\right.$ 'num2str(Row_number+2), 'c',num2str(Column_number+21)];

Pos6 $=\left[r^{\prime}\right.$, num $2 s t r\left(R o w \_n u m b e r+2\right),{ }^{\prime} c^{\prime}$, num $\left.2 s t r\left(C o l u m n \_n u m b e r+26\right)\right]$;

$\operatorname{Pos} 7=\left[{ }^{\prime} r\right.$, num $2 \operatorname{str}($ Row_number +2$),{ }^{\prime}{ }^{\prime}$, num $2 \operatorname{str}($ Column_number +31$\left.)\right]$;

channell=ddeinit('excel','frequ_analysis.xls');

pps=ddepoke (channel1,Pos1,a_bar);

pps $=$ ddepoke $($ channel1,Pos2,b_bar $)$;

pps $=$ ddepoke $($ channel1,Pos3,Mt);

pps $=$ ddepoke $($ channell,Pos4,Mp);

pps=ddepoke (channell,Pos5,Mf);

pps=ddepoke(channell,Pos6,base_nat_freq);

pps $=$ ddepoke $($ channell,Pos7,Ymf)

ddeterm(channell);

\section{C.7- The MATLAB .m File ellipse1.m}

\begin{tabular}{|c|c|}
\hline$\% *$ & This File Calculates the Mass of a Fluid Volume in a \\
\hline$\% *$ & Partially Filled Elliptical Tank and also finds the point \\
\hline$\% *$ & of intersection of the flat fluid surface with the tank walls \\
\hline$\% *$ & at Different Surface Angles and Different Fill Level \\
\hline$\% *$ & Written by Mohamed I. Salem in 12/11/97 \\
\hline
\end{tabular}

close all

clc

format long

$a=$ input('The value of " $a$ " in meters is $=$ ');

$b=$ input('The value of " $b$ " in meters is $=$ ');

\%rho=input('The density of Transported Fluid in $N / m^{\wedge} 3$ is=');

rho=9810;

$L=$ input('Length of compartment in meters $=$ ');

$\% L=1.5$;

$M o=p i * a * b * r h o * L ;$ 


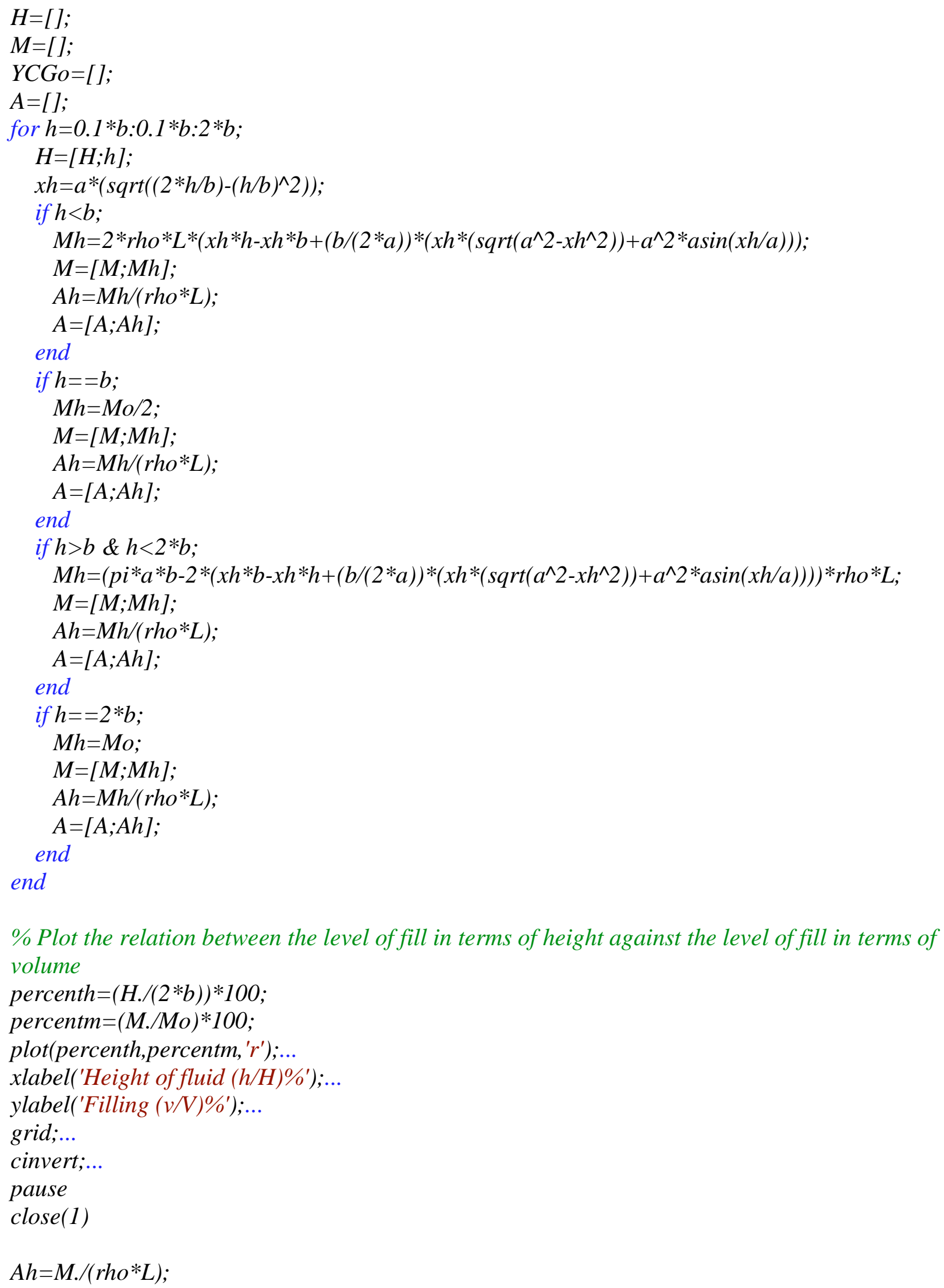




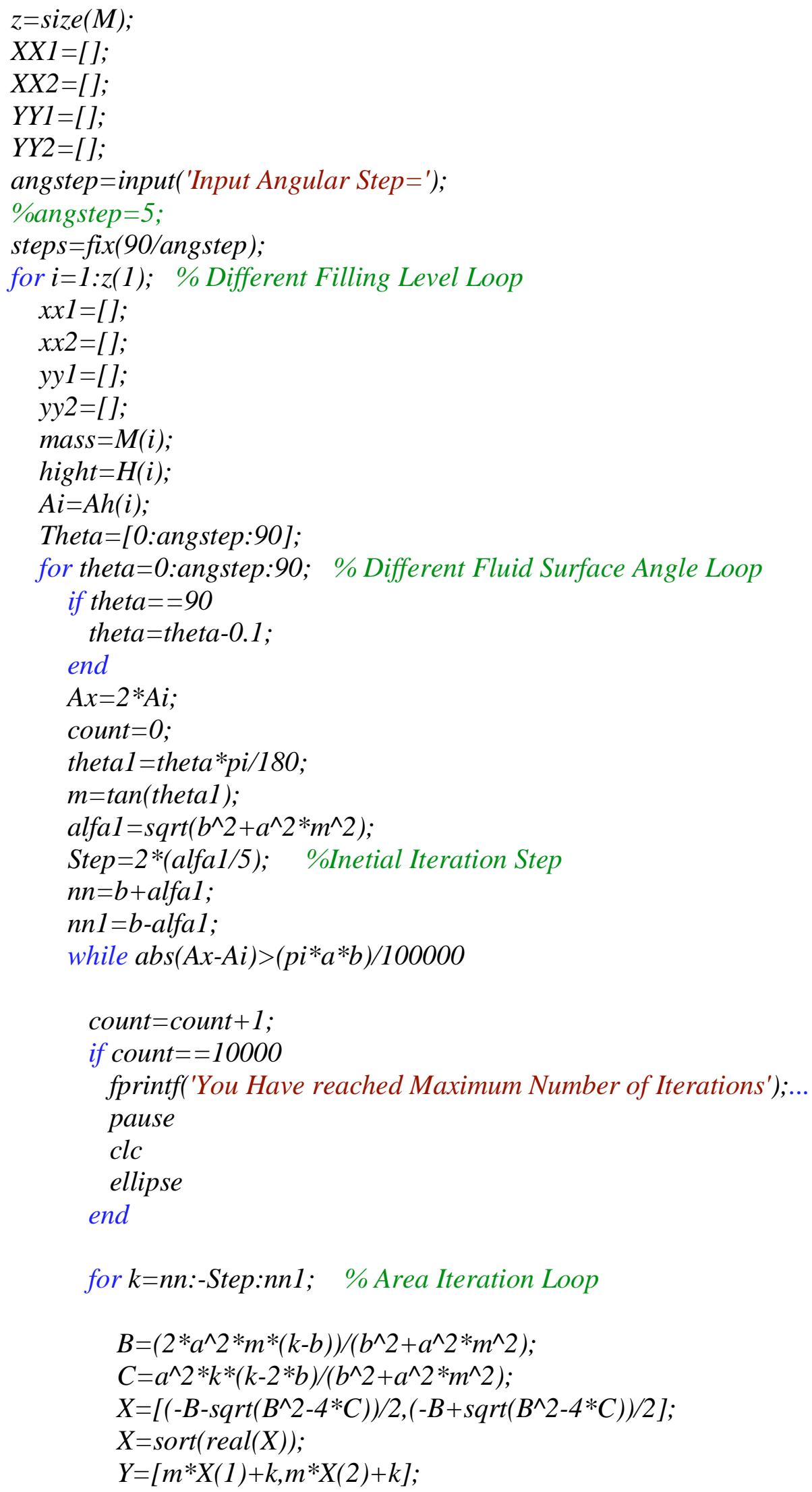




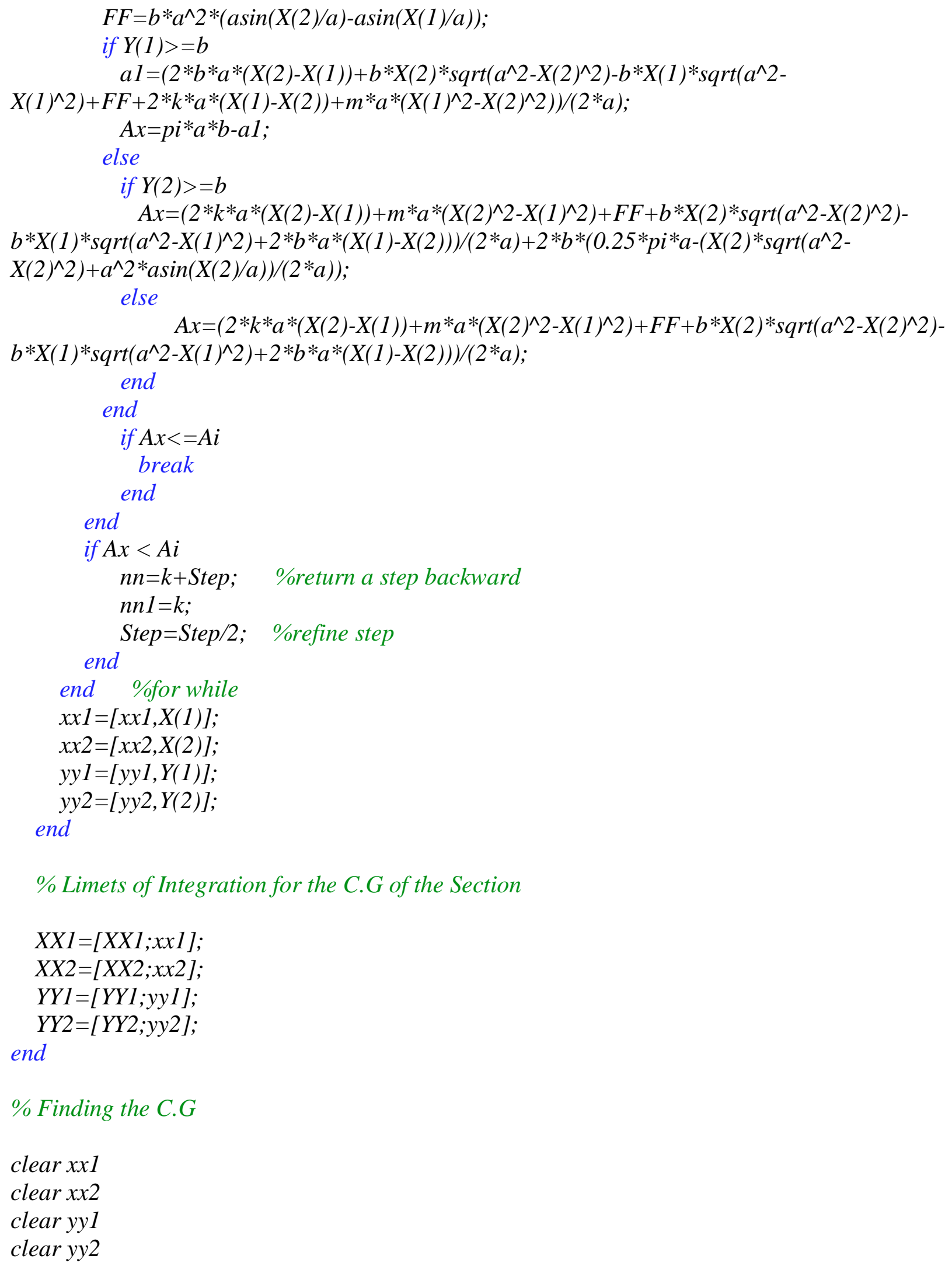




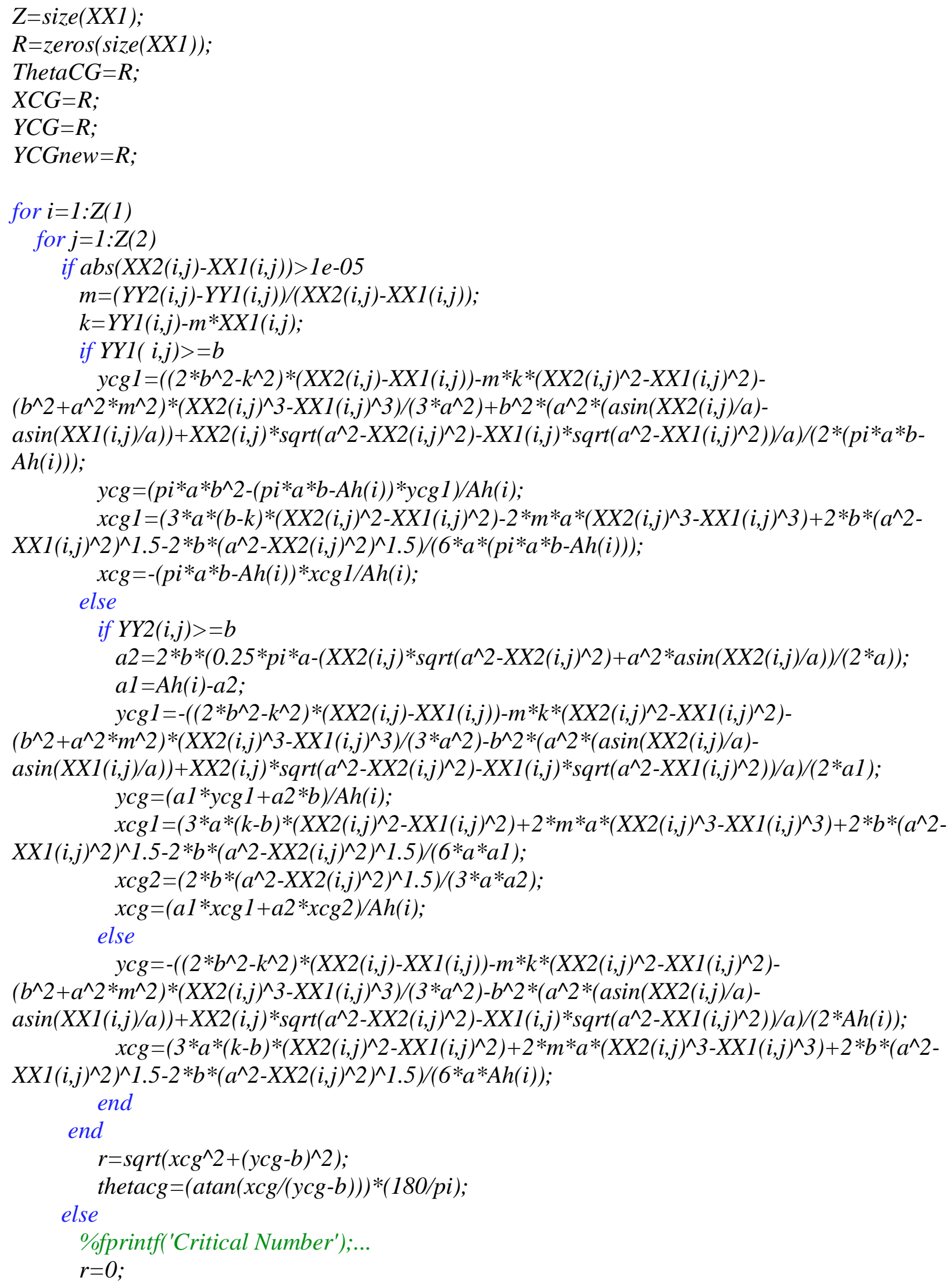




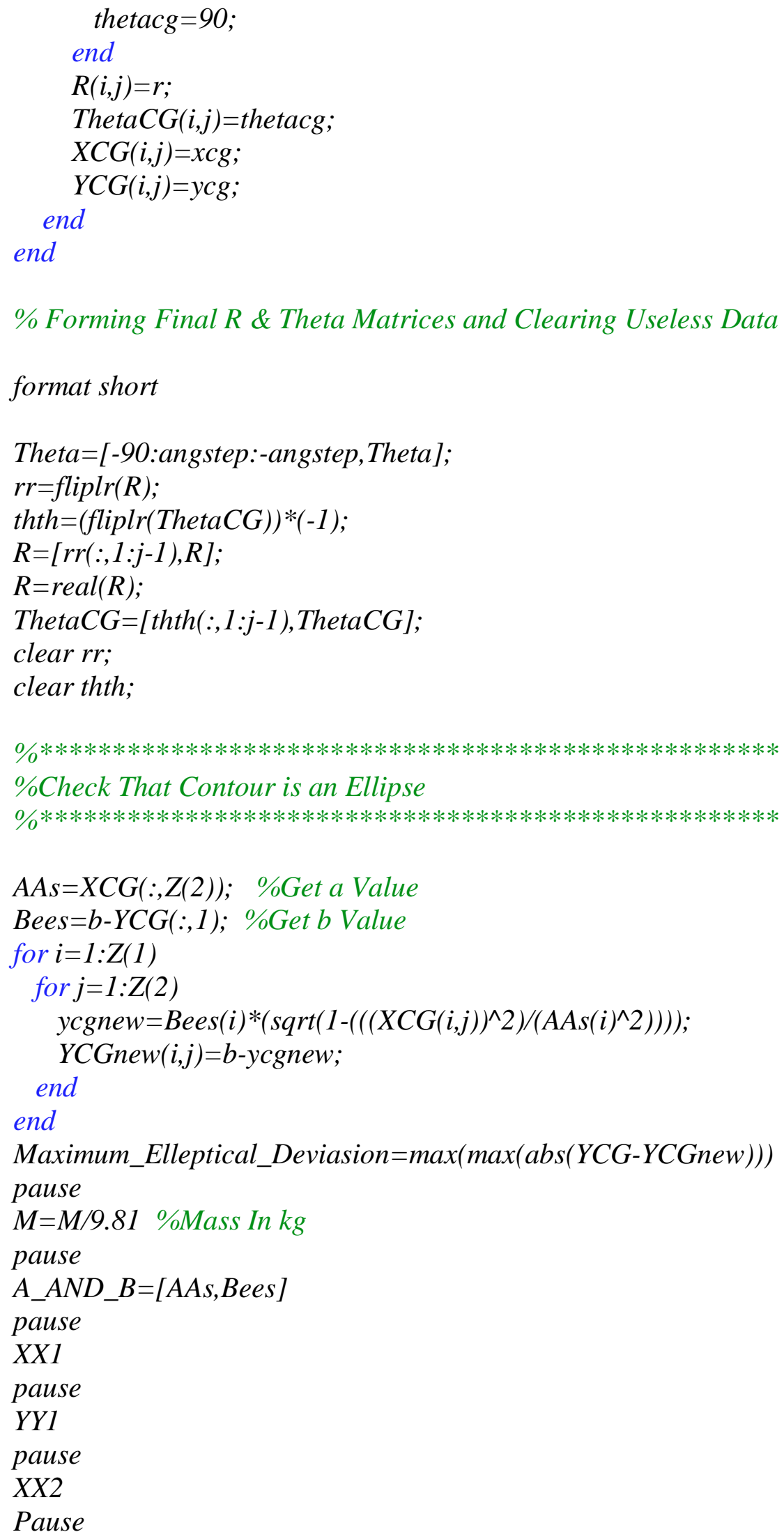




\section{C.8- File Basic3.m}

$\%$ This file calculates the angular position, velocity, and \%acceleration of a trammel pendulum $\%$ If one of the supports lifts off. It calls a file called \%mid.m, Right_s3.m and Left_s3.m $\%$ Written by Mohamed Salem

close all

clear all hidden

clear

clc

$\%$ Define Global Variables

global a_bar b_bar acc_ratio z YAB T M_tanker Mf Mp hl Ymf I_tanker_o I_fluid_o

$a=$ input('Input the Value of $a=$ ');

$b=$ input('Input the Value of $b=$ ');

Alfa $=a / b$;

fill_level=input('Input Fill Level $h / 2 b=')$;

Gama $=(0.7726259-1.304727 / A l f a) *\left(f i l l \_l e v e l\right)^{\wedge} 2+(-1.780896+1.542048 / A l f a) *($ fill_level $)+1$;

b_bar $=b * G a m a$;

$a \_b a r=b \_b a r * A l f a$;

rho=input('Input fluid density $\left[\mathrm{kg} / \mathrm{m}^{\wedge} 3\right]=$ ');

$L=$ input ('Tank Length $[m]=$ ');

$h=$ fill_level $* 2 * b$;

$x h=a *\left(\operatorname{sqrt}\left((2 * h / b)-(h / b)^{\wedge} 2\right)\right)$;

$A h o=2 *\left(x h^{*} h-x h^{*} b+(b /(2 * a)) *\left(x h^{*}\left(\operatorname{sqrt}\left(a^{\wedge} 2-x h^{\wedge} 2\right)\right)+a^{\wedge} 2 * a \sin (x h / a)\right)\right)$;

$Y c g o=\left(h^{\wedge} 2 * x h-2 * b^{\wedge} 2 * x h+\left(b^{\wedge} 2 *\left(x h^{*} \operatorname{sqrt}\left(a^{\wedge} 2-\right.\right.\right.\right.$

$\left.\left.\left.\left.x h^{\wedge} 2\right)+a^{\wedge} 2 * a \sin (x h / a)\right)\right) / a+\left(b^{\wedge} 2 * x h^{\wedge} 3\right) /\left(3 * a^{\wedge} 2\right)\right) / A h o$;

if $h<=b$;

$A h=A h o$;

$Y c g=Y c g o ; \%$ Note: Ycg is measured from the bottom of the tank

end

if $h>b \& h<2 * b$;

Alfal $=2 *\left(x h^{*} b-x h^{*} h+(b /(2 * a)) *\left(x h^{*}\left(\operatorname{sqrt}\left(a^{\wedge} 2-x h^{\wedge} 2\right)\right)+a^{\wedge} 2 * a \sin (x h / a)\right)\right)$;

$A h=p i * a * b-$ Alfal;

$Y c g=\left(p i * a * b^{\wedge} 2-A l f a 1 *(2 * b-Y c g o)\right) / A h$;

end

if $h>=2 * b$

$A h=p i * a * b$;

$Y c g=b$;

end

$M t=A h * L * r h o$;

Phay $=1+(-0.86299391+1.2372751 * \log ($ Alfa $)) *$ fill_level -

$\left(0.12260181+1.2488977 * \log (\right.$ Alfa $) *(\text { fill_level })^{\wedge} 2$;

$M p=M t^{*}$ Phay; 
$M f=M t-M p$;

$h o=\left(\right.$ Ycg-Phay $* b^{*}(1-$ Gama $\left.)\right) /(1-$ Phay $)$;

$\%$ Vehicle and fluid Parameters inputs for Reaction Calculations

T=input('Input Wheel Track $[m]=$ ');

$Y A B=$ input('Input Distance between Ground and CL of the Tank $[\mathrm{m}]=$ ');

M_tanker=input('Input Vehicle Curb Weight [Kg]=');

hl=input('Input Height of Vehicle Curb Weight CG from the Ground [kg]=');

$Y m f=Y A B-b+h o ; \%$ ight of fixed mass from the ground

I_tanker=('Input polar moment of inertia of the tanker around It's $\left.C G\left[\mathrm{~kg} \cdot \mathrm{m}^{\wedge} 2\right]={ }^{\prime}\right)$;

I_tanker_o=I_tanker $+M \_$tanker $*\left(h 1^{\wedge} 2+(T / 2)^{\wedge} 2\right)$;

$I \_$fluid=('Input polar moment of inertia per meter length of the fluid volume arround it's $C G$ [kg. $\left.\left.\mathrm{m}^{\wedge} 2\right]^{\prime}\right)$;

$Y c g \_$ground $=Y A B-b+Y c g$;

\%move polar moment of inertia to support location

I_fluid_o=I_fluid $+M f^{*}\left(Y c g \_g r o u n d \wedge 2+(T / 2)^{\wedge} 2\right)$;

simtime=input('Input Simulation Time [sec]=');

sim_t=[0:0.005:simtime];

$z=\operatorname{size}\left(\operatorname{sim} \_t, 2\right)$;

acc_ratio=input('Input Lateral Acceleration Amplitude as Ratio of $g=$ ');

$\operatorname{Acc}=$ ones $(z, 1) *$ acc_ratio $* 9.81$;

$\%$ set pendulum and vehicle initial condition

$Y=[270 *(p i / 180) ; 0 ; 0 ; 0]$;

$\%$ Set Numerical Tolerance Values

options=odeset('RelTol',1e-6, 'AbsTol',[1e-6 1e-6 1e-6 1e-6]);

$\%$ Call The equation of Motion to be Solved

dif $=[]$;

$t=[]$;

Thetta_2prime= [] ;

$F V=[]$;

$F A=[]$;

$F B=[]$;

$F_{-} A=$ ones $(3,1)$;

$F_{-} B=F_{-} A$;

$\operatorname{Dif}=z \operatorname{eros}(3,4)$;

$i=0$;

for simut=0:0.01: simtime-0.01; \%start simulation routine

$i=i+1$;

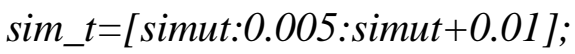

if $\min \left(F_{-} B\right)>0 \& \min \left(F_{-} A\right)>0 \& \operatorname{abs}(\operatorname{Dif}(:, 3))<0.0001 \quad \%$ Case both wheels on ground

[Tim,Dif]=ode45('mid',sim_t,Y,options); 
Pend_acc $=\left(-a \_b a r^{*} \cos (\operatorname{Dif}(:, 1)-1.5 * p i) . * A c c(i: i+2)+0.5 * \operatorname{Dif}(:, 2) . \wedge 2 *\left(a \_b a r^{\wedge} 2-b \_b a r^{\wedge} 2\right) . * \ldots\right.$ $\left.\sin (2 *(\operatorname{Dif}(., 1)-1.5 * p i))-9.81 * b \_b a r * \sin (\operatorname{Dif}(:, 1)-1.5 * p i)\right) . /\left(a \_b a r^{\wedge} 2 *(\cos (\operatorname{Dif}(., 1)-\ldots\right.$

$\left.\left.\left.1.5^{*} p i\right)\right) .^{\wedge} 2+b \_b a r^{\wedge} 2 * \sin ((\operatorname{Dif}(:, 1)-1.5 * p i)) . \wedge 2\right)$;

$\%$ Calculating reactions on both wheels

$\%$ Calculate total Reaction (1.5 pi added to change reference \%coordinate)

$F v=\left(M t+M \_t a n k e r\right) * 9.81+M p * b \_b a r *\left((\operatorname{Dif}(:, 2) . \wedge 2) *{ }^{*} \cos (\operatorname{Dif}(:, 1)-1.5 * p i)+\ldots\right.$

Pend_acc.*sin(Dif(:,1)-1.5*pi));

$\%$ Find reaction on Wheel $B$

compl $1=\left(M f+M \_\right.$tanker $) * 9.81 * T / 2$;

comp $2=M p *\left(\left(a \_b a r *\left((\operatorname{Dif}(:, 2) . \wedge 2) . * \sin (\operatorname{Dif}(:, 1)-1.5 * p i)-P e n d \_a c c . * .\right.\right.\right.$.

$\cos (\operatorname{Dif}(:, 1)-1.5 * p i))-A c c(i: i+2)) . *\left(Y A B-b \_b a r * \cos (\operatorname{Dif}(:, 1)-1.5 * p i)\right)+\ldots$

$\left(a \_b a r * \sin (\operatorname{Dif}(:, 1)-1.5 * p i)+T / 2\right) . *\left(b \_b a r *((\operatorname{Dif}(:, 2) . \wedge 2) . * \cos (\operatorname{Dif}(:, 1)-1.5 * p i)+\ldots\right.$

Pend_acc.*sin $(\operatorname{Dif}(:, 1)-1.5 * p i))+9.81))$;

comp3 $=-\left(M f * Y m f+M \_\right.$tanker $\left.* h 1\right) * A c c(i: i+2)$;

$F \_B=($ comp $1+$ comp $2+$ comp3 $) . / T$;

$\%$ Find reaction on Wheel $A$

$F \_A=F v-F \_B$;

elseif $\min \left(F \_B\right)<=0 \%$ case when right wheel lifts up

$Y=\operatorname{Dif}(3,:)$;

[Tim,Dif]=ode45('Left_s3',sim_t,Y,options);

if Dif(:,3)<-0.00001

$F \_B=$ ones $(3,1)$;

end

elseif $\min \left(F_{-} A\right)<=0 \%$ case when left wheel lifts up

$Y=\operatorname{Dif}(3,:)$;

$Y(3)=Y(3)+2 * p i$

[Tim,Dif]=ode45('Right_s3',sim_t,Y,options);

if Dif(:,3)-2*pi >0.0001

$F \_B=$ ones $(3,1)$;

end

end

$\operatorname{dif}=[\operatorname{dif} ; \operatorname{Dif}(1: 2,:)]$;

$t=[t ; \operatorname{Tim}(1: 2,:)]$;

Thetta_2prime $=[$ Thetta_2prime;Pend_acc $(1: 2,:)]$;

$F A=\left[F A ; F_{-} A(1: 2,:)\right]$;

$F B=\left[F B ; F \_B(1: 2,:)\right]$;

$F V=[F V ; F v(1: 2,:)]$;

$Y=\operatorname{Dif}(3,:)$;

end

$\%$ Calculate final results

Thetta $=\operatorname{dif}(:, 1)$; 


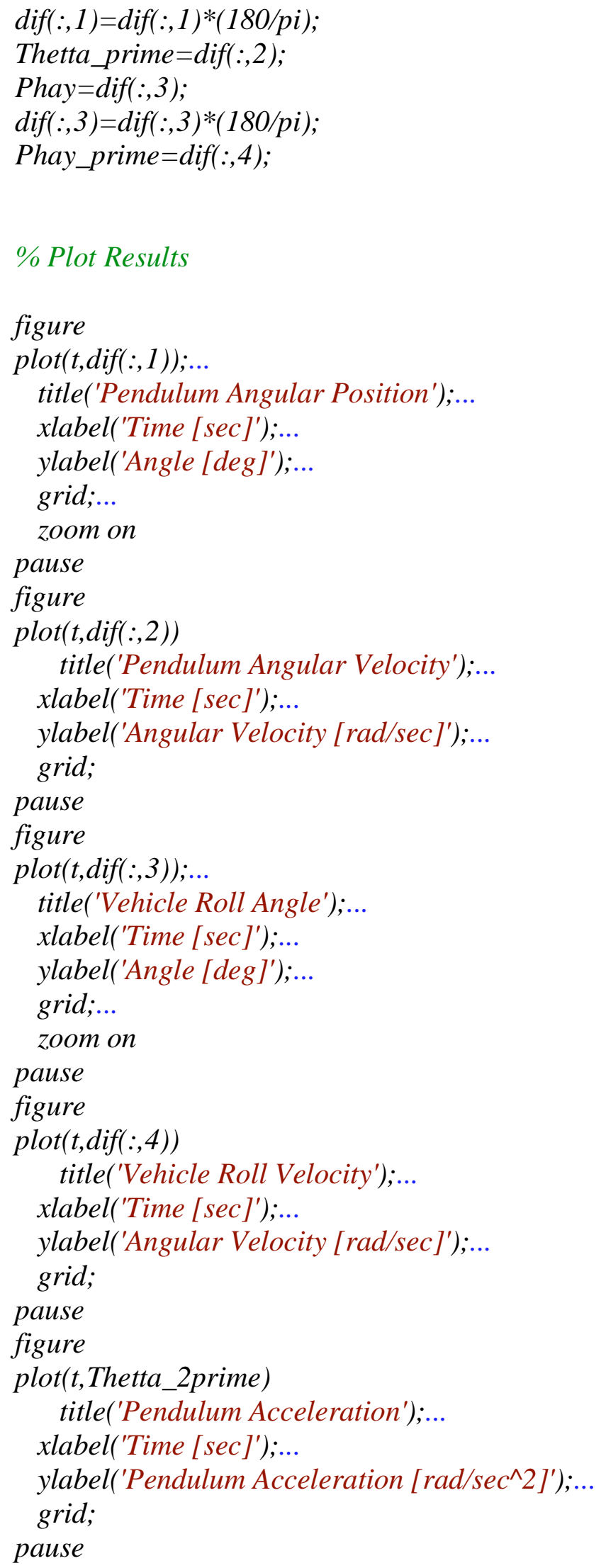




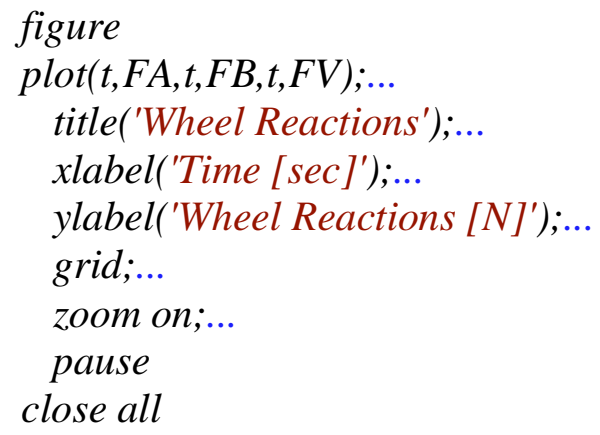

\section{C.9- Function File mid3.m}

$\%$ This file contains the equation of motion of the trammel

$\%$ pendulum when the two supports are in contact with the ground

function elliptical $=\operatorname{mid}(t, Y)$;

$\%$ Define Global Variables

global a_barb_bar acc_ratio

$a c c=$ acc_ratio $* 9.81$;

$\% 1.5$ * pi is added to rotate the frame of reference to be constant \% with Left_s3 and Right_s3 elliptical $(1,1)=Y(2)$;

elliptical $(2,1)=\left(-a \_b a r^{*} \cos (Y(1)-1.5 * p i) * a c c+0.5 * Y(2)^{\wedge} 2 *\left(a \_b a r^{\wedge} 2-b \_b a r^{\wedge} 2\right) * \sin (2 *(Y(1)-1.5 * p i))-\right.$

$\left.9.81^{*} b \_b a r^{*} \sin (Y(1)-1.5 * p i)\right) /\left(a \_b a r^{\wedge} 2 *(\cos (Y(1)-1.5 * p i))^{\wedge} 2+b \_b a r^{\wedge} 2 * \sin ((Y(1)-1.5 * p i))^{\wedge} 2\right)$;

elliptical $(3,1)=Y(3)$;

elliptical $(4,1)=Y(4)$;

\section{C.10- Function File Left s3.m}

$\%$ This file contains the equation of motion of the trammel

$\%$ pendulum When right support lifts off

function elliptical=Left_S2 $(t, Y)$;

$\%$ Define Global Variables

global a_bar b_bar acc_ratio YAB T M_tanker Mp Mfhl Ymf I_tanker_o I_fluid_o

$a c c=a c c \_r a t i o * 9.81$;

$Y \_1=-0.5 * Y(2)^{\wedge} 2 *\left(a \_b a r^{\wedge} 2-b \_b a r^{\wedge} 2\right) * \sin (2 * Y(1))$;

$Y \_2=-Y(4)^{\wedge} 2 *\left(a \_b a r^{*}(T / 2) * \sin (Y(1))-b \_b a r^{*} Y A B^{*} \cos (Y(1))+0.5 *\left(a \_b a r^{\wedge} 2-\right.\right.$

b_bar^2)*sin $(2 * Y(1)))$;

$Y \_3=-9.81 *\left(b \_b a r * \cos (Y(1)) * \cos (Y(3))-a \_b a r * \sin (Y(1)) * \sin (Y(3))\right)$;

$Y \_4=a c c *\left(a \_b a r * \sin (Y(1)) * \cos (Y(3))+b \_b a r * \cos (Y(1)) * \sin (Y(3))\right)$;

$Y \_5=-\left(a \_b a r * Y A B * \sin (Y(1))+b \_b a r^{*}(T / 2) * \cos (Y(1))+a \_b a r * b \_b a r\right)$;

$Y \_A=a \_b a r^{\wedge} 2 *(\sin (Y(1)))^{\wedge} 2+b \_b a r^{\wedge} 2 *(\cos (Y(1)))^{\wedge} 2$;

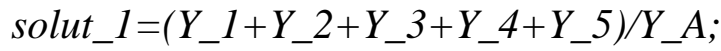


$Z \_1=a c c *\left(M p *\left(\left((T / 2)+a \_b a r * \cos (Y(1))\right) * \sin (Y(3))+\left(Y A B+b \_b a r * \sin (Y(1))\right) * \cos (Y(3))\right)+\ldots\right.$

$M \_$tanker $\left.*((T / 2) * \sin (Y(3))+h 1 * \cos (Y(3)))+M f^{*}\left((T / 2) * \sin (Y(3))+Y m f^{*} \cos (Y(3))\right)\right)$;

$Z \_2=2 * M p * Y(2) * Y(4) *\left(a \_b a r *(T / 2) * \sin (Y(1))-b \_b a r * Y A B * \cos (Y(1))+0.5 *\left(a \_b a r^{\wedge} 2-\right.\right.$

b_bar 2$)^{*} \ldots$

$\sin (2 * Y(1)))$;

$Z \_3=-9.81 *\left(M p *\left(\left((T / 2)+a \_b a r * \cos (Y(1))\right) * \cos (Y(3))-\left(Y A B+b \_b a r * \sin (Y(1))\right) * \sin (Y(3))\right)+\ldots\right.$

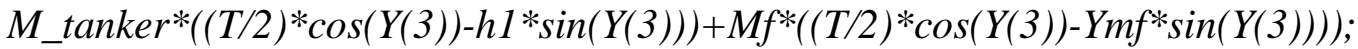

$Z \_4=-Y(4) \wedge 2 * M p *\left(a \_b a r * Y A B * \cos (Y(1))-b \_b a r *(T / 2) * \sin (Y(1))\right)$;

$Z \_5=M p * Y \_5$;

$Z \_B=M p^{*}\left((T / 2)+a \_b a r^{*} \cos (Y(1))\right)^{\wedge} 2+\left(Y A B+b \_b a r^{*} \sin (Y(1))\right)^{\wedge} 2+M \_$tanker $*\left(h 1^{\wedge} 2+(T / 2)^{\wedge} 2\right)+\ldots$

$M f^{*}\left(Y m f^{\wedge} 2+(T / 2)^{\wedge} 2\right)+I \_t a n k e r \_o+I f f l u i d \_o$;

solut_2=(Z_1 $\left.+Z \_2+Z \_3+Z \_4+Z \_5\right) / Z \_B$;

elliptical $(1,1)=Y(2)$;

elliptical $(2,1)=$ solut_1;

elliptical $(3,1)=Y(4)$;

elliptical $(4,1)=$ solut_2;

\section{C.11- Function File Right_s3.m}

$\%$ This file contains the equation of motion of the trammel

$\%$ pendulum when the left support lifts off

function elliptical=Right_s3(t,Y);

$\%$ Define Global Variables

global a_barb_bar acc_ratio YAB T M_tanker Mp Mfhl Ymf I_tanker_o,I_fluid_o

$a c c=a c c \_r a t i o * 9.81$;

$Y \_1=-0.5 * Y(2)^{\wedge} 2 *\left(a \_b a r^{\wedge} 2-b \_b a r^{\wedge} 2\right) * \sin (2 * Y(1))$;

$Y \_2=+Y(4)^{\wedge} 2 *\left(a \_b a r^{*}(T / 2) * \sin (Y(1))+b \_b a r^{*} Y A B^{*} \cos (Y(1))+0.5 *\left(a \_b a r^{\wedge} 2-\right.\right.$

b_bar 2$) * \sin (2 * Y(1)))$;

$Y \_3=-9.81 *\left(b \_b a r * \cos (Y(1)) * \cos (Y(3))-a \_b a r * \sin (Y(1)) * \sin (Y(3))\right)$;

$Y \_4=-a c c *\left(a \_b a r * \sin (Y(1)) * \cos (Y(3))+b \_b a r * \cos (Y(1)) * \sin (Y(3))\right)$;

$Y \_5=-\left(a \_b a r * Y A B^{*} \sin (Y(1))-b \_b a r *(T / 2) * \cos (Y(1))+a \_b a r * b \_b a r\right)$;

$Y \_A=a \_b a r^{\wedge} 2 *(\sin (Y(1)))^{\wedge} 2+b \_b a r^{\wedge} 2 *(\cos (Y(1)))^{\wedge} 2$;

solut_1 $=\left(Y \_1+Y \_2+Y \_3+Y \_4+Y \_5\right) / Y \_A$;

$Z \_1=a c c *\left(M p *\left(((T / 2))-a \_b a r * \cos (Y(1))\right) * \sin (Y(3))+\left(Y A B+b \_b a r * \sin (Y(1))\right) * \cos (Y(3))\right)+\ldots$

$M \_$tanker*((T/2)*sin( $\left.\left.(Y(3))-h 1 * \cos (Y(3))\right)+M f^{*}\left((T / 2) * \sin (Y(3))-Y m f^{*} \cos (Y(3))\right)\right)$;

$Z \_2=2 * M p * Y(2) * Y(4) *\left(a \_b a r *(T / 2) * \sin (Y(1))+b \_b a r * Y A B * \cos (Y(1))-0.5 *\left(a \_b a r^{\wedge} 2-\right.\right.$

b_bar 2$)^{*} \ldots$

$\sin (2 * Y(1)))$;

$Z \_3=-9.81 *\left(M p *\left(\left((T / 2)-a \_b a r * \cos (Y(1))\right) * \cos (Y(3))-\left(Y A B+b \_b a r * \sin (Y(1))\right) * \sin (Y(3))\right)+\ldots\right.$

M_tanker*((T/2)*cos $(Y(3))+h 1 * \sin (Y(3)))+M f *((T / 2) * \cos (Y(3))+Y m f * \sin (Y(3))))$; 
$Z \_4=-Y(4)^{\wedge} 2 * M p *\left(a \_b a r * Y A B * \cos (Y(1))+b \_b a r *(T / 2) * \sin (Y(1))\right)$;

$Z \_5=M p * Y$; ;

$Z \_B=M p^{*}\left((T / 2)-a \_b a r^{*} \cos (Y(1))\right)^{\wedge} 2+\left(Y A B+b \_b a r^{*} \sin (Y(1))\right)^{\wedge} 2+M \_$tanker ${ }^{*}\left(h 1^{\wedge} 2+(T / 2)^{\wedge} 2\right)+\ldots$

$M f^{*}\left(Y m f^{\wedge} 2+(T / 2)^{\wedge} 2\right)+I \_t a n k e r \_o+I \_f l u i d \_o$;

solut_2 $=\left(Z \_1+Z \_2+Z \_3+Z \_4+Z \_5\right) / Z \_B$;

elliptical $(1,1)=Y(2)$;

elliptical $(2,1)=$ solut_1;

elliptical $(3,1)=Y(4)$;

elliptical $(4,1)=$ solut_2;

\section{C.12- The 2D Model ANSYS Log File}

!2 DIMENSIONAL VALIDATION OF PENDULUM STRATEGY IN CONJUNCTION ! !WITH MATHEMATICAL PENDULUM MODELS.

!CASE OF 50 PERCENT FULL ELLIPTICAL PENDULUM WITH VARYING !VELOCITY

!Bold Font Indicates changes and/or Additions to Aquaro's Model

/FILNAM,ellsp50

!/CONFIG,NRES, 10000

/TITLE,ELLIPTICAL PENDULUM VALIDATION

/PREP7

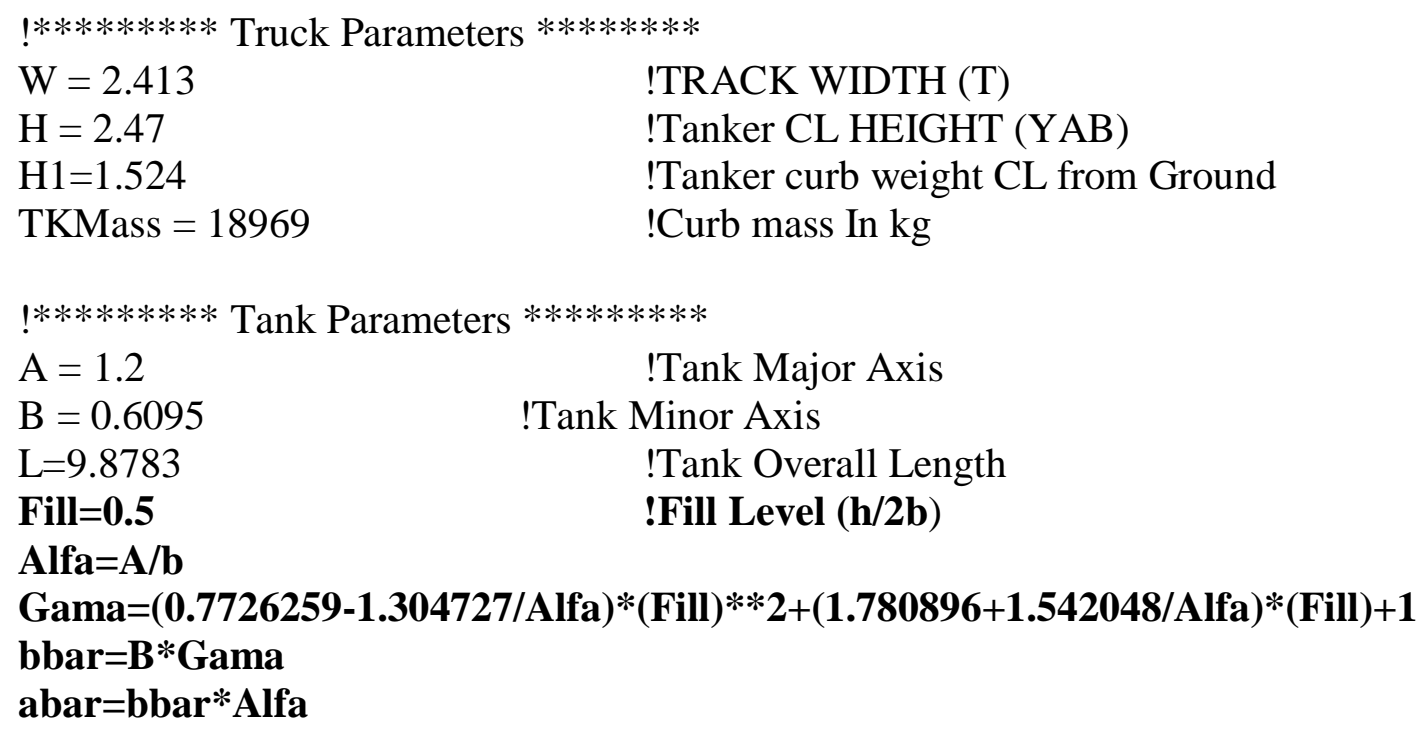

FTMass $=11349$

!Fluid total mass

Phay $=1+(-0.86299391+1.2372751 * \log ($ Alfa $)) *$ Fill-

$(0.12260181+1.2488977 * \log ($ Alfa $)) *($ Fill $) * * 2$

PENDMASS = FTMass*Phay

FIXMASS =FTMass-PENDMASS

$\mathrm{Ho}=0.5369$

!Fixed mass location from base of the tank

$! \mathrm{V}=27.825$

!VELOCITY OF TRUCK IN KM/HR 


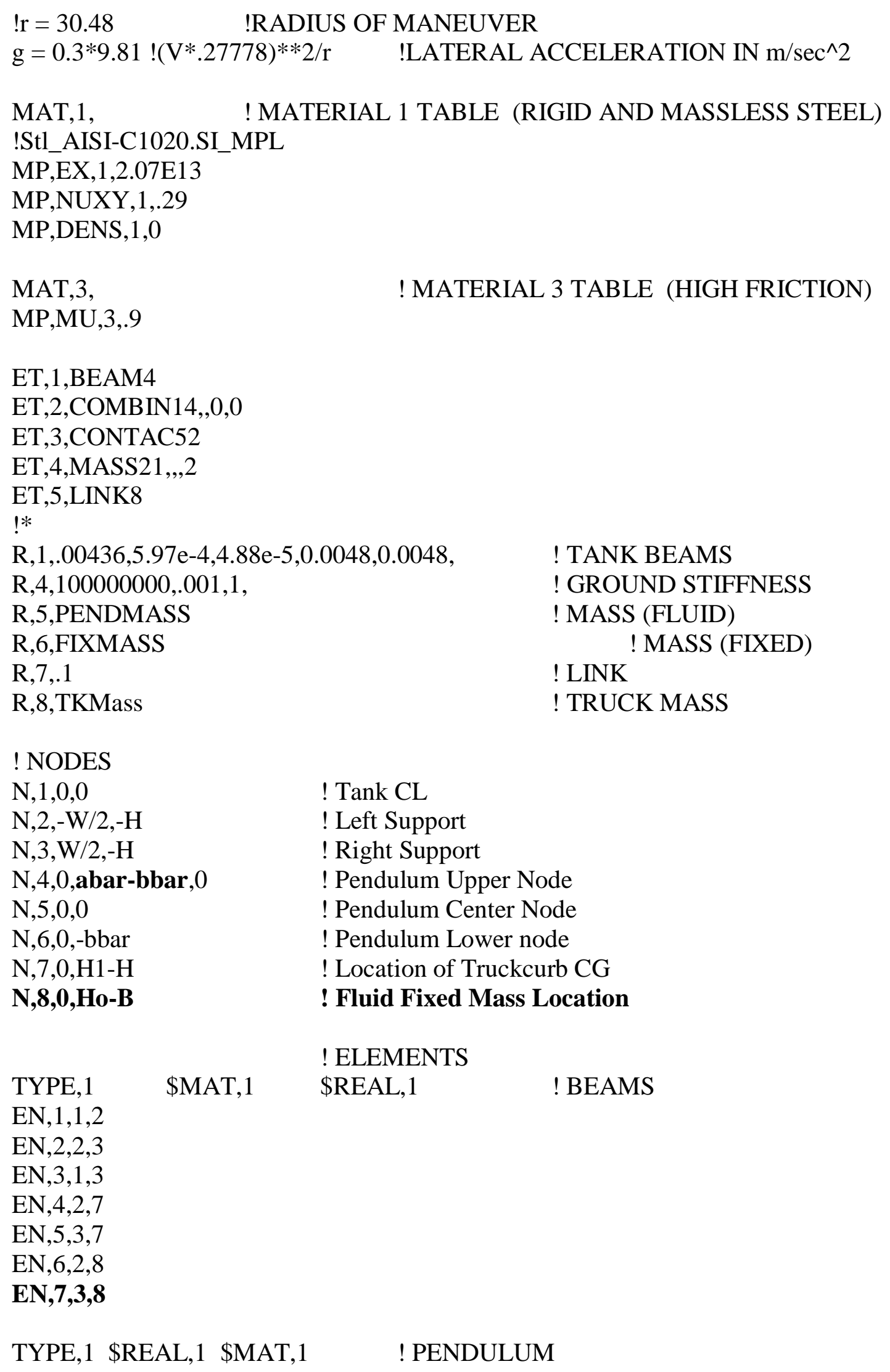

TYPE,1 \$REAL,1 \$MAT,1 ! PENDULUM 
EN, 8,5,6

EN, $9,4,5$

TYPE,4 \$REAL,5

! PENDULUM MASS

EN,10,6

REAL, 6

! FIXED MASS

EN, 11,8

REAL, 8

EN, 12,7

! Truck MASS

$\mathrm{CP}, 1, \mathrm{UX}, 4,1$

$\mathrm{CP}, 2, \mathrm{UY}, 5,1$

FINISH

/SOLU

D,2,UX,,,3,1,UY,UZ

D,ALL,UZ,

ANTYPE,TRANS

! NONLINEAR TRANSIENT DYNAMIC ANALYSIS

TRNOPT,FULL ! ANANYSIS OPTIONS

NLGEOM,ON

! LARGE DEFLECTION OPTION

LUMPM,ON

SSTIF,ON

NROPT,AUTO,

AUTOTS,OFF

EQSLV,FRONT,1e-08,0, ! USE FRONTAL SOLVER

$\mathrm{NCNV}, 0$,

$\mathrm{KBC}, 0$

OUTRES,,ALL

!*

TIME,.1

NSUBST, 25

ACEL, $0,9.81,0$

LSWRITE, 1

!*

TIME, .5

NSUBST, 100

ACEL, 0,9.81,0

LSWRITE, 2

!*

TIME,.51

ACEL,g,9.81,0

LSWRITE,3 


\section{!*}

TIME, 1

NSUBST,50

ACEL,g,9.81,0

LSWRITE, 4

!*

TIME, 2

NSUBST,100

ACEL,g,9.81,0

LSWRITE, 4

!*

TIME, 3

NSUBST, 100

ACEL,g,9.81,0

LSWRITE, 4

!*

TIME, 4

NSUBST, 100

ACEL,g,9.81,0

LSWRITE,4

!*

LSSOLVE, $1,7,1$

!*

FINISH

/POST26

RFORCE, 2,2,F,Y,LEFT

RFORCE,3,3,F,Y,RIGHT

ADD, 4,2,3, TOTAL, , ,1,1,1, PLVAR, $2,3,4$

PRVAR,2,3,4

\section{C.13- The 3D Model ANSYS Log File}

! ANSYS Code for Elliptical Full Truck Model

$!$

! RIGHT TRAMMEL PENDULUM PARAMETERS USED WITH MODEFIED AQUARO's ! WIREFRAME MODEL!

! Modifications and additions are shown in bold font

/FILNAM,stiffg

/CONFIG,NRES, 10000 !ALLOWS ANSYS TO READ ALL 835 LOAD CURVES

/TITLE,Tanker Truck Stability

/PREP7

$!^{*}$ 


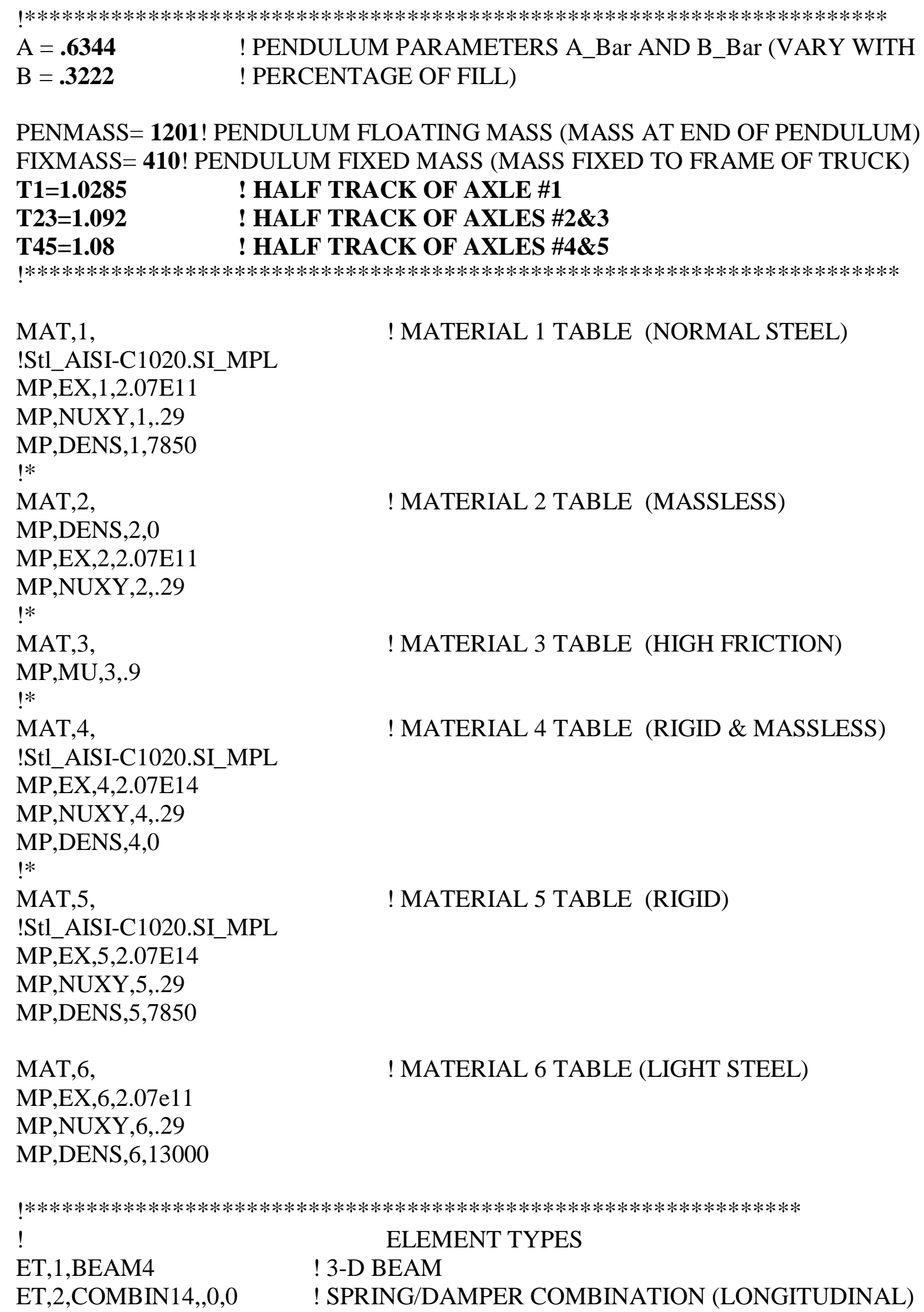

! MATERIAL 2 TABLE (MASSLESS)

! MATERIAL 3 TABLE (HIGH FRICTION)

! MATERIAL 4 TABLE (RIGID \& MASSLESS)

! MATERIAL 5 TABLE (RIGID)

! MATERIAL 6 TABLE (LIGHT STEEL) 
ET,3,CONTAC52

ET,4,MASS21,,2,

ET,5,LINK8

ET,6,COMBIN39, 0,0,0,1

ET,7,LINK8
! CONTACT ELEMENT

! LUMPED MASS

! 3-D SPAR (TENSION/COMPRESSION ONLY)

! NONLINEAR SPRING

! THIS IS ALSO A LINK WHICH CAN BE TURNED OFF

! TO DISPLAY THE MODELS MORE CLEARLY

!************************************************************************

$\mathrm{R}, 1, .004,1.1 \mathrm{e}-3,3 \mathrm{e}-3,0.0048,0.0048$, ! TANK BEAMS

$\mathrm{R}, 20, .0105,1.1 \mathrm{e}-3,3 \mathrm{e}-5,0.0048,0.0048,,,,,, 1$

R,2,2234027,12000, $\quad$ ! SPRING DAMPERS (REAR OF TRACTOR)

$\mathrm{R}, 3,371435, \mathbf{3 0 0 0}, \quad$ ! SPRING DAMPERS (FRONT OF TRACTOR)

$\mathrm{R}, 4,1000000, .0001,1$, ! GROUND STIFFNESS

R,5,PENMASS

! MASS (FLUID PENDULUM)

R,50,FIXMASS

$\mathrm{R}, 6,10100$

$\mathrm{R}, 60,50$

! MASS (FLUID FIXED)

! MASS (ENGINE \& CAB)

$\mathrm{R}, 61,255$

! MISC MASS

$\mathrm{R}, 62,150$

! DUAL WHEEL MASS

$\mathrm{R}, 63,75$

! SINGLE WHEEL MASS

$\mathrm{R}, 64,1073$

! DIFFERENTIAL MASS

$\mathrm{R}, 8, .01$

! TANK MASS

$\mathrm{R}, 10, .007887,7.98 \mathrm{e}-2,7.98 \mathrm{e}-2, .127, .127$,

$\mathrm{R}, 11, .007887,7.98 \mathrm{e}-2,7.98 \mathrm{e}-2, .127, .127$,

! TANDEM AXELS

$\mathrm{R}, 100, .00436,5.97 \mathrm{e}-4,4.88 \mathrm{e}-5,0.0048,0.0048, \quad$ ! CAB BEAMS

$\mathrm{R}, 101, .00436,5.97 \mathrm{e}-4,4.88 \mathrm{e}-5,0.0048,0.0048, \quad$ ! GROUND BEAMS

R,102,-0.188,-593925,-.1,-207708,-.075,-132725 !NONLINEAR SPRINGS

RMORE,-.0508,-74782,-.0381,-50136,-.0254,-29457

"

RMORE,-.0127,-12745,0,0,.0254,0.1 $\quad$ !Spring Lash Considered

RMORE,.0381,12153

$\mathrm{R}, 104,3.986 \mathrm{E}-8,5 \mathrm{E}-1,5 \mathrm{E}-1,005, .005$

! TRAILER TIRE SPRINGS

$\mathrm{R}, 105,3.986 \mathrm{E}-8,5 \mathrm{E}-1,5 \mathrm{E}-1,005, .005$

! CAB REAR TIRE SPRINGS

$\mathrm{R}, 106,1.993 \mathrm{E}-8, \underline{5 \mathrm{E}-1,5 \mathrm{E}-1, .005, .005}$

! CAB FRONT TIRE SPRINGS

!*************************************************************************

CLOCAL, 12,0,0,0,0,0,TILT, 0

CSYS, 12

!

TANK NODES

$\mathrm{N}, 1,0,0,1.175$

$\mathrm{N}, 2,0,0,-1.175 \quad \$ \mathrm{~N}, 3,0,1.62,0 \quad \$ \mathrm{~N}, 4,9.9,0.76,1.175$

$\mathrm{N}, 5,9.9,76,-1.175$

$\mathrm{N}, 6,9.9,1.62,0 \quad \$ \mathrm{~N}, 7,1.6,0,1.175 \quad \$ \mathrm{~N}, 8,1.6,0,-1.175$

$\mathrm{N}, 9,0.34,0,1.175$ 
$\mathrm{N}, 10,0.34,0,-1.175$

N,11,9.19,.76,0 ! KINGPIN

$\mathrm{N}, 12,9.19, .76,1.175 \$ \mathrm{~N}, 13,9.19, .76,-1.175$

$\mathrm{N}, 14,1.6,-0.4, \mathbf{T} 45 \quad \$ \mathrm{~N}, 15,1.6,-0.4,-\mathrm{T45} \quad \$ \mathrm{~N}, 16,0.34,-0.4, \mathbf{T 4 5}$

$\mathrm{N}, 17,0.34,-0.4,-\mathrm{T} 45 \quad \$ \mathrm{~N}, 20, .707,1.62,0 \quad \$ \mathrm{~N}, 21,2.12,1.62,0$

$\mathrm{N}, 22,3.54,1.62,0$

$\mathrm{N}, 23,4.95,1.62,0 \quad \$ \mathrm{~N}, 24,6.36,1.62,0 \quad \$ \mathrm{~N}, 25,7.78,1.62,0$

$\mathrm{N}, 26,9.19,1.62,0$

$\mathrm{N}, 30,4.13, .76,1.175 \$ \mathrm{~N}, 31,4.13, .76,-1.175 \$ \mathrm{~N}, 32,6.66, .76,1.175$

$\mathrm{N}, 33,6.66, .76,-1.175$

$\mathrm{N}, 34,-3,0,1.175 \quad \$ \mathrm{~N}, 35,-3,0,-1.175 \quad \$ \mathrm{~N}, 36,1.6,-0.88, \mathbf{T 4 5}$

N,37,1.6,-0.88,-T45 \$N,38,0.34,-0.88,T45 \$N,39,0.34,-0.88,-T45

$\mathrm{N}, 40,0.34,0, .485 \quad \$ \mathrm{~N}, 41,0.34,0,-.485 \quad \$ \mathrm{~N}, 42,1.6,0, .485$

$\mathrm{N}, 43,1.6,0,-.485$

$\mathrm{N}, 44,0.34,-.37, .485 \quad \$ \mathrm{~N}, 45,0.34,-.37,-.485 \$ \mathrm{~N}, 46,1.6,-.37, .485$

$\mathrm{N}, 47,1.6,-.37,-.485$

N,48,7.5,2.4,-.125 ! EXTRA BALANCING ELEMENT

$\mathrm{N}, 50, .707,1.62-\mathrm{B}, 0 \quad \$ \mathrm{~N}, 51,2.12,1.62-\mathrm{B}, 0 \quad \$ \mathrm{~N}, 52,3.54,1.62-\mathrm{B}, 0$

$\mathrm{N}, 53,4.95,1.62-\mathrm{B}, 0 \quad \$ \mathrm{~N}, 54,6.36,1.62-\mathrm{B}, 0 \quad \$ \mathrm{~N}, 55,7.78,1.62-\mathrm{B}, 0$

$\mathrm{N}, 56,9.19,1.62-\mathrm{B}, 0$

! CONTACT ELEMENT NODES ARE COMMENTED OUT

!N,60,1.6,-0.881,1.175 \$N,61, 1.6,-0.881,-1.175

$! \mathrm{N}, 62,0.34,-0.881,1.175 \quad \$ \mathrm{~N}, 63,0.34,-0.881,-1.175$

$\mathrm{N}, 64,0, .76,1.175 \quad \$ \mathrm{~N}, 65,0, .76,-1.175 \quad \$ \mathrm{~N}, 66,0.34, .76,1.175$

$\mathrm{N}, 67,0.34, .76,-1.175 \quad \$ \mathrm{~N}, 68,1.6, .76,1.175 \quad \$ \mathrm{~N}, 69,1.6, .76,-1.175$

$\mathrm{N}, 70, .707,1.62,0 \quad \$ \mathrm{~N}, 71,2.12,1.62,0 \quad \$ \mathrm{~N}, 72,3.54,1.62,0$

$\mathrm{N}, 73,4.95,1.62,0 \quad \$ \mathrm{~N}, 74,6.36,1.62,0 \quad \$ \mathrm{~N}, 75,7.78,1.62,0$

$\mathrm{N}, 76,9.19,1.62,0$

$\mathrm{N}, 80, .707,1.62+\mathrm{A}-\mathrm{B}, 0 \quad \$ \mathrm{~N}, 81,2.12,1.62+\mathrm{A}-\mathrm{B}, 0 \quad \$ \mathrm{~N}, 82,3.54,1.62+\mathrm{A}-\mathrm{B}, 0$

$\mathrm{N}, 83,4.95,1.62+\mathrm{A}-\mathrm{B}, 0 \quad \$ \mathrm{~N}, 84,6.36,1.62+\mathrm{A}-\mathrm{B}, 0 \quad \$ \mathrm{~N}, 85,7.78,1.62+\mathrm{A}-\mathrm{B}, 0$

$\mathrm{N}, 86,9.19,1.62+\mathrm{A}-\mathrm{B}, 0$

$\mathrm{N}, 100,9.19, .76,0$

! ADDED NODES FOR STIFFINING ELEMENTS

$\mathrm{N}, 220, .707, .76,1.175 \$ \mathrm{~N}, 320, .707, .76,-1.175$

$\mathrm{N}, 221,2.12, .76,1.175 \$ \mathrm{~N}, 321,2.12, .76,-1.175$ 


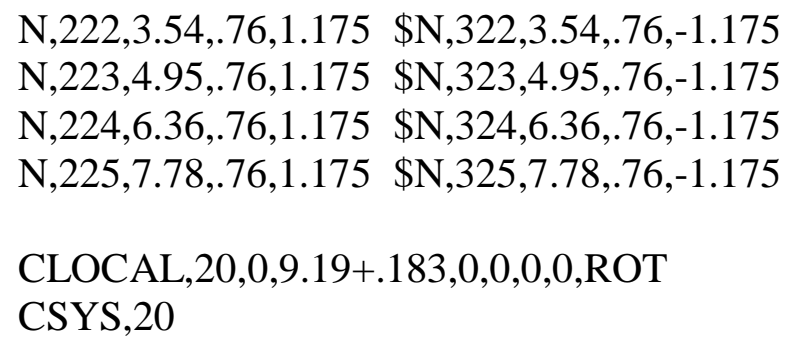

$\mathrm{N}, 101,-1.5, .26,1.22 \quad \$ \mathrm{~N}, 102,-1.5, .26,-1.22 \$ \mathrm{~N}, 103, .541, .26,1.22$

$\mathrm{N}, 104, .541, .26,-1.22 \$ \mathrm{~N}, 105,-.907, .26,1.22 \$ \mathrm{~N}, 106,-.907, .26,-1.22$

$\mathrm{N}, 107,-.183, .26,1.22 \$ \mathrm{~N}, 108,-.183, .26,-1.22 \$ \mathrm{~N}, 109, .183, .26, .4525$

$\mathrm{N}, 110,-.183, .26,-.4525 \$ \mathrm{~N}, 111,4.24, .26,1.16 \$ \mathrm{~N}, 112,4.24, .26,-1.16$

$\mathrm{N}, 113,5.88, .26,1.16 \quad \$ \mathrm{~N}, 114,5.88, .26,-1.16 \$ \mathrm{~N}, 115, .54,-0.37, \mathbf{T} 23$

$\mathrm{N}, 116, .54,-0.37,-\mathbf{T} 23 \$ \mathrm{~N}, 117,-.907,-0.37, \mathbf{T 2 3}$

$\mathrm{N}, 118,-.907,-0.37,-\mathbf{T} 23$

$\mathrm{N}, 119,4.24,-0.37, \mathbf{T 1} \quad \$ \mathrm{~N}, 120,4.24,-0.37,-\mathrm{T} 1$

$\mathrm{N}, 140,-.183,-0.37, .4525$

$\mathrm{N}, 141,-.183,-0.37,-.4525 \quad \$ \mathrm{~N}, 142,-.183,-0.37, \mathbf{T 2 3}$

$\mathrm{N}, 143,-.183,-0.37,-\mathbf{T} 23$

$\mathrm{N}, 144,4.24,-0.37, .419 \$ \mathrm{~N}, 145,4.24,-0.37,-.419 \$ \mathrm{~N}, 146,4.24, .26, .419$

$\mathrm{N}, 147,4.24, .26,-.419$

N,121,1.996+.2,-0.09768,.001 ! CENTER OF GRAVITY

N,125,-.907,-.88,T23\$N,126,-.907,-.88,-T23\$N,127,.541,-.88, T23

$\mathrm{N}, 128, .541,-.88,-\mathbf{T} 23 \$ \mathrm{~N}, 129,4.24,-.88, \mathbf{T 1} \$ \mathrm{~N}, 130,4.24,-.88,-\mathrm{T} 1$

!*

!N,131,-.907,-.881,1.22 \$N,132,-.907,--.881,-1.22

!N,133,.541,-..881,1.22

!N,134,.541,-.881,-1.22 \$N,135,4.24,-.881,1.16

!N,136,4.24,-.881,-1.16

!*********************************************************************** !

TYPE, 1

TANK BEAMS

MAT,6 ! DEFINES THE MATERIAL

REAL, 1 ! DEFINES THE REAL CONSTANT TABLE SET

EN,1,64,220

EN,5,222,30

\$EN,2,220,68 \$EN,3,68,221 \$EN,4,221,222

$\mathrm{EN}, 9,4,5$

\$EN,6,30,223 \$EN,7,223,224

\$EN,8,224,32

$\$ E N, 10,1,2 \quad \$ E N, 11,12,11$ \$EN,12,11,13

$\mathrm{EN}, 13,64,3$

EN, $17,64,65$

EN, 21,66,68

EN,203,69,321

$\$ E N, 14,65,3 \quad \$ E N, 15,6,4 \quad \$ E N, 16,6,5$

$\$ E N, 18,32,225 \quad \$ E N, 19,225,12 \quad \$ E N, 20,67,69$

\$EN,200,12,4 \$EN,201,65,320 \$EN,202,320,69

\$EN,204,321,322\$EN,205,322,31 \$EN,206,31,323 
EN,207,323,324

\$EN,208,324,33

\$EN,209,33,325

\$EN,210,325,13

EN,211,13,5

!*

REAL, 20

EN,22,1,9

$\$ \mathrm{EN}, 23,2,10 \quad \$ \mathrm{EN}, 24,7,9$

EN, 26, 1,34

$\$ E N, 27,2,35 \quad \$ E N, 28,9,40$

EN,30,41,10

\$EN,31,7,42 \$EN,32,42,43 \$EN,33,43,8

EN,34,1,64

$\$ E N, 35,2,65 \quad \$ E N, 36,7,68$

$\$ \mathrm{EN}, 37,8,69$

\begin{tabular}{|c|c|c|}
\hline $\begin{array}{l}\text { ! ADDED STIFF } \\
\text { MAT,4 }\end{array}$ & ELLEMENTS & \\
\hline REAL, 1 & & \\
\hline $\mathrm{EN}, 212,220,320$ & $\$ E N, 213,320,20$ & \$EN,214,20,220 \\
\hline $\mathrm{EN}, 215,221,321$ & \$EN,216,321,21 & \$EN,217,21,221 \\
\hline $\mathrm{EN}, 218,222,322$ & \$EN,219,322,22 & $\$ E N, 220,22,222$ \\
\hline $\mathrm{EN}, 221,223,323$ & $\$ E N, 222,323,23$ & $\$ E N, 223,23,223$ \\
\hline $\mathrm{EN}, 224,224,324$ & \$EN,225,324,24 & \$EN,226,24,224 \\
\hline $\mathrm{EN}, 227,225,325$ & \$EN,228,325,25 & $\$ E N, 229,25,225$ \\
\hline $\mathrm{EN}, 230,13,26 \$ E$ & 31,26,12 & \\
\hline
\end{tabular}

!

TYPE, 1

CAB BEAMS

REAL, 100

MAT, 4

EN, $38,101,102$

EN, $42,105,107$

EN, $46,108,110$

EN, 50,103,121

EN, 54,111,113

EN, $58,111,146$

EN,62,106,100

!*

\$EN,39,101,105 \$EN,40,102,106 \$EN,41,108,106

\$EN,43,104,103 \$EN,44,103,107 \$EN,45,107,109

\$EN,47,109,110 \$EN,48,104,108 \$EN,49,103,111

\$EN,51,104,121 \$EN,52,111,121 \$EN,53,112,121

\$EN,55,113,114 \$EN,56,112,114 \$EN,57,112,104

$\$ E N, 59,146,147 \quad \$ E N, 60,147,112 \quad \$ E N, 61,105,100$

TYPE, 2

$\$ \mathrm{EN}, 63,103,100 \quad \$ \mathrm{EN}, 64,104,100$

! SPRING-DAMPER COMBINATIONS

! WALKING BEAM SUSPENSION OF TRACTOR

REAL,2

$\mathrm{E}, 109,140 \quad \$ \mathrm{E}, 110,141$

TYPE,6

REAL, 102

$\mathrm{E}, 42,46 \quad \$ \mathrm{E}, 40,44 \quad \$ \mathrm{E}, 43,47 \quad \$ \mathrm{E}, 41,45$

TYPE, 2 ! FRONT SUSPENSION OF TRACTOR

REAL,3 


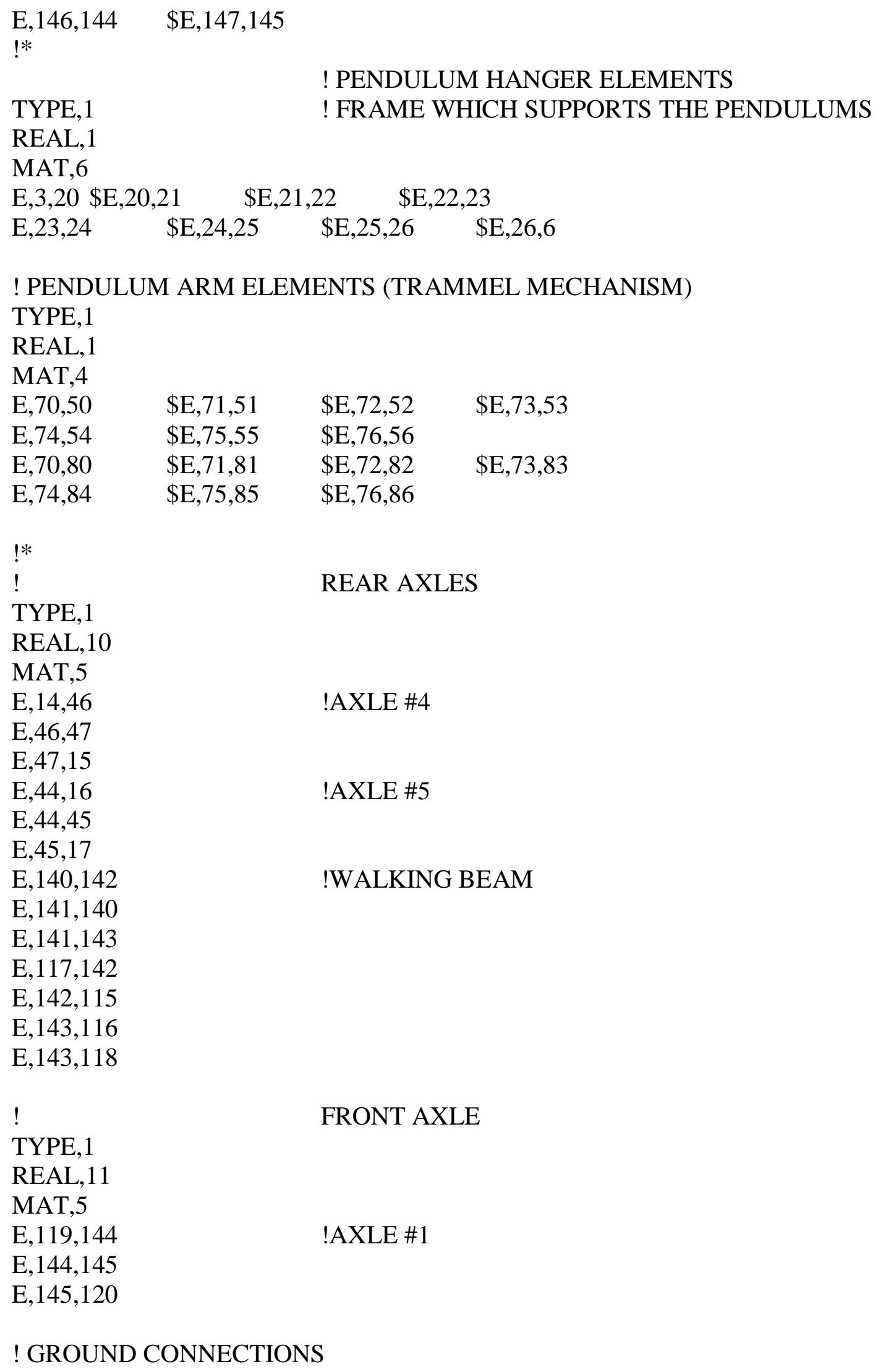


TYPE, 1

REAL, 105

MAT, 2

$\mathrm{E}, 117,125 \quad \$ \mathrm{E}, 118,126 \quad \$ \mathrm{E}, 115,127 \quad \$ \mathrm{E}, 116,128$

REAL, 104

E,119,129 \$E,120,130

REAL,106

$\mathrm{E}, 16,38$

$\$ \mathrm{E}, 14,36$

$\mathrm{E}, 15,37 \quad \$ \mathrm{E}, 17,39$

!*

! CONTACT ELEMENTS

TYPE, 3

! FOR THIS ANALYSIS THE CONTACT ELEMENTS ARE

OMMITTED

REAL, 4

MAT,3

!E, 38,62

!E, 39,63

!E,36,60

!E,37,61

!E, 129,135

!E, 130,136

!E, 125,131

!E, 126,132

!E, 127,133

!E, 128,134

!AXLE 5 RIGHT

!AXLE 5 LEFT

!AXLE 4 RIGHT

!AXLE 4 LEFT

!AXLE 1 RIGHT

!AXLE 1 LEFT

!AXLE 3 RIGHT

!AXLE 3 LEFT

!AXLE 2 RIGHT

!AXLE 2 LEFT

! MASS ELEMENTS

TYPE, 4

MAT, 1

REAL, 6

! CAB MASS LUMPED AT CG

E, 121

!*

REAL, 60

! MISC MASS (PUMP ON REAR OF TRAILER)

E,35

!*

TYPE, 4

REAL,5

MAT, 1

E,50 \$E,51 \$E,52

E,53 \$E,54 \$E,55

E,56

!*

TYPE, 4

! FIXED FLUID MASS 


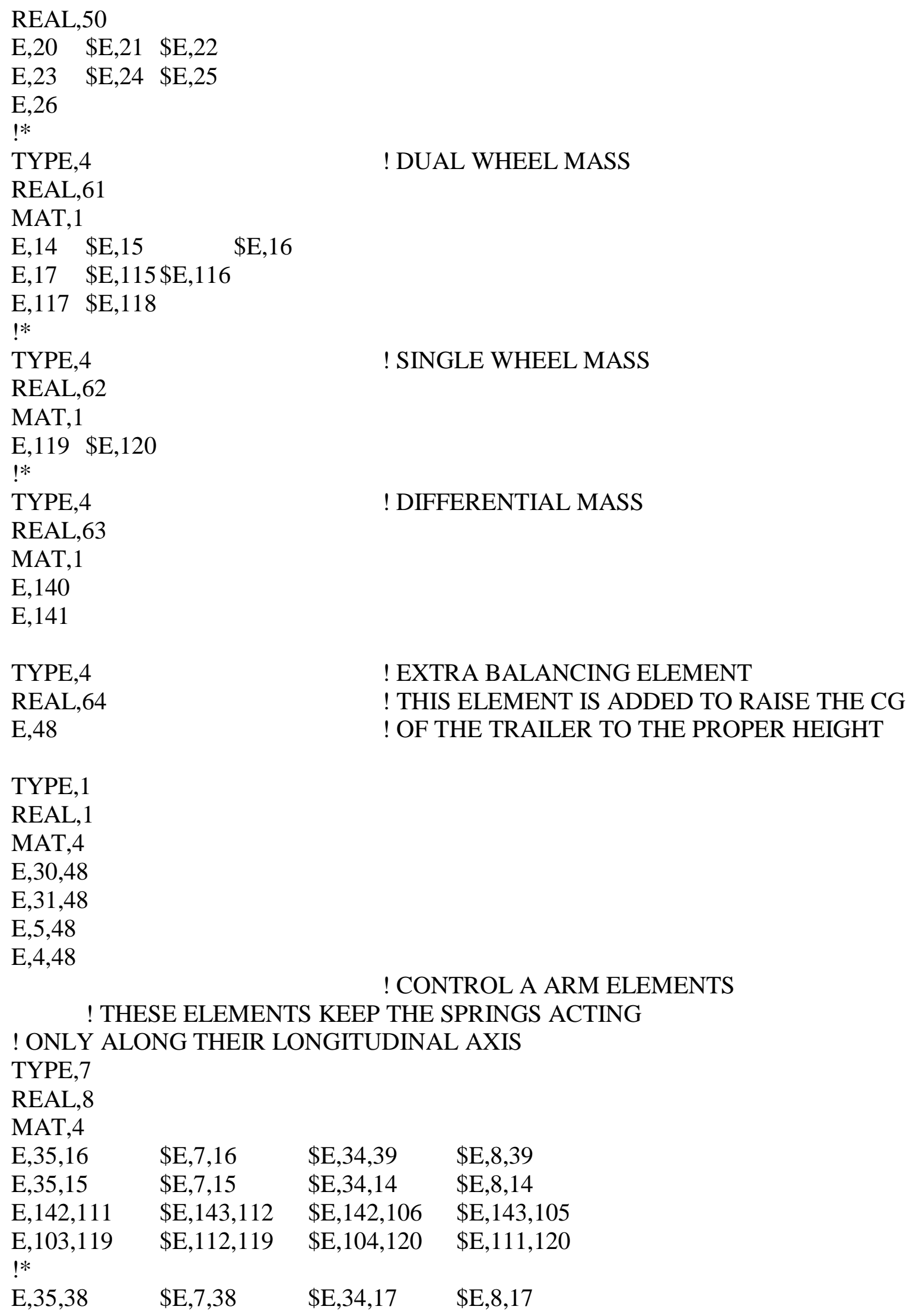




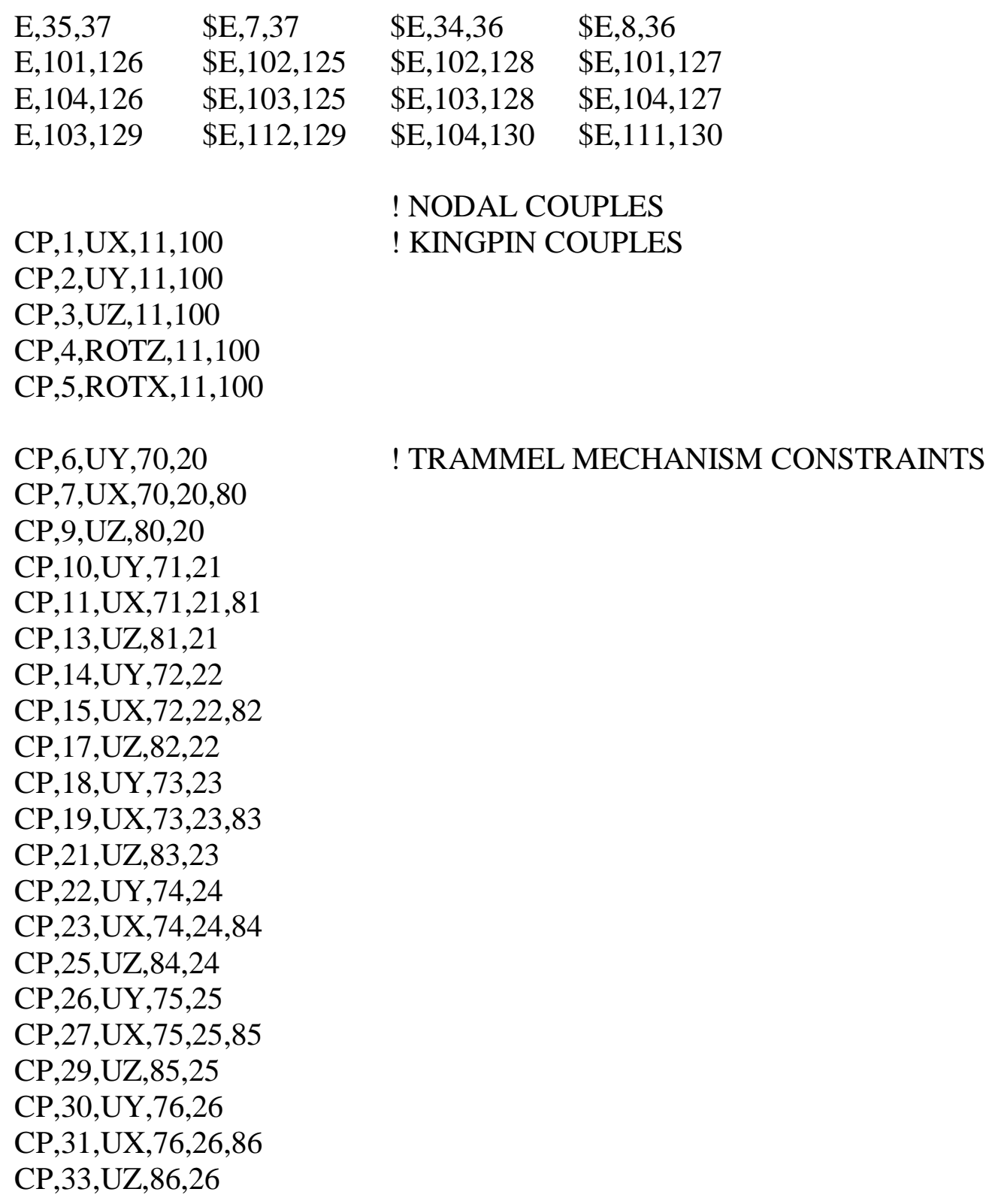

SAVE

FINISH

!FOR TRANSIENT DYNAMIC SOLUTIONS THE CODE IS WRITTEN AS FOLLOWS

/SOLU

$\mathrm{D}, 36, \mathrm{UX},, 39,1, \mathrm{UY}, \mathrm{UZ}$

$\mathrm{D}, 125, \mathrm{UY},, 130,1$

!*

ANTYPE,TRANS

! NONLINEAR TRANSIENT DYNAMIC ANALYSIS

TRNOPT,FULL ! ANANYSIS OPTIONS 


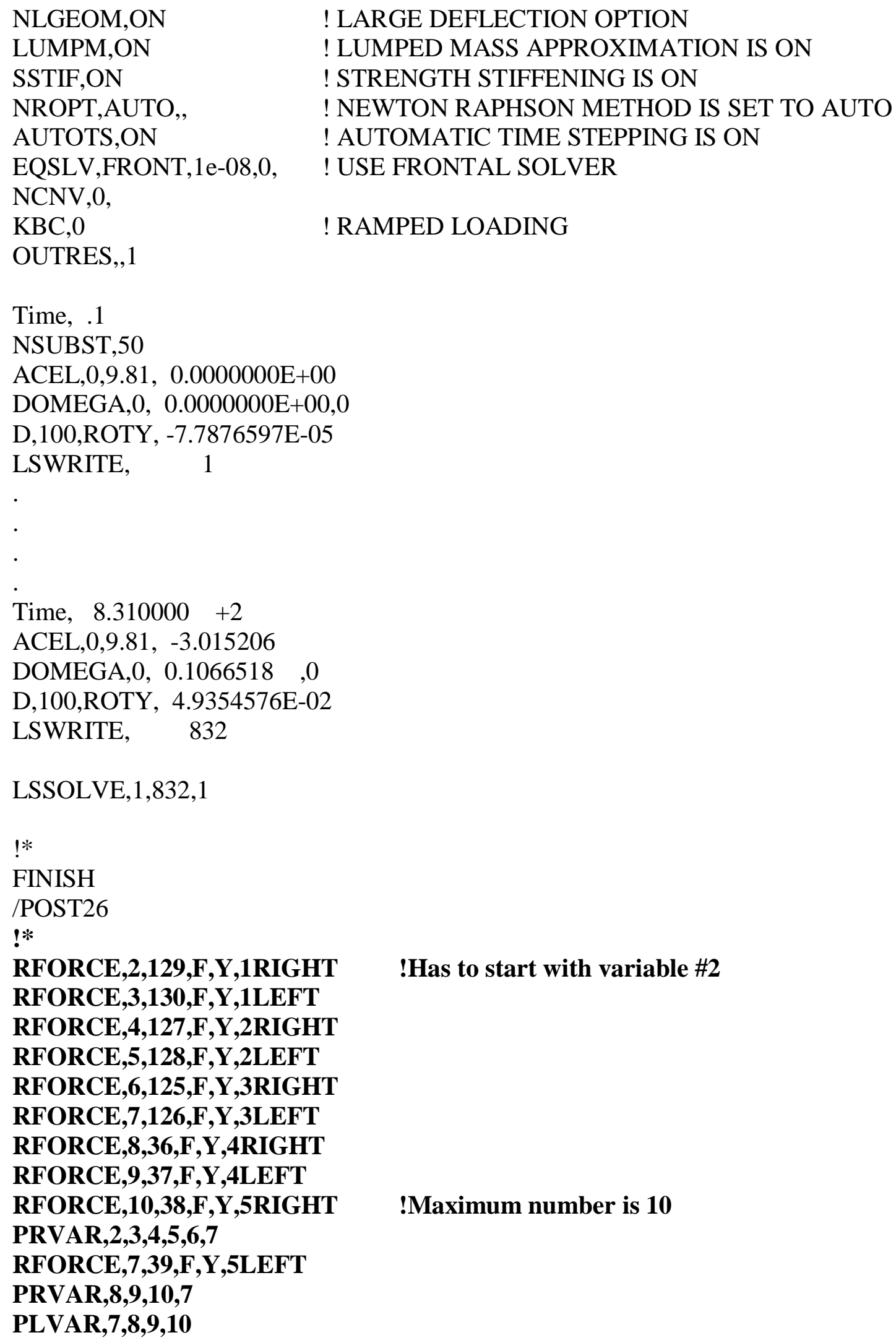

!Has to start with variable \#2

! LARGE DEFLECTION OPTION

! LUMPED MASS APPROXIMATION IS ON

! STRENGTH STIFFENING IS ON

! NEWTON RAPHSON METHOD IS SET TO AUTO

! AUTOMATIC TIME STEPPING IS ON

! USE FRONTAL SOLVER

! RAMPED LOADING 


\section{CURRICULUM VITA}

Mohamed I. Salem was born on June $23^{\text {rd }} 1969$ in Cairo, Egypt. He attended El Kobba secondary school from where he received his secondary school certificate in 1986. He joined Ain Shams University in Cairo, where he received his B.Sc. Degree in automotive engineering in 1991. After the completion of the obligatory military service in 1992, Salem worked as an instructor in Ain Shams University, as a teaching and a research assistant for the American University in Cairo from 1992 tell 1994. During this period, Salem worked as a consultant for several companies, as a design engineer, and handled many free lance projects. In 1995, Salem

joined the Masters program in West Virginia University, where he obtained his Master of Science in Mechanical Engineering (M.S.M.E.) in 1996. Then finally he joined the Ph.D. program in West Virginia University in 1997, where he wrote this dissertation in May 2000, as a partial fulfillment towards the degree of Doctor of Philosophy. 